\title{
Scavenging and Transport of Thorium Radioisotopes in the North Atlantic Ocean
}

\author{
by \\ Paul Lerner \\ B.A., University of California Berkeley (2012) \\ Submitted to the Joint Program in Oceanography and Applied Ocean Science \& \\ Engineering \\ in partial fulfillment of the requirements for the degree of \\ Doctor of Philosophy \\ at the \\ MASSACHUSETTS INSTITUTE OF TECHNOLOGY \\ and the \\ WOODS HOLE OCEANOGRAPHIC INSTITUTION
}

September 2018

(C)2018 Paul Lerner. All rights reserved.

The author hereby grants to MIT and WHOI permission to reproduce and to distribute publicly paper and electronic copies of this thesis document in whole or in part in any medium now known or hereafter created.

Author

Joint Program in Oceanography and Applied Ocean Science \& Engineering

Massachusetts Institute of Technology

\& Woods Hole Oceanographic Institution

Aug 13, 2018

Certified by

Olivier Marchal

Woods Hole Oceanographic Institution

Thesis Supervisor

Certified by

Phoebe Lam

University of California Santa Cruz

Thesis Supervisor

Accepted by

Shuhei Ono

Chairman, Joint Committee for Chemical Oceanography Massachusetts Institute of Technology

Woods Hole Oceanographic Institution 


\title{
Scavenging and Transport of Thorium Radioisotopes in the North Atlantic Ocean
}

by

Paul Lerner

\author{
Submitted to the Joint Program in Oceanography and Applied Ocean Science \& Engineering \\ Massachusetts Institute of Technology \\ \& Woods Hole Oceanographic Institution \\ on Aug 13, 2018, in partial fulfillment of the \\ requirements for the degree of \\ Doctor of Philosophy
}

\begin{abstract}
Many chemical constituents are removed from the ocean by attachment to settling particles, a process referred to as "scavenging." Radioisotopes of thorium, a highly particle-reactive element, have been used extensively to study scavenging in the ocean. However, this process is complicated by the highly variable chemical composition and concentration of particles in oceanic waters.

This thesis focuses on understanding the cycling of thorium as affected by particle concentration and particle composition in the North Atlantic. This objective is addressed using (i) the distributions ${ }^{228,230,234} \mathrm{Th}$, their radioactive parents, particle composition, and bulk particle concentration, as measured or estimated along the GEOTRACES North Atlantic Transect (GA03) and (ii) a model for the reversible exchange of thorium with particles. Model parameters are either estimated by inversion (chapter 2-4), or prescribed in order to simulate ${ }^{230} \mathrm{Th}$ in a circulation model (chapter 5).

The major findings of this thesis follow. In chapters 2 and 3, I find that the rate parameters of the reversible exchange model show systematic variations along GA03. In particular, $k_{1}$, the apparent first-order rate "constant" of Th adsorption onto particles, generally presents maxima in the mesopelagic zone and minima below. A positive correlation between $k_{1}$ and bulk particle concentration is found, consistent with the notion that the specific rate at which a metal in solution attaches to particles increases with the number of surface sites available for adsorption. In chapter 4, I show that Mn (oxyhydr)oxides and biogenic particles most strongly influence $k_{1}$ west of the Mauritanian upwelling, but that biogenic particles dominate $k_{1}$ in this region. In chapter 5 , I find that dissolved ${ }^{230} \mathrm{Th}$ data are best represented by a model that assumes enhanced values of $k_{1}$ near the seafloor. Collectively, my findings suggest that spatial variations in Th radioisotope activities observed in the North Atlantic reflect at least partly variations in the rate at which Th is removed from the water column.
\end{abstract}

Thesis Supervisor: Olivier Marchal

Title: Woods Hole Oceanographic Institution

Thesis Supervisor: Phoebe Lam

Title: University of California Santa Cruz 


\section{Acknowledgments}

First and foremost, I feel great appreciation to have worked with my advisors, Olivier Marchal and Phoebe Lam, over the last five years. The work presented in this thesis and my career as a young scientist would not have progressed nearly as far as it has without Olivier's constant willingness to share his deep and vast knowledge in mathematics, modeling, physical oceanography, paleoceanography, and thorium biogeochemistry. Similarly, Phoebe's expertise in marine particles and particulate trace metal chemistry lead to important and interesting discussions both in this thesis and in our meetings that otherwise would not have been considered. Moreover, her critical contributions to the GEOTRACES data used in this work were essential for all chapters, and her knowledge of the intricacies in the collection and analysis of her own and other GEOTRACES samples allowed for careful ground truthing of the model results presented here. Olivier and Phoebe's willingness to have weekly meetings to discuss my thesis research was immensely helpful. I must especially commend Phoebe to this end, as she continued her frequent interaction even after moving across the country to Santa Cruz.

I acknowledge the tremendous support of my thesis committee, including my advisors, Ken Buesseler, Andrew Solow, and Philip M. Gschwend. In their assistance, all members have gone beyond their roles as committee members. Ken has greatly contributed to the content in chapter 2 and 3 of this thesis, both in the generation of the ${ }^{228,234} \mathrm{Th}$ data and providing feedback on the discussion in these chapters. I also want to thank Ken for supporting my efforts in finding a postdoctoral position. The statistical framework used in interpreting the results of chapters 2, 3, and 4 stem largely from conversations with Andy. I also want to thank Andy for teaching applied statistics and providing numerous statistical textbooks, particularly since I still find both the coursework and these textbooks very useful references. Phil's enthusiasm in listening to my presentations and responding to my work and for marine geochemistry in general made me even more excited to continue my research, and our interactions greatly benefited the material presented in chapter 4. I would also like to acknowledge Matthew Charette for both agreeing to chair my proposal and doctoral defense, and for greatly contributing to chapters 2 and 3 through the generation of the ${ }^{228} \mathrm{Th}$ and ${ }^{228} \mathrm{Ra}$ data, and through feedback on the interpretation of results presented in both of these chapters.

I express my deepest gratitude to all the scientist and staff involved in the generation of the GEOTRACES data used in this study, without which this thesis would not exist. The following is only a partial list of people involved in this monumental collection effort, including those whom I have had the pleasure of interacting with. Ken Buesseler's lab, and particularly the work of Stephanie Owens, was responsible for the generation of the ${ }^{234} \mathrm{Th}$ data. The ${ }^{228} \mathrm{Th}$ and ${ }^{228} \mathrm{Ra}$ data was generated by the labs of Matt Charette, Ken Buesseler, and Willard S. Moore (University of South Carolina). At WHOI, the generation of both the ${ }^{228,234} \mathrm{Th}$ data benefited greatly from the help of many members of the scientific and technical staffs, including Paul Morris, Steven Pike, Paul Henderson, and Erin Black. The ${ }^{230}$ Th data were generated by the lab of Robert Anderson (Lamont-Doherty Earth Observatory), largely from the work of Christopher Hayes, and by the lab of R. Lawrence Edwards (University of Minnesota), including contributions from Yanbin Lu, and by Laura F. Robinson, Kuo-Fang (Denner) Huang, and Maureen Auro at WHOI. Bob, Chris, Larry, Laura, Yanbin, and Denner all contributed to the discussion in chapter 2. I would especially like to thank Bob and Chris for our multiple interactions at various GEOTRACES sessions at conferences and GEOTRACES workshops throughout the years, which has helped direct and focus my research and writing. Additionally, as head of the US GEOTRACES project office, Bob has been an essential force in providing funding, resources, and scientific direction for the GEOTRACES transects, including section GA03 from which most data used in this thesis are derived. I once again thank Phoebe who, in addition to being my co-advisor, generated the particle dataset used in this 
thesis. Finally, I would like to thank the captain and crew of the R/V Knorr for the 2010 and 2011 legs of section GA03, including the chief scientists Ed Boyle, Bill Jenkins, and Greg Cutter. The water mass analysis of Bill Jenkins also greatly contributed to chapters 2, 3, and 5 of this thesis.

This thesis also benefited from discussions with other scientists. I thank Adrian Burd for our discussions on particle aggregation at WHOI and Lamont. The analysis of the effect of particle composition on thorium adsorption in chapter 4 was improved based on discussions with both Phil Gschwend and Carl Lamborg (USCS). Comparison of model results with physical observations in chapter 5 was refined based on discussions with John Toole and Magdalena Andres (both at WHOI). I am also grateful to John Toole for providing daily velocity profiles derived from moored instruments along Line W. I thank Roger François for providing the ${ }^{230} \mathrm{Th}$ data used in Chapter 5. Finally, I thank Wilford Gardner and Mary Jo Richardson (Texas A\&M) for our helpful discussion on benthic nepheloid layers in the Northwest Atlantic.

I thank the Joint Committee for Chemical Oceanography for ensuring that I have remained on schedule. I also thank Jim Yoder and Meg Tivey, under whom the Joint Program has flourished through their promotion of a nurturing environment for young scientists like myself. I thank everyone in the Academic Programs Office, particularly Julia Westwater and Lea Fraser, both of who have greatly helped me navigate through MIT/WHOI courses, set up committee meetings as well as my thesis proposal and doctoral defense, and whose positive attitudes always made my day more pleasant. Thanks to Michelle McCafferty for her invaluable assistance in getting reimbursed for my frequent trips to MIT during my first year. I thank all instructors for the courses I have taken at WHOI, including Ben Van Mooy, Scott Doney, Jeff Seawald, Bernhard Peucker-Ehrenbrink, Bill Jenkins, Mak Saito, Dave Glover, and (again) Andy Solow. I thank Dave Glover, Bill Jenkins, and Scott Doney in particular for allowing me to TA 12.747 (also known as "the modeling course"), which I found to be a valuable experience. I also thank Bernhard for keeping an open door both as Education Coordinator and Department Chair, and in general for being incredibly supportive to all the chemical oceanography JP students. Thanks to Sheila Clifford, Mary Murphy, Donna Mortimer, and Mary Zawoysky for all their hard work in making life in the MC\&G department easier and enjoyable.

From the start of my time at WHOI, Tristan Horner has been a constant encouraging presence. Whether in his former capacity as a postdoc or in his current role as assistant scientist in the chemical oceanography department at WHOI, he has always been willing to listen to my various frustrations and successes, give advise, and talk about the science he and his lab are involved in (usually barium isotopes). Through our frequent interactions, I felt that Tristan was an unofficial third mentor. My conversations with Maureen Auro since the beginning of my graduate career were also greatly helpful in letting me decompress. I also thank everyone else involved in the Horner lab in the present and past for being positive presences, including Ann Dunlea, Dalton Hardisty, Julia Middleton, and Logan Tegler.

Thanks to all my friends and cohorts in the Joint Program. I feel great pride in belonging to the same class in the MIT/WHOI Joint Program in chemical oceanography as Chawalit (Net) Charoenpong, Gabriella Farfan, Lauren Kipp, Danielle Gruen, Tyler Rohr, and Cristina Schultz. Their brightness, enthusiasm, and friendliness not only were critical for my successful completion of the Joint Program, but made my time at WHOI one of the most wonderful and fulfilling times of my life. I especially want to thank Net, with whom I have been able to talk freely and openly since we met at the open house. Also a huge thanks to both Lauren and Erin for tolerating all the complaints and worries I conveyed to them over the years. Lauren and Erin, along with Nick Hawo, were also GEOTRACES cohorts and were always willing to share and discuss trace metal data. Thank you Cris and Alex Bergan for the huge number of parties you have hosted over the years, and Cris, Alex, and Greg Horning for both the homebrew and for introducing me to a wide 
variety of beers that I did not know existed. On a similar note, thanks again to Gabi for being our sommelier for multiple wine tasting events. I also thank Justin Ossolinski for coaching the chemistry softball team for my first four summers at WHOI, and everyone who participated in these games.

Many thanks to my former advisor, Jim Bishop (UC Berkeley), who deserves special recognition. My oceanographic journey began with him, and without his willingness to look out for me and allow me to participate in several research cruises as an undergraduate, I would not be at WHOI today.

Finally, my deepest thanks to my family for all their love, generosity, and motivation. My parents have been anchors through emotionally turbulent periods, and their encouragement has propelled me through my graduate career. My grandparents, cousins, aunts, and uncles have also lifted my spirits countless times, and my brother has always been someone I could vent my anxieties with. My ability to navigate through the Joint Program, and life in general, has been directly dependent on the abundance of support my family has provided.

This work was supported by the US National Science Foundation. Two US NSF grants have supported the research in this thesis (OCE-1232578 and OCE-155644). The US NSF funding also supported the generation of the GEOTRACES ${ }^{230} \mathrm{Th}$ data (OCE-0927064 to LDEO, OCE-O092860 to WHOI, and OCE-0927754 to UMN), ${ }^{228,234} \mathrm{Th}$ data and ${ }^{228,234} \mathrm{Th}$ data (OCE-0925158 to WHOI), and particle data (OCE-0963026 to WHOI) used in this thesis. 


\section{Contents}

1 Introduction $\quad 21$

1.1 Oceanic Trace Metal Cycling . . . . . . . . . . . . . . . . . . 21

1.2 Thorium Isotopes: Tracers of Trace Metal Cycling _ . . . . . . . . . . . . . 23

1.3 Adsorption mechanisms . . . . . . . . . . . . . . . . 27

1.4 Effects of Circulation . . . . . . . . . . . . . . . . . 30

1.5 New Insights from GEOTRACES . . . . . . . . . . . . . . . . . . 31

1.6 Inverse Methods . . . . . . . . . . . . . . . . . . . . . 32

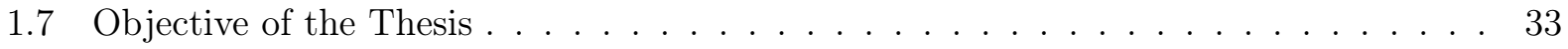

2 Testing models of thorium and particle cycling in the ocean using data from station GT11-22 of the U.S. GEOTRACES North Atlantic Section 37

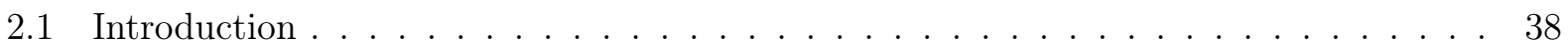

2.2 Methods . . . . . . . . . . . . . . . . . . . . . . 43

2.2.1 Hydrographic Setting . . . . . . . . . . . . . . . . . . . 43

2.2 .2 Data . . . . . . . . . . . . . . . . . . . . . . 44

2.2.3 Bulk Particulate ${ }^{228,230,234} \mathrm{Th} \ldots \ldots \ldots \ldots$. . . . . . . . . . 45

2.2 .4 Vertical Interpolation $\ldots \ldots \ldots \ldots$

2.2.5 Model of Thorium and Particle Cycling . . . . . . . . . . . . . . . 48

2.2 .6 Inverse Method . . . . . . . . . . . . . . . . . . . . . . . . . 49

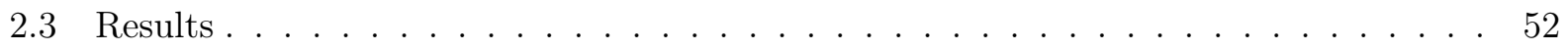

2.3 .1 Goodness of fit . . . . . . . . . . . . . . . . . . . 52

2.3 .2 Bootstrap Test . . . . . . . . . . . . . . . . . . . . 53 
2.3.3 Consistency with Prior Estimates . . . . . . . . . . . . . 54

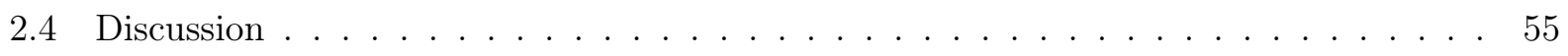

2.4 Robustness of the Test . . . . . . . . . . . . . . . . . 56

2.4.2 Implications for Oceanic Th Geochemistry . . . . . . . . . . . . . . . 58

2.4 .3 Diagnosis of Th Isotope Budgets . . . . . . . . . . . . . . . . . 63

2.4.4 Interpretation of Particle and Th isotope residuals . . . . . . . . . . . 64

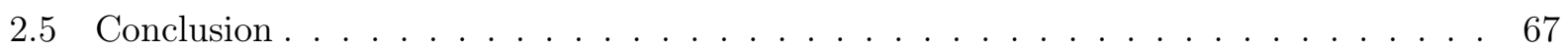

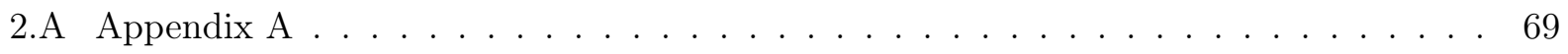

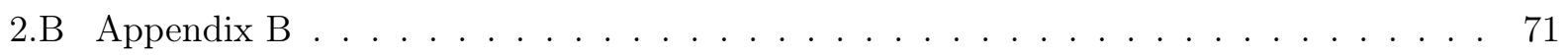

3 Kinetics of thorium and particle cycling along the U.S. GEOTRACES North $\begin{array}{ll}\text { Atlantic Transect } & 93\end{array}$

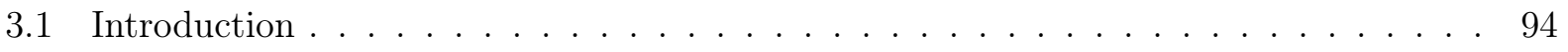

3.2 Methods . . . . . . . . . . . . . . . . . . . 98

3.2 .1 Hydrographic Setting . . . . . . . . . . . . . . . . . . . 98

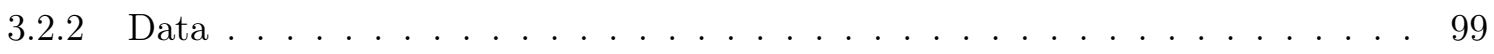

3.2 .3 Vertical Interpolation $\ldots \ldots \ldots$. . . . . . . . . . . . . . . . . . . . .

3.2.4 Model of Thorium and Particle Cycling . . . . . . . . . . . . . . . 103

3.2.5 Effects of Advection, Diffusion, and Unsteadiness . . . . . . . . . . . 104

3.2 .6 Inverse method . . . . . . . . . . . . . . . . . . . 106

3.3 Results . . . . . . . . . . . . . . . . . . . . 108

3.3 .1 Goodness of Fit . . . . . . . . . . . . . . . . . . 108

3.3.2 Radiochemical Activities and Particle Concentrations . . . . . . . . . . . 109

3.3.3 Rate Parameters . . . . . . . . . . . . . . . . . 110

3.4 Discussion . . . . . . . . . . . . . . . . . . . . . . 111

3.4 .1 Misfit to the interpolated values . . . . . . . . . . . . 111

3.4.2 Variation in Rate Parameters . . . . . . . . . . . . . . . . 113

3.4 .3 Importance of Missing Terms . . . . . . . . . . . . . . . . . . 122

3.5 Conclusion . . . . . . . . . . . . . . . . . . . . . 122

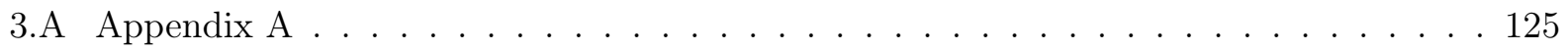




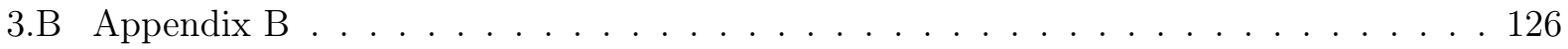

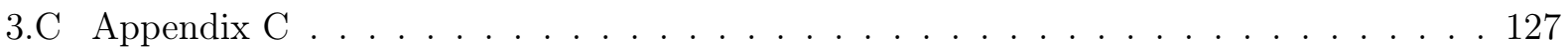

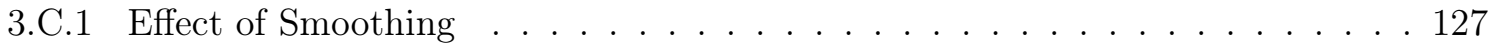

3.C.2 Effect of Algorithm . . . . . . . . . . . . . . . . . . . . . . 129

3.D Supplementary Material . . . . . . . . . . . . . . . . . . . . 152

4 Effects of Particle Composition on Thorium Scavenging in the North Atlantic 157

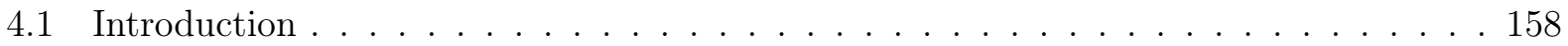

4.2 Methods . . . . . . . . . . . . . . . . . . . . . . . . 162

4.2.1 Particle and Radiochemical Data . . . . . . . . . . . . . . . . 162

4.2 .2 Principal Component Analysis _. . . . . . . . . . . . . . . . . . . 164

4.2 .3 Estimates of Th Adsorption Rate Constant . . . . . . . . . . . . . . 165

4.3 Results . . . . . . . . . . . . . . . . . . . . . . . . . . . . . . . 167

4.3.1 Dependence of $\boldsymbol{k}_{1}$ on Particle Composition: Additive Model . . . . . . . . 167

4.3.2 Dependence of $\boldsymbol{k}_{1}$ on Particle Composition: Multiplicative Model . . . . . . 170

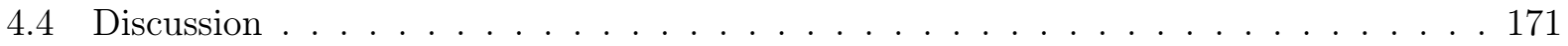

4.4.1 Relative Importance of Different Particulate Phases . . . . . . . . . . . . . 172

4.4.2 Kinetic Consistency of Additive and Multiplicative Models . . . . . . . . . . . 174

4.4 .3 Reduced Models . . . . . . . . . . . . . . . . . . . . . . . . . . . . . . . . . . 177

4.4 .4 Paleoceanographic Implications _ . . . . . . . . . . . . . . . . . . . 180

4.4 .5 Importance of Errors . . . . . . . . . . . . . . . . . . . . . . 182

4.4.6 Particle Concentration vs. Particle Composition _ . . . . . . . . . . . . . . 183

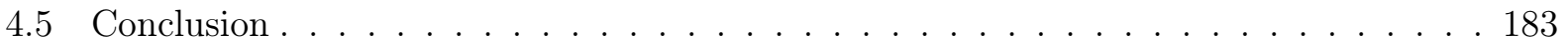

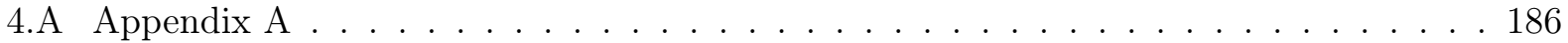

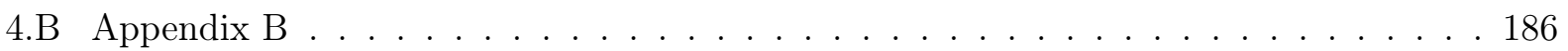

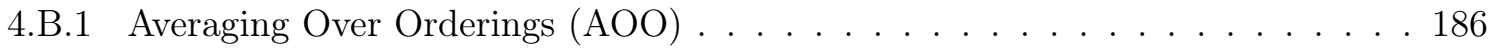

4.B.2 Dominance Analysis . . . . . . . . . . . . . . . . . . . . . . . . 187

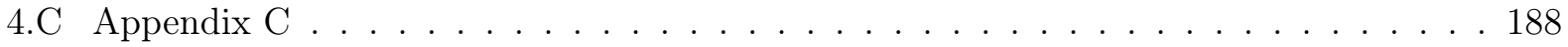

5 Combined Effects of Scavenging and Circulation in the Northwest Atlantic 201

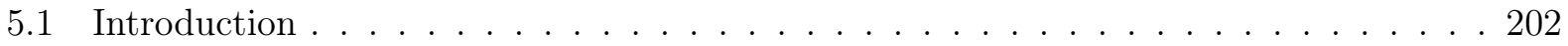


$5.1 .1{ }^{230} \mathrm{Th}$ in the Northwest Atlantic . . . . . . . . . . . . . . . . 203

5.1 .2 Influence of Scavenging in the Northwest Atlantic . . . . . . . . . . . . . . 203

5.1 .3 Influence of Circulation in the Northwest Atlantic . . . . . . . . . . . . . 205

5.1 .4 Objectives of The Study . . . . . . . . . . . . . . . . 206

5.2 Major Features of the General Circulation in the Northwest Atlantic . . . . . . . 207

5.2 .1 Gulf Stream and Recirculation Gyres . . . . . . . . . . . . 207

5.2 .2 Gulf Stream Meanders and Eddies . . . . . . . . . . . . . . . . . . 208

5.2 .3 Deep Western Boundary Current . . . . . . . . . . . . . . . . . 208

5.3 Data . . . . . . . . . . . . . . . . . . . . 210

5.3 .1 Hydrographic Data . . . . . . . . . . . . . . . . . . . . 210

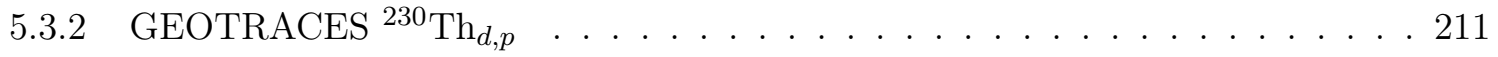

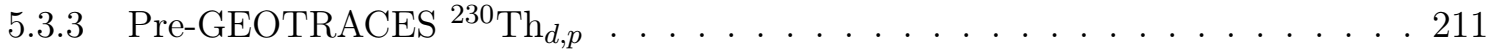

5.3.4 Beam Attenuation Coefficient, Dynamic Topography, and Bathymetry . . . . 212

5.4 Model of Ocean Circulation and ${ }^{230} \mathrm{Th}$ Transport . . . . . . . . . . . . . . 213

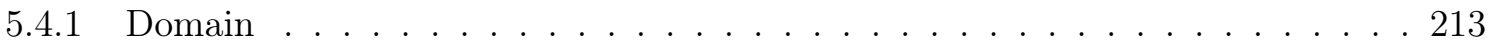

5.4 .2 Tracer Equations . . . . . . . . . . . . . . . . . . . . . 214

5.4 .3 Initial Conditions . . . . . . . . . . . . . . . . . . . . . 215

5.4 .4 Boundary Conditions . . . . . . . . . . . . . . . . . 215

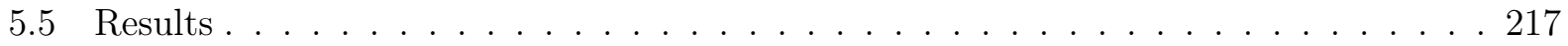

5.5.1 Reference Experiment: Circulation . . . . . . . . . . . . 217

5.5.2 Reference Experiment: ${ }^{230} \mathrm{Th}_{d, p}$ distributions . . . . . . . . . . . . . 219

5.5 .3 Sensitivity Experiments . . . . . . . . . . . . . . . 221

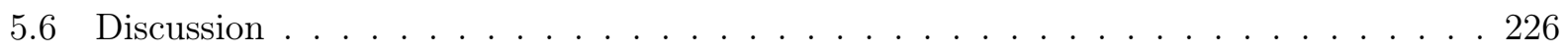

5.6 .1 Missing Processes Impacting ${ }^{230} \mathrm{Th}_{p} \ldots \ldots \ldots$. . . . . . . . . 227

5.6 .2 Paleoceanographic Implications . . . . . . . . . . . . . . . . 232

5.7 Conclusion . . . . . . . . . . . . . . . . . . . . . 234

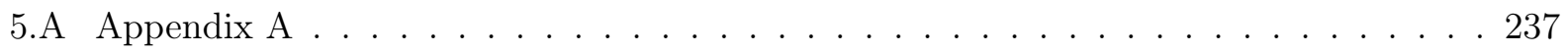

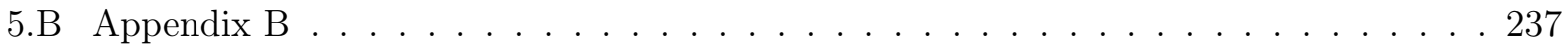

6 Conclusion $\quad 309$ 
6.1 Effect of Particle Concentration . . . . . . . . . . . . . . . . . . . . 309

6.2 Effect of Particle Composition . . . . . . . . . . . . . . . . . . . . . 312

6.3 Circulation vs. Scavenging Intensity in the Northwest Atlantic . . . . . . . . . . . . . 314

6.4 General Outlook . . . . . . . . . . . . . . . . . 317

6.4 Recommendations for Models . . . . . . . . . . . . . . . 317

6.4.2 Recommendations for Laboratory Experiments and Observations . . . . . . . 318 


\section{List of Figures}

\section{Chapter 1}

Fig. 1 (p. 36): Simplified schematic for trace metal cycling in the ocean.

\section{Chapter 2}

Fig. 1 (p. 72): Stations occupied by the R/V Knorr during the GEOTRACES North Atlantic section.

Fig. 2 (p. 73): Plot of potential temperature vs. salinity for station GT11-22.

Fig. 3 (p. 74): Measured and interpolated values of particle concentration $(P)$ at station GT11-22 for three different field property variances, and distribution function of the interpolation residuals normalized to measurement errors.

Fig. 4 (p. 75): Measured and interpolated values of particle concentration $(P)$ at station GT11-22 for three different vertical correlation scales, and distribution function of the interpolation residuals normalized to measurement errors.

Fig. 5 (p. 76): Profiles of Th isotope activities and their parent activities at station GT11-22.

Fig. 6 (p. 77): Schematic diagram of the single-particle class model of Th cycling and particle cycling.

Fig. 7 (p. 78): Radiochemical activities and particle concentrations at station GT11-22 for model with uniform rate constants.

Fig. 8 (p. 79): Radiochemical activities and particle concentrations at station GT11-22 for model with vertically varying rate constants.

Fig. 9 (p. 80): Histogram of the frequency of the test statistic $T$ obtained from the bootstrap.

Fig. 10 (p. 81): Rate constants of Th and particle cycling at station GT11-22 as inferred from the combination of data with a thorium and particle cycling model.

Fig. 11 (p. 82): Radiochemical activities and particle concentrations measured at station GT11-22 and obtained by inversion using a model with vertically uniform rate constants.

Fig. 12 (p. 83): Effect of smoothing on rate constants of Th and particle cycling at station GT11-22 as inferred from the combination of data with model with vertically varying rate constants.

Fig. 13 (p. 84): Profiles of the rate constant ratio and this ratio normalized to particle concentration at station GT11-22. 
Fig. 14 (p. 85): Variation of the adsorption rate constant, desorption rate constant, rate constant ratio, and this ratio normalized to particle concentration with particle concentration, as inferred from inversion of station GT11-22 data.

Fig. 15 (p. 86): ${ }^{234}$ Th budget at station GT11-22.

Fig. 16 (p. 87): ${ }^{228}$ Th budget at station GT11-22.

Fig. 17 (p. 88): ${ }^{230}$ Th budget at station GT11-22.

Fig. 18 (p. 89): Normalized residuals of the fit of a model with vertically varying rate constants to

station GT11-22 data for ${ }^{230} \mathrm{Th}_{d}$ and ${ }^{230} \mathrm{Th}_{p}$, and proportions of Upper Labrador Sea Water and Central Labrador Sea Water at this station.

Fig. 19 (p. 90): Normalized residuals for particle concentration, vertical particle concentration profile, and vertical profile of particle beam attenuation coefficient at station GT11-22.

Fig. B1 (p. 91): Normalized residuals of the fit of a model with vertically varying rate constants to station GT11-22 data for the reference inversion (black), and for the case where the prior ${ }^{228,234} \mathrm{Th}_{p}$ values are set to $20 \%$ less than their measured values.

\section{Chapter 3}

Fig. 1 (p. 131): Stations occupied by the R/V Knorr during the GEOTRACES North Atlantic section.

Fig. 2 (p. 132): Distribution function of residuals for the interpolation and the inversion normalized to the errors in the data.

Fig. 3 (p. 133): Schematic diagram of the single-particle class model of Th cycling and particle cycling.

Fig. 4 (p. 134): Estimates of the magnitude of the terms missing in the ${ }^{228,230,234}$ Th dissolved and particulate balance equations.

Fig. 5 (p. 135): Estimates of the magnitude of the terms missing in the bulk particle $(P)$ balance equations.

Fig. 6 (p. 136): Estimates of the magnitude of the missing terms in the ${ }^{230} \mathrm{Th}_{d}$ balance equations for station GT11-24.

Fig. 7 (p. 137): Section plots of ${ }^{234} \mathrm{Th}_{d, p}$ interpolated values and residuals normalized to interpolated values. 
Fig. 8 (p. 138): Section plots of ${ }^{230} \mathrm{Th}_{d, p}$ interpolated values and residuals normalized to interpolated values.

Fig. 9 (p. 139): Section plots of ${ }^{228} \mathrm{Th}_{d, p}$ interpolated values and residuals normalized to interpolated values.

Fig. 10 (p. 140): Section plots of $P$ interpolated values and residuals normalized to interpolated values.

Fig. 11 (p. 141): Posterior estimates of the rate constants of Th and particle cycling along GA03.

Fig. 12 (p. 142): Vertical profiles of the Th adsorption rate constant.

Fig. 13 (p. 143): ${ }^{228} \mathrm{Th}_{d}$ profiles at the five easternmost stations.

Fig. 14 (p. 144): ${ }^{234} \mathrm{Th}_{\text {tot }}$ and ${ }^{238} \mathrm{U}$ profiles at the crossover station GT11-24/GT10-12.

Fig. 15 (p. 145): Variation of Th adsorption rate constant with particle concentration.

Fig. 16 (p. 146): Posterior estimates of the rate constants at all selected stations of GA03 obtained from the inversion with and without smoothing.

Fig. 17 (p. 147): Posterior estimates of the rate constant ratio derived from the inversion with and without smoothing. Estimates of the rate constant ratio normalized to particle concentration derived from the inversion with and without smoothing.

Fig. 18 (p. 148): Variation of the rate constant ratio normalized to particle concentration and partition coefficient for ${ }^{230} \mathrm{Th}$ with particle concentration.

Fig. 19 (p. 149): Variation of the sum of the desorption and particle degradation rate constant estimated by inversion with particle concentration.

Fig. B1 (p. 150): Variation of the adsorption rate constant estimated by inversion with particle concentration, with the line of best fit obtained from the Algorithm of Total Inversion (ATI).

Fig. C1 (p. 151): Variation of the adsorption rate constant with particle concentration for station GT11-22 using the ATI with smoothing and total ${ }^{230} \mathrm{Th}_{p}$ (adsorbed $+{ }^{230} \mathrm{Th}$ locked in mineral lattices), the ATI with smoothing and adsorbed ${ }^{230} \mathrm{Th}_{p}$, FMINCON with smoothing, and FMINCON without smoothing.

Figure S1 (p. 153): Vertical profiles of the Th desorption rate constant.

Figure S2 (p. 154): Vertical profiles of the particle degradation rate constant.

Figure S3 (p. 155): Vertical profiles of the particle sinking speed. 


\section{Chapter 4}

Fig. 1 (p. 190): Stations occupied by the R/V Knorr during the GEOTRACES North Atlantic section.

Fig. 2 (p. 191): Pearson correlation coefficients between pairs of particle phases at selected stations of GA03.

Fig. 3 (p. 192): Principal component coefficients for each particle phase.

Fig. 4 (p. 193): Section plots of the principal components.

Fig. 5 (p. 194): Estimates of the adsorption rate constant at selected stations of the US GEOTRACES North Atlantic section.

Fig. 6 (p. 195): Regressions of the Th adsorption rate constant obtained from inversion of radiochemical data vs. the adsorption rate constant obtained from regression against particle composition using OLS with additive and multiplicative regression models.

Fig. 7 (p. 196): Relative Importance of particle phases for the additive and multiplicative models. Fig. 8 (p. 197): Regressions of the Th adsorption rate constant obtained from inversion of radiochemical data vs. the adsorption rate constant obtained from regression against particle composition using the ATI with additive and multiplicative regression models.

Fig. 9 (p. 198): Regressions of the natural logarithm of the Th adsorption rate constant estimated by inversion against the natural logarithm of measured bulk particle concentration.

Fig. A1 (p. 199): Th adsorption rate constant obtained from an inversion without bulk particle concentration data vs. the adsorption rate constant obtained from an inversion with bulk particle concentration data.

\section{Chapter 5}

Fig. 1 (p. 238): Vertical profiles of ${ }^{230} \mathrm{Th}_{d}$ activities in the North Atlantic. Fig. 2 (p. 239): Bathymetry (meters) and simplified schematic of the general circulation in the western North Atlantic.

Fig. 3 (p. 240): Potential temperature and salinity at eight stations occupied along the western segment of GA03.

Fig. 4 (p. 241): CFC-11 and dissolved $\mathrm{O}_{2}$ at eight stations occupied along the western segment of GA03.

Fig. 5 (p. 242): Along Line $\mathrm{W}$ and cross Line $\mathrm{W}$ velocities, respectively, obtained from velocity 
profiles from moored instruments.

Fig. 6 (p. 243): ${ }^{230} \mathrm{Th}_{d}$ activity profiles along the western end of section GA03.

Fig. 7 (p. 244): ${ }^{230} \mathrm{Th}_{p}$ activity profiles along the western end of section GA03.

Fig. 8 (p. 245): ${ }^{230} \mathrm{Th}_{p}$ activity profiles at station GT11-04 and GT11-08.

Fig. 9 (p. 246): ${ }^{230} \mathrm{Th}_{d, p}$ activity proifles from the pre-GEOTRACES era.

Fig. 10 (p. 247): ${ }^{230} \mathrm{Th}_{d, p}$ activity profiles at GT11-14, and exponential fits to these profiles.

Fig. 11 (p. 248): ${ }^{230} \mathrm{Th}_{d, p}$ activity profiles at a station in the Labrador Sea.

Fig. 12 (p. 249): Domain averages of kinetic energy, ${ }^{230} \mathrm{Th}_{d}$ activity, and ${ }^{230} \mathrm{Th}_{p}$ activity in the reference simulation.

Fig. 13 (p. 250): Horizontal velocities at the surface layer and $3000 \mathrm{~m}$ in the model.

Fig. 14 (p. 251): Mean and standard deviation in SSH from satellite altimetry and the model.

Fig. 15 (p. 252): Simulated and observed ${ }^{230} \mathrm{Th}_{d}$ activities at GEOTRACES stations.

Fig. 16 (p. 253): Simulated and observed ${ }^{230} \mathrm{Th}_{p}$ activities at GEOTRACES stations.

Fig. 17 (p. 254): Simulated and observed ${ }^{230} \mathrm{Th}_{d, p}$ activities at pre-GEOTRACES stations.

Fig. 18 (p. 255): Observed vs. simulated ${ }^{230} \mathrm{Th}_{d}$ and ${ }^{230} \mathrm{Th}_{p}$.

Fig. 19 (p. 256): ${ }^{230} \mathrm{Th}_{d, p}$ boundary values used in sensitivity experiments.

Fig. 20 (p. 257): Vertically averaged Th adsorption rate constant.

Fig. 21 (p. 258): Particle beam attenuation coefficient profiles along the western end of GA03, and the fit to these profiles used to constrain vertical variations in the Th adsorption rate constant.

Fig. 22 (p. 259): Vertical Th adsorption rate constant profiles within the region of bottom scavenging assumed in sensitivity experiments.

Fig. 23 (p. 260): $r^{2}$ for each experiment.

Fig. 24 (p. 261): Modeled and observed activities ${ }^{230} \mathrm{Th}_{d, p}$ at station GT11-08.

Fig. 25 (p. 262): Simulated and observed ${ }^{230} \mathrm{Th}_{p}$ activities at station GT11-04 and GT11-08.

Fig. 26 (pp. 263): Bathymetry obtained from the GMRT along Line W, and fraction of slopes that are critical.

Figure B1-B44 (p. 264-307): Every set of four figures corresponds to figures 13-16, but for a different sensitivity experiment. There are eleven sensitivity experiments in total. 


\section{List of Tables}

\section{Chapter 2}

Table 1 (p. 50): Prior estimates of rate constants of Th and particle cycling assumed in this study.

Table 2 (p. 53): Measures of goodness of fit of the thorium and particle cycling model with uniform and vertically varying rate constants to station GT11-22 data.

Table 3 (p. 57): Measures of goodness of fit of models with uniform and vertically varying rate constants to GT11-22 data for different initial estimate $\boldsymbol{x}_{k=0}$.

Table A1 (p. 70): Radiochemical and [article concentration Data at station GT11-22.

Table B1 (p. 71): $p$ values from Kolmogorov-Smirnov tests for differences between a reference inversion and inversions of different configurations.

\section{Chapter 3}

Table 1 (p. 103): Depth range of the radionuclide and particle concentration data considered in Chapter 3.

Table 2 (p. 107): Prior estimates of rate constants of Th and particle cycling assumed in Chapter 3.

Table 3 (p. 109): Measures of goodness of fit of the model to data at selected stations of GA03.

Table 4 (p. 114): Results from ordinary and weighted least squares regressions of the natural logarithm of the Th adsorption rate constant vs. the natural logarithm of bulk particle concentration. Table 5 (p. 117): Mean and standard deviation of the rate constants for thorium and particle cycling for the inversion without smoothing.

Table A1 (p. 126): Optional Inputs for Matlab's Constrained Nonlinear programming solver FMINCON.

Table C1 (p. 129): Mean and standard deviation of rate constants for thorium and particle cycling for the inversion with smoothing.

Table C2 (p. 130): Regression of the natural logarithm of the Th adsorption rate constant against the natural logarithm of bulk particle concentration, with the adsorption rate constant estimated from four different inversions at GT11-22. 


\section{Chapter 4}

Table 1 (p. 165): Percentages of total variance explained by principal components.

Table 2 (p. 168): Coefficients for regressions of the Th adsorption rate constant obtained by inversion vs. particle composition using the additive model (OLS).

Table 3 (p. 171): Coefficients for regressions of the Th adsorption rate constant obtained by inversion vs. particle composition using the multiplicative model (OLS).

Table 4 (p. 182): Coefficients for regressions of the Th adsorption rate constant obtained by inversion vs. particle composition using the additive model (ATI).

Table 5 (p. 182): Coefficients for regressions of the Th adsorption rate constant obtained by inversion vs. particle composition using the multiplicative model (ATI).

\section{Chapter 5}

Table 1 (p. 212): Water column measurements of ${ }^{230} \mathrm{Th}_{d, p}$ in the study region.

Table 2 (p. 217): Open boundary conditions of the model.

Table 3 (p. 219): Comparison of the reference experiment with physical observations.

Table 4 (p. 219): Parameter list for reference experiment.

Table 5 (p. 220): Comparison of the reference experiment with trace metal observations.

Table 6 (p. 221): Sensitivity experiment parameter changes.

Table 7 (p. 222): Comparison of sensitivity experiments with trace metal observations: Boundary values of ${ }^{230} \mathrm{Th}_{d, p}$.

Table 8 (p. 223): Comparison of sensitivity experiments with trace metal observations: Boundary values of DWBC and GS Transports.

Table 9 (p. 226): Comparison of sensitivity experiments with trace metal observations: Spatial variations in $k_{1}$.

Table A1 (p. 237): Parameters of the Princeton Ocean Model. 


\section{Chapter 1}

\section{Introduction}

\subsection{Oceanic Trace Metal Cycling}

Oceanic trace metals are metals that are present at low $\left(\leq 0.1 \mu \mathrm{mol} \mathrm{kg} \mathrm{kg}^{-1}\right)$ concentrations in seawater (Morel and Price, 2003). Despite these low concentrations, trace metals play important roles in the ocean as essential micronutrients (e.g., Fe, Mn, Zn, and Cd; Morel and Price (2003); Morel et al. (2003); Bruland and Lohan (2006), contaminants (e.g., Hg; Lamborg et al. (2014)),

and tracers of modern and past ocean processes, including biological productivity (e.g., ${ }^{234} \mathrm{Th}$, ${ }^{231} \mathrm{~Pa} /{ }^{230} \mathrm{Th}$, and $\mathrm{Ba}$; Buesseler et al. (2006); Henderson and Anderson (2003); Griffith and Paytan (2012)), sediment lateral redistribution $\left({ }^{230} \mathrm{Th}\right.$; François et al. (2004)), and ocean circulation (e.g., $\mathrm{Cd} / \mathrm{Ca},{ }^{231} \mathrm{~Pa} /{ }^{230} \mathrm{Th}$, and ${ }^{143 / 144} \mathrm{Nd} ;$ Marchitto and Broecker (2006); Henderson and Anderson (2003); Jeandel et al. (2007)). A thorough understanding of these respective roles requires intimate knowledge of the cycling of trace metals in the water column and sediment. The concentration of a given metal in the ocean is affected by inputs such as atmospheric dust deposition (Jickells et al., 2005), river runoff and submarine groundwater discharge (Moore, 2010), hydrothermalism (Tagliabue et al., 2010), and sedimentary supplies (Elrod et al., 2004). Moreover, as a metal atom moves in the ocean, it may be affected by a variety of chemical and biological transformations, such as biological uptake or adsorption onto the surfaces of particles (Fig. 1). Importantly, many of the processes depicted are simplistic encapsulations of one or multiple phenomena occurring at a variety of time and spatial scales. For example, particle degradation includes the remineralization of particles (Clegg et al., 1991a) as well as physical disaggregation of large to small particles (Jackson 
and Burd, 2015). Both of these processes can remove a trace metal from a sinking particle phase to either a non-sinking, soluble phase (remineralization) or a slower sinking particle phase (e.g., a colloidal phase, usually defined operationally as any particle smaller than $1 \mu \mathrm{m}$, the pore size of filters commonly used to collect particles; Morel and Gschwend (1987)).

In broadest terms, we may classify two types of processes affecting trace metal distributions in the ocean: exogenous inputs and internal cycling processes. Internal cycling includes (i) the transfer of metals between dissolved (colloidal + soluble) and particle phases (unless otherwise specified, particulate refers to material that is able to be collected on a filter of some nominal pore size, e.g. $>1 \mu \mathrm{m}$ ), (ii) the sinking of particle-bound metals along the water column, and (iii) the transport of dissolved and particulate metals via the ocean circulation (advection + mixing). The transfer from the dissolved to the particulate phase may arise from active biological uptake (e.g., utilization of micronutrients by phytoplankton), passive absorption, aggregation of colloids, or adsorption onto a particle surface. The transfer from the particulate to the dissolved phase may occur via particle degradation and trace metal desorption from particles. It is these internal cycling processes that are the main focus of the research presented in this thesis.

Trace metals attached to particles may eventually reach the sediments, and hence decrease the total ocean inventory of that metal. Particle sinking then acts as a link between the internal processes affecting trace metal distributions in different phases, and the removal of trace metals to the sediment. This combined process of adsorption (more generally, transfer to the particulate phase) and sinking has been termed "scavenging" (Goldberg, 1954), and has been implicated as the primary phenomenon responsible for the extremely low concentrations and short residence times ( 20-100 yrs) of trace metals in the ocean (Bhat et al., 1969; Lal, 1977; Turekian, 1977).

The GEOSECS program, a near-global survey of the distribution of chemical, radiochemical, and isotopic tracers undertaken between 1972-1978, was the first major project highlighting the importance of radionuclides in quantifying rates of trace metal scavenging and particle sinking. For example, Craig et al. (1973) applied a one dimensional (vertical) advection-diffusion model with scavenging to ${ }^{210} \mathrm{~Pb}$ and ${ }^{226} \mathrm{Ra}$ data collected from the North Atlantic and North Pacific to estimate the scavenging residence time of ${ }^{210} \mathrm{~Pb}$ to be 54 yrs. Using particulate ${ }^{55} \mathrm{Fe}$ and ${ }^{14} \mathrm{C}$ collected from the subtropical North Pacific, Lal (1977) estimated the settling speed of particles carrying these radionuclides to be $0.5 \times 10^{-3}$ and $2 \times 10^{-3} \mathrm{~cm} \mathrm{~s}^{-1}$, respectively, while Krishnaswami et al. (1976) 
estimated the settling speed of particles containing ${ }^{239} \mathrm{Pu}$ to be $0.3 \times 10^{-3} \mathrm{~cm} \mathrm{~s}^{-1}$. GEOSECS also highlighted the differences in the affinity of different trace metals for particles, which can complicate the use of radionuclides to quantify scavenging rates of other, non-radioactive trace metals. For example, using a similar model as applied by Craig et al. (1973), Craig (1974) found the scavenging residence times of $\mathrm{Cu}, \mathrm{Sn}, \mathrm{Sb}$, and $\mathrm{Ni}$ all to be $O(1000 \mathrm{yr})$, suggesting that scavenging removes these elements at much slower rates than for ${ }^{210} \mathrm{~Pb}$. One complication of applying a scavenging model to the study of a particular trace element or isotope (TEI) is the uncertainty in sources and sinks, such as the supply from dust deposition at the sea surface. The scavenging of trace metals in the ocean may be better constrained using TEIs whose sources and sinks (besides those associated with scavenging) are well understood.

\subsection{Thorium Isotopes: Tracers of Trace Metal Cycling}

The radioactive isotopes of thorium have for a long time been used to study the internal cycling of trace metals in the ocean. Among oceanic trace metals, thorium presents several advantages as a tracer of sorption and particle processes. One advantage is that thorium is highly particle reactive in seawater, so that a dominant sink of Th appears to be the attachment of this element to particles and the removal of these particles to the seafloor. Another advantage is that thorium has multiple isotopes characterized by widely different half-lives: $t_{1 / 2}=24.101 \pm 0.025 \mathrm{~d}$ for ${ }^{234} \mathrm{Th}$ (Knight and Macklin, 1948), 75, $584 \pm 110 \mathrm{yr}$ for ${ }^{230} \mathrm{Th}$ (Cheng et al., 2013), and $1.910 \pm 0.002 \mathrm{yr}$ for ${ }^{228} \mathrm{Th}$ (Kirby et al., 2002). The availability of the long-lived radioisotope ${ }^{230} \mathrm{Th}$, for example, implies that ${ }^{230} \mathrm{Th}$ occurs at detectable concentrations in old sediments and can be used for paleoceanographic reconstructions. A third advantage is that the sources of these thorium isotopes in the ocean are relatively well understood. ${ }^{234} \mathrm{Th},{ }^{230} \mathrm{Th}$, and ${ }^{228} \mathrm{Th}$ are produced in situ by radioactive decay of ${ }^{238} \mathrm{U},{ }^{234} \mathrm{U}$, and ${ }^{228} \mathrm{Ra}$, respectively. Since uranium seems to behave quasi-conservatively in the ocean (Ku et al., 1977; Delanghe et al., 2002), ${ }^{234} \mathrm{U}$ and ${ }^{238} \mathrm{U}$ activities are often estimated empirically from salinity (Chen et al., 1986; Owens et al., 2011). On the other hand, both ${ }^{228} \mathrm{Ra}$ and its daughter isotope ${ }^{228} \mathrm{Th}$ can be collected concurrently using manganese cartridges, and measured simultaneously using conventional $\beta$ counting techniques (Henderson et al., 2013). The high particle reactivity of Th isotopes, with a wide range of half-lives, makes them particularly well suited to study a variety of processes that affect particles, both today and in the past. In the following 
paragraphs, a brief summary describing three of these applications is provided.

(i) ${ }^{234} \mathrm{Th}$ is commonly used to estimate the vertical settling speed or flux of particles from the surface mixed layer from the secular disequilibrium with its radioactive parent, ${ }^{238} \mathrm{U}$. This approach was pioneered by Bhat et al. (1969), who estimated particle sinking speeds ranging between 220 to $631 \mathrm{~m} \mathrm{yr}^{-1}$ using ${ }^{234} \mathrm{Th}$ data obtained from the Arabian Sea, Java Sea, Australian coast, Wharton Sea, and the Tasmania coast. Their model, however, was based on the assumption that all ${ }^{234} \mathrm{Th}$ is adsorbed onto particles. Krishnaswami et al. (1976), using ${ }^{234}$ Th data from the Pacific GEOSECS expedition, estimated ${ }^{234} \mathrm{Th}$ to be only $10-20 \%$ of the total activity of ${ }^{234} \mathrm{Th}$ (note that dissolved and particulate activities are referred throughout this thesis with subscripts "d" and "p", respectively). Based on this observation, as well as the observation that particulate ${ }^{230} \mathrm{Th}_{p}$ increased with depth in the water column, they proposed a one-dimensional (vertical) scavenging model for particulate thorium, similar to that of Bhat et al. (1969) but with an added scavenging term to account for the existence of both dissolved and particulate phases. In their analysis, Krishnaswami et al. (1976) considered both particulate ${ }^{234} \mathrm{Th}_{p}$ and ${ }^{230} \mathrm{Th}_{p}$ data together with an estimate of ${ }^{234} \mathrm{Th}_{d}$ based on an assumed activity ratio ${ }^{238} \mathrm{U} /{ }^{234} \mathrm{Th}_{d}$ of 0.8 and a "typical" seawater activity of $2200 \mathrm{dpm} \mathrm{m}{ }^{-3}$ for ${ }^{238} \mathrm{U}$. They reported a mean particulate sinking speed of $630 \mathrm{~m} \mathrm{yr}^{-1}$, on the high end of the range found by Bhat et al. (1969).

Since then, ${ }^{238} \mathrm{U}_{-}{ }^{234} \mathrm{Th}$ disequilibria has evolved as a popular method for estimating the flux of particulate material from the surface mixed layer (Bruland and Coale, 1986; Savoye et al., 2006; Buesseler et al., 2006). More specifically, this method has been used to estimate the vertical flux of various elements in particulate form from the surface ocean by multiplying the vertical ${ }^{234} \mathrm{Th}$ flux by the element to ${ }^{234} \mathrm{Th}$ ratio in particles collected below the mixed layer. For example, using $\mathrm{C} /{ }^{234} \mathrm{Th}$ measured in suspended organic particles, this technique has been used to estimate fluxes of particulate organic carbon (POC) between 5-70 mmol C m${ }^{-2} \mathrm{~d}^{-1}$ during the onset of the spring bloom in the North Atlantic Bloom Experiment of the JGOFS program (Buesseler et al., 1992). Planquette et al. (2011) estimated fluxes in total and labile Fe fluxes below the mixed layer between 0.003-0.145 $\mathrm{mmol} \mathrm{m}^{-2} \mathrm{~d}^{-1}$ and 0.004-0.301 $\mathrm{mmol} \mathrm{m}^{2} \mathrm{~d}^{-1}$, respectively, from particulate Fe and total ${ }^{234} \mathrm{Th}$ data collected near the Crozet Islands, in the Indian sector of the Southern Ocean. Importantly, these authors only focused on the net flux of ${ }^{234} \mathrm{Th}$ from the mixed layer, and were not concerned with the exchange between dissolved and particulate material in the water column. 
(ii) Thorium isotopes have also been extensively used to constrain various aspects of trace metal and particle cycling. The first applications of thorium isotopes in dissolved and particulate forms to estimate rates of trace metal and particle cycling relied on data from the North Pacific (Nozaki et al., 1981) and from the Panama and Guatemala Basins (Bacon and Anderson, 1982). Both studies reported a general increase with depth in the activities of ${ }^{230} \mathrm{Th}$ in both dissolved and particulate forms. To explain this feature, these authors constructed a model that allowed for the reversible exchange of thorium between the dissolved and particulate forms by including a term for the loss of thorium from particles (one particle class) through Th desorption and (or) particle degradation, in addition to the adsorption term. These processes were assumed to be first order with apparent rate constants $k_{1}, k_{-1}$, and $\beta_{-1}$ for thorium adsorption, thorium desorption, and particle degradation, respectively, a symbolism that will be used throughout the remainder of this thesis. The estimates of $k_{1}$ were $1.5 \mathrm{yr}^{-1}$ (Nozaki et al., 1981) and 0.3 to $1.3 \mathrm{yr}^{-1}$ (Bacon and Anderson, 1982), and the estimates of $k_{-1}+\beta_{-1}$ were $6.3 \mathrm{yr}^{-1}$ from Nozaki et al. (1981), and 1.3 to $6.3 \mathrm{yr}^{-1}$ from Bacon and Anderson (1982)). Nozaki et al. (1987) modified the model of Nozaki et al. (1981) by including particles in two size classes which interact through particle aggregation and disaggregation. By combining dissolved and particulate ${ }^{228} \mathrm{Th}$ and ${ }^{230} \mathrm{Th}$ measurements from large volume pumps, particulate ${ }^{230} \mathrm{Th}$ from sediments traps deployed in the western North Pacific and the Japan Sea, and different estimates of particle settling speeds, Nozaki et al. (1987) inferred specific rates of particle aggregation and disaggregation ranging from 2.4 to $12.2 \mathrm{yr}^{-1}$, and 148.2 to $2.37 \times 10^{4} \mathrm{yr}^{-1}$, respectively. Similar models have been developed to constrain rates of thorium and vertical particle cycling from measurements of ${ }^{234} \mathrm{Th}_{p},{ }^{234} \mathrm{Th}_{d}$, and suspended particle concentration obtained north of Hawaii (Clegg et al., 1991b), and from measurements of dissolved and particulate ${ }^{228,234} \mathrm{Th}$, suspended particle concentration, and particle flux obtained during the North Atlantic Bloom Experiment (Clegg and Whitfield, 1993; Cochran et al., 1993; Murnane et al., 1996).

(iii) ${ }^{230} \mathrm{Th}$ has been extensively used in paleoceanography. One of its earliest applications was to estimate the sedimentation rates of terrigenous clays and calcium carbonate from deep-sea cores obtained from the Mid-Atlantic Ridge, North American Basin, Equatorial Atlantic, and Caribbean Sea (Bacon, 1984). This study, and numerous others that have followed (e.g., François et al. (1993); François and Bacon (1994); Rühlemann et al. (2001); Anderson et al. (2006)) measured the ${ }^{230} \mathrm{Th}$ activity in sediments, and assumed that all ${ }^{230} \mathrm{Th}$ produced in the water column by ${ }^{234} \mathrm{U}$ is deposited 
in underlying sediments, to reconstruct the flux of particle mass to the sediments, a technique referred to as ${ }^{230}$ Th-normalization (e.g., François et al. (2004). However, one potential limitation of this approach is that the ratio of particle mass to ${ }^{230} \mathrm{Th}$ in sediments is susceptible to the dissolution of the particulate material following deposition. Kumar et al. $(1993,1995)$ combined ${ }^{231} \mathrm{~Pa}$ with ${ }^{230} \mathrm{Th}$ data measured in sediments to reconstruct past variations in biological productivity in the Southern Ocean. According to these authors, using the ${ }^{231} \mathrm{~Pa} /{ }^{230} \mathrm{Th}$ ratio as a tracer of vertical particle flux has the advantage of being less sensitive to sedimentary diagenesis.

${ }^{230} \mathrm{Th}$ has also been used to estimate the lateral redistribution of particulate material desposited on the seafloor. Assuming that the vertical supply of particulate ${ }^{230} \mathrm{Th}$ to the sediment is entirely balanced by water-column production from its radioactive parent, Suman and Bacon (1989) combined the radiocarbon chronology and ${ }^{230} \mathrm{Th}$ activities from a core raised from 4583-m water depth in the North American Basin to estimate the degree to which sedimentary material is augmented by lateral supply: the sediment focusing factor. These authors found focusing factors as high at 13.2, suggesting that up to 13 times more sediment was supplied by lateral vs. vertical transport to the core site. Since then, extensive sediment focusing, estimated using ${ }^{230} \mathrm{Th}$, has been found in cores sampled in different oceanic regions, including the northeast Atlantic (Thomson et al., 1993), the Southern Ocean (Frank et al., 1996, 1999), the equatorial Pacific Ocean (Marcantonio et al., 2001; Kienast et al., 2007), and the northeast Pacific (Costa et al., 2016).

Aside from estimating changes in lateral sedimentary redistribution, ${ }^{230} \mathrm{Th}$ has also been used in combination with ${ }^{231} \mathrm{~Pa}$ to constrain changes in the intensity of the Atlantic meridional overturning circulation (AMOC). Yu et al. (1996) first proposed this application by taking advantage of the fact that $\mathrm{Th}$ is generally more particle reactive than $\mathrm{Pa}$. By assuming that ${ }^{230} \mathrm{Th}$ is primarily removed by sedimentation, whereas a significant fraction of ${ }^{231} \mathrm{~Pa}$ is exported laterally by the circulation to the Southern Ocean, Yu et al. (1996) suggested that periods of stronger AMOC would result in lower ${ }^{231} \mathrm{~Pa} /{ }^{230} \mathrm{Th}$ ratios in bulk sediments, and that periods of weaker AMOC would result in higher ${ }^{231} \mathrm{~Pa} /{ }^{230} \mathrm{Th}$ (note that these authors more precisely invoked the Atlantic thermohaline circulation, which is now generally referred to as the AMOC). Based on ${ }^{231} \mathrm{~Pa} /{ }^{230} \mathrm{Th}$ ratios from sediment cores throughout the Atlantic Ocean, Yu et al. (1996) concluded that there was no significant change in the strength of the AMOC between the Last Glacial Maximum (LGM) and the Holocene, the present interglacial period. Subsequent studies extended the approach to draw inferences about AMOC 
changes from ${ }^{231} \mathrm{~Pa} /{ }^{230} \mathrm{Th}$ changes observed along a single core. For instance, from a core sampled at Bermuda Rise, McManus et al. (2004) found that the sediment ${ }^{231} \mathrm{~Pa} /{ }^{230} \mathrm{Th}$ ratio approached the production rate of the two radionuclides in the water column during Heinrich Stadial I of the last deglacial, which was interpreted as an almost complete shutdown of AMOC. More recently, an analysis of cores throughout the Atlantic revealed that ${ }^{231} \mathrm{~Pa} /{ }^{230} \mathrm{Th}$ values for Heinrich Stadial I were generally larger than those from the LGM and the Holocene, though not large enough to cause a complete shutdown in overturning (Bradtmiller et al., 2014).

Importantly, Burke et al. (2011) showed that when uncertainties in the data, scavenging, and circulation are formally considered, the bulk of the Atlantic ${ }^{231} \mathrm{~Pa} /{ }^{230} \mathrm{Th}$ data for from Heinrich Stadial I, the LGM, and the Holocene are all compatible with the modern ocean circulation. Spatial variations in scavenging intensity confound the interpretation of ${ }^{231} \mathrm{~Pa} /{ }^{230} \mathrm{Th}$ records from deepsea sediments (Hayes et al., 2015a). In the face of such uncertainties, it is still unclear whether ${ }^{231} \mathrm{~Pa} /{ }^{230} \mathrm{Th}$ can provide a rigorous constraint on the strength of the Atlantic's meridional circulation in the geologic past.

\subsection{Adsorption mechanisms}

Our ability to use thorium isotopes to make inferences about oceanic processes today and in the past depends heavily on our understanding of how thorium attaches to the surfaces of marine particles. Balistrieri et al. (1981) proposed one of the first surface chemistry models for the adsorption of metal ions onto marine particulate matter. They constrained apparent stability constants for a wide variety of metal-particle surface interactions (including $\mathrm{Th}$ ) from particulate material collected in sediment traps deployed in the subtropical North Atlantic (Parflux E site, $13^{\circ} \mathrm{N}, 54^{\circ} \mathrm{W}$ ). They discussed two types of sorption models. A first model considers the reaction between a hydrolyzed metal ion and the surface of a metal oxide:

$$
\begin{aligned}
& \mathrm{Me}^{\mathrm{z}+}+\mathrm{H}_{2} \mathrm{O} \longleftrightarrow \mathrm{MeOH}^{(\mathrm{z}-1)+}+\mathrm{H}^{+}, \\
& \mathrm{SOH}+\mathrm{MeOH}^{(\mathrm{z}-1)+}+\mathrm{H}^{+} \longleftrightarrow \mathrm{SOMeOH}^{(\mathrm{z}-2)+}+2 \mathrm{H}^{+}
\end{aligned}
$$

where $\mathrm{SOH}$ represents the functional group $\mathrm{OH}$ associated with the surface of a metal oxide, $\mathrm{Me}^{\mathrm{z}+}$ a metal ion in solution, and $\mathrm{SOMeOH}^{(\mathrm{z}-2)+}$ a hydrolysis complex. The second model considers the reaction between a free-metal ion and an organically-coated surface: 


$$
\mathrm{SOH}+\mathrm{Me}^{\mathrm{z}+} \longleftrightarrow \mathrm{SOMe}^{(\mathrm{z}-1)+}+\mathrm{H}^{+},
$$

where $\mathrm{SOMe}^{(\mathrm{z}-1)+}$ represents the complex formed by the association of the free metal ion with the organically coated particle surface.

Balistrieri et al. (1981) fit these two models of metal ion-particle surface reactions to sediment trap data, assuming in both cases competition between inorganic ligands $\left(\mathrm{OH}^{-}, \mathrm{Cl}^{-}, \mathrm{SO}_{4}{ }^{2-}\right.$, and $\left.\mathrm{CO}_{3}{ }^{2-}\right)$ and particle surfaces. They found that the stability constants for the reaction describing the adsorption of trace metals onto organically coated particles (1.3) were orders of magnitude larger than those describing the adsorption of these metals onto metal oxides (1.1-1.2). They also found that the stability constants for metal ion-organic coating reactions fell within the range of values found for organic substances in laboratory studies from Sillen et al. (1964), Wilson and Kinney (1977), Mantoura et al. (1978), and Huizenga and Kester (1979), while their estimated stability constants for metal ion-metal oxide reactions consistently exceeded constants for similar systems obtained in laboratory experiments from Schindler (1975) and Davis et al. (1978a,b).

Formally, the derivation of the stability constants estimated by Balistrieri et al. (1981) do not consider the effect of particle surface charge. Hunter et al. (1988) considered a triple layer model (Yates et al., 1974; Davis et al., 1978a,b) for the behavior of thorium near the solid-solution interface. In this model, a metal is envisioned as having to go through two outer layers; a diffuse outer layer of non-specifically adsorbed ions and a compact layer of specifically adsorbed ions, where specificallly adsorbed ions are ions adsorbed in excess of what is expected based on simple coulombic considerations. Both types of ions have their own electrical potential and surface charge, before arriving at an inner layer of surface hydroxyl groups which can undergo proton exchange with the metal. Applying this model to synthetic goethite and $\delta-\mathrm{MnO}_{2}$, Hunter et al. (1988) found that at the $\mathrm{pH}$ of seawater, thorium adsorbed to synthetic goethite was mostly in the form of $\mathrm{Th}(\mathrm{OH})_{2}{ }^{2+}$, $\mathrm{Th}(\mathrm{OH})_{3}{ }^{+}$, and $\mathrm{Th}(\mathrm{OH})_{4}$, while thorium adsorbed to $\delta-\mathrm{MnO}_{2}$ was mostly in the form of $\mathrm{Th}(\mathrm{OH})_{4}$. This study thus highlighted the importance of considering the hydrolyzed species of thorium in estimating the equilibrium constants associated with thorium adsorption. Moreover, Hunter et al. (1988) found that the thorium adsorbed was largely unaffected when including in the solution the major cations and anions of seawater. In contrast, when including organic ligands (EDTA 
and CDTA) in their thorium $\delta-\mathrm{MnO}_{2}$ adsorption experiments, they found that the proportion of adsorbed thorium shifted from $\geq 80 \%$ to under $15 \%$. This dramatic shift further highlights the potential importance of the interaction between thorium and organic molecules, whether they be ligands or coatings on marine particles, in studies on the solid-solution partitioning of this element.

When applying their derived equilibrium constants to $\mathrm{MnO}_{2}$ at concentrations more typical of the deep North Atlantic $\left(\sim 9 \mu \mathrm{g} / \mathrm{m}^{3}\right)$, Hunter et al. (1988) predicted the particulate/dissolved Th ratio to be $10^{-5} \mathrm{~g} / \mathrm{g}$, which is three orders of magnitude smaller than the ratio found for samples collected in the Panama and Guatemala Basins (Bacon and Anderson, 1982). While the apparent lack of importance of $\mathrm{MnO}_{2}$ was partly due to the low concentration of this particulate phase in the deep ocean, Hunter et al. (1988) also speculated that the large negative charge on the surface of Mn (oxyhydr)oxide created an electrostatic attraction for hydrogen ions in the inner layer that was greater than the attraction for thorium in the compact outer layer, thereby inhibiting the ability of thorium to attach to the particle surface at the inner layer. They further speculated that iron (oxyhydr)oxides, having a circumneutral charge at seawater $\mathrm{pH}$, might be superior scavengers of thorium in the deep sea.

The aforementioned studies only considered the equilibrium sorption of thorium onto particle surfaces, and not the kinetics of sorption reactions. Nyffeler et al. (1984) constructed a model for the kinetics of metal adsorption and desorption (eqs. 1.2-1.3):

$$
d[M e] / d t=-k_{1, c}[M e][S O H]-+k_{-1}[S O M e]
$$

Here, $k_{1, c}$ is a second-order rate constant for the adsorption of the metal onto particles per unit particle concentration $\left(\mathrm{m}^{3} \mathrm{mg}^{-1} \mathrm{yr}^{-1}\right), k_{-1}$ is a first-order rate constant for the desorption of the metal from particles, and $[M e]$ is the concentration of the metal in solution. From particulate ${ }^{228} \mathrm{Th}$ collected by sediment traps deployed in the northwest Atlantic, Nyffeler et al. (1984) found $k_{1, c}$ to be $0.07 \mathrm{~m}^{3} \mathrm{mg}^{-1} \mathrm{yr}^{-1}$, which yielded values of the apparent first-order rate constant, $k_{1, c}[S O H]$, similar to those found by Bacon and Anderson (1982) when using a particle concentration of $10 \mathrm{mg}$ $\mathrm{m}^{-3}$.

Notably, the kinetic model by Nyffeler et al. (1984) implies that $k_{1}$ increases linearly with the number of surfaces sites available for adsorption. Using a variety of estuarine and coastal observa- 
tions of ${ }^{228,230,234} \mathrm{Th}$, Honeyman et al. (1988) found that $k_{1}$ varies with bulk particle concentration, $P$, as $k_{1}=k_{1, c} P^{b}$, where $k_{1, c}$ is a constant and $b<1$. To explain this power law, they hypothesized that coagulation of colloids was the rate limiting step for the transfer of particles from the nonfilterable to filterable particle pools. Honeyman and Santschi (1989) later constructed a Brownian pumping model which showed that $k_{1}$ can be less than expected from a linear relationship with $P$ if colloidal coagulation is considered as the rate limiting step. However, the influence of colloids appears to be widely varying in the ocean. For example, Baskaran et al. (1992) found a strong correlation between $k_{1}$ for ${ }^{234} \mathrm{Th}$ and the fraction of ${ }^{234} \mathrm{Th}$ in the colloidal material sampled from the Gulf of Mexico, with $k_{1}$ exceeding $18 \mathrm{yr}^{-1}$ when the fraction of total ${ }^{234} \mathrm{Th}$ in the colloidal pool exceeded 20\%, while Hayes et al. (2017) found that colloids at 8 stations south of Bermuda represent only $2-6 \%$ of ${ }^{230} \mathrm{Th}$ present in "dissolved" form. Such a discrepancy may reflect a differing influence of colloids between oligotrophic, open-ocean environments and biologically more productive, coastal environments. Nevertheless, the influence of colloids on thorium scavenging, and on the removal of particle-reactive elements more generally, remains an outstanding and open question.

\subsection{Effects of Circulation}

Aside from variations in scavenging intensity, the distribution of thorium radioisotopes in the ocean may be influenced by ocean circulation. For example, Moran et al. (1997) found that in order to explain the general deficit in ${ }^{230} \mathrm{Th}_{d}$ (relative to values expected from reversible exchange) observed in deep waters throughout the western North Atlantic, including at the Bermuda Atlantic Time-Series (BATS) site, the Nares Abyssal Plain, and the Hatteras Abyssal Plain, the influence of North Atlantic Deep Water (specifically, deep waters from the Norwegian and Labrador Seas, containing relatively low activities of ${ }^{230} \mathrm{Th}$ ) had to be considered. Similarly, below 1000-m water depth, a scavenging-mixing model was found to better reproduce ${ }^{230} \mathrm{Th}_{t o t}$ (dissolved + particulate) data at two stations $\left(47.4^{\circ} \mathrm{N}, 19.5^{\circ} \mathrm{W}\right.$, and $\left.54.4^{\circ} \mathrm{N}, 21.1^{\circ} \mathrm{W}\right)$ in the eastern North Atlantic compared to a model that only considered reversible exchange (Vogler et al., 1998). From a compilation of ${ }^{230} \mathrm{Th}$ measurements in the North Atlantic, Marchal et al. (2007) found that the activities of ${ }^{230} \mathrm{Th}_{t o t}$ at different locations in the top $1000 \mathrm{~m}$ tended to exhibit a similar linear increase with depth, but below $1000 \mathrm{~m}^{230} \mathrm{Th}_{t o t}$ showed large geographic variations. These authors also considered two fits of a model describing the circulation of the North Atlantic: a fit to density observations and volume 
transport estimates, and a fit to the same dataset in combination with ${ }^{230} \mathrm{Th}_{t o t}$. They found that the second fit resulted in an increase in the estimated meridional transport, suggesting that regional variations in ${ }^{230} \mathrm{Th}_{t o t}$ contain information about deep water circulation in the North Atlantic.

\subsection{New Insights from GEOTRACES}

In recent years, an unprecedented collection of trace element and isotope (TEI) data from a variety of ocean basins, including the North Atlantic, has been gathered under the GEOTRACES project. The overarching goal of GEOTRACES is "to identify processes and quantify fluxes that control the distribution of key trace elements and isotopes in the ocean, and to establish the sensitivity of these distribution to changing environmental conditions" (GEOTRACES Planning Group, 2006). To this end, the GEOTRACES scientific community has participated in a number of trans-oceanic cruises, as well as regional process studies. Thorium isotopes (particularly ${ }^{230} \mathrm{Th}$ ) are listed among the key parameters to be collected during GEOTRACES campaigns, and the recently completed U.S. GEOTRACES North Atlantic section (section GA03) has vastly increased the horizontal and vertical coverage of available measurements of these radionuclides in this region.

Thorium radioisotope data collected along GA03 have already provided important insights into the cycling of trace metals in the ocean. For example, Hayes et al. (2015b) found that deviations from reversible exchange theory are prevalent, and suggested that a combination of deep water ventilation and scavenging intensity must be invoked to explain ${ }^{230} \mathrm{Th}_{d}$ data along the section. In a parallel study, Hayes et al. (2015a) also found that the partitioning of ${ }^{230} \mathrm{Th}$ between particulate and dissolved forms shows geographic variations in along the section. Using multiple linear regression, they showed that these variations can be explained, at least partly, by variations in particle composition. Throughout GA03, Owens et al. (2015) found deficits and excesses in ${ }^{234} \mathrm{Th}$ below $1000 \mathrm{~m}$ from activities expected from secular equilibrium with ${ }^{238} \mathrm{U}$. Such deviations may be expected in the upper water column where particles are more abundant and particle scavenging and remineralization are rapid (Maiti et al., 2012), but are more surprising at mid-depths, away from regions of intense particle production, export, and degradation, where secular equilibrium is expected to be re-established. 


\subsection{Inverse Methods}

It may be challenging to understand the variability in thorium adsorption in the ocean using kinetic and equilibrium models, each of which make different assumptions about how $k_{1}$ depends on particle concentration and composition. An alternative approach, taken by Bacon and Anderson (1982), is to estimate apparent rates of thorium adsorption onto and release from particles by combining in situ data with equations describing the mass balance for thorium isotopes in dissolved and particulate forms in the water column:

$$
\begin{aligned}
0 & =\lambda A_{\pi}+k_{-1}^{*} A_{p}-\left(k_{1}+\lambda\right) A_{d}, \\
w \frac{\partial A_{p}}{\partial z} & =k_{1} A_{d}-\left(k_{-1}^{*}+\lambda\right) A_{p} .
\end{aligned}
$$

Here $A_{d}\left(A_{p}\right)$ is the thorium isotope activity in dissolved (filterable particulate) form $\left(\mathrm{dpm} \mathrm{m}^{-3}\right)$, $A_{\pi}$ is the activity of the radioactive parent $\left(\mathrm{dpm} \mathrm{m}^{-3}\right)$, and $\lambda$ is the radioactive decay constant $\left(\mathrm{yr}^{-1}\right)$. The (apparent) rate parameters of the model are the adsorption rate constant $\left(k_{1}, \mathrm{yr}^{-1}\right)$, the combine rate constant for thorium desorption and particle degradation $\left(k_{-1}^{*}, \mathrm{yr}^{-1}\right)$, and the particle sinking speed $\left(w, \mathrm{~m} \mathrm{yr}^{-1}\right)$. Clearly, $z$ is depth (m). Bacon and Anderson (1982) derived estimates of these rate constants by fitting eqs. (1.5a-1.5b) to ${ }^{228,230} \mathrm{Th}_{d, p}$ data from the Panama and Guatemala Basins. However, this and other early attempts using ordinary least squares to estimate apparent rates of thorium and particle cycling in-situ did not account neither for the uncertainties in the data nor for the uncertainties in the model equations.

Aside from ordinary least squares, other inverse methods can formally consider uncertainties in the derivation of model parameters. Wunsch (1978) pioneered the use of inverse methods for estimating geostrophic velocities across trans-oceanic sections, which involved weighting the thermal wind equations by a covariance matrix containing information about the error statistics. While multiple studies have used this approach to estimate the fluxes of mass, heat, and salt across transoceanic sections (e.g., Roemmich and Wunsch (1985); Rintoul and Wunsch (1991); Macdonald and Wunsch (1996); Ganachaud and Wunsch (2002); Lumpkin and Speer (2003, 2007)), these studies formally treat the density field used to constrain the velocities as perfectly known (specifically, density errors are considered in the thermal wind relations but not in the density conservation equations). Departing from prior work, Mercier (1989) proposed to treat the density field as another unknown of the inverse problem, which resulted in the constraint equations being non-linear in the 
unknowns (velocities and densities). He applied a generalized nonlinear least squares technique (the Algorithm of Total Inversion or ATI), developed by Tarantola and Valette (1982), to combined constraint equations with hydrographic and current meter data in order to estimate the velocity field in the northwest Atlantic.

Nonlinear inverse methods have also been used by R. Murnane and colleagues to constrain the oceanic cycling of thorium, including rate constants for thorium adsorption $\left(k_{1}\right)$, thorium desorption $\left(k_{-1}\right)$, particle degradation $\left(\beta_{-1}\right)$, particle sinking speed $(w)$, particle aggregation, and particle disaggregation (Murnane et al., 1990; Murnane, 1994a; Murnane et al., 1994b, 1996). Murnane (1994a) applied different inverse procedures to estimate rate constants of Th and particle cycling from Th and particle data from Station Papa in the Gulf of Alaska: the ATI, ordinary least squares, and a regression procedure by Wolberg (1967). He found that the solution obtained from the ATI was both more realistic and consistent with prior estimates of the rate constants and with station $\mathrm{P}$ data than solutions obtained from the other techniques. He also emphasized an important advantage of the ATI: the ability of this method to include prior information about the value and uncertainty of the variables being sought. More recently, Marchal and Lam (2012) inferred rate parameters using the ATI from simulated Th and particle data in two size classes. Their study revealed that measurements of particle and ${ }^{228,230,234} \mathrm{Th}$ concentrations in different size fractions, such as those generated from the GEOTRACES campaigns, can provide important constraints on the kinetics of sorption reactions and particle processes in the ocean.

\subsection{Objective of the Thesis}

The overarching objective of this thesis is to examine the effect of particle concentration, particle composition, and ocean circulation on the cycling of thorium isotopes in the North Atlantic. To this end, the unprecedented dataset collected along the North Atlantic section GA03 is analyzed, using both inverse methods and an ocean circulation model. For each investigation reported in this study, thorium is assumed to exchange reversibly with settling particles (Bacon and Anderson, 1982). The remainder of this thesis is divided into four chapters and a Conclusion.

In chapter 2, I apply a nonlinear inverse method (the ATI) to analyze radionuclide and particle data at an oligotrophic station along section GA03. The inverse method is used to test two different models of Th and particle cycling: a model that considers vertically uniform rate parameters, and 
a model in which vertical variations in the rate parameters are permitted. In both models, the rate parameters are the rate constants for adsorption of thorium onto particles $\left(k_{1}\right)$, desorption of thorium from particles $\left(k_{-1}\right)$, particle degradation $\left(\beta_{-1}\right)$, as well as the particle sinking speed $(w)$. In the second model, no explicit formulation for the depth dependence of the rate parameters is imposed. For both models, the inversion finds estimates of rate parameters that are most consistent with the data and the model equations, given the error covariance(s) in the data. Moreover, error estimates for the rate parameters derived by inversion are provided.

In chapter 3, I apply a nonlinear inverse technique to constrain the vertical distribution of the same rate parameters at 11 stations occupied along GA03. Although the technique differs from that used in Chapter 2, it is shown that the conclusions drawn in this chapter are independent of the inverse technique used. Evidence is provided to show that the budgets of ${ }^{228,230,234} \mathrm{Th}$ and bulk stations at the stations considered are dominated by sorption reactions, particle degradation, and particle sinking, rather than by advection and mixing. Moreover, the relationship between $k_{1}$ and particle concentration is documented, and compared to relationships found by previous studies. The question of whether the $k_{1}$ values found in this study are consistent with a general particle concentration effect due to colloidal coagulation is addressed. Notice that, this study analyzes jointly multiple thorium isotopes $\left({ }^{228,230,234} \mathrm{Th}\right)$ in different size fractions, an effort only previously performed at individual stations (Murnane, 1994a; Murnane et al., 1994b). In this manner, this study presents the first estimate of spatial variability in the kinetics of thorium and particle cycling in the North Atlantic as constrained by in-situ data of multiple thorium isotopes, their parents, and particle concentrations.

In chapter 4 , I investigate the relationship between the Th adsorption rate constants estimated by inversion and the particle composition measured along the GA03 section. Here particle composition means the sum of the biogenic particle phases (particulate organic carbon + particulate inorganic carbon + biogenic silica), lithogenic material, Mn (oxyhydr)oxides, and Fe (oxyhydr)oxides. A principal component analysis is conducted to document the dominant modes of variance in the particle composition dataset. Then, our $k_{1}$ estimates derived by inversion are regressed against the particle composition data under two models: an additive model in which $k_{1}$ is considered as a weighted sum of the particle phases (with an additive error), and a multiplicative model in which $k_{1}$ is considered as a product of the particle phases (with a multiplicative error). A relative importance 
analysis is used to determine which phases most contribute to the explained variance in $k_{1}$ according to each model, and the implications of our results for the kinetics of thorium adsorption and use of ${ }^{230} \mathrm{Th}$ in paleoceanography are discussed.

In chapter 5 , the combined effects of ocean circulation and scavenging on ${ }^{230} \mathrm{Th}$ in the Northwest Atlantic are investigated by embedding a description of the reversible exchange into the Princeton Ocean Model (POM), a regional circulation model based on the primitive equations. The simulated dissolved and particulate ${ }^{230} \mathrm{Th}$ activities are compared to a compilation of radionuclide data from the pre-GEOTRACES era and the western segment of GA03. Sensitivities of the simulated radionuclide activities to the strength of the DWBC, the strength of the Gulf Stream, ${ }^{230} \mathrm{Th}$ activities at open boundaries, and spatial variations in the Th adsorption rate constant are documented. In this manner, this chapter addresses the question of whether elements of the general circulation or particle scavenging exert a strong control on the distributions of ${ }^{230} \mathrm{Th}_{d, p}$ in the Northwest Atlantic.

Finally, in the Conclusion section, I summarize the insights gathered during the thesis about the effects of particle concentration, particle composition, and ocean circulation on the distribution of thorium radioisotopes in the North Atlantic. The section then culminates with a number of ideas for further research. 


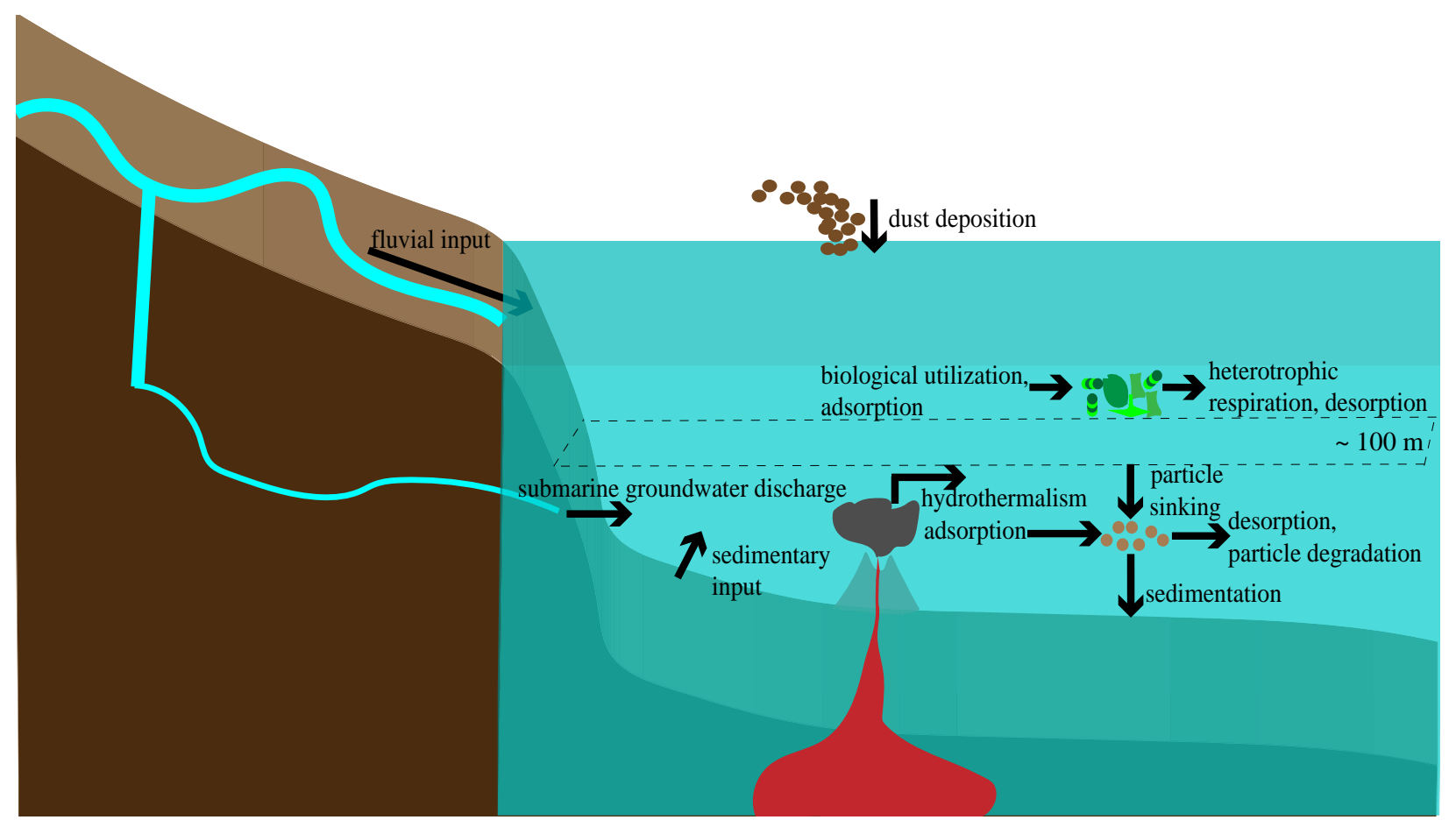

Figure 1: Simplified schematic for trace metal cycling in the ocean. The dashed lines are the outline of a plane separating the surface and deep ocean. The vertical scale is nonlinear, so that the ratio of the surface ocean to deep ocean width is $\sim 1 / 50$ Oceanic sources for trace metals include dust deposition, fluvial discharge, submarine groundwater discharge, sedimentary input, and hydrothermal fluxes. Deposition is the main oceanic sink for many trace metals although some metals may be removed at hydrothermal vents. The internal cycling of trace metals in the open ocean is represented here by a multitude of processes, including biological utilization and adsorption which transfer thorium from the dissolved to particulate pools, and trace metal desorption, heterotrophic respiration, and particle degradation which transfer trace metals back to the dissolved pool. 


\section{Chapter 2}

\section{Testing models of thorium and particle}

cycling in the ocean using data from

station GT11-22 of the U.S.

\section{GEOTRACES North Atlantic Section}

This article was originally published in Deep-Sea Research Part I in 2017 by Elsevier, and has been slightly modified here. Elsevier permits the reproduction of this content in a dissertation/thesis.

Lerner, P., Marchal, O., Lam, P.J., Anderson, R.F., Buesseler, K., Charette, M.A., Edwards, R.L., Hayes, C.T., Huang, K., Lu, Y., Robinson, L.F., and Solow, A., 2016, "Testing models of thorium and particle cycling in the ocean using data from station GT11-22 of the U.S. GEOTRACES North Atlantic section," Deep-Sea Research Part I, 113, 57-79, doi: 10.1016/j.dsr.2016.03.008 


\begin{abstract}
Thorium is a highly particle-reactive element that possesses different measurable radio-isotopes in seawater, with well-constrained production rates and very distinct half-lives. As a result, Th has emerged as a key tracer for the cycling of marine particles and of their chemical constituents, including particulate organic carbon.

Here two different versions of a model of Th and particle cycling in the ocean are tested using an unprecedented data set from station GT11-22 of the U.S. GEOTRACES North Atlantic Section: (i) ${ }^{228,230,234} \mathrm{Th}$ activities of dissolved and particulate fractions, (ii) ${ }^{228} \mathrm{Ra}$ activities, (iii) ${ }^{234,238} \mathrm{U}$ activities estimated from salinity data and an assumed ${ }^{234} \mathrm{U} /{ }^{238} \mathrm{U}$ ratio, and (iv) particle concentrations, below a depth of $125 \mathrm{~m}$. The two model versions assume a single class of particles but rely on different assumptions about the rate parameters for sorption reactions and particle processes: a first version (V1) assumes vertically uniform parameters (a popular description), whereas the second (V2) does not. Both versions are tested by fitting to the GT11-22 data using generalized nonlinear least squares and by analyzing residuals normalized to the data errors.

We find that model V2 displays a significantly better fit to the data than model V1. Thus, the mere allowance of vertical variations in the rate parameters can lead to a significantly better fit to the data, without the need to modify the structure or add any new processes to the model. To understand how the better fit is achieved we consider two parameters, $K=k_{1} /\left(k_{-1}+\beta_{-1}\right)$ and $K / P$, where $k_{1}$ is the adsorption rate constant, $k_{-1}$ the desorption rate constant, $\beta_{-1}$ the remineralization rate constant, and $P$ the particle concentration. We find that the rate constant ratio $K$ is large $(\geq 0.2)$ in the upper $1000 \mathrm{~m}$ and decreases to a nearly uniform value of ca. 0.12 below $2000 \mathrm{~m}$, implying that the specific rate at which Th attaches to particles relative to that at which it is released from particles is higher in the upper ocean than in the deep ocean. In contrast, $K / P$ increases with depth below $500 \mathrm{~m}$. The parameters $K$ and $K / P$ display significant positive and negative monotonic relationship with $P$, respectively, which is collectively consistent with a particle concentration effect.
\end{abstract}

\title{
$2.1 \quad$ Introduction
}

Roughly $20-25 \%$ of carbon fixed photosynthetically by phytoplankton in near surface-waters is estimated to sink as particles to depths below $100 \mathrm{~m}$, with approximately $10 \%$ of this sinking material reaching the sediments (Bishop, 2009). The sinking and subsequent remineralization of particulate organic matter strongly influence the vertical concentration gradients of chemical constituents in the ocean, including dissolved inorganic carbon, nutrients, and dissolved oxygen. Therefore, understanding the processes that control the cycling of particles and the exchange of elements between the dissolved and particulate phases is essential in order to understand the distribution of these constituents in the ocean.

The processes that impact marine particles include, e.g., (dis)aggregation, remineralization, dissolution, and gravitational sinking. The radioactive isotopes of thorium have for a long time been used to study these processes (for reviews see Savoye et al. (2006); Lam and Marchal (2014)). Thorium is highly particle reactive in seawater, and its isotopes are characterized by widely different 
half-lives: $t_{1 / 2}=24.101 \pm 0.025$ days for ${ }^{234} \mathrm{Th}$ (Knight and Macklin, 1948), 75, 584 $\pm 110 \mathrm{yr}$ for ${ }^{230} \mathrm{Th}$ (Cheng et al., 2013), and $1.910 \pm 0.002 \mathrm{yr}$ for ${ }^{228} \mathrm{Th}$ (Kirby et al., 2002). Additionally, the sources of these thorium isotopes in the ocean are relatively well understood. ${ }^{234} \mathrm{Th},{ }^{230} \mathrm{Th}$, and ${ }^{228} \mathrm{Th}$ are produced in situ by radioactive decay of ${ }^{238} \mathrm{U},{ }^{234} \mathrm{U}$, and ${ }^{228} \mathrm{Ra}$, respectively. Since uranium seems to behave quasi-conservatively in the ocean (Ku et al., 1977; Delanghe et al., 2002), the ${ }^{234} \mathrm{U}$ and ${ }^{238} \mathrm{U}$ activities are often estimated from salinity (Chen et al., 1986; Owens et al., 2011), whereas the ${ }^{228} \mathrm{Ra}$ activity is generally measured directly (Henderson et al., 2013). Another potential source of ${ }^{230} \mathrm{Th}$ is the dissolution of lithogenic materials, although this contribution appears negligible except in surface waters close to mineral dust sources (Hayes et al., 2013). The high particle reactivity of Th combined with multiple isotopes that have a wide range of half-lives makes it particularly well suited to study the variety of processes that affect particles. For example, ${ }^{230} \mathrm{Th}$ has found several applications in paleo-oceanography. These include using ${ }^{230} \mathrm{Th}$ to correct for sediment lateral redistribution (e.g., François et al. (2004)) and (in concert with ${ }^{231} \mathrm{~Pa}$ ) to estimate biological productivity (e.g., Kumar et al. $(1993,1995))$ and aspects of ocean circulation (e.g., Yu et al. (1996)).

The concept of scavenging, i.e., the attachment of trace metals to sinking particles and their subsequent removal to the sea floor, was proposed by Goldberg (1954). Subsequently, there has been widespread recognition of the importance of scavenging in controlling the distribution of trace metals in the ocean (Krauskopf, 1956; Turekian, 1977). Bhat et al. (1969) concluded from ${ }^{234} \mathrm{Th}$ data obtained from the Arabian Sea, Java sea, Australian coast, Wharton Sea, and the Tasmania coast, that the distribution of ${ }^{234} \mathrm{Th}$ is controlled by adsorption of thorium onto particles. They considered an irreversible scavenging model for particulate ${ }^{234} \mathrm{Th}$, which explained the deficit of ${ }^{234} \mathrm{Th}$ relative to ${ }^{238} \mathrm{U}$ in the surface mixed layer. Their model, however, was based on the assumption that all ${ }^{234} \mathrm{Th}$ is adsorbed onto particles. Krishnaswami et al. (1976), using ${ }^{234}$ Th data from the Pacific GEOSECS expedition, estimated particulate ${ }^{234} \mathrm{Th}$ to be only $10-20 \%$ of the total activity of ${ }^{234} \mathrm{Th}$ (dissolved and particulate). They also found ${ }^{230} \mathrm{Th}$ in the particulate phase to increase approximately linearly with depth in the water column. Based on their observations, they proposed a one-dimensional (vertical) scavenging model for particulate thorium, similar to that of Bhat et al. (1969) but with an added scavenging term to account for the existence of both dissolved and particulate phases.

Nozaki et al. (1981), using data from the western North Pacific, and Bacon and Anderson (1982), using data from the Panama and Guatemala Basins, observed that the activities of ${ }^{230} \mathrm{Th}$ in 
both dissolved and particulate forms increase generally with depth. This observation necessitated a revision of the scavenging model and prompted the authors to develop a reversible exchange model to account for the observed vertical distributions of dissolved and particulate ${ }^{230} \mathrm{Th}$. The innovation in this model was a term for the loss of thorium from the particles (one particle class) through desorption and (or) remineralization. Since then, the reversible exchange model has become a popular description of thorium isotope cycling in the oceanic water column, and many studies used this model in combination with a "ventilation" term in the interpretation of ${ }^{230} \mathrm{Th}$ and ${ }^{231} \mathrm{~Pa}$ data (e.g., Rutgers van der Loeff and Berger (1993); Scholten et al. (1995); Vogler et al. (1998); Moran et al. (2002); Scholten et al. (2008); Hayes et al. (2015a)).

Nevertheless, there has been extensive modifications to the reversible exchange model with one particle class. Clegg and Whitfield (1990) modeled thorium and particles in both small and large size classes. Included in their model are terms for the aggregation of small particles and disaggregation of large particles. More recently, Burd et al. (2000) presented a "coupled adsorption-aggregation" model, in which a particle size spectrum (particle size ranging from less than $10^{-2}$ to $53 \mu \mathrm{m}$ ) is represented in order to interpret field observations of the particulate organic carbon (POC) to ${ }^{234} \mathrm{Th}$ ratio.

Observational estimates of the rate constants of thorium and particle cycling in the ocean display large variations. They range from $0.1-1 \mathrm{yr}^{-1}$ for adsorption and 1-10 $\mathrm{yr}^{-1}$ for desorption (Nozaki et al., 1987; Bacon and Anderson, 1982; Murnane et al., 1990; Murnane, 1994a; Murnane et al., 1994b), 1-100 $\mathrm{yr}^{-1}$ for remineralization (Clegg et al., 1991b), 0.1-100 $\mathrm{yr}^{-1}$ for aggregation, and 1-2500 $\mathrm{yr}^{-1}$ for disaggregation (Nozaki et al., 1987; Murnane et al., 1990; Cochran et al., 1993; Murnane et al., 1996; Cochran et al., 2000). Likewise, estimates of the sinking speed of bulk particles, including all sizes, vary widely, from 300-1100 $\mathrm{m} \mathrm{yr}^{-1}$ (Krishnaswami et al., 1976, 1981; Rutgers van der Loeff and Berger, 1993; Scholten et al., 1995; Venchiarutti et al., 2008). For the average sinking speed of particles greater than $45 \mu \mathrm{m}$ in diameter, McDonnell and Buesseler (2010) found values from 10 to $150 \mathrm{~m} \mathrm{~d}^{-1}$ in waters near the west Antarctic Peninsula. Turner (2002) reported an even larger range, from less than tens to over thousands $\mathrm{m} \mathrm{d}^{-1}$, for the sinking velocity of fecal pellets. These large variations are a current impediment to any attempt to develop large-scale models of particle and biogeochemical processes in the ocean.

In a series of studies, R. Murnane and colleagues pioneered the use of inverse methods in order 
to estimate rate constants of particle and thorium cycling in the ocean (Murnane et al., 1990; Murnane, 1994a; Murnane et al., 1994b, 1996). Using a generalized nonlinear least squares technique (Algorithm of Total Inversion or ATI) (Tarantola and Valette, 1982), Murnane (1994a) performed an inversion of $\mathrm{Th}$ and particle data from Station $\mathrm{P}\left(50^{\circ} \mathrm{N}, 145^{\circ} \mathrm{W}\right)$ in the Gulf of Alaska. He compared solutions obtained from the ATI with two other regression techniques: ordinary least squares and a regression procedures by Wolberg (1967). He found that the solution obtained from the ATI was both more realistic and consistent with prior estimates of the rate constants and with data from station $\mathrm{P}$ than solutions obtained from the other techniques.

The adequacy of the ATI to infer rate constants of sorption reactions, however, was questioned by Athias et al. (2000a) and Athias et al. (2000b). These authors reported that a least squares approach could not recover rate parameters of a model of $\mathrm{Al}$ (another relatively insoluble trace metal) cycling from a simulated data set generated by the same model. They concluded that the generalized least squares approach of Tarantola and Valette (1982) could not be applied to their problem. In contrast, Marchal and Lam (2012) succeeded in inferring rate parameters using the ATI from simulated Th and particle data. Their study suggests that field observations could be used to constrain rate parameters of Th and particle processes in the ocean. Furthermore, they concluded that measurements of particle and ${ }^{228,230,234} \mathrm{Th}$ concentrations in different size fractions, such as generated during GEOTRACES, should significantly improve the precision of the rate parameters inferred relative to a priori estimates.

The differences between the results of Athias et al. (2000b) and Marchal and Lam (2012) were discussed by Marchal and Lam (2012). These authors found that relatively large prior errors in the rate parameters in combination with the constraint that the model equations be imposed exactly can prevent the ATI from converging to a stable solution. Thus differences in assumptions about the prior errors between these two studies may have led to different results regarding the adequacy of the ATI to infer rate parameters. Besides Murnane (1994a), Murnane et al. (1994b), Murnane et al. (1996), and Marchal and Lam (2012), other studies have successfully applied the ATI to oceanographic problems (e.g., Mercier (1986), Mercier (1989), Mercier et al. (1993), Paillet and Mercier (1997), Marchal et al. (2007)).

Here we rejuvenate the approach first applied by Murnane (1994a) to constrain aspects of thorium and particle cycling from the extensive data set collected at station GT11-22 of the U.S. 
GEOTRACES North Atlantic Section (GA03) (Boyle et al., 2015). Station GT11-22 was chosen because it is an open ocean station that appears to have relatively little influence from hydrothermal vents near the Mid-Atlantic Ridge and from the Mauritanian Upwelling, both of which may be regions that exhibit enhanced scavenging due to processes not encapsulated by the reversible exchange model (e.g., Hayes et al. (2015a); Lam et al. (2015a)). The GT11-22 data include ${ }^{234,230,228}$ Th activities in dissolved $(<0.8 \mu \mathrm{m})$ and particulate $(0.8-51 \mu \mathrm{m})$ phases, particle concentration in the small $(<51 \mu \mathrm{m})$ and large $(>51 \mu \mathrm{m})$ size fractions, measurements of dissolved ${ }^{228} \mathrm{Ra}$, and ${ }^{234,238} \mathrm{U}$ activities estimated from salinity data. We feel that there is as of yet insufficient data to constrain a model that deals with Th and particles in both small and large particle fractions. Thus, we use the data (collected at and below $125 \mathrm{~m}$ ) in order to test two versions of a 1-D (vertical) model of Th and particle cycling that considers one particle class with either vertically uniform (model V1) or nonuniform (model V2) parameters. Specifically, we test whether the fit of model V2 to radiochemical and particle data is significantly better than that of model V1 given the larger number of degrees of freedom of a model with variable parameters. The model is similar to that used by Bacon and Anderson (1982), except that remineralization and desorption are treated separately. The model therefore neglects potentially important effects such as lateral transport. The purpose of this study is to quantify the improvement of the model when the rate parameters are allowed to vary with depth. This way, we provide a test to the commonly held assumption that the rate parameters of Th and particle cycling are uniform along the oceanic water column (e.g., Nozaki et al. (1987); Cochran et al. (1993, 2000); Hayes et al. (2015b).

This paper is organized as follows. In section 2.2, the GT11-22 data, the vertical interpolation, the model of particle and Th cycling, and the inverse method used to combine the data and the model are described. In section 2.3, each version of the model is fitted to the data, considering errors in the data and in their vertical interpolation. A range of measures of goodness of fit are calculated in order to test the consistency of each model version with the data. In section 2.4 , we discuss the robustness of the tests, their implications for oceanic Th geochemistry, and the depth-dependent budgets of ${ }^{228,230,234} \mathrm{Th}$ at station GT11-22. An attempt to interpret particle and Th isotope residuals of the fit in terms of processes missing in the model is also undertaken. Conclusions follow in section 2.5 . 


\subsection{Methods}

\subsubsection{Hydrographic Setting}

We use the data collected aboard the R/V Knorr in November 2011 at station GT11-22 (19 $26^{\prime}$ N, $29^{\circ} 22^{\prime} \mathrm{W}$, water depth of $5014 \mathrm{~m}$ ), approximately $700 \mathrm{~km}$ northwest of Cape Verde (Figure 1). This station is situated in the southeast portion of the North Atlantic subtropical gyre, which is under the influence of the southwestward flow of the Northern Equatorial Current (NEC, Stramma et al. (2005)). The potential temperature $(\theta)$ and salinity $(S)$ measured by the CTD at station GT11-22 (Figure 2) reveal the presence of distinct water masses, defined here according to Jenkins et al. (2015). Between 80 and 554 m, the North Atlantic Central Water (NACW) and Atlantic Equatorial Water (AEW) are carried to station GT11-22 by the NEC (Schmitz and McCartney, 1993; Stramma et al., 2005). In this depth range, NACW represents between $71 \%$ and $98 \%$ of the total mass according to the water property analysis of Jenkins et al. (2015). The "kink" in the $\theta-S$ diagram at about $230 \mathrm{~m}\left(\theta \simeq 15^{\circ} \mathrm{C}, S \simeq 36\right)$ may be due to the replacement of NACW by AEW, decreasing the relative abundance of NACW from about $88 \%$ to $71 \%$ at that depth. Jenkins et al. (2015) estimated that east of $22^{\circ} \mathrm{W}$, nearly all water down to $500 \mathrm{~m}$ is AEW. While station GT11-22 is near $30^{\circ}$ $\mathrm{W}$, it is well within the influence of AEW, which contributes about $30 \%$ of the thermocline waters at this site. Between 609 and $904 \mathrm{~m}$, the Mediterranean Outflow Water (MOW), the Antarctic Intermediate Water (AAIW), and the Irminger Sea Water (ISW) become dominant. The transition between the thermocline (NACW and $\mathrm{AEW}$ ) and intermediate waters is not conspicuous in the $\theta-S$ diagram, largely because the effect of the relatively salty and warm MOW tends to be offset by the fresher and colder AAIW. Between 940 and 1200 m, the Upper Circumpolar Deepwater (UCDW) and the Upper Labrador Sea Water (ULSW) become important. Salinity increases with depth because the relatively fresh AAIW, the dominant component at shallower depths, decreases to about $0 \%$ at $1200 \mathrm{~m}$ (Jenkins et al., 2015). Nearly all water is comprised of ULSW and UCDW between 1200 and $1900 \mathrm{~m}$. The deep water masses below 2000 m include the Classical Labrador Sea Water (CLSW) and two components of North Atlantic Deep Water: the Denmark Straits Overflow Water (DSOW) and the Iceland-Scotland Overflow Water (ISOW). Finally, the lower $2000 \mathrm{~m}$ of the water column at station GT11-22 is bathed by Antarctic Bottom Water (AABW). This water flows northward in the western South Atlantic, crosses the equator, and penetrates into the eastern 
basins of the North Atlantic through the Vema Fracture Zone cutting the Mid-Atlantic Ridge near $11^{\circ} \mathrm{N}$ (Schmitz and McCartney, 1993).

\subsubsection{Data}

The following systems were used to collect the samples. The particulate, dissolved ${ }^{228} \mathrm{Th}$, and dissolved ${ }^{228} \mathrm{Ra}$ data used in this paper were obtained from samples collected by large volume in-situ filtration. The dissolved ${ }^{230} \mathrm{Th}$ and ${ }^{234} \mathrm{Th}$ measurements were gathered using $30-\mathrm{L}$ Niskin bottles. For the collection of ${ }^{234} \mathrm{Th}$ samples below $1000 \mathrm{~m}$, these bottles were attached individually to the pump wire at the depths of the in-situ pumps. For the collection of dissolved ${ }^{230} \mathrm{Th}$ samples at all depths and ${ }^{234} \mathrm{Th}$ samples above $1000 \mathrm{~m}$, these bottles were mounted on the ODF/SIO rosette on a separate cast. All Niskin and in-situ pump casts were operated over the course of the 26hour occupation of the station. These data can be found in the GEOTRACES Intermediate Data Product (The GEOTRACES group, 2015).

\section{Particle concentration}

In this paper, $P$ denotes particle concentration in units of $\mu \mathrm{g} \mathrm{m}^{-3}$. Size fractionated particles were collected by large volume in-situ filtration using a modified dual-flow WTS-LV McLane research pump equipped with 142-mm "mini-MULVFS" filter holders (Bishop et al., 2012). One filter holder was loaded with a 51- $\mu$ m Sefar polyester mesh prefilter followed by paired Whatman QMA quartz fiber filters $(1 \mu \mathrm{m}$ nominal pore size). The other filter holder was also loaded with another $51-\mu \mathrm{m}$ polyester prefilter, but followed by paired $0.8-\mu \mathrm{m}$ Pall Supor800 polyethersulfone filters. The particles retained on the quartz filters were used to analyze total particulate carbon and particulate inorganic carbon, while those retained on the polyethersulfone filters were used to analyze biogenic silica and particulate trace metals (Lam et al., 2015a; Ohnemus and Lam, 2015). Particle concentrations were determined from the sum of the chemical dry weight of the major particles phases: particulate organic matter, particulate inorganic carbon, biogenic silica, lithogenic material estimated from total particulate aluminum, and Fe and Mn oxyhyroxides (Lam et al., 2015a).

\section{${ }^{234}$ Th and ${ }^{238} \mathbf{U}$}

In this paper, the subscript $d(p)$ designates the Th isotope activity in dissolved (particulate) form in units of $\mathrm{dpm} \mathrm{m}^{-3}$, e.g., ${ }^{234} \mathrm{Th}_{d}$ denotes the activity of ${ }^{234} \mathrm{Th}$ in the $<1 \mu \mathrm{m}$ size fraction (QMA) and ${ }^{234} \mathrm{Th}_{p}$ denotes the activity of ${ }^{234} \mathrm{Th}$ in the $>1 \mu \mathrm{m}$ size fraction (similar designations are adopted for ${ }^{230} \mathrm{Th}$ and ${ }^{228} \mathrm{Th}$ ). ${ }^{234} \mathrm{Th}_{\text {tot }}$ designates total (dissolved + particulate) ${ }^{234} \mathrm{Th}$. Particulate 
${ }^{234} \mathrm{Th}$ activities on the 1-51 $\mu \mathrm{m}$ (QMA filter) and $>51 \mu \mathrm{m}$ size fractions from in-situ filtration were determined by beta counting (Maiti et al., 2012; Owens et al., 2015). Small volume (4 L) samples for ${ }^{234} \mathrm{Th}_{\text {tot }}$ were obtained using an ODF/SIO Rosette above $1000 \mathrm{~m}$ or Niskin bottles hung above the McLane pump below $1000 \mathrm{~m}$, and were also analyzed using beta counting (Owens et al., 2015). ${ }^{238} \mathrm{U}$ is estimated from salinity using the empirical equation derived by Owens et al. (2011),

$$
{ }^{238} \mathrm{U}=0.0786( \pm .00446) S-0.315( \pm 0.158)
$$

where ${ }^{238} \mathrm{U}$ is in dpm $\mathrm{m}^{-3}$ and $S$ is on the Practical Salinity Scale of 1978 . The uncertainties of ${ }^{238} \mathrm{U}$ are estimated by the root mean square error of the linear regression of ${ }^{238} \mathrm{U}$ with salinity (Owens et al., 2011).

\section{${ }^{230}$ Th and ${ }^{234} \mathbf{U}$}

Subsamples of polyethersulfone filters were acid digested and co-precipitated with Fe after complete dissolution. Particulate ${ }^{230} \mathrm{Th}$ in the small $(0.8-51 \mu \mathrm{m})$ size fraction was determined on the subsamples by inductively coupled plasma mass spectrometry (ICP-MS) (Hayes et al., 2015a). Subsamples for dissolved ${ }^{230} \mathrm{Th}$ were obtained using Niskin bottles attached to an ODF/SIO Rosette, and gravity filtered through Pall Acropak 500 filters containing a $0.8-\mu \mathrm{m}$ prefilter followed by a 0.45 $\mu \mathrm{m}$ filter. Dissolved ${ }^{230}$ Th was also measured by ICP-MS (Anderson et al., 2012; Shen et al., 2012; Hayes et al., 2015b). Finally, ${ }^{234} \mathrm{U}$ is estimated from ${ }^{238} \mathrm{U}$ by assuming a ${ }^{234} \mathrm{U} /{ }^{238} \mathrm{U}$ ratio of 1.147 (Andersen et al., 2010). The uncertainties in ${ }^{234} \mathrm{U}$ are obtained by multiplying the uncertainties in ${ }^{238} \mathrm{U}$ by 1.147 , i.e., the effect of the uncertainty in ${ }^{234} \mathrm{U} /{ }^{238} \mathrm{U}$ is neglected.

\section{${ }^{228}$ Th and ${ }^{228} \mathbf{R a}$}

Particulate ${ }^{228} \mathrm{Th}$ in the small $(1-51 \mu \mathrm{m})$ size fraction sampled by in-situ filtration was measured by alpha delayed coincidence counting of QMA filters (Maiti et al., 2015). Dissolved ${ }^{228} \mathrm{Ra}$ and ${ }^{228} \mathrm{Th}$ were collected simultaneously with particles by sorption on $\mathrm{MnO}_{2}$ impregnated acrylic cartridges located downstream of the two filter holders. They were analyzed by alpha delayed coincidence $\left({ }^{228} \mathrm{Th}\right)$ and gamma counting $\left({ }^{228} \mathrm{Ra}\right)$ (Henderson et al., 2013; Charette et al., 2015).

\subsubsection{Bulk Particulate ${ }^{228,230,234}$ Th}

Observational estimates of ${ }^{228,230,234}$ Th activities for the whole particulate fraction (sizes $>1 \mu \mathrm{m}$ ) are obtained as follows. ${ }^{228,230,234} \mathrm{Th}$ activities have been measured on the small particles $(1-51 \mu \mathrm{m})$. ${ }^{234} \mathrm{Th}$ data for large particles are available only in the top $900 \mathrm{~m}$, and ${ }^{228,230} \mathrm{Th}$ data for large 
particles are currently not available. In order to obtain bulk particle data for each Th isotope, the ratio of large $\left({ }^{234} \mathrm{Th}_{p, l}\right)$ to small particulate ${ }^{234} \mathrm{Th}\left({ }^{234} \mathrm{Th}_{p, s}\right)$ is calculated from the ${ }^{234} \mathrm{Th}_{p, l}$ and ${ }^{234} \mathrm{Th}_{p, s}$ data available for the upper $900 \mathrm{~m}$ and below the euphotic zone (below $125 \mathrm{~m}, n=5$ ). This ratio (mean of 0.19 with a standard deviation of 0.01 ) is then applied to derive ${ }^{234} \mathrm{Th}_{p, l}$ below $900 \mathrm{~m}$ and ${ }^{228,230} \mathrm{Th}_{p, l}$ at all depths, from the measured activities on the small size fraction. The measured or calculated ${ }^{228,230,234} \mathrm{Th}_{p, l}$ is added to the measured ${ }^{228,230,234} \mathrm{Th}_{p, s}$ to obtain total particulate Th for each isotope (e.g., ${ }^{228} \mathrm{Th}_{p}={ }^{228} \mathrm{Th}_{p, s}+{ }^{228} \mathrm{Th}_{p, l}$ ). Additionally, dissolved ${ }^{234} \mathrm{Th}$ (not measured at station GT11-22) is obtained by subtracting ${ }^{234} \mathrm{Th}_{p}$ from total ${ }^{234} \mathrm{Th}$ and its error is derived by error propagation neglecting error covariance (Bevington and Robinson, 1992).

\subsubsection{Vertical Interpolation}

The depths at which $P,{ }^{228,230,234} \mathrm{Th}_{d, p},{ }^{234,238} \mathrm{U}$, and ${ }^{228} \mathrm{Ra}$ data are available do not generally coincide exactly (Appendix A). In order to facilitate the data analysis, the measured (or calculated) values of $P,{ }^{228,230,234} \mathrm{Th}_{d, p},{ }^{234,238} \mathrm{U}$, and ${ }^{228} \mathrm{Ra}$ are interpolated onto an irregular grid in which each grid point is at a depth where at least one measurement is available. The shallowest and deepest points of the grid are at 125 and 4243 meters, respectively. Such a grid is chosen in order (i) to exclude surface waters where processes of particle production, which are outside the scope of this study, occur, and (ii) to avoid the need for data extrapolation. We use an objective interpolation technique (e.g., Wunsch (2006)): an estimate of property $x$ at depth level $i, \hat{x}_{i}$, is taken as a linear combination of the measurements of that property at all depths, $x_{k}(k=1,2, \cdots, n)$,

$$
\hat{x}_{i}=\sum_{k=1}^{n} w_{i k} x_{k},
$$

where the weighting factors $w_{i k}$ are determined such that the $\hat{x}_{i}$ errors have minimum variance.

The interpolation requires the prescription of two matrices: a covariance matrix for the measurement errors and a matrix describing the vertical covariance of the property being interpolated. The first matrix is taken as diagonal, where the diagonal elements are the squared errors in the measurements. The $(i, j)$ element of the second matrix is taken as $\sigma_{M}^{2} e^{-\left|z_{i}-z_{j}\right| / l_{z}}$, where $\sigma_{M}^{2}$ is the variance of the property and $l_{z}$ is a length scale characterizing its vertical covariance. The quantities $\sigma_{M}^{2}$ and $l_{z}$ have the following interpretation. When the interpolation depth is far from the measurement depth, the error in the interpolated value approaches the square root of the field property variance $\sigma_{M}^{2}$. So, $\sigma_{M}^{2}$ is the maximum tolerable variance in the gridded (interpolated) data. On the 
other hand, $l_{z}$ is an e-folding length scale: if the distance between two depths in the water column increases by $l_{z}$, the property covariance between both depths is reduced by a factor of $1 / e$.

Figure 3 shows three interpolation scenarios obtained with $\sigma_{M}^{2}=0.25 \sigma_{d}^{2}, 0.5 \sigma_{d}^{2}$, or $\sigma_{d}^{2}\left(l_{z}=1000\right.$ $\mathrm{m}$ in all cases), where $\sigma_{d}^{2}$ is the variance in a particular data set (e.g., the variance in the ${ }^{230} \mathrm{Th}_{d}$ data). Besides differences in uncertainty in the interpolated values (Figure 3a-c), the agreement between the interpolated and measured values at the measurement depths deteriorates as $\sigma_{M}^{2}$ decreases (Figure 3d-f). This is because $\sigma_{M}^{2}$ influences the weight of each measurement in the interpolated values: the larger the variance, the better the ability of the interpolated values to approach the measured values. Thus, increasing $\sigma_{M}^{2}$ improves the agreement of the interpolated values with the measured values, although each scenario tends to overfit the data according to the normal distribution (Figure 3d-f). Decreasing $\sigma_{M}^{2}$ to less than $0.25 \sigma_{d}^{2}$ (not shown) results in interpolated data errors that are smaller than the uncertainties in the measurements, which is not desirable. Therefore, we retain $\sigma_{M}^{2} \geq 0.25 \sigma_{d}^{2}$ in this study.

Figure 4 shows three other interpolation scenarios with the same values of $\sigma_{M}^{2}=0.5 \sigma_{d}^{2}$ but with different values of $l_{z}=500 \mathrm{~m}, 1000 \mathrm{~m}$, or $2000 \mathrm{~m}$. These different length scales capture scenarios in which there is varying vertical correlation between property values at different depths. As $l_{z}$ increases, the error of the interpolated values decreases (Figure 4a-c). For the three values of $l_{z}$, the interpolated values are consistent with the measured values, although each scenario displays an overfit to the measurements according to the normal distribution (Figure $4 \mathrm{~d}-\mathrm{f}$ ). In this paper, we choose $\sigma_{d}^{2}=0.5 \sigma_{M}^{2}$ and $l_{z}=1000 \mathrm{~m}$ as our reference interpolation. Different sets of interpolated values of $P,{ }^{228,230,234} \mathrm{Th}_{d, p},{ }^{234,238} \mathrm{U}$, and ${ }^{228} \mathrm{Ra}$ are considered in section 2.3 .

Figure 5 shows the measured and interpolated values of the various radiochemical activities at station GT11-22 $\left(\sigma_{d}^{2}=0.5 \sigma_{M}^{2}, l_{z}=1000 \mathrm{~m}\right)$. The measured and interpolated values of ${ }^{230} \mathrm{Th}_{d}$ and ${ }^{230} \mathrm{Th}_{p}$ show increases with depth. However, ${ }^{230} \mathrm{Th}_{p}$ is in general relatively uniform below $2000 \mathrm{~m}$, as is dissolved ${ }^{230} \mathrm{Th}$ below $3500 \mathrm{~m}$. Particulate ${ }^{234} \mathrm{Th}$ decreases generally with depth to $2000 \mathrm{~m}$, and shows reduced variations below. Total ${ }^{234} \mathrm{Th}$ varies between 2100 and $2700 \mathrm{dpm} \mathrm{m}{ }^{-3}$, with no clear systematic changes with depth. Finally, the profiles of dissolved and particulate ${ }^{228} \mathrm{Th}$ generally resemble that of the parent isotope, ${ }^{228} \mathrm{Ra}$, with maxima in surface and bottom waters and minima at mid-depth. 


\subsubsection{Model of Thorium and Particle Cycling}

We consider a model of thorium and particle cycling that includes a balance equation for each thorium isotope in each phase (dissolved and particulate), and a balance equation for bulk (large + small) particles (Figure 6). The model accounts for adsorption of thorium to particles, desorption of Th from particles, radioactive production and decay, remineralization of particles, and sinking of particles. The balance equations are (Nozaki et al., 1981; Bacon and Anderson, 1982)

$$
\begin{aligned}
0 & =\lambda A_{\pi}+\left(k_{-1}+\beta_{-1}\right) A_{p}-\left(k_{1}+\lambda\right) A_{d}, \\
w \frac{\partial A_{p}}{\partial z} & =k_{1} A_{d}-\left(\beta_{-1}+k_{-1}+\lambda\right) A_{p}, \\
w \frac{\partial P}{\partial z} & =-\beta_{-1} P .
\end{aligned}
$$

Here $A_{d}\left(A_{p}\right)$ is the thorium isotope activity in dissolved (particulate) form ( $\left.\mathrm{dpm} \mathrm{m}^{-3}\right), A_{\pi}$ is the activity of the radioactive parent $\left(\mathrm{dpm} \mathrm{m}^{-3}\right), P$ is the particle concentration $\left(\mu \mathrm{g} \mathrm{m}^{-3}\right)$, and $\lambda$ is the radioactive decay constant $\left(\mathrm{yr}^{-1}\right)$. The rate parameters of the model are the adsorption rate constant $\left(k_{1}, \mathrm{yr}^{-1}\right)$, the desorption rate constant $\left(k_{-1}, \mathrm{yr}^{-1}\right)$, the remineralization rate constant $\left(\beta_{-1}, \mathrm{yr}^{-1}\right)$, and the particle sinking speed $\left(w, \mathrm{~m} \mathrm{yr}^{-1}\right)$. The presence of the vertical derivative in equations $(2.3 \mathrm{~b}-2.3 \mathrm{c})$ requires the prescription of boundary conditions, which we take as the values of $A_{p}$ and $P$ at $z=125 \mathrm{~m}$. Two model versions are considered: version V1 assumes uniform rate parameters, whereas version V2 allows these parameters to vary with depth.

Note the various assumptions in the Th and particle cycling model (besides the assumption of uniform rate parameters in model V1). Equations (2.3a-2.3c) rely on steady state and omit the effects of water transport by advection and diffusion. They assume that sorption and remineralization processes obey first-order kinetics. The potential sources of dissolved and particulate Th from lithogenic contributions are taken as negligible. This assumption should be valid for ${ }^{228,234} \mathrm{Th}$, at least in the surface ocean where the activities of both isotopes are generally large. Following the procedures documented in Roy-Barman et al. (2002) and Hayes et al. (2013), we calculate the percent contribution of lithogenic ${ }^{230} \mathrm{Th}$ to the measured ${ }^{230} \mathrm{Th}_{d, p}$ using dissolved and particulate ${ }^{232}$ Th data at station GT11-22 (Anderson et al., 2012; Hayes et al., 2015a). We find that lithogenic ${ }^{230} \mathrm{Th}_{d}$ always accounts for less than $10 \%$ of total ${ }^{230} \mathrm{Th}_{d}$. Lithogenic ${ }^{230} \mathrm{Th}_{p}$ accounts for up to $30 \%$ of total ${ }^{230} \mathrm{Th}_{p}$ in the upper $500 \mathrm{~m}$, but less than $10 \%$ below $500 \mathrm{~m}$. Unless stipulated otherwise, we do not correct ${ }^{230} \mathrm{Th}$ for a lithogenic contribution (the sensitivity of our results to such a correction 
is examined in section 2.4.1.2).

\subsubsection{Inverse Method}

The ATI (Tarantola and Valette, 1982) is used to combine the Th and particle cycling model (section 2.2.5) with the radiochemical and particle measurements at station GT11-22 (sections 2.2.2-2.2.4): model V1 and V2 are fitted to the data, and their respective ability to explain the data is assessed from the residuals of the fit. The rate parameters $\left(k_{1}, k_{-1}, \beta_{-1}, w\right)$ as well as the radiochemical activities and particle concentrations are adjusted so as to obtain the best fit. Thus the particle and radiochemical data are not fixed to their measured (interpolated) values but are allowed to change in the inversion within a range consistent with their estimated uncertainties. This approach allows us to give due consideration to the errors in the radiochemical and particle data when testing model V1 and V2 (see discussion in Lam and Marchal (2014)).

A brief description of the ATI follows. Let $\boldsymbol{x}$ be a vector describing the state of the Th and particle cycles according to the model. The elements of $\boldsymbol{x}$ are the Th isotope activities in the dissolved and particulate phases, the particle concentrations, the parent activities $\left({ }^{234} \mathrm{U},{ }^{238} \mathrm{U},{ }^{228} \mathrm{Ra}\right)$, as well as the rate parameters $\left(k_{1}, k_{-1}, \beta_{-1}, w\right)$. In model V1, $\boldsymbol{x}$ includes these variables at all depths of the grid at station GT11-22 (section 2.2.4), except for the uniform rate parameters. In model V2, $\boldsymbol{x}$ includes these variables at all depths of the grid. The objective is to find a vector $\boldsymbol{x}$ that fits station GT11-22 (interpolated) data given their error statistics, while satisfying the model equations (2.3a-2.3c) perfectly. This vector is found at a stationary point of the objective function:

$$
J=\left(\boldsymbol{x}-\boldsymbol{x}_{0}\right)^{T} \boldsymbol{C}_{0}^{-1}\left(\boldsymbol{x}-\boldsymbol{x}_{0}\right)+\boldsymbol{x}^{T} \boldsymbol{S}^{-1} \boldsymbol{x}-2 \boldsymbol{u}^{T} \boldsymbol{f}(\boldsymbol{x}) .
$$

Here, $\boldsymbol{x}_{0}$ is a vector including prior estimates of the elements in $\boldsymbol{x}$ (in our study, the interpolated data and prior estimates of rate parameters), $\boldsymbol{C}_{0}$ is the error covariance matrix for the prior estimates (the diagonal elements of $\boldsymbol{C}_{0}$ are the squared errors in the estimates and the off-diagonal elements of $\boldsymbol{C}_{0}$ are the covariances between the errors), $\boldsymbol{S}^{-1}$ is another square matrix, $\boldsymbol{u}$ is a vector of Lagrange multipliers, and $\boldsymbol{f}(\boldsymbol{x})=\mathbf{0}$ is a vector including the difference equations derived from (2.3a-2.3c), with $w \partial A_{p} / \partial z$ and $w \partial P / \partial z$ discretized with a first-order backward scheme.

The three terms of the objective function (3.5) have the following interpretations. The first term represents the deviation of the state vector from its prior estimate, where $\boldsymbol{C}_{0}$ plays the role of a weighting factor: the elements of $\boldsymbol{x}_{0}$ with small (large) uncertainties contribute strongly (modestly) to the objective function. The second term prescribes a certain amount of smoothing on the vertical 
variation of some elements of $\boldsymbol{x}$ (see below). Finally, the third term is the hard constraint imposed in the search for a stationary point of $J$. Thus, we search for a minimum of the sum of the first two terms, subject to the hard constraint $\boldsymbol{f}(\boldsymbol{x})=\mathbf{0}$. The prior estimates of the rate parameters and their errors are based on observational estimates published in the literature (Table 1; section 2.1).

\begin{tabular}{|l|c|c|}
\hline parameter & prior estimate & prior estimate error \\
\hline$k_{1}\left(\mathrm{y}^{-1}\right)$ & 0.5 & 5 \\
\hline$k_{-1}\left(\mathrm{y}^{-1}\right)$ & 2 & 5 \\
\hline$\beta_{-1}\left(\mathrm{y}^{-1}\right)$ & 1 & 10 \\
\hline $\mathrm{w}\left(\mathrm{m} \mathrm{y}^{-1}\right)$ & 700 & $300-1100$ \\
\hline
\end{tabular}

Table 1: Prior estimates of rate parameters of Th and particle cycling assumed in this study

The motive for including the smoothing term $\boldsymbol{x}^{T} \boldsymbol{S}^{-1} \boldsymbol{x}$ in $J$ is twofold. First, note that errors in the prior estimates represent \pm 1 standard deviation of a normal distribution, which means that there is a possibility of estimating negative values of these parameters in the solution, especially when the error is larger than the prior estimate of the parameter (as is the case for $k_{1}, k_{-1}$, and $\left.\beta_{-1}\right)$. However, radionuclide and particle measurements and all estimates of the rate parameters found in the literature are positive, and in fact negative values of the elements in $\boldsymbol{x}$ are nonsensical in our study. Preliminary inversions have shown that negative values tend to not occur when some smoothing is imposed to the solution. Second, the imposition of smoothing tends to prevent large variations of solution elements on small vertical scales, which do not appear geochemically plausible. Here $\boldsymbol{S}^{-1}$ only acts on the rate parameters $\left(k_{1}, k_{-1}, \beta_{-1}, w\right)$. The non-vanishing elements of $\boldsymbol{x}^{T} \boldsymbol{S}^{-1} \boldsymbol{x}$ have the generic form

$$
\gamma\left(x\left(z_{2}\right)-x\left(z_{1}\right)\right)^{2}
$$

where $x$ represents $k_{1}, k_{-1}, \beta_{-1}$, or $w, z_{1}$ and $z_{2}$ are two different depths, and the parameter $\gamma$ trades smoothness of the solution against its proximity to the data. In our study, $\gamma$ is set equal to 1, unless stipulated otherwise. The effect of $\gamma$ on our results is examined in section 2.4.1.

The dimensions of the vector $\boldsymbol{x}$ and $\boldsymbol{f}(\boldsymbol{x})$ are as follows. The number of grid points is 41, but since the shallowest grid point is where the boundary conditions of the model are imposed, the ${ }^{234,238} \mathrm{U},{ }^{228} \mathrm{Ra}$, and ${ }^{228,230,234} \mathrm{Th}_{d}$ activities are defined at 40 depths, while the ${ }^{228,230,234} \mathrm{Th}_{p}$ activities and $P$ concentrations are defined at all depths. Furthermore, there are 40 equations for each Th isotope in each phase (dissolved and particulate), as well as 40 equations for the particle 
concentration. As a result, there are $M=280$ equations (elements of $\boldsymbol{f}(\boldsymbol{x})$ ) and $N=408$ unknowns (elements of $\boldsymbol{x}$ ) for model V1, and there are $M=280$ equations and $N=564$ unknowns for model V2. In both cases, the fit of the model equations to the data is an underdetermined problem.

The ATI can be derived as follows. The model equations $\boldsymbol{f}(\boldsymbol{x})$ are linearized, i.e., $\boldsymbol{f}\left(\boldsymbol{x}_{k+1}\right)=$ $\boldsymbol{f}\left(\boldsymbol{x}_{k}\right)+\boldsymbol{F}_{k}\left(\boldsymbol{x}_{k+1}-\boldsymbol{x}_{k}\right)$, where $\boldsymbol{x}_{k+1}$ and $\boldsymbol{x}_{k}$ denote two different values of $\boldsymbol{x}$, and $\boldsymbol{F}_{k}$ is a matrix whose elements are the partial derivatives of the model equations with respect to the elements of $\boldsymbol{x}$, i.e., the element in the $i$ th row and $j$ th column of $\boldsymbol{F}_{k}$ is $\partial f_{i} / \partial x_{j}$. Setting $\partial J / \partial \boldsymbol{x}=\mathbf{0}$ and $\partial J / \partial \boldsymbol{u}=\mathbf{0}$ then leads to a system of linear algebraic equations from which an iterative procedure to find $\boldsymbol{x}$ can be derived. The solution for $\boldsymbol{x}$ at the $(k+1)$ th iteration and its error covariance matrix are given by, respectively:

$$
\begin{aligned}
\hat{\boldsymbol{x}}_{k+1} & =\boldsymbol{P}_{k} \boldsymbol{x}_{0}+\boldsymbol{C}_{o s} \boldsymbol{F}_{k}^{T}\left(\boldsymbol{F}_{k} \boldsymbol{C}_{o s} \boldsymbol{F}_{k}^{T}\right)^{-1} \boldsymbol{b}_{k}, \\
\boldsymbol{C}_{k+1} & =\boldsymbol{P}_{k} \boldsymbol{C}_{0} \boldsymbol{P}_{k}^{T}
\end{aligned}
$$

where

$$
\begin{aligned}
\boldsymbol{P}_{k} & =\boldsymbol{C}_{o s} \boldsymbol{C}_{0}^{-1}-\boldsymbol{C}_{o s} \boldsymbol{F}_{k}^{T}\left(\boldsymbol{F}_{k} \boldsymbol{C}_{o s} \boldsymbol{F}_{k}^{T}\right)^{-1} \boldsymbol{F}_{k} \boldsymbol{C}_{o s} \boldsymbol{C}_{0}^{-1}, \\
\boldsymbol{b}_{k} & =\boldsymbol{F}_{k} \hat{\boldsymbol{x}}_{k}-\boldsymbol{f}\left(\boldsymbol{x}_{k}\right), \\
\boldsymbol{C}_{o s} & =\left(\boldsymbol{C}_{0}^{-1}+\boldsymbol{S}^{-1}\right)^{-1} .
\end{aligned}
$$

The iterative procedure is initiated at $\hat{\boldsymbol{x}}_{k=0}=\boldsymbol{x}_{0}$ and terminated when the relative difference between two subsequent values of each element of $\hat{\boldsymbol{x}}$ is less than $1 \%$ in absolute magnitude. The estimate of $\boldsymbol{x}$ obtained at this stage is noted $\hat{\boldsymbol{x}}$ and referred to as the solution of the fit of model V1 or V2 to GT11-22 data.

To ensure that the Th and particle equations in $\boldsymbol{f}(\boldsymbol{x})=\mathbf{0}$ have a commensurate effect in the inversion, independent of the choice of units, the elements of $\boldsymbol{x}$ are normalized by their prior values in $\boldsymbol{x}_{\mathbf{0}}$ prior to the operation of the ATI. Accordingly, all elements of $\boldsymbol{x}_{0}$ are set equal to 1 and all elements of $\boldsymbol{C}_{0}$ are scaled by the squared errors in the prior estimates. Additionally, each model equation is normalized by the root of the sum of the squared terms in that equation. This normalization scheme forces the leading terms in each equation to be roughly on the same order of magnitude, so that the effect of each equation should be similar in the inversion. 


\subsection{Results}

In this section, the two versions of the Th and particle cycling model (V1 and V2; section 2.2.4) are fitted to the station GT11-22 data (sections 2.2.2-2.2.4) using the ATI (section 2.2.5). The fitted values of ${ }^{234,238} \mathrm{U},{ }^{228} \mathrm{Ra},{ }^{228,230,234} \mathrm{Th}_{d, p}$ and particle concentration are plotted in Figure 7 for model V1 and Figure 8 for model V2. For both versions, convergence to a stable solution is reached after 14 iterations. In order to check whether the model equations are satisfied by the solution $\hat{\boldsymbol{x}}$ given the inevitable numerical errors involved in the matrix operations (2.6-2.10), the residual of each equation, $f_{i}(\hat{\boldsymbol{x}})=\epsilon_{i}$, is calculated and compared with the maximum term in the corresponding equation. For each fit (V1 or V2), we find that the residual $\epsilon_{i}$ amounts to less that $10^{-3}$ (in absolute magnitude) of the maximum term for each equation, indicating that the model equations are satisfied to at least the 3rd order.

\subsubsection{Goodness of fit}

Two measures of goodness of fit of model V1 and V2 to GT11-22 data are considered. A first measure is the fraction ( $\phi$ below) of the normalized residuals that are less than 2 in absolute magnitude, where a normalized residual is defined as

$$
r_{i}=\frac{\hat{x}_{i}-x_{d, i}}{\sigma_{d, i}}, i=1,2, \ldots, n .
$$

Here $\hat{x}_{i}$ is the estimated value of the $i$ th variable $\left({ }^{228,230,234} \mathrm{Th}_{d, p},{ }^{234,238} \mathrm{U},{ }^{228} \mathrm{Ra}\right.$, and $\left.P\right)$ in the vector $\hat{\boldsymbol{x}}$ that is obtained from the fit of model V1 or V2 to GT11-22 data, $x_{d, i}$ is the measured (not interpolated) value of this variable, $\sigma_{d, i}$ is the error in this measured value, and $n$ is the number of measured values. The interpretation of $\phi$ is straightforward. For example, a value of 0.95 for $\phi$ would mean that the model version being considered can be brought into consistency with $95 \%$ of the GT11-22 data given their errors.

The second measure of goodness of fit is the arithmetic average of the difference between the estimated and measured values, normalized by the measurement error:

$$
B=\frac{1}{n} \sum_{i=1}^{n} \frac{\hat{x}_{i}-x_{d, i}}{\sigma_{d, i}} .
$$

In general, a relatively large absolute value of $B$ would indicate a relatively large bias of the corresponding model version in describing the GT11-22 data. The quantities $\phi$ and $B$ are complementary measures of goodness of fit: $\phi$ describes the overall ability of model V1 or V2 to fit GT11-22 data with no regard for possible under- or over-estimation, whereas $B$ should be indicative 
of systematic errors in the model versions.

We find that the version of the Th and particle cycling model that assumes vertically uniform rate parameters (model V1) can fit $52 \%-55 \%$ of the GT11-22 data $(0.52 \leq \phi \leq 0.55)$, where the range reflects varying assumptions for vertical interpolation (Table 2). In contrast, the model version that permits vertical variations in $k_{1}, k_{-1}, \beta_{-1}, w$ (model V2) can explain 73\%-78\% of the GT11-22 data, where the range reflects again varying assumptions about $\sigma_{M}^{2}$ and $l_{z}$.

\begin{tabular}{|l|c|c|c|c|c|}
\hline & $\sigma_{M}^{2}=0.5 \sigma_{d}^{2}$ & $\sigma_{M}^{2}=\sigma_{d}^{2}$ & $\sigma_{M}^{2}=0.25 \sigma_{d}^{2}$ & $l_{z}=2000 \mathrm{~m}$ & $l_{z}=500 \mathrm{~m}$ \\
\hline$\phi$ for V1 & 0.54 & 0.55 & 0.52 & 0.52 & 0.55 \\
\hline$\phi$ for V2 & 0.74 & 0.76 & 0.73 & 0.73 & 0.78 \\
\hline$B$ for V1 & -1.91 & -1.86 & -2.00 & -1.96 & -1.89 \\
\hline$B$ for V2 & -0.45 & -0.40 & -0.53 & -0.49 & -0.41 \\
\hline
\end{tabular}

a. For each listed $\sigma_{M}^{2}, l_{z}=1000 \mathrm{~m}$. For each listed $\mathrm{l}_{z}, \sigma_{M}^{2}=0.5 \sigma_{d}^{2}$

Table 2: Measures of goodness of fit of model V1 and V2 to station GT11-22 data ${ }^{a}$.

The model version that allows vertical variations in the rate parameters produces a better fit to the observations than the version that does not. This result holds as the interpolation parameters are varied within plausible ranges, e.g., $0.25 \sigma_{d}^{2} \leq \sigma_{M}^{2} \leq \sigma_{d}^{2}$ and $500 \mathrm{~m} \leq l_{z} \leq 2000 \mathrm{~m}$. Moreover, the bias of model V2 $(-0.53 \leq B \leq-0.40)$ is always less than for model V1 $(-2.00 \leq B \leq-1.86)$, indicating that allowance of vertical variations of $k_{1}, k_{-1}, \beta_{-1}$, and $w$ reduces the systematic errors of the Th and particle cycling model (Table 2).

\subsubsection{Bootstrap Test}

To compare models V1 and V2, we perform a parametric bootstrap test (Efron and Tibshirani, 1993) of the null hypothesis $H_{o}$ that V1 is correct against the alternative hypothesis $H_{1}$ that V2 is correct. Let $x_{d, i}$ be a measured value with standard deviation $\sigma_{d, i}$ and let $\hat{x}_{1, i}$ be the corresponding fitted value for V1. The goodness of fit of V1 to all $n$ measurements can be determined by:

$$
J_{d}(V 1)=\sum_{i=1}^{n}\left(\frac{\ln \left(x_{d, i}\right)-\ln \left(\hat{x}_{1, i}\right)}{\sigma_{\ln \left(x_{d, i}\right)}}\right)^{2},
$$

where

$$
\sigma_{\ln \left(x_{d, i}\right)}=\sqrt{\ln \left(1+\frac{\sigma_{d, i}^{2}}{x_{d, i}^{2}}\right)}
$$


is an estimate of the standard deviation of $\ln \left(x_{d, i}\right)$ (Vanmarcke, 1983). The test statistic used in the parametric bootstrap procedure is:

$$
T=J_{d}(V 1)-J_{d}(V 2)
$$

where $J_{d}(V 2)$ is the analogue of (13) for V2. The quantity $T$ measures the improvement in fit by relaxing the constraint of parameter uniformity in model V1.

The parametric bootstrap test proceeds by (i) simulating a set of measurements from the fitted model V1,

$$
x_{d, i}^{*}=e^{\ln \left(\hat{x}_{1, i}\right)+\epsilon_{i}}, \quad i=1,2, \ldots n
$$

where $\epsilon_{i}$ is normally distributed with mean 0 and standard deviation $\sigma_{\ln \left(x_{d, i}\right)}$, (ii) re-fitting V1 and V2 to the simulated observations, and (iii) forming the corresponding value of the test statistic $T$. The procedure is repeated a total of 200 times and the observed significance level (or $p$ value) is approximated by the proportion of occurrences the simulated value of $\mathrm{T}$ exceeds the value for the original data $(T=7170)$.

A histogram of the values of $\mathrm{T}$ generated by this bootstrap procedure is shown in Figure 9 along with the observed value. In this case, none of these values exceeds the observed value, so model V1 can be rejected in favor of model V2.

\subsubsection{Consistency with Prior Estimates}

A question of geochemical interest is whether the rate parameters $\left(k_{1}, k_{-1}, \beta_{-1}, w\right)$ that are obtained from the fit of model V2 to GT11-22 data vary within plausible ranges. To address this question, we compare the vertical variations of $\left(k_{1}, k_{-1}, \beta_{-1}, w\right)$ obtained from the fit with the prior estimates of these parameters (Figure 10). The posterior estimates of $k_{1}, k_{-1}, \beta_{-1}$, and $w$ are all within two standard deviations of the prior estimates, indicating that the rate parameters inferred from the fit of model V2 to GT11-22 data are consistent with prior knowledge. The most significant differences between the prior and posterior values occur for the particle sinking speed (Figure 10d), a result elaborated upon below (section 2.4.2). Note also the dramatic reduction in the prior un-

certainties in $\left(k_{1}, k_{-1}, \beta_{-1}, w\right)$ that results from the combination of model V2 with station GT11-22 data (Figure 10). 


\subsection{Discussion}

Our results indicate that a model with depth-dependent rate parameters provides a significantly better description of particle concentration and thorium activity data at station GT11-22 than a model with uniform rate parameters. While non-negative rate parameters are obtained for both model V1 and V2, there is a disconcerting feature in both solutions: ${ }^{228} \mathrm{Ra}$ values inferred by inversion near $500 \mathrm{~m}$ are negative, which is nonsensical (Figure 7 and 8 ). These values differ from 0 $\mathrm{dpm} \mathrm{m}^{-3}$ by more than one standard deviation. While these negative values are evidence that the model is not consistent with the data, we think that by themselves they do not warrant definitive rejection of the model. In testing model V1 and V2, any solution elements that deviate significantly from the data, not only negative values, should be interpreted as a failure to explain the entire data set. Generally, one does not definitively reject a model because a few observations cannot be replicated. Indeed, the model may still replicate most of the observations, and thus provide a useful (albeit clearly not exact) description. The negative ${ }^{228} \mathrm{Ra}$ values are a reflection of inconsistencies between the data and the model, which should be understood before a decision is made about the plausibility of the model.

The importance of using a data set consisting of multiple thorium isotope and particle measurements is highlighted in Figure 11. In this figure, we consider the more usual situation where data for only one Th isotope (here ${ }^{230} \mathrm{Th}$ ) are available. In order to test model $\mathrm{V} 1$, the ${ }^{230} \mathrm{Th}_{d}$ and ${ }^{230} \mathrm{Th}_{p}$ data would be individually regressed linearly versus depth (Nozaki and Nakanishi, 1985; Edmonds et al., 1998; Okubo et al., 2012) (here a weighted least squares regression is used). The ${ }^{230} \mathrm{Th}_{p}$ values estimated by regression are comparable with those estimated from the entire data set (compare solid line with grey circles in Figure 11b). In contrast, the ${ }^{230} \mathrm{Th}_{d}$ values from the regression are systematically larger than those inferred from the entire data set and much closer to the measurements (Figure 11a). With only ${ }^{230}$ Th data available, one might perhaps conclude that model V1 provides an adequate description of the data, which contrasts with the conclusion drawn from the multiple thorium isotope and particle concentration data set. This result illustrates the pitfall of using data for only one Th isotope when making inferences about the appropriateness of a Th cycling model. 


\subsubsection{Robustness of the Test}

\section{Sensitivity to Vertical Smoothing}

In section 2.3, we tested model V2 using a smoothing parameter $\gamma=1$. In order to document the effect of $\gamma$ on our results, we fit model V2 to GT11-22 data (interpolated values obtained using $\sigma_{M}^{2}=0.5 \sigma_{d}^{2}$ and $\left.l_{z}=1000 \mathrm{~m}\right)$ using $\gamma=0.01$ or $\gamma=100$. For $\gamma=0.01$, a solution is found after 66 iterations and the model equations are satisfied to the third order. For $\gamma=100$, a solution is found after 8 iterations and the model equations are also satisfied to the third order. When $\gamma=0.01$, the fraction of normalized residuals less than 2 in absolute magnitude $(\phi)$ reaches 0.86 , and the bias $(B)$ shrinks to -0.27 . When $\gamma=100, \phi=0.62$ and $B=-0.60$. Thus decreasing $\gamma$ improves the fit of model V2 to the data, though in each case model V2 displays a better fit to the data than model V1 (see Table 3). The resulting vertical profiles of $\left(k_{1}, k_{-1}, \beta_{-1}, w\right)$ are compared in Figure 12. As expected, the posterior variances in the rate parameters are larger when $\gamma$ is smaller. Moreover, some $\beta_{-1}$ and $w$ values are negative if $\gamma=0.01$. The intermediate value of $\gamma=1$ provides the rate parameters some ability to vary with depth while preventing them from taking on negative values.

\section{Sensitivity to Initial Estimates}

In section 2.3, our initial estimate $\hat{\boldsymbol{x}}_{k=0}$ was constructed so as to satisfy the measurements and the interpolation assumptions, i.e., $\hat{\boldsymbol{x}}_{k=0}=\boldsymbol{x}_{0}$. However, due to the nonlinearity of $\boldsymbol{f}(\boldsymbol{x})$, the ATI may converge to a solution that depends on $\hat{\boldsymbol{x}}_{k=0}$ (Tarantola and Valette, 1982). In particular, with $\boldsymbol{f}(\boldsymbol{x})$ being nonlinear, initial estimates of $\boldsymbol{x}$ that are far from the "true" solution may not lead to this solution but to a secondary minimum of $J$. Here we examine whether our results hold for a different initial estimate of the state vector, i.e. $\hat{\boldsymbol{x}}_{k=0} \neq \boldsymbol{x}_{0}$. Specifically, $\hat{\boldsymbol{x}}_{k=0}$ is constructed so as to satisfy the model equations perfectly: the parent activities $\left({ }^{234} \mathrm{U},{ }^{238} \mathrm{U},{ }^{228} \mathrm{Ra}\right)$, the boundary values of ${ }^{228,230,234} \mathrm{Th}_{p}$ and $P$, and the rate parameters are equal to their prior values as in section 2.3, but the other elements of $\hat{\boldsymbol{x}}_{k=0}\left({ }^{228,230,234} \mathrm{Th}_{d, p}\right.$ and $P$ at all depths save at the boundary point) are obtained as the solution of the model with these prior values.

We find that the results of the inversions for the two different estimates $\hat{\boldsymbol{x}}_{k=0}$ are very similar (Table 3). The ATI converges to a stable solution after 12 iterations and all model equations are satisfied to at least the third order. Model V2 provides a superior fit to the data $(0.73 \leq \phi \leq$ 0.74) compared to model V1 $(\phi=0.54)$. The bias of the fit is also similar between both inversions for model V2 $(-0.45 \leq B \leq-0.4)$ and both inversions for model V1 $(B=-1.91)$. Thus, our 


\begin{tabular}{|l|c|c|c|c|c|}
\hline & 'reference' $\hat{\boldsymbol{x}}_{k=0}$ & model-based $\hat{\boldsymbol{x}}_{k=0}$ & $\mathrm{Th}_{p, l} / \mathrm{Th}_{p, s}=0.07$ & corrected ${ }^{230} \mathrm{Th}(\mathrm{a})$ & QMA bias 20\% \\
\hline$\phi$ for V1 & 0.54 & 0.54 & 0.53 & 0.53 & 0.55 \\
\hline$\phi$ for V2 & 0.74 & 0.73 & 0.73 & 0.73 & 0.76 \\
\hline$B$ for V1 & -1.91 & -1.91 & -1.85 & -1.84 & -1.64 \\
\hline$B$ for V2 & -0.45 & -0.40 & -0.49 & -0.46 & -0.38 \\
\hline
\end{tabular}

a.measured ${ }^{230} \mathrm{Th}_{d, p}$ corrected for lithogenic ${ }^{230} \mathrm{Th}$.

Table 3: Measures of goodness of fit of model V1 and V2 to GT11-22 data for different initial estimate $\boldsymbol{x}_{k=0}$.

test of models V1 and V2 does not depend on whether the initial state estimate satisfies the entire set of prior values, or only a fraction of the prior values and the model equations. This result is encouraging, although we cannot rule out that other plausible choices of $\hat{\boldsymbol{x}}_{k=0}$ would lead to different results.

\section{Sensitivity to Bulk Particle Activities}

Our observational estimates of bulk particulate ${ }^{234,230,228} \mathrm{Th}$ assumed a ratio between large and small particulate Th activities of 0.19 (see section 2.2.4). This value is large compared to the ${ }^{234} \mathrm{Th}_{p, l} /{ }^{234} \mathrm{Th}_{p, s}$ ratio of 0.07 found by Buesseler et al. (2001) in the Southern Ocean. In order to test the effects of a smaller ratio, we repeat our inversion using bulk particulate Th data based on a large to small particulate Th activity of 0.07 (Buesseler et al., 2001). We find that, in this case, the model equations are satisfied to the third order, and the objective function again converges after 14 iterations. The results are very similar to those of our reference solution (Table 3).

\section{Sensitivity to Lithogenic Sources}

Our model does not consider the contribution of a lithogenic source to ${ }^{230} \mathrm{Th}_{p}$. In order to test the sensitivity of our results to the inclusion of this source, we repeat our inversion by correcting ${ }^{230} \mathrm{Th}_{p}$ data for a contribution from lithogenic material (section 2.2.5). In this case, we find that the model equations are satisfied to the third order, and the objective function converges after 15 iterations. No notable difference between these results and those from the reference inversion occurs (Table 3). Despite the relatively large $(>30 \%)$ contribution to ${ }^{230} \mathrm{Th}_{p}$ from lithogenic particles in the upper $500 \mathrm{~m}$, the solution is insensitive to this correction, presumably because the lithogenic source is small compared to other sources and sinks of ${ }^{230} \mathrm{Th}_{p}$.

\section{Sensitivity to Filtering Bias}

Finally, we consider the effects of a potential bias due to the filters chosen to extract Th isotopes. Maiti et al. (2012) found no significant differences in ${ }^{234} \mathrm{Th}$ activities between different filter types and pore sizes (between $0.2-0.8 \mu \mathrm{m}$ ), except for the quartz filters: the ${ }^{234}$ Th activities measured on 
QMA filters were found to be $10 \%$ to $20 \%$ higher than those measured on Supor filters. The higher activities on QMA filters were attributed mainly to sorption (Maiti et al., 2012). In order to test a potential bias due to ${ }^{228,234} \mathrm{Th}$ sorption on QMA filters, we repeat our inversions by reducing the particulate ${ }^{234} \mathrm{Th}$ and ${ }^{228} \mathrm{Th}$ data values by $20 \%$. In this case, the model equations are satisfied to the third order and the objective function converges after 14 iterations. The values of $\phi$ and $B$ are close to those of our reference solution (Table 3), suggesting our results are not very sensitive to a potential bias due to the use of different types of filters.

\section{Kolmogorov-Smirnov Test}

In order to further test whether our results are robust against the changes discussed in sections 2.4.1.2-2.4.1.5, we employ a Kolmogorov-Smirnov test (Appendix B). The tests show that these changes do not significantly alter the results compared to those of our reference solution (section $2.3)$.

\subsubsection{Implications for Oceanic Th Geochemistry}

\section{Effect of Particle Concentration}

In this section, we examine the roles of adsorption $\left(k_{1}\right)$, desorption $\left(k_{-1}\right)$, and remineralization $\left(\beta_{-1}\right)$ in the fit of model V2 to GT11-22 data. For simplicity we consider a single parameter, $K=k_{1} /\left(k_{-1}+\beta_{-1}\right)$. Thus, a value of $K<1$, for example, would imply that the specific rate at which Th attaches to particles is smaller than those at which it is released from particles by desorption and remineralization. Interestingly, the vertical profile of $K$ in our reference solution (section 2.3) shows a decrease with depth in the upper $2000 \mathrm{~m}$ and relatively uniform values below (Figure 13a). Using data from the Guatemala and Panama Basins, Bacon and Anderson (1982) estimated that the $k_{1} / k_{-1}$ ratio ranged from 0.078 to 0.462 , where their $k_{-1}$ included both remineralization and desorption. These values are consistent with our estimates of $K$ at station GT11-22. However, the $k_{1} / k_{-1}$ ratio of Bacon and Anderson (1982) did not show similar variability with depth across stations, so it is unclear whether the profile of $K$ derived here (Figure 13a) is consistent with their findings.

We find a higher $K$ value in the upper $2000 \mathrm{~m}$, mainly because $k_{1}$ is enhanced in these waters (Figure 9). A potential cause of the increased adsorption rate constant in the upper $2000 \mathrm{~m}$ is the higher particle concentration in this region of the water column, because of the increase in the number of surface sites available for attachment (Honeyman et al., 1988). We assess the strength of 
the association between $k_{1}$ and $P$ (Figure 14a), as well as between $K$ and $P$ (Figure 14b), using the Kendall tau $(\tau)$ rank correlation coefficient (Kendall and Gibbons, 1990). This coefficient ranges between -1 and 1 , where a value of $1(-1)$ implies a perfect positive (negative) monotonic relationship between $k_{1}$ (or $\left.K\right)$ and $P$. We find that $\tau=0.73(p<0.01)$ for the relationship between $k_{1}$ and $P$, and $\tau=0.70(p<0.01)$ for the relationship between $K$ and $P$ (the Pearson correlation coefficient for both of these relationships amount to $r=0.89$ ). Thus both $k_{1}$ and $K$ increase significantly with $P$.

Honeyman et al. (1988) and Honeyman and Santschi (1989) have calculated an equilibrium distribution coefficient, $\bar{K}_{D}=k_{1} /\left(k_{-1} P\right)$ to describe the affinity of trace metals for particles. Echoing previous studies, Honeyman et al. (1988) found that $\bar{K}_{D}$ decreases with increasing particle concentration, a phenomenon referred to as the "particle concentration effect". According to these authors, one potential cause for this effect is that the rate of adsorption of trace metals onto filterable particles depends on the rate of coagulation of colloidal $(<0.8 \mu \mathrm{m})$ particles. They showed that $\bar{K}_{D}$ should decrease with $P$, even though $k_{1}$ increases with particle concentration, because $\bar{K}_{D}$ varies explicitly as $P^{-1}$ while $k_{1}$ has a power law dependence with particle concentration, $k_{1}=k_{1, c} P^{b}$, where $k_{1, c}$ is constant and $b<1$.

We examine the possibility of a particle concentration effect at station GT11-22 from the values of $\bar{K}_{D, \beta_{-1}}=k_{1} /\left(\left(k_{-1}+\beta_{-1}\right) P\right)$ obtained from the fit of model V2 to the data gathered at that station. The profile of $\bar{K}_{D, \beta_{-1}}$ shows a general increase with depth (Figure 13b). Hayes et al. (2015a) estimated $K_{D}=A_{p} /\left(A_{d} P\right)$ from ${ }^{230} \mathrm{Th}$ and filtered particle concentration data from the GA03 transect (eq. (2.3b) shows that $A_{p} / A_{d}$ equals $k_{1} /\left(k_{-1}+\beta_{-1}\right)$ if sinking and radioactive decay are neglected). Their method involved dividing ${ }^{230} \mathrm{Th}$ adsorbed onto particles by ${ }^{230} \mathrm{Th}$ in the dissolved phase and normalizing this ratio to the filtered particulate matter concentration $(>0.8$ $\mu \mathrm{m})$. Our values for $\bar{K}_{D, \beta_{-1}}$, between $1.5 \times 10^{-5}$ and $5 \times 10^{-5} \mathrm{~m}^{3} \mu \mathrm{g}^{-1}$, are within the range found by Hayes et al. (2015a) below $500 \mathrm{~m}$, from about $1 \times 10^{-5}$ to about $1 \times 10^{-4} \mathrm{~m}^{3} \mu \mathrm{g}^{-1}$. Plotting $\ln \left(\bar{K}_{D, \beta_{-1}}\right)$ against $\ln (P)$ suggests that, in general, $\bar{K}_{D, \beta_{-1}}$ decreases with $P$ (Figure $14 \mathrm{~d}$ ). The rank correlation between $\ln \left(\bar{K}_{D, \beta_{-1}}\right)$ and $\ln (P)$ amounts to $\tau=-0.62$ with $p<0.01(r=-0.75)$, which indicates a significant negative monotonic relationship between both variables and hence the possibility of a particle concentration effect. Note that below about $3500 \mathrm{~m}$, the vertical gradient of $\bar{K}_{D, \beta_{-1}}$ is particularly large. However, this feature is due at least partly to the decrease in the 
estimated particle concentrations below $3500 \mathrm{~m}$, which is not observed (Figure 8).

If a particle concentration effect exists, $k_{1}$ should vary as $P^{b}$, where $b$ is less than 1 (see dashed line in Figure 14a). Conversely, in the absence of such an effect, $\bar{K}_{D, \beta_{-1}}$ should be independent of particle concentration (see dashed line in Figure 14d). A least-squares fit of $\ln \left(k_{1}\right)$ vs. $\ln (P)$ yields a slope of $0.60 \pm 0.05$. This contrasts with the slope obtained by Honeyman et al. (1988) of 0.51-0.58 from field data spanning a much larger particle concentration range from $O\left(10^{4} \mu \mathrm{g} \mathrm{m}^{-3}\right)$ to $O\left(10^{12}\right.$ $\left.\mu \mathrm{g} \mathrm{m}^{-3}\right)$. For the regression of $\ln \left(\bar{K}_{D, \beta_{-1}}\right)$ against $\ln (P)$, we obtain a slope of $-0.36 \pm 0.06$, smaller in magnitude than the slope of -0.42 found by Honeyman et al. (1988) from the same field data used to obtain their $\ln (k 1)$ vs. $\ln (P)$ slope. These results suggest that the particle concentration effect we obtain from our analysis of station GT11-22 data is not as strong as that reported by Honeyman et al. (1988) for a much larger particle concentration range. This discrepancy may arise because the particle concentrations reported at station GT11-22 are lower than those considered by Honeyman et al. (1988). Honeyman and Santschi (1989) found that $\bar{K}_{D}$ appears independent of particle concentration below $10^{4} \mu \mathrm{g} \mathrm{m}^{-3}$, a value larger than $P$ at any depth investigated here. Interestingly, using ${ }^{230} \mathrm{Th}$ activity and particle concentration data across the entire North Atlantic section, Hayes et al. (2015a) found a much larger slope of $\log _{10}\left(K_{D}\right)$ vs. $\log _{10}(P)$ of $-0.66\left(\mathrm{r}^{2}=0.53\right)$. Such a discrepancy may be due to station GT11-22 being an oligotrophic site with low colloidal concentrations relative to stations closer to the margins. Nevertheless, our findings suggest that a particle concentration effect does remain present at this station, albeit reduced compared to the one found at higher particle concentrations.

Bacon and Anderson (1982) reported that $k_{-1}$ does not depend on particle concentration from data collected in the Panama and Guatemala Basins. We test whether $k_{-1}$ depends on particle concentration according to our analysis of GT11-22 data. We find that for the relationship between $\ln \left(k_{-1}\right)$ and $\ln (P), \tau=-0.18(p=0.11)$ and the slope of the least squares fit is $-0.06 \pm 0.04$ $(r=-0.19)$. Thus, we find no significant evidence that $P$ affects $k_{-1}$, consistent with Bacon and Anderson (1982).

\section{Vertical Variation in Particle Settling Speed}

In this section, we discuss the profile of particle sinking speed obtained from the fit of model V2 to GT11-22 data. Particle concentration in model V2 shows a decrease below $2000 \mathrm{~m}$ that is not as pronounced as in model V1. Associated with this feature is the inference of relatively large 
particle settling speeds below $2000 \mathrm{~m}$. The particle sinking term can be either a loss or a source in the particle equation (eq. 2.3c). For example, if there are more particles at the depth above a grid point than at the depth of the grid point, that grid point will "gain" particles from sinking. In this case, increasing the sinking velocity tends to increase the particle concentration and to better offset the loss due to particle remineralization. As a result, particle concentration in deep water is higher in model V2 compared to model V1 (compare Figure 7j to Figure 8j).

It is difficult to determine possible mechanisms underlying the vertical variation in our particle sinking speed profile, in particular because our model does not discriminate between large and small particles. One of the most noticeable features in this profile is an apparent increase in sinking speed below $2000 \mathrm{~m}$. One potential cause for the apparent increase in particle sinking speed is a local increase in the rate of aggregation of suspended particles, resulting in an increase in large, more quickly sinking particles and hence in bulk $w$. However, this appears unlikely given the nearly constant partitioning of particles between the small and large size fractions below $2000 \mathrm{~m}$ at station GT11-22 (Lam et al., 2015a). The apparent increase in particle sinking speed may reflect a bias due to the exclusion of other processes that could increase particle concentration below $2000 \mathrm{~m}$, such as lateral transport of suspended particles.

Below $2000 \mathrm{~m}$, the particle sinking speed inferred by inversion exhibits significant variability on short vertical scales (Fig. 10). Interestingly, similar variability is not apparent in the inferred vertical profiles of the parent isotope activities, Th isotope activities, and particle concentration. Below $2000 \mathrm{~m}$, the particle sinking term is a minor term in the balance of the particulate Th isotopes (Section 2.4.3), except for ${ }^{228} \mathrm{Th}_{p}$. Speculatively, the large variability of $w$ at small vertical scales would be due to the small vertical gradients of particulate ${ }^{228} \mathrm{Th}_{p}$ between about 2000 and $3500 \mathrm{~m}$ : small changes in these gradients would require large changes in $w$ to achieve a balance between the particulate ${ }^{228} \mathrm{Th}_{p}$ sources and sinks at different levels within this depth interval. Whereas further inversions may help to isolate the specific measurements that are responsible for the inference of a large variability of $w$ below $2000 \mathrm{~m}$, such effort is beyond the scope of this study.

To our knowledge, there are no reported depth-varying estimates of the sinking speed of bulk particles (small + large) in the current literature. Armstrong et al. (2009) and Lee et al. (2009) collected sinking particles using indented rotating-sphere settling velocity (IRS-SV) traps placed at various depths at a location in the northwestern Mediterranean Sea. These traps have the ability 
to sort the mass flux density of particles into sinking speed bins. The time-integrated mass flux density is defined as the mass of any constituent per square meter of trap area, integrated over the trap deployment time and divided by $\log _{10}\left(S V_{\max } / S V_{\min }\right)$, where $S V_{\max }\left(S V_{\min }\right)$ is the maximum (minimum) particle settling speed in a given SV range. Armstrong et al. (2009) and Lee et al. (2009) found that, at all depths, (i) the majority of the time-integrated mass flux density of particles occurs within a rapidly sinking $\left(200-500 \mathrm{~m} \mathrm{~d}^{-1}\right)$ speed interval, and (ii) the time-integrated mass flux density presents an exponential "tail" within a much slower (0.68-2.7 $\left.\mathrm{m} \mathrm{d}^{-1}\right)$ sinking speed interval. Closer to station GT11-22, Alonso-González et al. (2010) conducted a study south of the Canary Islands using IRS-SV traps placed at a depth of $260 \mathrm{~m}$. They found that the time-integrated mass flux density was highest in the low sinking speed range $\left(0.7-11 \mathrm{~m} \mathrm{~d}^{-1}\right)$, which overlaps with the range of sinking speeds obtained from the fit of model V2 to GT11-22 data (0.19-3.86 $\left.\mathrm{m} \mathrm{d}^{-1}\right)$. Therefore, it could be concluded that the vast majority of particles below $125 \mathrm{~m}$ at station GT11-22 settle very slow. However, comparison to our study is difficult. Whereas Armstrong et al. (2009), Lee et al. (2009), and Alonso-González et al. (2010) consider particles within specific sinking speed ranges, the sinking speeds reported here are average values for the bulk particle concentration (all particles) at a given depth. The sinking speeds of the different particles may vary greatly, making the interpretation of our estimated sinking speeds difficult. Moreover, the sinking speed of bulk particulate thorium may not be the same as the speeds of bulk particulate material. For example, one may expect the settling velocity of bulk particulate thorium to be smaller than for bulk particles, as smaller particles with generally slower sinking speeds have higher surface area to volume ratios and therefore a greater capacity to adsorb thorium (Buesseler et al., 2006). Interestingly, the carbon to thorium $(\mathrm{C} / \mathrm{Th})$ ratio measured in particles collected in IRS-SV traps at both the Mediterranean Sea (Stewart et al., 2007; Szlosek et al., 2009) and the North Pacific (Buesseler et al., 2009) show no apparent relationship with sinking speed, somewhat at odds with what is expected based on simple surface area to volume ratio considerations (again, assuming slower sinking particles are generally smaller and have larger surface area to volume ratios). Regardless, caution must be applied when comparing our bulk particle sinking speeds to those obtained from sediment or settling velocity traps. 


\subsubsection{Diagnosis of Th Isotope Budgets}

In this section, we examine the budget of each Th isotope at station GT11-22, as determined by inversion of the particle and radiochemical data. The different terms of the budget are, for each Th isotope, the adsorption flux $\left(k_{1} A_{d}\right)$, the desorption flux $\left(k_{-1} A_{p}\right)$, the remineralization flux $\left(\beta_{-1} A_{p}\right)$, the particle sinking flux $\left(w \partial A_{p} / \partial z\right)$, the decay fluxes $\left(\lambda A_{d}, \lambda A_{p}\right)$, and the production flux $\left(\lambda A_{\pi}\right)$. Since our estimation of the flux terms accounts for the data uncertainties but not for the model uncertainties, it should only be considered as suggestive.

${ }^{234} \mathbf{T h}$

The dominant terms in the ${ }^{234} \mathrm{Th}_{d}$ budget (Figure 15) are the production and decay terms. These terms are the largest because ${ }^{234} \mathrm{Th}_{d}$ and ${ }^{238} \mathrm{U}$ are one order of magnitude larger than ${ }^{234} \mathrm{Th}_{p}$, and the decay constant for ${ }^{234} \mathrm{Th}$ is about one order of magnitude larger than $k_{1}, k_{-1}$, and $\beta_{-1}$. For the ${ }^{234} \mathrm{Th}_{p}$ budget, the first-order terms are the adsorption flux and radioactive decay. The adsorption term dominates because it includes ${ }^{234} \mathrm{Th}_{d}$, and the decay term is the main loss because the decay constant is much larger than the other rate constants. The particle sinking term is particularly small below $800 \mathrm{~m}$, where ${ }^{234} \mathrm{Th}_{p}$ shows small vertical variations (Figure 5). The average fluxes, taken by integrating the source and loss terms and dividing by the vertical extent of our domain (from 125 to $4243 \mathrm{~m}$ ), are shown in Figure 15a. To obtain kinetic measures in the budget, we calculate residence times by dividing the depth-averaged Th isotope activity by the dominant depth-averaged volumetric flux associated with that isotope. We find that the residence time with respect to radioactive production is 34 days for ${ }^{234} \mathrm{Th}_{d}$, and the residence time with respect to adsorption is 29 days for ${ }^{234} \mathrm{Th}_{p}$.

${ }^{228} \mathbf{T h}$

Unlike for ${ }^{234} \mathrm{Th}_{d}$, the dominant terms in the ${ }^{228} \mathrm{Th}_{d}$ budget vary with depth. Near the surface, radioactive production is the dominant source, and is balanced mostly by adsorption of ${ }^{228} \mathrm{Th}_{d}$. However, throughout most of the water column, adsorption, decay, desorption, and production are nearly equivalent in magnitude. Below $3500 \mathrm{~m}$, adsorption and decay are comparable as losses of ${ }^{228} \mathrm{Th}_{d}$, and production once again becomes the dominant source. This pattern is consistent with the vertical profile of ${ }^{228} \mathrm{Ra}$, which has surface and deepwater maxima and is minimum near the middle of the water column. Thus production is highest in near-surface and deep waters. Near the surface, the adsorption term is larger than decay of ${ }^{228} \mathrm{Th}_{d}$, even though both scale with ${ }^{228} \mathrm{Th}_{d}$, 
because $k_{1}$ is highest in surface waters (Figure 10).

The dominant terms in the ${ }^{228} \mathrm{Th}_{p}$ budget similarly vary with depth. Near the shallowest depth, adsorption is the dominant source, balanced mostly by desorption. Near the surface, the particle sinking flux is a loss term for ${ }^{228} \mathrm{Th}_{p}$, but becomes a source equivalent in magnitude to adsorption below about $500 \mathrm{~m}$. Below about $3500 \mathrm{~m},{ }^{228} \mathrm{Th}_{p}$ is lost in about equal measure through particle sinking and desorption, and is gained solely through adsorption. We show the average fluxes for ${ }^{228} \mathrm{Th}_{d, p}$ in Figure 16a. The residence time of ${ }^{228} \mathrm{Th}_{d}$ and ${ }^{228} \mathrm{Th}_{p}$ with respect to adsorption is 891 days (2.44 yr) and 125 days, respectively.

\section{${ }^{230} \mathrm{Th}$}

Since the radioactive decay constant for ${ }^{230} \mathrm{Th}$ is about 5 orders of magnitude smaller than the rate parameters $k_{1}, k_{-1}$, and $\beta_{-1}$, production and decay generally no longer dominate the budget for the dissolved and particulate phases. Instead, desorption is the first-order source of ${ }^{230} \mathrm{Th}_{d}$ and adsorption is the first-order loss, with production and remineralization being second-order gains except nearest to the surface. The primary mechanisms influencing ${ }^{230} \mathrm{Th}_{p}$ are also adsorption and desorption, with remineralization and sinking being generally losses of second-order importance. We show the average fluxes for ${ }^{230} \mathrm{Th}_{d, p}$ in Figure 17a. The residence time of ${ }^{230} \mathrm{Th}_{d}$ and ${ }^{230} \mathrm{Th}_{p}$ with respect to adsorption is 1090 days (2.98 yr) and 130 days, respectively.

\subsubsection{Interpretation of Particle and Th isotope residuals Model with Uniform Rate Parameters}

The values of ${ }^{234} \mathrm{Th}_{d}$ estimated from the fit of model V1 to GT11-22 data show vertical variations associated with vertical variations in ${ }^{238} \mathrm{U}$ (Figure 7 ). Some of the ${ }^{238} \mathrm{U}$ values inferred from the fit differ from prior values estimated from salinity by more than one standard deviation. These values seem to stem from the attempt by the algorithm to produce the best fit to the ${ }^{234} \mathrm{Th}_{d}$ data: since rate parameters cannot vary with depth in model V1, the algorithm allows the parent isotope to change in order to fit the daughter. In any case, it is clear that model V1 cannot fit uranium and thorium activities very well (e.g. Figure 7e), with the exception of ${ }^{228} \mathrm{Th}$ and ${ }^{228} \mathrm{Ra}$. Unlike the ${ }^{238,234} \mathrm{U}$ estimates derived from salinity, ${ }^{228} \mathrm{Ra}$ measurements show consistent variability with depth (maxima in near surface and deep waters, minima at mid-depth), which is largely reflected in the ${ }^{228} \mathrm{Th}_{d}$ and ${ }^{228} \mathrm{Th}_{p}$ data. Therefore, whereas the algorithm produces ${ }^{234,238} \mathrm{U}$ values that deviate significantly from prior estimates, such large deviations from the prior estimates are generally not 
as necessary for ${ }^{228} \mathrm{Ra}$ to reach consistency with the model. Finally, the particle concentrations inferred by inversion show an exponential decrease with depth, in contrast to the data which shows a slight increase below $2000 \mathrm{~m}$. Below this depth, model V1 is unable to replicate the particle data within two standard deviations (Figure 7).

\section{Model with Non-Uniform Rate Parameters}

Model V2 produces enhanced agreement with radiochemical and particle data from station GT11-22 (Figure 8). This can be viewed most clearly by the much improved fit of model V2 to ${ }^{234,238} \mathrm{U},{ }^{230} \mathrm{Th}_{d}$, and particle data. However, even allowing the rate parameters to vary with depth does not allow the model to fit the entire data set. In particular, ${ }^{234} \mathrm{Th}_{d},{ }^{230} \mathrm{Th}_{p}$, and particle concentration contain regions of relatively large normalized residuals (Figure 8). The vertical variations in the ${ }^{234} \mathrm{Th}_{t o t}$ data (used to derive ${ }^{234} \mathrm{Th}_{d}$ ) are large compared to those in the salinitybased estimates of ${ }^{238} \mathrm{U}$ (compare Figure 5a with Figure 5b). The half-life of ${ }^{234} \mathrm{Th}_{d}$ (24.1 days) implies that processes responsible for departure from secular equilibrium should be characterized by a timescale of a few weeks or less. It is worthwhile to note that such departures from secular equilibrium in deep-water ${ }^{234} \mathrm{Th}_{\text {tot }}$ are not unique to station GT11-22. Owens et al. (2015) have shown how ${ }^{234} \mathrm{Th}_{\text {tot }}$ disequilibrium is prevalent in deep waters throughout the section. For instance, cross-over station GT11-24 (Figure 1) exhibits ${ }^{234} \mathrm{Th}_{\text {tot }}$ deficits, while station GT11-20 (the second station to the west of GT11-22; Figure 1) exhibits ${ }^{234} \mathrm{Th}_{\text {tot }}$ excess, like station GT11-22. While a subsurface excess in ${ }^{234} \mathrm{Th}_{\text {tot }}$ relative to secular equilibrium may be explained by a deficit in ${ }^{234} \mathrm{Th}$ in the surface due to enhanced scavenging and subsequent remineralization of Th-laden particles just beneath the surface (Maiti et al., 2015), a mechanism for maintaining a ${ }^{234} \mathrm{Th}_{\text {tot }}$ excess below 1000 $\mathrm{m}$ has yet to be elucidated. Therefore, the variations in ${ }^{234} \mathrm{Th}_{d, t o t}$ remain a confounding element of the GEOTRACES North Atlantic data set.

On the other hand, ${ }^{230} \mathrm{Th}$ appears more likely to be influenced by the transport of water masses. Due to its long half-life, ${ }^{230} \mathrm{Th}$ anomalies can be transported over large distances in the ocean, provided that these anomalies are not erased by the effects of particle scavenging and water mixing. For example, ventilation by ${ }^{230} \mathrm{Th}_{d}$-poor water from the northern North Atlantic has been postulated to influence ${ }^{230} \mathrm{Th}$ in deep water in the North Atlantic (Moran et al., 1997; Vogler et al., 1998). Here, we examine the potential influence of two water masses, Upper Labrador Sea Water (ULSW) and Central Labrador Sea Water (CLSW), on the misfits of model V2 to ${ }^{230}$ Th data for station GT11-22. 
Figure 18 compares the normalized ${ }^{230} \mathrm{Th}_{d}$ and ${ }^{230} \mathrm{Th}_{p}$ residuals with the estimated proportion of ULSW and CLSW at this station (Jenkins et al., 2015). Since LSW is characterized by low ${ }^{230} \mathrm{Th}_{d}$ (Moran et al., 2002), an intrusion of LSW at station GT11-22 should be reflected in low ${ }^{230} \mathrm{Th}_{d}$ activities between 1500 and $3000 \mathrm{~m}$. Such an intrusion would also result in low ${ }^{230} \mathrm{Th}_{p}$ if ${ }^{230} \mathrm{Th}_{d}$ continuously undergoes reversible exchange in transit. However, the ${ }^{230} \mathrm{Th}_{d}$ normalized residuals are only large (and negative) in the top $500 \mathrm{~m}$, and do not show any systematic change below $1500 \mathrm{~m}$, where Labrador Sea Waters are inferred to be present (section 2.2.1). Therefore, it seems unlikely that the ${ }^{230} \mathrm{Th}_{d, p}$ residuals arise from the omission of the effect of LSWs in the model. Other processes missing in model V2 must be responsible for the significant ${ }^{230} \mathrm{Th}_{d, p}$ residuals (see section 2.2.4 for model limitations).

Both model V1 and V2 underpredict particle concentrations compared to observations below $2000 \mathrm{~m}$. Since our interpolation grid only extends down to $4243 \mathrm{~m}$, only 2 measurements of particle concentration were considered in the data interpolation below $2000 \mathrm{~m}$. Inspection of the entire particle concentration profile from $125 \mathrm{~m}$ to $4989 \mathrm{~m}$ (Figure 19) confirms that particle concentration remained constant or increased slightly with depth below $2000 \mathrm{~m}$, consistent with beam attenuation coefficient data at station GT11-22 (Anderson et al., 2013). Although a slight bottom nepheloid layer might have been present at station GT11-22, as was observed at other stations along the section (Lam et al., 2015a), the particle residuals below $2000 \mathrm{~m}$ (Figure 19) should probably not be interpreted as due to the omission of such a layer in the model. Another potential source of misfit of model V2 to particle concentration data is lateral transport of suspended particles (Alonso-González et al., 2010), although it is unclear whether there can be particle enriched waters moving laterally to station GT11-22 below 2000 m. McCartney et al. (1991) and Schmitz and McCartney (1993) have presented a schematic of the AABW circulation in the North Atlantic in which the bottom water flowing through the Vema Fracture Zone enters the eastern North Atlantic basins and becomes part of the NADW. Speculatively, this water may be enriched in suspended particles due to intense turbulent mixing within the fracture zone (Polzin et al., 1996; Hayes et al., 2015b).

Finally, notice that we have not shown that model V2 is a "good" model, in the sense that it is both parsimonious with parameters and does not over-fit the data. Rather our study evaluates whether the mere allowance of vertical variations in model parameters, with no change in model structure (e.g., assumption of second-order instead of first-order kinetics for Th adsorption flux) 
and with no additional terms in the model (e.g., horizontal transport), can significantly improve the fit to radiochemical and particle data for an open-ocean station. We show that a significantly better fit is obtained in this case (in fact, model V2 leads generally to an over-fit compared to the normal distribution) and, moreover, that some of the inferred parameters may feature systematic vertical variations suggestive of a particle concentration effect in situ.

\subsection{Conclusion}

A suite of radiochemical and particle data from station GT11-22 of the U.S. GEOTRACES North Atlantic section are used to test two versions of a particle and Th cycling model that assumes a single class of particles. Model V2, with depth-dependent rate parameters, shows a significant improvement in fit to the data set than model V1 with uniform rate parameters. In contrast to the reversible exchange model described by Bacon and Anderson (1982), we are able to successfully tease apart desorption $\left(k_{-1}\right)$ and remineralization $\left(\beta_{-1}\right)$ by invoking a model that describes thorium as well as particle cycling in the deep ocean. We find that $k_{-1}$ is much larger than $\beta_{-1}$, suggesting that remineralization does not result in a major loss of particulate thorium. Our analysis illustrates the pitfall of using data for a single Th isotope when testing particle and Th cycling models. It suggests the occurrence of systematic vertical variations in some of the rate constants (most notably $k_{1}$ and $w)$ and in some of their combinations $\left(K=k_{1} /\left(k_{-1}+\beta_{-1}\right)\right.$ and $\left.\bar{K}_{D, \beta_{-1}}=K / P\right)$ in the mesopelagic and bathypelagic zones. It points to a significant effect of particle concentration on $K$ and $K / P$ in situ, i.e., $k_{1}$ and $K$ increase with $P$ whereas $K / P$ decreases with $P$. Finally, it suggests that deviations of Th profiles from those predicted by reversible exchange, which are often interpreted in terms of an effect of ocean circulation, could be due, at least partly, to vertical variations in rate constants.

While model V2 can describe most of the GT11-22 data, it does not explain the entire data set. Features in the data that remain unexplained by model V2 include the large vertical variations of total (and hence dissolved) ${ }^{234} \mathrm{Th}$, the kink in the particulate ${ }^{230} \mathrm{Th}$ profile near $2000 \mathrm{~m}$, and the relatively uniform particle concentration below that depth. Finally, one should exercise caution when interpreting the rate parameters obtained from this study. The parameters we estimate are apparent ones that may, at least partially, mask the effects of processes not encapsulated in the model used here. We cannot rule out the presence of a bias in the inferred rate parameters, because 
the processes not described by the model may be the source of systematic errors. The fact that we can produce a reasonable fit to station GT11-22 data does not imply that there is no influence from other processes, such as the circulation of deep water masses, on the isotope activities and (or) particle concentrations. The parameter estimated obtained in this study are only as reliable as the assumptions about the data errors $\left(\boldsymbol{C}_{0}\right)$ and parameter distribution $(\boldsymbol{S})$ from which these estimates have been derived.

\section{Acknowledgements}

We acknowledge the U.S. National Science Foundation for providing funding for this study (grant OCE-1232578) and for U.S. GEOTRACES North Atlantic section ship time, sampling, and data analysis. The U.S. NSF also supported the generation of ${ }^{230}$ Th data (OCE-0927064 to LDEO, OCEO092860 to WHOI, and OCE-0927754 to UMN) and ${ }^{228,234}$ Th data (OCE-0925158 to WHOI). We thank the two chief scientists of the GA03 section (Ed Boyle and Bill Jenkins) as well as the captain, the crew, and the scientific party on the $\mathrm{R} / \mathrm{V}$ Knorr, which completed this section. We are also grateful to the scientists and staff involved in the collection and analysis of the thorium isotope and particle data. 
2.A Appendix A 


\begin{tabular}{|c|c|c|c|c|c|c|c|c|c|c|c|c|}
\hline depth $(\mathrm{m})$ & $\mathrm{U}$ & ${ }^{228} \mathrm{Ra}$ & ${ }^{234} \mathrm{Th}_{t o t}$ & ${ }^{234} \mathrm{Th}_{p, s}$ & ${ }^{234} \mathrm{Th}_{p, l}$ & ${ }^{230} \mathrm{Th}_{d}$ & ${ }^{230} \mathrm{Th}_{p, s}$ & ${ }^{228} \mathrm{Th}_{d}$ & ${ }^{228} \mathrm{Th}_{p, s}$ & $P_{s}$ & $P_{l}$ & reference \\
\hline $1,25,49$ & $\checkmark$ & & $\checkmark$ & & & & & & & & & $\mathrm{a}$ \\
\hline 50 & & $\checkmark$ & & $\checkmark$ & $\checkmark$ & $\checkmark$ & & $\checkmark$ & $\checkmark$ & $\checkmark$ & $\checkmark$ & $\mathrm{a}, \mathrm{b}, \mathrm{c}, \mathrm{d}$ \\
\hline 75 & & & & & & $\checkmark$ & & & & & & $\mathrm{b}$ \\
\hline 84 & & & & & & $\checkmark$ & & & & & & $\mathrm{b}$ \\
\hline 90 & & $\checkmark$ & & $\checkmark$ & $\checkmark$ & & & $\checkmark$ & $\checkmark$ & $\checkmark$ & $\checkmark$ & $a, c, d$ \\
\hline 124 & & & & & & $\checkmark$ & & & & & & $\mathrm{b}$ \\
\hline 125 & $\checkmark$ & $\checkmark$ & $\checkmark$ & $\checkmark$ & $\checkmark$ & & $\checkmark$ & $\checkmark$ & $\checkmark$ & $\checkmark$ & $\checkmark$ & $\mathrm{a}, \mathrm{b}, \mathrm{c}, \mathrm{d}$ \\
\hline 149 & $\checkmark$ & & $\checkmark$ & & & & & & & & & $\mathrm{a}$ \\
\hline 184 & & & & & & $\checkmark$ & & & & & & $\mathrm{b}$ \\
\hline 185 & $\checkmark$ & 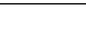 & $\checkmark$ & & & & & & & & & $\mathrm{a}$ \\
\hline 187 & & $\checkmark$ & & $\checkmark$ & $\checkmark$ & & & $\checkmark$ & $\checkmark$ & & & $\mathrm{a}, \mathrm{c}$ \\
\hline 233 & . & & & & & $\checkmark$ & & & & & & $\mathrm{b}$ \\
\hline 234 & $\checkmark$ & & $\checkmark$ & & & & & & & & & $\mathrm{a}$ \\
\hline 237 & & $\checkmark$ & & $\checkmark$ & $\checkmark$ & & $\checkmark$ & $\checkmark$ & $\checkmark$ & $\checkmark$ & $\checkmark$ & $\mathrm{a}, \mathrm{b}, \mathrm{c}, \mathrm{d}$ \\
\hline 283 & & & & & & $\checkmark$ & & & & & & $\mathrm{b}$ \\
\hline 285,389 & $\checkmark$ & 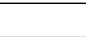 & $\checkmark$ & & & & & & & & & $\mathrm{a}$ \\
\hline 392 & & $\checkmark$ & & $\checkmark$ & $\checkmark$ & & & $\checkmark$ & $\checkmark$ & $\checkmark$ & $\checkmark$ & $a, c, d$ \\
\hline 549 & $\checkmark$ & & $\checkmark$ & & & & & & & & & $\mathrm{a}$ \\
\hline 551 & & $\checkmark$ & & $\checkmark$ & $\checkmark$ & & & $\checkmark$ & $\checkmark$ & $\checkmark$ & $\checkmark$ & $\mathrm{a}, \mathrm{c}, \mathrm{d}$ \\
\hline 568,663 & 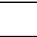 & & & & & $\checkmark$ & & & & & & $\mathrm{b}$ \\
\hline 664 & $\checkmark$ & & $\checkmark$ & & & & & & & & & $\mathrm{a}$ \\
\hline 751 & $\checkmark$ & & $\checkmark$ & & & & & & & & & $\mathrm{a}$ \\
\hline 896 & $\checkmark$ & & $\checkmark$ & & & & & & & & & $\mathrm{a}$ \\
\hline 897 & & $\checkmark$ & & $\checkmark$ & $\checkmark$ & & $\checkmark$ & $\checkmark$ & $\checkmark$ & $\checkmark$ & $\checkmark$ & $\mathrm{a}, \mathrm{b}, \mathrm{c}, \mathrm{d}$ \\
\hline 898,1195 & . & & & & & $\checkmark$ & & & & & & $\mathrm{b}$ \\
\hline 1351 & $\checkmark$ & & $\checkmark$ & & & & & & & & & $a$ \\
\hline 1492 & & & & & & $\checkmark$ & & & & & & $\mathrm{b}$ \\
\hline 1498 & & $\checkmark$ & & $\checkmark$ & & & $\checkmark$ & $\checkmark$ & $\checkmark$ & & & $\mathrm{a}, \mathrm{b}, \mathrm{c}$ \\
\hline 1793,2092 & & & & & & $\checkmark$ & & & & & & $\mathrm{b}$ \\
\hline 2098 & & $\checkmark$ & & $\checkmark$ & & & $\checkmark$ & $\checkmark$ & $\checkmark$ & $\checkmark$ & $\checkmark$ & $\mathrm{a}, \mathrm{b}, \mathrm{c}, \mathrm{d}$ \\
\hline 2251,2851 & $\checkmark$ & & $\checkmark$ & & & & & & & & & $\mathrm{a}$ \\
\hline 2988 & & $\checkmark$ & & & & $\checkmark$ & & $\checkmark$ & $\checkmark$ & & & $\mathrm{b}, \mathrm{c}$ \\
\hline 2998 & & & & $\checkmark$ & & & $\checkmark$ & & & & & $\mathrm{a}, \mathrm{b}$ \\
\hline 3451 & $\checkmark$ & & $\checkmark$ & & & & & & & & & $\mathrm{a}$ \\
\hline 3568 & & & & & & $\checkmark$ & & & & & & $\mathrm{b}$ \\
\hline 3600 & & $\checkmark$ & & $\checkmark$ & & & $\checkmark$ & $\checkmark$ & $\checkmark$ & $\checkmark$ & $\checkmark$ & $\mathrm{a}, \mathrm{b}, \mathrm{c}, \mathrm{d}$ \\
\hline 3851,4051 & $\checkmark$ & & $\checkmark$ & & & & & & & & & $\mathrm{a}$ \\
\hline 4184 & & & & & & $\checkmark$ & & & & & & $\mathrm{b}$ \\
\hline 4200 & & $\checkmark$ & & $\checkmark$ & & & $\checkmark$ & $\checkmark$ & $\checkmark$ & & & $\mathrm{a}, \mathrm{b}, \mathrm{c}$ \\
\hline 4243 & $\checkmark$ & & $\checkmark$ & & & & & & & & & $\mathrm{a}$ \\
\hline 4581 & & & & & & $\checkmark$ & & & & & & $\mathrm{b}$ \\
\hline 4600 & & $\checkmark$ & & $\checkmark$ & & & $\checkmark$ & $\checkmark$ & $\checkmark$ & $\checkmark$ & $\checkmark$ & $\mathrm{a}, \mathrm{b}, \mathrm{c}, \mathrm{d}$ \\
\hline 4802 & & $\checkmark$ & & $\checkmark$ & & & $\checkmark$ & $\checkmark$ & $\checkmark$ & & & $\mathrm{a}, \mathrm{b}, \mathrm{c}$ \\
\hline 4970 & & & & & & $\checkmark$ & & & & & & $\mathrm{b}$ \\
\hline 4989 & & $\checkmark$ & & $\checkmark$ & & & $\checkmark$ & $\checkmark$ & $\checkmark$ & $\checkmark$ & $\checkmark$ & $\mathrm{a}, \mathrm{b}, \mathrm{c}, \mathrm{d}$ \\
\hline
\end{tabular}
a. ${ }^{234}$ Th data from Owens et al. (2015)
b. ${ }^{230}$ Th data from Anderson et al. (2012); Hayes et al. (2015b).
c. ${ }^{228}$ Th data from Charette et al. (2015)
d. Particle concentration data from Lam et al. (2015a)

Table A1: Radiochemical and Particle Concentration Data at Station GT11-22. 


\section{B Appendix B}

We use a Kolmogorov-Smirnov (KS) test (Dudewicz and Mishra, 1988) in order to test the null hypothesis that the results of our reference solution (section 2.3) are not significantly different from the results derived from the changes discussed in sections 2.4.1.2-2.4.1.5. The two-sample KS test compares the maximum vertical distance between two empirical distribution functions (DFs). This distance is used to construct the probability ( $p$ value) of rejecting a null hypothesis that is correct. The $p$ values for each test are reported in Table B1.

\begin{tabular}{|c|c|c|c|c|}
\hline & model-based $\hat{\boldsymbol{x}}_{k=0}$ & $\mathrm{Th}_{p, l} / \mathrm{Th}_{p, s}=0.07$ & corrected ${ }^{230} \mathrm{Th}(\mathrm{a})$ & QMA bias $20 \%$ \\
\hline$p$ for V1 & $>0.99$ & $>0.99$ & $>0.99$ & 0.98 \\
\hline$p$ for V2 & $>0.99$ & 0.52 & $>0.99$ & 0.02 \\
\hline
\end{tabular}

a.measured ${ }^{230} \mathrm{Th}_{d, p}$ corrected for lithogenic ${ }^{230} \mathrm{Th}$.

Table B1: $p$ values from Kolmogorov-Smirnov test.

With one exception, the $p$ values all show that the two considered solutions are not significantly different. The exception is the test between our reference solution and the solution obtained from correcting for a potential bias in the QMA filters. Although the KS test suggests that the normalized residuals of the fit of model V2 to GT11-22 data do not have the same underlying distribution at the $5 \%$ significance level ( $p=0.02)$, the DFs of the two solutions look very similar (Figure B1). Thus, in fact, the solution with reduced ${ }^{234,228} \mathrm{Th}_{p}$ data values and the reference solution generally lead to similar results, in particular regarding the relative merits of model V1 and V2 (Table 3). 


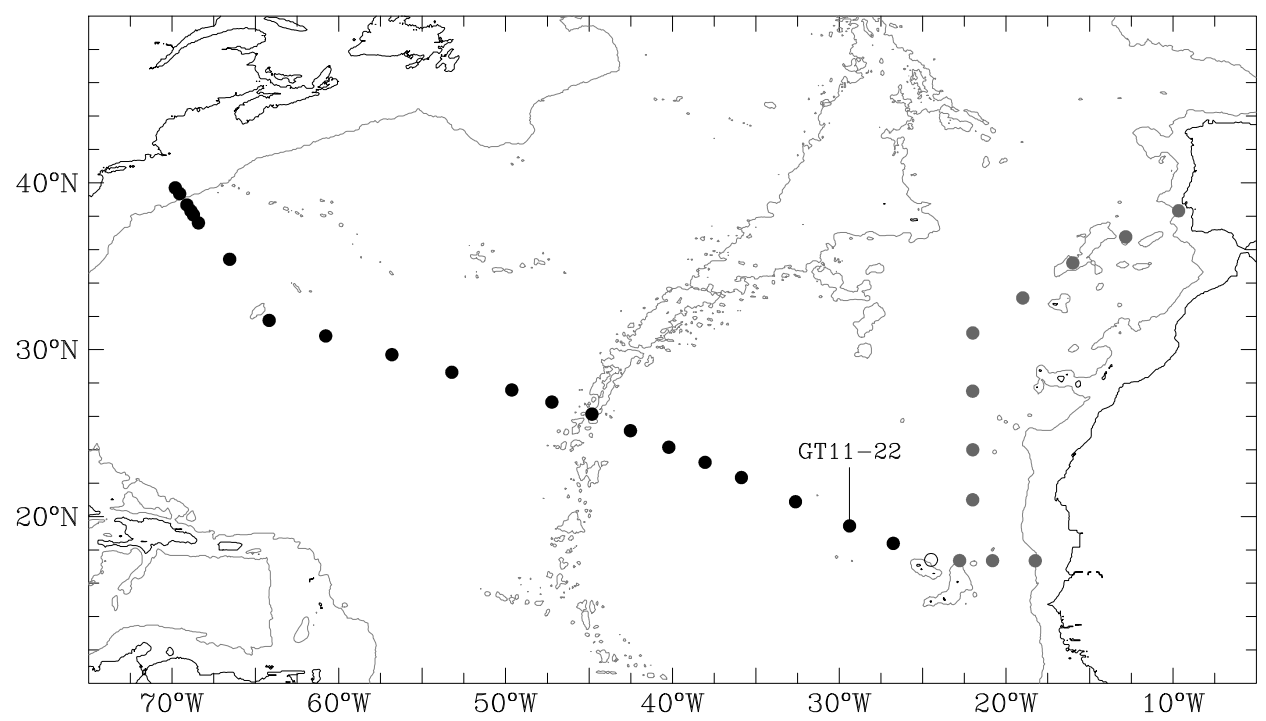

Figure 1: Stations occupied by the $\mathrm{R} / \mathrm{V}$ Knorr during the GEOTRACES North Atlantic section. The latitude and longitude of the stations are from http://data.bcodmo.org/jg/dir/BCO/GEOTRACES/NorthAtlanticTransect/. The grey dots show the stations occupied during the first leg (October 2010) and the black dots show the stations occupied during the second leg (November 2011). The open circle is both station 12 of the first leg and station 24 of the second leg. The data analyzed in this paper originate from station GT11-22, northwest of Cape Verde. The solid lines show the coastline (dark) and the 3000-m isobath (light) (bathymetric data are from http://www.ngdc.noaa.gov/mgg/global). 

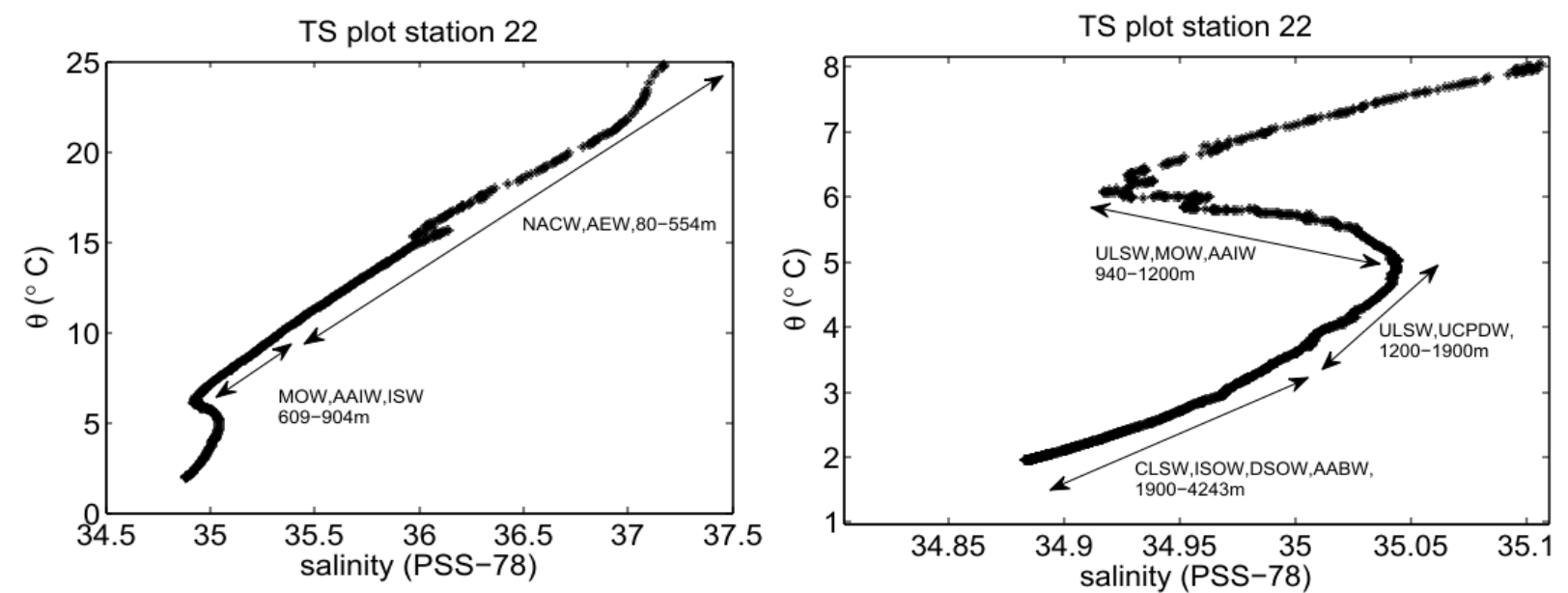

Figure 2: Plot of potential temperature vs. salinity for station GT11-22. Arrows indicate the estimated range of water masses. Shown are the labels and the depth range for the water masses. The left panel shows the $\theta-S$ plot from the surface to a depth of $904 \mathrm{~m}$. The right panel shows the $\theta-S$ plot below $8^{\circ} \mathrm{C}$. Data from Jenkins et al. (2015) 

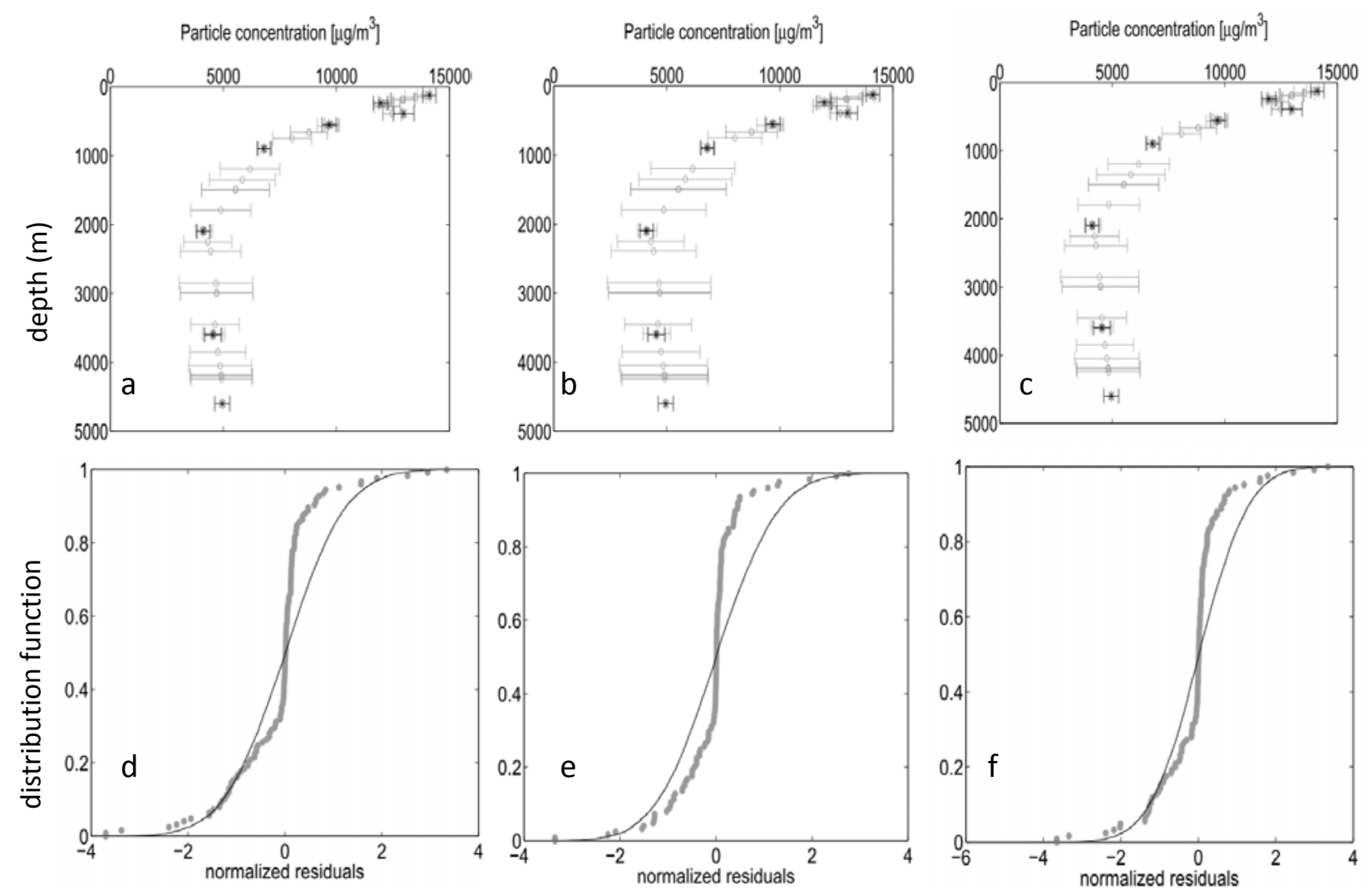

Figure 3: Top row (a-c): Measured (black asterisks) and interpolated (open circles) values of particle concentration $(P)$ at station GT11-22 for three different field property variances: $\sigma_{M}^{2}=0.25 \sigma_{d}^{2}(\mathrm{a}), \sigma_{M}^{2}=0.5 \sigma_{d}^{2}(\mathrm{~b})$, and $\sigma_{M}^{2}=\sigma_{d}^{2}(\mathrm{c})$, where $\sigma_{d}^{2}$ is the variance of $P$ measurements at station GT11-22. A length scale of $l_{z}=1000 \mathrm{~m}$ is used for each panel. Measured particle concentrations are from Lam et al. (2015a). The deepest measured value used in the interpolation of particle concentration is at $4600 \mathrm{~m}$. Bottom row (d-f): Distribution function (DF) of the interpolation residuals normalized to measurement errors for all GT11-22 data used in this study. A normalized residual is defined as $\left(\hat{x}_{i}-\right.$ $\left.x_{d, i}\right) / \sigma_{d, i}$, where $\hat{x}_{i}$ is the interpolated value at measurement depth level i, $x_{d, i}$ is the measured value at this level, and $\sigma_{d, i}$ is the error in the measurement at this level. For the three values of $\sigma_{M}^{2}$, the difference $\left|\hat{x}_{i}-x_{d, i}\right|$ is less than $2 \sigma_{d, i}$ for more than $95 \%$ of the data, which suggests an overfit (the solid line shows the normal DF). 

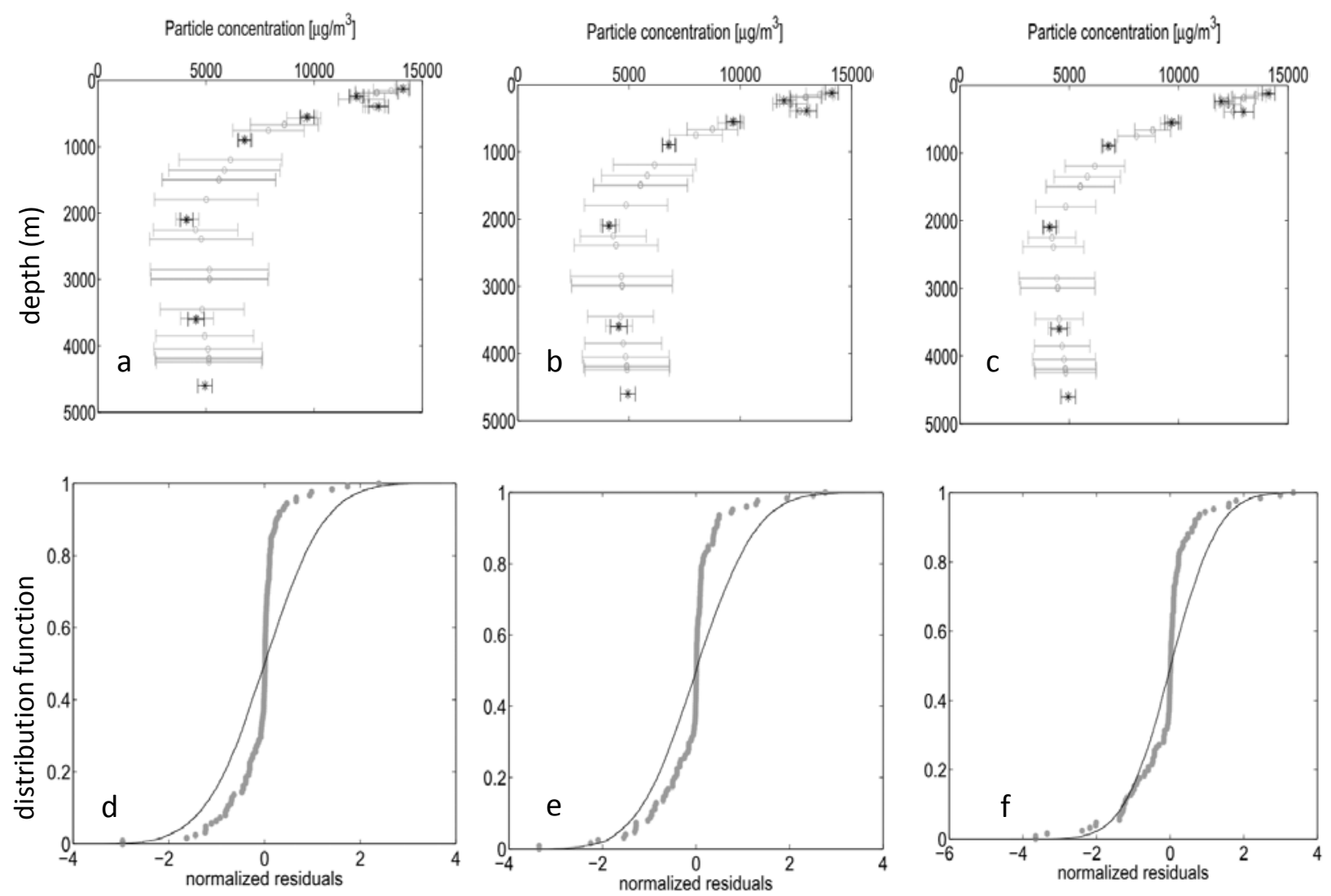

Figure 4: Same as Figure 3, but for three different vertical correlation scales:

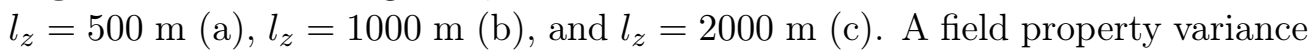
of $\sigma_{M}^{2}=0.5 \sigma_{d}^{2}$ is used for each panel. Measured particle concentrations are from Lam et al. (2015a). 

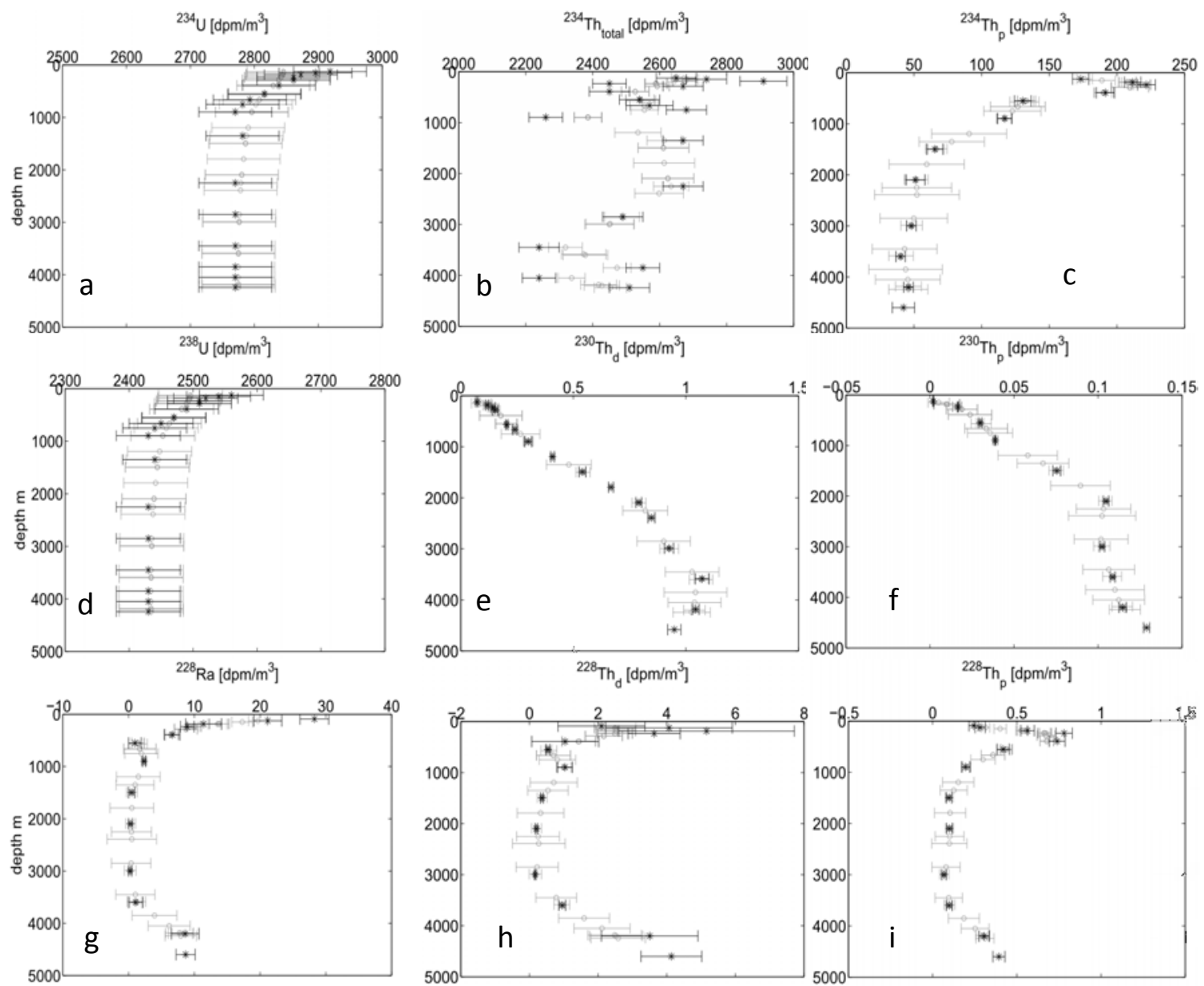

Figure 5: Profiles of Th isotope activities and their parent activities at station GT11-22. The black asterisks are the measured values. The open circles are the interpolated values obtained with $\sigma_{M}^{2}=0.5 \sigma^{2}, l_{z}=1000 \mathrm{~m}$. 


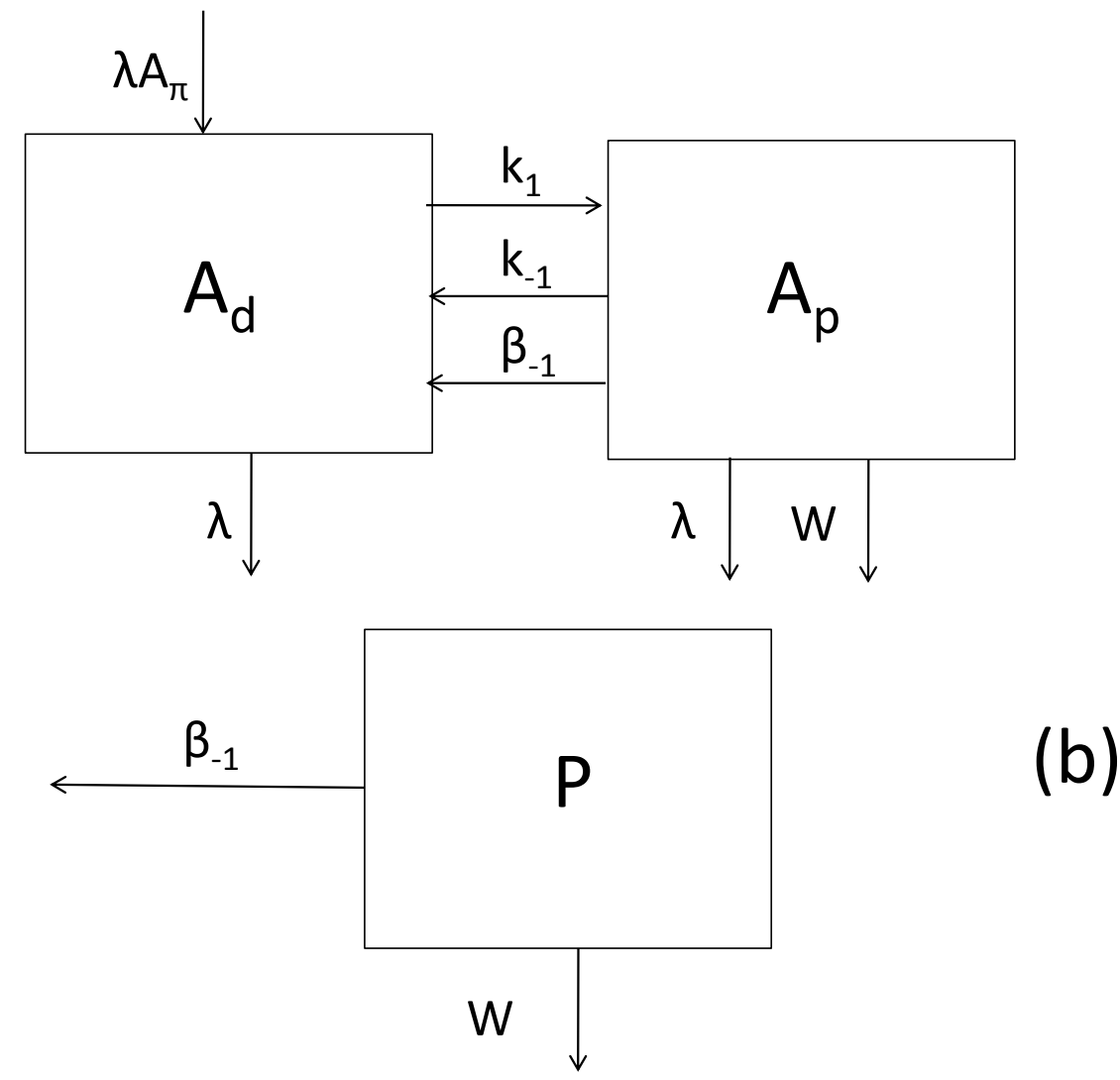

Figure 6: Schematic diagram of the single-particle class model of Th cycling (a) and particle cycling (b). ' $A$ ' and ' $\mathrm{P}$ ' represent, respectively, the Th isotope activity and the particle concentration in the dissolved fraction ('d') or the particulate fraction ('p'). $A_{\pi}$ is the activity of the parent isotope. The other symbols represent the rate parameters of solid-solution exchange ( $k_{1}$ for adsorption, $k_{-1}$ for desorption) and particle processes $\left(\beta_{-1}\right.$ for remineralization, and $w$ for particle sinking). $\lambda$ is the radioactive decay constant. 


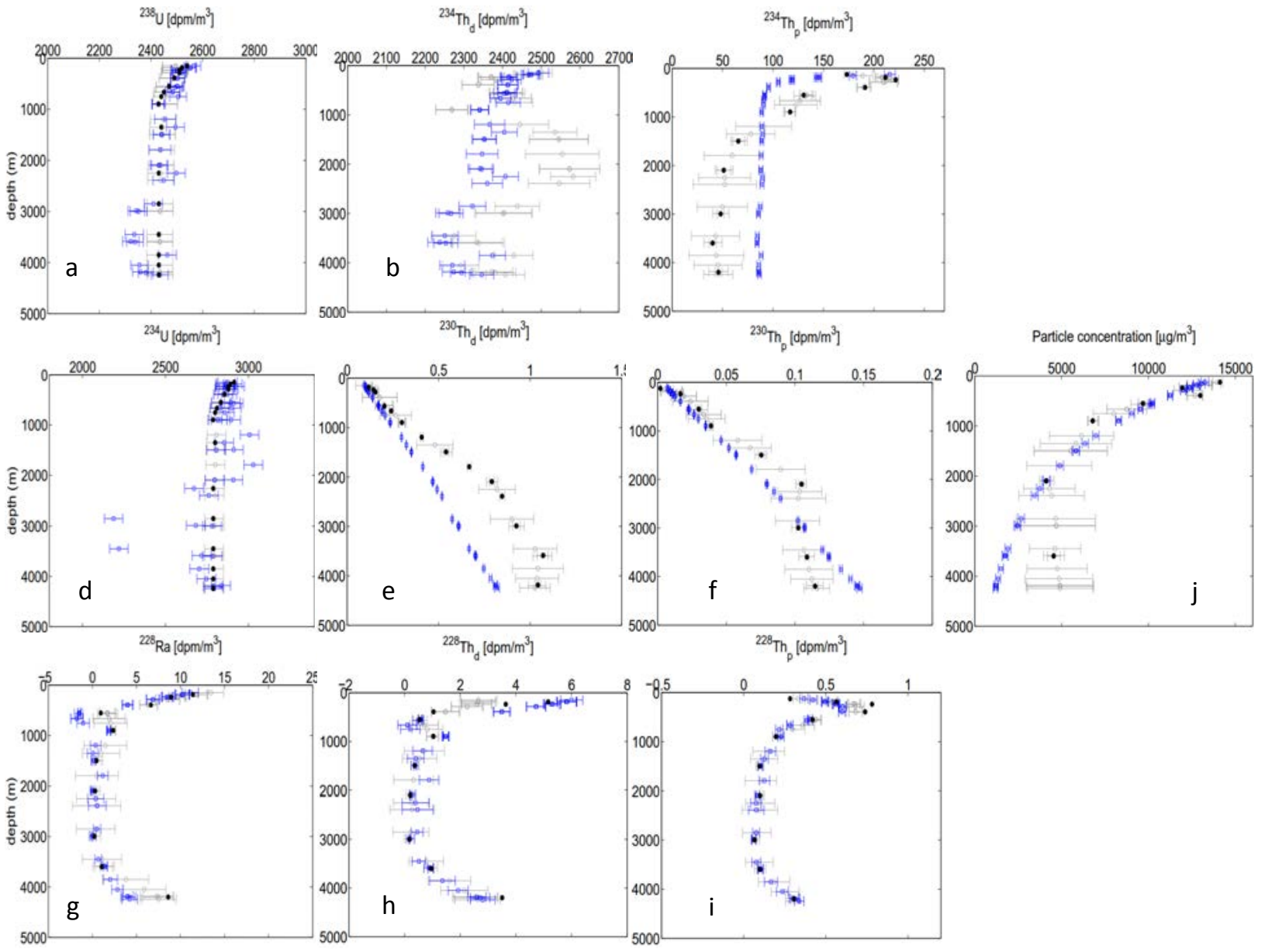

Figure 7: Radiochemical activities and particle concentrations at station GT11-22. The black circles are the measured values, the open circles are the interpolated values, and the blue squares are the fitted values for model V1. Horizontal bars represent \pm 1 standard deviation. 

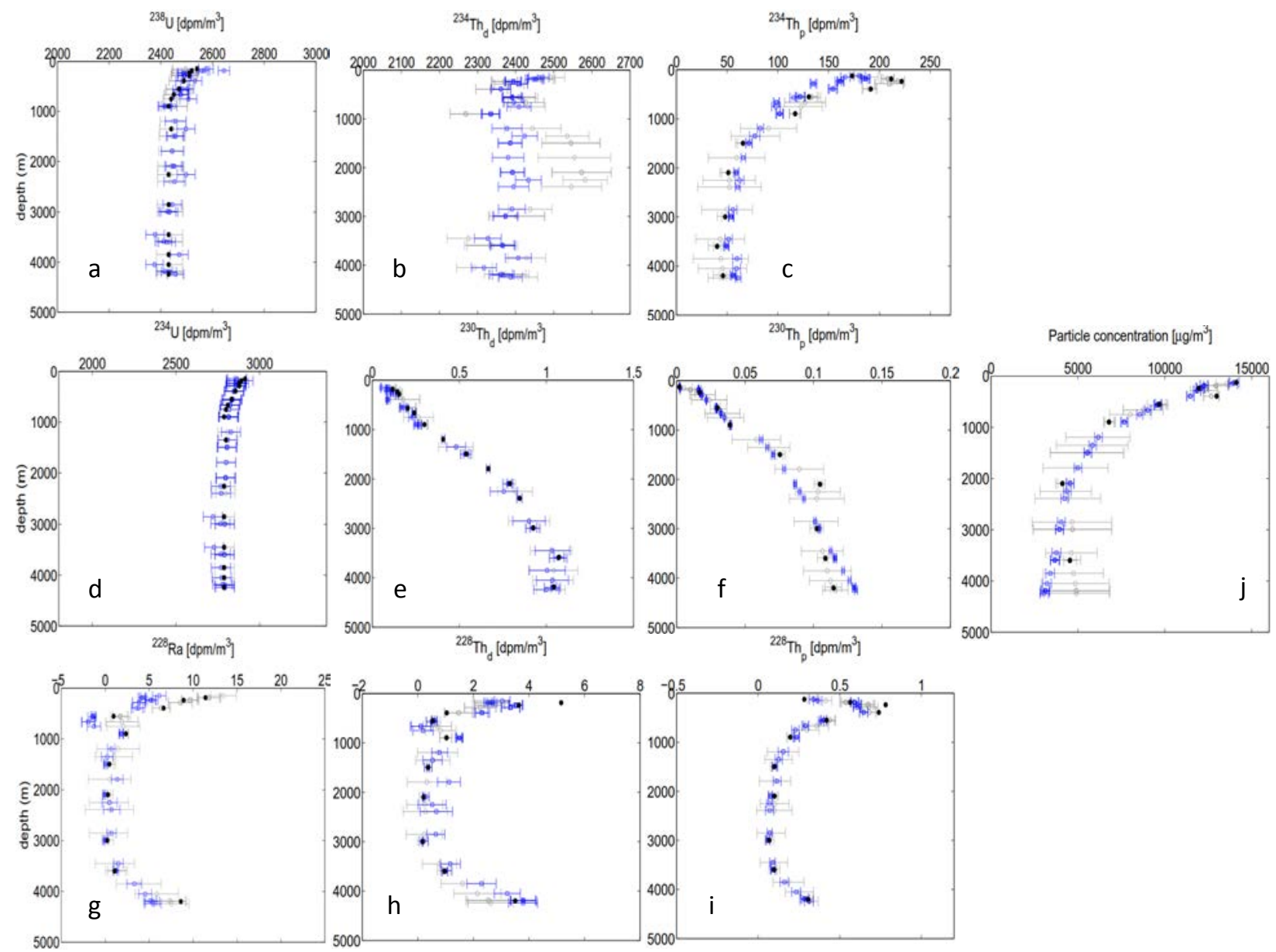

Figure 8: Same as figure 7, but for model V2. 


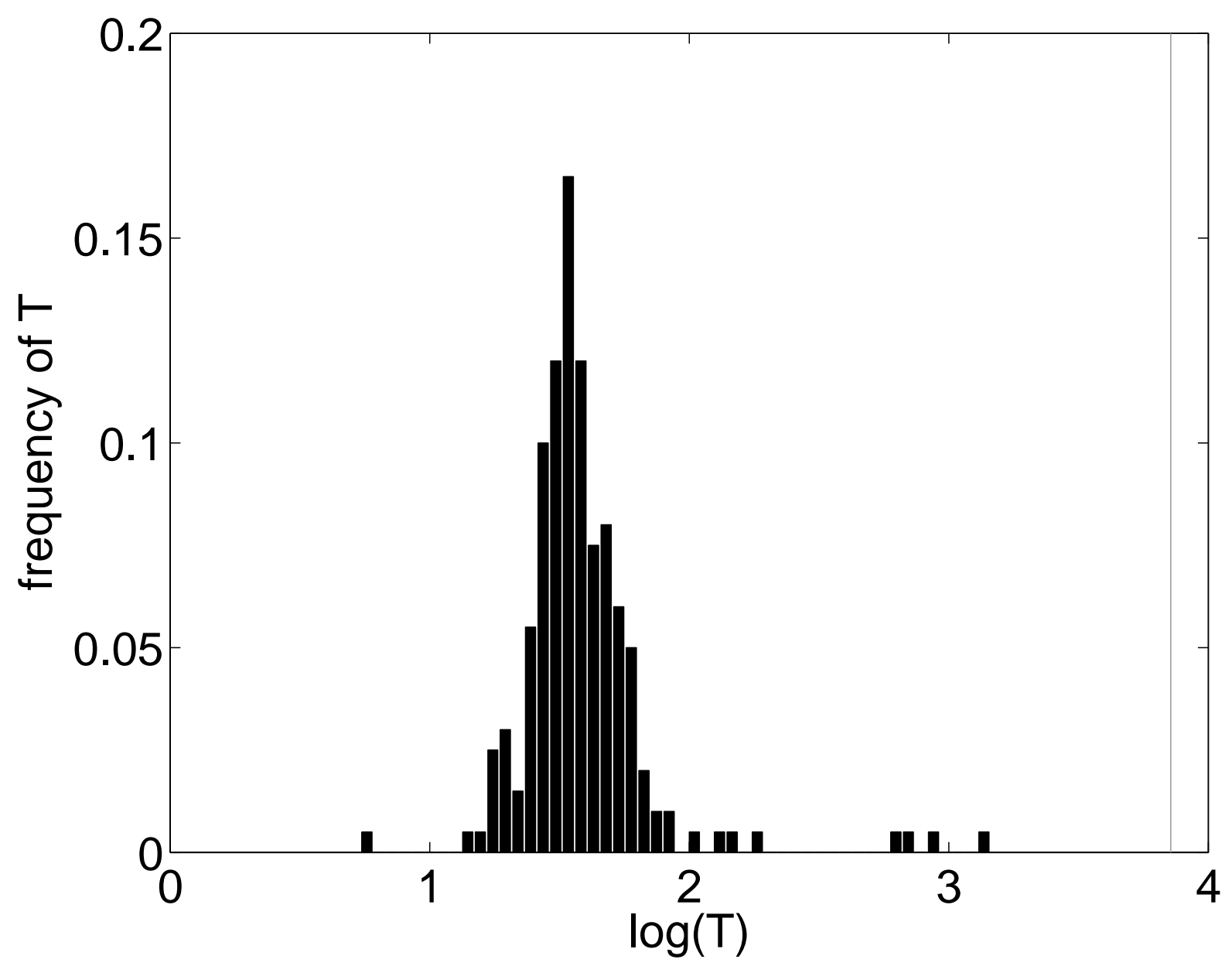

Figure 9: Histogram of the frequency of the test statistic $T$ obtained from the bootstrap. The grey line shows the value of $T$ obtained from our reference solution (section 2.3). The abscissa is the logarithm of the test statistic. Note that two negative values of $\mathrm{T}$ were found (-67 and -905), which are not shown in the figure 

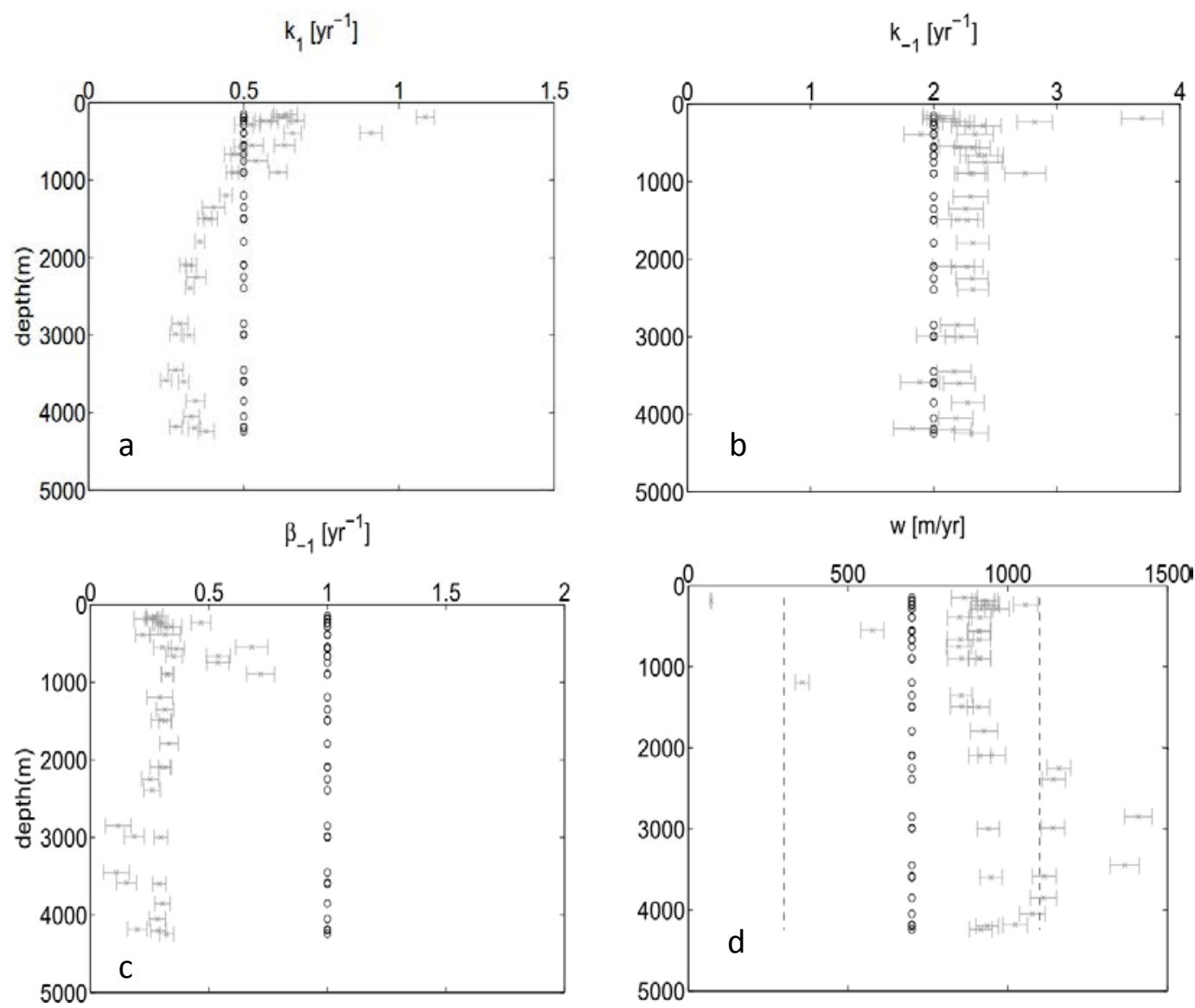

Figure 10: Rate parameters of Th and particle cycling at station GT11-22 as inferred from the combination of data with model V2. The open circles are the prior values $\left(k_{1}=0.5 \pm 5 \mathrm{yr}^{-1}, k_{-1}=2 \pm 5 \mathrm{yr}^{-1}, \beta_{1}=1 \pm 10 \mathrm{yr}^{-1}\right.$, $w=700 \pm 400 \mathrm{~m} \mathrm{yr}^{-1}$ ). The vertical dashed lines in (d) show the range [3001100] $\mathrm{m} \mathrm{yr}^{-1}$ for the prior estimate of the particle sinking speed. The grey crosses are the posterior estimates and their errors (obtained by inversion). For comparison, the rate parameters inferred from the combination of data with model V1 are $k_{1}=0.49 \pm 0.01 \mathrm{yr}^{-1}, k_{-1}=2.11 \pm 0.07 \mathrm{yr}^{-1}, \beta_{1}=0.46 \pm 0.02$ $\mathrm{yr}^{-1}, w=725 \pm 7 \mathrm{~m} \mathrm{yr}^{-1}$ 

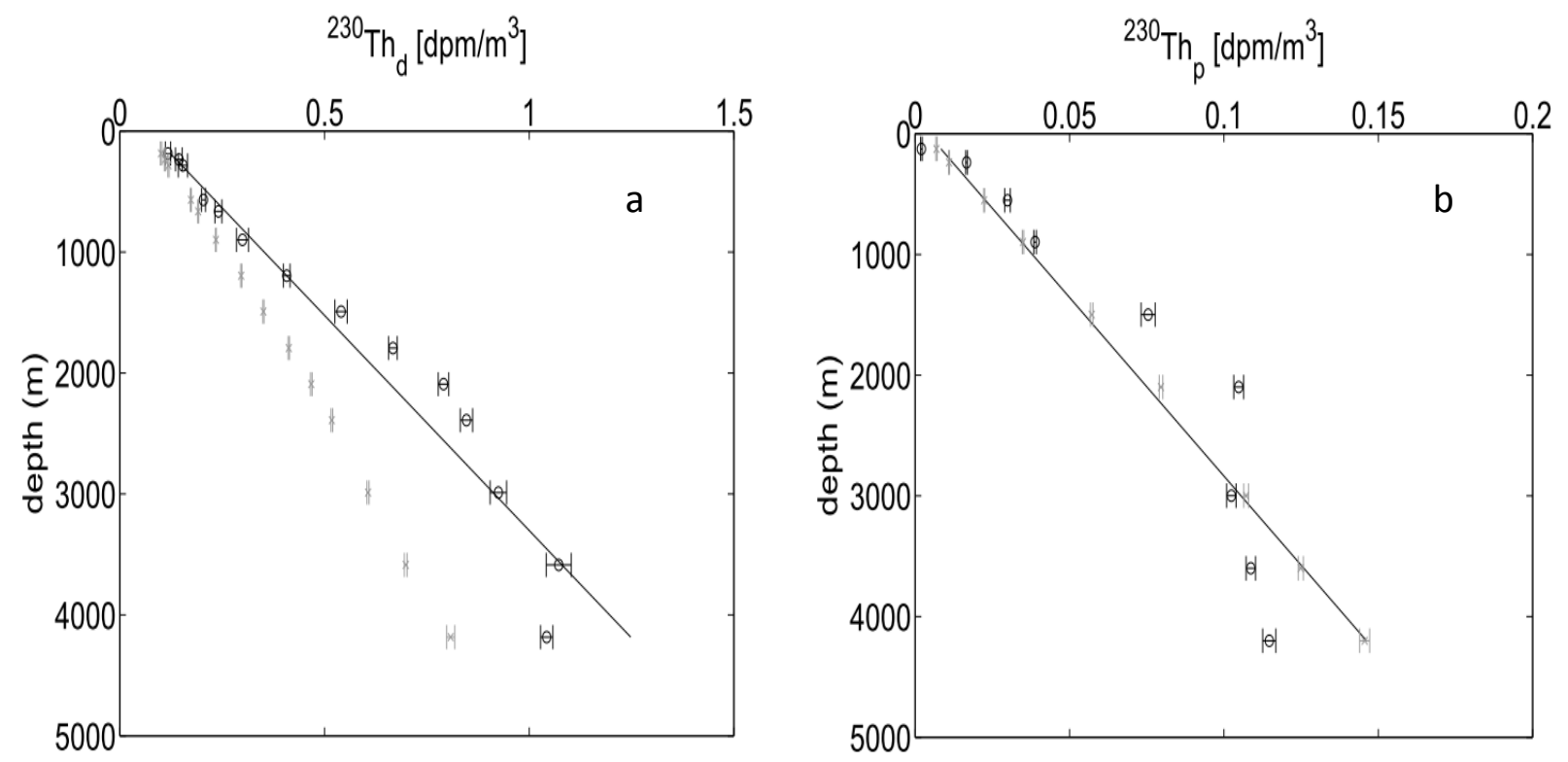

Figure 11: The left panel shows the ${ }^{230} \mathrm{Th}_{d}$ measurements at station GT1122 (open circles), the posterior estimates of ${ }^{230} \mathrm{Th}_{d}$ and their errors from the fit of model V1 (grey crosses) to the entire dataset $\left({ }^{234,238} \mathrm{U},{ }^{228,230,234} \mathrm{Th}_{d, p}, P\right)$, and the line from fit of model V1 to only dissolved ${ }^{230} \mathrm{Th}$ data (derived from weighted least squares regression of ${ }^{230} \mathrm{Th}_{d}$ with depth). The right panel is the same as the left panel, but for ${ }^{230} \mathrm{Th}_{p}$ 

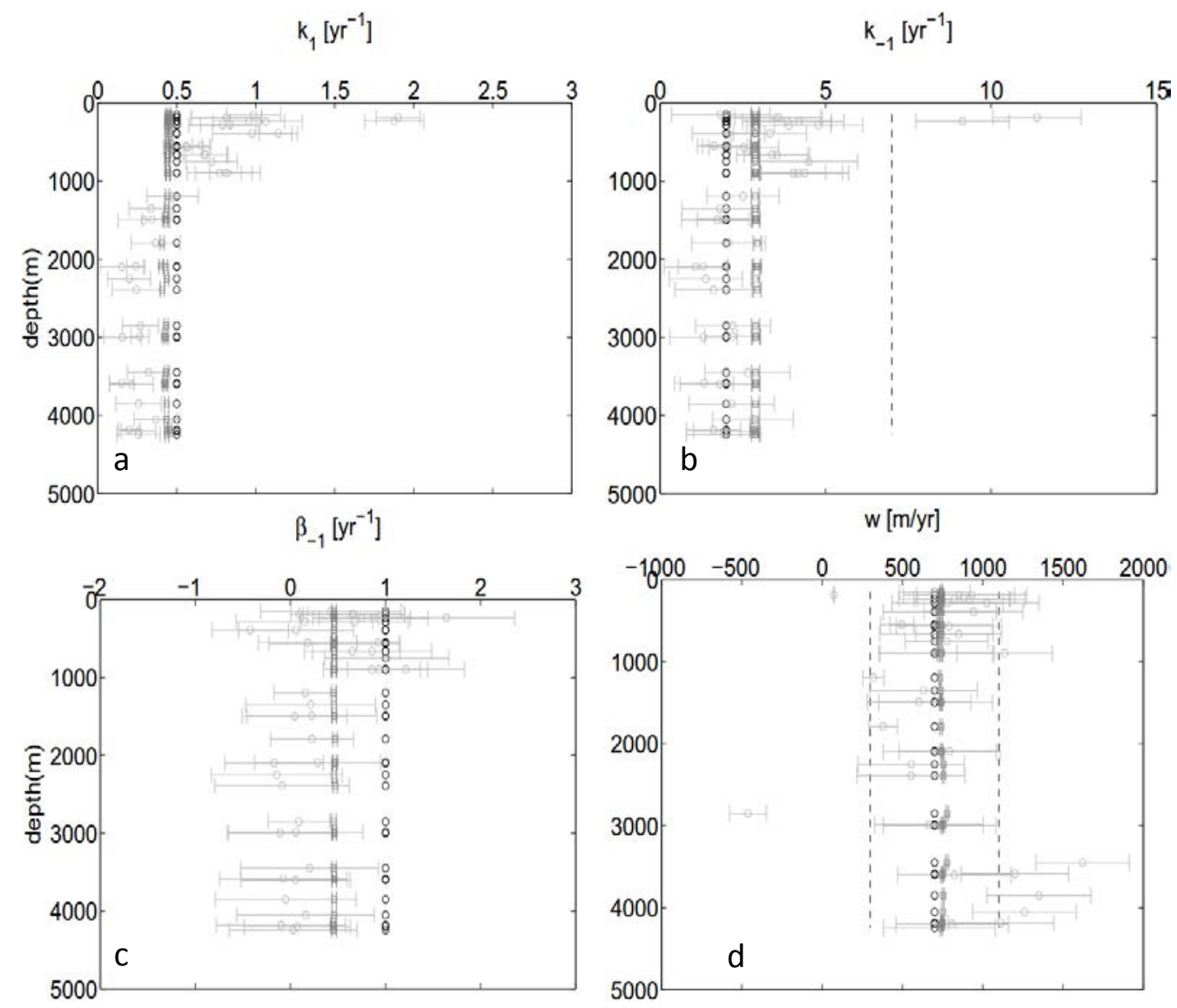

Figure 12: Rate parameters of Th and particle cycling at station GT11-22 as inferred from the combination of data with model V2. The vertical dashed lines are the prior estimates, and the posterior estimates for $\gamma=0.01$ and $\gamma=100$ are represented by open grey circles and black squares, respectively. The solid lines show the range [value \pm one standard deviation] for the prior estimates. 

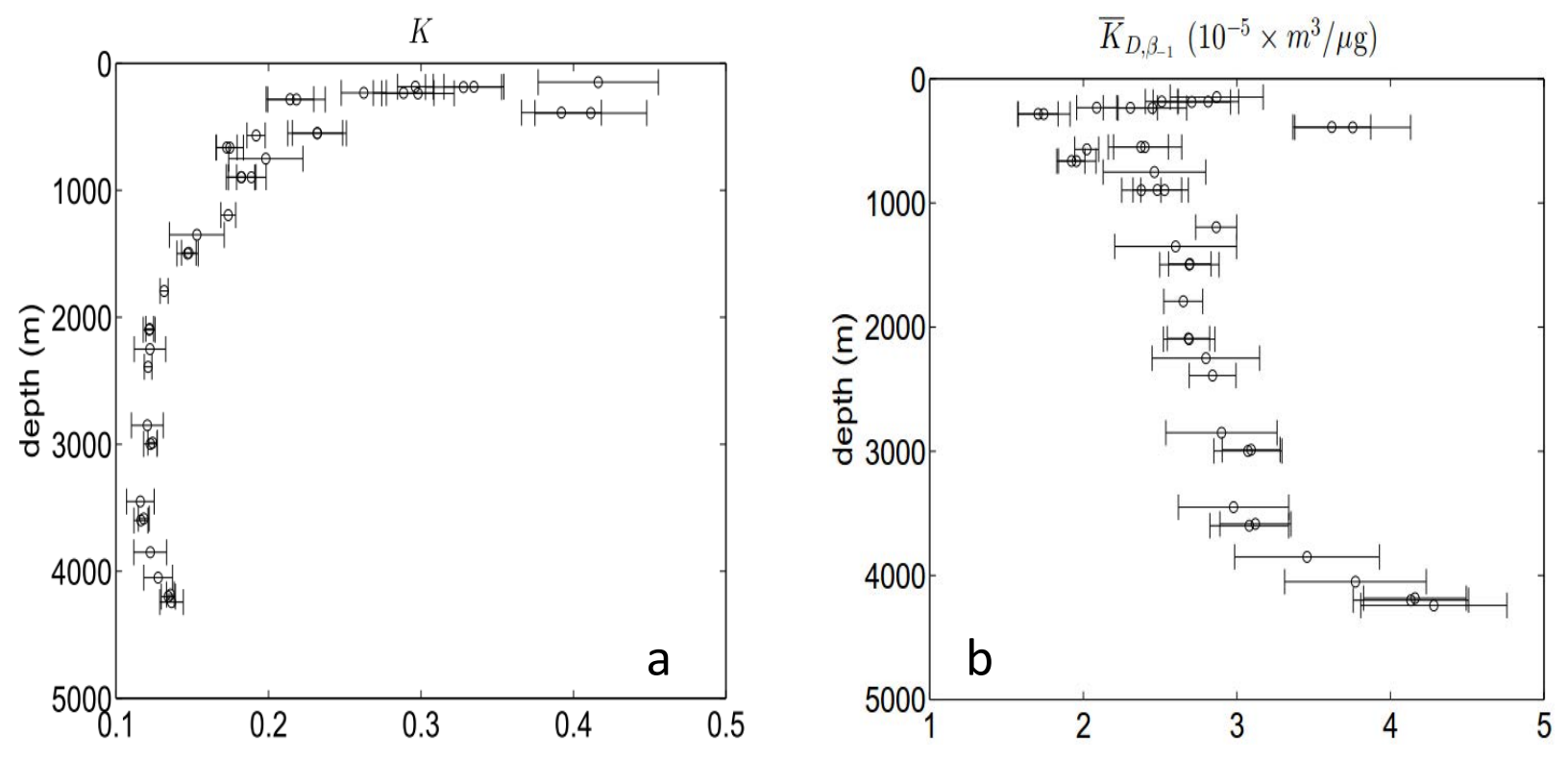

Figure 13: Profiles of $K=k_{1} /\left(k_{-1}+\beta_{-1}\right)$ (a) and $\bar{K}_{D, \beta_{-1}}=K / P$ (b) at station GT11-22, as inferred from the combination of data with model V2. The horizontal bars show the errors (one standard deviation) derived from propagating the posterior errors of $k_{1}, k_{-1}, \beta_{-1}$ (and $P$ for $\bar{K}_{D, \beta_{-1}}$ ), with due regard for the error covariances. 

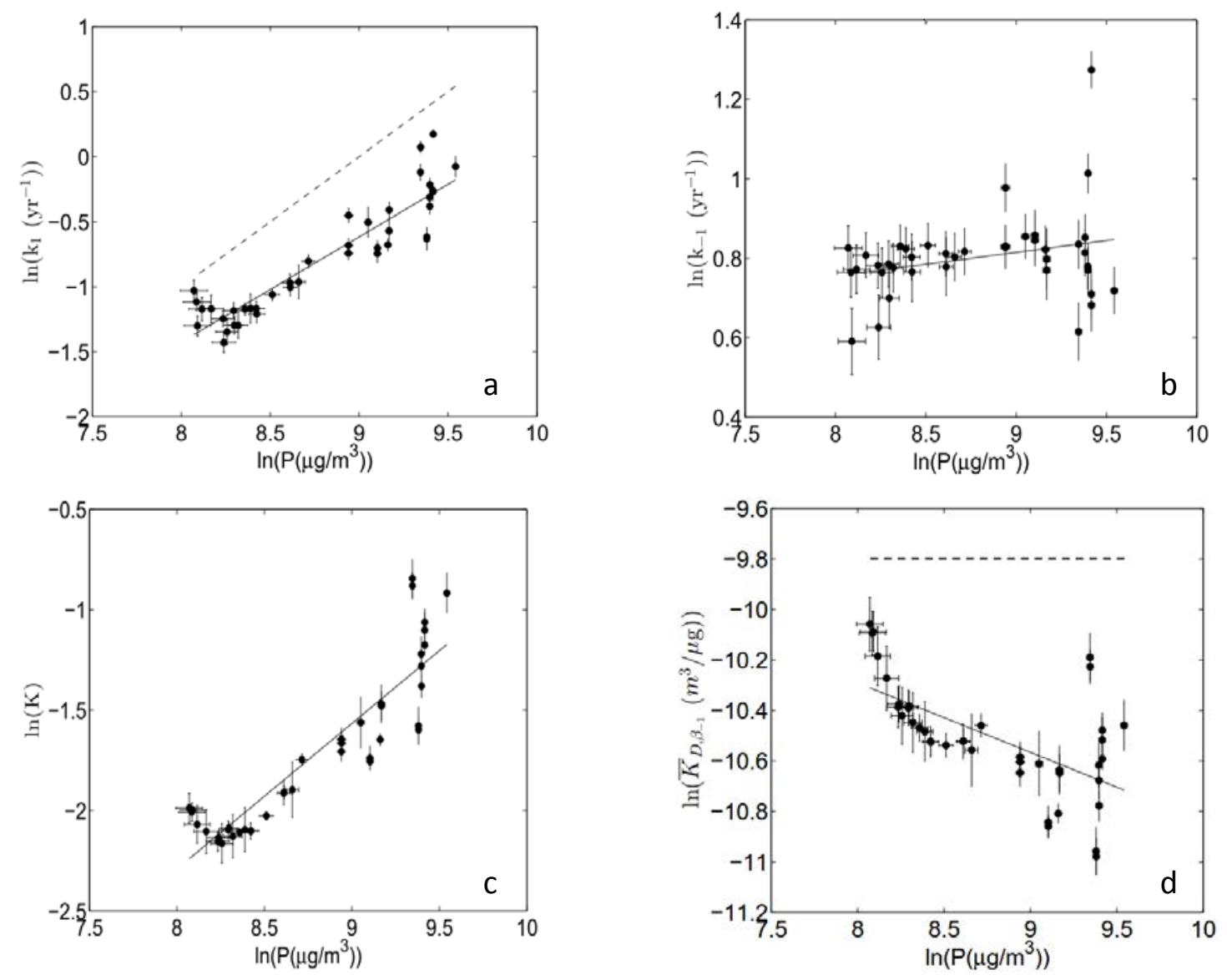

Figure 14: Variation of $k_{1}$ (a), $k_{-1}$ (b), $K=k_{1} /\left(k_{-1}+\beta_{-1}\right)$ (c), and $\bar{K}_{D, \beta_{-1}}=$ $K / P(\mathrm{~d})$ with particle concentration, as inferred from inversion of station GT1122 data. In each panel, the solid line is the ordinary least squares fit. The slopes of these lines are (a) $0.60 \pm 0.05$, (b) $-0.06 \pm 0.04$, (c) $0.64 \pm 0.06$, and (d) $-0.36 \pm 0.06$. In panels (a) and (d), the dashed line represents the slope expected in the absence of a particle concentration effect (the slope of this dashed line is 1 in panel a, and 0 in panel $d$ ). 

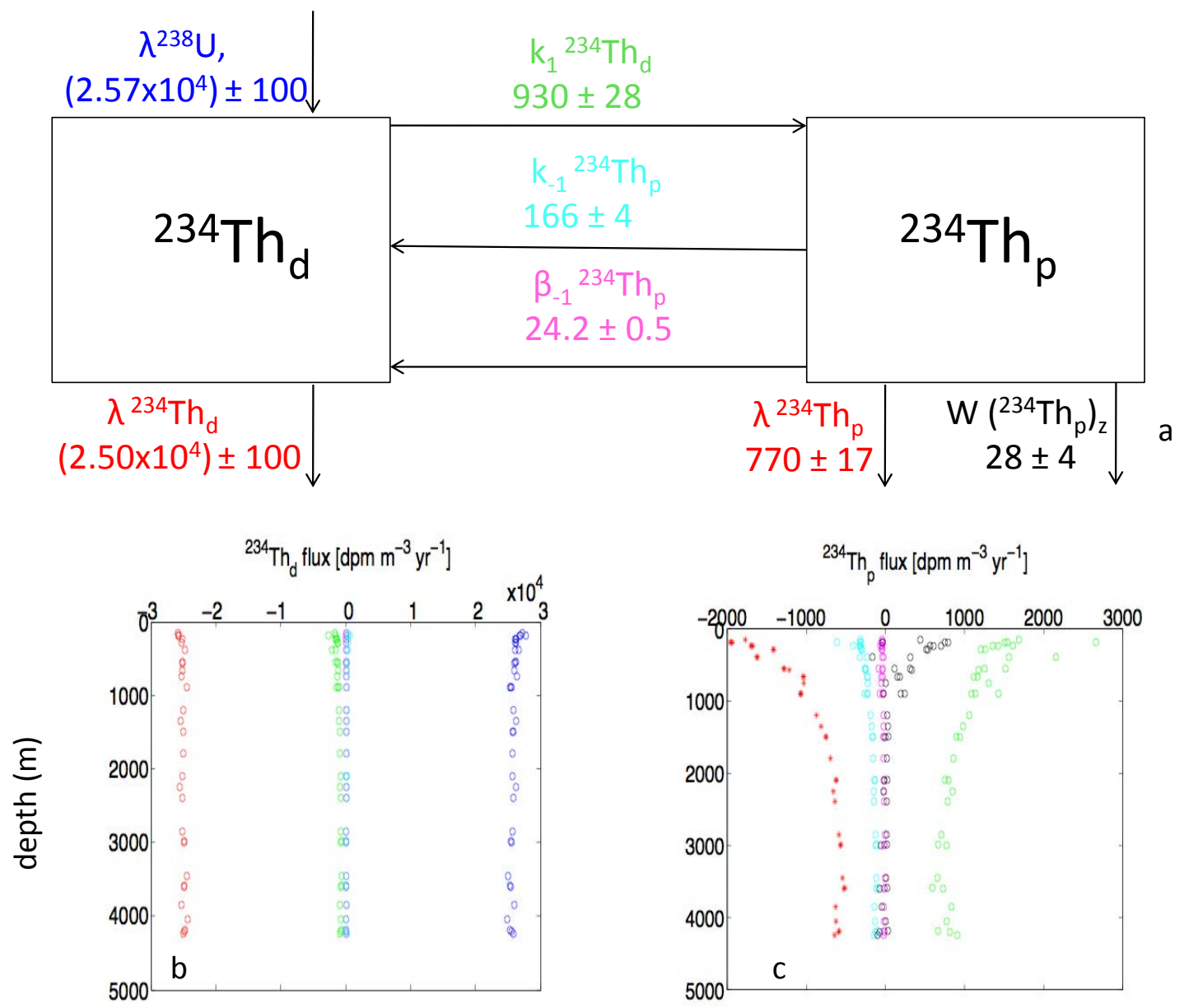

Figure 15: Panel (a) shows the ${ }^{234} \mathrm{Th}$ budget at station GT11-22. The displayed values are vertical averages (125-4243 m) in dpm m $\mathrm{m}^{-3} \mathrm{yr}^{-1}$. Panel (b) and (c) show the vertical distribution of the ${ }^{234}$ Th fluxes. In all panels, red for radioactive decay, blue for radioactive production, green for adsorption, cyan for desorption, magenta for remineralization, and black for the sinking flux. 


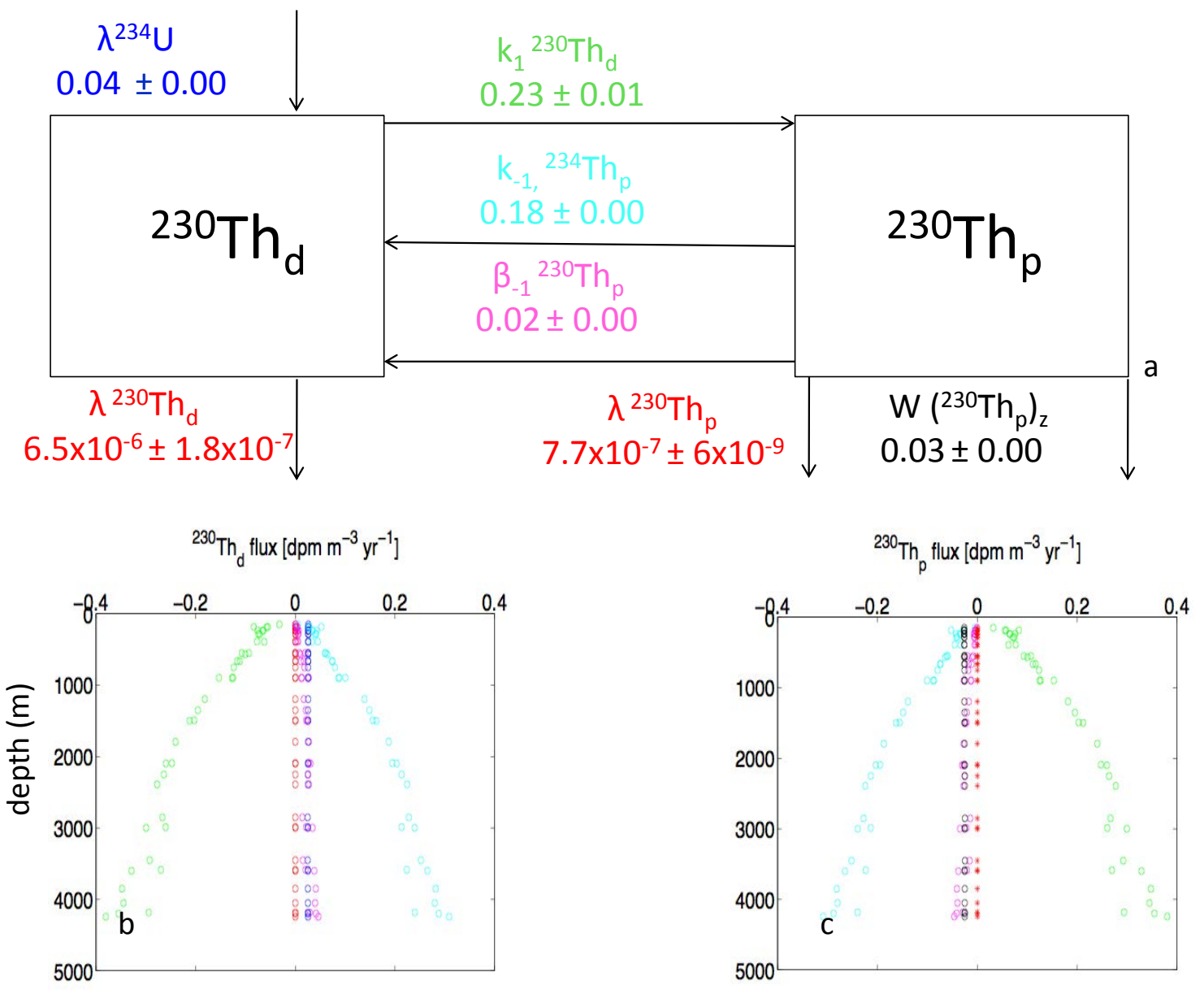

Figure 16: Same as Figure 15, but for ${ }^{228} \mathrm{Th}$. 


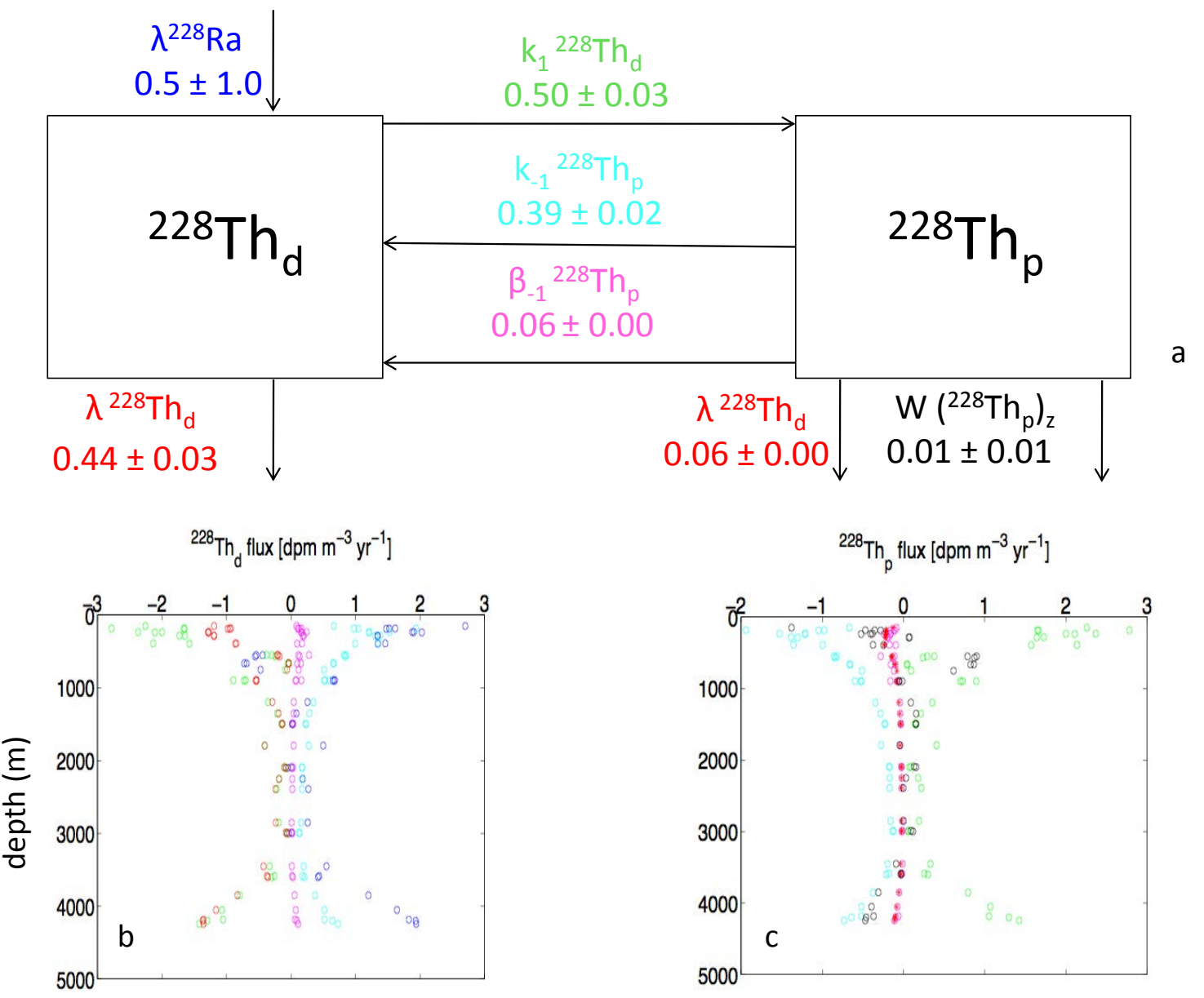

Figure 17: Same as Figure 15, but for ${ }^{230} \mathrm{Th}$. 


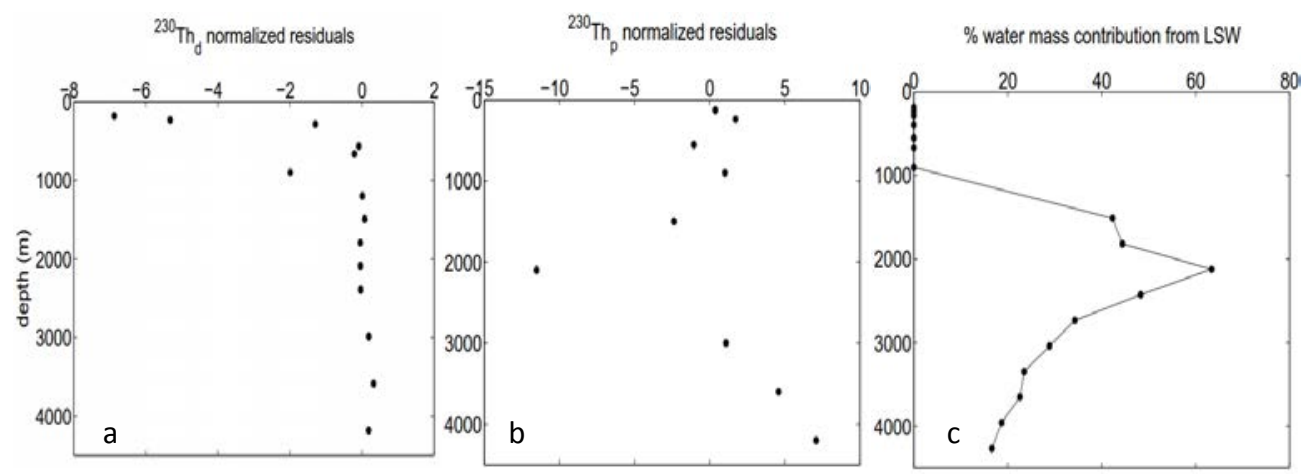

Figure 18: Panel (a) and (b) show the normalized residuals (eq. 2.11) of ${ }^{230} \mathrm{Th}_{d}$ and ${ }^{230} \mathrm{Th}_{p}$, respectively, for our reference solution (section 2.3). Panel (c) shows the sum of the proportions of Upper Labrador Sea Water and Central Labrador Sea Water at station GT11-22 (estimates from Jenkins et al. (2015)). 

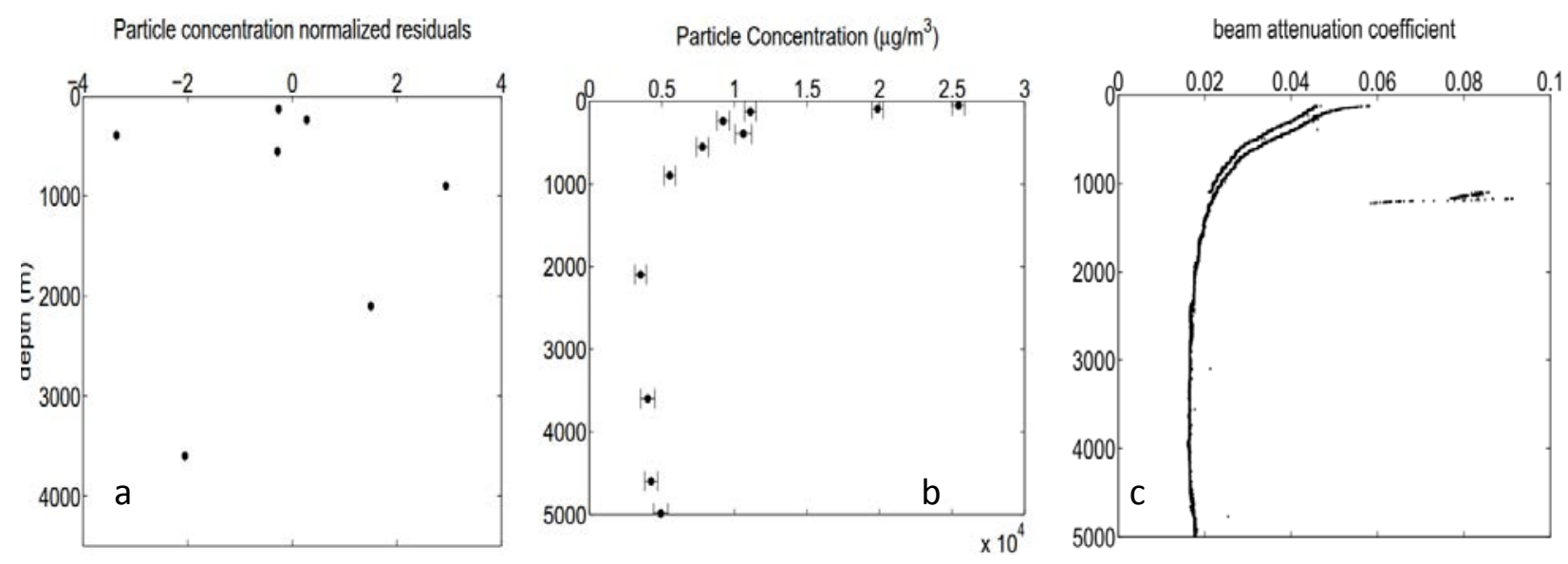

Figure 19: Panel (a) portrays the normalized residuals for particle concentration (eq. 2.11). Panel (b) portrays the particle concentration profile from $125 \mathrm{~m}$ to $4989 \mathrm{~m}$, the deepest depth at which small and large particles were sampled (data from Lam et al. (2015a)). Panel (c) shows the beam attenuation coefficient measured using a WET Labs $25 \mathrm{~cm}$ pathlength C-Star transmissometer $(660 \mathrm{~nm})$ (data from Anderson et al. (2013)). The very large values between 1000 and $1500 \mathrm{~m}$ are real measurements. 


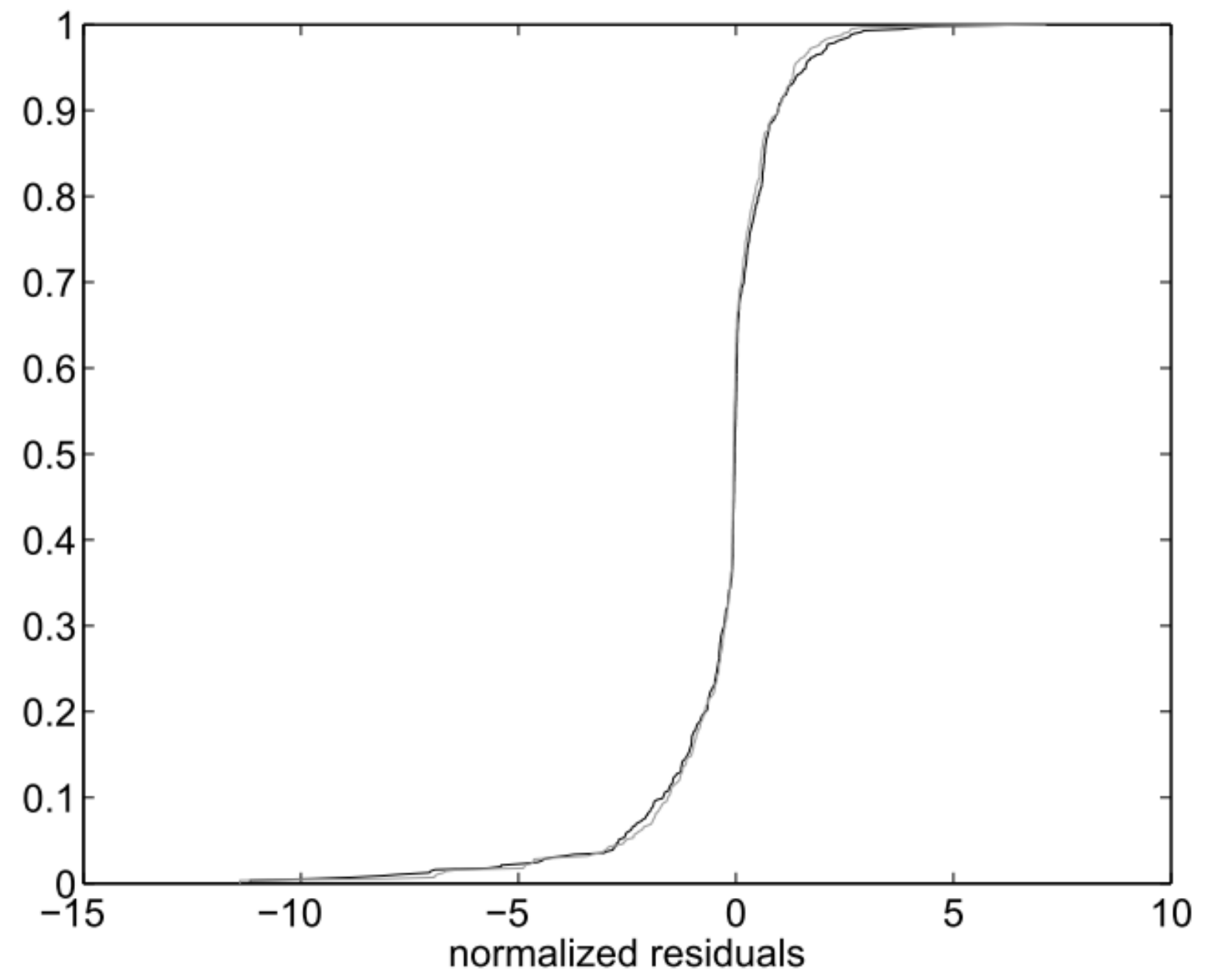

Figure B1: Normalized residuals of the fit of model V2 to station GT11-22 data for the reference case $\left(l_{z}=1000 \mathrm{~m}, \sigma_{M}^{2}=0.5 \sigma_{d}^{2}\right)$ (black), and for the case where the prior ${ }^{228,234} \mathrm{Th}_{p}$ values are set to $20 \%$ less than their measured values (grey). 


\section{Chapter 3}

\section{Kinetics of thorium and particle cycling}

\section{along the U.S. GEOTRACES North}

\section{Atlantic Transect}

This article was originally published in Deep-Sea Research Part I in 2017 by Elsevier, and has been slightly modified here. Elsevier permits the reproduction of this content in a dissertation/thesis.

Lerner, P., Marchal, O., Lam, P.J., Buesseler, K., and Solow, A., 2017, "Kinetics of thorium and particle cycling along the U.S. GEOTRACES North Atlantic Transect," Deep-Sea Research Part I, 125, 106-128, doi: 10.1016/j.dsr.2017.05.003 


\begin{abstract}
The high particle reactivity of thorium has resulted in its widespread use in tracing processes impacting marine particles and their chemical constituents. The use of thorium isotopes as tracers of particle dynamics, however, largely relies on our understanding of how the element scavenges onto particles. Here, we estimate apparent rate constants of Th adsorption $\left(k_{1}\right)$, Th desorption $\left(k_{-1}\right)$, bulk particle degradation $\left(\beta_{-1}\right)$, and bulk particle sinking speed $(w)$ along the water column at 11 open-ocean stations occupied during the GEOTRACES North Atlantic Section (GA03). First, we provide evidence that the budgets of Th isotopes and particles at these stations appear to be generally dominated by sorption reactions, particle degradation, and particle sinking. Rate parameters are then estimated by fitting a Th and particle cycling model to data of dissolved and particulate ${ }^{228,230,234} \mathrm{Th},{ }^{228} \mathrm{Ra}$, particle concentrations , and ${ }^{234,238} \mathrm{U}$ estimates based on salinity, using a nonlinear programming technique.

We find that the adsorption rate constant $\left(k_{1}\right)$ generally decreases with depth across the section: broadly, the time scale $1 / k_{1}$ averages $1.0 \mathrm{yr}$ in the upper $1000 \mathrm{~m}$ and (1.4-1.5) yr below. A positive relationship between $k_{1}$ and particle concentration $(P)$ is found, i.e., $k_{1} \propto P^{b}$, where $b \geq 1$, consistent with the notion that $k_{1}$ increases with the number of surface sites available for adsorption. The rate constant ratio, $K=k_{1} /\left(k_{-1}+\beta_{-1}\right)$, which measures the collective influence of rate parameters on Th scavenging, averages 0.2 for most stations and most depths. We clarify the conditions under which $K / P$ is equivalent to the distribution coefficient, $K_{D}$, test that the conditions are met at the stations, and find that $K(P) / P$ decreases with $P$ (where $K(P)$ indicates that $K$ itself can be a function of particle concentration), in line with a particle concentration effect $\left(d K_{D} / d P<0\right)$. In contrast to the influence of colloids as envisioned by the Brownian pumping hypothesis, we provide evidence that the particle concentration effect arises from the joint effect of $P$ on the rate constants for thorium attachment to, and detachment from, particles.
\end{abstract}

\title{
3.1 Introduction
}

One of the major questions in marine biogeochemistry concerns the processes controlling the formation, transport, and destruction of marine particles. Thorium can be useful for investigating these processes: thorium is highly particle reactive in seawater, with radioactive isotopes characterized by a wide range of half-lives: $t_{1 / 2}=24.101 \pm 0.025$ days for ${ }^{234} \mathrm{Th}$ (Knight and Macklin, 1948), $1.910 \pm 0.002 \mathrm{yr}$ for ${ }^{228} \mathrm{Th}$ (Kirby et al., 2002), and 75, 584 $\pm 110 \mathrm{yr}$ for ${ }^{230} \mathrm{Th}$ (Cheng et al., 2013). Additionally, the sources of these isotopes are well known. ${ }^{234} \mathrm{Th},{ }^{230} \mathrm{Th}$, and ${ }^{228} \mathrm{Th}$ are produced in situ by radioactive decay of ${ }^{238} \mathrm{U},{ }^{234} \mathrm{U}$, and ${ }^{228} \mathrm{Ra}$, respectively. The apparent quasi-conservative behavior of uranium ( Ku et al., 1977; Delanghe et al., 2002) allows the ${ }^{234} \mathrm{U}$ and ${ }^{238} \mathrm{U}$ activities to be estimated from salinity (Chen et al., 1986; Owens et al., 2011), whereas ${ }^{228}$ Ra is generally measured (Henderson et al., 2013). The high particle reactivity, widely different half-lives, and relatively well understood sources of thorium isotopes have led to their extensive use in tracing processes that affect particles and their chemical constituents such as particulate organic carbon.

The use of thorium isotopes to trace particle dynamics in the North Atlantic has a rich history. 
Brewer et al. (1980) relied on ${ }^{228,230,234} \mathrm{Th}$ (multiple mass numbers written as left superscript denote several isotopes) measurements on particulate material caught in sediment traps deployed between 414-5332 $\mathrm{m}$ in the Sargasso Sea $\left(31^{\circ} 32.5^{\prime} \mathrm{N}, 55^{\circ} 55.4^{\prime} \mathrm{W}\right)$ and between 389-5068 $\mathrm{m}$ off the coast of Barbados $\left(13^{\circ} 30.2^{\prime} \mathrm{N}, 54^{\circ} 00.1^{\prime} \mathrm{W}\right)$ to estimate a residence time of Th with respect to scavenging 22 yr. Bacon et al. (1985) found that radionuclides, particularly ${ }^{230} \mathrm{Th}$ and ${ }^{231} \mathrm{~Pa}$, on particles collected in a sediment trap at $3200 \mathrm{~m}$ in the Sargasso Sea showed seasonal variations consistent with the annual cycle of primary production in the overlying surface water. They concluded that, since small particles reside on average for several years in the deep ocean, there must be a continuous exchange of material between the small and large size fractions.

More recently, Murnane (1994a) analyzed thorium isotope and particle concentration data from the (i) Nares Abyssal Plain $\left(23^{\circ} 11^{\prime} \mathrm{N}, 63^{\circ} 28^{\prime} \mathrm{W}\right)$, (ii) station $20\left(23^{\circ} 17^{\prime} \mathrm{N}, 64^{\circ} 09^{\prime} \mathrm{W}\right.$ ) of the Transient Tracers in the Ocean-North Atlantic Study, and (iii) station 31 ( $27^{\circ} \mathrm{N}, 53^{\circ} 32^{\prime} \mathrm{W}$ ) of the Geochemical Oceans Section Study (GEOSECS). By fitting to the water column data models for the cycling of inert and biogenic particles in small and large size classes, they estimated depthdependent rate constants for the remineralization of biogenic particles and for the aggregation and disaggregation of both biogenic and inert detrital particles. They reported median values between 2.1 and $3.6 \mathrm{yr}^{-1}$ for the aggregation rate constant, 135 and $195 \mathrm{yr}^{-1}$ for the disaggregation rate constant, and 0.2 and $75 \mathrm{yr}^{-1}$ for the remineralization rate constant. However, the errors in the rate parameters were large compared to the estimates themselves, and the authors concluded that the data do not strongly constrain the particle cycling rate constants. The authors also estimated the rate constant for Th adsorption normalized to particle concentration to be $5.0 \pm 1.0 \times 10^{4} \mathrm{~m}^{3} \mathrm{~kg}^{-1}$ $\mathrm{yr}^{-1}$, and the rate constant for Th desorption to be $k_{-1}=3.1 \pm 1.5 \mathrm{yr}^{-1}$. In contrast to the other model parameters, the rate constants for adsorption and desorption were assumed to be vertically uniform in their analysis.

Colley et al. (1995) collected particulate ${ }^{230} \mathrm{Th}$ and ${ }^{210} \mathrm{~Pb}$ intercepted by sediment traps at 3100 $\mathrm{m}$ and $4465 \mathrm{~m}$ in the Porcupine Abyssal Plain $\left(47^{\circ} \mathrm{N}, 19^{\circ} \mathrm{W}\right)$. They found that, below a mass flux of $120 \mathrm{mg} \mathrm{m}^{-2} \mathrm{~d}^{-1}$, the fluxes of these radionuclides were linearly related to the mass flux, and above $120 \mathrm{mg} \mathrm{m}^{-2} \mathrm{~d}^{-1}$, the radionuclide fluxes leveled off. The authors ascribed this lack of correlation at high mass flux values to an incomplete interaction of radionuclides with faster settling particulate material. Furthermore, they found that the trap material had the ${ }^{210} \mathrm{~Pb} /{ }^{230} \mathrm{Th}$ signature of the 
entire water column, suggesting the surface material does not reach the trap unaltered.

Studies from the JGOFS North Atlantic Bloom Experiment (24 April-30 May, 1989) provided insight into various processes affecting particles in the top $300 \mathrm{~m}$ in a mid-latitude oceanic environment. These studies used ${ }^{228,234} \mathrm{Th}$ measured on particles intercepted by in-situ pumps outfitted with $0.5-\mu \mathrm{m}$ nominal pore size filters (Buesseler et al., 1992) to determine rate constants for Th adsorption (Clegg and Whitfield, 1993), and for particle aggregation, disaggregation, and degradation (Cochran et al., 1993; Murnane et al., 1996). A key finding of both Cochran et al. (1993) and Murnane et al. (1996) was that, over the course of the bloom, the particle aggregation rate constant increased from about 0 to $30 \mathrm{yr}^{-1}$, while the particle disaggregation rate constant increased from about 100 to $500 \mathrm{yr}^{-1}$.

More recently, a large amount of measurements of trace elements and isotopes, including thorium isotopes, have been generated along the US GEOTRACES North Atlantic (GA03) transect (Fig. 1). These measurements, concurrently with measurements of particle concentration, provide a unique opportunity to study trace metal and particle cycling in a range of environments in the North Atlantic. Specifically, the GA03 transect includes stations from near the western margin, the Trans-Atlantic Geotraverse (TAG) hydrothermal vent at the Mid-Atlantic Ridge, the subtropical gyre interior, the Mauritanian upwelling, and the region influenced by Mediterranean Outflow Water near the Iberian Peninsula.

The collection and analysis of radionuclides from the U-Th decay series at GA03 have already been described in several studies. These include studies on the distribution of dissolved and particulate ${ }^{230} \mathrm{Th}$ (Hayes et al., 2015b,a), ${ }^{234} \mathrm{Th}$ (Owens et al., 2015), and dissolved ${ }^{228} \mathrm{Ra}$ (Charette

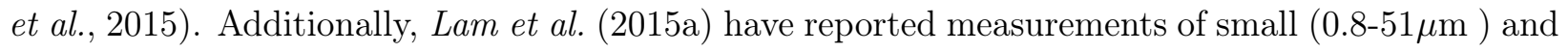
large $(\geq 51 \mu \mathrm{m})$ bulk particle concentrations as well as particle phase concentrations (particulate organic carbon, particulate inorganic carbon, biogenic opal, lithogenic material, $\mathrm{MnO}_{2}$, and iron (oxyhydr)oxides obtained along GA03.

Particularly relevant to the present study, Hayes et al. (2015a) used ${ }^{230}$ Th data for the dissolved and particulate fractions collected along GA03 to estimate the apparent distribution coefficient $K_{D}=A_{p} /\left(A_{d} P\right)$, where $A_{d}\left(A_{p}\right)$ is the activity measured in the dissolved (particulate) phase and $P$ is particle concentration. They found that $K_{D}$ generally increased with depth and that $\log _{10}\left(K_{D}\right)$ decreased with $\log _{10}(P)$ (slope $=-0.66, r=-0.72$ ). They also noticed that $K_{D}$ was 
highest for $\mathrm{MnO}_{2}\left(116.5 \pm 54.7 \times 10^{7} \mathrm{~g} / \mathrm{g}\right)$ and relatively small for particulate organic matter (POM) $\left(0.3 \pm 0.2 \times 10^{7} \mathrm{~g} / \mathrm{g}\right)$. A possible reason for the low $K_{D}$ values for POM was that the operationally defined "dissolved" phase $(\leq 0.8 \mu \mathrm{m})$ includes colloidal particles, too fine to be caught by conventional filters but onto which much of the thorium is expected to adsorb (Hayes et al., 2015a). Thus, the fraction of thorium that is adsorbed onto particulate material (both colloidal and larger particles) may potentially be underestimated.

A recent study by Lerner et al. (2016) demonstrated that, at open ocean station GT11-22 of GA03, a model with vertically varying rate parameters fits Th isotope and particle data significantly better than a model with vertically uniform rate parameters. They also found that the adsorption rate constant has a significant positive relationship with particle concentration. Outstanding questions are (i) whether a similar result holds at other stations along GA03 and, more generally, (ii) the extent to which rate parameters of Th and particle cycling vary along this section, and what processes are controlling them.

In this paper, we extend previous studies by documenting the vertical and horizontal variations in apparent kinetic parameters of Th sorption reactions and particle processes in the North Atlantic. A nonlinear least-squares technique is used to fit a 1-D (vertical) single-particle class model of thorium and particle cycling to ${ }^{228,230,234} \mathrm{Th}$ isotope activities in the dissolved and particulate phases, ${ }^{234,238} \mathrm{U}$ activities, ${ }^{228}$ Ra activities, and particle concentrations, at a number of open-ocean stations occupied during GA03. Particular emphasis is placed on the Th adsorption rate constant $\left(k_{1}\right)$ and on its relationship with particle concentration, thereby complementing the analysis of Hayes et al. (2015a): whereas $K_{D}$ is a measure of the amount of thorium bound to particles relative to the amount of thorium dissolved in seawater, $k_{1}$ is a measure of the specific rate at which thorium attaches to particles. Both concepts, $K_{D}$ and $k_{1}$, are thus different and provide distinct perspectives on the scavenging process. Furthermore, our study analyzes jointly three thorium isotopes $\left({ }^{228,230,234} \mathrm{Th}\right)$ in different size fractions, an effort only previously performed by Murnane (1994a) and Murnane et al. (1994b). In this manner, we provide the first look into the spatial variations in the kinetics of thorium and particle cycling in the North Atlantic as constrained by multiple thorium isotopes, their parents, and particle concentrations.

This paper is organized as follows. In section 3.2, we present (i) the data from the two legs of GA03, (ii) the vertical interpolation of the data that is assumed in our analysis, (iii) the model of 
particle and Th cycling, (iv) an assessment of the effects of advection, diffusion, and unsteadiness (all neglected in the model), and (v) the inverse method used to combine the data and the model. In section 3.3, the model is fitted to the data, the consistency of the model with the data is tested, and the rate parameters estimated from the fit are reported. In section 3.4, the rate parameters are interpreted in the context of the different oceanographic environments spanned by our selected stations, and the relationship of the rate parameters, particularly $k_{1}$, with particle concentration, is explored. Attention is paid to the implications of our results for the interpretation of $K_{D}$ in terms of the kinetics of Th sorption reactions. Moreover, we examine the robustness of the estimated rate parameters to various assumptions in our analysis. Conclusions follow in section 3.5.

\subsection{Methods}

\subsubsection{Hydrographic Setting}

We use data collected aboard the R/V Knorr in October 2010 (leg GT10) and NovemberDecember 2011 (leg GT11; Figure 1). The first leg (GT10) of the transect went from Lisbon to Cape Verde, while the second leg (GT11) ran from Woods Hole to Cape Verde. We consider only the stations highlighted in red (Figure 1). These stations, situated in the open-ocean, are chosen on the assumption that they suffer from a relatively small influence of lateral and vertical transport of thorium isotopes and particles. This assumption is tested in section 3.2.5.

The hydrography along GA03 is thoroughly described by Jenkins et al. (2015) and briefly summarized here. Across our selected stations, the main thermocline gradually shoals equatorward to station GT10-10, at which point deep isotherms below the main thermocline rise to about $200 \mathrm{~m}$ in the region of the Mauritanian upwelling (Figure 2a in Jenkins et al. (2015)). Evidence for upwelling is also seen by the incursion of deep, low salinity water to the near-surface at stations GT10-10 and GT10-09. Additionally, an intrusion of low salinity water at intermediate depths (about $1000 \mathrm{~m}$ ) is apparent in the southeastern part of leg GT11 (Figure 2b in Jenkins et al. (2015)). This feature has been ascribed to Antarctic Intermediate Water shoaling to the southeastern part of the leg, where the Northern Equatorial Current abuts the Northern Equatorial Counter Current (Schmitz and McCartney, 1993; Jenkins et al., 2015). Another noteworthy feature is the presence of high salinity water at intermediate depths of stations GT10-01 and GT10-03, presumably associated with the Mediterranean Outflow Water emanating from the Straits of Gibraltar (Bozec et al., 2011). 
Jenkins et al. (2015) conducted a multiple regression analysis to estimate the relative contributions of various water masses along GA03. They show that most of the thermocline waters (above $600 \mathrm{~m})$ are comprised of North Atlantic Central Waters. However, a significant proportion ( $\geq 40 \%)$ of thermocline waters in the southeastern segment of leg GT-11 consists of Atlantic Equatorial Water flowing from the south of the subtropical gyre (Schmitz and McCartney, 1993; Stramma et al., 2005; Jenkins et al., 2015). The intermediate waters at the stations considered here are estimated to include Irminger Sea Water, Antarctic Intermediate Water, Mediterranean Outflow Water, Upper Circumpolar Deep Water, and Upper Labrador Sea Water. Stations GT11-22, GT11-24, GT10-10, GT10-11, and GT10-12 are dominated (> 50\%) by Antarctic Intermediate Water between 600-1000 m, and Upper Circumpolar Deep Water between 1000-2000 m. West of the Mid-Atlantic Ridge, the intermediate depths are dominated by Irminger Sea Water (between 800-1000 m) and Upper Labrador Sea Water (between 1000-2000 m). The deepwater masses at our selected stations are Classical Labrador Sea Water, Denmark Strait Overflow Water, Iceland-Scotland Overflow Water, and Antarctic Bottom Water. Throughout both legs, Classical Labrador Sea Water is estimated to be the main contributor $(\geq 60 \%)$ to the total water mass between $2000-3000 \mathrm{~m}$. Below $3000 \mathrm{~m}$, Iceland Scotland Overflow water is the main contributor, although Antarctic Bottom Water also constitutes a significant portion of the total water mass (around 30\%).

\subsubsection{Data}

The samples were collected using the following procedures. Particles fractionated into small (1-51 $\mu \mathrm{m})$ and large $(>51 \mu \mathrm{m})$ size classes were obtained by large volume in-situ filtration using a modified dual-flow WTS-LV McLane research pump equipped with 142-mm "mini-MULVFS" filter holders (Bishop et al., 2012). Samples for particulate ${ }^{228} \mathrm{Th}$, dissolved ${ }^{228} \mathrm{Th}$, and dissolved ${ }^{228} \mathrm{Ra}$ were collected by large volume in-situ filtration. The dissolved ${ }^{230} \mathrm{Th}$ and ${ }^{234} \mathrm{Th}$ samples were gathered using 30-L Niskin bottles. Bottles collecting ${ }^{234} \mathrm{Th}$ samples below $1000 \mathrm{~m}$ were attached individually to the pump wire at the depths of the in-situ pumps. Bottles collecting dissolved ${ }^{230} \mathrm{Th}$ samples at all depths and ${ }^{234} \mathrm{Th}$ samples above $1000 \mathrm{~m}$ were mounted on the ODF/SIO rosette on a separate cast. These data can be found in the GEOTRACES Intermediate Data Product (The GEOTRACES group, 2015).

In this paper, we use data collected below 85-187 m; data from shallower depths are not used because the model considered in this study does not account for particle production. The shallowest 
depth varies with station $(85-187 \mathrm{~m})$, and is chosen so that data extrapolation is avoided (Table 1). We restrict our analysis to depths below the primary production zone, defined as the upper layer in the water column where net primary production (the difference between gross primary production and phytoplankton respiration) is positive. Following Owens et al. (2015), it is defined as the depth where in vivo fluorescence, as measured by the CTD, reaches $10 \%$ of its maximum value. This definition is based on a study by Marra et al. (2014) in the Northwest Atlantic, which found that the compensation depth generally occurred at or below the in vivo fluorescence maximum.

For convenience, the subscript $d(p)$ is used to designate Th isotope activity in dissolved (small and large particulate) form, and $P$ designates bulk particle concentration. The following studies have reported and discussed methods and (or) data for radionuclide activities and particle concentrations used here: Lam et al. (2015a) for particle concentrations, Maiti et al. (2012) and Owens et al. (2015) for total ${ }^{234} \mathrm{Th}$ and ${ }^{234} \mathrm{Th}_{p}$, Anderson et al. (2012), Shen et al. (2012), and Hayes et al. (2015b) for ${ }^{230} \mathrm{Th}_{d, p}$, Maiti et al. (2015) for ${ }^{228} \mathrm{Th}$, and Henderson et al. (2013) and Charette et al. (2015) for ${ }^{228} \mathrm{Ra}$. The tabulated ${ }^{228} \mathrm{Th}_{d, p}$ can be found on BCO-DMO (Charette et al., 2014). Finally, ${ }^{234} \mathrm{Th}_{d}$, which was not measured directly, is calculated by subtracting ${ }^{234} \mathrm{Th}_{p}$ from total ${ }^{234} \mathrm{Th}$, and its error is derived by error propagation neglecting error covariances (Bevington and Robinson, 1992).

The radioactive parents of ${ }^{234} \mathrm{Th}$ and ${ }^{230} \mathrm{Th}$, which were also not measured, are estimated as follows. The parent ${ }^{238} \mathrm{U}$ is estimated from salinity, measured at the Scripps Oceanographic Data Facility, using the empirical equation derived by Owens et al. (2011),

$$
{ }^{238} \mathrm{U}=0.0786( \pm 0.00446) S-0.315( \pm 0.158)
$$

where ${ }^{238} \mathrm{U}$ is in dpm $\mathrm{L}^{-1}$ and $S$ is on the Practical Salinity Scale of 1978. The uncertainties of ${ }^{238} \mathrm{U}$ are taken as the root mean square error of the linear regression of ${ }^{238} \mathrm{U}$ against salinity (Owens et al., 2011). On the other hand, ${ }^{234} \mathrm{U}$ is estimated from ${ }^{238} \mathrm{U}$ by assuming a ${ }^{234} \mathrm{U} /{ }^{238} \mathrm{U}$ ratio of 1.147 (Andersen et al., 2010), and its uncertainty is obtained by multiplying the uncertainties in ${ }^{238} \mathrm{U}$ by 1.147 .

Notice that ${ }^{230} \mathrm{Th}_{p}$ present in marine particulate material may have two distinct origins: ${ }^{230} \mathrm{Th}$ adsorbed in-situ onto the surfaces of particles and ${ }^{230} \mathrm{Th}$ locked in the mineral lattices of particles (Roy-Barman et al., 2009; Hayes et al., 2013, 2015b). Since we are only interested in the adsorption 
of thorium onto particles, we estimate the fraction of ${ }^{230} \mathrm{Th}$ adsorbed onto particles using equations (3.2-3.3) in Hayes et al. (2015a). For these estimates, measurements of dissolved and particulate ${ }^{232} \mathrm{Th}$ are required. As for ${ }^{230} \mathrm{Th}_{p}$, particulate ${ }^{232} \mathrm{Th}$ was measured on particles collected with a modified dual-flow WTS-LV McLane research pump. Subsamples of filters fixed on the pump were acid digested, co-precipitated with Fe after dissolution, and subject to inductively coupled plasma mass spectrometry (ICP-MS). Dissolved ${ }^{232} \mathrm{Th}$ collected in Niskin bottles was also measured by ICP-MS. Within our selected stations and depths (Table 1), these corrections never amount to more than $30 \%$ of total ${ }^{230} \mathrm{Th}_{p}$, the largest correction accounting to $30 \%$ near the surface at station GT11-24. For simplicity, estimates of ${ }^{230} \mathrm{Th}_{p}$ in the adsorbed fraction are simply referred to as ${ }^{230} \mathrm{Th}_{p}$ in the remainder of this paper.

Whereas particle and radionuclide samples were collected in both small $(1-51 \mu \mathrm{m})$ and large $(>51$ $\mu \mathrm{m})$ size classes, only data for bulk particles $(P)$ and bulk particulate radionuclides $\left({ }^{228,230,234}{ }^{T h} h_{p}\right)$ are used in this paper. At some stations, ${ }^{234} \mathrm{Th}$ in the large size fraction is only available above about $1000 \mathrm{~m}$ (the precise depth varies between stations). ${ }^{230} \mathrm{Th}$ in the large size fraction is only available at a few depths at stations GT11-14, GT11-22, GT10-12, and GT10-11. Finally, ${ }^{228} \mathrm{Th}$ in the large size fraction is only available at a few depth ranges at stations GT10-10, GT10-11, and GT10-12 (samples from multiple depths were combined for better detectability). In order to obtain bulk particle data for each Th isotope at a given station, the ratio of large $\left({ }^{234} \mathrm{Th}_{p, l}\right)$ to small particulate ${ }^{234} \mathrm{Th}\left({ }^{234} \mathrm{Th}_{p, s}\right)$ is calculated from the ${ }^{234} \mathrm{Th}_{p, l}$ and ${ }^{234} \mathrm{Th}_{p, s}$ data that are available at that station. Then, from the measured activities on the small size fraction, this ratio is applied to derive (i) ${ }^{234} \mathrm{Th}_{p, l}$ at depths where it was not measured and (ii) ${ }^{228,230} \mathrm{Th}_{p, l}$ at stations where it was not measured. At stations where ${ }^{230} \mathrm{Th}_{p, l}$ was measured, the average ratio of ${ }^{230} \mathrm{Th}_{p, l}$ to ${ }^{230} \mathrm{Th}_{p, s}$ is applied to the ${ }^{230} \mathrm{Th}$ activities measured on the small size fraction to derive ${ }^{230} \mathrm{Th}_{p, l}$ at depths where it was not measured. Similarly, at stations where ${ }^{228} \mathrm{Th}_{p, l}$ within certain depth ranges was measured, the average ratio of ${ }^{228} \mathrm{Th}_{p, l}$ to ${ }^{228} \mathrm{Th}_{p, s}$ is used to estimate ${ }^{228} \mathrm{Th}_{p, l}$ at all depths. At all stations and depths, the measured or calculated ${ }^{228,230,234} \mathrm{Th}_{p, l}$ is added to the measured ${ }^{228,230,234} \mathrm{Th}_{p, s}$ to obtain total particulate $\mathrm{Th}$ for each isotope (e.g., ${ }^{228} \mathrm{Th}_{p}={ }^{228} \mathrm{Th}_{p, s}+{ }^{228} \mathrm{Th}_{p, l}$ ). Errors in ${ }^{228,230,234} \mathrm{Th}_{p}$ were calculated by error propagation omitting error covariances. 


\subsubsection{Vertical Interpolation}

The depths at which radiochemical and particle data are available do not generally coincide. To facilitate our analysis, the measured (or calculated) values of $P,{ }^{228,230,234} \mathrm{Th}_{d, p},{ }^{234,238} \mathrm{U}$, and ${ }^{228} \mathrm{Ra}$ are interpolated onto an irregular grid for which each grid point is at a depth where at least one measurement is available (see Table 1 for the depth range at each station). The grid is defined such that no data extrapolation is needed. That is, at each station, the shallowest depth of the grid is chosen such that neither thorium isotope activities nor particle concentrations need to be extrapolated beyond that depth (and likewise for the deepest depth).

We use a minimum variance interpolation technique (e.g., Wunsch (2006)) which is described in detail in Lerner et al. (2016). The parameters of the interpolation procedure are the variance of the water property being interpolated, $\sigma_{M}^{2}$, and the length scale characterizing its vertical covariance along the water column, $l_{z}$. The parameter $\sigma_{M}^{2}$ is the maximum tolerable variance in the gridded (interpolated) data: when the interpolation depth is far from the measurement depth, the error in the interpolated value approaches $\sigma_{M}$. On the other hand, $l_{z}$ is a length scale that determines the property covariance along the water column. Specifically, if the distance between two depths increases by $l_{z}$, the property covariance between both depths is reduced by a factor of $1 / e$. Following Lerner et al. (2016), we set $\sigma_{M}^{2}=0.5 \sigma_{D}^{2}$ and $l_{z}=1000 \mathrm{~m}$, where $\sigma_{D}^{2}$ is the variance in a particular data set (e.g., the variance in the ${ }^{230} \mathrm{Th}_{d}$ data) at a given station.

To assess the vertical interpolation, we interpolate the data at the sampling depths and calculate the interpolation residuals normalized to measurement errors for all data used in this study (Figure 2a). A normalized residual is defined as

$$
r_{i}=\left(\hat{x}_{i}-x_{d, i}\right) / \sigma_{d, i}
$$

where $\hat{x}_{i}$ is the gridded value at sampling depth level $i, x_{d, i}$ is the measured value at this level, and $\sigma_{d, i}$ is the error in the measurement at this level. For $\sigma_{M}^{2}=0.5 \sigma_{D}^{2}$ and $l_{z}=1000 \mathrm{~m}$, the fraction of normalized residuals less than 2 in absolute magnitude is 0.96 (Figure 2a). Thus, over 95\% of the gridded values agree with the data within $2 \sigma_{d, i}$, which supports the interpolation. 


\begin{tabular}{|l|c|}
\hline Station & Depth range $(\mathrm{m})$ \\
\hline GT11-10 & $185-3006$ \\
\hline GT11-12 & $185-5485$ \\
\hline GT11-14 & $135-4000$ \\
\hline GT11-16 & $114-3600$ \\
\hline GT11-18 & $187-4362$ \\
\hline GT11-20 & $186-5420$ \\
\hline GT11-22 & $125-4243$ \\
\hline GT11-24 & $90-3458$ \\
\hline GT10-10 & $100-3200$ \\
\hline GT10-11 & $85-3200$ \\
\hline GT10-12 & $90-3500$ \\
\hline
\end{tabular}

Table 1: Depth range of the radionuclide and particle concentration data considered in this study.

\subsubsection{Model of Thorium and Particle Cycling}

We consider a single-particle class model for thorium and particle cycling that includes balance equations for ${ }^{228,230,234} \mathrm{Th}_{d}$, bulk ${ }^{228,230,234} \mathrm{Th}_{p}$, and bulk particles (Figure 3 ). The model equations for the thorium isotopes include the production by their radioactive parents, the radioactive decay, the adsorption onto particles, the desorption from particles, the release from particles via particle degradation, and the effect of particle sinking. The equations for particle concentration include the degradation of particles and the effect of particle sinking. The equations are (Nozaki et al., 1981; Bacon and Anderson, 1982):

$$
\begin{aligned}
T\left(A_{d}\right) & =\lambda A_{\pi}+\left(k_{-1}+\beta_{-1}\right) A_{p}-\left(k_{1}+\lambda\right) A_{d} \\
T\left(A_{p}\right)+w \frac{\partial A_{p}}{\partial z} & =k_{1} A_{d}-\left(\beta_{-1}+k_{-1}+\lambda\right) A_{p} \\
T(P)+w \frac{\partial P}{\partial z} & =-\beta_{-1} P .
\end{aligned}
$$

Here, $A_{d}\left(A_{p}\right)$ represents the thorium isotope activity in the dissolved (particulate) phase (in units of $\left.\mathrm{dpm} \mathrm{m}^{-3}\right), A_{\pi}$ is the activity of the parent isotope $\left(\mathrm{dpm} \mathrm{m} \mathrm{m}^{-3}\right), P$ is the particle concentration $\left(\mathrm{mg} \mathrm{m} \mathrm{m}^{-3}\right), \lambda$ is the radioactive decay constant $\left(\mathrm{yr}^{-1}\right)$, and $k_{1}, k_{-1}$, and $\beta_{-1}$ are the rate constants for Th adsorption, Th desorption, and particle degradation, respectively $\left(\mathrm{yr}^{-1}\right)$. The particle sinking speed is $w\left(\mathrm{~m} \mathrm{yr}^{-1}\right)$, and $z$ is depth $(\mathrm{m})$. Notice that the presence of vertical derivatives require boundary conditions, which we take as the values of $P$ and $A_{p}$ at the shallowest depth of the grid at each station (Table 1). Finally, the term $T(\cdot)$ in each equation represents the effects of unsteadiness, advection, and diffusion, i.e.,

$$
T\left(A_{d}\right) \equiv \frac{\partial A_{d}}{\partial t}+\boldsymbol{u} \cdot \nabla A_{d}-\nabla \cdot\left(\boldsymbol{k} \nabla A_{d}\right)
$$


where $\mathbf{u}$ is the vector velocity and $\boldsymbol{k}$ a diffusion tensor.

Several assumptions are made in the governing equations (3.3a-3.3c). We assume steady state and omit the effects of transport by advection and diffusion, i.e., $T(\cdot)=0$. Furthermore, Th sorption reactions and particle degradation are described using first order kinetics. Some models also assume no significant contribution to the dissolved and particulate pools of thorium isotopes from lithogenic sources. While this assumption should be reasonable for ${ }^{228,234} \mathrm{Th}$ far from sedimentary sources, ${ }^{230} \mathrm{Th}$ may have a sizeable lithogenic contribution in the vicinity of mineral dust sources (Hayes et al., 2013). Here, the lithogenic contribution is removed from the data (see section 3.2.2), and our model (equations 3.3a-3.3c) only considers the ${ }^{230} \mathrm{Th}$ fraction that takes part in sorption reactions.

\subsubsection{Effects of Advection, Diffusion, and Unsteadiness}

In this section, we assess our assumption of a negligible contribution of advection, diffusion, and unsteadiness in the Th isotope and particle budgets at our selected stations along GA03. To this end, we rely on the Th and particle data along legs GT10 and GT11 together with other observational estimates in the North Atlantic. The advection fluxes are estimated from (i) the horizontal and vertical velocities inferred for November 2011 from a least-squares fit of an ocean general circulation model to oceanic and atmospheric observations (ECCO v4 non-linear inverse solution; Forget et al. (2015)), and (ii) the horizontal and vertical concentration gradients measured along the two legs. For example, the vertical advection flux of $A_{d}$ at depth $z$ of the ECCO grid and at station GT11-24 is estimated as $u \partial A_{d} / \partial l$, where $\partial A_{d} / \partial l$ is the concentration gradient evaluated using an upstream scheme, $A_{d}$ is obtained by vertically interpolating data on the ECCO grid at station GT11-24 and its upstream station (GT11-22), $l$ is the geodesic distance between the two stations, and $u$ is the magnitude of the horizontal velocity component at depth $z$, closest to station GT11-22, and along the line joining the two stations. Similarly we estimate the vertical advection flux of $A_{d}$ at a given depth of the ECCO grid and at a given station as $\omega \partial A_{d} / \partial z$, where $\partial A_{d} / \partial z$ is the vertical concentration gradient evaluated from $A_{d}$ interpolated at that depth and the depth immediately above, and $\omega$ is the vertical velocity at that depth. The horizontal diffusion flux of $A_{d}$ is estimated by evaluating $K_{h} \partial^{2} A_{d} / \partial l^{2}$ using a 2nd-order central difference scheme, where the horizontal diffusivity $K_{h}=1000 \mathrm{~m}^{2} \mathrm{~s}^{-1}$ is taken from a tracer release experiment in the North Atlantic (Ledwell et al., 1998). Note that our horizontal flux estimates only consider fluxes along the transect, and do not take into account fluxes of Th isotopes or particles in cross-transect directions. 
The vertical diffusion of $A_{d}, K_{z} \partial^{2} A_{d} / \partial z^{2}$, where $K_{z}$ is the vertical diffusivity, is evaluated using a similar scheme. We restrict our estimates of vertical diffusion to stations GT11-24, GT10-11, and GT10-10, which are nearby the African continental rise (Figure 1), where Toole et al. (1994) reported diffusivities $K_{z}=10^{-5} \mathrm{~m}^{2} \mathrm{~s}^{-1}$ using microstructure data. Finally, we assess the unsteadiness terms $\partial(\cdot) / \partial t$ at the cross-over station of GA03 (station GT10-12 of leg 1 and station GT11-24 of leg 2). Specifically, data from these stations are interpolated on the ECCO grid, and differences between interpolated values at the same depth are divided by the time interval between the two occupations of the cross-over station. Note that our estimates of $\partial(\cdot) / \partial t$ have limited usefulness, particularly for the short-lived ${ }^{234} \mathrm{Th}$ as this time interval exceeds the ${ }^{234} \mathrm{Th}$ half-life by one order of magnitude.

Figures 4 and 5 show our estimates of the magnitude of the various terms in $T(\cdot)$ for the Th isotopes and the particles (depth averages are shown). For reference, these figures also include prior estimates of terms that are retained in the balance equations (3.3a-3.3c) (horizontal lines in Figures 4 and 5). These terms are the production flux $\lambda A_{\pi}$ for ${ }^{228,234} \mathrm{Th}_{d}$, the adsorption flux $k_{1} A_{d}$ for ${ }^{230} \mathrm{Th}_{d}$ and ${ }^{228,230,234} \mathrm{Th}_{p}$, and the degradation flux $\beta_{-1} P$ for $P$. They are estimated using the interpolated thorium isotope and particle concentrations $\left({ }^{234,238} \mathrm{U},{ }^{228} \mathrm{Ra},{ }^{228,230,234} \mathrm{Th}_{d, p}\right.$, and $P)$ and, where appropriate, prior estimates of the rate parameters. For the prior adsorption and degradation fluxes, we take the smallest and largest values of $k_{1}\left(0.1\right.$ and $\left.1 \mathrm{yr}^{-1}\right)$ and $\beta_{-1}(1$ and $100 \mathrm{yr}^{-1}$ ) reported in previous syntheses (Marchal and Lam, 2012; Lerner et al., 2016). A term in $T(\cdot)$ would appear negligible, in the depth-average sense, if its vertical average has an order of magnitude less than that of the retained term in the same equation. We find that (horizontal and vertical ) diffusion and advection, as well as the unsteadiness term, appear generally negligible for the balance equations of ${ }^{228,230,234} \mathrm{Th}_{d, p}$ and $P$ (Figures 4 and 5).

We illustrate the vertical variations in the missing terms for ${ }^{230} \mathrm{Th}_{d}$ at the crossover station (Figure 6), the only station for which all terms in the ${ }^{230} \mathrm{Th}_{d}$ governing equations can be estimated. Consistent with the vertical averages (Figure $4 \mathrm{~b}$ ), it is seen that the missing terms appear to be generally small compared to prior estimates of $k_{1}^{230} \mathrm{Th}_{d}$. Some of these terms exceed the minimum estimate of the adsorption flux in the top $300 \mathrm{~m}$. Between 300 and $500 \mathrm{~m}$, horizontal diffusion appears to be significant. Between 500 and $1500 \mathrm{~m}$, the unsteadiness term occasionally exceeds the prior estimates of the minimum adsorption rate. Finally, below $1500 \mathrm{~m}$, only the unsteadiness term appears significant compared to the minimum adsorption rate. 
In summary, our results show that, at most of our selected stations, advection and diffusion fluxes appear to be generally smaller by at least one order of magnitude than retained terms in the Th isotope and particle budgets. Likewise, the unsteadiness term appears generally negligible at the cross-over station. Although our estimates of missing terms are very crude, they do suggest that a 1-D (vertical) model that neglects advection, diffusion, and unsteadiness may provide a generally plausible description of Th and particle cycling at our selected stations. As a result, we proceed in the remainder of this paper by neglecting $T(\cdot)$ in the model equations (3.3a-3.3c). At least at some stations and in some depth intervals, the effects of transport and unsteadiness do seem to be important, and they will be discussed in Section 3.4.

\subsubsection{Inverse method}

We fit the single-particle class model (equations 3.3a-3.3c with $T(\cdot)=0$ ) to the radiochemical and particle data (interpolated values) at our selected stations (Figure 1) using an inverse method. The fit is obtained by adjusting the rate parameters of the model $\left(k_{1}, k_{-1}, \beta_{-1}\right.$, and $\left.w\right)$ as well as the radiochemical and particle concentration data themselves, given the range in the prior estimates of the rate parameters as well as uncertainties in the data. Prior estimates of $k_{1}, k_{-1}, \beta_{-1}$ and $w$ are obtained from previous observational estimates of these rate parameters in different oceanic environments (Table 2). The errors in the prior estimates represent \pm 1 standard deviation of a normal distribution, which means that there is a possibility of estimating negative values of these parameters in the solution, especially when the error is larger than the prior estimate of the parameter (as is the case for $k_{1}, k_{-1}$, and $\beta_{-1}$ ). As described in Appendix A, our inversions prevents the occurrence of negative values, which are nonsensical in our study. By allowing the interpolated values to change within a range consistent with their uncertainties, this approach aims to prevent, or at least reduce, a possible bias in the rate parameter estimates (for a discussion, see Lam and Marchal (2014)).

The inverse method used to fit the model to the data is a nonlinear programming technique (Waltz et al., 2006), which operates as follows. Let $\boldsymbol{x}$ be a vector describing the state of the Th and particle cycles at a given (selected) station of GA03 according to the model. The elements of $\boldsymbol{x}$ are the Th isotope activities in dissolved and particulate forms, the parent activities $\left({ }^{234} \mathrm{U},{ }^{238} \mathrm{U},{ }^{228} \mathrm{Ra}\right)$, the particle concentration, as well as the rate parameters $\left(k_{1}, k_{-1}, \beta_{-1}, w\right)$, at all depths of the interpolation grid for that station. We seek a vector $\boldsymbol{x}$ that is consistent with the data and the prior 


\begin{tabular}{|l|c|c|c|}
\hline parameter & prior estimate & prior estimate error & sources \\
\hline$k_{1}\left(\mathrm{y}^{-1}\right)$ & 0.5 & 5 & $\mathrm{a}, \mathrm{b}, \mathrm{c}, \mathrm{d}, \mathrm{e}$ \\
\hline$k_{-1}\left(\mathrm{y}^{-1}\right)$ & 2 & 5 & $\mathrm{a}, \mathrm{b}, \mathrm{c}, \mathrm{d}, \mathrm{e}$ \\
\hline$\beta_{-1}\left(\mathrm{y}^{-1}\right)$ & 1 & 10 & $\mathrm{a}, \mathrm{b}, \mathrm{f}, \mathrm{g}$ \\
\hline $\mathrm{w}\left(\mathrm{m} \mathrm{y}^{-1}\right)$ & 700 & 400 & $\mathrm{~h}, \mathrm{i}, \mathrm{j}, \mathrm{k}, \mathrm{l}, \mathrm{m}$ \\
\hline
\end{tabular}

a. Nozaki et al. (1987). b. Bacon and Anderson (1982). c. Murnane et al. (1990). d. Murnane (1994a). e. Murnane et al. (1994b).

f. Clegg et al. (1991b). g. Cochran et al. (1993). h. Krishnaswami et al. (1976). i. Krishnaswami et al. (1981).

j. Rutgers van der Loeff and Berger (1993). k. Rutgers van der Loeff and Berger (1993). 1.Scholten et al. (1995). m. Venchiarutti et al. (2008).

Table 2: Prior estimates of rate parameters of Th and particle cycling assumed in this study.

estimates of the rate parameters given their respective error statistics, while satisfying the model equations (3.3a-3.3c) perfectly. This vector is found at a minimum of the objective function:

$$
J(\boldsymbol{x})=\left(\boldsymbol{x}-\boldsymbol{x}_{0}\right)^{\prime} \boldsymbol{C}_{0}^{-1}\left(\boldsymbol{x}-\boldsymbol{x}_{0}\right)
$$

subject to the hard constraint $\boldsymbol{f}(\boldsymbol{x})=\mathbf{0}$, where $\boldsymbol{f}(\boldsymbol{x})=\mathbf{0}$ represents equations (3a-3c) (with $T(\cdot)=0$ ) discretized at all depths of the grid (a first-order backward finite-difference scheme is used to discretize $w \partial A_{p} / \partial z$ and $\left.w \partial P / \partial z\right)$. Here, $\boldsymbol{x}_{0}$ is a vector including prior estimates of the elements in $\boldsymbol{x}$ (in our study, $\boldsymbol{x}_{0}$ includes the interpolated values obtained from the data and prior estimates of the rate parameters), $\boldsymbol{C}_{0}$ is the error covariance matrix for the prior estimates (the diagonal elements of $\boldsymbol{C}_{0}$ are the squared errors in the estimates and the off-diagonal elements of $\boldsymbol{C}_{0}$ are the covariances between the errors), and the prime designates the transpose. The function (3.5) therefore represents the deviation of the state vector from its prior estimate, where $\boldsymbol{C}_{0}$ plays the role of a weighting factor: the elements of $\boldsymbol{x}_{0}$ with small (large) uncertainties contribute strongly (modestly) to the objective function.

We ensure that the Th and particle equations in $\boldsymbol{f}(\boldsymbol{x})=\mathbf{0}$ have a comparable effect in the inversion, independent of the choice of units, by normalizing the elements of $\boldsymbol{x}$ by their prior values in $\boldsymbol{x}_{\mathbf{0}}$ before the inversion; $\boldsymbol{C}_{\mathbf{0}}$ is modified accordingly. Additionally, each model equation is normalized by the root of the sum of the squared terms in that equation (row scaling; Wunsch (2006)). Details about the iterative method used to find a minimum of $J(\boldsymbol{x})$ subject to $\boldsymbol{f}(\boldsymbol{x})=\mathbf{0}$ and about the estimation of the solution error are reported in appendix A. 


\subsection{Results}

In this section, we present the solution of the inverse problem stated in section 3.2.6. We first describe the goodness of fit of the Th and particle cycling model to the radiochemical and particle data at our selected stations along the GA03 transect. Emphasis is then placed on estimated radiochemical activities and particle concentrations and on the estimated rate parameters at these stations.

We find that at each station, a converging solution $(\hat{\boldsymbol{x}})$ is obtained: at the solution point, the gradient of $\boldsymbol{J}(\boldsymbol{x})$ vanishes, and the change in $\hat{\boldsymbol{x}}$ (normalized values) between subsequent iterations has an Euclidean norm of less than $10^{-10}$. In order to check whether the model equations are reasonably satisfied by the solution $\hat{\boldsymbol{x}}$, the residual of each equation, $f_{i}(\hat{\boldsymbol{x}})=\epsilon_{i}$, is calculated and compared with the maximum term in the corresponding equation. We find that the residual $\epsilon_{i}$ amounts to less that $10^{-4}$ (in absolute magnitude) of the maximum term for each equation, indicating that the model equations are satisfied to at least the 4 th order.

\subsubsection{Goodness of Fit}

Two measures of goodness of fit of the model to the data are considered for each station. One measure is the fraction (noted $\phi$ below) of the fit residuals normalized to the measurement error that are lower than 2 in absolute magnitude. A normalized fit residual is defined as

$$
r_{i}=\frac{\hat{x}_{i}-x_{d, i}}{\sigma_{d, i}}, i=1,2, \ldots, n
$$

Here $\hat{x}_{i}$ is the estimated value of the $i$ th variable $\left({ }^{228,230,234} \mathrm{Th}_{d, p},{ }^{234,238} \mathrm{U},{ }^{228} \mathrm{Ra}\right.$, or $\left.P\right)$ in the vector $\hat{\boldsymbol{x}}$ that is obtained from the fit, $x_{d, i}$ is the measured (not interpolated) value of this variable, $\sigma_{d, i}$ is the error in this measured value, and $n$ is the number of measured values. The second measure considered is the arithmetic average of the normalized fit residuals:

$$
B=\frac{1}{n} \sum_{i=1}^{n} \frac{\hat{x_{i}}-x_{d, i}}{\sigma_{d, i}} .
$$

Note that $\phi$ and $B$ provide different information about the model fit to the data: $\phi$ reflects the ability of the model to explain the data with no regard for possible over- or under-estimation, while $B$ is an indicator of systematic errors in the model. 
We find that the model fits $82 \%$ of the data at our selected stations within 2 standard deviations $(\phi=0.82$ ), with an overall bias $B=0.07$ (Figure $2 \mathrm{~b}$ ). Among our selected stations, the value of $\phi$ ranges from 0.76 to 0.84 , and the value of $B$ ranges from -0.24 to 0.32 (Table 3 ). Note the remarkably small range of $\phi$ across the stations, which suggests that the model has about the same aptitude to explain the data. Moreover, both negative and positive values of $B$ are found, i.e., no noticeable bias of the model seems to occur across the stations.

\begin{tabular}{|l|c|c|}
\hline & $\phi$ & $B$ \\
\hline all stations & 0.82 & 0.07 \\
\hline GT11-10 & 0.76 & -0.1 \\
\hline GT11-12 & 0.84 & 0.04 \\
\hline GT11-14 & 0.81 & -0.22 \\
\hline GT11-16 & 0.81 & 0.29 \\
\hline GT11-18 & 0.82 & 0.19 \\
\hline GT11-20 & 0.82 & 0.13 \\
\hline GT11-22 & 0.82 & -0.24 \\
\hline GT11-24 & 0.82 & 0.32 \\
\hline GT10-12 & 0.82 & 0.06 \\
\hline GT10-11 & 0.82 & 0.07 \\
\hline GT10-10 & 0.82 & 0.07 \\
\hline
\end{tabular}

Table 3: Measures of goodness of fit of the model to data at selected stations of GA03.

\subsubsection{Radiochemical Activities and Particle Concentrations}

In this section we present the interpolated Th isotope activities and particle concentrations as well as the residuals between the posterior estimates $\left(\hat{x}_{i}\right)$ and interpolated values $\left(x_{I, i}\right)$, normalized to the error in the interpolated values $\left(\sigma_{I, i}\right)$.

$$
R_{i}=\frac{\hat{x}_{i}-x_{I, i}}{\sigma_{I, i}}, i=1,2, \ldots, n,
$$

where $n$ is the number of estimated or interpolated values. These normalized residuals (Figure 7a-10a and 7c-10c) are generally less than 2 in absolute magnitude, indicating that the general features in the (interpolated) data (Figure $7 \mathrm{~b}-10 \mathrm{~b}$ and $7 \mathrm{~d}-10 \mathrm{~d}$ ) are broadly replicated within 2 $\sigma_{I, i}$. For ${ }^{230} \mathrm{Th}_{d, p}$, these features include the general increase with depth, and the ${ }^{230} \mathrm{Th}_{d}$ deficit and ${ }^{230} \mathrm{Th}_{p}$ excess near the TAG hydrothermal vent at station GT11-16. For ${ }^{228} \mathrm{Th}_{p}$, they include maxima at the surface and near the seafloor, and minima at mid-depth. Similarly, the posterior estimates successfully capture the general decrease of ${ }^{234} \mathrm{Th}_{p}$ with depth and an excess in deep 
water at station GT11-16. Finally, both the data and posterior estimates show a general decrease of particle concentration with depth, and a larger concentration of particles near the surface waters at the easternmost stations (GT11-24, GT10-10, GT10-11, and GT10-12) relative to other stations.

Nonetheless, notable differences between the posterior estimates and the interpolated data are also apparent. For ${ }^{228} \mathrm{Th}_{d}$, the number of posterior estimates that deviate notably from the interpolated values is remarkably large compared to the other thorium isotopes. Most of these large deviations occur at stations GT11-22, GT11-24, GT10-10, GT10-11 and GT10-12. At each of these stations, over $40 \%$ of the posterior estimates exceed the interpolated data by more than 2 standard deviations. In addition, at the crossover station GT11-24/GT10-12, over $30 \%$ of the ${ }^{234} \mathrm{Th}_{d}$ normalized residuals are greater than 2 in absolute magnitude. These deviations are discussed in section 3.4.1. For ${ }^{228} \mathrm{Th}_{p},{ }^{230} \mathrm{Th}_{d, p}$, and ${ }^{234} \mathrm{Th}_{p}$, less than $20 \%$ of the normalized residuals are greater than 2 in absolute magnitude at each station. Thus, despite inconsistencies, both measures of goodness of fit $(\phi$ and $B)$ and the distribution of the normalized residuals (Figure $2 \mathrm{~b}$ ) show that the model generally is successful at reproducing the data gathered at our selected stations.

\subsubsection{Rate Parameters}

In this section we present the posterior estimates of the rate parameters $\left(k_{1}, k_{-1}, \beta_{-1}\right.$, and $\left.w\right)$ at our selected stations (Figure 11). Conspicuously, the adsorption rate constant $k_{1}$ tends to decrease with depth at most of the stations. Some of the highest values of $k_{1}$ are estimated above $500 \mathrm{~m}$ at the four easternmost stations (station GT11-22, GT11-24, GT10-10, and GT10-11). Furthermore, $k_{1}$ displays a spectacular enhancement in deep water near the TAG hydrothermal vent (station GT11-16). The desorption rate constant, $k_{-1}$, on the other hand, does not exhibit a consistent vertical trend, except at stations GT11-24, GT10-12, GT10-10, and GT10-11, where $k_{-1}$ appears to decrease with depth. As with $k_{1}$, near-surface values of $k_{-1}$ at stations GT11-24, GT10-12, GT10-10, and GT10-11 are high relative to the other stations. The rate constant of particle degradation, $\beta_{-1}$, shows generally both higher values and higher vertical variability above $1000 \mathrm{~m}$. Finally, estimates of particle sinking speed, $w$, tend to be larger and display enhanced vertical variability below 2000 m. One notable exception is at the TAG hydrothermal vent, where estimates of $w$ are low relative to those at the other stations.

The vertical profiles of estimated $k_{1}$ at our selected stations (Figure 12; vertical profile of other profiles included in online supplement) reveal features that are more difficult to discern in the section 
plot (Figure 11). For example, it is seen that stations GT11-22, GT11-24, GT10-12, GT10-11, and GT10-10 exhibit the highest values of $k_{1}$ near the surface. These stations also portray the steepest vertical gradients in $k_{1}$ in the upper $1000 \mathrm{~m}$. Additionally, at the easternmost stations GT11-24, GT10-12, GT10-11, and GT10-10, $k_{1}$ values below $1000 \mathrm{~m}$ are higher than those below the same depth at all other stations. There is a notable exception to this pattern at station GT11-16, where a very large value of $k_{1}\left(21 \mathrm{yr}^{-1}\right)$ is estimated below $3000 \mathrm{~m}$, near the TAG hydrothermal vent.

\subsection{Discussion}

\subsubsection{Misfit to the interpolated values}

In this section, we discuss the model misfits to the data, with emphasis on ${ }^{228} \mathrm{Th}$ and ${ }^{234} \mathrm{Th}$. Consider first ${ }^{228} \mathrm{Th}$. Many of the normalized residuals greater than 2 in absolute magnitude stem from the misfit to the ${ }^{228} \mathrm{Th}_{d}$ data. Particularly, at each station east of GT11-20, over $40 \%$ of the ${ }^{228} \mathrm{Th}_{d}$ data are overestimated by more than 2 standard deviations. To examine the depths where these large residuals occur, we show the vertical profiles of the interpolated and posterior estimates of ${ }^{228} \mathrm{Th}_{d}$ in Figure 13. At each of these stations, most of the posterior estimates differ by more than two standard deviations from the interpolated values above $1000 \mathrm{~m}$ as well as near the seafloor. The greatest misfit between data and model clearly occurs in the top $1000 \mathrm{~m}$ at station GT10-10. Speculatively, these misfits arise from the missing terms in the ${ }^{228} \mathrm{Th}_{d}$ balance equations (Figure 3 ), since our estimates of the magnitude of these terms (depth averages) are close to the posterior estimates of radioactive production of ${ }^{228} \mathrm{Th}_{d}$ by ${ }^{228} \mathrm{Ra}$. At station GT10-10, where the misfits in the top $1000 \mathrm{~m}$ are the most pronounced (Figure 13), the largest estimated missing term is vertical advection (our missing term estimates are depth-averages, and terms that appear smaller (Figure 3) may still influence of ${ }^{228} \mathrm{Th}_{d}$ at certain depths). That the posterior ${ }^{228} \mathrm{Th}$ values overestimate the ${ }^{228} \mathrm{Th}_{d}$ data (interpolated values in the upper $1000 \mathrm{~m}$ at GT10-10) suggests that a process which tends to decrease ${ }^{228} \mathrm{Th}_{d}$ is missing in the model. Such a process could be, for example, the upwelling of ${ }^{228} \mathrm{Th}_{d}$-poor waters below 1000-2000 $\mathrm{m}$ to above $1000 \mathrm{~m}$ (as suggested by the relatively shallow ${ }^{228} \mathrm{Th}_{d}$ maximum at GT10-10), or unsteadiness. An alternative explanation for these misfits is that measured ${ }^{228} \mathrm{Th}_{d}$ activities in the upper $1000 \mathrm{~m}$ are biased such that they underestimate their "true" value. ${ }^{228} \mathrm{Th}$ was collected onto $\mathrm{MnO}_{2}$ impregnated acrylic cartridges using an in-situ pump. These cartridges are known to have extraction efficiencies for Ra and Th that are less than $100 \%$. 
However, the extraction efficiency for Th is generally estimated and corrected for by sampling ${ }^{234} \mathrm{Th}$ using Niskin bottles and in-situ pumps co-located at the same depth, and using the bottle-derived ${ }^{234} \mathrm{Th}$ to estimate the extraction efficiency of both ${ }^{234} \mathrm{Th}$ and ${ }^{228} \mathrm{Th}$ (Maiti et al., 2015). Assuming the extraction efficiency of ${ }^{234} \mathrm{Th}$ equals the extraction efficiency of ${ }^{228} \mathrm{Th}$, then, a bias in the ${ }^{228} \mathrm{Th}_{d}$ due to inefficient extraction is unlikely.

Consider then ${ }^{234} \mathrm{Th}$. Figure 14 shows profiles of ${ }^{238} \mathrm{U}$ and total ${ }^{234} \mathrm{Th}\left({ }^{234} \mathrm{Th}_{t o t}={ }^{234} \mathrm{Th}_{d}+{ }^{234} \mathrm{Th}_{p}\right)$ at the crossover station GT10-12/GT11-24. These profiles suggest the source of misfits to the ${ }^{234} \mathrm{Th}_{d}$ data. At both stations, the data indicate deviations of total ${ }^{234} \mathrm{Th}_{\text {tot }}$ from secular equilibrium (Owens et al., 2015). For example, at station GT10-12, ${ }^{234} \mathrm{Th}_{t o t}$ in the top $700 \mathrm{~m}$ exceeds the value expected from secular equilibrium, in contrast to the model which produce ${ }^{234} \mathrm{Th}_{\text {tot }}$ activities close to ${ }^{238} \mathrm{U}$ activities. To understand the misfits consider the sum of equations (3.3a-3.3b) (section 3.2.4):

$$
T\left(A_{t o t}\right)+w \frac{\partial A_{p}}{\partial z}=\lambda\left(A_{\pi}-A_{t o t}\right)
$$

Since the model does not consider terms in $T\left(A_{t o t}\right)$, this equation reduces to $w \frac{\partial A_{p}}{\partial z}=\lambda\left(A_{\pi}-\right.$ $A_{\text {tot }}$ ). If the vertical gradient in ${ }^{234} \mathrm{Th}_{p}$ is small, then $w$ must be relatively large in order to allow departure from secular equilibrium and hence to allow the model to fit the (interpolated) ${ }^{234} \mathrm{Th}_{t o t}$ (and ${ }^{238} \mathrm{U}$ ) data. Yet, the posterior estimates of $w$ are also determined from the other data (e.g., ${ }^{228,230} \mathrm{Th}_{p}$ and $P$ ). Rather than increase $w$ the posterior estimates of ${ }^{234} \mathrm{Th}_{d}$ and ${ }^{238} \mathrm{U}$ are modified compared to their prior values, so that secular equilibrium is obtained. This interpretation would explain the relatively large misfits to ${ }^{234} \mathrm{Th}_{\text {tot }}$ (and thus ${ }^{234} \mathrm{Th}_{d}$ ) and ${ }^{238} \mathrm{U}$ data at the crossover station.

While systematic errors in ${ }^{234} \mathrm{Th}_{t o t}$ measurements may also contribute to the model-data misfit, the misfit to this data is not systematic, as the model overestimates ${ }^{234} \mathrm{Th}_{\text {tot }}$ in the top $700 \mathrm{~m}$ at station GT10-12 and underestimates ${ }^{234} \mathrm{Th}_{\text {tot }}$ at most depths at station GT11-24. Thus the deviation between the model and data cannot be explained by a bias in the measurement of ${ }^{234} \mathrm{Th}_{t o t}$. Of course, the rate parameter estimates may also be affected by errors in the radionuclide and particle concentration measurements. For example, if the particulate Th and particle measurements are positively biased compared to their "true" values in the ocean, this could impact the estimated particle sinking speed and, by extension, the ability of the model to allow for departures from secular 
equilibrium. ${ }^{228,234} \mathrm{Th}$ were collected on paired Whatman QMA quartz fiber filters $(1 \mu \mathrm{m}$ nominal pore size) filters, and the activities measured on these filters may be inflated by $10-20 \%$ due to the adsorption of dissolved ${ }^{228,234} \mathrm{Th}$ on the filters (Maiti et al., 2012). ${ }^{230} \mathrm{Th}_{p}$ was measured on paired Supor filters (0.8 $\mu \mathrm{m}$ nominal pore size). Total digest of these filters was required for the analysis of ${ }^{230} \mathrm{Th}_{p}$ by ICP-MS. However, Supor filters are difficult to digest, and errors in the measured ${ }^{230} \mathrm{Th}_{p}$ activities may result from the solution obtained from the digest clogging the introduction system of analytical instruments (Ohnemus and Lam, 2015; Lam et al., 2015b). Additionally, any filter clogging during seawater filtration may result in positively biased measurements of particulate thorium and particles, although QMA and Supor filters are thought to be able to process large volumes of water without clogging (Maiti et al., 2012).

\subsubsection{Variation in Rate Parameters}

\section{Th Adsorption}

Across all selected stations of GA03, with the exception of station GT11-16, $k_{1}$ tends to decrease with depth (Figure 11). One possible explanation for this trend is that particle concentration also generally decreases with depth (see Figure 10). A decrease in particle concentration reflects a decrease in the number of surface sites onto which thorium can adsorb. To describe this process, Honeyman et al. (1988) developed a simple kinetic model of the interaction between metals and the surface sites of particles. Their model assumes that $k_{1}$ is proportional to the sum of all surface sites not associated with the metal. It also assumes that particle concentration can be taken as a surrogate for the number of surface sites. These assumptions suggest the following, more general relationship between $k_{1}$ and $P$ :

$$
k_{1}=k_{1, c} P^{b}
$$

where $k_{1, c}$ and $b$ are positive constants. Clearly, $b=1$ would indicate a linear dependence of $k_{1}$ on particle concentration.

To test (3.9), we examine the relationship between the adsorption rate constant and particle concentration at our selected stations. Figure $15 \mathrm{a}-\mathrm{c}$ shows scatter plots of $\ln k_{1}$ vs. $\ln P$ interpolated, inverted, or measured. The light dashed lines are 1:1 lines, corresponding to $k_{1} \propto P(b=1)$. In Figure 15c, the solid line is the best fit derived by ordinary least squares (OLS), and the dark 


\begin{tabular}{|l|c|c|c|c|c|}
\hline & $\hat{b} \pm \sigma_{\hat{b}}\left(^{a}\right)$ & $r$ & $p$ for $r$ & $\tau$ & $p$ for $\tau$ \\
\hline OLS & $1.44 \pm 0.18$ & 0.71 & $<0.001$ & 0.58 & $<0.001$ \\
\hline WLS & $1.62 \pm 0.24$ & 0.70 & $<0.001$ & 0.58 & $<0.001$ \\
\hline OLS w/ smoothing & $0.95 \pm 0.13$ & 0.69 & $<0.001$ & 0.54 & $<0.001$ \\
\hline WLS w/ smoothing & $1.02 \pm 0.12$ & 0.69 & $<0.001$ & 0.54 & $<0.001$ \\
\hline
\end{tabular}

$a . \hat{b}$ is the slope estimate and $\sigma_{\hat{b}}$ is its standard error

Table 4: Results from ordinary (OLS) and weighted (WLS) least squares regressions of $\ln \left(k_{1}\right)$ against $\ln \left(P_{m}\right)$

dashed line is the best fit derived by weighted least squares (WLS), which accounts for the error (co)variances in the $k_{1}$ posterior estimates (since our interpolation procedure introduces correlation among interpolated or inverted $P$ values, we apply OLS and WLS only to the relationship between $\ln k_{1}$ and $\ln P_{m}$, where $P_{m}$ denotes measured particle concentration). We exclude station GT11-16 in order to prevent the large values of $k_{1}$ at that station from influencing the regressions. Table 4 lists the slope, the linear Pearson correlation coefficient $(r)$ and associated $p$ value for OLS and WLS $(r$ for WLS estimated following Buse (1973)), and the Kendall tau rank correlation coefficient $(\tau)$ and associated $p$ value (Kendall and Gibbons, 1990). For both OLS and WLS, the slope is significantly greater than 1 by at least 1 standard error, and the coefficient $r$ indicates that the (positive) relationship between $k_{1}$ and $P_{m}$ is highly significant $(p<0.001)$. Similarly, the nonparametric coefficient $\tau$ and its $p$-value indicate a significant positive monotonic relationship between $k_{1}$ and $P_{m}$. While Bacon and Anderson (1982) did not report a slope, the positive, linear relationship between $k_{1}$ and $P$ reported here is consistent with that found by these authors using data from the Panama and Guatemala basins. On the other hand, our slope estimates contrast with the value of $0.58\left(r^{2}=0.93\right)$ found by Honeyman et al. (1988) using field data spanning a large range in particle concentration from $O\left(10 \mathrm{mg} \mathrm{m}^{-3}\right)$ to $O\left(10^{9} \mathrm{mg} \mathrm{m}^{-3}\right)$. We speculate on the reasons for this difference in Section 4.2.5. The effect of the errors in $P_{m}$ on the regression of $\ln k_{1} \mathrm{vs}$. $\ln P_{m}$, which are accounted for neither in OLS nor in WLS, is discussed in Appendix B. We also examine the effects of smoothing on the regression of $\ln k_{1}$ vs. $\ln P_{m}$ in Appendix C.

Particle composition may also influence $k_{1}$. For example at the easternmost stations (stations GT11-22 and GT11-24 of leg 1, and stations GT10-12, GT10-11, and GT10-10 of leg 2), the particles are composed mostly of organic matter above $500 \mathrm{~m}$, whereas nearly equal proportions of POM and particulate lithogenic material are observed below $500 \mathrm{~m}$ (Lam et al., 2015a). Multiple studies have suggested that marine POM contains organic ligands for which thorium has a high affinity (Hirose and Tanoue, 1998; Quigley et al., 2002; Hirose, 2004). If POM adsorbs Th more strongly than 
lithogenic particles, then the decrease in $k_{1}$ from near surface waters, where POM is highest, to deep waters, where the lithogenic fraction is higher, may even be larger than expected from just a decrease in particle concentration (i.e., not taking into account any change in particle composition).

Another apparent example of an effect of particle composition on $k_{1}$ arises from the large values of $k_{1}$ in deep waters at station GT11-16 (Figure 12d). This station is located in the vicinity of the TAG hydrothermal vent, where the fraction of iron (oxyhydr)oxides in the particles is the highest across the entire GA03 transect (Lam et al., 2015a). Thorium has been shown to strongly bind to iron oxides. For instance, Quigley et al. (2002) showed that the distribution coefficient for thorium bound to $\mathrm{Fe}_{2} \mathrm{O}_{3}, 10^{5.1} \mathrm{~L} \mathrm{~kg}^{-1}$, was within the range $\left(10^{5}-10^{6.8} \mathrm{~L} \mathrm{~kg}^{-1}\right)$ of the distribution coefficients for Th onto colloidal organic matter collected in the Gulf of Mexico (Baskaran et al., 1992), Chesapeake and Galveston Bay (Guo et al., 1997), and off the continental shelf of New England (Moran and Buesseler, 1993). Similarly, Guo et al. (2002a) found that the distribution coefficient for ${ }^{234} \mathrm{Th}$ bound to $\mathrm{Fe}_{2} \mathrm{O}_{3}$ was $10^{5.83} \mathrm{~L} \mathrm{~kg}^{-1}$, larger than the coefficients for $\mathrm{CaCO}_{3}$, humic material, chitin, and $\mathrm{SiO}_{2}$, and exceeded only by $\mathrm{MnO}_{2}$ and acid polysaccharides. In experiments with natural seawater, Lin et al. (2014) found the distribution coefficient for $\mathrm{Fe}_{2} \mathrm{O}_{3}$ to be $10^{5.59}$ $\mathrm{L} \mathrm{kg}{ }^{-1}$, greater than the coefficients for $\mathrm{CaCO}_{3}, \mathrm{Al}_{2} \mathrm{O}_{3}, \mathrm{TiO}_{2}$, and $\mathrm{SiO}_{2}$, and comparable to the coefficient for colloidal organic matter from the Gulf of Mexico added to natural seawater $\left(10^{5.86} \mathrm{~L}\right.$ $\mathrm{kg}^{-1}$ ). In their analysis of samples collected along GA03, Hayes et al. (2015a) found that $K_{D}$ for iron (oxyhydr)oxides is two orders of magnitude higher than for $\mathrm{POM}, \mathrm{CaCO}_{3}$, opal, or lithogenic particles. Fe K-edge-ray absorption spectroscopy of the TAG particles confirmed that the high iron was in the form of 6-line ferrihydrite (Ohnemus and Lam, 2015), a poorly crystalline iron oxyhydroxide with specific surface area and scavenging capacity 10-20 times higher than for $\mathrm{Fe}_{2} \mathrm{O}_{3}$ (Borggaard, 1983). Therefore, it seems that the large values of $k_{1}$ near the TAG hydrothermal vent are due to the high concentrations of ferrihydrite at this site.

\section{Th Desorption and Particle Degradation}

In general, the rate constants for Th desorption do not appear to exhibit vertical trends as pronounced as $k_{1}$ (Figures 11), although estimates of $k_{-1}$ do seem larger near the surface at some stations (e.g., GT11-24, GT10-12, GT10-11, and GT10-10; Figure 11). Nonetheless, both the mean and standard deviation of $k_{-1}$ across all stations and depths appear larger in the mesopelagic zone (ca. $100-1000 \mathrm{~m}$ ) than below (Figure $16 \mathrm{~b}$; Table 5). As with $k_{1}$, a potentially important influence 
on $k_{-1}$ is particle composition. Speculatively, the large values in $k_{-1}$ in near-surface waters of the easternmost stations (GT10-11 and GT10-10) may be due to the tendency for biogenic opal to contribute to a larger fraction of total particle concentration than at other stations (Lam et al., 2015a): assuming biogenic opal has a smaller affinity for thorium than particulate organic matter (Östhols, 1995; Chase et al., 2002; Quigley et al., 2002; Guo et al., 2002a; Luo and Ku, 2004; Roberts et al., 2009; Chuang et al., 2014; Lin et al., 2014), thorium might more easily desorb from biogenic opal than POM.

The particle degradation rate constant, $\beta_{-1}$, shows generally larger values above $1000 \mathrm{~m}$ (1.87 $\left.\mathrm{yr}^{-1}\right)$ than below $\left(0.21 \mathrm{yr}^{-1}\right)$, although the errors preclude a strong inference (Figure 16c; Table 5). These values may occur due to increased microbial respiration of particles in the mesopelagic zone, where the highest rates of bacterial production and particle degradation rates have been found (Reinthaler et al., 2006; Dehairs et al., 2008; Steinberg et al., 2008; McDonnell et al., 2015). Zooplankton feeding may also contribute to particle loss, although this process is likely to mostly affect aggregates greater that $0.5 \mathrm{~mm}$ in size (Lampitt et al., 1993; Dilling et al., 1998; Stemmann et al., 2004). Besides, $\beta_{-1}$ exhibits increased vertical variability in the top $1000 \mathrm{~m}$ : the standard deviation of our $\beta_{-1}$ estimates amounts to $2.67 \mathrm{yr}^{-1}$ above $1000 \mathrm{~m}$ and $0.40 \mathrm{yr}^{-1}$ below (Table 5). Whether this apparent variability is due to variability in microbial activity, particle lability, or biases from processes not incorporated in the model remains unclear.

\section{Rate Constant Ratio}

We describe the collective effect of the rate parameters $k_{1}, k_{-1}$, and $\beta_{-1}$ on Th scavenging using the rate constant ratio

$$
K=k_{1} /\left(k_{-1}+\beta_{-1}\right) .
$$

This ratio reflects the importance of the specific rate at which thorium attaches to particles relative to the specific rate at which thorium is removed from particles through Th desorption and particle degradation (Lerner et al., 2016). It is calculated using the estimates of $k_{1}, k_{-1}$, and $\beta_{-1}$ obtained by inversion at each station, and its error is determined by error propagation considering error covariances. To prevent the occurrence of very large $K$ values, we restrict our calculations to cases where $k_{-1}+\beta_{-1}>0.1 \mathrm{yr}^{-1}$. This value is determined from the probability density function of 


\begin{tabular}{|l|c|c|c|c|c|c|c|c|}
\hline & $k_{1}\left(\mathrm{yr}^{-1}\right)$ & $k_{-1}\left(\mathrm{yr}^{-1}\right)$ & $\beta_{-1}\left(\mathrm{yr}^{-1}\right)$ & $K$ & $K^{a}$ & $K^{b}$ & $K / P_{m}(\mathrm{~g} / \mathrm{g})$ & $\mathrm{w}\left(\mathrm{m} \mathrm{yr}^{-1}\right)^{c}$ \\
\hline mean above $1000 \mathrm{~m}$ & 1.11 & 5.10 & 1.87 & 0.20 & 0.18 & 0.20 & $2 \times 10^{7}$ & 851 \\
\hline s.d. above $1000 \mathrm{~m}$ & 0.84 & 4.57 & 2.67 & 0.30 & 0.08 & 0.31 & $1.1 \times 10^{7}$ & 389 \\
\hline mean below $1000 \mathrm{~m}$ & 0.71 & 2.45 & 0.21 & 0.19 & 0.15 & 0.15 & $3 \times 10^{7}$ & 990 \\
\hline s.d. below $1000 \mathrm{~m}$ & 2.21 & 1.81 & 0.40 & 0.20 & 0.06 & 0.05 & $8.5 \times 10^{6}$ & 656 \\
\hline
\end{tabular}

a. Excluding $K$ values $>1$.

b. Excluding station GT11-16.

c. For $w$, means and standard deviations are those above and below $2000 \mathrm{~m}$

Table 5: Mean and standard deviation (s.d.) of $k_{1}, k_{-1}, \beta_{-1}, K$, and $w$ for the inversion without smoothing.

our estimates of $\left(k_{-1}+\beta_{-1}\right)$, which reveals two modes: one mode below $10^{-2} \mathrm{yr}^{-1}$ containing $7 \%$ of our estimates, and one mode above $1 \mathrm{yr}^{-1}$ containing $93 \%$ of our estimates (not shown). Thus, by retaining cases where $k_{-1}+\beta_{-1}>0.1 \mathrm{yr}^{-1}$, the majority of $(K)$ values are considered.

We find that $K$ is generally less than 1 (Figure 17a), suggesting that the specific rate at which thorium is attached to particles is generally less than that at which it is lost from particles due to desorption and (or) particle degradation. We cannot discern a vertical trend in $K$ and its variability: the mean of $K$ in the top $1000 \mathrm{~m}$ is 0.20 with a standard deviation of 0.30 , while the mean of $K$ below $1000 \mathrm{~m}$ is 0.19 with a standard deviation of 0.20 (Table 5). To remove the potential influence of the few $K$ values larger than 1, we also estimate these means and standard deviations for $K<1$ (Figure 17a). For this subset of values, $K$ has a mean of 0.20 and a standard deviation of 0.11 above $1000 \mathrm{~m}$, and a mean of 0.15 and standard deviation of 0.06 below $1000 \mathrm{~m}$. In addition, we account for the influence of the TAG hydrothermal vent by estimating these means and standard deviations excluding station GT11-16. This subset of values yields a mean $K$ of 0.20 and standard deviation of 0.31 above $1000 \mathrm{~m}$, and a mean of 0.15 and standard deviation of 0.05 below $1000 \mathrm{~m}$ (Table 5). Considering these two subsets of $K$, a vertical pattern is suggested, with $K$ in the mesopelagic zone being larger and displaying stronger variability than below.

The choice of whether to apply a smoothing constraint also impacts the estimates of the rate parameters and $K$. The effects of smoothing on the estimates of $k_{-1}, \beta_{-1}$, and $K$ are examined in Appendix C.

\section{Relationship to Distribution Coefficient}

The rate constant ratio $K$, introduced in the previous section, can be related under certain circumstances to the distribution coefficient, $K_{D}=A_{p} /\left(A_{d} P\right)$, which is generally used to describe the partitioning of metals between the particulate and dissolved forms (Section 3.1). To elucidate the conditions under which $K$ and $K_{D}$ are related, consider the balance equation for dissolved 
Th (eq. 3.3a). Assuming that radioactive production, radioactive decay, and $T(\cdot)$ are negligible terms (for future reference, the first two assumptions are labeled A1 and A2, respectively), we get $0=\left(k_{-1}+\beta_{-1}\right) A_{p}-k_{1} A_{d}$, i.e.,

$$
A_{p} / A_{d}=k_{1} /\left(k_{-1}+\beta_{-1}\right)
$$

Thus, under assumptions A1, A2, and negligible $T(\cdot)$, the distribution coefficient, $K_{D}$, would be equivalent to $K$ normalized to particle concentration, $K / P$.

Alternatively, consider the balance equation for particulate Th (eq. 3.3b). Assuming that the effects of particle sinking, radioactive decay, and $T(\cdot)$ are negligible (first two assumptions labeled as $\mathrm{A} 3$ and $\mathrm{A} 4$, respectively), we obtain $0=k_{1} A_{d}-\left(k_{-1}+\beta_{-1}\right) A_{p}$, so $K_{D}$ would again be equivalent to $K / P$. Clearly, the approximate expression (3.11) could only hold when both sets of assumptions, (A1-A2) and (A3-A4) (together with negligible $T(\cdot))$ simultaneously hold (otherwise, a mathematical inconsistency would arise). Under such circumstances, expression (3.11) would provide a framework for interpreting the partitioning of Th between the solid and dissolved phases in terms of the relative importance of kinetic rate constants (for a related discussion see Honeyman et al. (1988)).

Stimulated by this development, we compare $K_{D}$ obtained from ${ }^{230} \mathrm{Th}$ and $P$ data (interpolated values are used since ${ }^{230}$ Th and $P$ data do not generally occur at the same depth) with $K$ normalized by measured particle concentration $\left(K / P_{m}\right)$. We calculate $K_{D}$ values from ${ }^{230} \mathrm{Th}_{d, p}$ data because (i) Hayes et al. (2015a) estimated $K_{D}$ using ${ }^{230} \mathrm{Th}_{d, p}$ and $P_{m}$ from all stations and depths along GA03, providing a basis for comparison, and (ii) assumptions (A1-A4) are most likely to hold for ${ }^{230} \mathrm{Th}$, since radioactive decay of ${ }^{230} \mathrm{Th}_{d, p}$ and production of ${ }^{230} \mathrm{Th}$ by ${ }^{234} \mathrm{U}$ are generally small compared to the effects due to ${ }^{230} \mathrm{Th}_{d}$ adsorption, ${ }^{230} \mathrm{Th}_{p}$ desorption, and (or) particle degradation (not shown; Lerner et al. (2016)). Likewise, we estimate $K / P_{m}$ at our selected stations, where the influences of advection and diffusion appear generally negligible (Section 3.2.5). Whereas assumptions A1, A2, and A4 also hold at all these stations, the effect of ${ }^{230} \mathrm{Th}_{p}$ sinking (A3) may not be negligible below $3000 \mathrm{~m}$ at station GT11-16 (not shown). Therefore, we restrict our estimation of $K / P_{m}$ to all our selected stations except GT11-16. With this restriction, we find that values of $K_{D}$ and $K / P_{m}$ are generally not significantly different given their respective error estimates (Figure 18a-b). Although our estimates of $K_{D}$ and $K / P_{m}$ are not independent (both are constrained from the same ${ }^{230} \mathrm{Th}_{d, p}$ 
and $P$ data), the comparison is encouraging.

Previous studies were also able to successfully relate a rate constant ratio to $K_{D}$ for thorium. Jannasch et al. (1988), using ${ }^{230} \mathrm{Th}$ from Puget Sound, found a strong correlation between $k_{1} / P$ and $K_{D}\left(r^{2}=0.88\right)$. Honeyman and Santschi (1989) expressed $K_{D}$ as $k_{1, c} P^{b} /\left(k_{-1} P\right)$, and found that this relationship holds for a constant $k_{-1}$, with $b$ varying from 1 at low particle concentration $\left(<10^{2} \mathrm{mg} \mathrm{m}^{-3}\right)$ to 0.3 at high particle concentration $\left(>10^{4} \mathrm{mg} \mathrm{m}^{-3}\right)$.

We examine the potential differences in $K / P_{m}$ between the mesopelagic zone and below across our selected stations, except GT11-16 (Table 5; Figure 17(c)). The mean $K / P_{m}$ in the top 1000 $\mathrm{m}$ is $2 \times 10^{7} \mathrm{~g} / \mathrm{g}$ with a standard deviation of $1.1 \times 10^{7} \mathrm{~g} / \mathrm{g}$, while the mean $K / P_{m}$ below $1000 \mathrm{~m}$ is $3 \times 10^{7} \mathrm{~g} / \mathrm{g}$ with a standard deviation of $8.5 \times 10^{6} \mathrm{~g} / \mathrm{g}$. These values, combined with the vertical profile in Figures 17 (c), do not appear to suggest consistent vertical patterns in $K / P_{m}$ and its variance.

\section{Particle Concentration Effect}

The particle concentration effect usually refers to a negative relationship between the distribution coefficient, $K_{D}$, and particle concentration, $P$ (e.g., Honeyman et al. (1988)). For our selected stations, we find a rank correlation coefficient $\tau=-0.64$ with $p<0.001$ for the relationship between $K_{D}$ and $P_{m}$ (Figure 18c). A comparable result holds for the relationship between $K / P_{m}$ and $P_{m}$ $(\tau=-0.51, p<0.001$, Figure 18d). These negative relationships are qualitatively consistent with

the negative correlation $r=-0.72$ between $K_{D}$ and $P$ found by Hayes et al. (2015a) using ${ }^{230} \mathrm{Th}_{d, p}$ and $P$ data from all GA03 stations and depths, excluding samples (i) with $>60 \%$ POM, (ii) with significant Fe and Mn (oxyhydr)oxide content, and (ii) from benthic nepheloid layers (note that $\tau$ and $r$ cannot be easily compared; we refrain from calculating a value of $r$ here given the indication of a variable relationship between $\log _{10} K_{D}$ and $\log _{10} P_{m}$ for $P_{m}<9 \mathrm{mg} \mathrm{m}^{-3}$ and $P_{m}>9 \mathrm{mg} \mathrm{m}^{-3}$; Figure 18c).

Thus, both $K_{D}$ and $K / P_{m}$ exhibit a significantly negative relationship with particle concentration at open-ocean stations of GA03. A proposed explanation for the particle concentration effect is that the rate of adsorption of trace metals onto filterable particles depends on the rate of coagulation of colloidal (e.g., $<0.8 \mu \mathrm{m}$ ) particles (Honeyman and Santschi, 1989; Baskaran et al., 1992, 1996; Hayes et al., 2015a). This hypothesis posits that $k_{1} \propto P^{b}$ with $b<1$ and that Th desorption and particle degradation are independent of particle concentration. That is, if colloidal coagulation 
limits the rate of thorium adsorption onto particles, then a given increase in particle concentration would result in an increase in $k_{1}$ that is less than that expected from a linear relationship between $k_{1}$ and $P$ (Honeyman et al., 1988). However, our results suggest that $k_{1} \propto P^{b}$ with $b \geq 1$ (section 3.4.2.1), although $K / P_{m}$ decreases with $P$. The cause of this conundrum obviously lies in the fact that the specific rate of Th loss from particles due to Th desorption and particle degradation is not independent of particle concentration, as previously postulated (e.g., Honeyman et al. (1988)), but may also vary, in this case increase, with particle concentration. To test this possibility, we examine the relationship between $\ln \left(k_{-1}+\beta_{-1}\right)$ and $\ln P_{m}$, neglecting again values of $\left(k_{-1}+\beta_{-1}\right)$ less than $0.1 \mathrm{yr}^{-1}$ (Figure 19). A positive relationship is apparent, with a slope of $0.99 \pm 0.18$ for OLS and $1.21 \pm 0.13$ for WLS, confirming that the specific rate of Th loss from particles due to Th desorption and particle degradation tends to increase with particle abundance.

Thus, although we observe a particle concentration effect at our selected stations, we find that this effect may not strictly comply to the Brownian-pumping model (Honeyman and Santschi, 1989). Rather, the variation of $K_{D}$ or $K / P$ with $P$ would arise from two distinct relationships. The first relationship, $k_{1} \propto P^{b}$ with $b \geq 1$, contradicts (i) the proposal by Honeyman et al. (1988) that $b<1$ if there is a particle concentration effect due to colloidal coagulation limiting the rate at which thorium appears on filterable $(>0.8 \mu \mathrm{m})$ particles, as well as (ii) their value $b=0.51$ derived from oceanic field data corresponding to $P \geq 10 \mathrm{mg} \mathrm{m}^{-3}$. Recall that their proposal relies on the notion that the number of surface sites available for metal attachment can be approximated by the particle concentration (section 3.4.2.1). This assumption may not hold universally as, for example, particles with the same concentration may be characterized by different surface-to-volume ratios or different shapes. The lack of a particle concentration effect in the relationship between $k_{1}$ and measured $P$ (i.e., $b \geq 1$ ) may suggest a low abundance of colloids, relatively low colloidal reactivity of Th compared to its filterable particle reactivity, or a short residence time of colloids with respect to aggregation, such that colloidal aggregation does not limit the rate of appearance of thorium onto filterable particles (Moran and Buesseler, 1993). In fact, Honeyman and Santschi (1989) suggested that a particle concentration effect should not exist for particle concentration below $10^{2} \mathrm{mg} \mathrm{m}^{-3}$, a concentration level which is much larger than those considered in this study (Figure 10).

While Th isotope measurements on the colloidal fraction are not available for the GA03 transect, Hayes et al. (2017) found that colloidal ${ }^{230} \mathrm{Th}$ was a small portion $(<6 \%)$ of "dissolved" ${ }^{230} \mathrm{Th}$ and 
${ }^{232} \mathrm{Th}$ in the upper $1000 \mathrm{~m}$ at $27^{\circ} 12^{\prime} \mathrm{N}, 62^{\circ} 58^{\prime} \mathrm{W}$, about $500 \mathrm{~km}$ south of Bermuda. The presence of small concentrations of colloidal ${ }^{230,232} \mathrm{Th}$ appears consistent with the relationship between $k_{1}$ and $P$ found in our study, which does not require an influence of colloids as described in the Brownianpumping model. However, it is unclear whether the low colloidal ${ }^{230,232} \mathrm{Th}$ activities reported by Hayes et al. (2017) are due to a low abundance of colloids, or to a relatively small affinity of Th for colloids compared to filterable particles. Furthermore, extrapolation of their results to the entire set of stations considered here may be inappropriate.

The second relationship is $\left(k_{-1}+\beta_{-1}\right) \propto P^{d}$ (Figure 19) with $d>0$, again contrasting with the assumption of Honeyman et al. (1988) that the rate constant for Th loss from particles is independent of $P$. This apparent association of $\left(k_{-1}+\beta_{-1}\right)$ with $P_{m}$ pertains to $93 \%$ of the rate constants estimated in this paper, as it relies on $\left(k_{-1}+\beta_{-1}\right)$ estimates higher than $0.1 \mathrm{yr}^{-1}$. A positive relationship between $\left(k_{-1}+\beta_{-1}\right)$ and $P_{m}$ is somewhat surprising, given that neither of these rate parameters is expected to be directly influenced by particle concentration. For example, Honeyman et al. (1988) proposed that the apparent desorption rate constant of a particle-reactive metal could be expressed as

$$
k_{-1}=k_{-1, c}[X]
$$

where $k_{-1, c}$ is a positive constant and $[\mathrm{X}]$ is the seawater concentration of the ion with which thorium is exchanging on the surface of a particle. In this proposed relationship (and in contrast to $k_{1}$ ), $k_{-1}$ is independent of particle concentration.

The apparent relationship between $\left(k_{-1}+\beta_{-1}\right)$ and particle concentration found in this study may reflect the covariance of particle concentration with particle composition in the upper 1000 m. For example, it has been shown that the fraction of total particulate matter composed of labile POM rapidly attenuates with depth in the mesopelagic zone, which mimics the downward decrease of particle concentration (Wakeham et al., 1997; Hedges et al., 2000; Lutz et al., 2002; Sheridan et al., 2002; Collins et al., 2015). A decrease with depth in the relative abundance of labile POM would then result in a corresponding decrease in $\beta_{-1}$. However such reasoning is speculative, and the apparent relationship between $\left(k_{-1}+\beta_{-1}\right)$ and particle concentration remains an intriguing result of this study. 


\subsubsection{Importance of Missing Terms}

In section 3.2.4, we assessed the influence of (lateral and vertical) advection and diffusion (as well as non-steady state conditions for the cross-over station) on the Th isotope and particle budgets at our selected stations along GA03. Here we revisit this assessment based on the dominant posterior flux in the corresponding balance equation as obtained by inversion (section 3.3). As for the prior fluxes, the posterior fluxes are the adsorption rate of thorium onto particles, the production rate from the radioactive parent, and the degradation rate of particles (Figures 4-5). Reassuringly, the posterior fluxes are always larger than the estimates of the missing terms. At some stations, horizontal diffusion and unsteadiness terms are within or close to within 1 standard deviation of the posterior estimates of radioactive ${ }^{228} \mathrm{Th}_{d}$ production. These missing terms, then, may contribute to the budget of ${ }^{228} \mathrm{Th}_{d}$.

One should probably re-iterate that the rate parameters reported in this study are estimated under the assumption that the effects of advection, diffusion, and unsteadiness are all negligible. Hence, these parameters may suffer from some bias due to the exclusion of these terms. While our results, with the possible exception of ${ }^{228} \mathrm{Th}_{d}$, suggest that the neglected processes do not, to the first-order, influence thorium isotopes and particles at our selected stations (Figures 4-5), a significant impact of these terms cannot be ruled out with complete confidence.

\subsection{Conclusion}

Radiochemical and particle data from the GEOTRACES section GA03 are combined with a single-particle class model in order to estimate the lateral and vertical distributions of $\left(k_{1}, k_{-1}, \beta_{-1}\right.$, $w)$ in the open North Atlantic. We provide evidence that advection by the mean flow, turbulent diffusion, and unsteadiness have generally a small influence on the Th and particle budgets at the stations considered in this paper. The model displays a good fit to the data $(\phi=0.82, B=0.07)$, and the posterior estimates of the radiochemical activities and particle concentrations generally replicate the (vertically interpolated) data. The rate parameters exhibit some discernible spatial patterns. Most notably, $k_{1}$, the adsorption rate constant, tends to decrease with depth, and many of its highest values occur in the top $500 \mathrm{~m}$ of the water column at the easternmost stations (GT11-24, GT10-12, GT10-11, and GT10-10). One exception is at station GT11-16 near the TAG hydrothermal vent, where $k_{1}$ reaches its absolute maximum of $21 \mathrm{yr}^{-1}$. Notable features in the 
distributions of the other rate parameters include (i) larger values of $k_{-1}$ in the top $500 \mathrm{~m}$ of the easternmost stations and (ii) larger variability of $\beta_{-1}$ in the top $1000 \mathrm{~m}$. We speculate on a number of processes that may influence $k_{-1}$ and $\beta_{-1}$, including microbial activity and changes in particle composition.

We find a positive relationship between $k_{1}$ and particle concentration, $P, k_{1}=k_{1, c} P^{b}$ with $b \geq 1$, supporting the notion that $k_{1}$ increases with the number of surface sites available for adsorption, but opposing the assertion that colloids affect the apparent rate at which Th attaches to filterable particles as envisioned by the Brownian-pumping model (Honeyman and Santschi, 1989). We also find a negative relationship between the distribution coefficient for ${ }^{230} \mathrm{Th}, K_{D}$, and particle concentration, indicative of a particle concentration effect. We clarify the conditions under which $K_{D}$ is equivalent to the rate constant ratio, $K=k_{1} /\left(k_{-1}+\beta_{-1}\right)$, normalized to particle concentration, and find that these conditions are met at all our selected stations except GT11-16. We think that this result is significant, for it suggests that a kinetic interpretation of $K_{D}$ is generally warranted at these stations. Interestingly, the relationship between $K / P$ and $P$ appears to be partly driven by a positive relationship between $\left(k_{-1}+\beta_{-1}\right)$ and $P$, in contrast with a previous interpretation of the particle concentration effect (e.g. Honeyman et al. (1988); Honeyman and Santschi (1989)).

Importantly, if these relationships between $k_{1}$ and $\left(k_{-1}+\beta_{1}\right)$ on the one hand and $P$ on the other hand are robust, then they suggest that Th adsorption onto particles and Th release from particles, due to the combined effects of desorption and particle degradation, are not first order processes with respect to particle concentration. Notice that these findings do not challenge the model considered in this study (which systematically relies on first order kinetics), since the specific rate constants for sorption and particle processes inferred here are apparent ones (e.g. Honeyman et al. (1988)). In our study, they are allowed to vary with both location and depth. A dependence of $k_{1}$ on $P$, for example, is not formally incorporated into the model but emerges from the quantitative combination of the model and the data. It is established a posteriori, not posited a priori.

While we emphasize relationships between rate parameters and particle concentration, we stress that these parameters may also be influenced by other properties. Future studies should focus on (i) quantifying the effects of particle composition on rate parameters for thorium and particle cycling, and (ii) examining the effect of microbial and zooplankton activities on these rate parameters. Finally, the approach of data analysis used in this study should be applicable to study the geochem- 
ical behavior of other particle-reactive metals in the ocean, such as protactinium, whose long-lived isotope ${ }^{231} \mathrm{~Pa}$, paired with ${ }^{230} \mathrm{Th}$ and measured on sediment samples, is taken as as an indicator of paleo-productivity or paleo-circulation(Henderson and Anderson, 2003). A potential complication with ${ }^{231} \mathrm{~Pa}$ is that its $\sim 130 \mathrm{yr}$ residence time in the ocean makes it more susceptible to the effects of advection and diffusion (Henderson and Anderson, 2003). Therefore, an assessment of these effects, similar to that carried out for ${ }^{230} \mathrm{Th}$ in this study, is critical for ${ }^{231} \mathrm{~Pa}$. A better understanding of the scavenging of both radionuclides is needed in order to properly interpret bulk sediment ${ }^{231} \mathrm{~Pa} /{ }^{230} \mathrm{Th}$ records in terms of palaeoceanographic phenomena (e.g., Burke et al. (2011); Hayes et al. (2015b)) and to improve their representation in ocean circulation-biogeochemistry models (e.g., Siddall et al. (2005); Dutay et al. (2009)).

\section{Acknowledgement}

We acknowledge the U.S. National Science Foundation for providing funding for this study (grant OCE-1232578) and for U.S. GEOTRACES North Atlantic section ship time, sampling, and data analysis. The U.S. NSF also supported the generation of ${ }^{230} \mathrm{Th}$ data (OCE-0927064 to LDEO, OCEO092860 to WHOI, and OCE-0927754 to UMN) and ${ }^{228,234}$ Th data (OCE-0925158 to WHOI). We thank the chief scientists of the GA03 section (Ed Boyle, Bill Jenkins, and Greg Cutter) as well as the captain, the crew, and the scientific party on the R/V Knorr, which completed this section. We are also grateful to the scientists and staff involved in the collection and analysis of the thorium isotope and particle data. We also thank Andrew Solow (WHOI) for directing us to MATLAB constrained optimization routine FMINCON. 


\section{A Appendix A}

This appendix provides further details about our method of data inversion. The vector $\boldsymbol{x}$ that yields a minimum of $J(\boldsymbol{x})$ subject to $\boldsymbol{f}(\boldsymbol{x})=\mathbf{0}$ is derived as follows (Byrd et al., 2000; Waltz et al., 2006). Equation (3.5) is replaced by a sequence of subproblems of the form:

$$
J_{\mu}(\boldsymbol{x}, \boldsymbol{s})=J(\boldsymbol{x})-\mu \sum_{i=1}^{m} \ln \left(s_{i}\right) .
$$

Here, $\mu>0$ is a barrier parameter, ln is the natural logarithm, and $s>0$ is a vector of "slack" variables. The function (A.1) is minimized subject to the equality constraints $\boldsymbol{f}(\boldsymbol{x})=\mathbf{0}$ and the inequality constrains $\boldsymbol{g}(\boldsymbol{x}) \geq \mathbf{0}$. In our study, $\boldsymbol{f}(\boldsymbol{x})=\mathbf{0}$ represents the model equations, whereas $\boldsymbol{g}(\boldsymbol{x})=\boldsymbol{x}$ represents our desire to avoid the inference of negative values for the radiochemical activities, the particle concentrations, and the rate parameters. The slack variables are used to replace the inequality constraints with equality constraints, i.e., $\boldsymbol{g}(\boldsymbol{x})+\boldsymbol{s}=\mathbf{0}$. A solution is then obtained by finding a stationary point of the following function:

$$
L(\boldsymbol{x}, \boldsymbol{s}, \boldsymbol{\lambda})=J_{\mu}(\boldsymbol{x}, \boldsymbol{s})+\boldsymbol{\lambda}_{f}^{\prime} \boldsymbol{f}(\boldsymbol{x})+\boldsymbol{\lambda}_{g}^{\prime}(\boldsymbol{g}(\boldsymbol{x})+\boldsymbol{s}) .
$$

Here, $L(\boldsymbol{x}, \boldsymbol{s}, \boldsymbol{\lambda})$ is the Lagrangian associated with (A.1), and $\boldsymbol{\lambda}=\left[\boldsymbol{\lambda}_{f}^{\prime}, \boldsymbol{\lambda}_{h}^{\prime}\right]^{\prime}$ is a vector of Lagrange multipliers. The solution is found using an Interior Point algorithm, which relies on one of two methods. The first method is the "direct step" (Waltz et al., 2006), whereby a solution is found by linearizing equation (A.2) at some reference point $\left(\boldsymbol{x}_{k}, \boldsymbol{s}_{k}\right)$, where $k$ is an iterate index. This method may fail due to nonconvexity or rank deficiencies in the (i) Jacobians (matrices of first-order partial derivatives of $\boldsymbol{f}(\boldsymbol{x})$ and $\boldsymbol{g}(\boldsymbol{x})$ with respect to $\boldsymbol{x}$ ) or (ii) the Hessian (matrix of second-order partial derivatives of $L(\boldsymbol{x}, \boldsymbol{s}, \boldsymbol{\lambda})$ with respect to $\boldsymbol{x})$. In this case, the Interior Point algorithm relies on a second method, which involves a trust region. For details regarding these methods, we refer the reader to the relevant literature (Byrd et al., 1999, 2000; Waltz et al., 2006).

We apply the Interior Point algorithm using the constrained nonlinear programming solver FMINCON, which is part of Matlab's optimization toolbox (Matlab, 2016). Among the optional inputs to the algorithm is the gradient of the objective function (3.5), which we provide as:

$$
\nabla_{\boldsymbol{x}} J(\boldsymbol{x})=2 \boldsymbol{C}_{0}^{-1}\left(\boldsymbol{x}-\boldsymbol{x}_{0}\right)
$$


The other user-defined inputs are listed in table A.1. These options are used for all inversions discussed in this paper.

The posterior uncertainties in $\boldsymbol{x}$ are estimated from the square root of the diagonal elements of the posterior error covariance matrix (Tarantola and Valette, 1982)

$$
\boldsymbol{C}_{k+1}=\boldsymbol{C}_{0}-\boldsymbol{C}_{0} \boldsymbol{F}_{k}^{\prime}\left(\boldsymbol{F}_{k} \boldsymbol{C}_{0} \boldsymbol{F}_{k}^{\prime}\right)^{-1} \boldsymbol{F}_{k} \boldsymbol{C}_{0}
$$

Here, $\boldsymbol{F}_{k}$ is a matrix whose elements are the partial derivatives of the model equations with respect to the elements of $\boldsymbol{x}$ at the solution point, i.e., the element in the $i$ th row and $j$ th column of $\boldsymbol{F}_{k}$ is $\partial f_{i} / \partial x_{j}$ evaluated at $\boldsymbol{x}=\hat{\boldsymbol{x}}_{k}$, where $\hat{\boldsymbol{x}}_{k}$ denotes the solution (subscript $k$ is dropped in the text). The matrix inversions in (A.3-A.4) are performed using LU decomposition.

\begin{tabular}{|l|c|}
\hline & Input \\
\hline Maximum iterations & 10,000 \\
\hline Maximum Objective Function Evaluations & $40,000,000$ \\
\hline User supplied objective function gradient & On \\
\hline Objective Function Tolerance & $10^{-3}$ \\
\hline Tolerance on hard constraint & False \\
\hline CheckGradients & Inf \\
\hline DiffMaxChange & 0 \\
\hline DiffMinChange & $\left(\sqrt{2^{-52}}\right)$ \\
\hline FiniteDifferenceStepSize & forward \\
\hline FiniteDifferenceType & $10^{-6}$ \\
\hline OptimalityTolerance & false \\
\hline SpecifyConstraintGradient & $10^{-10}$ \\
\hline StepTolerance & false the number of elements in $\boldsymbol{x}$ \\
\hline TypicalX & true \\
\hline UseParallel & 0.1 \\
\hline Hessian Approximation & Bector $[1 \ldots .1]$ with length equal to \\
\hline HonorBounds & $\sqrt{N}$, where $N$ is the number of elements in $\boldsymbol{x}$ \\
\hline Initial Barrier Parameterithm & factorization \\
\hline Initial Trust Region Radius & $10^{-10}$ \\
\hline Maximum Projected Conjugate Gradients & 0.01 \\
\hline Subproblem Algorithm & $2 \times(N-E)$, where $E$ is the number of inequality constraints \\
\hline Tolerance for Projected Conjugate Gradient & \\
\hline Relative Tolerance for Projected Conjugate Gradient & \\
\hline
\end{tabular}

Table A1: Optional Inputs for Matlab's Constrained Nonlinear programming solver FMINCON

\section{B Appendix B}

The OLS and WLS regressions of $\ln k_{1}$ against $\ln P_{m}$ do not account for the uncertainties in the measurements of particle concentration. Specifically, in the regression problem, 


$$
\ln \left(k_{1}\right)=a+b \ln \left(P_{m}\right)+\epsilon
$$

both $b$ and $P_{m}$ should be treated as unknowns. Here, $a=\ln k_{1, c}$ is the intercept, $b$ is the slope, and $\epsilon$ is a regression error. In order to estimate $(a, b)$ taking uncertainties in $P_{m}$ into account, we use the Algorithm of Total Inversion (ATI; Tarantola and Valette (1982)). Thus, we construct a prior estimate of a vector $\boldsymbol{x}$ of unknowns, which is $\boldsymbol{x}_{0}$. The elements of $\boldsymbol{x}_{0}$ contain measurements of $P_{m}$ (more specifically, $\ln \left(P_{m}\right)$ ) and prior estimates of $a$ and $b$. We then minimize the objective function

$$
J(\boldsymbol{x})=\left(\boldsymbol{x}-\boldsymbol{x}_{0}\right)^{\prime} \boldsymbol{C}_{0}^{-1}\left(\boldsymbol{x}-\boldsymbol{x}_{0}\right)+\boldsymbol{f}(\boldsymbol{x})^{\prime} \boldsymbol{C}_{f}^{-1} \boldsymbol{f}(\boldsymbol{x}) .
$$

Here, the error covariance matrix $C_{0}$ is taken as diagonal, and its diagonal elements are based on the variances in the measurements of $P$ (Aitchison and Brown, 1957) and in the prior estimates of $a$ and $b$. The vector $\boldsymbol{f}(\boldsymbol{x})$ contains the regression equations (B.1), and $\boldsymbol{C}_{f}$ is a matrix whose elements are based on the posterior error statistics of $\ln k_{1}$ derived by inversion (section 3.2.5).

The prior estimates of $a$ and $b$ are taken from Honeyman et al. (1988): $a_{0}=-9.2 \pm 9.2$ and $b_{0}=0.51 \pm 0.51$, where we subjectively assumed $100 \%$ relative error for both coefficients. We find that the slope of $\ln k_{1}$ vs. $\ln P_{m}$ is $\hat{b}=3.1 \pm 0.1$ (Figure B.1). Although this value is significantly larger than the slopes derived from OLS and WLS (Table 4), the slopes estimated from the three regression methods (OLS, WLS, and ATI) are all $\mathcal{O}(1)$.

\section{C Appendix C}

\section{C.1 Effect of Smoothing}

In our recent study (Lerner et al., 2016), a vertical smoothing constraint on the rate parameters, $\gamma \boldsymbol{x}^{\prime} \boldsymbol{S}^{-1} \boldsymbol{x}$, was added to the objective function (5). The motivation for adding a vertical smoothing constraint was twofold: (i) to reduce the occurrence of negative values in the solution, since the method used to fit the model to the data did not prevent inference of negative values, and (ii) to reduce large variations of the rate parameters on small vertical scales, which do not appear geochemically plausible. In this section, we describe the influence of smoothing on the rate parameter estimates at our selected stations. 
Adsorption Rate Constant We consider the results of regressing $\ln k_{1}$ against $\ln P_{m}$ for the case where $\gamma \boldsymbol{x}^{\prime} \boldsymbol{S}^{-1} \boldsymbol{x}$ (with $\gamma=1$ ) is added to the objective function (5) (Figure 15d-f). Table 4 lists the slopes of OLS and WLS, as well as the Pearson and Kendall correlation coefficients.

The regressions of $\ln k_{1}$ obtained by inversion with smoothing vs. $\ln P_{m}$ consistently yield smaller slopes compared with the corresponding regressions based on $\ln k_{1}$ obtained by inversion without smoothing. Given that vertical smoothing tends to reduce the vertical variations in the rate parameters, this result is not surprising. On the other hand, the regressions based on $\ln k_{1}$ obtained by inversion with and without smoothing are comparable in that all slopes are on the order of 1 (Table 4).

Other Rate Parameters Figure 16e-h show $k_{-1}, \beta_{-1}$ and $w$ estimates at all depths and all selected stations obtained by inversion with the smoothing constraint $(\gamma=1)$. Compared with the solution without smoothing, the vertical variability in $\beta_{-1}$ and $w$ is drastically reduced. Without smoothing, the standard deviation of our posterior estimates of $k_{-1}, \beta_{-1}$, and $w$ (all selected stations and all depths) is $3.76 \mathrm{yr}^{-1}, 2.12 \mathrm{yr}^{-1}$, and $548 \mathrm{~m} \mathrm{yr}^{-1}$, respectively. With smoothing, these values amount to $1.81 \mathrm{yr}^{-1}, 0.15 \mathrm{yr}^{-1}$, and $383 \mathrm{~m} \mathrm{yr}^{-1}$, respectively. The large vertical variations of $\beta_{-1}$ apparent in the upper $1000 \mathrm{~m}$ inferred from the inversion without smoothing (2.67 $\mathrm{yr}^{-1}$; Table 5) are reduced for the inversion with smoothing $\left(0.15 \mathrm{yr}^{-1}\right.$; Table C.1). Similarly, the large vertical variations of $w$ below $2000 \mathrm{~m}$ inferred without smoothing (656 $\mathrm{m} \mathrm{yr}^{-1}$; Table 5) are smaller for the inversion with smoothing (465 $\mathrm{m} \mathrm{yr}^{-1}$; Table C.1).

We consider the effect of smoothing on the rate constant ratio $K$ (Figure 17b). With smoothing, the standard deviation of $K$ decreases from 0.25 to 0.13 (all selected stations and depths). Interestingly, the mean values of $K$ above and below 1000 m estimated without smoothing (respectively, 0.20 and 0.19 for $K$; Table 5) show little change from those estimated with smoothing (respectively, 0.21 and 0.17; Table C.1). In our previous analysis of station GT11-22 data (Lerner et al., 2016), we found that $K$ exhibits a mean of 0.27 (standard deviation of 0.09 ) above $1000 \mathrm{~m}$, and a mean of 0.14 (standard deviation of 0.02 ) below $1000 \mathrm{~m}$. We compare these values with those obtained from our inversion without smoothing. We find that $K$ averages 0.18 (standard deviation of 0.10) above $1000 \mathrm{~m}$, and 0.12 (standard deviation of 0.02) below $1000 \mathrm{~m}$. These results show that our previous inference of higher $K$ in the mesopelagic zone than below at GT11-22 (Lerner et al., 2016) 
was due, at least in part, to the effects of smoothing. Finally, notice that the absence a of clear vertical pattern in $K / P_{m}$ persist with smoothing (Fig $17 \mathrm{~d}$ ), as expected.

The decision to apply vertical smoothing (or not) in field data inversion is largely driven by prior knowledge of the property gradients along the water column. Presumably, the availability of measurements with high vertical resolution (e.g., tens of meters) would preclude the need for smoothing, since in this scenario the sampling would be dense enough that adding further assumptions about vertical property gradients would be superfluous. Trace metal and particle measurements at stations occupied along GEOTRACES transects are $O(100-1000) \mathrm{m}$ apart along the water column. Absent prior knowledge about thorium isotope activity or particle concentration gradients on shorter spatial scales, it is difficult to recommend whether or not to use smoothing in the analysis of GEOTRACES data.

\begin{tabular}{|l|c|c|c|c|c|c|c|c|}
\hline & $k_{1}\left(\mathrm{yr}^{-1}\right)$ & $k_{-1}\left(\mathrm{yr}^{-1}\right)$ & $\beta_{-1}\left(\mathrm{yr}^{-1}\right)$ & $K$ & $K^{a}$ & $K^{b}$ & $K / P_{m}(\mathrm{~g} / \mathrm{g})$ & $\mathrm{w}\left(\mathrm{m} \mathrm{yr}^{-1}\right)^{b}$ \\
\hline mean above $1000 \mathrm{~m}$ & 0.73 & 3.22 & 0.33 & 0.21 & 0.21 & 0.21 & $2.3 \times 10^{7}$ & 942 \\
\hline s.d. above $1000 \mathrm{~m}$ & 0.39 & 1.87 & 0.15 & 0.08 & 0.08 & 0.08 & $9.5 \times 10^{6}$ & 274 \\
\hline mean below $1000 \mathrm{~m}$ & 0.46 & 2.76 & 0.24 & 0.17 & 0.17 & 0.14 & $2.8 \times 10^{7}$ & 1051 \\
\hline s.d. below $1000 \mathrm{~m}$ & 0.33 & 1.73 & 0.13 & 0.16 & 0.08 & 0.04 & $7.5 \times 10^{6}$ & 465 \\
\hline
\end{tabular}

a. Excluding $K$ values $>1$.

a. Excluding station GT11-16

$c$. For $w$, means and standard deviations are those above and below $2000 \mathrm{~m}$.

Table C1: Mean and standard deviation (s.d.) of $k_{1}, k_{-1}, \beta_{-1}, K$, and $w$ for the inversion with smoothing.

\section{C.2 Effect of Algorithm}

Previous studies have reported that estimates of rate constants of Th and particle cycling may depend on the specific method used to fit the model to the data (Murnane, 1994a; Athias et al., 2000b,a). Lerner et al. (2016) combined Th isotope and particle data at station GT11-22 with the Th and particle cycling model (3) (with $T(\cdot)=0$ ) using a different algorithm (Algorithm of Total Inversion, or ATI) than implemented here (FMINCON; Appendix A). In order to document the effect of the nature of the algorithm, we compare the results of regressing $\ln k_{1}$ vs. $\ln P$ ( $P$ obtained from the inversion) for the case where $k_{1}$ (as well as other variables in $\boldsymbol{x}$ for station GT11-22) are estimated from ATI and FMINCON, both with smoothing (Figure C.1; Table C.2). Whereas the present study uses adsorbed ${ }^{230} \mathrm{Th}$, Lerner et al. (2016) used particulate ${ }^{230} \mathrm{Th}$ (which also comprises ${ }^{230} \mathrm{Th}$ locked in the mineral lattices of lithogenic particles). Thus, for this comparison, we run the inversion using FMINCON with particulate ${ }^{230} \mathrm{Th}$ as well as adsorbed ${ }^{230} \mathrm{Th}$, so that changes in the 


\begin{tabular}{|l|c|c|c|c|c|}
\hline & $\hat{b} \pm \sigma_{\hat{b}}$ & $r$ & $p$ for $r$ & $\tau$ & $p$ for $\tau$ \\
\hline ATI w/ smoothing, total ${ }^{230} \mathrm{Th}_{p}$ & $0.60 \pm 0.05$ & 0.80 & $<0.001$ & 0.73 & $<0.001$ \\
\hline ATI w/ smoothing, adsorbed ${ }^{230} \mathrm{Th}_{p}$ & $0.58 \pm 0.06$ & 0.81 & $<0.001$ & 0.71 & $<0.001$ \\
\hline FMINCON w/ smoothing & $0.58 \pm 0.06$ & 0.84 & $<0.001$ & 0.68 & $<0.001$ \\
\hline FMINCON no smoothing & $1.6 \pm 0.09$ & 0.78 & $<0.001$ & 0.62 & $<0.001$ \\
\hline
\end{tabular}

Table C2: Regression of $\ln k_{1}$ against $\ln P$, with $k_{1}$ estimated from four different inversions at GT11-22.

regression results due to changes in the data used may also be documented.

For the results with smoothing, the slopes of $\ln k_{1}$ vs. $\ln P$ are not significantly different at the level of 1 standard error (Table 7). The result obtained using FMINCON without smoothing (section 3.3) is also included for reference. These findings suggest that the nature of the algorithm used to invert the data does not significantly influence our inference about a dependence of $k_{1}$ upon particle concentration. 


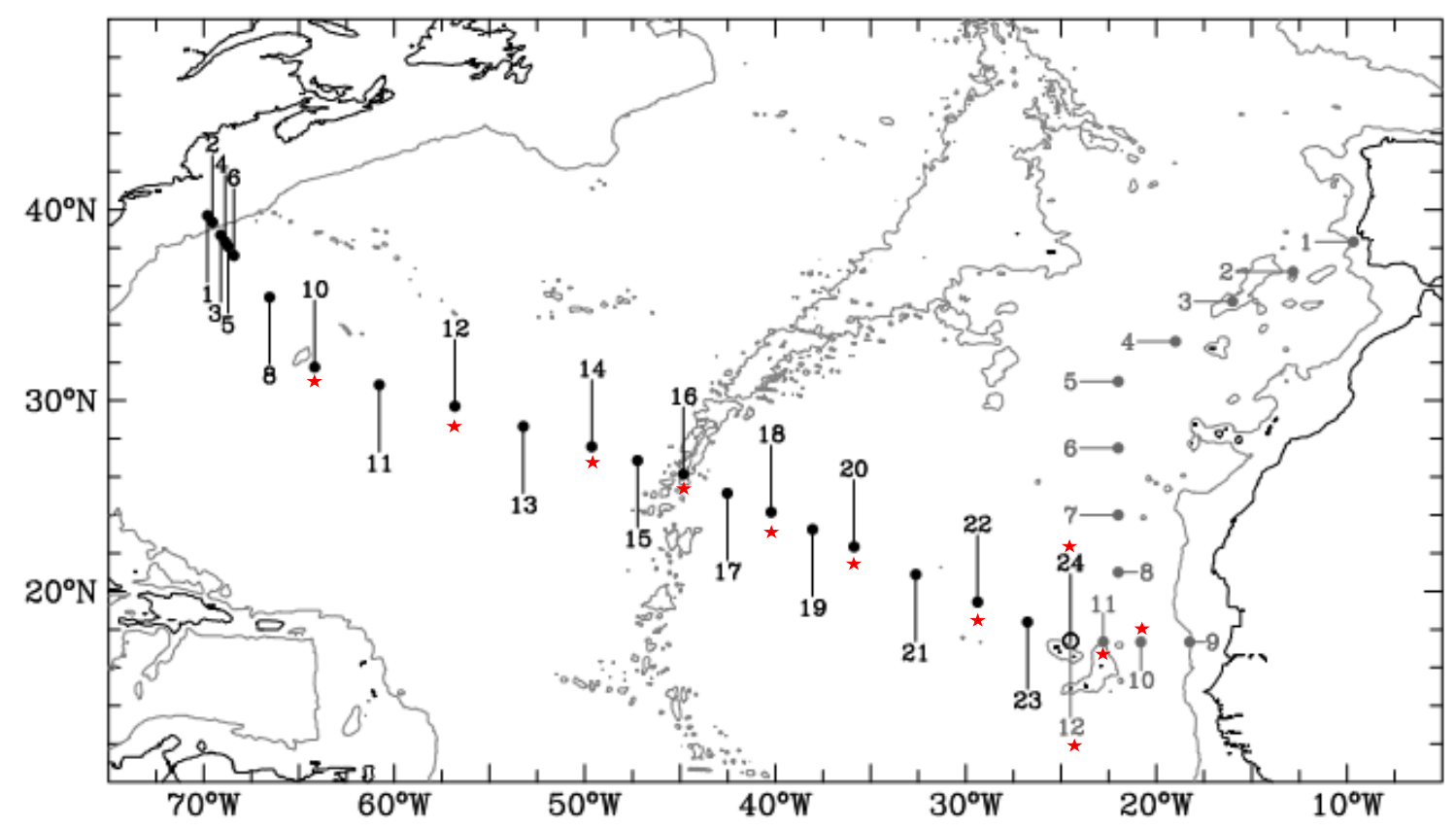

Figure 1: Stations occupied by the R/V Knorr during the GEOTRACES North Atlantic section (GA03). The grey dots show the stations occupied during the first leg (October 2010) and the black dots show the stations occupied during the second leg (November-December 2011). The open circle is both station GT1012 of the first leg and station GT11-24 of the second leg. The data analyzed in this paper occur at stations marked by red asterisks. The solid lines show the coastline (black) and the 3000-m isobath (grey). 

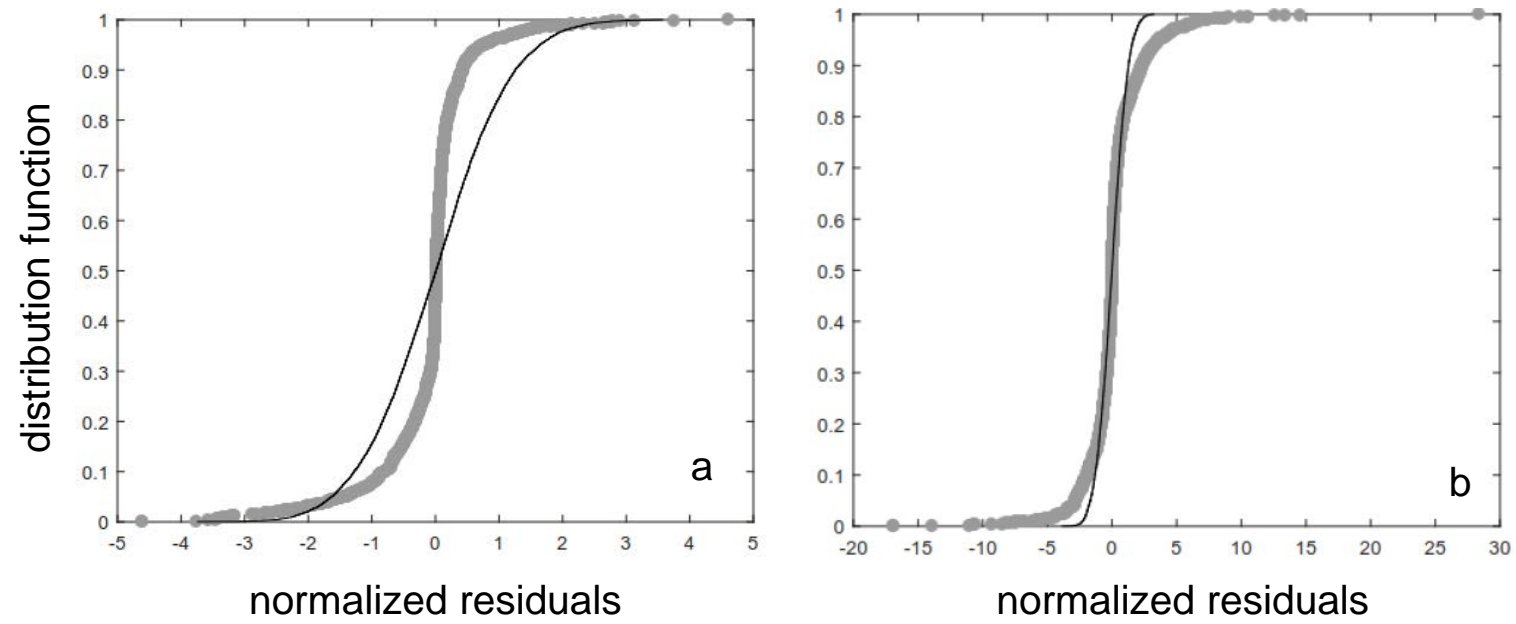

Figure 2: Distribution function of residuals normalized to the errors in the data. The gray circles are the normalized residuals, and the black curve is the standard normal distribution. Panel (a) shows the residuals for the interpolation (Section 3.2.3), and panel (b) shows the residuals for the inversion (Section 3.3.1). 


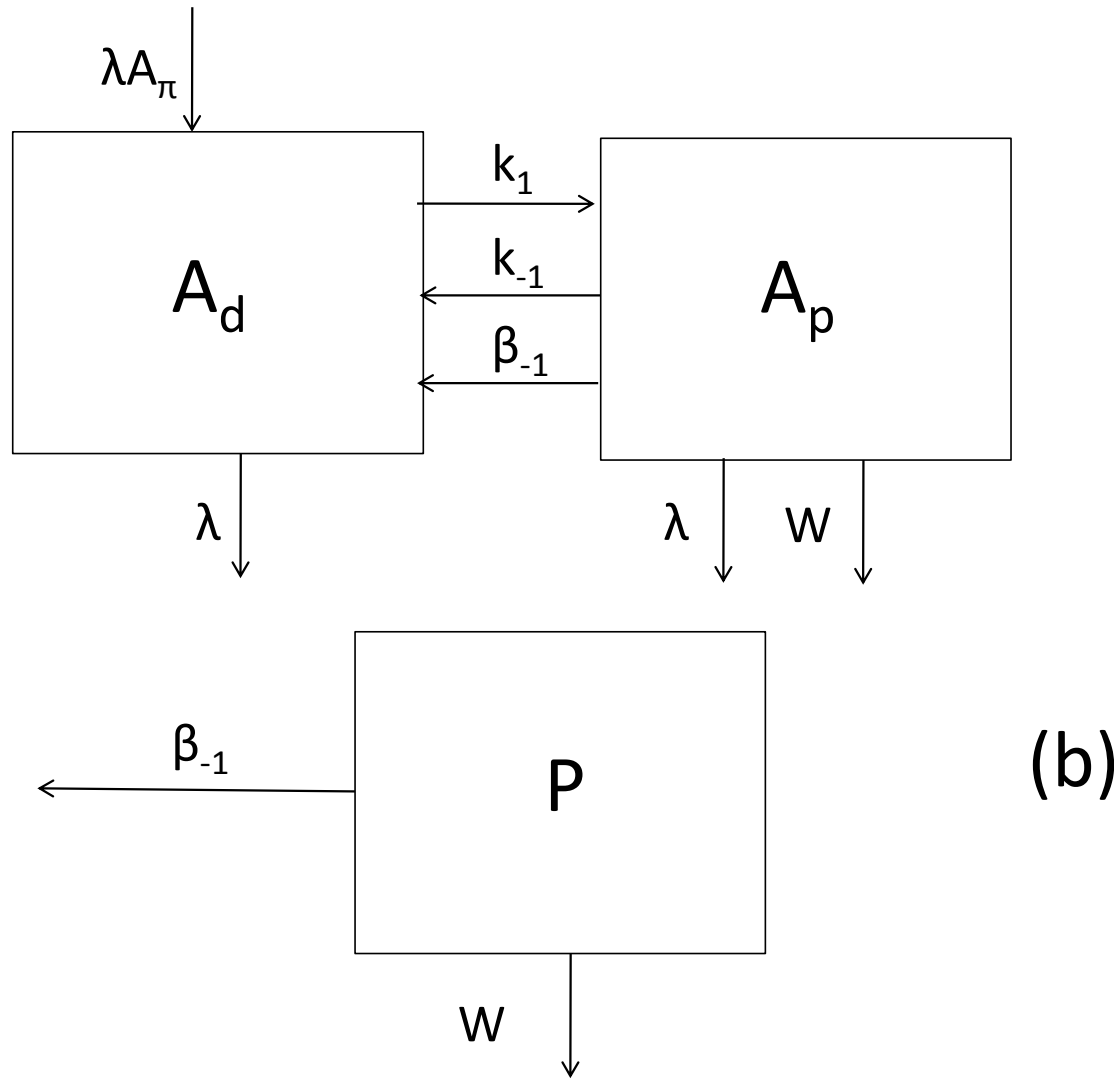

Figure 3: Schematic diagram of the single-particle class model of Th cycling (a) and particle cycling (b). 'A' and 'P' represent, respectively, the Th isotope activity and the particle concentration. Subscript 'd' designates the dissolved fraction and subscript 'p' designates the particulate fraction. $A_{\pi}$ is the activity of the parent isotope. The other symbols represent the rate parameters of solidsolution exchange ( $k_{1}$ for adsorption, $k_{-1}$ for desorption) and particle processes ( $\beta_{-1}$ for particle degradation, and $w$ for particle sinking). $\lambda$ is the radioactive decay constant. 

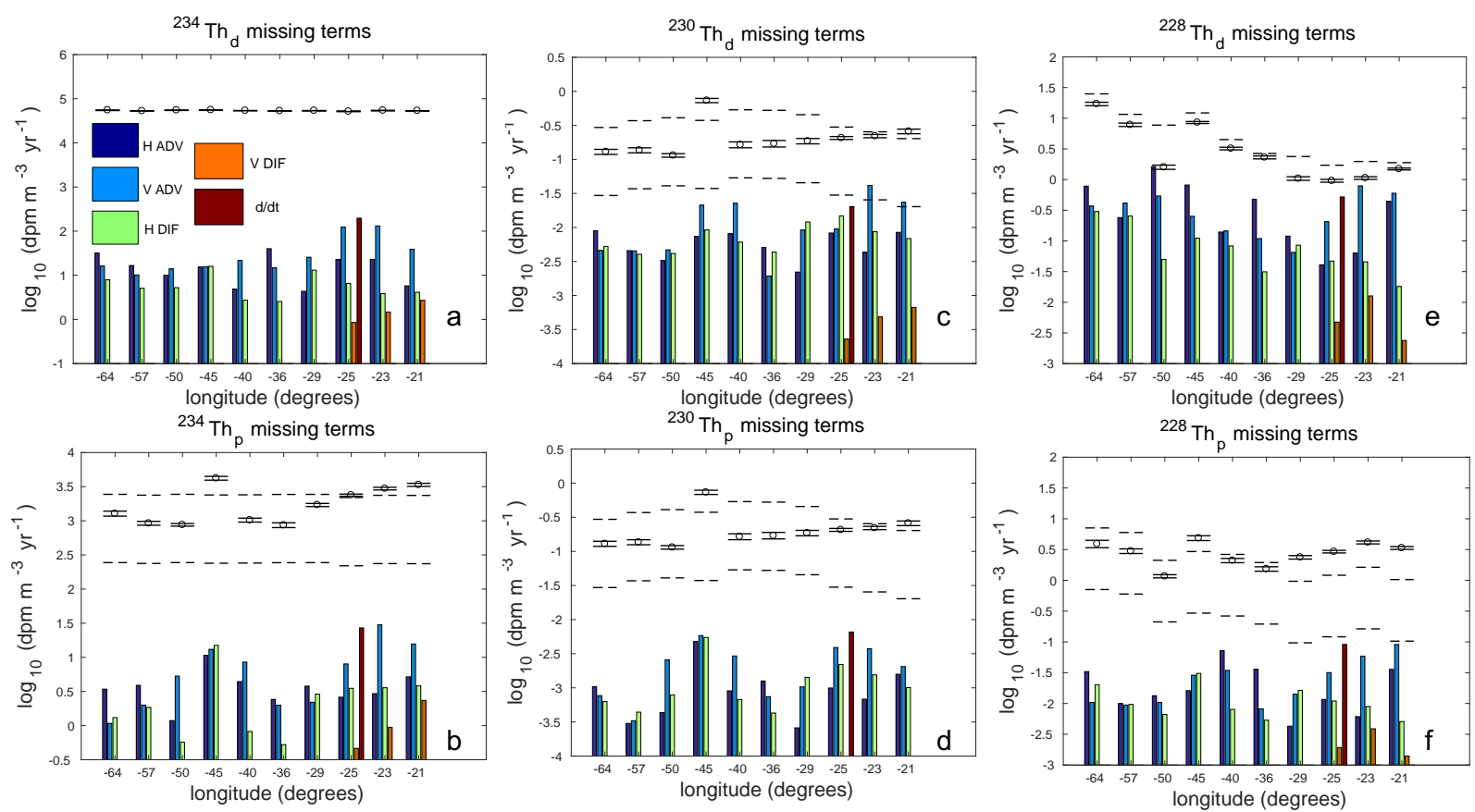

Figure 4: Estimates of the magnitude of the terms missing in the ${ }^{228,230,234} \mathrm{Th}$ dissolved (a-c) and particulate ( $\mathrm{d}-\mathrm{f}$ ) balance equations (vertical averages). The $\mathrm{x}$-axis is longitude, and the $\mathrm{y}$-axis is the base- 10 logarithm of the magnitude of the missing term. The legends in panel (a) apply to all panels. In panels (a, e), the dashed lines are the prior estimates of the radioactive production rates, and the open circles are the posterior estimates of these rates ( \pm 1 standard deviation). Note that in (a), prior and posterior estimates of production rates are barely distinguishable. In panel (b, c, d, f), the dashed lines are prior estimates of the adsorption rate, and the open circles are posterior estimates of the adsorption rate ( \pm 1 standard deviation). In all panels, the following abbreviations are used:"H ADV" for horizontal advection,"V ADV" for vertical advection,"H DIF" for horizontal diffusion, "V DIF" for vertical diffusion, and "d/dt" for the unsteadiness. 


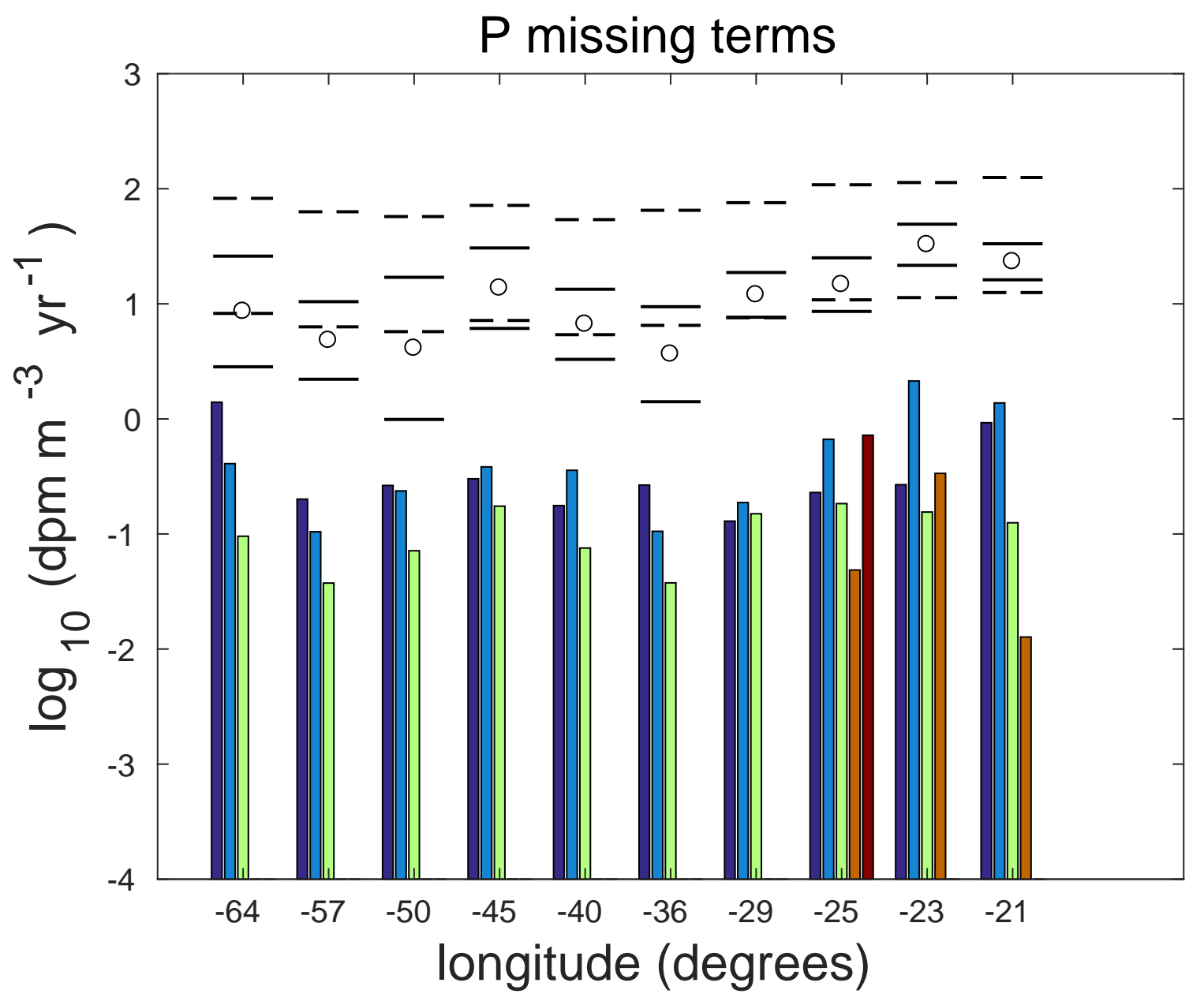

Figure 5: Same as Figure 4, but for $P$. The dashed lines are prior estimates of particle degradation rates, and the open cirlces are the posterior estimates of these rates ( \pm 1 standard deviation). 


\section{${ }^{230} \mathrm{Th}_{\mathrm{d}}$ flux $\left(\mathrm{dpm} / \mathrm{m}^{3} / \mathrm{yr}\right)$}

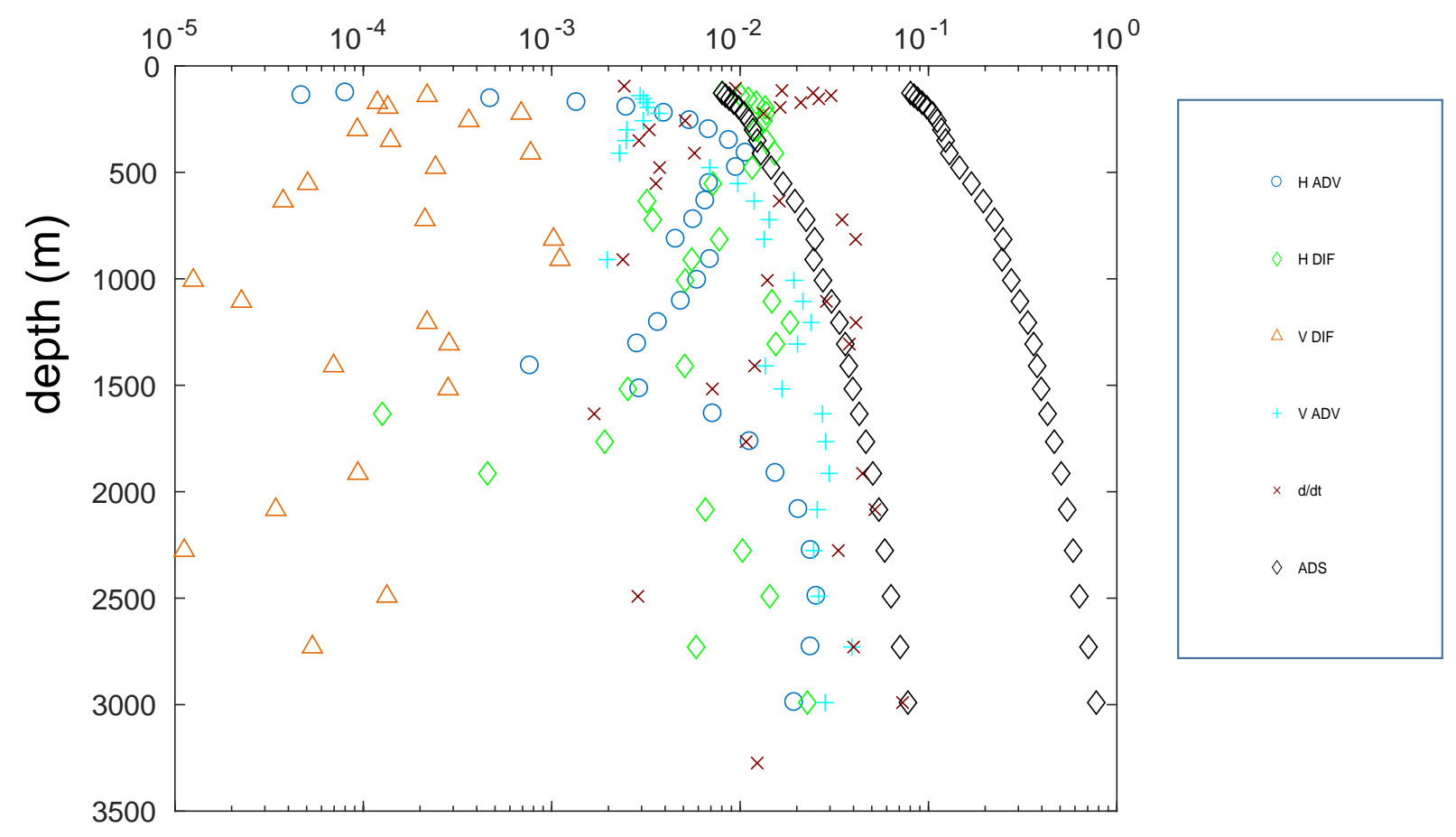

Figure 6: Estimates of the magnitude of the missing terms in the ${ }^{230} \mathrm{Th}_{d}$ balance equations for station GT11-24. In the legend, the following abbreviations are used: "H ADV" for horizontal advection (dark blue circles), "V DIF" for vertical diffusion (light blue crosses), "H DIF" for horizontal diffusion (green squares), "V ADV" for vertical advection (orange triangles), and "d/dt" for unsteadiness (brown crosses). For comparison, "ADS" denotes the prior estimates of Th adsorption rates (minimum and maximum estimates, black diamonds). 
${ }^{234} \mathrm{Th}_{\mathrm{d}}$ normalized residuals

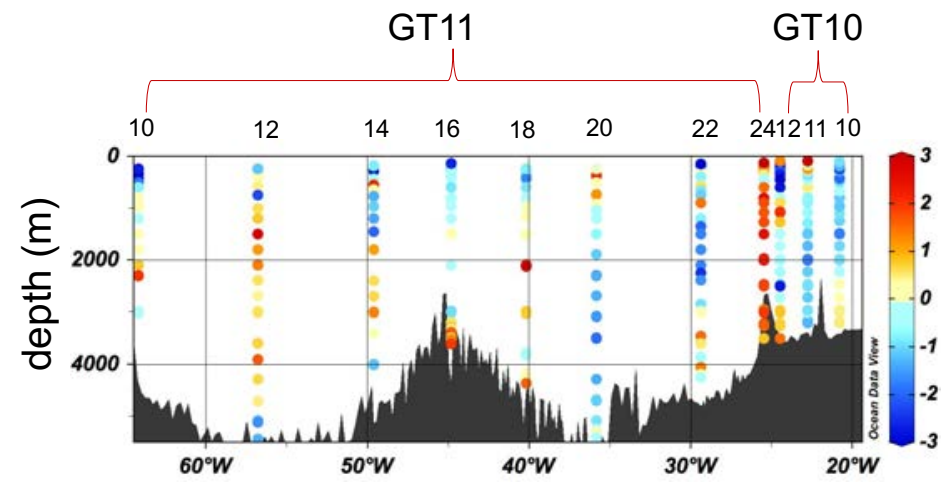

a

${ }^{234} \mathrm{Th}_{\mathrm{p}}$ normalized residuals

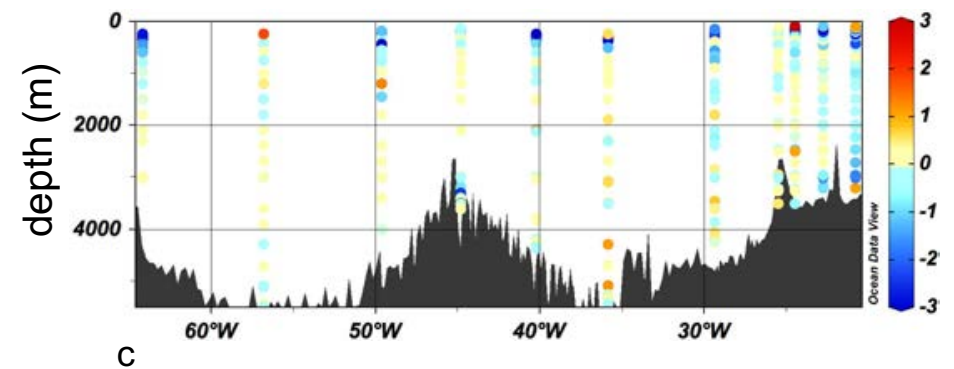

${ }^{234} \mathrm{Th}_{\mathrm{d}}$ (interpolated from data)

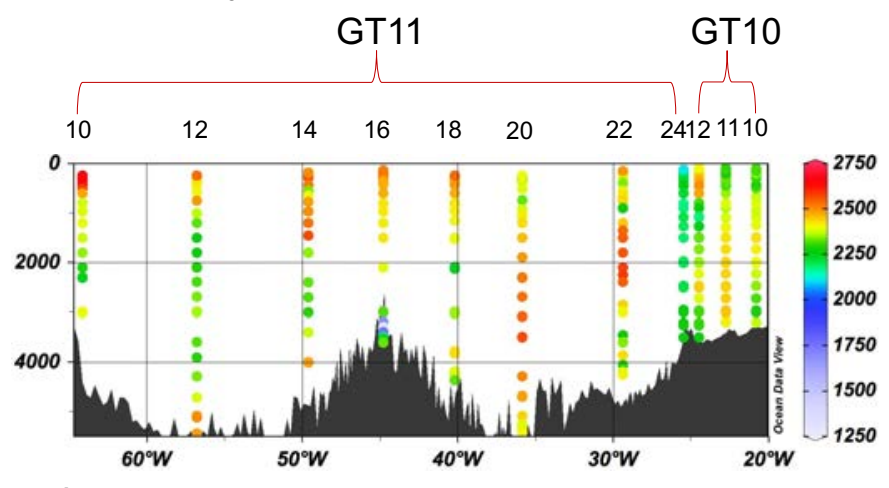

$\mathrm{b}$

${ }^{234} \mathrm{Th}_{\mathrm{p}}$ (interpolated from data)

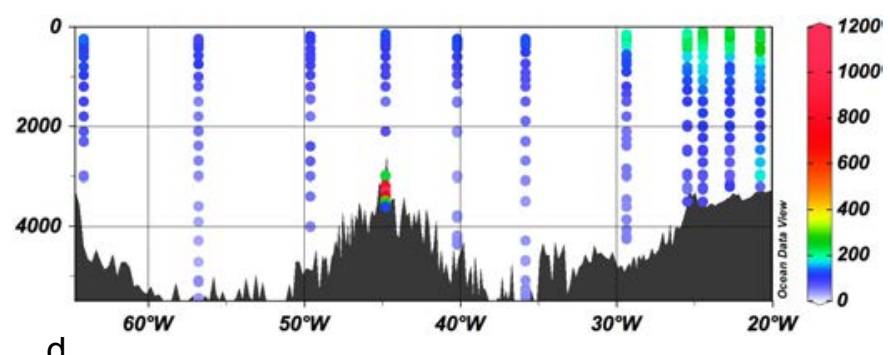

Figure 7: Section plots of ${ }^{234} \mathrm{Th}_{d, p}$. In panels (a, c), the colors show the difference of the posterior estimates and interpolated values, normalized to the interpolated values. Note that the minimum and maximum of the colorbar represent all values $\leq 3$ and all values $\geq 3$, respectively. In panels (b, d), the colors show the activities of interpolated ${ }^{234} \mathrm{Th}$ in $\mathrm{dpm} \mathrm{m} \mathrm{m}^{-3}$. The third and fourth stations from the right of each panel (GT10-12 and GT11-24, respectively) are actually at the same geographic location $\left(17^{\circ} 23^{\prime} \mathrm{N}, 24^{\circ} 30^{\prime} \mathrm{W}\right)$. 
${ }^{230} \mathrm{Th}_{\mathrm{d}}$ normalized residuals
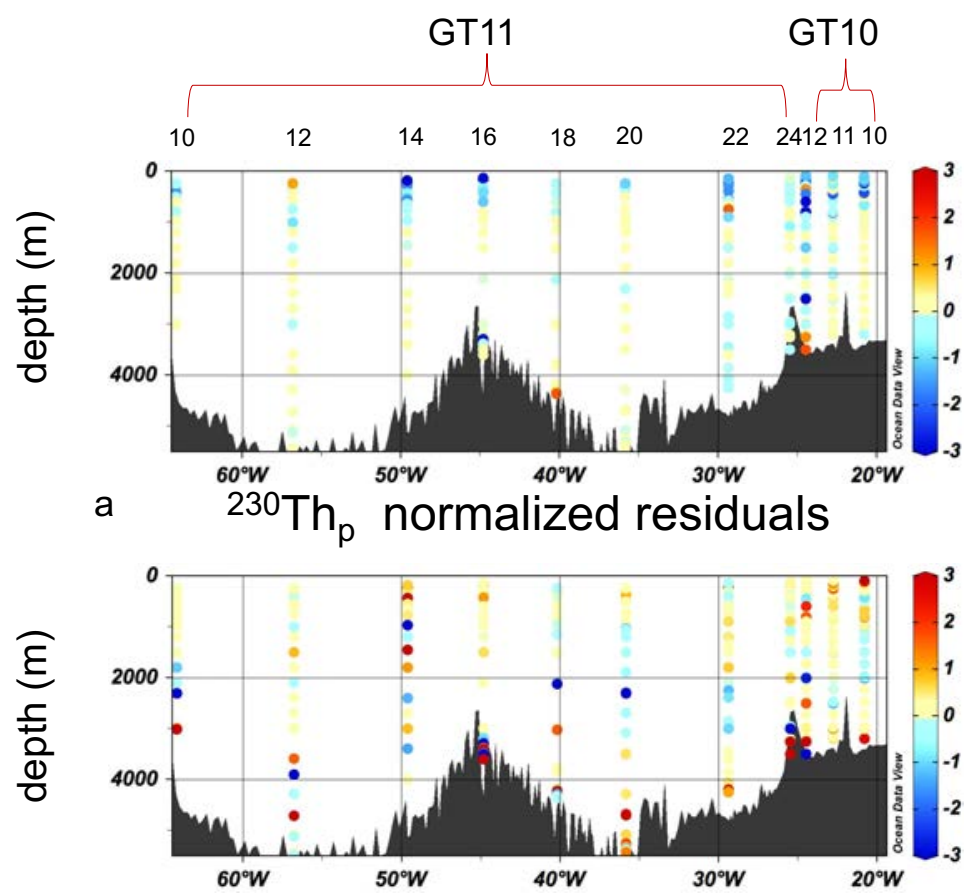

C
${ }^{230} \mathrm{Th}_{\mathrm{d}}$ (interpolated from data)
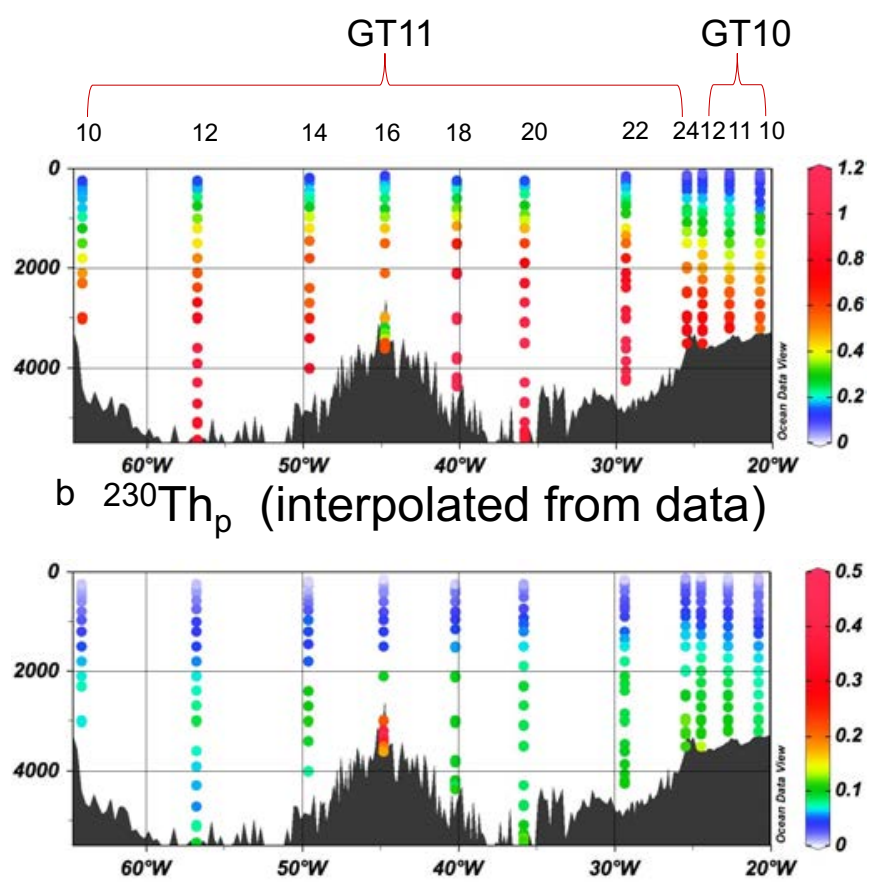

d

Figure 8: Same as Figure 7, but for ${ }^{230} \mathrm{Th}\left(\mathrm{dpm} \mathrm{m}^{-3}\right)$. 
${ }^{228} \mathrm{Th}_{\mathrm{d}}$ normalized residuals
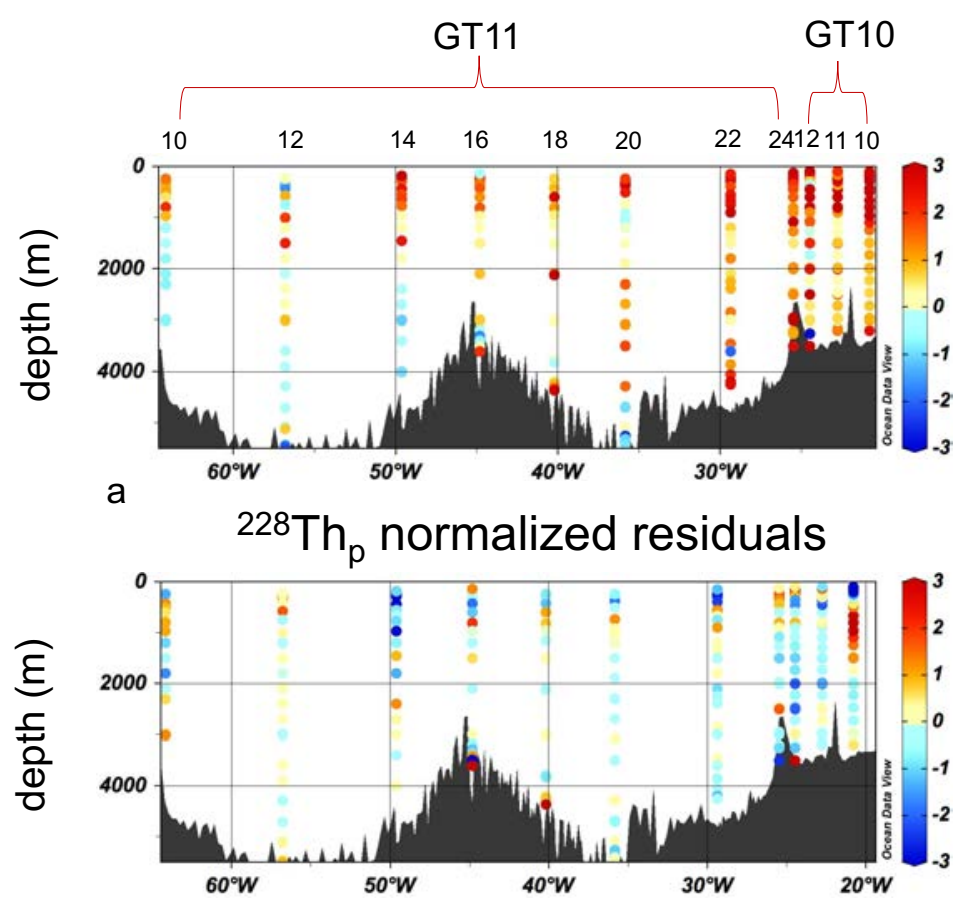

C
${ }^{228} \mathrm{Th}_{\mathrm{d}}$ (interpolated from data)
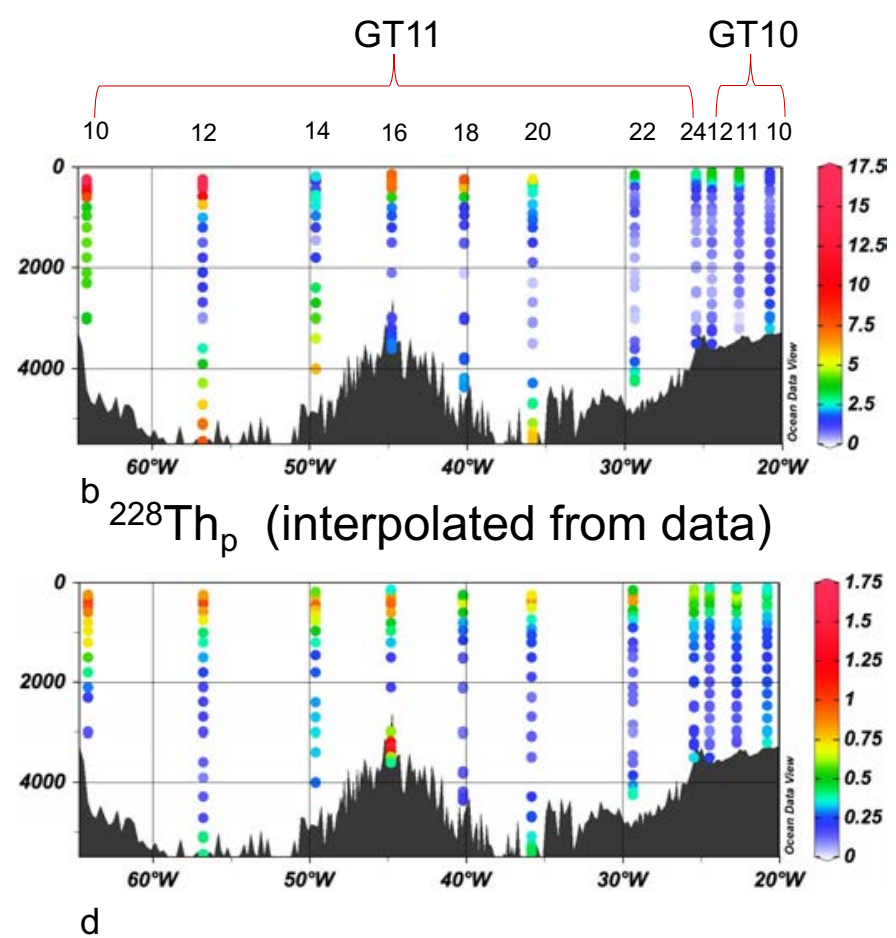

Figure 9: Same as Figure 7, but for ${ }^{228} \mathrm{Th}\left(\mathrm{dpm} \mathrm{m}^{-3}\right)$. 

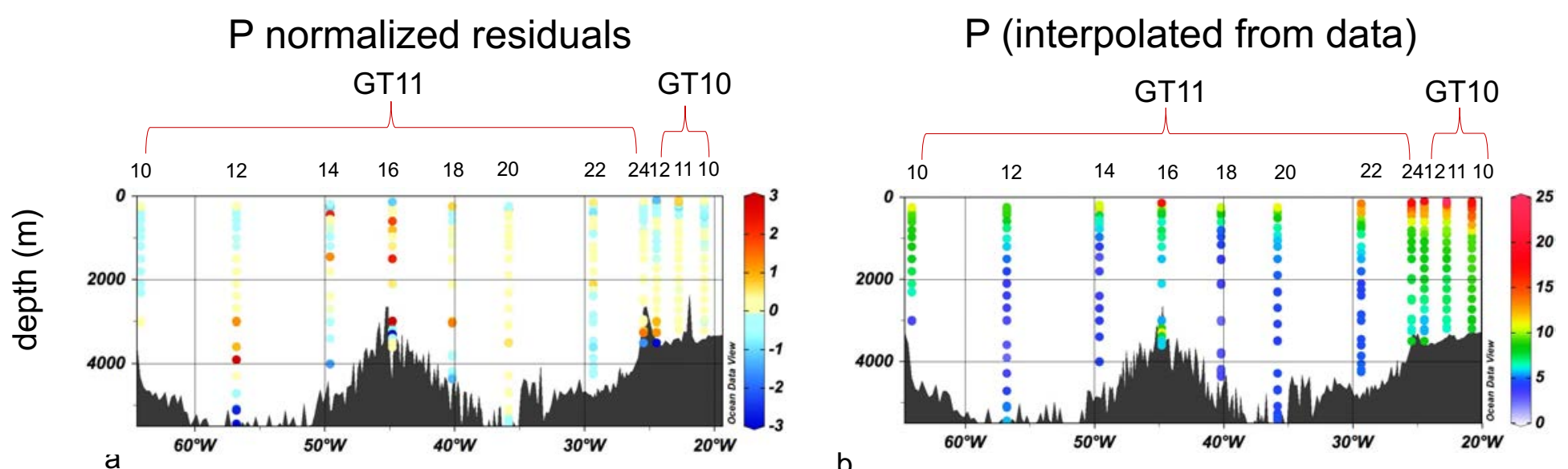

Figure 10: Same as Figure 7, but for $P\left(\mathrm{mg} \mathrm{m}^{-3}\right)$. 

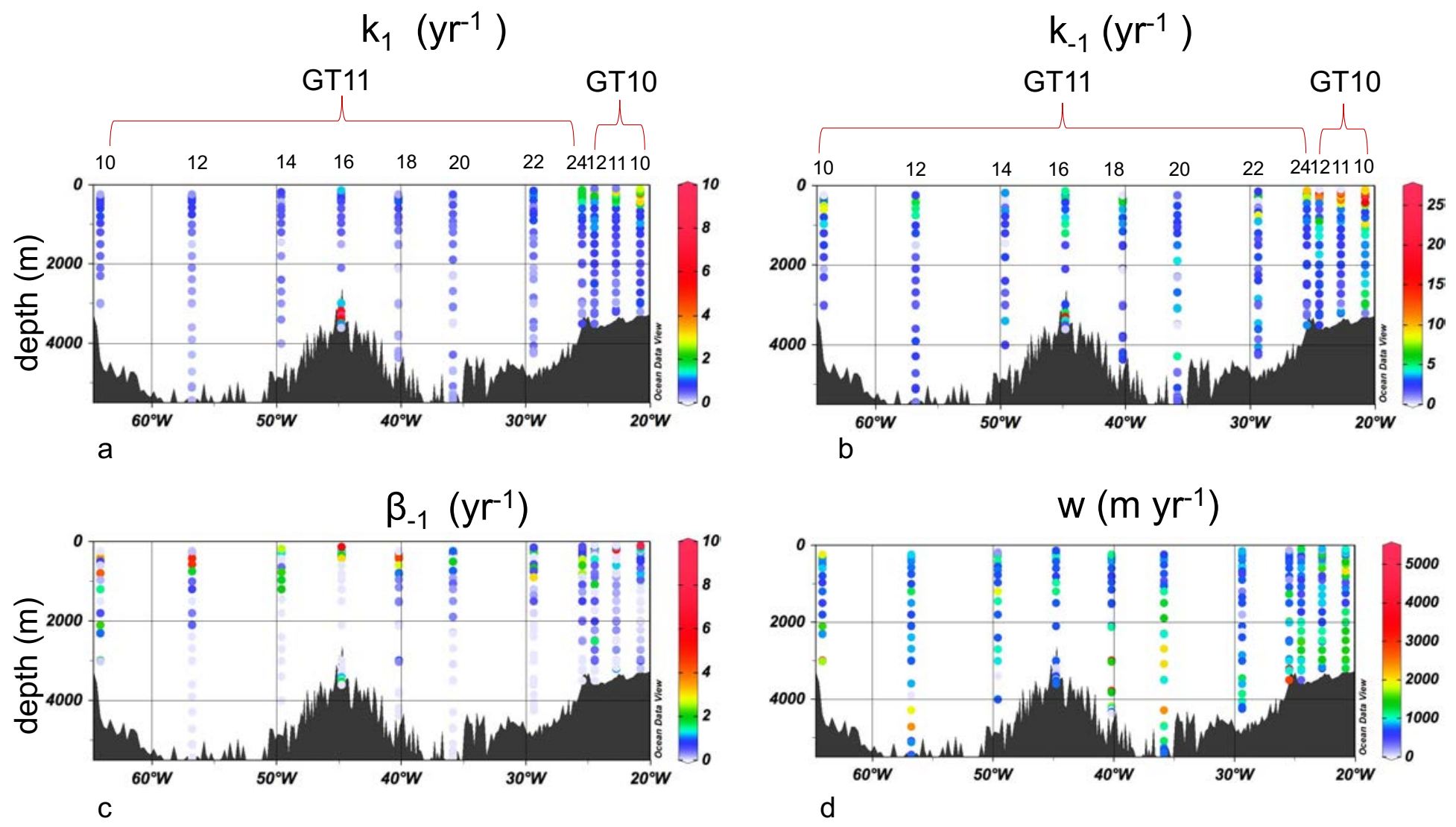

Figure 11: Posterior estimates of the rate parameters of Th and particle cycling along GA03 (legs 1-2). The rate constants for Th adsorption $\left(k_{1}\right)$, Th desorption $\left(k_{-1}\right)$, and particle degradation $\left(\beta_{-1}\right)$ are in $\mathrm{yr}^{-1}$, and the particle sinking speed $(w)$ is in $\mathrm{m} \mathrm{yr}^{-1}$. Notice that the largest value inferred for $k_{1}\left(21 \mathrm{yr}^{-1}\right.$ at $3200 \mathrm{~m}$ at station GT11-16) is not shown so that individual values of $k_{1}$ are more easily distinguished. The third and fourth stations from the right of each panel (GT10-12 and GT11-24, respectively) are actually at the same geographic location $\left(17^{\circ} 23^{\prime} \mathrm{N}, 24^{\circ} 30^{\prime} \mathrm{W}\right)$. 

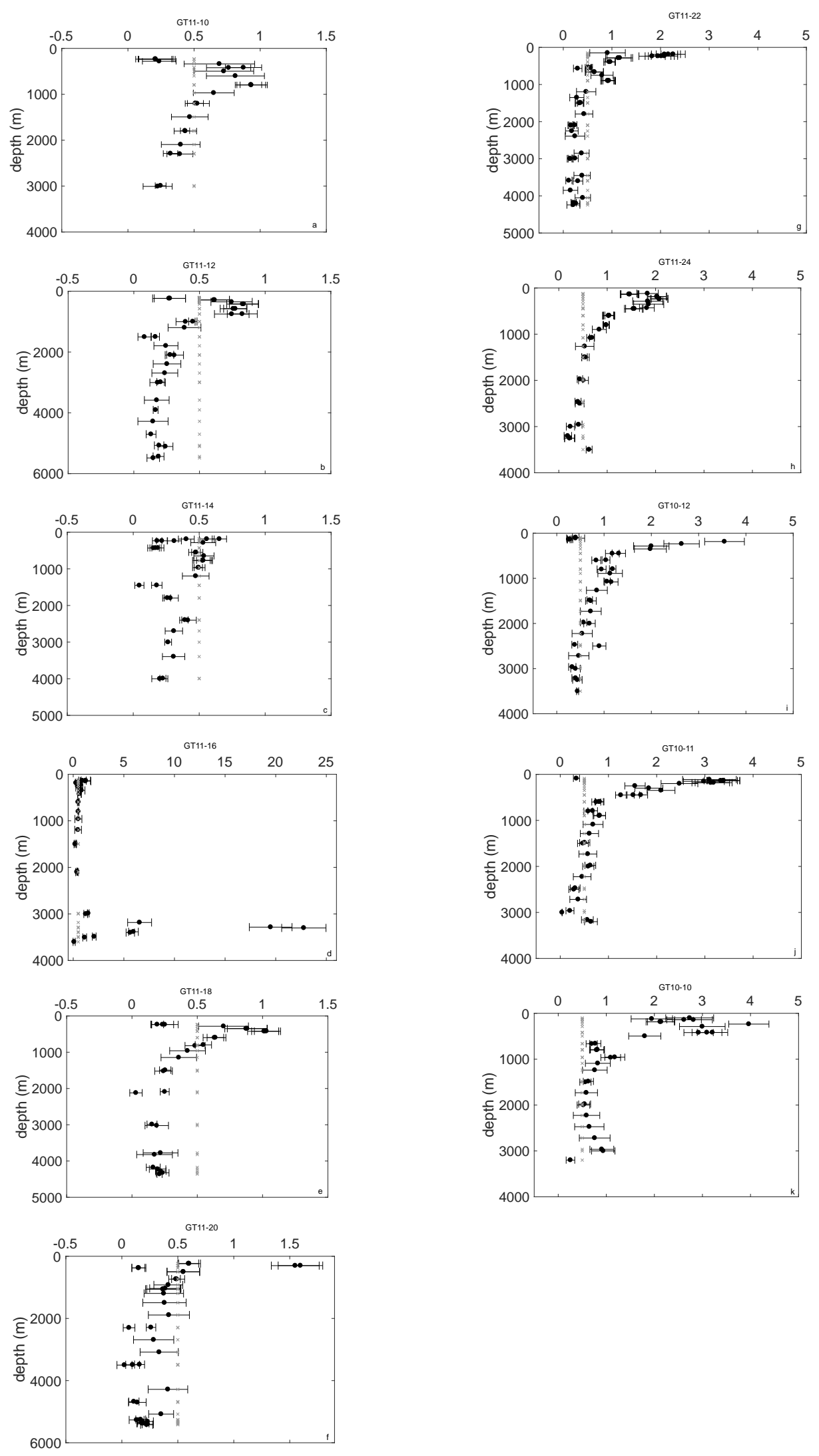

Figure 12: Vertical profile of the Th adsorption rate constant, $k_{1}$, in $\mathrm{yr}^{-1}$ (solid circles, error bars indicate \pm 1 standard deviation) at our selected stations along GA03:(a) GT11-10, (b) GT11-12, (c) GT11-14, (d) GT11-16, (e) GT11-18, (f) GT11-20, (g) GT11-22, (h) GT11-24, (i) GT10-12, (j) GT10-11, and (k) GT10-10. The grey asterisks show the prior estimate of $k_{1}=0.5 \pm 5 \mathrm{yr}^{-1}$. 

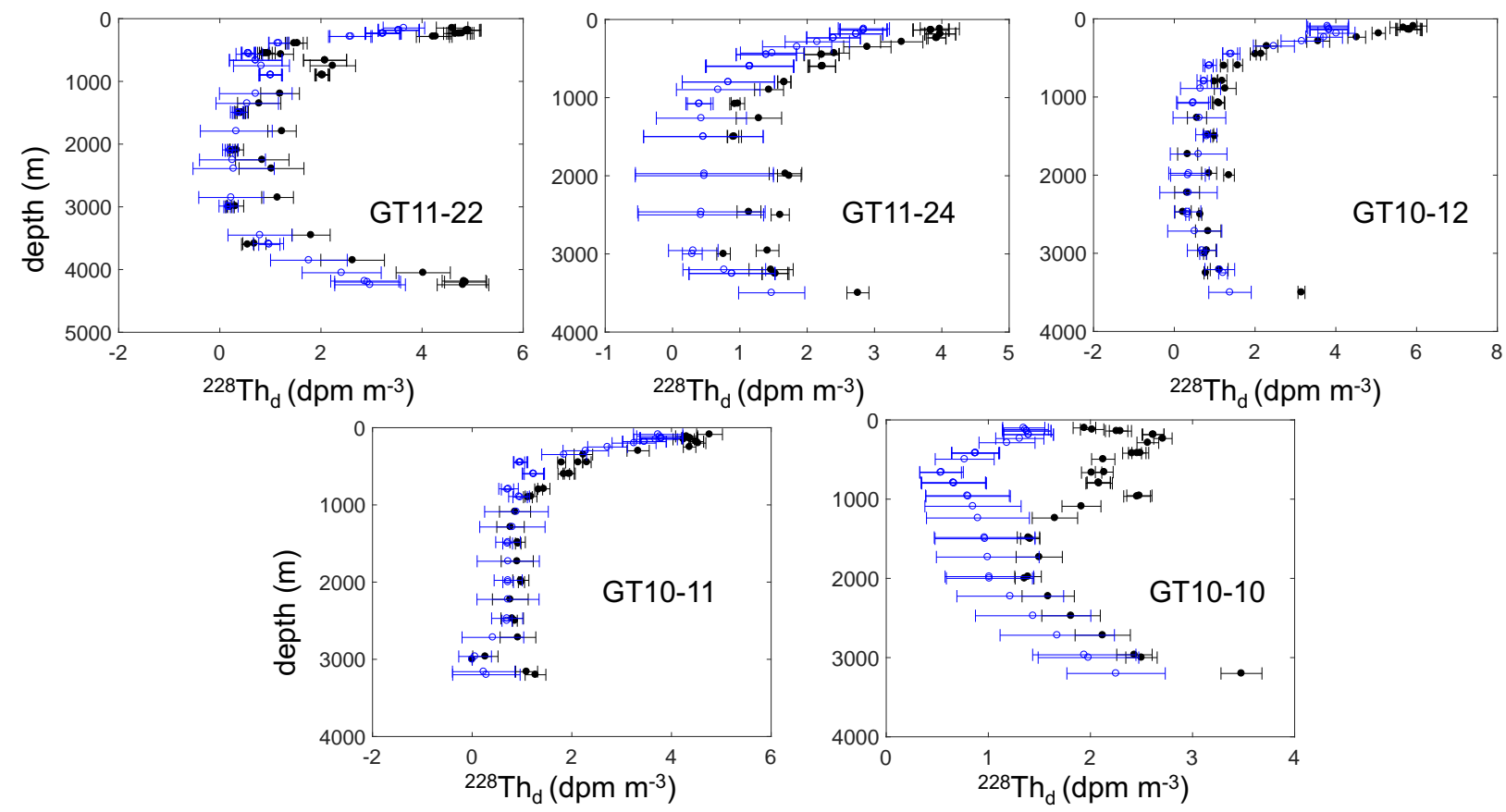

Figure 13: ${ }^{228} \mathrm{Th}_{d}$ profiles at the five easternmost stations. The open circles represent the interpolated values, and the solid circles represent the posterior estimates (error bars are \pm 1 standard deviation). 

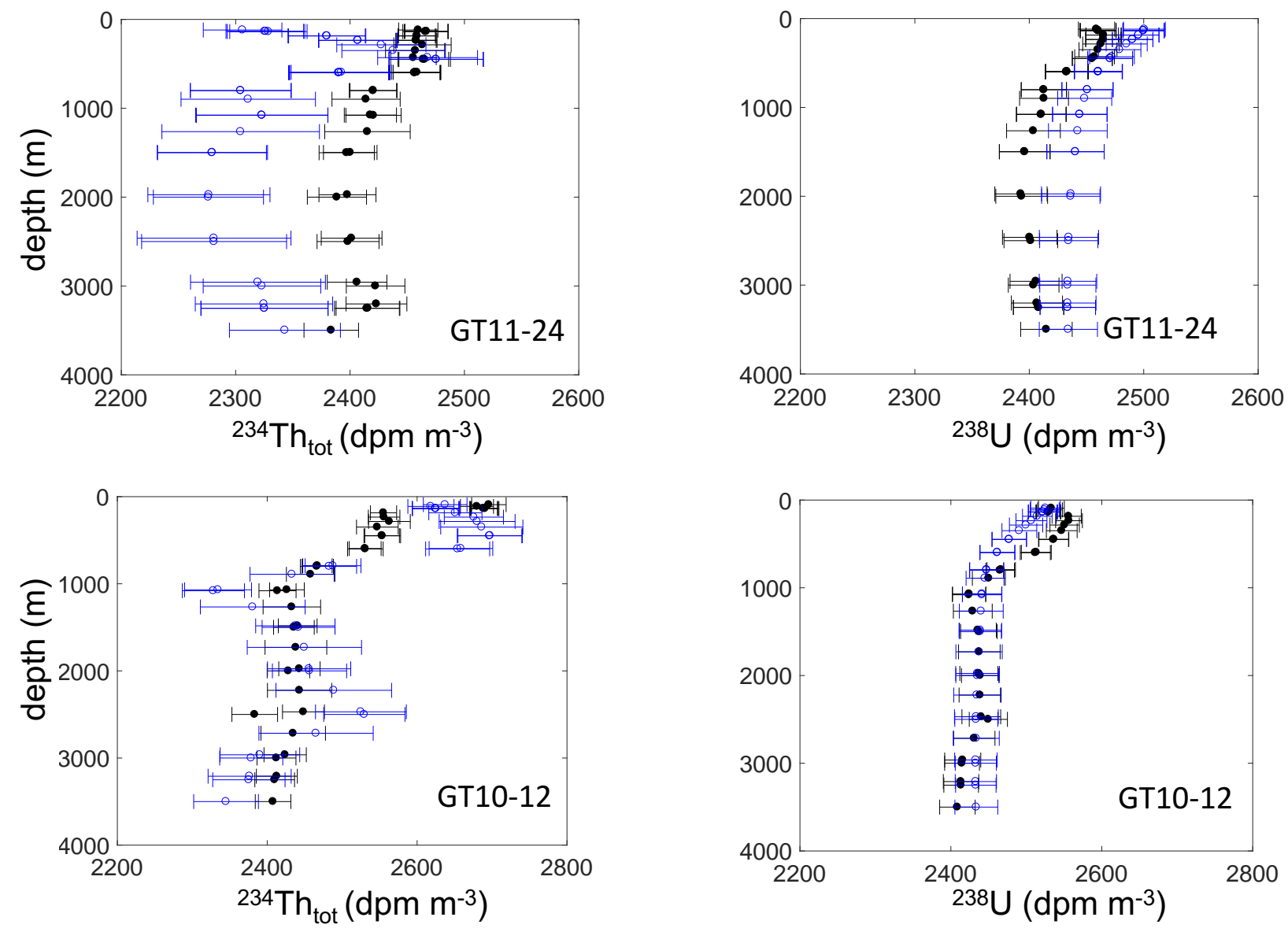

Figure 14: ${ }^{234} \mathrm{Th}_{\text {tot }}$ and ${ }^{238} \mathrm{U}$ profiles at the crossover station GT11-24/GT1012. The open circles represent the interpolated values, and the solid circles represent the posterior estimates (error bars are \pm 1 standard deviation). 

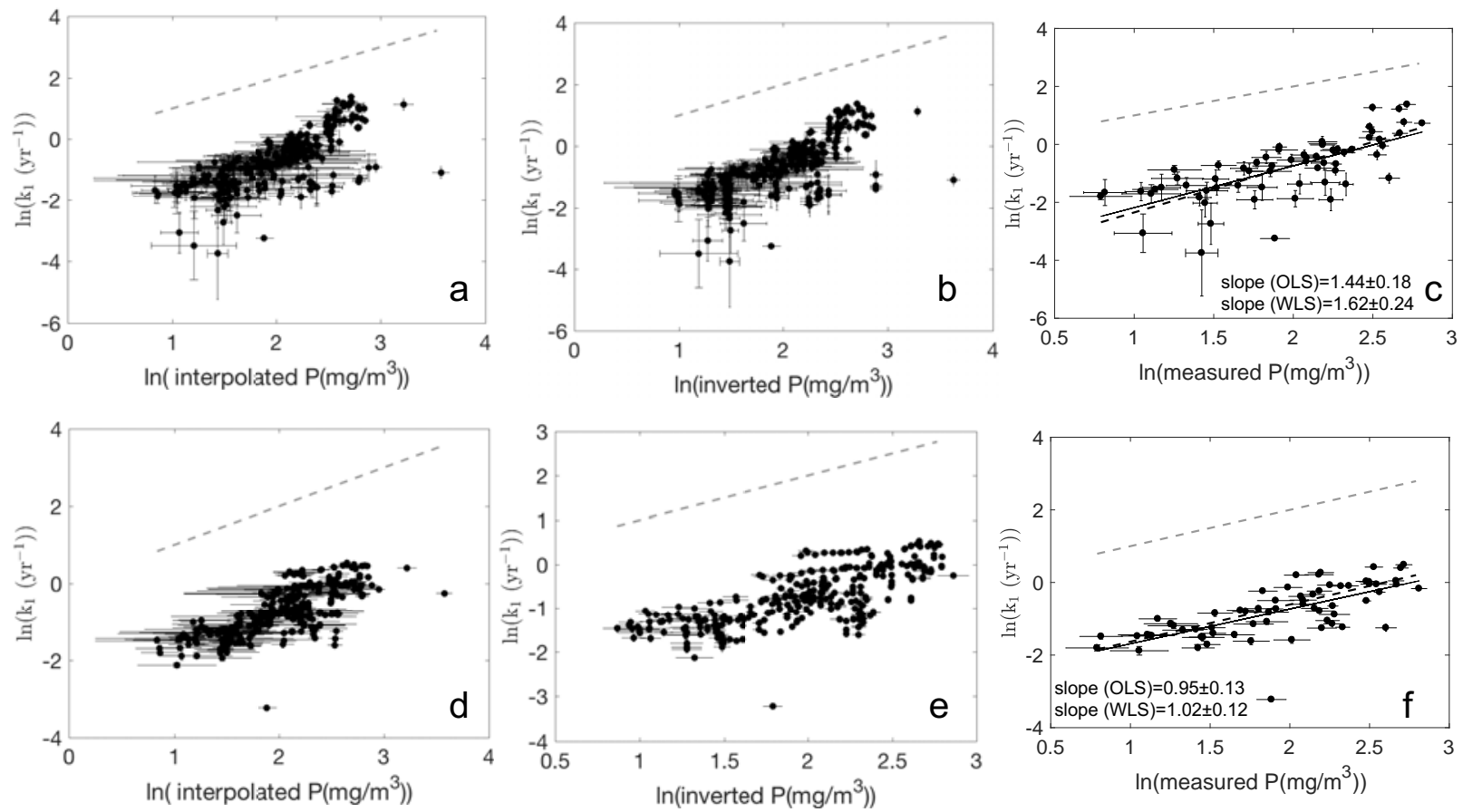

Figure 15: Variation of Th adsorption rate constant with particle concentration. Panels (a-c) show plots of $\ln k_{1}$ estimated by inversion vs. $\ln P$ obtained from interpolation (a), inversion (b), and measurements (c). The error bars are \pm 1 standard deviation, and the light dashed line is the 1:1 line. In panel (c), the solid (dark dashed) line is the best fit obtained by OLS (WLS). Panels (d-f) are the same as panels (a-c), except that $k_{1}$ is estimated from an inversion that assumes a smooth vertical distribution of the rate parameters $(\gamma=1$; see text). Note that none of the panels include the very large $k_{1}$ values from station GT11-16. 

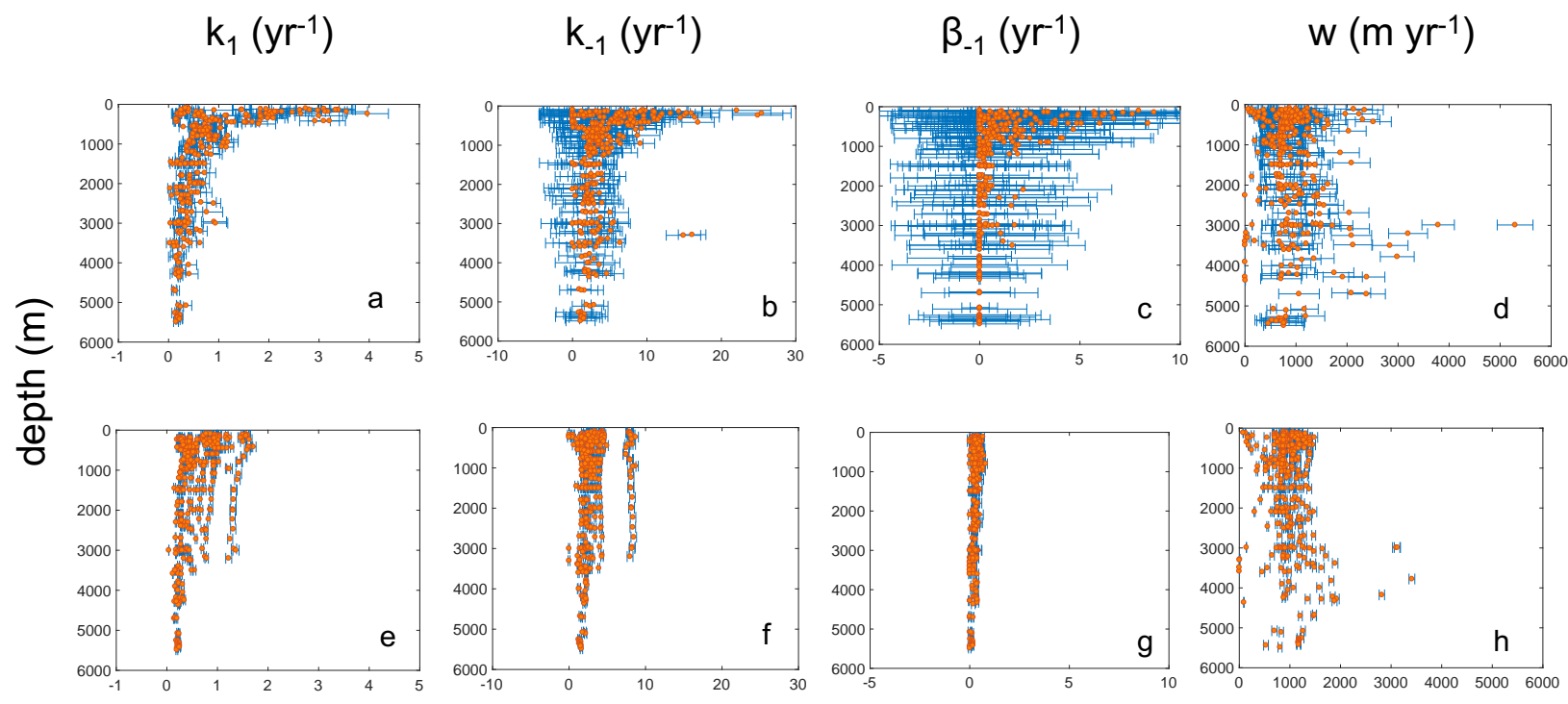

Figure 16: Posterior estimates of $k_{1}, k_{-1}, \beta_{-1}$, and $w$ at all selected stations of GA03 obtained from the inversion without smoothing (panels a-d) and with smoothing with $\gamma=1$ (panels e-h). Panels (a,e) do not include $k_{1}$ values from station GT11-16, which include extremely large values of $k_{1}$ present near the TAG hydrothermal vent. Different colors are used to denote the estimates (orange) and their errors (blue; \pm 1 standard deviation). The separate cluster of large $k_{-1}$ values in panel (f) (around $8 \mathrm{yr}^{-1}$ ) includes values mostly from stations GT11-24, GT10-12, GT10-11, and GT10-10. 

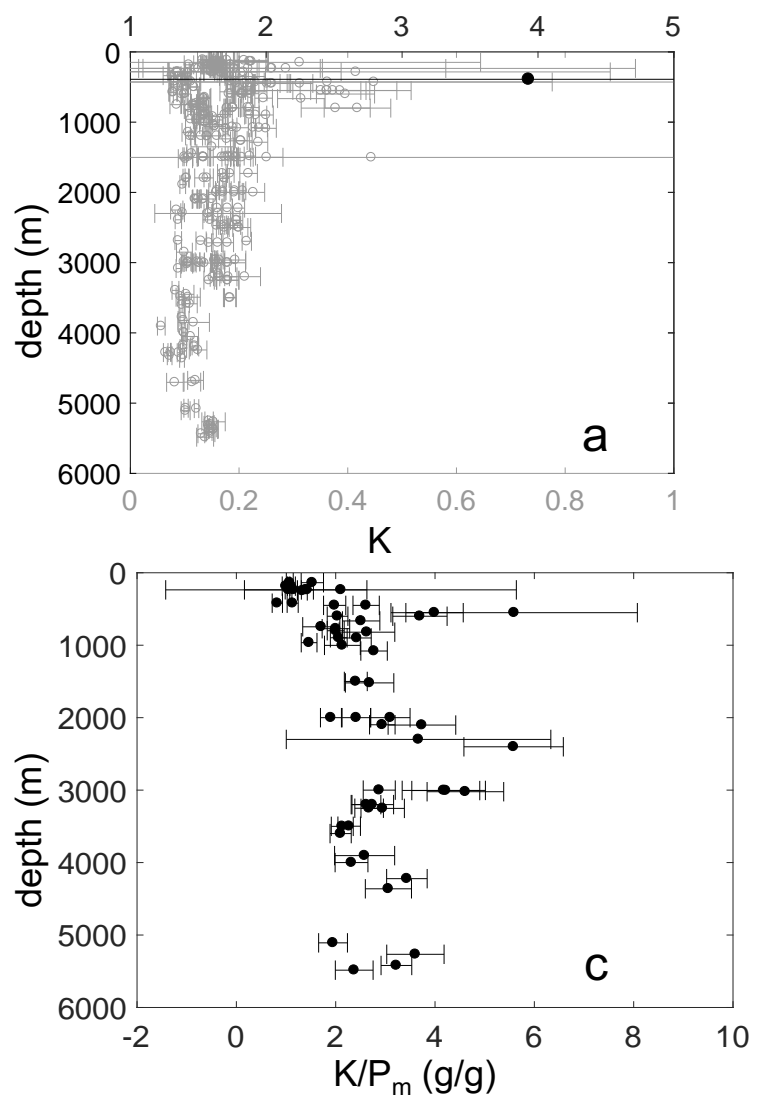
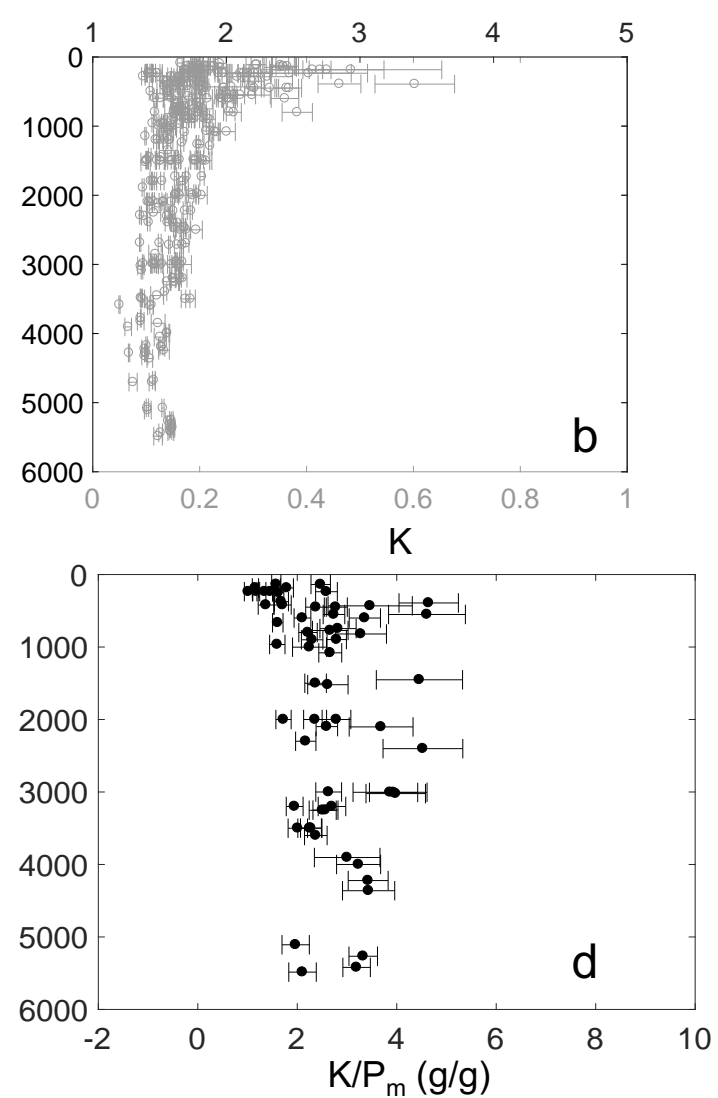

Figure 17: Panels (a,b): posterior estimates of the rate constant ratio $K$ derived from the inversion (a) without smoothing and (b) with smoothing $(\gamma=$ 1 ), at all selected stations and all depths where $k_{-1}+\beta_{-1}>0.1 \mathrm{yr}^{-1}$. Two scales are used to isolate the large values of $K>1$ and better show the vertical structure of $K$. The black circles (top axis) show $K$ values $>1$, while the grey circles (bottom axis) show the $K$ values $\leq 1$. Panels $(\mathrm{c}, \mathrm{d})$ : Estimates of $K / P_{m}$, where $K$ is derived from the inversion (a) without smoothing and (b) with smoothing $(\gamma=1)$, at all selected stations and depths where $P$ is measured and where $k_{-1}+\beta_{-1}>0.1 \mathrm{yr}^{-1}$. None of the panels include values estimated at station GT11-16. 

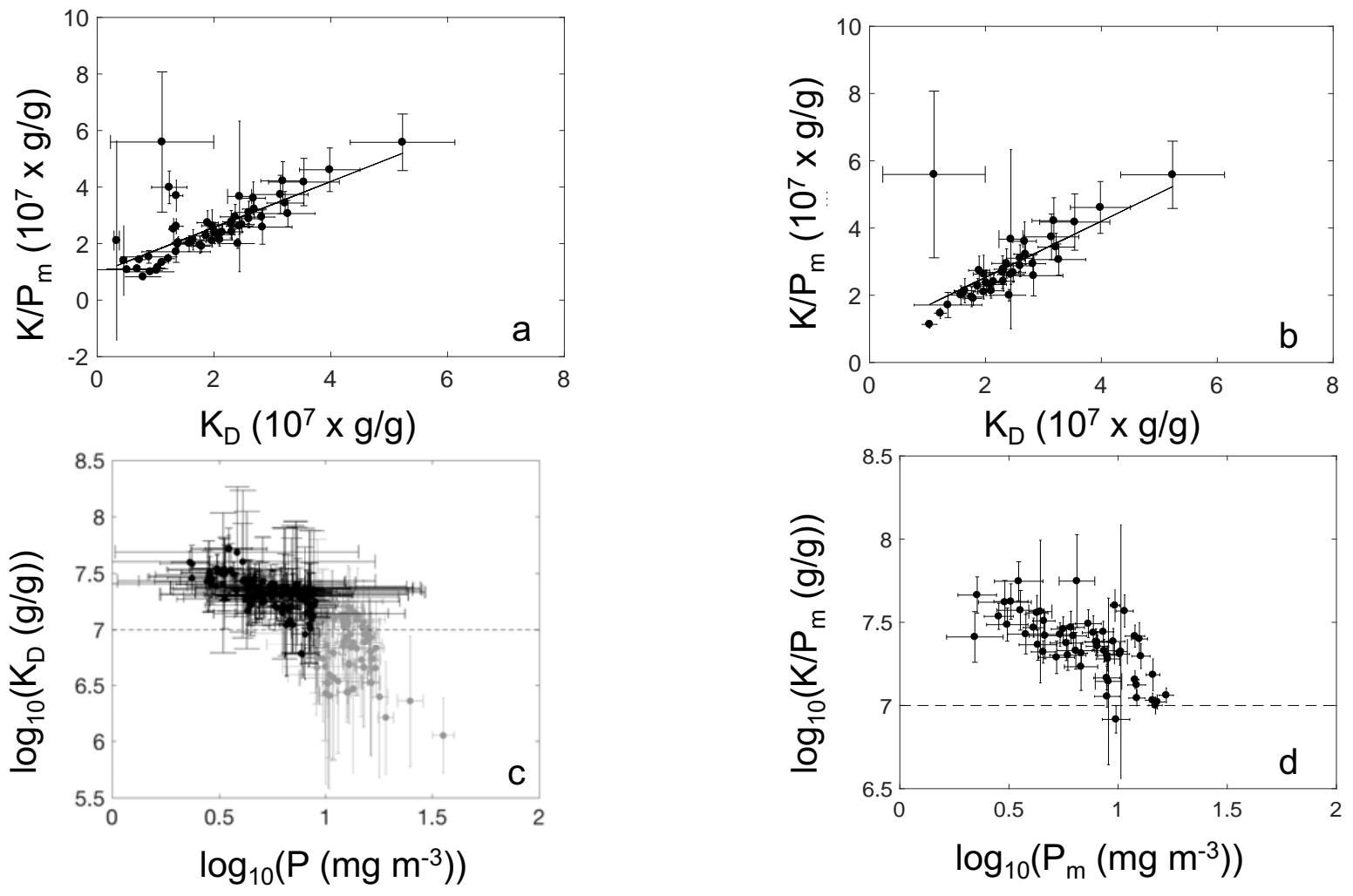

Figure 18: (a) $\log _{10}\left(K / P_{m}\right)$ vs. $\log _{10} K_{D}$. The dashed line is the 1:1 line. (b) Same as (a), except excluding $\left(K_{D}, K / P_{m}\right)$ pairs for which $P_{m}<9 \mathrm{mg} \mathrm{m}^{-3}$. (c) $\log _{10} K_{D}$ vs. $\log _{10} P$ for interpolated ${ }^{230} \mathrm{Th}$ and $P$. The grey circles show values for which $P_{m} \geq 9 \mathrm{mg} \mathrm{m}^{-3}$, and the black circles show values for which $P_{m}<9$ $\mathrm{mg} \mathrm{m}^{-3}$. (d) $\log _{10}\left(K / P_{m}\right)$ vs. $\log _{10} P_{m}$, where $K$ is estimated by inversion and $P_{m}$ is measured particle concentration. For panels (c) and (d), the dashed line is the slope expected in the absence of a particle concentration effect (slope is 0 ). In all panels, error bars are \pm 1 standard deviation, and values of $K_{D}$ and $K / P_{m}$ at station GT11-16 are not included. 


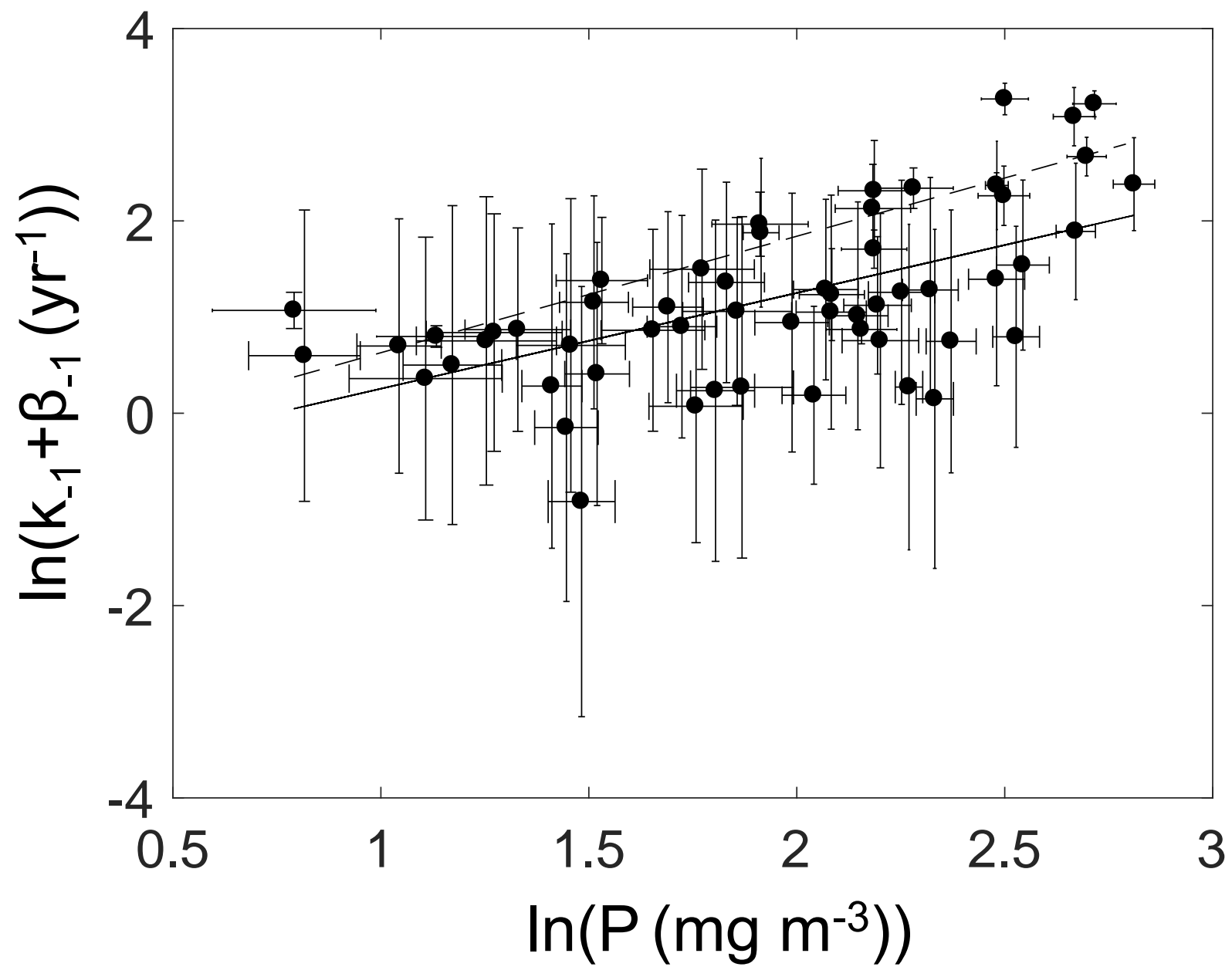

Figure 19: $\ln \left(k_{-1}+\beta_{-1}\right)$ estimated by inversion vs. $\ln P_{m}$, excluding $\left(k_{-1}+\right.$ $\left.\beta_{-1}, P\right)$ pairs for which $k_{-1}+\beta_{-1}<0.1 \mathrm{yr}^{-1}$. The error bars are \pm 1 standard deviation and the solid (dashed) line is the best fit using OLS (WLS). 


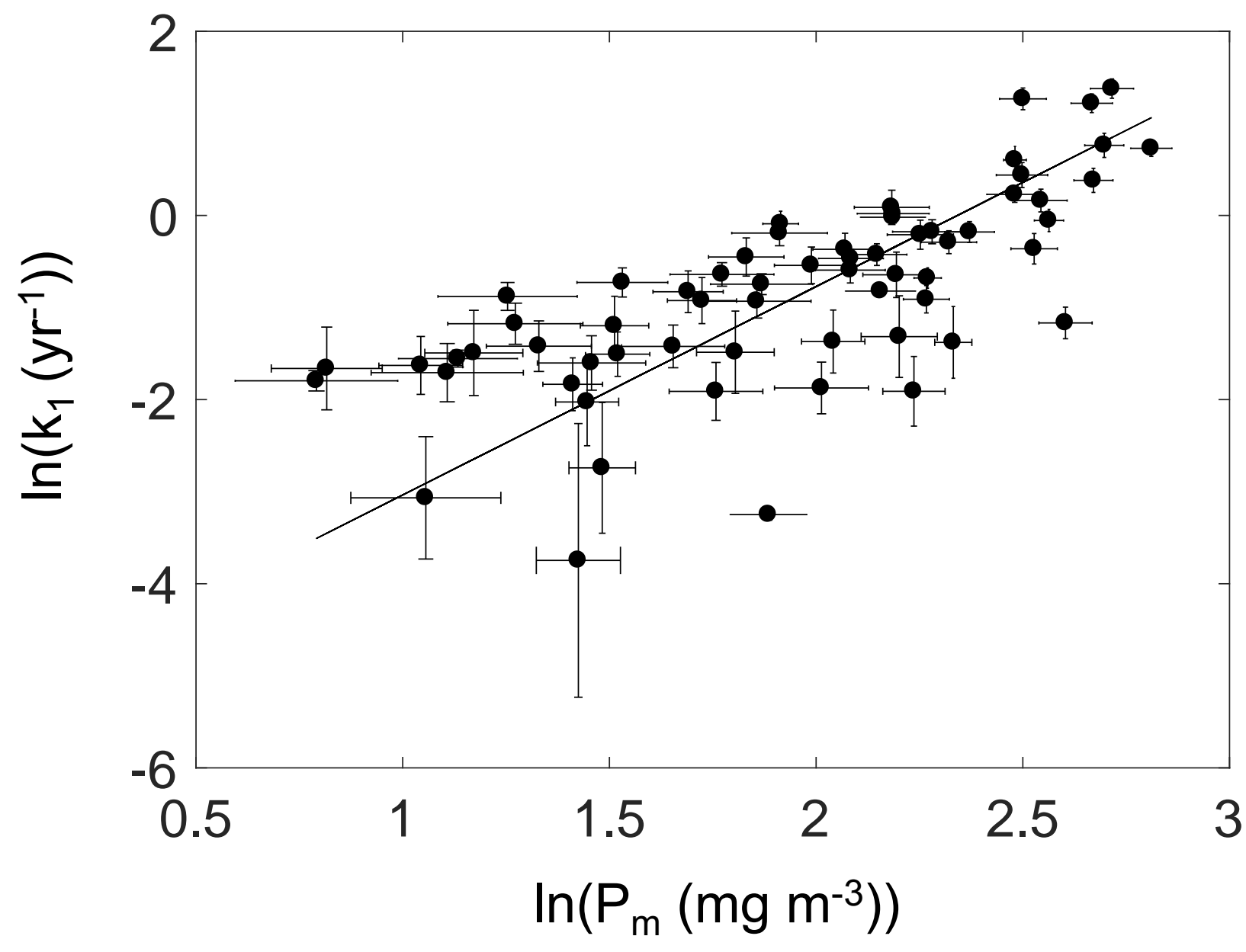

Figure B1: $\ln k_{1}$ estimated by inversion vs. $\ln P_{m}$. The error bars are \pm 1 standard deviation and the solid line is the best fit obtained from the ATI. The figure does include very large $k_{1}$ values from station GT11-16. 

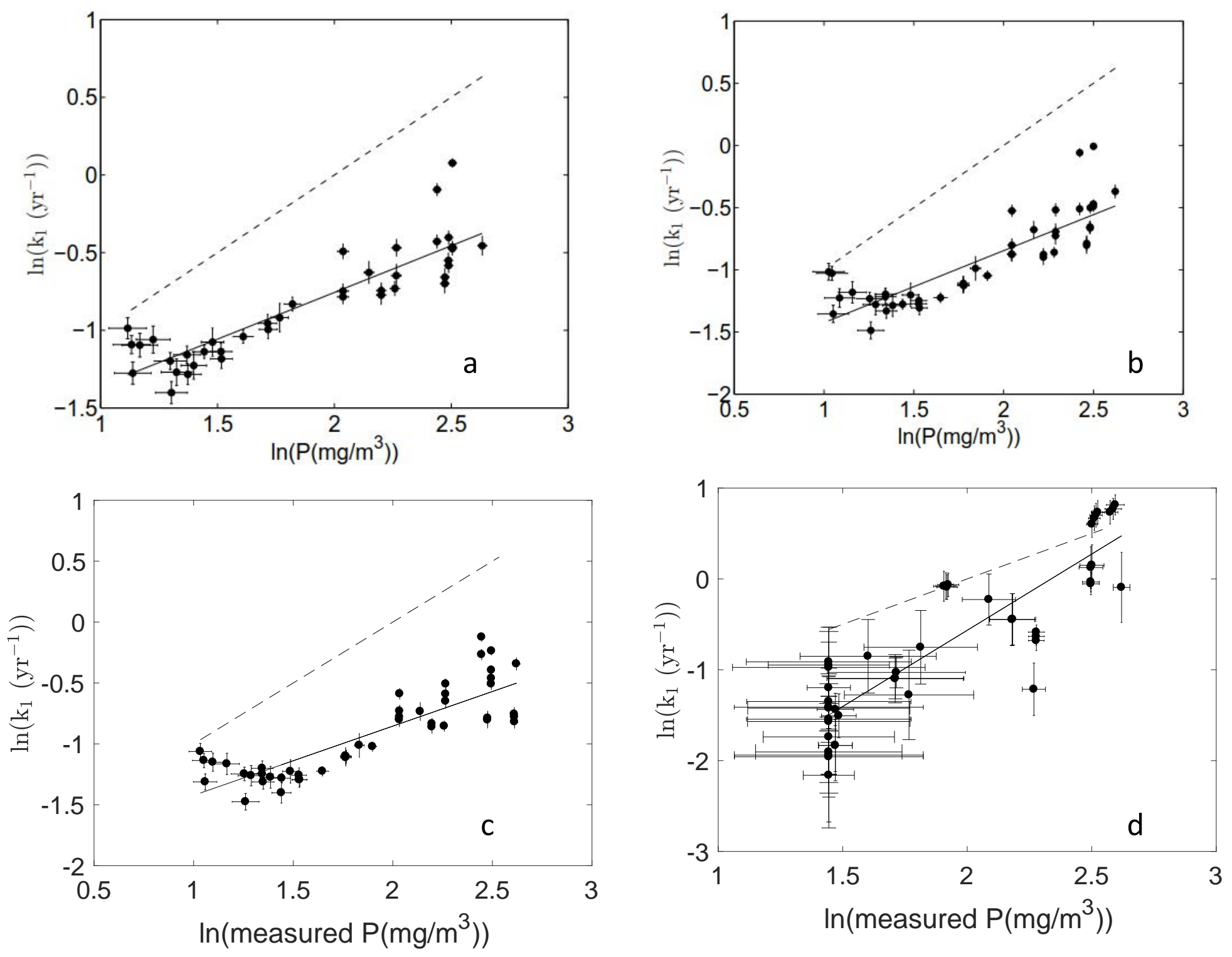

Figure C1: $\ln k_{1}$ vs. $\ln P$ for station GT11-22 using (a) ATI with smoothing and total ${ }^{230} \mathrm{Th}_{p}$ (adsorbed $+{ }^{230} \mathrm{Th}$ locked in mineral lattices; Lerner et al. (2016)), (b) ATI with smoothing and adsorbed ${ }^{230} \mathrm{Th}_{p}$, (c) FMINCON with smoothing, and (d) FMINCON without smoothing. In each panel, the solid line is the best fit using OLS, the dashed line is the 1:1 line (no particle concentration effect), and error bars are \pm 1 standard deviation. 


\section{D Supplementary Material}

In this supplement, we present the vertical profiles of the rate parameters for Th desorption

$\left(k_{-1}\right)$, particle degradation $\left(\beta_{-1}\right)$, and particle sinking speed $(w)$. These profiles are shown in the same format as Figure 12 in the main text. 

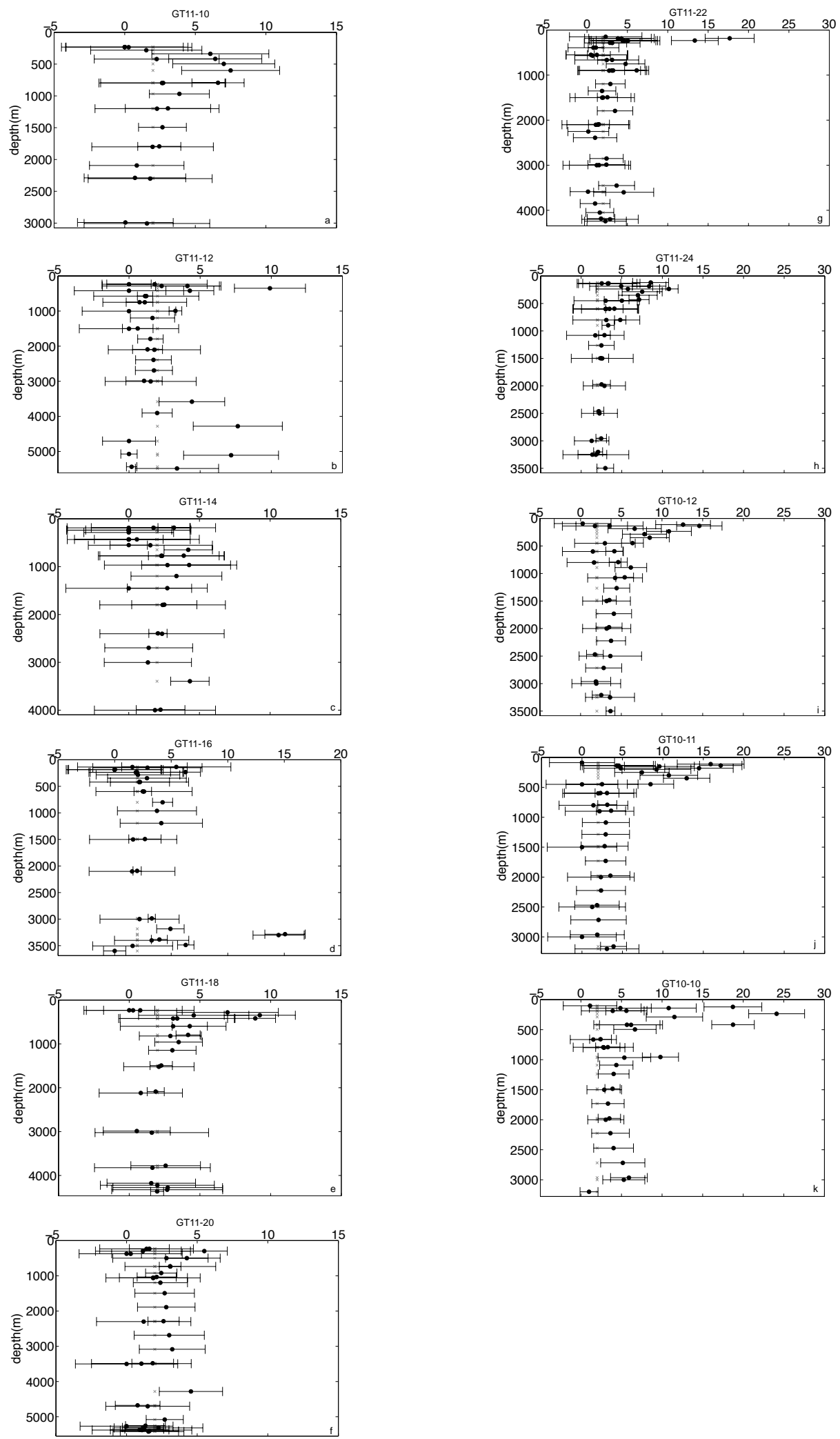

Figure S1: Vertical profile of the Th desorption rate constant, $k_{-1}$, in $\mathrm{yr}^{-1}$ (solid circles, error bars indicate \pm 1 standard deviation) at our selected stations along GA03:(a) GT11-10, (b) GT11-12, (c) GT11-14, (d) GT11-16, (e) GT11-18, (f) GT11-20, (g) GT11-22, (h) GT11-24, (i) GT10-12, (j) GT10-11, and (k) GT10-10. The grey asterisks show the prior estimate of $k_{-1}=2 \pm 5 \mathrm{yr}^{-1}$. 

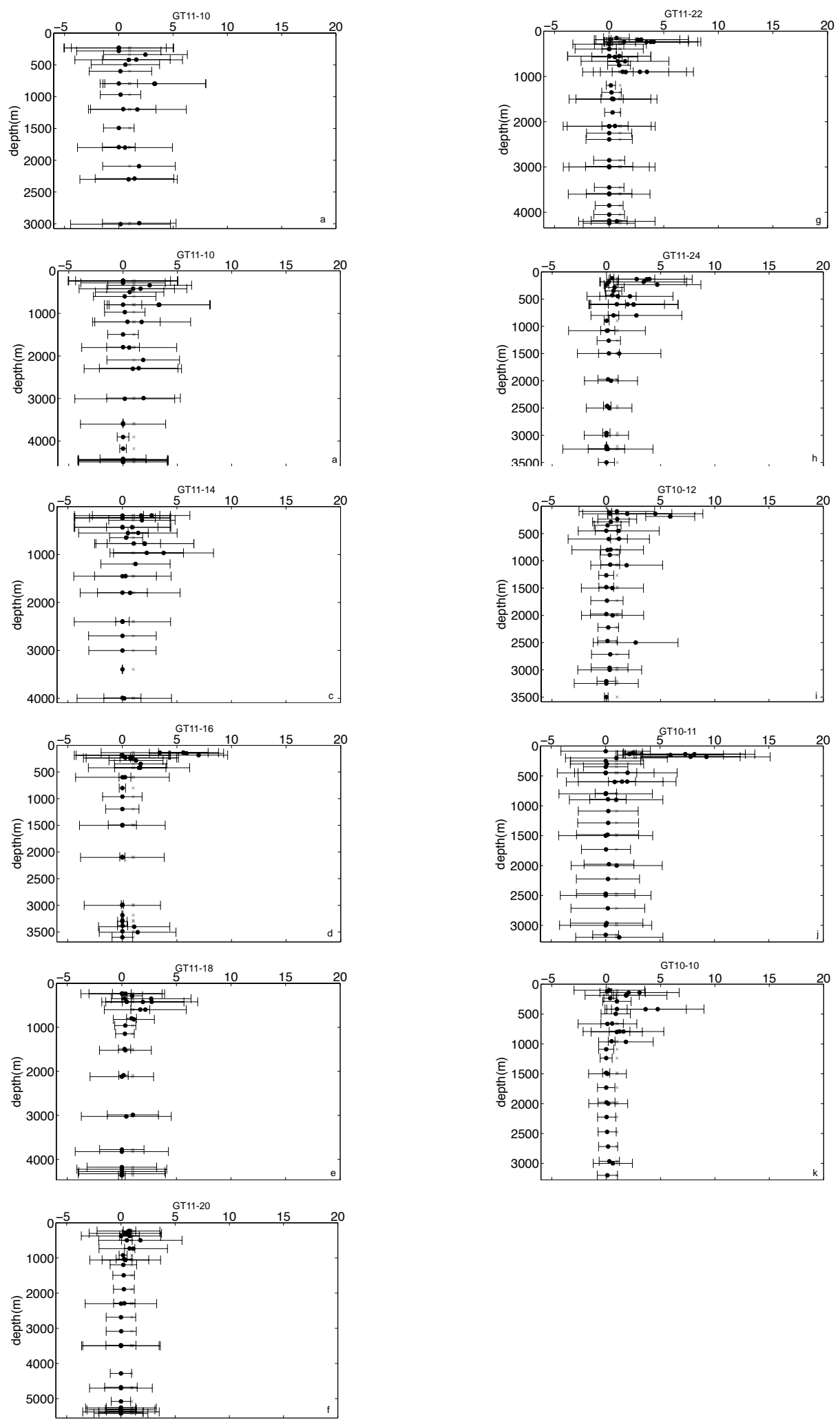

Figure S2: Vertical profile of the particle degradation rate constant, $\beta_{-1}$, in $\mathrm{yr}^{-1}$ (solid circles, error bars indicate \pm 1 standard deviation) at our selected stations along GA03:(a) GT11-10, (b) GT1112, (c) GT11-14, (d) GT11-16, (e) GT11-18, (f) GT11-20, (g) GT11-22, (h) GT11-24, (i) GT10-12, (j) GT10-11, and (k) GT10-10. The grey asterisks show the prior estimate of $\beta_{-1}=1 \pm 10 \mathrm{yr}^{-1}$. 

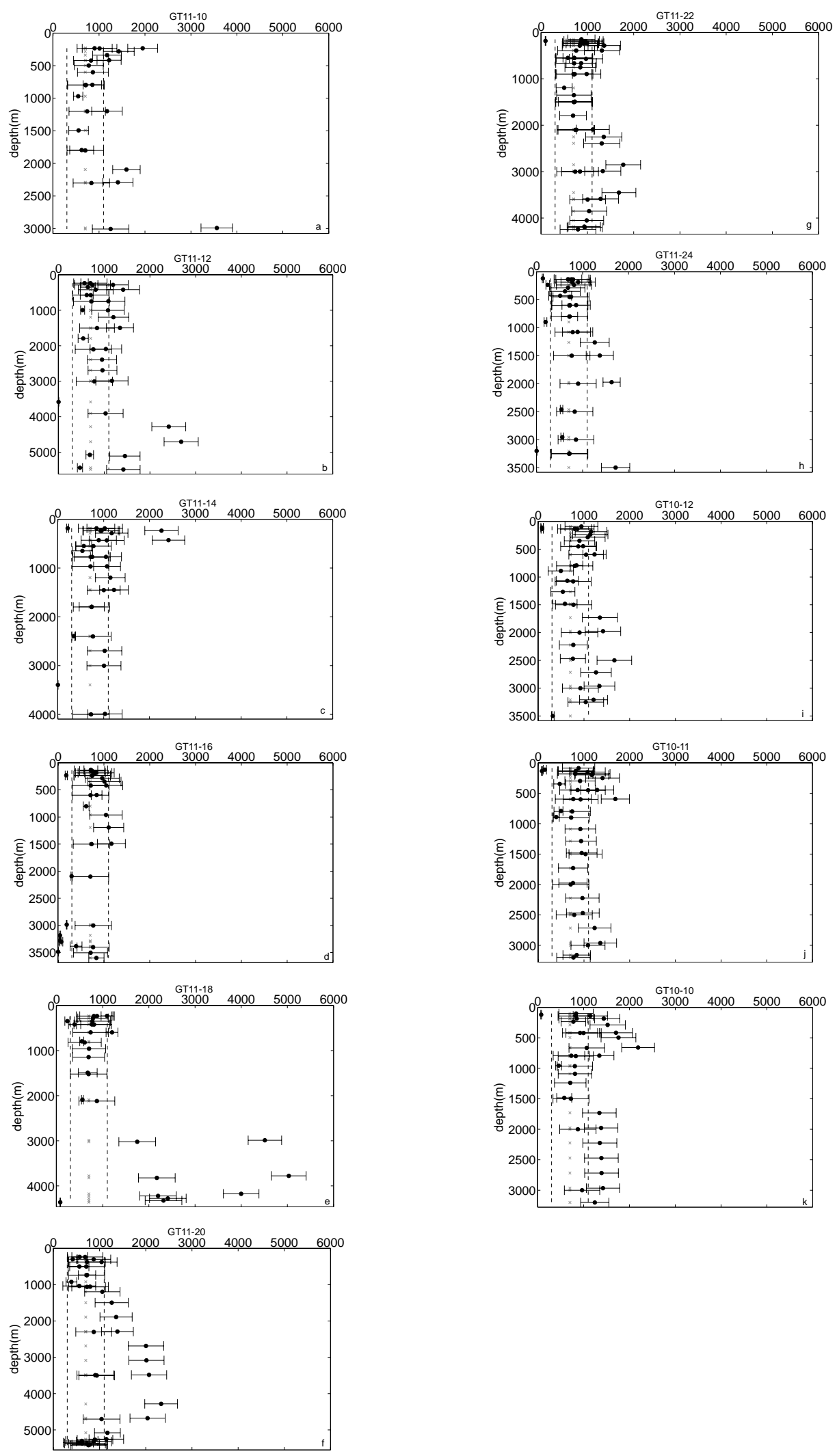

Figure S3: Vertical profile of the particle sinking speed, $w$, in $\mathrm{yr}^{-1}$ (solid circles, error bars indicate \pm 1 standard deviation) at our selected stations along GA03:(a) GT11-10, (b) GT11-12, (c) GT1114, (d) GT11-16, (e) GT11-18, (f) GT11-20, (g) GT11-22, (h) GT11-24, (i) GT10-12, (j) GT10-11, and (k) GT10-10. The grey asterisks show the prior estimate of $w=700 \pm 500 \mathrm{~m} \mathrm{yr}^{-1}$. 


\section{Chapter 4}

\section{Effects of Particle Composition on}

\section{Thorium Scavenging in the North}

\section{Atlantic}

This article was originally published in Geochimica et Cosmochimica Acta in 2018 by Elsevier. Elsevier permits the reproduction of this content in a dissertation/thesis.

Lerner, P., Marchal, O., Lam, P.J., and Solow, A., 2018, "Effects of Particle Composition and Thorium Scavenging in the North Atlantic," Geochimica et Cosmochimica Acta, 233, 113-134, doi: 10.1016/j.gca.2018.04.035 


\begin{abstract}
The dependence of thorium scavenging by particles on particle composition is examined at selected stations of the U.S. GEOTRACES North Atlantic Section (GA03). Scavenging is here described by the apparent, first-order rate constant of Th adsorption onto particles $\left(k_{1}\right)$, as estimated from an inversion of $\mathrm{Th}$ radioisotope and radioactive parent data. Our $k_{1}$ estimates are regressed against particle phase data using two different models. Model I considers biogenic particles (POC $+\mathrm{PIC}+\mathrm{bSi}$ ), lithogenic particles, Mn (oxyhydr)oxides, and Fe (oxyhydr)oxides as regressors, and $k_{1}$ as the regressand. Model II considers $\ln (\mathrm{POC}+\mathrm{PIC}+\mathrm{bSi}), \ln ($ lithogenic particles $), \ln (\mathrm{Mn}$ (oxyhydr)oxides), and $\ln$ (Fe (oxyhydr)oxides) as regressors, and $\ln \left(k_{1}\right)$ as the regressand, where $\ln ($ ) denotes the natural logarithm. Thus, models I and II posit that the effects of particle phases on $k_{1}$ are, respectively, additive and multiplicative. These models are applied to three groups of stations: (i) all selected stations, (ii) stations west of the Mauritanian upwelling region ("western stations"), and (iii) stations within that region ("eastern stations").

We find that model II appears to better describe the effect of particle composition on $k_{1}$ than model I. Particle composition explains a larger fraction of the variance of $k_{1}$ for the eastern stations $\left(R^{2}=0.60\right.$ for model I and 0.67 for model II) than for the western stations $\left(R^{2}=0.26\right.$ for model I and 0.39 for model II). When considering all stations, the variance of $k_{1}$ explained by particle composition is intermediate $\left(R^{2}=0.50\right.$ for model I and 0.51 for model II). According to model II, the variance of $k_{1}$ explained by particle composition is predominantly due to biogenic particles at the eastern stations and to Mn (oxyhydr)oxides at the western stations. Additionally, we find that particle composition does not explain a significantly different proportion of variance of $k_{1}$ than particle concentration. It is thus concluded that, at our selected stations, (i) biogenic particles and Mn (oxyhydr)oxides more strongly influence Th scavenging than any other phases considered, and (ii) particle composition and particle concentration have comparable effects on this process.
\end{abstract}

\title{
4.1 Introduction
}

The high particle reactivity of radioactive thorium isotopes in seawater has resulted in their widespread use in evaluating processes affecting marine particles. Examples include the use of ${ }^{234} \mathrm{Th}$ to estimate the export of particulate matter from the surface ocean (Bhat et al., 1969; Coale and Bruland, 1987; Buesseler et al., 1992, 2006), and the applications of ${ }^{230}$ Th to paleo-oceanography, including for estimating past changes in sediment redistribution (François et al., 2004). However, the use of thorium isotopes to investigate these processes depends partly on our understanding of how the metal attaches to particles. In seawater, thorium exists in the $+\mathrm{IV}$ oxidation state (Choppin and Wong, 1998) and has a strong binding affinity to oxygen containing compounds (Santschi et al., 2006). Thus, one may expect the affinity of thorium for particles to depend on their chemical composition and surface charge.

Previous studies have examined the dependence of Th scavenging on particle composition. Balistrieri et al. (1981) found that stability constants for the adsorption of thorium onto particles in the deep subtropical North Atlantic were closer in magnitude to those for the adsorption of Th onto or- 
ganic compounds than to those for metal oxides. Using sediment trap data from a variety of regions including the Equatorial Pacific, the Southern Ocean, and the North Atlantic, Chase et al. (2002) explored the relationship between the partition coefficient for ${ }^{230} \mathrm{Th}, K_{D}$, and particle composition (in their study, $K_{D}$ was defined as $A_{p *} / A_{d}$, where $A_{p *}$ is the ${ }^{230} \mathrm{Th}$ activity per gram of particles in the sediment trap, and $A_{d}$ is the ${ }^{230} \mathrm{Th}$ activity per gram of seawater $1000 \mathrm{~m}$ above the trap). They found that $K_{D}$ is positively correlated with $\mathrm{CaCO}_{3}$ weight fraction $\left(\% \mathrm{CaCO}_{3}\right)\left(r^{2}=0.66\right)$, weakly and positively correlated $\%$ lithogenic material $\left(r^{2}=0.15\right)$, negatively correlated with $\%$ biogenic silica $\left(r^{2}=0.59\right)$, and not significantly correlated with $\%$ particulate organic matter. Using the data of Chase et al. (2002) from the North Atlantic, Equatorial Pacific, and Southern Ocean, together with data from the Arabian Sea, Scholten et al. (2005) reported that $K_{D}$ exhibits significant positive correlation with $\% \mathrm{CaCO}_{3}\left(r^{2}=0.37\right)$, \% particulate organic carbon $\left(r^{2}=0.11\right)$, and $\%$ lithogenic material $\left(r^{2}=0.34\right)$, and a significant negative correlation with $\%$ biogenic silica $\left(r^{2}=0.40\right)$. However, each of these correlations were only significant when including data from the Southern Ocean; upon removing this dataset, only a weak, negative relationship between $K_{D}$ and $\%$ biogenic silica was apparent $\left(r^{2}=0.08\right)$ (Scholten et al., 2005). Further restricting the analysis to datasets from the North Atlantic, including the Middle Atlantic Bight (Biscaye et al., 1988), the Sargasso Sea (Anderson et al., 1983), and stations near the Canary Islands (Scholten et al., 2001), $K_{D}$ shows a significant, but weak, negative relationship with $\% \mathrm{CaCO}_{3}\left(r^{2}=0.05\right)$, positive relationship with $\%$ lithogenic material $\left(r^{2}=0.11\right)$, and no significant relationship with \% particulate organic carbon and $\%$ biogenic silica (Figure S1; $K_{D}$ and particle composition data from Chase et al. (2002)).

Roy-Barman et al. (2005) found that the relationship between the fraction of thorium present in particles and the particle composition varies between Th isotopes. From ${ }^{230,234} \mathrm{Th}$ data collected by sediment traps deployed in the Northeast Atlantic, they found particulate ${ }^{230} \mathrm{Th}$ (in units of dpm/gram of particles) to show the strongest (positive) correlation with lithogenic material and particulate Mn, whereas particulate ${ }^{234} \mathrm{Th}$ (dpm/gram of particles) showed the strongest (positive) correlation with particulate organic carbon. They also found biogenic opal to have little or no correlation with either particulate ${ }^{230} \mathrm{Th}$ or ${ }^{234} \mathrm{Th}$. From sediment traps deployed from 2000 to 2003 as part of the Oceanic Flux Program off Bermuda (at 500, 1500, and 3200 m), Roberts et al. (2009) reported only a significant positive relationship between $K_{D}$ and $\%$ total carbohydrate content of particles. However, from data collected from the same program between 2005 and 2007, Chuang 
et al. (2013) found the strongest positive relationship between $K_{D}$ and $\% \mathrm{CaCO}_{3}$, although they speculated that such a relationship was caused by the coating of this mineral phase by various biopolymers.

The recent radionuclide and particle concentration dataset obtained along the U.S. GEOTRACES North Atlantic transect (GA03) provides a unique opportunity to study the dependence of Th scavenging on particle composition in oceanic waters. Lam et al. (2015a) have reported measurements of small $(0.8-51 \mu \mathrm{m})$ and large $(\geq 51 \mu \mathrm{m})$ bulk particle concentrations as well as particle phase concentrations, including particulate organic carbon (POC), particulate inorganic carbon (PIC), biogenic opal (bSi), lithogenic material, and manganese and iron (oxyhydr)oxides, obtained along GA03. Hayes et al. (2015a) have used the ${ }^{230} \mathrm{Th}$ (Hayes et al., 2015b) and particle (Lam et al., 2015a) data from GA03 to determine how particle composition affects the partitioning of Th between particulate and dissolved phases. They estimated the distribution coefficient for ${ }^{230} \mathrm{Th}$ from $K_{D}=A_{p} /\left(A_{d} P\right)$, where $A_{p}$ is the ${ }^{230} \mathrm{Th}$ activity in the particulate phase $\left(\mathrm{dpm} / \mathrm{m}^{3}\right), A_{d}$ is the ${ }^{230} \mathrm{Th}$ activity in the dissolved phase $\left(\mathrm{dpm} / \mathrm{m}^{3}\right)$, and $P$ is bulk particle concentration $(\mu \mathrm{g} / \mathrm{kg})$. They found that $K_{D}$ is largest for $\mathrm{Mn}$ and Fe (oxyhydr)oxides $(116.8 \mathrm{~g} / \mathrm{g}$ and $32.8 \mathrm{~g} / \mathrm{g}$, respectively), and smallest for particulate organic matter $(0.3 \mathrm{~g} / \mathrm{g})$. They could not derive significant values of $K_{D}$ for opal, consistent with a similar result obtained for trap particles (Roy-Barman et al., 2005).

Importantly, most of pre-existing studies focused on the effects of particle composition on the partitioning of thorium between the dissolved and particulate phases, rather than on the kinetics of sorption reactions. Below the euphotic zone, thorium is generally thought to undergo a reversible exchange with slowly sinking particles (Nozaki et al., 1981; Bacon and Anderson, 1982). In this model, thorium cycling is governed by three parameters: the rate constant of Th adsorption onto particles $\left(k_{1}\right)$, the rate constant of Th desorption from particles $\left(k_{-1}\right)$, and the particle sinking speed $(w)$. A more complete treatment includes in addition the rate constant of Th release from particles during particle degradation ( $\beta_{-1}$; Clegg et al. (1991a)). The quantities $k_{1}, k_{-1}$, and $\beta_{-1}$ should be viewed as apparent, first-order rate constants given the various assumptions in the model, such as the consideration of only one particle class. Under a set of assumptions, including steady state, three of these rate parameters are related to $K_{D}$ by the expression $K_{D}=k_{1} /\left(\left(k_{-1}+\beta_{-1}\right) P\right)$ (Honeyman et al., 1988; Lerner et al., 2017). Thus, the processes of thorium adsorption, thorium desorption, and particle degradation may each influence the observed partitioning of the metal between the 
dissolved and particulate forms.

Several studies have investigated the effects of particle concentration on estimates of $k_{1}$ in oceanic waters. Bacon and Anderson (1982) found a linear relationship between $k_{1}$ and $P$ from samples taken from the Guatemala and Panama Basins. Honeyman et al. (1988) proposed that $k_{1}$ could be related to particle concentration as $k_{1}=k_{1, c} P^{b}$, where $k_{1, c}$ and $b$ are positive constants. If $b<1$, then $K_{D}$ would decrease with increasing particle concentration (assuming invariant $k_{-1}$ and $\beta_{-1}$ ), the so-called "particle concentration effect" (Honeyman and Santschi, 1989). In this case, the thorium activity in filterable particles would be controlled by colloidal coagulation. In contrast, if $b=1$, the influence of colloids on Th partitioning between the solution and filterable particles would be absent (Honeyman et al., 1988). Numerous model studies have relied on this relationship between $k_{1}$ and $P$, generally assuming $b=1$ (Clegg and Sarmiento, 1989; Clegg et al., 1991b; Clegg and Whitfield, 1993; Burd et al., 2000). In recent work, Lerner et al. (2017) estimated the vertical distribution of $k_{1}$ at selected stations of GA03 from an inversion of Th radioisotope, Th radioactive parent, and bulk particle concentration data. They found that $k_{1}$ appears to be proportional to $P^{b}$ with $b \geq 1$, suggesting that colloidal coagulation due to Brownian pumping (Honeyman et al., 1988) does not noticeably affect on Th removal at these stations. On the other hand, to our knowledge, no previous studies have considered the relationship between $k_{1}$ and particle composition in oceanic waters.

The objective of this paper is to examine the influence of particle composition on the rate constant of Th adsorption $\left(k_{1}\right)$ onto particles obtained along the GA03 transect in the North Atlantic. We consider two distinct regression models to describe this influence: in a first model, the effects of different particle types on $k_{1}$ are additive, whereas in a second model they are multiplicative. We examine the influence of particle composition on $k_{1}$ for three groups of stations: (i) stations both west and east of the Mauritanian upwelling region, (ii) stations west of the Mauritanian upwelling region, and (iii) stations within the Mauritanian upwelling region. The effect of particle composition on $k_{1}$ is contrasted with that of particle concentration in order to determine whether it is the chemical nature of the particles or the number of surface sites available for adsorption which dominates the specific rate at which Th is removed from solution. Our study therefore complements previous works on the effect of particle composition on thorium scavenging by identifying the particle phases that appear to govern the kinetics of Th adsorption onto particles. 
This paper is organized as follows. The particle and radiochemical data considered in this study are summarized, and the estimates of $k_{1}$ obtained by data inversion are presented in section 2 . Results from the regression analyses are contained within section 3. In section 4, we determine the relative importance of different particulate phases to the explained variance in $k_{1}$, attempt to interpret our results kinetically, clarify the paleoceanographic implications of our study, discuss the influence of observational errors, and contrast the effects of particle composition and particle concentration on our $k_{1}$ estimates. Conclusions follow in section 5 .

\subsection{Methods}

\subsubsection{Particle and Radiochemical Data}

We use data collected aboard the R/V Knorr along the GA03 transect in October 2010 (leg GT10) and November-December 2011 (leg GT11; red stars in Figure 1). The first leg (GT10) of the transect went from Lisbon to Cape Verde, while the second leg (GT11) ran from Woods Hole to Cape Verde. We consider only the eleven stations highlighted in red (Fig. 1). At these stations (referred to below as the "selected" stations), the impact of lateral and vertical transport on thorium isotope and particle budgets appear to be small compared to the impact of sorption reactions and particle processes (Lerner et al., 2017). In addition, we rely only on data collected at depths located outside nepheloid layers, where processes not considered in this study presumably take place. Station GT11-16 near the TAG hydrothermal vent is also excluded from the present analysis because of the very high concentrations of Fe (oxyhydr)oxides measured at that station (Lam et al., 2015a).

Particle phase concentrations considered in this work are the sum of the "small" and "large" size classes. Particles in the small $(1-51 \mu \mathrm{m})$ and large $(>51 \mu \mathrm{m})$ size fractions were sampled using a WTS-LV McLane pump modified to include dual flow paths and equipped with 142-mm "miniMULVFS" filter holders (Bishop et al., 2012). One filter holder was loaded with a 51- $\mu \mathrm{m}$ Sefar polyester mesh prefilter followed by paired Whatman QMA quartz fiber filters $(1-\mu \mathrm{m}$ nominal pore size). The other filter was loaded with another $51-\mu \mathrm{m}$ polyester prefilter followed by paired $0.8-\mu \mathrm{m}$ Pall Supor800 polyethersulfone filters. Further details about the method of particle collection can be found in Lam et al. (2015a).

The bulk chemical composition of the collected particles was determined as follows (notice that 
particle composition data used in this study are all in units of $\mathrm{mg}$ per $\mathrm{m}^{3}$ of seawater). For PIC, subsamples were taken from the QMA filters for analysis in the small size fraction, while subsamples were taken from the polyester prefilters for analysis in the large size fraction. PIC was measured using coulometry or salt-corrected $[\mathrm{Ca}]$. Total particulate carbon in the small (large) size fraction was analyzed from subsamples taken from QMA filters (polyesther prefilters) and was measured using a Dynamic Flash Combustion technique. POC was taken as the difference between total particulate carbon and particulate inorganic carbon.

Biogenic silica in the small and large size fractions were measured from subsamples taken from the Supor and polyester prefilters, respectively. Silica (in $\mathrm{mol} / \mathrm{m}^{3}$ ) was determined using spectrophotometric detection of a blue silico-molybdate complex, and converted to units of $\mathrm{mg} / \mathrm{m}^{3}$ assuming a molar mass of biogenic silica of $67.2 \mathrm{~g} / \mathrm{mol}$. The concentrations of lithogenic particles were estimated by measuring the concentration of particulate $\mathrm{Al}$ and assuming an $\mathrm{Al} /$ lithogenic mass ratio of 0.0804 . Whereas $\mathrm{Al} /$ lithogenic mass ratios vary only slightly with particle source, by about $8 \%$ for upper and lower continental crust (Taylor and McLennan, 1995), dissolved Al is susceptible to scavenging, which could lead to overestimates of lithogenic material. However, overestimation of lithogenic particle concentration based on $\mathrm{Al}$ scavenging is mostly a concern for coastal samples Lam et al. (2015a). Since we restrict our study to open-ocean stations, we estimate lithogenic mass based on the $\mathrm{Al} /$ lithogenic mass ratio. Total particulate $\mathrm{Al}$ (and $\mathrm{Ti}, \mathrm{Mn}$, and $\mathrm{Fe}$, all in units of $\mathrm{nM}$ ) were measured by complete digestion of the supor filters and polyester prefilters, followed by ICP-MS analysis.

Iron and manganese (oxyhydr)oxides were calculated by subtracting total particulate Mn and Fe from their respective lithogenic components. The lithogenic components were estimated using $\mathrm{Fe} / \mathrm{Ti}$ and $\mathrm{Mn} / \mathrm{Ti}$ mass ratios measured on aerosols collected along GA03 between Cape Verde and Mauritania $(\mathrm{Fe} / \mathrm{Ti}=8.7$ and $\mathrm{Mn} / \mathrm{Ti}=0.13$; Shelley et al. $(2015))$, and by multiplying these ratios by the concentration of $\mathrm{Ti}$ (assumed to be purely lithogenic) measured on oceanic particles. The difference between total particulate $\mathrm{Mn}$ and its lithogenic component was multiplied by the molar mass for birnessite $\left(\mathrm{MnO}_{2}=96.9 \mathrm{ng} / \mathrm{nmol}\right)$ to obtain the mass of $\mathrm{Mn}$ (oxyhydr)oxides per unit water volume. Likewise, the difference between total particulate Fe and its lithogenic component was multiplied by the molar mass for iron hydroxide $\left(\mathrm{Fe}(\mathrm{OH})_{3}=106.9 \mathrm{ng} / \mathrm{nmol}\right)$ to obtain the mass of Fe (oxyhydr)oxides per unit water volume. Hereafter, the Mn and Fe particulate concentrations 
obtained from these ratios are referred to as "Fe and Mn (oxyhydr)oxides".

The Th isotope data used in this study consist of measurements of ${ }^{228}$ Th (Charette et al., 2014), ${ }^{230} \mathrm{Th}$ (Hayes et al., 2015b,a), and ${ }^{234} \mathrm{Th}$ (Owens et al., 2015) in both the dissolved $(<1 \mu \mathrm{m})$ and total particulate phase $(>1 \mu \mathrm{m})$. We also use measurements of ${ }^{228} \mathrm{Ra}$ activity (radioactive parent of ${ }^{228} \mathrm{Th} ;{ }^{228} \mathrm{Ra}$ data from Charette et al. (2015)) as well as estimates of ${ }^{234} \mathrm{U}$ (parent of ${ }^{230} \mathrm{Th}$ ) and ${ }^{238} \mathrm{U}$ (parent of ${ }^{234} \mathrm{Th}$ ) derived from salinity data and a fixed ${ }^{234} \mathrm{U} /{ }^{238} \mathrm{U}$ activity ratio for seawater. Details about the methods of sample collection and analysis and about the estimation of ${ }^{234,238} \mathrm{U}$ activities can be found in Lerner et al. (2017) and in references therein.

\subsubsection{Principal Component Analysis}

In this section, we explore whether the variability in the particulate phase data at our selected stations of GA03 can be summarized by only a few spatial patterns using principal component analysis (PCA). In broadest terms, PCA aims to find a few principal components (PCs), or linear combinations of the original variables, that explain a large proportion of the total variance in the dataset being considered (Rencher, 1998). This goal can be reached if the original variables are highly correlated, which is the case for our particle phase data (Fig. 2). Particularly, we find strong positive correlations between POC and PIC $(r=0.74)$, and between POC and bSi $(r=0.70)$, which suggest that the variability in the particle composition data along GA03 could be approximated by a few PCs.

A PCA is conducted on the following particle phase data gathered at our selected stations: POC, PIC, bSi, lithogenic material, Mn (oxyhydr)oxides, and Fe (oxyhydr)oxides. Since the data occur at different geographic locations and depths, the PCs extracted from these data represent different spatial patterns along the section. PCs are extracted from both the covariance matrix and the correlation matrix of the data in order to document the effect of variable variance among different particulate phases (e.g., POC is typically present in seawater at much higher concentrations than Mn (oxyhydr)oxides). In both cases, the coefficients in a given PC are taken as measures of the importance of the different particulate phases for the corresponding pattern.

We find that the first two principal components explain $98 \%$ of the total variance in the particulate phase dataset if the PCs are extracted from the covariance matrix; if extracted from the correlation matrix, then the first two PCs explain over $69 \%$ of the total variance (Table 1). PC1, the first PC, is largely dominated by POC when the PCs are extracted from the covariance matrix; 
if extracted from the correlation matrix, the coefficients for POC, PIC, and bSi are all much closer in magnitude, though the coefficient for POC is still the largest (Fig. 3). Compared to PC1, PC2 represents a clear contrast between particles of different composition. PC2 extracted from both matrices is dominated by lithogenic material. If extracted from the correlation matrix, then bSi, Mn, and Fe (oxyhydr)oxides have coefficients closer in magnitude to that for lithogenic particles.

\begin{tabular}{|l|c|c|c|c|c|c|}
\hline & PC1 & PC2 & PC3 & PC4 & PC5 & PC6 \\
\hline covariance & $57.3 \%$ & $41.2 \%$ & $1.2 \%$ & $0.3 \%$ & $0.0 \%$ & $0.0 \%$ \\
\hline correlation & $42.7 \%$ & $26.7 \%$ & $15.8 \%$ & $7.8 \%$ & $4.8 \%$ & $2.2 \%$ \\
\hline
\end{tabular}

Table 1: Percentages of total variance explained by principal components.

Figure 4 shows the distribution along the GA03 section of the first two leading principal components. Interestingly, $\mathrm{PC} 1$ from both the covariance matrix and correlation matrix show systematic vertical variations at a given station, which likely reflects the effects of particle recycling along the water column. In addition, the values of PC1 at the four easternmost stations (GT11-24, GT1012, GT10-11, GT10-10) are in general noticeably different than those at the remaining stations. The salinity distribution along GA03 portrays pronounced upward bowing of isohalines near the crossover station GT11-24/GT10-12 (Jenkins et al., 2015), suggesting that these four stations are under the influence of the Mauritanian upwelling. In contrast to PC1, PC2 does not exhibit clear systematic variations with depth. On the other hand, both PC1 and PC2 tend to show a gradient between the four easternmost stations and the remaining stations along GA03.

In summary, the particle composition data collected along GA03 can be summarized by two principal components presenting systematic geographic and (or) vertical patterns along the section (Fig. 4). Although a precise interpretation of these patterns appears difficult, they seem to reflect the influences of particle recycling and of the Mauritanian upwelling or, more generally, of the proximity to the western African coast.

\subsubsection{Estimates of Th Adsorption Rate Constant}

We estimate the vertical distribution of $k_{1}$ (below $\sim 100 \mathrm{~m}$ ) at each of the selected stations of GA03 by fitting a Th cycling model to radionuclide measurements (Fig. 1). The model as well as the inverse method used to fit the model to the data are briefly described here: details about the method can be found in Lerner et al. (2017). For convenience, subscripts $p$ and $d$ denote thorium activities in the particulate and dissolved phases, respectively. 
We use a single-particle class model for thorium cycling that includes balance equations for ${ }^{228,230,234} \mathrm{Th}_{d}$ and bulk ${ }^{228,230,234} \mathrm{Th}_{p}$, i.e., ${ }^{228,230,234} \mathrm{Th}$ in both the small and large size particulate fractions. The thorium balance equations account for radioactive production, radioactive decay, Th adsorption onto particles, Th release from particles due to desorption and particle degradation, and particle sinking:

$$
\begin{aligned}
T\left(A_{d}\right) & =\lambda A_{\pi}+\left(k_{-1}+\beta_{-1}\right) A_{p}-\left(k_{1}+\lambda\right) A_{d}, \\
T\left(A_{p}\right)+w \frac{\partial A_{p}}{\partial z} & =k_{1} A_{d}-\left(\beta_{-1}+k_{-1}+\lambda\right) A_{p} .
\end{aligned}
$$

Here, $A_{d}\left(A_{p}\right)$ represents the thorium isotope activity in the dissolved (particulate) phase (in units of $\left.\mathrm{dpm} \mathrm{m}^{-3}\right), A_{\pi}$ is the activity of the radioactive parent $\left(\mathrm{dpm} \mathrm{m}^{-3}\right), \lambda$ is the radioactive decay constant $\left(\mathrm{yr}^{-1}\right), k_{1}, k_{-1}$, and $\beta_{-1}$ are the apparent rate constants for Th adsorption, Th desorption, and particle degradation, respectively $\left(\mathrm{yr}^{-1}\right), w$ is the particle settling speed $\left(\mathrm{m} \mathrm{yr}^{-1}\right)$, and $z$ is depth (m). In accordance with previous models (e.g., Nozaki et al. (1981); Bacon and Anderson (1982)), we assume first-order kinetics for thorium adsorption, thorium desorption, and particle degradation. The lithogenic components of ${ }^{228,234} \mathrm{Th}$ are taken as negligible, while particulate ${ }^{230} \mathrm{Th}$ data are corrected for a contribution from a lithogenic source (for details, see Lerner et al. (2017)). Finally, the term $T(\cdot)$ in each equation represents the effects of unsteadiness, advection, and diffusion, i.e.,

$$
T\left(A_{d}\right) \equiv \frac{\partial A_{d}}{\partial t}+\boldsymbol{u} \cdot \nabla A_{d}-\nabla \cdot\left(\boldsymbol{k} \nabla A_{d}\right)
$$

where $t$ is time, $\mathbf{u}$ the vector velocity, and $\boldsymbol{k}$ a diffusion tensor. In this study, we assume $T(\cdot)=0$. Evidence for negligible effects of advection, diffusion, and, to some extent, unsteadiness at the stations analyzed here is provided in Lerner et al. (2017).

Equations (1a-1b) with $T(\cdot)=0$ are fit to the radiochemical data $\left({ }^{228,230,234} \mathrm{Th}_{d},{ }^{228,230,234} \mathrm{Th}_{p}\right.$, ${ }^{228} \mathrm{Ra}$, and ${ }^{234,238} \mathrm{U}$ ) below about $100 \mathrm{~m}$ at each selected station (see Table 1 of Lerner et al. (2017)) by adjusting the rate parameters $\left(k_{1}, k_{-1}, \beta_{-1}\right.$, and $\left.w\right)$ and the data themselves, given the uncertainties in the prior estimates of the rate parameters and in the data (more specifically, finite-difference analogs of equations (1a-b) with $T(\cdot)=0$ are fit to vertically interpolated data obtained from a minimum variance estimation procedure). The adjustments in the rate parameters and in the data can be different at different depths, such that a vertical profile of $k_{1}$, for example, 
is inferred at each station. Prior estimates of $k_{1}, k_{-1}, \beta_{-1}$ and $w$ are obtained from observational estimates of these parameters in distinct oceanic environments (see Table 2 of Lerner et al. (2017)). Note that, in contrast to Lerner et al. (2017), the present estimates of the rate parameters (e.g., $\left.k_{1}\right)$ are derived with no consideration of data of total particle concentration, calculated as the sum of the particle phase data (Lam et al., 2015a), to ensure that the $k_{1}$ estimates are independent of these data. We show in Appendix A that both sets of $k_{1}$ estimates are very strongly correlated. The errors in the rate parameters and in the data derived by inversion are also estimated (Lerner et al., 2017).

The estimates of $k_{1}$ obtained by the inversion performed in this study are presented in Figure 5 . It is seen that $k_{1}$ is generally larger in the upper $1000 \mathrm{~m}$ of the water column than below, and that $k_{1}$ values in the Mauritanian upwelling region (stations GT11-24, GT10-12, GT10-11, and GT10-10) exceed those to the west of this region. These vertical and lateral variations in $k_{1}$ are consistent with those found by Lerner et al. (2017) and generally exceed the errors in the $k_{1}$ estimates (not shown).

\subsection{Results}

\subsubsection{Dependence of $k_{1}$ on Particle Composition: Additive Model}

In this section, we use multiple linear regression to determine the dependence of $k_{1}$ upon particle composition. Before proceeding with the regression analysis, two questions regarding the particle composition data arise. A first question is whether to normalize the particle composition data to bulk particle concentration. Previous studies on the relationship between $K_{D}$ and particle composition have related $K_{D}$ to one particulate phase, or a linear combination of particulate phases, normalized to the total particle concentration (e.g., Chase et al. (2002); Li (2005); Hayes et al. (2015a)). Consideration of particle phase data normalized to total particle concentration $(P)$ appears to have been motivated by the presence of $P$ in the defining expression for $K_{D}$, i.e., $K_{D}=A_{p} /\left(A_{d} P\right)$. However, since our estimates of $k_{1}$ do not consider $P$ data (section 2.2), there is no clear reason to normalize the particle phases by bulk particle concentration in this study. Hence, we explore the dependence of $k_{1}$ on particle phase concentrations expressed in units of mass of particles of a certain type divided by water volume $\left(\mathrm{mg} \mathrm{m}^{-3}\right)$.

A second question is whether each particle phase should be treated as an individual regressor. 
As shown in Figure 2a, there is strong correlation among the biogenic phases. If strongly correlated variables are used as regressors in multiple regression, a situation called multicollinearity, then the individual estimates of the regression coefficients can be unstable (i.e., overly sensitive to the data values) and suffer from excessive variance (Rencher, 1998; Keith, 2014). Multicollinearity can thus lead to difficulties in the interpretation of regression coefficients. To reduce the influence of multicollinearity from the regression of $k_{1}$ against the particle phases, we lower the number of regressors to four. These include (i) the sum of the biogenic phases $(\mathrm{POC}+\mathrm{PIC}+\mathrm{bSi}$, hereafter referred to as biogenic particles or "bio" for brevity), (ii) lithogenic material ("litho"), (iii) Mn (oxyhydr)oxides ("Mn"), and (iv) Fe (oxyhydr)oxides ("Fe"). As shown in Figure 2b, the correlation coefficients for this group of regressors do not exceed 0.5. Therefore, we proceed with a model of $k_{1}$ as a linear combination biogenic particles, lithogenic material, and Mn and Fe (oxyhydr)oxides:

$$
k_{1}=a_{0}+a_{1}[b i o]+a_{2}[\text { litho }]+a_{3}[M n]+a_{4}[F e]+\epsilon,
$$

where $a_{i}(i=0,1,2,3,4)$ are regression coefficients and $\epsilon$ is an error term representing the error in $k_{1}$ and the unmodeled variability. The regression model (4.3), referred to below as model I, posits that the effects of the particle phases on $k_{1}$ are additive. We regress $k_{1}$ against particle composition using ordinary least squares (OLS), which does not consider the error (co)variances for $k_{1}$ and the regressors ([bio], [litho], $[\mathrm{Mn}]$, and $[\mathrm{Fe}]$ ). In section 4.3, we document the effect of these errors on the regression.

The estimates of the regression coefficients $\left(a_{1}, a_{2}, a_{3}, a_{4}\right)$ and their standard errors are listed in Table 2 (first row; see also Table S1), and the best fit is shown in Figure 6a. We find that the

\begin{tabular}{|l|c|c|c|c|}
\hline & bio & litho & Mn & Fe \\
\hline all stations $(\mathrm{n}=63)$ & $0.52 \pm 0.08(<0.01)^{a}$ & $0.05 \pm 0.10(0.65)$ & $-44.62 \pm 49.11(0.36)$ & $14.56 \pm 18.01(0.42)$ \\
\hline western stations $(\mathrm{n}=35)$ & $0.14 \pm 0.07(0.06)$ & $0.03 \pm 0.10(0.76)$ & $21.24 \pm 34.92(0.54)$ & $-1.24 \pm 11.82(0.92)$ \\
\hline eastern stations $(\mathrm{n}=28)$ & $0.58 \pm 0.15(<0.01)$ & $-0.31 \pm 0.29(0.29)$ & $-3.31 \pm 107.94(0.98)$ & $10.38 \pm 36.21(0.77)$ \\
\hline
\end{tabular}

a. Values in parentheses are $p$-values.

Table 2: Regression coefficients \pm 1 standard error $\left(\mathrm{yr}^{-1} \mathrm{~m}^{3} \mathrm{mg}^{-1}\right)$ for model $\mathrm{I}$.

multiple correlation $R$ is 0.71 , with $p<0.01$, i.e., up to $0.71^{2}=50 \%$ of the variance in $k_{1}$ can be explained by particle composition $(n=63)$. Moreover, only the regression coefficients for biogenic particles is significant at the 0.05 level. 
While a strong relationship between $k_{1}$ and particle composition is observed $(R=0.71$ with $p<0.01$ ), this relationship may not hold across all our selected stations. Stations in the Mauritanian upwelling region (GT11-24, GT10-12, GT10-11, and GT10-10) show large concentrations of lithogenic material, POC, and bSi compared to those at the other stations of GA03 (Lam et al., 2015a). Our PCA of the particle data reveals a compositional contrast between stations west and east of GT11-24 (section 2.2). To test whether different regions sampled along GA03 are characterized by different relationships between $k_{1}$ and particle composition, we perform two multiple linear regressions: one for stations west of GT11-24 (Fig. 1), and another for stations east of, and including, station GT11-24. For simplicity, these two groups of stations are referred below to as "western" and "eastern" stations, respectively.

\section{Western and Eastern Stations}

For the western stations, the multiple correlation between $k_{1}$ and the particle phase data (Fig. $6 \mathrm{~b})$ amounts to $R=0.51$, with $p=0.05(n=35)$. The regression coefficient for the biogenic particles is the most significant among the different particulate phases, although none of the coefficients for this regression are significant at the 0.05 level (Table 2).

For the eastern stations, the multiple correlation between $k_{1}$ and particle phase data (Fig. 6c) reaches a value of $R=0.77$, with $p<0.01(n=28)$. Here, the regression coefficient for the biogenic phases is significant at the 0.05 level and the remaining regression coefficients are not significant at this level (Table 2). Overall, these results indicate that the strength of the correlation between the Th adsorption rate constant and particle composition varies along the GA03 section, i.e., the correlation is relatively weak for the western stations and stronger for the eastern stations.

\section{Critique of Model I}

Two issues emerge with the application of the additive model (model I) to describe the dependence of $k_{1}$ on particle composition at our selected stations of GA03. First, the presence of negative regression coefficients for some of the particle types (Table 2) is troubling: one would not expect the specific rate of adsorption of a metal onto particles to decrease with increasing particle concentration, regardless of particle phase. Indeed, adding particles of any type should increase the number of surface sites for thorium to attach to, and thus increase $k_{1}$. On the other hand, this reasoning does not consider the correlations between particle phases (Fig. 2b), i.e., a negative relationship between $k_{1}$ and a particle type (e.g., Mn (oxyhydr)oxides) may not be causal but rather reflect the 
simultaneous removal of another particle type (e.g., biogenic particles) characterized by a positive regression coefficient. Moreover, none of the negative regression coefficients differ significantly from $0 \mathrm{yr}^{-1} \mathrm{~m}^{3} \mathrm{mg}^{-1}$ (Table 2). Nevertheless, the inference of negative coefficients is intriguing, for it implies that $k_{1}$ can, at least in principle, be negative for some combinations of the particle phase concentrations.

Another issue with the application of the additive model is the appearance of variable variance (heteroscedasticity) in some of the plots of $k_{1}$ derived by inversion against $k_{1}$ derived by regression (Fig. 6a-c). Using a Breusch-Pagan test, we find evidence for significant heteroscedasticity for each group of stations $(p<0.05 ;$ Madansky, 1988; p.81). When heteroscedasticity is present, the standard errors of the regression coefficients may be unreliable (Greene, 2012; p.299).

\subsubsection{Dependence of $k_{1}$ on Particle Composition: Multiplicative Model}

A possible remedy to the two foregoing issues is to assume the following relationship between $k_{1}$ and the particle phases:

$$
\ln \left(k_{1}\right)=b_{0}+b_{1} \ln [b i o]+b_{2} \ln [\text { litho }]+b_{3} \ln [M n]+b_{4} \ln [F e]+\epsilon,
$$

where $b_{i}(i=0,1,2,3,4)$ are another set of regression coefficients and $\epsilon$ accounts for the error in $\ln \left(k_{1}\right)$ and for unmodeled variability. Using logarithms for the regressand and the regressors both prevents the inference of negative regressand estimates and reduces variations in variance. Taking the exponential of each side of equation (4.4) yields

$$
k_{1} \propto[\text { bio }]^{b_{1}}[l i t h o]^{b_{2}}[M n]^{b_{3}}[F e]^{b_{4}} \epsilon
$$

That is, the effects of the particle phases on $k_{1}$ are now considered as multiplicative with a multiplicative error. Hereafter, the regression model (4.4) is referred to as model II.

The estimates of the regression coefficients $\left(b_{1}, b_{2}, b_{3}, b_{4}\right)$ and their standard errors for all stations are listed in Table 3 (first row; see also Table S2), and the best fit is shown in Figure 6d. We find that, for the multiplicative model, the multiple correlation for all stations is $R=0.71$, with $p<0.01$ $(n=63)$. The regression coefficient for biogenic particles is significant at the 0.05 level, while the remaining regression coefficients are not significant at this level. These results are similar to those obtained from model I (section 3.1). 


\begin{tabular}{|l|c|c|c|c|}
\hline & bio & litho & Mn & Fe \\
\hline all stations $(\mathrm{n}=63)$ & $1.03 \pm 0.20(<0.01)^{a}$ & $0.35 \pm 0.20(0.08)$ & $0.13 \pm 0.31(0.68)$ & $0.01 \pm 0.03(0.61)$ \\
\hline western stations $(\mathrm{n}=35)$ & $0.32 \pm 0.27(0.22)$ & $-0.17 \pm 0.31(0.58)$ & $0.92 \pm 0.42(0.03)$ & $0.03 \pm 0.03(0.35)$ \\
\hline eastern stations $(\mathrm{n}=28)$ & $1.45 \pm 0.29(<0.01)$ & $-0.46 \pm 0.47(0.32)$ & $-0.20 \pm 0.41(0.62)$ & $-0.01 \pm 0.04(0.86)$ \\
\hline
\end{tabular}

a. Values in parentheses are $p$-values.

Table 3: Regression coefficients \pm 1 standard error for model II

\section{Western and Eastern Stations}

For the western stations, the multiple correlation between $k_{1}$ and the particle phase data (Fig. $6 \mathrm{e})$ amounts to $R=0.62$, with $p<0.01(n=35)$. Notably, only the regression coefficient for $\mathrm{Mn}$ (oxyhydr)oxides is significant at the 0.05 level.

For the eastern stations, the multiple correlation between $k_{1}$ and the particle phase data (Fig. 6 f) reaches a value of $R=0.82$, with $p<0.01(n=28)$. In contrast to the western stations, only the regression coefficient for biogenic particles is significant at the 0.05 level. Thus, as for model I, model II shows that the correlation between Th adsorption rate constant and particle composition is stronger for the eastern stations than for the western stations. On the other hand, in contrast to model I, model II shows that Mn (oxyhydr)oxides are the only significant regressor for the western stations, whereas in both models the biogenic particles are the only significant regressor for the eastern stations.

\section{Resolution of Model I Issues}

As for model I, some of the regression coefficients for model II are negative, although none of them are significantly so (Table 3). However, unlike for model I, negative regression coefficients for the multiplicative model do not imply that $k_{1}$ can be negative, since a regression based on logarithms ensures that regressand estimates remain positive definite for any combination of regressor values. Moreover, heteroscedasticity is less apparent (compare Figs. 6a-6c with Figs. 6d-6f) and not significant $(p>0.05)$ when the relationship between $k_{1}$ and the particle phases is described using a multiplicative model. Hence, the regression and correlation statistics seem more robust for model II than for model I.

\subsection{Discussion}

Our results show that a multiplicative model appears to provide a better description than an additive model of the effects of particle phases on the apparent rate constant of $\mathrm{Th}$ adsorption onto particles along GA03. They also show that biogenic particles are a significant regressor in this model, except for the western stations where Mn-rich particles are the only significant regressor. In 
fact, the fits of the multiplicative model to the particle data are significantly different for both the eastern and western stations according to an F test ( $p=0.03$; Seber and Lee, 1992; p.100).

\subsubsection{Relative Importance of Different Particulate Phases}

In this section, we attempt to elucidate the relative contribution of different particle phases to the variability in our $k_{1}$ estimates along GA03. The difficulty in this task lies in the remaining correlation between the particle types used as regressors (Fig. 2b), preventing a confident assignment of the phases that exert a dominant influence on $k_{1}$. To address this issue, we conduct a relative importance analysis, a technique which can provide estimates of the contribution of correlated regressors to the explained variance of a regressand.

In his review on techniques used to evaluate the relative importance of regressors, Grömping (2007, p. 140) noted that there is a "lack of an accepted mainstream methodology for the important task of relative importance investigations." Given an apparent lack of consensus, we apply two methods for evaluating the relative importance of the particle phases. One method, referred to as averaging over orderings (AOO) (Kruskal, 1987), averages partial correlation coefficients obtained from every possible ordering of the regressors. The other method, termed dominance analysis (DA) (Azen and Budescu, 2003), averages the contribution to the squared multiple correlation $R^{2}$ by a regressor over models encapsulating every possible subset of regressors. Both of these analyses are explained briefly in Appendix B.

Figure 7 shows values of relative importance $(R I)$ from both analyses for each particle phase and for each group of stations. Notably, the ranking of the particle phases is the same for the two methods. It is also the same for models I and II, with the exception that, at the western stations, Mn (oxyhydr)oxides have the highest $R I$ value for model II but the second highest value for model I. However, since model I appears questionable (section 3.2.2), we focus our discussion on the relative importance values for model II.

For all stations and stations in the Mauritanian upwelling region, biogenic particles are the most important regressor $(R I=0.41$ for $\mathrm{AOO}$ and $R I=0.36$ for $\mathrm{DA})$. In contrast, for the western stations, the most important regressor is Mn (oxyhydr)oxides, with $R I=0.24$ for $\mathrm{AOO}$ and $R I=$ 0.22 for DA, i.e., about double the $R I$ value for biogenic particles according to both methods. Thus, the results from relative importance analysis suggest that the biogenic particles (POC, PIC, and bSi) dominate Th scavenging in the Mauritanian upwelling region, whereas Mn (oxyhydr)oxides 
dominate Th scavenging west of this region along GA03.

An interesting observation is that the groups of stations where biogenic particles dominate the explained variance in $k_{1}$ are also those displaying the largest $R^{2}$ values. Indeed, as shown in section 3 , the variance of $k_{1}$ explained by particle composition is higher for the eastern stations $\left(R^{2}=0.67\right)$ and for all selected stations $\left(R^{2}=0.51\right)$ than for the western stations $\left(R^{2}=0.39\right)$. Moreover, the concentrations of two of the three types of biogenic particles, POC and bSi, increase eastward in the upper $500 \mathrm{~m}$ towards the Mauritanian upwelling region (Lam et al., 2015a). This finding suggests that the influence of the biogenic phases on the specific rate of Th adsorption onto particles is relatively large in waters where these types of particles are abundant.

To our knowledge, no previous studies exist on the effect of particle composition on the adsorption rate constant of Th onto marine particles $\left(k_{1}\right)$. In the absence of such studies, we compare our results to previous investigations on the distribution coefficient, $K_{D}$, derived for different particle phases, with the understanding that $k_{1}$ and $K_{D}$ are different concepts. Our results appear qualitatively consistent with the positive relationship between $K_{D}$ and $\% \mathrm{CaCO}_{3}$ found by Chase et al. (2002), though these authors also found that $K_{D}$ decreases with increasing \% opal. $L i$ (2005), using the composition of bulk particles in sediment traps deployed in the Middle Atlantic Bight, the Southern Ocean, and the Equatorial Pacific, showed that $K_{D}$ was generally larger for organic carbon than for lithogenic material. The values of $K_{D}$ for each particle phase, however, varied across oceanic regions: $K_{D}$ was larger for lithogenic material than for organic matter in the Southern Ocean and Equatorial Pacific, whereas the opposite result was observed in the Middle Atlantic Bight (Li, 2005). Quigley et al. (2002) found that partition coefficients for Th were larger for colloidal organic matter than for Mn and Fe (oxyhydr)oxides. They also found, from measurements of Th on colloids collected from the Gulf of Mexico and Galveston Bay, that the partition coefficient for colloids generally increases with the weight fraction of carbohydrate present in the colloidal fraction.

Using data from the GA03 section, Hayes et al. (2015a) estimated distribution coefficients for ${ }^{230} \mathrm{Th}$ and regressed these estimates against fractional contributions of particle composition (POC, bSi, lithogenic material, Fe, and Mn, all expressed in \% particulate mass fraction). They found that $K_{D}$ values for $\mathrm{Mn}$ and $\mathrm{Fe}$ (oxyhydr)oxides exceed those for the other particle types by one to two orders of magnitude. Among the remaining particle phases, they found that $K_{D}$ for $\mathrm{CaCO}_{3}$ was largest. Here, we find (for model II) that the biogenic phases $(\mathrm{POC}+\mathrm{PIC}+\mathrm{bSi})$ are the most 
important regressors for $k_{1}$ at all stations and eastern stations, while Mn-rich particles are the most important at western stations (Fig. 7). We also find that the lithogenic material is not important compared to the biogenic phases (Fig. 7), whereas Hayes et al. (2015a) found lithogenic material to have $K_{D}$ values of a similar magnitude to $\mathrm{CaCO}_{3}$.

The comparison of our results with those of Hayes et al. (2015a) is difficult for several reasons, three of which are listed below. First, whereas $K_{D}$ values for Th are generally higher for (oxyhydr)oxides than for the biogenic phases present in oceanic waters (Anderson et al., 1992; Guo et al., 2002b; Geibert and Usbeck, 2004; Lin et al., 2014; Hayes et al., 2015a), our analysis excludes marginal, near-bottom, and hydrothermal plume regions where Mn and Fe (oxyhydr)oxides significantly contribute to total particle concentration (Lam et al., 2015a). For example, the largest $k_{1}$ estimate reported by Lerner et al. (2017) (see their Figure 12), near the TAG hydrothermal vent at station GT11-16 (excluded from this analysis), coincides with the largest concentrations of Fe (oxyhydr)oxides (Lam et al., 2015a). Thus, it is likely that the relatively low importance of the (oxyhydr)oxide phases for Th scavenging, inferred here for all stations and the eastern stations, stems from the exclusion of samples presenting high concentrations of these phases.

Second, if several assumptions are made in the Th isotope budget, including steady state, then the relationship between $k_{1}$ and $K_{D}$ involves the rate constants for Th desorption $\left(k_{-1}\right)$ and particle degradation $\left(\beta_{-1}\right)$, i.e., $K_{D}=k_{1} /\left(\left(k_{-1}+\beta_{-1}\right) P\right)$. Lerner et al. (2017) showed that, among the selected stations along GA03, $k_{-1}$ and $\beta_{-1}$ vary geographically, and generally increase with bulk particle concentration. Thus, even restricting the analyses of $k_{1}$ and $K_{D}$ values to the same set of stations would not necessarily lead to the same ordering of regression coefficients.

Finally, whereas Hayes et al. (2015a) derived $K_{D}$ values for each of the particle phases reported by Lam et al. (2015a), we regressed $k_{1}$ against the sum of the biogenic phases, lithogenic material, and $\mathrm{Mn}$ and $\mathrm{Fe}$ (oxyhydr)oxides (model I), or we regressed $\ln \left(k_{1}\right)$ against the natural logarithms of these phases (model II). As a result the sensitivity of $k_{1}$ to the particle phases as estimated in this study cannot easily be compared to the variation of $K_{D}$ among the six particle phases (POC, PIC, bSi, lithogenic material, and Mn and Fe (oxyhydr)oxides) as reported by Hayes et al. (2015a).

\subsubsection{Kinetic Consistency of Additive and Multiplicative Models}

In this section, we discuss the ability of the additive and multiplicative models for $k_{1}$ to realistically describe the kinetics of Th sorption onto marine particles. Consider first model I, where the 
effects of different particle phases on $k_{1}$ are assumed to be additive. This model can be rationalized by considering a set of sorption reactions for Th onto different particle phases, each consisting of one elementary step:

$$
\begin{gathered}
S(1) X+T h \longrightarrow S(1) T h+X \\
\vdots \\
S(n) X+T h \longrightarrow S(n) T h+X
\end{gathered}
$$

Here, $S(1), \ldots, S(n)$ represent particle surfaces for $n$ different particle phases, and $X$ represents any chemical species that exchanges with thorium (e.g., $\mathrm{H}^{+}, \mathrm{Mg}^{2+}$, or $\mathrm{Na}^{+}$). For simplicity, we have ignored the electrical charges on thorium and $X$. The rate of disappearance of Th from solution in the $i$ th reaction of the set $(4.6)$ is:

$$
\left(\frac{d[T h]}{d t}\right)_{i}=-k_{1, i}[S(i) X][T h]
$$

where $k_{1, i}$ is a second-order rate constant. Summing the rates of thorium disappearance from solution for all sorption reactions in (4.6) yields:

$$
\frac{d[T h]}{d t}=-\left(k_{1,1}[S(1) X]+\ldots+k_{1, n}[S(n) X]\right)[T h]
$$

From this equation, a pseudo, first-order rate constant can be defined:

$$
k_{1}=k_{1,1}[S(1) X]+\ldots+k_{1, n}[S(n) X]
$$

which is formally analogous to the additive regression model (equation (4.3)). Thus, the additive model for $k_{1}$ can be kinetically grounded by considering a set of simultaneous sorption reactions, with the important and obvious caveat that these reactions do not consider any potential interaction among the particle phases.

Consider now model II, where the effects of different particle phases on $k_{1}$ are assumed to be multiplicative. Here we envision a situation in which thorium and $n$ different particle phases are interacting simultaneously (Stumm and Morgan, 1996, p. 65): 


$$
\begin{aligned}
& v_{1} \mathrm{~S}(1) \mathrm{X}+\ldots+v_{n} \mathrm{~S}(\mathrm{n}) \mathrm{X}+\mathrm{Th} \longrightarrow \\
& w_{1} \mathrm{~S}(1) \mathrm{Th}+\ldots+w_{n} \mathrm{~S}(\mathrm{n}) \mathrm{Th}+ \\
& w_{n+1}[\mathrm{~S}(1)+\mathrm{S}(2)] \mathrm{Th}+\ldots+w_{n+m}[\mathrm{~S}(1)+\mathrm{S}(2)+\ldots \mathrm{S}(\mathrm{n})] \mathrm{Th}+\left(v_{1}+\ldots v_{n}\right) \mathrm{X}
\end{aligned}
$$

In this reaction, $\left(v_{1}, \ldots, v_{n}\right)$ and $\left(w_{1}, \ldots, w_{n+m}\right)$ are stoichiometric coefficients, and $m$ is the number of possible combinations of particle surfaces. Assuming for the moment that the chemical equation (4.10) is an elementary step, the rate of disappearance of thorium from solution is:

$$
\frac{d[T h]}{d t}=-\left\{k_{1, c}[S(1) X]^{v_{1}} \ldots[S(n) X]^{v_{n}}\right\}[T h]
$$

where $k_{1, c}$ is a high-order rate constant. The factor between curly brackets can be used to define another pseudo first-order rate constant $k_{1}$,

$$
k_{1}=k_{1, c}[S(1) X]^{v_{1}} \ldots[S(n) X]^{v_{n}}
$$

This alternative expression of $k_{1}$ is formally analogous to the multiplicative model (equation $4.5)$.

Thus, like the additive model, the multiplicative model for $k_{1}$ could also be justified kinetically at first glance. However, the assumption that the chemical equation (4.10) is an elementary step may be severely questioned, as the probability of a simultaneous encounter of four different types of particles and a thorium cation is extremely low. For example, Stumm and Morgan (1996) noted that even an elementary reaction involving three species is infrequent in solution. Thus, although both the additive model and the multiplicative model could on first consideration be justified kinetically, each of these models is questionable - the first because it neglects interactions between different particulate phases and the second because it implies the simultaneous collision of large number of reactants in solution.

In summary, the additive and multiplicative models of Th adsorption onto particles seem to represent two limiting cases. Whereas model I neglects particle interactions, model II posits the simultaneous interaction of all particle phases with thorium in solution. A hybrid model that expresses $k_{1}$ as a sum of terms, with some of the terms proportional to a product of concentrations of 
different particle phases, might provide a more credible description of the kinetics of Th attachment to particles. However, identifying and testing the appropriateness of such a model is beyond the scope of this study. It should also be stressed that multiple phenomena could lead to formulations of models for $k_{1}$ that are fundamentally different than those considered here. Such phenomena include, for example, the coagulation of colloidal phases (Morel and Gschwend, 1987; Honeyman and Santschi, 1989), changes in the chemical composition of POM, and the coating of particles by organic material

\subsubsection{Reduced Models}

Although a multiplicative model can be challenged, it should be noted that equation (4.5) includes the possibility that several exponents vanish; should only one or two exponents be significantly different from zero, then model II would provide a more plausible description of Th sorption onto particles. Table 3 shows that, when considering results derived for all stations or the eastern stations, only the exponents for the biogenic phases are significantly different from zero. For both groups of stations, then, we may approximate equation (4.5) as:

$$
k_{1}=k_{1, c}[b i o]^{b_{1}} .
$$

For all stations and the eastern stations, we estimate that $b_{1} \geq 1$ (Table 3 ). Thus equation (4.13) is similar to the dependence of $k_{1}$ on bulk particle concentration $(P)$ found by Lerner et al. (2017), $k_{1} \propto P^{b}$ with $b \geq 1$. A nonlinear relationship between biogenic particle concentration and surface site concentration may explain an exponent $b_{1} \neq 1$. The chemical quality of particulate organic matter (POM) may also play a role. If labile POM is assumed to more efficiently adsorb thorium than semi-labile or refractory POM, then changes in POM lability with depth, as has been reported in multiple studies (Wakeham et al., 1997; Hedges et al., 2000; Lutz et al., 2002; Sheridan et al., 2002; Collins et al., 2015), could conceivably also result in a nonlinear relationship between $k_{1}$ and the concentration of biogenic particles. For example, organic molecules with carboxylic acid funtional groups, in particular acid polysaccharides, have a strong affinity for thorium (Quigley et al., 2001, 2002; Santschi et al., 2003; Quiroz et al., 2006; Santschi et al., 2006). Sheridan et al. (2002) reported that the weight fractions of amino acids and fatty acids, which can include carboxylic acid functional groups, in suspended particles decrease with depth along the mesopelagic zone $(\sim 200-1000 \mathrm{~m})$ in 
the Equatorial Pacific.

Although the nature of POC along GA03 has not been characterized, a change in the composition of POC may also explain differences in the sensitivity of $k_{1}$ to the biogenic particles at the eastern vs. western stations (Table 3). POC at stations from the Mauritanian upwelling region may comprise a larger fraction of fresh and labile material with different surface functional groups than POC at the western stations. If POC at the western stations was characterized by fewer reactive functional groups than POC at the eastern stations, then the biogenic particle phases would become relatively less important for Th adsorption at the western stations.

At the western stations under model II, we find that the regression coefficient for Mn (oxyhydr)oxides is the only one that is significant at the 0.05 level (Table 3 ). Thus, for this group of stations, we may approximate equation (4.5) as:

$$
k_{1}=k_{1, c}[M n]^{b_{3}} .
$$

For the western stations, we estimate that $b_{3}=0.92 \pm 0.42$, which is close to and not significantly different from 1 (Table 3). The shift in the dominant phase influencing $k_{1}$ from biogenic particles at the eastern stations to Mn (oxyhydr)oxides at the western stations coincides with an increase in the average contribution of $\mathrm{Mn}$ (oxyhydr)oxides to total particulate material from $=0.06 \pm 0.01 \%$ at the eastern stations to $=0.12 \pm 0.02 \%$ at the western stations, where averages and standard errors are calculated from the particle phase data used in this study (below $\sim 100 \mathrm{~m}$; Lam et al. (2015a)). Thus, $k_{1}$ appears more sensitive to Mn (oxyhydr)oxides when the fraction of this particle phase, relative to bulk particles, is increased. Below, we speculate on two potential reasons for the increased importance of Mn (oxyhydr)oxides at the western stations: the effect of surface area of the particles and the effect of surface charge of the particles.

Thorium may preferentially scavenge onto particles with a high surface area to mass ratio. Mn (oxyhydr)oxides have specific surface areas (SSAs) ranging from tens to hundreds of $\mathrm{m}^{2} / \mathrm{g}$ (Toner et al., 2005), whereas the SSAs of biogenic particles appear to vary widely with particle type. Laboratory studies have shown that SSAs of biogenic carbonate particles are low, ranging between 1-10 $\mathrm{m}^{2} / \mathrm{g}$ (Keir, 1990) while SSAs of biogenic silica vary between $10-250 \mathrm{~m}^{2} / \mathrm{g}$ (Dixit et al., 2001). It is unclear what the relevant range of SSAs for POC is in our study area. Thus 
particles with high Mn (oxyhydr)oxides and biogenic silica content would be expected to have more available surface area than particles with high carbonate content. Notice that, similarly to Mn (oxyhydr)oxides, Fe (oxyhydr)oxides can have SSAs up to hundreds of $\mathrm{m}^{2} / \mathrm{g}$ (Borggaard (1983); Hiemstra and Van Riemsdijk (2009)). Yet, this particle phase does not appear to significantly influence $k_{1}$ at the western or eastern stations (Table 3). One reason for the low influence of Fe (oxyhydr)oxides at both groups of stations may be this phase's lower contribution to total particle concentration at both the western $(0.07 \pm 0.00 \%)$ and eastern $(0.02 \pm 0.00 \%)$ stations (averages and standard errors calculated from data below $100 \mathrm{~m}$; Lam et al. (2015a)). Speculatively, then, the increased importance of $\mathrm{Mn}$ (oxyhydr)oxides at the western stations might be due to a combination of its high specific surface area and its greater contribution to bulk particle concentrations, so that the encounter rate between Th-bearing molecules or ions in solution and Mn-rich particles site would be higher at these stations.

Mn (oxyhydr)oxides may also increase in importance at the western stations because of the electrical charge present at the surfaces of Mn-enriched particles. For example, while both Mn and Fe (oxyhydr)oxides have large SSAs, their surface charge at seawater $\mathrm{pH}$ differs. Potentiometric titrations performed at a variety of ionic strengths have shown that synthetic ferrihydrite (Fe oxydroxide) has near neutral surface charge whereas synthetic birnessite $\left(\mathrm{MnO}_{2}\right)$ is negatively charged (Peacock and Sherman, 2007; Moon and Peacock, 2013). Thorium, which exists in the +IV oxidation state in seawater (Choppin and Wong, 1998; Santschi et al., 2006), may have a stronger affinity towards negatively charged Mn (oxyhydr)oxides than neutrally charged Fe (oxyhydr)oxides.

Note that the increase in the fraction of Mn (oxyhydr)oxides from the eastern to western stations also coincides with a change in the composition of biogenic particles between these stations. The contribution of biogenic silica to bulk particle concentration amounts to $5.0 \pm 0.4 \%$ at the eastern stations and $2.5 \pm 0.3 \%$ at the western stations (averages and standard errors calculated from phase data below $100 \mathrm{~m}$; Lam et al. (2015a)). In contrast, the contribution of carbonate to bulk particle concentration does not change significantly from the eastern stations $(3.3 \pm 0.4 \%)$ to the western stations $(3.9 \pm 0.9 \%)$. In addition to the larger SSAs of biogenic silica compared to those of biogenic carbonate particles, laboratory studies have shown that, at seawater $\mathrm{pH}$, carbonate particles are positively charged (Morse, 1986), but biogenic silica and organic matter are negatively charged (Niehof and Loeb, 1972; Hunter and Liss, 1979; Davis, 1982; Dixit et al., 2001). Assuming $k_{1}$ 
increases with either SSA or negative charge of particles, the effect on $k_{1}$ of non-biogenic particles relative to that of biogenic particles would thus be stronger at the western vs. the eastern stations, consistent with our results (Table 3 ). Thus, our study suggests the hypothesis that $k_{1}$ in the North Atlantic is at least partly controlled by particle phases with negatively charged surfaces: biogenic particles at the eastern stations and Mn (oxyhydr)oxides at the western stations.

\subsubsection{Paleoceanographic Implications}

In this section, the potential implications of our results for the interpretation of ${ }^{230} \mathrm{Th}$ measurements on bulk sediment samples are briefly discussed. Such measurements have found different applications in paleoceanography. For example, they are used to correct accumulation rates of constituents for the effects of sediment redistribution on the seafloor by bottom currents (for a review see, e.g., François et al. (2004)). In this approach, the accumulation rates are normalized to the flux

of ${ }^{230} \mathrm{Th}$ scavenged from seawater, $F$, which is assumed to be equal to the rate of ${ }^{230} \mathrm{Th}$ radioactive production in the overlying water column,

$$
F=\lambda A_{\pi} Z
$$

Here, $\lambda$ is the ${ }^{230} \mathrm{Th}$ radioactive decay constant, $A_{\pi}$ is the ${ }^{234} \mathrm{U}$ activity, and $\mathrm{Z}$ is the local water depth. Expression (4.15) can be derived by summing equations (4.1-4.2) with $\mathrm{T}(\cdot)=0$, which yields an equation for total ${ }^{230} \mathrm{Th}$ activity,

$$
w \frac{d A_{p}}{d z}=\lambda A_{\pi}
$$

Integration of equation (4.16) from the surface $(z=0)$ to the bottom $(z=Z)$ and with the boundary condition $A_{p}=0 \mathrm{dpm} \mathrm{m}^{-3}$ at $z=0$ leads to (4.15), since $F=w A_{p}(Z)$. Naturally, the reversible exchange terms in (4.1a) and (4.1b) cancel out in the derivation of the equation for total ${ }^{230} \mathrm{Th}(4.16)$. As a result, at least under the assumptions under which (4.15) holds, the ${ }^{230} \mathrm{Th}$ normalization approach appears to be immune to variations in $k_{1}$ such as caused by variations in particle concentration and particle composition.

Such a conclusion, however, would not hold if any of the assumptions used to derived (4.15) are violated. For example, if the residence time of thorium with respect to scavenging (average residence time $\sim 20$ yrs, Henderson and Anderson (2003)) is close to or greater than the time scale 
for Th advection, then the assumption that $T(\cdot)=0$ would not hold, and the vertical flux of ${ }^{230}{ }^{2} h_{p}$ may be sensitive to variations in $k_{1}$. This can be seen by including horizontal advection in equations (4.1a) and (4.1b), and by assuming the other terms in $T\left(A_{d}\right)$ and $T\left(A_{p}\right)$ are negligible:

$$
\begin{aligned}
u \frac{\partial A_{d}}{\partial x} & =\lambda A_{\pi}+k_{-1}^{*} A_{p}-k_{1} A_{d}, \\
u \frac{\partial A_{p}}{\partial x}+w \frac{\partial A_{p}}{\partial z} & =k_{1} A_{d}-k_{-1}^{*} A_{p},
\end{aligned}
$$

where $u$ is the velocity component along the horizontal coordinate $x$, and $k_{-1}^{*}=k_{-1}+\beta_{1}$. Note that radioactive decay rates have been omitted in $(4.17 \mathrm{a}-4.17 \mathrm{~b})$, since they are very small compared to adsorption and desorption rates for the long-lived ${ }^{230} \mathrm{Th}$. Multiplying (4.17a) by $k_{1}$, applying the operator $u \frac{\partial}{\partial x}+k_{1}$ to $(4.17 \mathrm{~b})$, and summing the two resulting equations, yields the following equation for $A_{p}$ :

$$
u^{2} \frac{\partial^{2} A_{p}}{\partial x^{2}}+u w \frac{\partial^{2} A_{p}}{\partial x \partial z}+u\left(k_{1}+k_{-1}^{*}\right) \frac{\partial A_{p}}{\partial x}+w k_{1} \frac{\partial A_{p}}{\partial z}=k_{1} \lambda A_{\pi}
$$

Equation (4.18) is a second order linear partial differential equation with three terms that depend on the horizontal velocity $u$. If these terms are ignored, we recover equation (4.16), which does not include $k_{1}$. However, if these terms are retained, then $k_{1}$ would not cancel out from (4.18), i.e., adsorption onto particles would influence the distribution of ${ }^{230} \mathrm{Th}_{p}$ and hence the vertical settling flux of particulate ${ }^{230} \mathrm{Th}$ reaching the sediment.

Paired measurements of ${ }^{230} \mathrm{Th}$ and ${ }^{231} \mathrm{~Pa}$ on bulk sediment samples have been used to draw inferences about past changes in biological productivity (Kumar et al., 1993) and ocean circulation ( Yu et al., 1996). The interpretation of sediment ${ }^{231} \mathrm{~Pa} /{ }^{230} \mathrm{Th}$ data in terms of ocean circulation is complicated by the uncertainties in the analysis of these data (Burke et al., 2011) and by the spatial variability in scavenging intensity (Hayes et al., 2015b). Since the present paper addresses the effects of particle composition on Th scavenging only, it appears inappropriate to comment on implications for the interpretation of sediment ${ }^{231} \mathrm{~Pa} /{ }^{230} \mathrm{Th}$ records. Nevertheless, by providing evidence that the specific rate at which Th attaches to particles varies along GA03, apparently in relation to particle concentration and particle composition, our study suggests that such variations may play a role in the distribution of sedimentary indicators based on ${ }^{230} \mathrm{Th}$. 


\subsubsection{Importance of Errors}

Our previous results are based on a regression technique (OLS) that does not consider the error (co)variances in the particle data and $k_{1}$ estimates. However, these errors may significantly influence estimates of the regression coefficients. To document this influence, we use the Algorithm of Total Inversion (ATI). Whereas OLS is a standard procedure, the ATI is less commonly used and is described in detail in Appendix B. The regression coefficients estimated by ATI are reported in Tables 4 and 5, and the best fits are shown in Figure 8.

\begin{tabular}{|l|c|c|c|c|}
\hline & bio & litho & Mn & Fe \\
\hline all stations $(\mathrm{n}=63)$ & $0.35 \pm 0.07$ & $0.25 \pm 0.11$ & $-38.5 \pm 20.8$ & $103.5 \pm 14.4$ \\
\hline western stations $(\mathrm{n}=35)$ & $0.19 \pm 0.07$ & $0.17 \pm 0.16$ & $-27.4 \pm 20.1$ & $73.64 \pm 14.67$ \\
\hline eastern stations $(\mathrm{n}=28)$ & $0.58 \pm 0.24$ & $0.30 \pm 0.58$ & $-182.4 \pm 144.2$ & $176.5 \pm 62.7$ \\
\hline
\end{tabular}

Table 4: Regression coefficients \pm 1 standard deviation $\left(\mathrm{yr}^{-1} \mathrm{~m}^{3} \mathrm{mg}^{-1}\right)$ for model I (ATI)

\begin{tabular}{|l|c|c|c|c|}
\hline & bio & litho & Mn & Fe \\
\hline all stations $(\mathrm{n}=63)$ & $1.81 \pm 0.18$ & $0.16 \pm 0.20$ & $-2.03 \pm 0.53$ & $0.46 \pm 0.09$ \\
\hline western stations $(\mathrm{n}=35)$ & $1.27 \pm 0.20$ & $-0.37 \pm 0.20$ & $-0.40 \pm 0.38$ & $0.26 \pm 0.06$ \\
\hline eastern station $(\mathrm{n}=28)$ & $1.69 \pm 0.34$ & $-1.09 \pm 0.46$ & $1.03 \pm 0.54$ & $-0.22 \pm 0.06$ \\
\hline
\end{tabular}

Table 5: Regression coefficients \pm 1 standard deviation for model II (ATI)

For the biogenic phases, the coefficients obtained by the ATI differ from 0 by more than 2 standard deviations in all cases. This result is consistent with the finding from OLS that across all regression models (I and II ) and groups of stations (all stations, western stations, and eastern stations), $k_{1}$ has a positive relationship with biogenic particles which is generally significant at the 0.05 level. The sole exception to this result arises when OLS is used to fit model II at the western stations, for which the biogenic phase regression coefficient is not significant $(p=0.32)$. For the other particle types, the choice of regression technique significantly changes the estimated regression coefficients (compare Tables 2-3 with Tables 4-5). The coefficient for Fe (oxyhydr)oxides at all stations provides an extreme example, amounting to $14.56 \pm 18.01 \mathrm{yr}^{-1} \mathrm{~m}^{3} \mathrm{mg}^{-1}$ for OLS and to $103.5 \pm 14.4 \mathrm{yr}^{-1} \mathrm{~m}^{3} \mathrm{mg}^{-1}$ for the ATI (all stations, model I).

Notice that there is no a priori reason to prefer one regression method over another, since each has advantages and disadvantages. Ordinary least squares, while not considering the error (co)variances of the data and $k_{1}$ estimates, finds the best, unbiased linear fit to the data. In contrast to OLS, the ATI accounts for estimated errors in $k_{1}$ and the particle data. However, it can 
produce solutions which are (i) questionable if the regression problem is strongly nonlinear (i.e., if the errors in the regressors are substantial), (ii) biased, and (iii) very sensitive to prior statistics assumed for the regression coefficients (Appendix C).

\subsubsection{Particle Concentration vs. Particle Composition}

In this section, we test whether particle concentration and particle composition explain significantly different fractions of the variance in our $k_{1}$ estimates at the selected GA03 stations. We first perform this analysis for model II. Figure 9 shows the $k_{1}$ estimates derived in this paper vs. bulk particle concentration. A log-log scale is used to be consistent with model II. The Pearson correlation coefficient between $\ln \left(k_{1}\right)$ and $\ln (P)$ amounts to $r=0.71$ for all stations, 0.54 for stations west of GT11-24, and 0.77 for stations east of and including GT11-24. In comparison, the multiple correlation between $\ln \left(k_{1}\right)$ and a linear combination of the logarithms of particle phases for these groups of stations amounts to $R=0.71,0.62$, and 0.82 , respectively (section 3.1 ). To compare both sets of correlations, we conduct an $F$ test for the equality of the variance in $\ln \left(k_{1}\right)$ explained by $\ln (P)$ and by a linear combination of the logarithms of particle phases. The $p$ values are 0.95 for all selected stations, 0.23 for stations west of GT11-24, and 0.82 for stations east of and including GT11-24. A similar analysis for model I yields $p$ values of 0.09 for all selected stations, 0.63 for stations west of GT11-24, and 0.15 for stations east of and including GT11-24. Thus, for each group of stations and for each model, particle composition explains a proportion of variance in $k_{1}$ or $\ln k_{1}$ that is statistically indistinguishable at the 0.05 level from that explained by particle concentration.

However, the particle composition analysis brings additional insight into the mechanisms by which thorium adsorbs onto particles. For example, the better performance of the multiplicative compared to the additive model highlights the importance of interactions between particle phases. Moreover, the regression against particle composition illustrates the regional variability in the effectiveness of different particle phases at adsorbing thorium. For example, the importance of Mn (oxyhydr)oxides at the oligotrophic western stations, but not in the more productive, Mauritanian upwelling regions, suggests a difference in the interactions and/or chemical quality of particles between these two regions.

\subsection{Conclusion}

In this paper, we document the influence of particle composition on estimates of the rate constant of thorium adsorption onto particles $\left(k_{1}\right)$ at selected stations of the U.S. GEOTRACES North 
Atlantic section. Multiple linear regression analysis is applied to determine the sensitivity of $k_{1}$ to various particle phases (biogenic particles, lithogenic particles, Mn and Fe (oxyhydr)oxides). Two models for the dependence of $k_{1}$ on particle composition are considered: model I assumes that the effects of the different particle phases on $k_{1}$ are additive, whereas model II assumes that these effects are multiplicative. We apply these regression models to three groups of stations: (i) all selected stations, (ii) stations west of the Mauritanian upwelling, and (iii) stations within the Mauritanian upwelling.

We find that the variations in $k_{1}$ explained by the particle phase data depends on the group of stations considered and the model applied: $R^{2}=0.60$ (0.67) for model I (II) applied to the eastern stations, and $R^{2}=0.26(0.39)$ for model I (II) applied to the western stations. The estimates of the regression coefficients and their standard errors are sensitive to the consideration of errors in the particle data and $k_{1}$ estimates. Nonetheless, the regression coefficient estimate for biogenic particles is generally significant at the 0.05 level for both models I and II. A relative importance analysis reveals that the biogenic particles dominate the variability in $k_{1}$ explained by particle composition in the Mauritanian upwelling region, but that Mn (oxyhydr)oxides account for most of the explained variability in $k_{1}$ west of this region. We also find that the correlation between $k_{1}$ and particle composition is not significantly different from that between $k_{1}$ and particle concentration at the 0.05 level.

Our results thus suggest that the apparent rate constant $k_{1}$ may largely be driven by one particle type: biogenic phases for the stations in the Mauritanian upwelling region and $\mathrm{Mn}$ (oxyhydr)oxides for the stations east of this region. While this finding may appear to be at variance with Hayes et al. (2015a) and earlier studies on the relationship between $K_{D}$ and particle composition, it is important to emphasize that $K_{D}$ and $k_{1}$ are different concepts. On the one hand, $K_{D}$ is an empirical measure of the proportion of the metal bound to particles, given the concentrations of the metal in solution and the concentration of particles. On the other hand, $k_{1}$ represents an apparent first order rate constant for the adsorption of the metal onto particles. The expression $K_{D}=k_{1} /\left(\left(k_{-1}+\beta_{-1}\right) P\right)$, valid only under a set of assumptions (Honeyman et al., 1988; Lerner et al., 2017), shows that $K_{D}$ would carry information about the relative intensity of metal attachment to, and detachment from, particles, whereas $k_{1}$ would carry information about the rate of only one of these processes. In this perspective, $K_{D}$ and $k_{1}$ would play the same role as equilibrium and rate constants in the study of 
chemical reactions and provide complementary information about the scavenging of particle-reactive metals in oceanic waters.

Finally the analysis presented here may grant us some insight into the nature of thorium adsorption onto particles in the North Atlantic. The importance of biogenic particles at the eastern stations may stem from the preferential adsorption of Th onto particulate matter with large amounts of carboxyl functional groups, while the importance of Mn (oxyhydr)oxides at the western stations may reflect preferential adsorption on Mn-rich particles with high specific surface area and/or negative surface charges. Whether similar results hold for other metals in the ocean, and their implications for the interpretation of thorium isotope measurements in the seawater and the sediments, remain to be investigated.

\section{Acknowledgement}

We acknowledge the U.S. National Science Foundation for supporting this study (grant OCE1232578) and the U.S. GEOTRACES North Atlantic section ship time, sampling, and data analysis.

The U.S. NSF also supported the generation of ${ }^{230}$ Th data (OCE-0927064 to LDEO, OCE-O092860 to WHOI, and OCE-0927754 to UMN) and ${ }^{228,234}$ Th data (OCE-0925158 to WHOI). We thank the chief scientists of the GA03 section (Ed Boyle, Bill Jenkins, and Greg Cutter) as well as the captain, the crew, and the scientific party on the R/V Knorr, which completed this section. We are also grateful to the scientists and staff involved in the analysis of the thorium isotope and particle data. We also benefited from very useful discussions with Carl Lamborg (UCSC) and Philip M. Gschwend (MIT) on the kinetics of sorption reactions in aqueous media. 


\section{A Appendix A}

In this study, we derive estimates of $k_{1}$ from the fit of a thorium cycling model (eqs. 5.2a-5.2b) to radionuclide data. The fit is achieved using a nonlinear programming technique using parameter values reported in Table A.1 of Lerner et al. (2017). We refer the reader to this previous work for details. In contrast to the present study, Lerner et al. (2017) obtained estimates of $k_{1}$ by fitting a model considering both thorium and particle dynamics to radionuclide and particle concentration data. In order to check for consistency between the two sets of estimates of $k_{1}$, we regress $k_{1}$ values obtained in this study against those obtained in Lerner et al. (2017) (Figure A1). We find that the slope of the OLS fit amounts to $1.25 \pm 0.09$. Thus, the $k_{1}$ estimates derived in this paper are in general slightly larger than those derived by Lerner et al. (2017), but the two sets of estimates are highly correlated $\left(R^{2}=0.99\right)$.

\section{B Appendix B}

In this section, we briefly review the relative importance techniques of Kruskal (1987) and Azen and Budescu (2003); further details on these techniques can be found in these two papers.

\section{B.1 Averaging Over Orderings (AOO)}

To evaluate the importance of different regressors, Kruskal (1987) addressed the scenario in which there is no "natural ordering" of importance of the regressors. In this case, he suggested to take an average over all orderings. Using partial correlation coefficients as measures of importance, the method of AOO averages these coefficients obtained from every possible permutation of the regressors. When calculating the partial correlation between a regressor and regressand, the order of regressors determines whether the correlation takes into account the variance in the regressand explained by the other regressors. As an example, consider a case with only two particulate phases, $P_{1}$ and $P_{2}$. In that case, $\ln k_{1}$ is modeled as a linear combination of these two regressors:

$$
\ln \left(k_{1}\right)=a_{0}+a_{1} \ln \left(P_{1}\right)+a_{2} \ln \left(P_{2}\right)+\epsilon
$$

To estimate the relative importance of $\ln P_{1}$, one averages the partial correlation coefficients between $\ln P_{1}$ and $\ln k_{1}$ over every possible order of $P_{1}$ and $P_{2}$. In this case, the regressors have only two possible orders: $P_{1}, P_{2}$ and $P_{2}, P_{1}$. For the first ordering, $P_{1}, P_{2}$, the partial correlation coefficient between $\ln k_{1}$ and $\ln P_{1}$ is simply the Pearson correlation coefficient between $\ln k_{1}$ and 
$\ln P_{1}, R_{k_{1}, P_{1}}$. For the second ordering, $P_{2}, P_{1}$, the partial correlation coefficient must take the variance in $\ln k_{1}$ explained by $\ln P_{2}$ into account:

$$
R_{k_{1}, P_{1} \cdot P_{2}}=\left(R_{k_{1}, P_{1}}-R_{k_{1}, P_{2}} R_{P_{1}, P_{2}}\right) / \sqrt{\left(1-R_{k_{1}, P_{2}}^{2}\right)\left(1-R_{P_{1}, P_{2}}^{2}\right)} .
$$

Here, $R_{k_{1}, P_{2}}$ is the Pearson correlation coefficient between $\ln k_{1}$ and $\ln P_{2}$, and $R_{k_{1}, P_{2}}$ is the Pearson correlation coefficient between $\ln P_{1}$ and $\ln P_{2}$. The relative importance of $P_{1}$ is then taken as the average of the two squared partial correlation coefficients,

$$
R I=0.5\left(R_{k_{1}, P_{1}}^{2}+R_{k_{1}, P_{1} \cdot P_{2}}^{2}\right)
$$

\section{B.2 Dominance Analysis}

Dominance analysis accounts for the correlation between regressors by averaging the contribution to $R^{2}$ by a regressor over models encapsulating every possible subset of regressors (Azen and Budescu, 2003). As an example, consider again the case with only two particulate phases:

$$
\ln \left(k_{1}\right)=a_{0}+a_{1} \ln \left(P_{1}\right)+a_{2} \ln \left(P_{2}\right)+\epsilon
$$

To estimate the relative importance of $\ln P_{1}$, take the average additional contribution of $\ln P_{1}$ to the explained variance over two models: (1) a null model with no contributions from $\ln P_{1}$ and $\ln P_{2}$, and (2) a model considering $\ln k_{1}$ as a linear function of $\ln P_{2}$. Respectively:

$$
\begin{array}{r}
\ln \left(k_{1}\right)=a_{0}+\epsilon, \\
\ln \left(k_{1}\right)=a_{0}+a_{2} \ln \left(P_{2}\right)+\epsilon .
\end{array}
$$

Denote $R_{\text {null }}^{2}$ and $R_{P 2}^{2}$ as the squared correlation coefficients for models (B.5a) and (B.5b), respectively. Adding $\ln P_{1}$ to each model results in the following extended models,

$$
\begin{array}{r}
\ln \left(k_{1}\right)=a_{0}+a_{1} \ln \left(P_{1}\right)+\epsilon, \\
\ln \left(k_{1}\right)=a_{0}+a_{1} \ln \left(P_{1}\right)+a_{2} \ln \left(P_{2}\right)+\epsilon .
\end{array}
$$

Denote $R_{P 1}^{2}$ and $R_{\text {full }}^{2}$ as the squared correlation coefficients for models (B.6a) and (B.6b), respectively. The relative importance is then defined as the average contribution of $P_{1}$ to the 
explained variance, i.e.,

$$
R I_{P 1}=0.5\left(\left(R_{\text {full }}^{2}-R_{P 2}^{2}\right)+\left(R_{P 1}^{2}-R_{\text {null }}^{2}\right)\right) .
$$

A similar expression is used for $R I_{P 2}$. Thus, dominance analysis breaks down the squared correlation of a multiple linear regression into contributions from the different regressors.

\section{C Appendix C}

We account for the error (co)variances of the particle composition data and $k_{1}$ estimates using the Algorithm of Total Inversion (ATI; Tarantola and Valette, 1982). The ATI proceeds as follows. In equations (4.3) and (4.4) (section 3.2), the regression coefficients as well as the particle phase data are treated as unknowns. We construct a prior estimate of a vector $\boldsymbol{x}$ of unknowns, which is $\boldsymbol{x}_{0}$. The elements of $\boldsymbol{x}_{0}$ contain (i) prior estimates of the regression coefficients, and (ii) the particle measurements (bio, litho, $M n, F e$ ). We also construct a vector equation $\boldsymbol{f}(\boldsymbol{x})=\mathbf{0}$ which contains the regression equations (4.3) or (4.4). We then minimize the objective function

$$
J(\boldsymbol{x})=\left(\boldsymbol{x}-\boldsymbol{x}_{0}\right)^{\prime} \boldsymbol{C}_{0}^{-1}\left(\boldsymbol{x}-\boldsymbol{x}_{0}\right)+\boldsymbol{f}(\boldsymbol{x})^{\prime} \boldsymbol{C}_{f}^{-1} \boldsymbol{f}(\boldsymbol{x}) .
$$

Here $\boldsymbol{C}_{\mathbf{0}}$ and $\boldsymbol{C}_{f}$ are covariance matrices for the errors in $\boldsymbol{x}_{0}$ and in the model equations (eq. 4.3 or 4.4 ), respectively, and the primes are vector transposes. The error covariance matrix $\boldsymbol{C}_{0}$ is taken as diagonal. Its diagonal elements are the squared errors in the particle measurements (or the squared errors of the natural logarithm of these elements) and in the prior estimates of the regression coefficients. The matrix $\boldsymbol{C}_{f}$ is based on the error (co)variances of $k_{1}$ derived by inversion (section 2.3). Thus we seek an estimate of $\boldsymbol{x}$ that is consistent with (i) its prior estimates, $\boldsymbol{x}_{0}$, given the error variances in $\boldsymbol{C}_{0}$, and (ii) the regression model (eq. 4.3 or 4.4), given the error covariances in $\boldsymbol{C}_{f}$.

The prior estimates of the regression coefficients in equation (4.3) or (4.4) are taken from Honeyman et al. (1988). These authors fit a power law, $k_{1}=k_{1, c} P^{b}$, to field data spanning a wide range of particle concentrations from $O\left(10 \mathrm{mg} \mathrm{m}^{-3}\right)$ to $O\left(10^{9} \mathrm{mg} \mathrm{m}^{-3}\right)$. The prior estimates for the regression coefficients $\left(a_{1}, \ldots, a_{4}\right)$ for model I are set to the value of $k_{1, c}=0.024 \mathrm{yr}^{-1} \mathrm{~m}^{3} \mathrm{mg}^{-1}$ found by Honeyman et al. (1988), and the prior estimate for $a_{0}$ under model I is set to $0 \mathrm{yr}^{-1}$. 
For model II, the prior estimates of the regression coefficients $\left(b_{1}, \ldots, b_{4}\right)$ are set to the value of $b=0.58$ found by Honeyman et al. (1988), and the prior estimate for $b_{0}$ under model II is set to $\ln \left(k_{1, c}\right)=\ln \left(0.024 \mathrm{yr}^{-1}\left(\mathrm{~m}^{3} \mathrm{mg}^{-1}\right)^{0.58}\right)$. Since these prior estimates are poorly constrained, being based on a single study that considers the effect of particle concentration (not composition) on $k_{1}$, we set the prior estimates of their errors to be large, i.e., the prior estimates of the errors in the regression coefficients are three orders of magnitude greater than their absolute value (for $a_{0}$ under model $\mathrm{I}$, the error is set to $1000 \mathrm{yr}^{-1}$ ). With this choice, we find that the regression coefficient estimates are very poorly sensitive to the prior values, a desirable result.

The ATI solution at iteration $k_{i+1}$ is:

$$
\hat{\boldsymbol{x}}_{k+1}=\boldsymbol{x}_{0}+\boldsymbol{C}_{\mathbf{0}} \boldsymbol{F}_{k}^{\prime}\left(\boldsymbol{F}_{k} \boldsymbol{C}_{0} \boldsymbol{F}_{k}^{\prime}\right)^{-1}\left(\boldsymbol{F}_{k}\left(\hat{\boldsymbol{x}}_{k}-\boldsymbol{x}_{\mathbf{0}}\right)-\boldsymbol{f}\left(\hat{\boldsymbol{x}}_{\mathrm{k}}\right)\right)
$$

where $\boldsymbol{F}$ is a matrix whose elements are the partial derivatives of equations (4.3) or (4.4) with respect to the elements of $\boldsymbol{x}$, i.e., the element in the $i$ th row and $j$ th column of $\boldsymbol{F}_{k}$ is $\partial f_{i} / \partial x_{j}$. The solution error covariance matrix is estimated from

$$
\boldsymbol{C}_{k+1}=\boldsymbol{C}_{0}-\boldsymbol{C}_{0} \boldsymbol{F}_{k}^{\prime}\left(\boldsymbol{F}_{k} \boldsymbol{C}_{0} \boldsymbol{F}_{k}^{\prime}\right)^{-1} \boldsymbol{F}_{k} \boldsymbol{C}_{0}
$$

The matrix inversions in (A.2-A.3) are performed using LU decomposition.

We use two measures of goodness of fit. One is the fraction of the regression equations in $\boldsymbol{f}(\boldsymbol{x})=\mathbf{0}$ that are satisfied to within $\pm 2 \sigma_{k_{1}}$, where $\sigma_{k_{1}}$ is the estimated error in $k_{1}$ obtained by inversion. The other is the number of particle phase measurements that are fit to within 2 standard deviations by the model. We count a particle phase measurement as fit by the model I or II if the corresponding normalized residual is less than 2 in absolute magnitude. A normalized residual is defined as $\left(\hat{x}_{i}-x_{0, i}\right) / \sigma_{0, i}$, where index $i$ refers to a particular sample and $\sigma_{0, i}$ is the corresponding error (Lam et al., 2015a). We find that, when the error (co)variance in the particle data and $k_{1}$ estimates are considered, between 96 and $100 \%$ of the particle phase data are fit to within two standard deviations in the data, and that between 97 and $100 \%$ of the regression equations are satisfied to within two standard deviations in the $k_{1}$ or $\ln \left(k_{1}\right)$ estimates, where the ranges reflect the different models and different groups of stations. Thus, the regression coefficients obtained from ATI appear generally consistent with both the particle data and the $k_{1}$ or $\ln \left(k_{1}\right)$ estimates given their respective error estimates. 


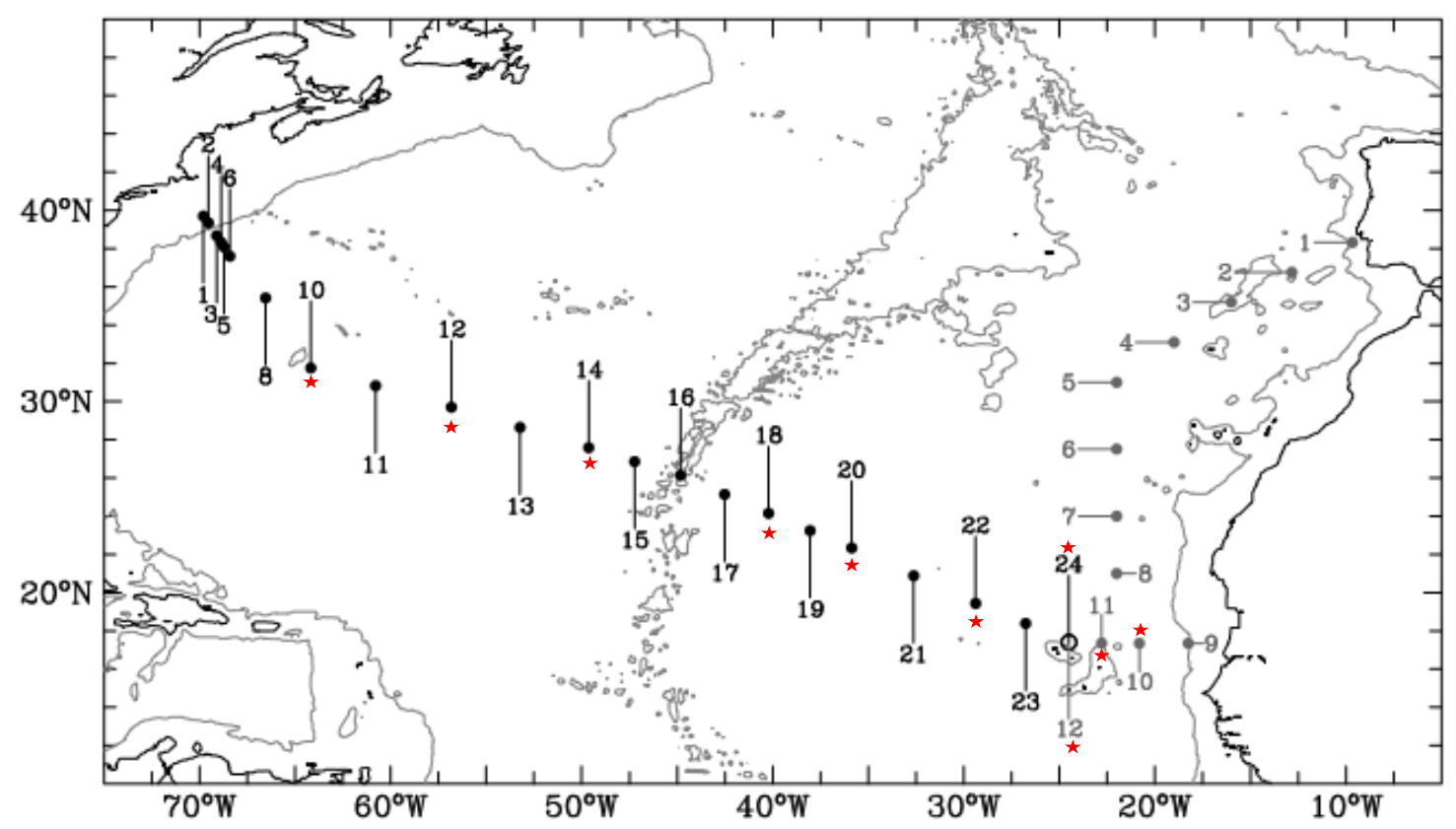

Figure 1: Stations occupied by the R/V Knorr during the GEOTRACES North Atlantic section (GA03). The grey dots show the stations occupied during the first leg (October 2010) and the black dots show the stations occupied during the second leg (November-December 2011). The open circle is both station GT1012 of the first leg and station GT11-24 of the second leg. The data analyzed in this paper occur at stations marked by red asterisks. The solid lines show the coastline (black) and the 3000-m isobath (grey). 

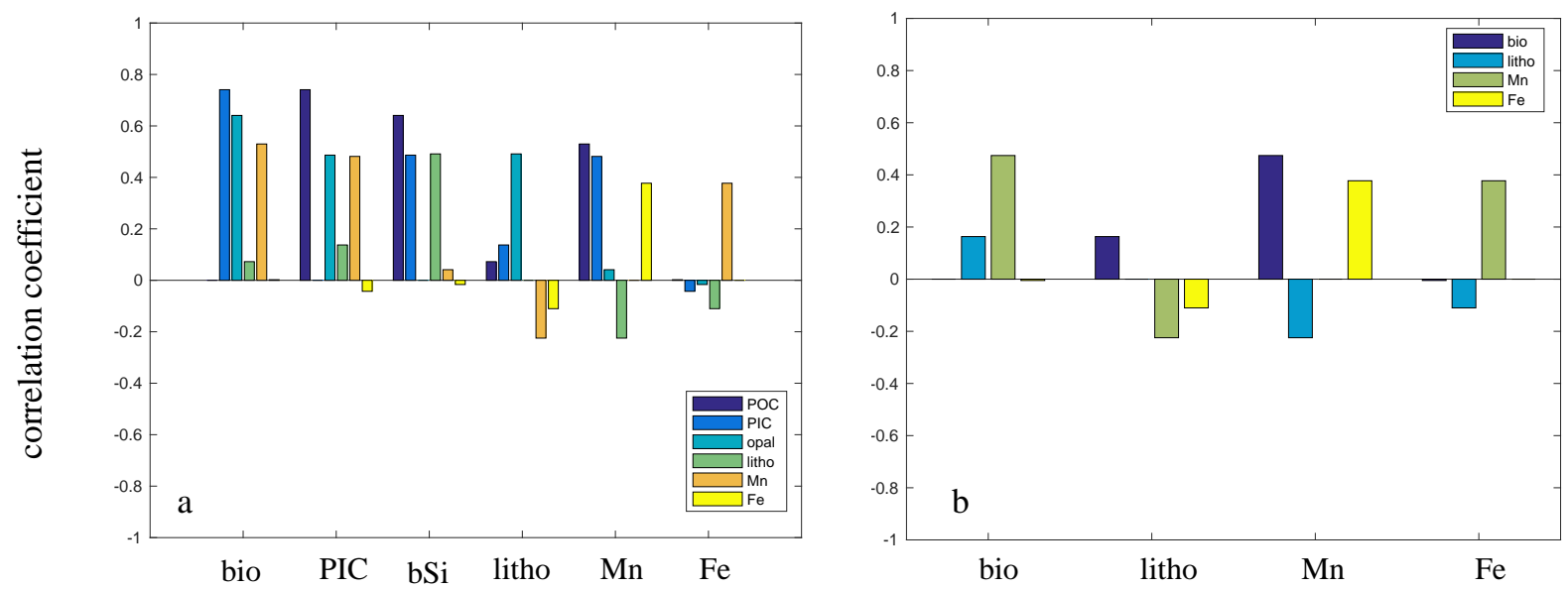

Figure 2: Pearson correlation coefficients between pairs of particle phases at selected stations of GA03. (a) Correlations between POC, PIC, bSi, lithogenic material, Mn (oxyhydr)oxides, and Fe (oxyhydr)oxides. (b) Correlations between the sum of biogenic particles $(\mathrm{POC}+\mathrm{PIC}+\mathrm{bSi}$ ), lithogenic material, $\mathrm{Mn}$ (oxyhydr)oxides, and $\mathrm{Fe}$ (oxyhydr)oxides. 

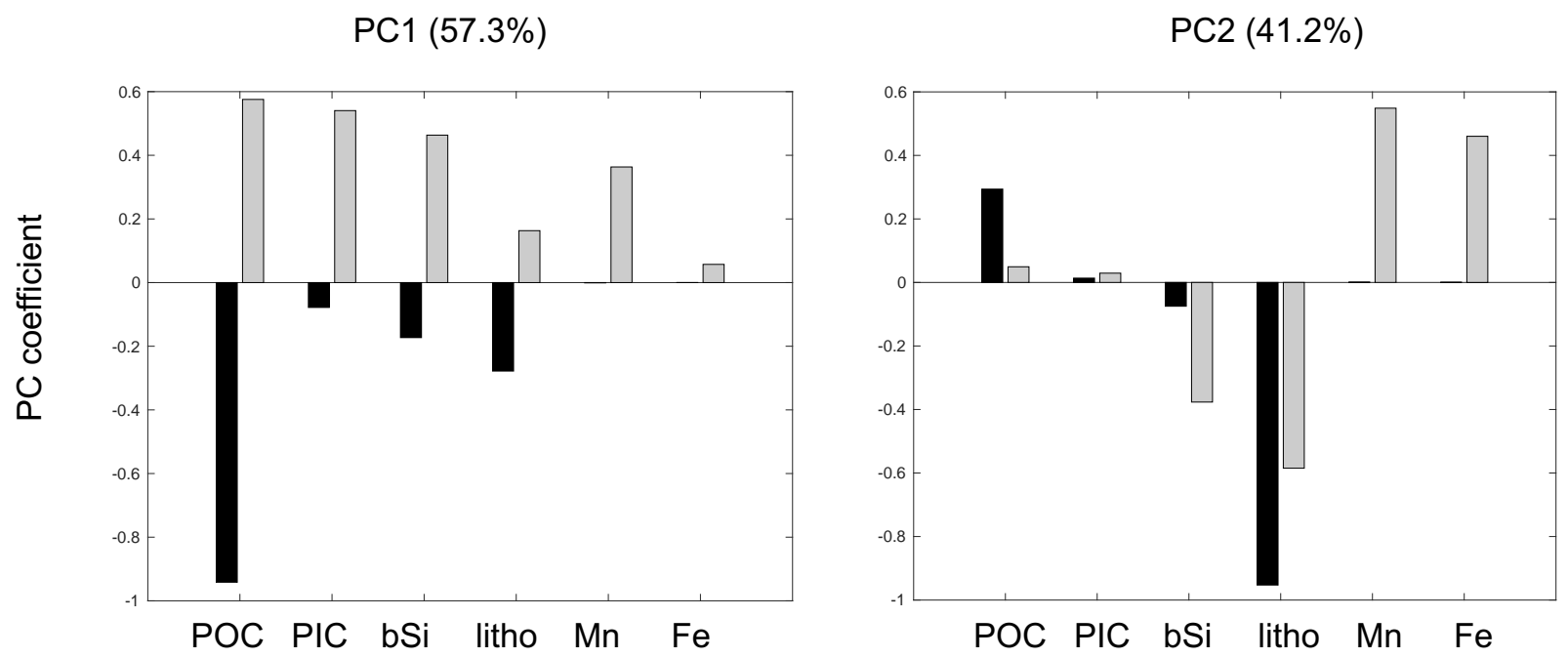

Figure 3: Principal component coefficients for each particle phase, with the percentage of total particle variance captured put into parentheses. The left panel corresponds to PC1, and the right panel corresponds to PC2. In each panel, the black (grey) bars show results obtained from the covariance (correlation) matrix. 

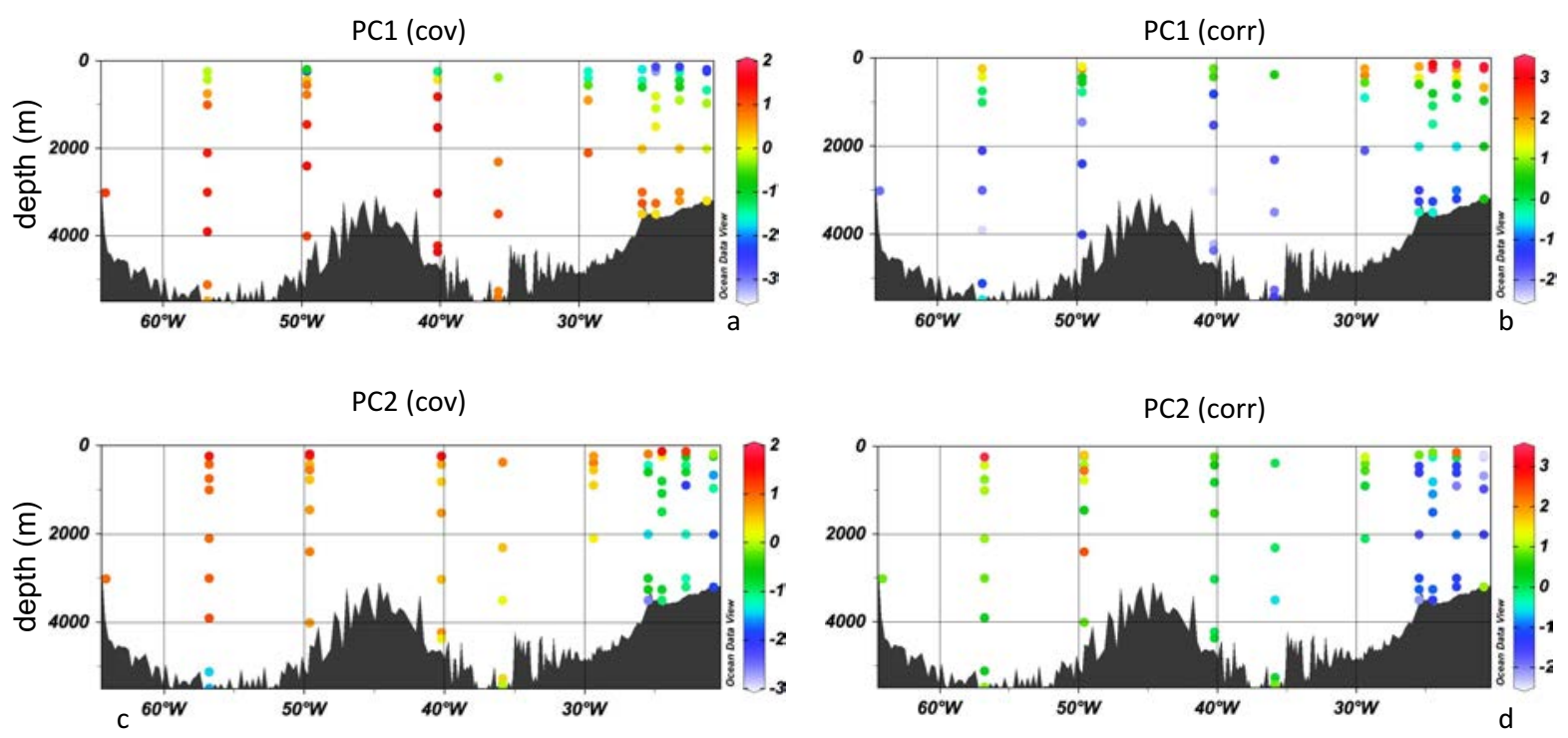

Figure 4: Section plots of the principal components. Left (right) panels show the PCs calculated from the covariance (correlation) matrix. Note that the third and fourth stations from the right of each panel (GT10-12 and GT11-24, respectively) are actually at the same geographic location $\left(17^{\circ} 23^{\prime} \mathrm{N}, 24^{\circ} 30^{\prime} \mathrm{W}\right)$. 


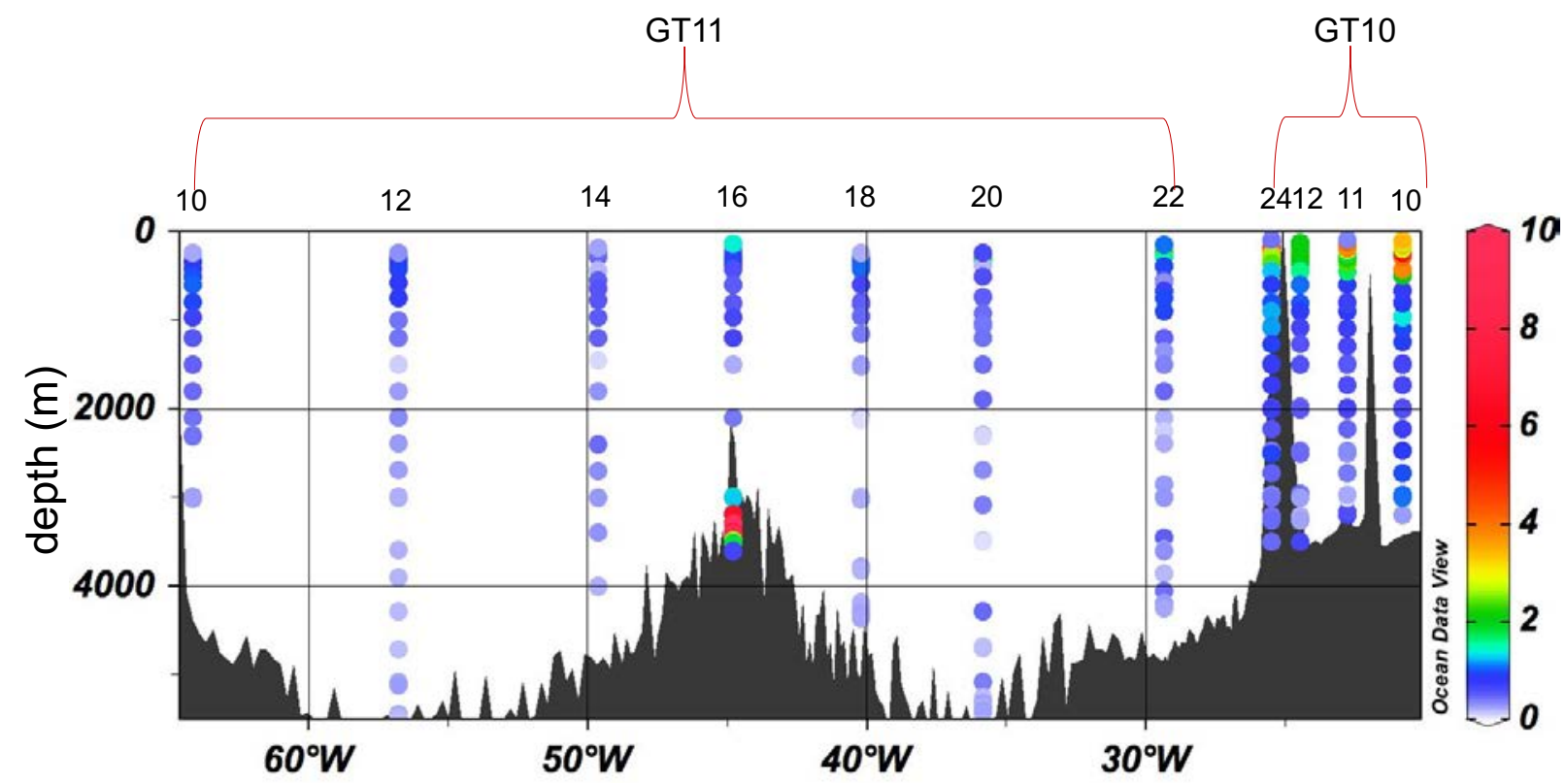

Figure 5: Estimates of the adsorption rate constant, $k_{1}\left(\mathrm{yr}^{-1}\right)$, at selected stations of the US GEOTRACES North Atlantic section. The third and fourth stations from the right of each panel (GT10-12 and GT11-24, respectively) are actually at the same geographic location $\left(17^{\circ} 23^{\prime} \mathrm{N}, 24^{\circ} 30^{\prime} \mathrm{W}\right)$. The largest value inferred for $k_{1}\left(21 \mathrm{yr}^{-1}\right.$ at $3200 \mathrm{~m}$ at station GT11-16) is not shown so that individual values of $k_{1}$ are more easily distinguished. 

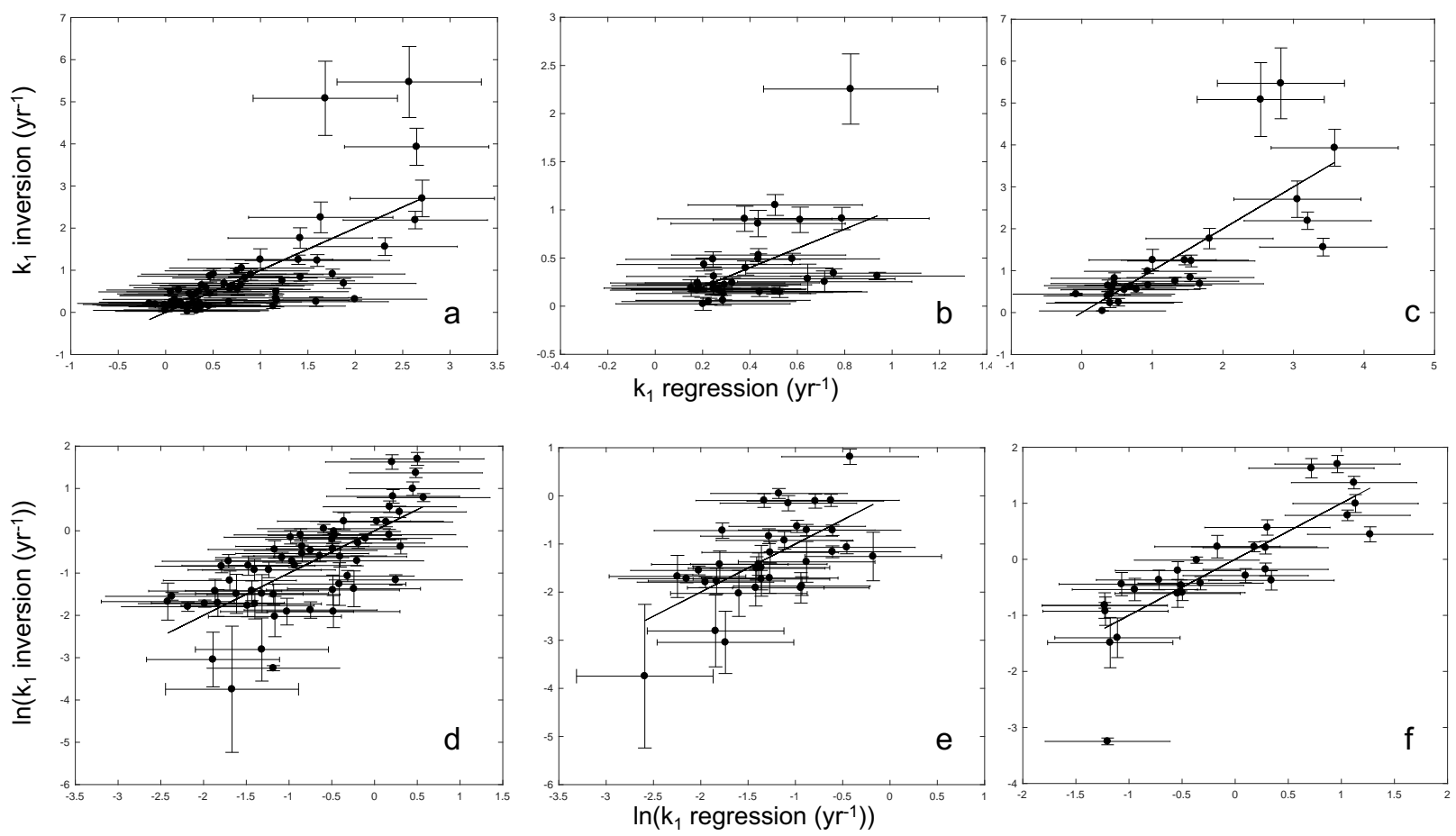

Figure 6: Regressions performed using OLS. Panels (a), (b), and (c) correspond to the regression for all stations, stations west of GT11-24, and stations east of and including GT11-24, respectively. Panels (d-f) correspond, respectively, to the same groups of stations and panels (a-c), but for the regression of $\ln \left(k_{1}\right)$ vs the natural logarithm of the particle composition data. The solid line is the best fit, and the error bars are \pm 1 standard deviation 

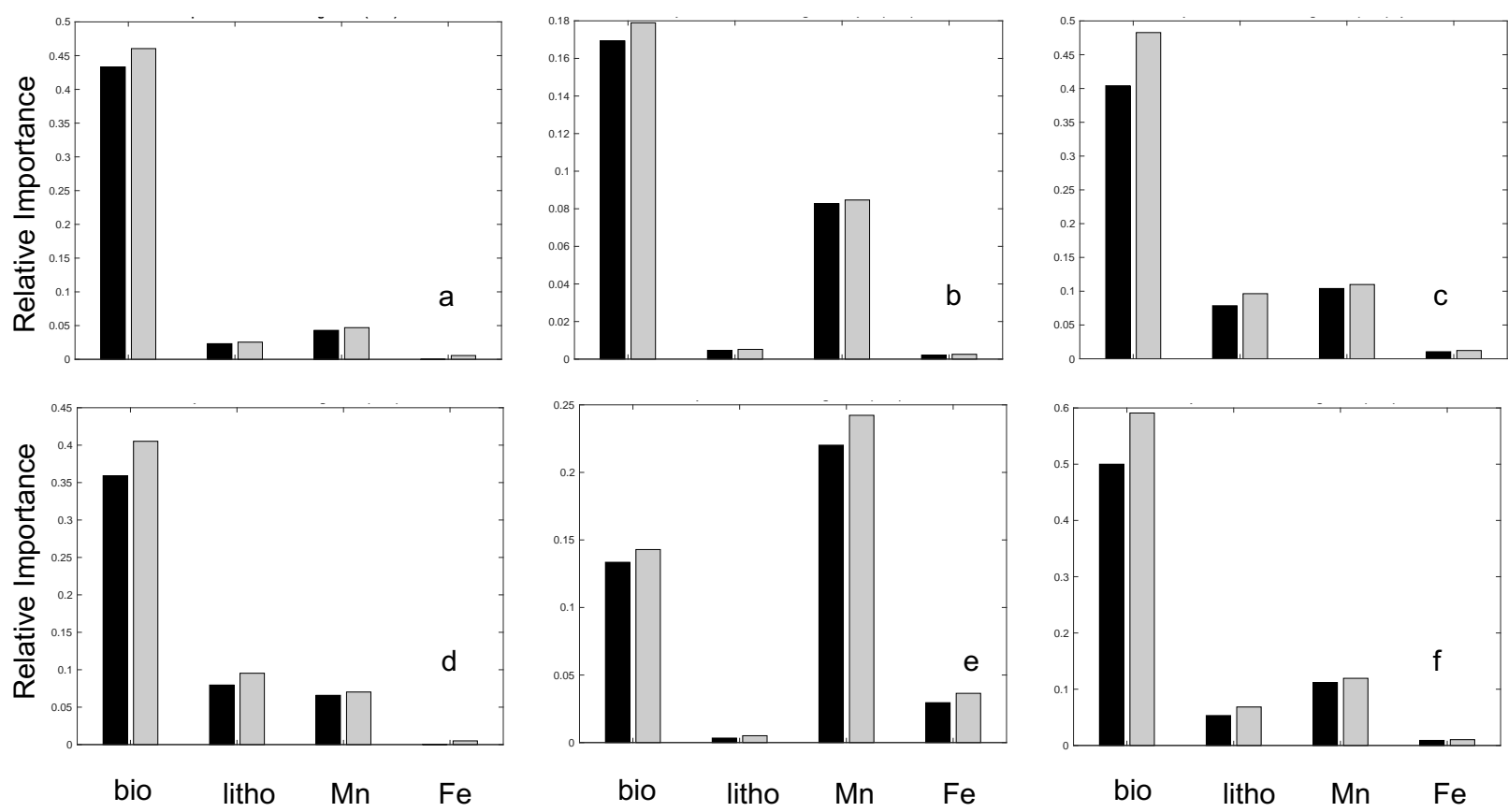

Figure 7: Relative Importance $(R I)$ of particle phases for model I (upper panels) and model II (lower panels). Panels (a,d), (b,e), and (c,f) correspond to all stations, western stations, and eastern stations, respectively. The black and grey bars are the $R I$ values obtained by respectively, dominance analysis and averaging over orders. 

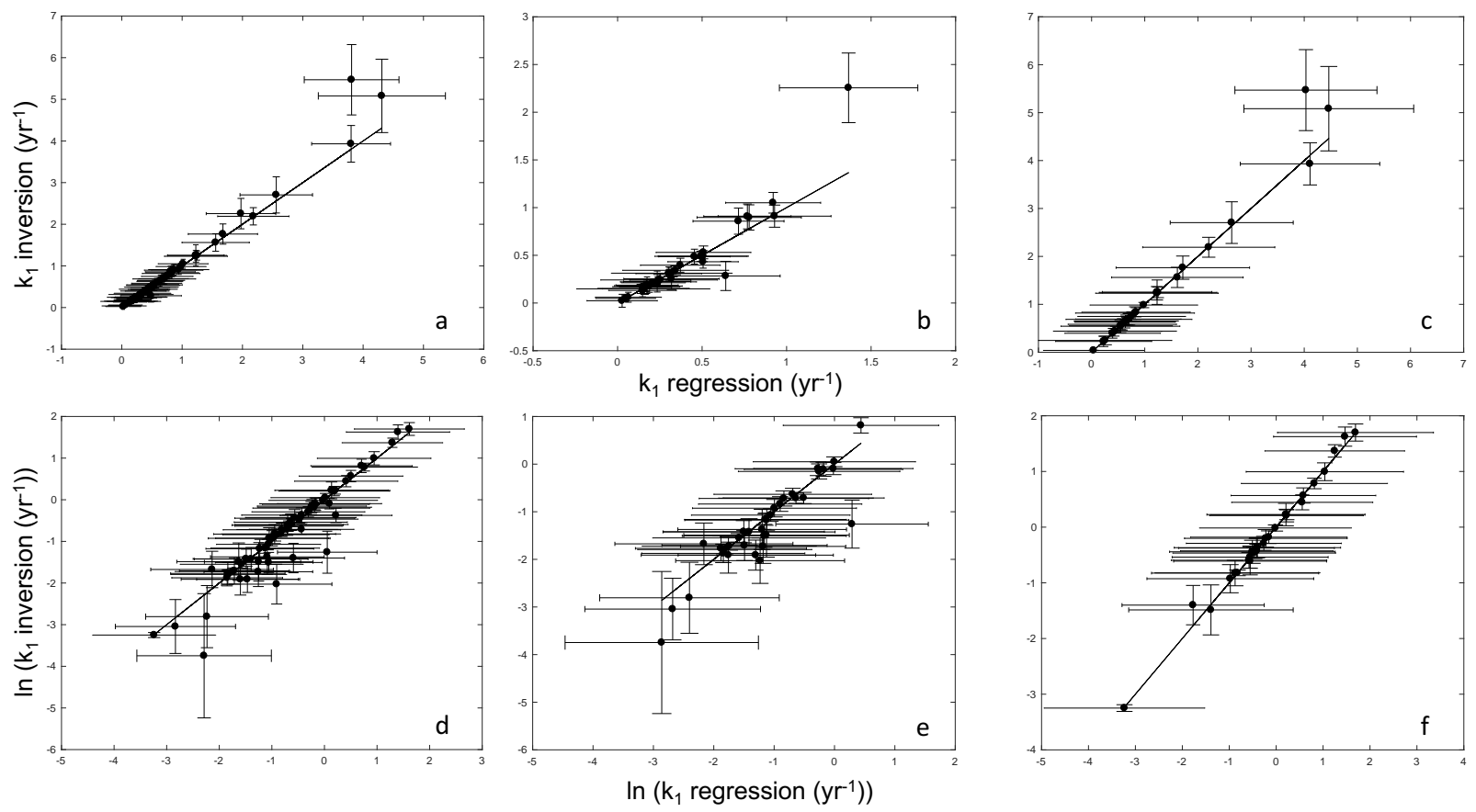

Figure 8: Panels a, b, and c show $k_{1}$ obtained from inversion of radiochemical data vs. $k_{1}$ obtained from regression against particle composition data using the ATI. Panels d, e, and $\mathrm{f}$ are similar to panels a, b, and c, except show $\ln \left(k_{1}\right)$ rather than $k_{1}$. Panels $(\mathrm{a}, \mathrm{d}),(\mathrm{b}, \mathrm{e})$, and $(\mathrm{c}, \mathrm{f})$ correspond to the regression for all stations, stations west of GT11-24, and stations east of and including GT11-24, respectively. The solid line is the best fit, and the error bars are \pm 1 standard deviation. 

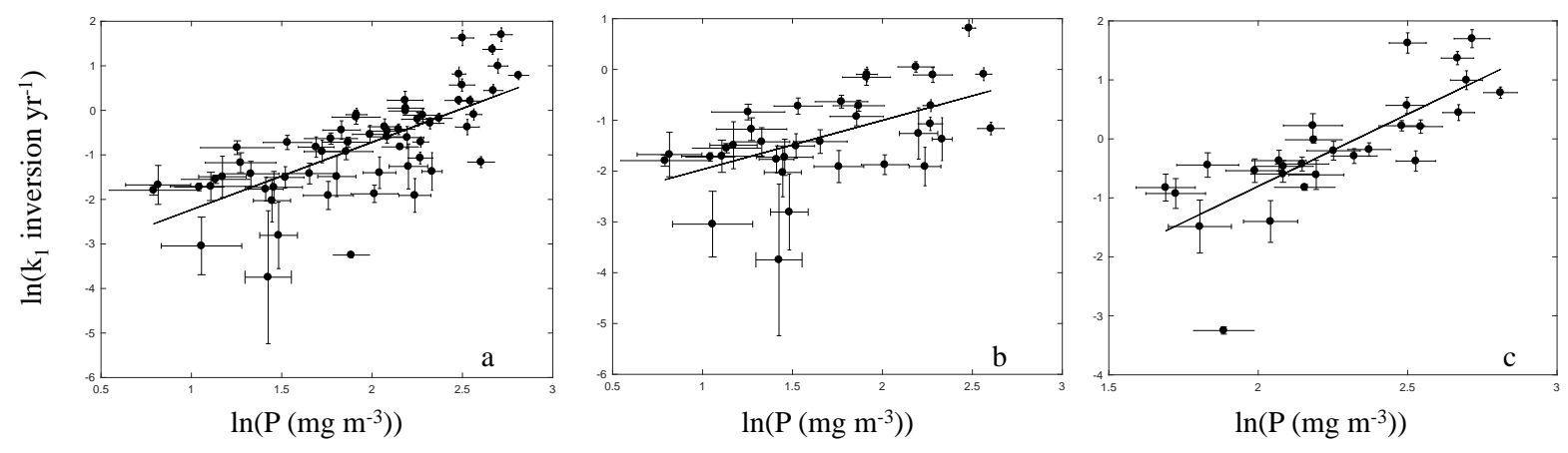

Figure 9: Regressions of $\ln \left(k_{1}\right)$ estimated by inversion against the natural logarithm of measured bulk particle concentration at (a) all selected stations, (b) stations west of GT11-24, and (c) stations east of and including GT11-24. The solid line is the best fit and error bars are \pm 1 standard deviation. 


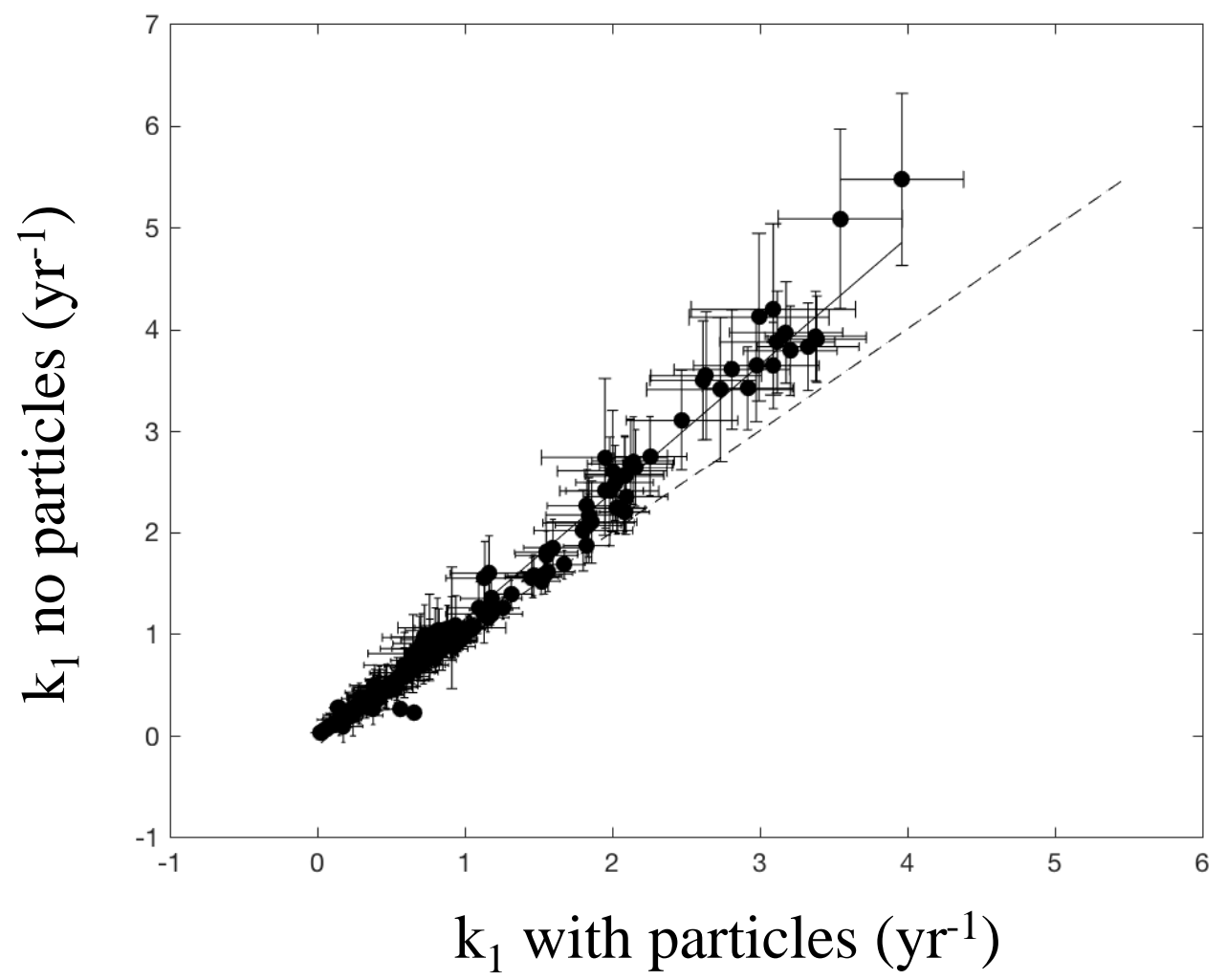

Figure A1: $k_{1}$ obtained from an inversion without bulk particle concentration data vs. $k_{1}$ obtained from an inversion with bulk particle concentration data. The error bars are \pm 2 standard deviations. The solid line is the OLS fit, while the dashed line is the line of perfect agreement. 
Chapter 5

Combined Effects of Scavenging and

Circulation in the Northwest Atlantic 


\begin{abstract}
Processes operating along ocean margins on ${ }^{230} \mathrm{Th}$ activities are of great importance for the application of this radioisotope in oceanography and paleoceanography. ${ }^{230} \mathrm{Th}$ activities measured along sediment cores are used to correct sediment records for the effect of sediment lateral redistribution and to infer, in conjunction with ${ }^{231} \mathrm{~Pa}$ measurements, changes in ocean circulation on millennial and longer time scales. However, in the vicinity of ocean margins, the rate at which trace metals are removed from the water column by attachment to settling particles can be enhanced relative to the open ocean, due to some combination of changes in particle concentration and particles composition. These changes may confound the interpretation of sediment ${ }^{230} \mathrm{Th}$ and ${ }^{231} \mathrm{~Pa}$ records.

In this chapter, I use a regional circulation model to examine the influence of particle scavenging and ocean circulation on dissolved (d) and particulate $(\mathrm{p}){ }^{230} \mathrm{Th}\left({ }^{230} \mathrm{Th}_{d, p}\right)$ in the Northwest Atlantic. This task is accomplished by conducting a variety of numerical experiments which isolate the influence of (i) ${ }^{230} \mathrm{Th}_{d, p}$ at the lateral boundaries of the regional domain, (ii) the Deep Western Boundary Current (DWBC) inflow and Gulf Stream (GS) outflow, and (iii) spatial variations in scavenging intensity. We find that changing the boundary values of ${ }^{230} \mathrm{Th}_{d, p}$ can improve or reduce the model fit to the dissolved ${ }^{230} \mathrm{Th}$ data, depending on the sensitivity experiment considered. Changing the DWBC inflow and GS inflow results in small increases in the ability of the model to replicate dissolved ${ }^{230} \mathrm{Th}$ data below $3000 \mathrm{~m}$ and ${ }^{230} \mathrm{Th}_{p}$ data above $3000 \mathrm{~m}$. The largest improvement in fit to dissolved ${ }^{230} \mathrm{Th}$ data below $3000 \mathrm{~m}$ is obtained using a scavenging rate that mimics the presence of bottom nepheloid layers observed in the Northwest Atlantic. This study therefore points to the importance of variable scavenging intensity over ocean transport in controlling the ${ }^{230} \mathrm{Th}$ distribution in the Northwest Atlantic.
\end{abstract}

\title{
$5.1 \quad$ Introduction
}

Our ability to use ${ }^{230} \mathrm{Th}$ as a tracer of ocean processes is hampered by our limited understanding of the influences of particle scavenging and ocean circulation on this radioisotope. In this chapter, I examine how both of these processes affect the distributions of dissolved (d) and particulate (p) ${ }^{230} \mathrm{Th}\left({ }^{230} \mathrm{Th}_{d, p}\right)$ in the Northwest Atlantic. This task is accomplished by embedding a reversible exchange model within a three-dimensional regional circulation model that is based on the primitive equations. The simulated ${ }^{230} \mathrm{Th}_{d, p}$ activities are compared to a compilation of ${ }^{230} \mathrm{Th}_{d, p}$ measurements from the pre-GEOTRACES era and the western end of the U.S. GEOTRACES North Atlantic section (section GA03). The comparison is first performed for a "reference" simulation, which serves as a basis for comparison to a variety of sensitivity experiments. These experiments explore the sensitivity of the simulated ${ }^{230} \mathrm{Th}_{d, p}$ to the ${ }^{230} \mathrm{Th}_{d, p}$ activities at lateral boundaries of the regional domain, the Deep Western Boundary Current (DWBC) inflow and Gulf Stream (GS) outflow, and spatial variations in scavenging intensity, as represented by an apparent first order rate constant for the adsorption of thorium onto particles. The ability of each simulation to fit the observed ${ }^{230} \mathrm{Th}_{d, p}$ activities is then compared. In this way, this chapter addresses the extent to which a model that 
accounts for both circulation and spatial variations in scavenging intensity can explain the available ${ }^{230} \mathrm{Th}_{d, p}$ data in the Northwest Atlantic.

\subsection{1 ${ }^{230} \mathrm{Th}$ in the Northwest Atlantic}

The radioactive isotope ${ }^{230} \mathrm{Th}\left(t_{1 / 2}=75,584 \mathrm{yr}\right)$ has been extensively utilized to investigate a variety of marine processes, including vertical particle fluxes (Kumar et al., 1993, 1995; François et al., 2004), lateral redistribution of sediment (Suman and Bacon, 1989; François et al., 2004), and aspects of modern and past circulations ( Yu et al., 1996; Moran et al., 2002). For each of these applications, the flux of ${ }^{230} \mathrm{Th}$ due to radioactive production by ${ }^{234} \mathrm{U}$ is assumed to be balanced by the flux of ${ }^{230} \mathrm{Th}$ to the sediments. This balance between water column production and sediment supply is predicted by a one dimensional (vertical) model describing the reversible exchange of thorium between dissolved and particulate phases (Nozaki et al., 1981; Bacon and Anderson, 1982). The reversible exchange model also predicts a linear increase of ${ }^{230} \mathrm{Th}$ activity in both the dissolved $\left({ }^{230} \mathrm{Th}_{d}\right)$ and particulate $\left({ }^{230} \mathrm{Th}_{p}\right)$ phases with depth. However, deviations from linearity are apparent throughout the North Atlantic (Fig 1; Moran et al. (1997); Hayes et al. (2015b)). These deviations are particularly pronounced near the ocean margin of the Northwest Atlantic, where ${ }^{230} \mathrm{Th}_{d}$ deficits relative to values expected from reversible exchange are observed (Fig 1a). Understanding the causes of such deviations is critical for the applications of ${ }^{230} \mathrm{Th}$ to (paleo-)oceanographic research in this region.

\subsubsection{Influence of Scavenging in the Northwest Atlantic}

Discrepancies of ${ }^{230} \mathrm{Th}_{d, p}$ data from values expected from reversible exchange in the Northwest Atlantic may be driven partly by enhanced particle scavenging (adsorption onto particles followed by particle sinking) in deep waters in this region. Indeed, "boundary scavenging", or the higher rate removal of particle reactive elements at ocean margins relative to the open ocean, has been observed in multiple studies in the Northwest Atlantic. These studies are largely based on data collected from the Shelf Edge Exchange Processes programs (SEEP). Bacon et al. (1988) reported dissolved ${ }^{210} \mathrm{~Pb}$ activities along the continental shelf and slope off Cape Cod (SEEP-I) that were greatly depleted in the upper $1000 \mathrm{~m}$ relative to offshore waters. Biscaye et al. (1994) found that the flux of ${ }^{210} \mathrm{~Pb}$ as measured by sediment traps deployed along the continental slope off the coast of Virginia (SEEP-II) increased in the onshore direction. From surface sediments sampled in the Middle Atlantic Bight between 384 and $2736 \mathrm{~m}$ water depths, as well as from material collected by sediment traps deployed in the same regions between 500 and $2800 \mathrm{~m}$, Anderson et al. (1994) found that the deposition of 
${ }^{230} \mathrm{Th},{ }^{231} \mathrm{~Pa}$, and ${ }^{10} \mathrm{Be}$ exceeded their local supply from radioactive production $\left({ }^{230} \mathrm{Th}\right.$ and $\left.{ }^{231} \mathrm{~Pa}\right)$ and atmospheric deposition $\left({ }^{10} \mathrm{Be}\right)$. In the case of ${ }^{230} \mathrm{Th}$, is was found that deposition exceeded the local supply by a factor of 12-15 near 1000-m water depth. This finding suggested the presence of both enhanced scavenging and significant lateral transport of ${ }^{230} \mathrm{Th}$ near the margin.

The recent dissolved and particulate ${ }^{230} \mathrm{Th}$ dataset from the US GEOTRACES North Atlantic Section (GA03) has further illuminated the role of scavenging on ${ }^{230} \mathrm{Th}$ in the Northwest Atlantic. Hayes et al. (2015b) found depletion of dissolved ${ }^{230} \mathrm{Th}$ below $3500 \mathrm{~m}$ in regions characterized by intense bottom nepheloid layers (BNLs). Within these layers, Hayes et al. (2015a) found that partition coefficients for ${ }^{230} \mathrm{Th}, K_{D}=A_{p} /\left(A_{d} P\right)$, where $A_{d}\left(A_{p}\right)$ is the radioisotope activity in the dissolved (particulate) phase, and $P$ is bulk particle concentration, were larger than those predicted from a linear, negative relationship between $K_{D}$ and $P$ based on data collected at other locations along GA03. These elevated values of $K_{D}$ were speculated, among other possibilities, to be caused by a lack of organic colloidal material within regions of high sediment resuspension, based on the apparent contribution of colloids to the negative relationship between $K_{D}$ and $P$ observed in estuarine and coastal environments (e.g. Honeyman et al. (1988); Honeyman and Santschi (1989)).

Two factors responsible for the enhanced scavenging of ${ }^{230} \mathrm{Th}$ near the Northwest Atlantic margin are sediment resuspension and differences in particle composition from that of particles found further from the margin. A major finding from the SEEP experiments was that a sizeable portion of the vertical fluxes of particles and particulate radioisotopes $\left({ }^{210} \mathrm{~Pa},{ }^{230} \mathrm{Th},{ }^{231} \mathrm{~Pa},{ }^{10} \mathrm{Be}\right)$ caught by along-slope sediment traps and found in surface sediments could not be accounted for by vertical supply, i.e., these fluxes must have been supported by lateral transport from the nearby continental shelf (Biscaye et al., 1988; Churchill et al., 1988; Walsh et al., 1988; Palanques and Biscaye, 1992; Biscaye et al., 1994; Anderson et al., 1994). The lateral supply of resuspended material from the continental shelf may contribute to enhanced scavenging of ${ }^{230} \mathrm{Th}$ in waters overlying the continental slope and perhaps even further offshore. In addition, BNLs with suspended sediment concentrations $>1000 \mathrm{mg} \mathrm{m}^{-3}$ have been documented during the High Energy Benthic Boundary Layer Experiment (HEBBLE), a field study conducted on the Scotian Rise and focused on the response of surficial sediments to near-bottom shear stresses (Nowell et al., 1982). McCave (1983) proposed that the lateral transport of resuspended material from the upper Scotian Rise could explain high levels of suspended sediment above the bottom mixed layers which were observed on the lower Rise. 
Periods of high turbidity in near-bottom waters of the HEBBLE area were found to be associated with periods of exceptionally strong $\left(>0.2 \mathrm{~m} \mathrm{~s}^{-1}\right.$ ) near-bottom flows (Hollister and McCave, 1984). Recently, Gardner et al. (2017) compiled observations from bottom-tethered instruments (including nephelometers, transmissometers, and current meters) deployed at various locations along the lower continental rise of eastern North America (HEBBLE area, near the Hatteras Abyssal Plain, south of New England), the Bermuda Rise, the Eastward Scarp northeast of the Bermuda Rise, and the Greater Antilles Outer Ridge. They found that BNLs as well as "benthic storms" (periods of strong abyssal currents and intense BNL development) are associated with high levels of eddy kinetic energy near the surface from the meandering Gulf Stream, perhaps as a result of deep cyclones, deep anti-cylones, and (or) topographic Rossby Waves.

The chemically reducing environment of continental margin sediments allows for the reductive dissolution and upward flux of dissolved Mn and Fe, which can oxidize and form Mn and Fe (oxyhydr)oxides in the water column (Burdige, 1993). Mn (oxyhydr)oxides have been shown to enhance the removal of particle-reactive elements from seawater (Balistrieri and Murray, 1986),

and at the Mid-Atlantic Ridge Fe precipitates have been shown to intensely scavenge ${ }^{228,230,234} \mathrm{Th}$ (German et al., 1991; Hayes et al., 2015b). More recently, Hayes et al. (2015a) estimated the $K_{D}$ values for ${ }^{230} \mathrm{Th}$ associated with $\mathrm{Mn}$ and Fe(oxyhydr)oxides along section GA03 to be two orders of magnitude greater than for biogenic or lithogenic particles. Also along section GA03, Lam et al. (2015a) showed that concentrations of Mn and Fe (oxyhydr)oxides measured within BNLs near the Northwest Atlantic margin exceeded by a factor of 2-3 those further from the margin.

\subsubsection{Influence of Circulation in the Northwest Atlantic}

The Deep Western Boundary Current (DWBC) is a major component of the Atlantic's meridional circulation, transporting recently formed North Atlantic Deep Water (NADW) towards the equator along the western boundary (Bower and Hunt, 2000). Near the source regions of NADW, the vertical distribution of ${ }^{230} \mathrm{Th}$ have been shown to depart significantly from linear profiles expected from reversible exchange theory. Moran et al. (1997) and Moran et al. (2002) have reported that at a station in the Labrador Sea and below $1000 \mathrm{~m}$, the vertical profiles of ${ }^{230} \mathrm{Th}_{d, p}$ are rather uniform. Distributions of ${ }^{230} \mathrm{Th}_{d, p}$ simulated by a two-dimensional (latitude-depth) transport model showed departures from linearity in both phases due to the intrusion of northern sourced waters between $60^{\circ} \mathrm{N}$ and $30^{\circ} \mathrm{N}$ ( Luo et al., 2010). On the other hand, no clear conclusion has been drawn on the 
influence of NADW on ${ }^{230} \mathrm{Th}$ data collected along GA03 (Hayes et al., 2015b). The systematic depletions in ${ }^{230}$ Th observed between 1000-3000 m depth at the western end of the section, relative to ${ }^{230}$ Th values observed at similar depths further east along GA03 (Fig. 1), suggest that transport of northern sourced waters plays a role in the distribution ${ }^{230} \mathrm{Th}$ in the western North Atlantic. However, between 2000 and $3000 \mathrm{~m}$ (where the components of NADW are predominant), no zonal gradient was observed in the dissolved ${ }^{231} \mathrm{~Pa} /{ }^{230} \mathrm{Th}$ ratio between 500 and $4200 \mathrm{~km}$ off the continental shelf, despite an estimated increase in the mean water mass age from 80 to 400 yrs across this distance (Hayes et al., 2015a) ${ }^{231} \mathrm{~Pa}$ is a radioisotope that, like ${ }^{230} \mathrm{Th}$, is produced radioactively in the water column and is long-lived, but that in general is less particle reactive than ${ }^{230} \mathrm{Th}$ ). More recently, based on ${ }^{230} \mathrm{Th}_{d, p}$ activities simulated from a reversible exchange model embedded within a simplified (geostrophic-frictional) model of ocean circulation, Rempfer et al. (2017) found that bottom scavenging and Atlantic meridional overturning circulation (AMOC) were both important for explaining the observed distributions of ${ }^{230} \mathrm{Th}$. These authors included a simple representation of bottom scavenging by assuming a globally uniform concentration of resuspended sediment (on which the adsorption rate constant for ${ }^{230} \mathrm{Th}$ was assumed to depend) near the bottom. They found that a model that includes bottom scavenging provided the best fit to a compilation of ${ }^{230} \mathrm{Th}_{d, p}$ water column measurements. They also simulated ${ }^{230} \mathrm{Th}_{d, p}$ for "on" and "off" states of AMOC, and found that the fit to the observations was improved when an "on" state was used. While an improvement in fit was obtained by considering bottom scavenging, the mechanisms leading to enhanced scavenging near the bottom were not represented in the model (Rempfer et al., 2017). Moreover, this study was performed with very coarse spatial resolution $(36 \times 36$ grid cells for the entire world ocean), and the relative importance of scavenging and circulation within specific ocean regions was not investigated.

\subsubsection{Objectives of The Study}

It is commonly assumed that the radioactively produced ${ }^{230} \mathrm{Th}$ is primarily removed by settling particles to the underlying sediment, with lateral transport having little to no effect ( $Y u$ et al., 1996; François, 2007). However, deviations of ${ }^{230} \mathrm{Th}_{d, p}$ activities from linear increases with depth predicted from reversible exchange with no circulation effects (Bacon and Anderson, 1982) are commonly

observed in the North Atlantic (Hayes et al., 2015b,a). Regional variability in $K_{D}$ for ${ }^{230} \mathrm{Th}$ found by Hayes et al. (2015a) may contribute to these deviations and complicate the interpretation of 
sedimentary ${ }^{230} \mathrm{Th}$ records. Additionally, Lerner et al. (2017) provided evidence that the vertical distribution of ${ }^{230} \mathrm{Th}$ in the North Atlantic may deviate from that expected from reversible exchange due to vertical variations in the rate parameters for Th adsorption, Th desorption, particle settling, and (or) particle degradation. How the combined influences of geographically varying scavenging rates and ocean circulation affect the distribution of dissolved and particulate ${ }^{230} \mathrm{Th}$ in the Northwest Atlantic (and elsewhere) remains an open question.

In this chapter, I investigate the effects of circulation and scavenging on ${ }^{230} \mathrm{Th}$ in the Northwest Atlantic by conducting numerical experiments with a regional ocean circulation model including ${ }^{230} \mathrm{Th}_{d, p}$. This chapter is organized as follows. In section 5.2, the major features of the circulation within our model domain are summarized. In section 5.3, an overview of the bathymetry and hydrography along line W, running between the New England shelf and Bermuda and along which current velocities have been repeatedly measured is provided, and a compilated of (pre-)GEOTRACES data is briefly presented. In section 5.4, details of the model, including tracer equations, initial conditions, and boundary conditions, are given. The experimental design and results of the various model experiments are described in section 5.5. In section 5.6, we examine which, if any, of the model experiments lead to a better description of the data. Among other results we find that the large ${ }^{230} \mathrm{Th}_{p}$ activities observed near the bottom of two stations along the western segment of GA03 cannot be captured by our model. Thus, section 5.6 also explores a potential mechanism, not included in the model, that could account for these very large activities. Conclusions follow in section 5.7.

\subsection{Major Features of the General Circulation in the Northwest Atlantic}

\subsubsection{Gulf Stream and Recirculation Gyres}

Our study region extends meridionally from $28^{\circ} \mathrm{N}$ to $44^{\circ} \mathrm{N}$ and zonally from $80^{\circ} \mathrm{W}$ to $55^{\circ} \mathrm{W}$, offshore of the Southern Atlantic Bight, the Middle Atlantic Bight, and the Gulf of Maine. Two of the major circulation features in this region include the Gulf Stream (GS) and the western limb of the Northern Recirculation Gyre (NRG), north of the GS (Fig. 2). The western limb of the NRG contribute to the transport of Slope Water to the southwest along the continental slope. The Gulf Stream enters our study region from the southwest as the Florida Current, follows the western boundary until Cape Hatteras, where the stream detaches from the boundary, flows northeastward, 
and exits our study region at approximately $40^{\circ} \mathrm{N}, 55^{\circ} \mathrm{W}$ (Schmitz and McCartney, 1993).

The volume transports at the boundaries of our study region as reported in different observational studies are given in figure 2. For example, Hogg (1992) found that of $20-30 \mathrm{~Sv}\left(1 \mathrm{~Sv}=10^{6} \mathrm{~m}^{3} \mathrm{~s}^{-1}\right)$ from the NRG contributed to the increased GS transport from the Straits of Florida (30 Sv; Schmitz and McCartney (1993)) to $40^{\circ} \mathrm{N}, 60^{\circ} \mathrm{W}$, the site of the Abyssal Circulation Experiment, (Hogg, 1992). The remainder of this increased transport is estimated to come from the "Worthington Gyre" (WG), an anti-cyclonic recirculation gyre south of the GS and largely confined within our model domain (Schmitz and McCartney, 1993). Due to its confinement within our study region, we do not assign an inflow or outflow of water to our domain associated with this gyre. For the western limb of the NRG, we assume an inflow of $20 \mathrm{~Sv}$ (Hogg et al., 1986).

\subsubsection{Gulf Stream Meanders and Eddies}

The energetic Gulf Stream System in the Northwest Atlantic is characterized by swiftly fluctuating currents and eddies. Among the well-known aspects of the eddy field in the Northwest Atlantic are its Warm Core Rings, which bring warm water to the north of the GS, and its Cold Core Rings, which bring cold to the south of the GS (Lai and Richardson, 1977). The diameter of these rings typically varies between 100-300 km (Richardson et al., 1977). Both Warm and Cold Core Rings are thought to be formed by the meandering of the unstable Gulf Stream jet. These meanders can display periodicities between 4 and 50 days and wavelengths between 180 and $600 \mathrm{~km}$ (Watts and Johns, 1982). In the area west of $55^{\circ} \mathrm{W}$ and north of $30^{\circ} \mathrm{W}$, there may be 3 Warm Core Rings to the north of the GS and 10 cold-core rings to the south at any given time during the year (Richardson, 1983). Warm Core Rings impinging on continental shelves can induce cross-shelf water exchange, both pulling colder shelf water offshore and moving warmer ring water onshore (Zhang and Gawarkiewicz, 2015). This process may also contribute to a flux of shelf material, such as resuspended sediments, to offshore waters regions (Bishop and Fleisher, 1987). Aside from forming rings, large amplitude GS meanders may also result in cyclones that can penetrate thousands of meters below the surface of the ocean (Savidge and Bane, 1999).

\subsubsection{Deep Western Boundary Current}

Along with the NRG, the DWBC also contributes to the transport of Slope Water, and is the third major circulation feature in the study region. The DWBC transports dense waters formed at high latitudes in the Labrador and Nordic Seas and flowing subsequently towards the equator 
(McCartney, 1992). The DWBC enters our study region along the continental slope south of Nova Scotia (Schmitz and McCartney, 1993), extending from the seafloor to approximately $700 \mathrm{~m}$ water depth (Pickart, 1992). The DWBC flows approximately along the $3400 \mathrm{~m}$ isobath until it passes below the GS just north of Cape Hatteras (Hogg et al., 1986). At this point, the DWBC veers slightly from its former path to deeper isobaths (Hogg and Stommel, 1985; Pickart and Watts, 1990), and then continues its southward along-isobath flow out of the study region (Schmitz and McCartney, 1993).

The DWBC may be modified by interaction with the GS where the two flows cross over near Cape Hatteras. Pickart and Smethie (1993) found that the DWBC near this region could be split into three layers, each of which interacts with the GS differently. Based on a 17-day hydrographic survey of the GS-DWBC crossover, Pickart and Smethie (1993) found that south of the crossover region, the shallowest layer of the $\mathrm{DWBC}$ was depleted in $\mathrm{CFC}$ relative to north of the crossover region, while the deeper layers had similar $\mathrm{CFC}$ concentration in each region, suggesting that the shallow layer of the DWBC becomes entrained in the subsurface GS flow. However, recirculation may replace the waters lost by GS entrainment, so that the net volume transport of the DWBC is only slightly modified at the crossover.

The inflow and outflow of the DWBC represented in Figure. 2 are based on daily transport estimates obtained between 2004 and 2014 along line W, a hydrographic section combining moored instruments and shipboard observations to monitor the DWBC on the continental slope south of New England (Toole et al., 2017). Integrating the transports of Upper and Lower Labrador Sea Waters, Iceland Scotland Overflow Water, and Denmark Straits Overflow Water, Toole et al. (2017) obtained a mean cross-section transport of $-22.76 \pm 1.38 \mathrm{~Sv}$ (mean \pm 1 standard error), where the negative value indicates transport to the south. This value is similar to the southwestward transport of $20 \mathrm{~Sv}$ along the continental slope at $70^{\circ} \mathrm{W}$ (west of the NRG), estimated from moored current meter data from the Rise Array (Hogg, 1983; Hogg et al., 1986). For this study, we assume a value of $20 \mathrm{~Sv}$ for the DWBC, and set the outflow of the DWBC at the southern boundary of the domain to its inflow from the northeast. Note that the inflow in the northeast is $40 \mathrm{~Sv}$ (Fig. 2) because it represents the combined fluxes from the DWBC and the western branch of the NRG (Hogg et al., 1986).

Finally, our schematic (Fig. 2) includes a postulated Subtropical Gyre inflow (STG) at the 
eastern boundary, south of the GS outflow, in order to balance the net outflow of $40 \mathrm{~Sv}$ out of the domain (see also Ezer (2017)).

\subsection{Data}

\subsubsection{Hydrographic Data}

Section GA03 was completed during November 2011, and the hydrography along the transect has been previously documented by Jenkins et al. (2015). Figures 3-4 show profiles of temperature, salinity, dissolved oxygen, and CFC-11 measured at eight stations along the western segment of GA03. The high concentrations of dissolved oxygen in deep waters near the western end of the section are partly due to the intrusion of (low salinity, cold) well-oxygenated waters from high latitudes (Jenkins et al., 2015). The high oxygen concentrations below $\sim 3000 \mathrm{~m}$ at stations GT11-03 and GT11-04 also coincide with relatively high concentrations of CFC-11, a transient (anthropogenic) tracer that is enriched in northern sourced waters (Smith et al., 2016). Taken together, these observations suggest a pronounced intrusion of NADW components at the western end of GA03, particularly at stations GT11-03 and GT11-04.

The western segment of GA03, encompassing the stations for which profiles are shown in Figures 3-4, was sampled along line W. Data from the line includes velocity measurements obtained from shipboard deployments of Lowered Accoustic Doppler Current Profilers (LADCPs) and from moored profiler and current meters (Toole et al., 2017) between 2004 and 2014. Figure 5a-b shows the 10-yr average velocities obtained by averaging daily profiles of subinertial-filtered velocity (the easternmost mooring only contains data between 2008 and 2014, and only a 6-yr average is used for that mooring). The daily profiles were constructed by combining moored profiler and current meter data from six moorings deployed along line $\mathrm{W}$ and have been provided by John Toole at WHOI (Toole et al., 2017). The velocity components displayed in Figure 5a-b are those along (eastward positive) and across line W. The high near-surface velocities associated with the GS are clearly represented in the cross-section at a distance of approximately $250 \mathrm{~km}$. The core of the DWBC is barely delineated with velocity extrema near the bottom at a distance of $\sim 200 \mathrm{~km}$. This position of the core roughly aligns with the velocity maximum associated with Denmark Strait Overflow Water and obtained from a 10-yr average of shipboard LADCP measurements (Andres et al., 2016). Notably, the positions of the GS and DWBC at any instant can deviate significantly from their 
mean positions. For example, Toole et al. (2017) found that only $16 \%$ of moored observations were from days when the GS core was close to its 10-yr mean positions.

\subsubsection{GEOTRACES ${ }^{230} \mathrm{Th}_{d, p}$}

The ${ }^{230} \mathrm{Th}_{d, p}$ data collected along GA03 (Table 1) have been described by Hayes et al. (2015b,a), and only a brief review of them is provided (Figs. 6 and 7). ${ }^{230} \mathrm{Th}_{d, p}$ data from the upper $1000 \mathrm{~m}$ of the water column appear generally consistent with reversible exchange theory (Bacon and Anderson, 1982), which predicts that the radionuclide activities in both phases linearly increase with depth. Below $1000 \mathrm{~m},{ }^{230} \mathrm{Th}_{d, p}$ data continue to increase with depth, although the vertical gradients in ${ }^{230} \mathrm{Th}_{d}$ activity appear reduced at stations GT11-01 to GT11-03 compared with the other stations. However, vertical ${ }^{230} \mathrm{Th}_{d, p}$ gradients change sign at about $3000 \mathrm{~m}$ at deeper stations, where their behavior changes from monotonically increasing to monotonically decreasing with depth. These inversions are particularly noticeable at stations GT11-04 to GT11-12 for ${ }^{230} \mathrm{Th}_{d}$, and at stations GT11-06 to GT11-12 for ${ }^{230} \mathrm{Th}_{p}$.

\subsubsection{Pre-GEOTRACES ${ }^{230} \mathrm{Th}_{d, p}$}

Consistent with the GA03 data, dissolved ${ }^{230}$ Th data from the pre-GEOTRACES era (Table 1) show a general increase with depth in the top $1000 \mathrm{~m}$, with the exception of station CMME13 in the Hatteras Abyssal Plain (Cochran et al., 1987) and station S1 close to the coast of Cape Hatteras (Guo et al., 1995) (Fig. 9). Most stations show continued increases in ${ }^{230} \mathrm{Th}_{d}$ with depth down to $3000 \mathrm{~m}$, though there is an obvious departure from this gradient at station EN407-3 (close to station GT11-03 along line W). Below $3000 \mathrm{~m}$, most stations sampled during pre-GEOTRACES cruises display the ${ }^{230} \mathrm{Th}_{d}$ inversion shown in the ${ }^{230} \mathrm{Th}_{d}$ data for the western segment of GA03 data, with the exception of station CMME13. For example, at or near station BATS southeast of Bermuda, both GA03 and pre-GEOTRACES ${ }^{230} \mathrm{Th}_{d}$ data display an inversion near $3000 \mathrm{~m}$, although ${ }^{230} \mathrm{Th}_{d}$ present lower values at $4000 \mathrm{~m}\left(0.3 \mathrm{dpm} \mathrm{m}{ }^{-3}\right)$ at the GA03 station than at the pre-GEOTRACES station $\left(0.4 \mathrm{dpm} \mathrm{m}^{-3}\right)$.

To our knowledge, prior to GEOTRACES, only two studies reported measurements of ${ }^{230} \mathrm{Th}_{p}$ in the study region (Fig. 9). Cochran et al. (1987) published measurements of ${ }^{230} \mathrm{Th}_{p}$ at station CMME13 in the Hatteras Abyssal Plain. The ${ }^{230} \mathrm{Th}_{p}$ activities at this station generally increase with depth. The larger activities of ${ }^{230} \mathrm{Th}_{p}$ near the surface were attributed to atmospheric and fluvial inputs, and the larger ${ }^{230} \mathrm{Th}_{p}$ near the bottom was attributed to sediment resuspension (Cochran 
et al., 1987). Guo et al. (1995) reported observations of ${ }^{230} \mathrm{Th}_{p}$ at another station near Cape Hatteras. The large ${ }^{230} \mathrm{Th}_{p}$ near the surface and bottom, greater than those expected from reversible exchange theory, were also attributed to atmospheric deposition and sediment resuspension. However, Guo et al. (1995) also noted that ${ }^{230} \mathrm{Th}_{p}$ values measured far from the surface and the bottom were elevated compared to those expected from reversible exchange, and attributed this elevation to lateral injection of sediments from the nearby continental shelf and slope.

\begin{tabular}{|c|c|c|c|c|}
\hline location & station ID & $\#^{230} \mathrm{Th}_{d}$ & $\#^{230} \mathrm{Th}_{p}$ & reference \\
\hline $32.76^{\circ} \mathrm{N}, 70.78^{\circ} \mathrm{W}$ & CMME-13 (a) ${ }^{a}$ & 8 & 8 & Cochran et al. (1987) \\
\hline $36.05^{\circ} \mathrm{N}, 74.43^{\circ} \mathrm{W}$ & Cap.Hat.S1 (b) & 11 & 10 & Guo et al. (1995) \\
\hline $39.47^{\circ} \mathrm{N}, 68.37^{\circ} \mathrm{W}$ & EN407-3 (c) & 11 & 0 & Luo et al. (2010) \\
\hline $38.6^{\circ} \mathrm{N}, 68.89^{\circ} \mathrm{W}$ & EN407-4 (d) & 19 & 0 & Luo et al. (2010) \\
\hline $39.73^{\circ} \mathrm{N}, 69.75^{\circ} \mathrm{W}$ & EN407-6 (e) & 10 & 0 & R. François, P.C. ${ }^{b}$ \\
\hline $32^{\circ} \mathrm{N}, 64^{\circ} \mathrm{W}$ & BATS (f) & 19 & 0 & R. François, P.C. \\
\hline $37^{\circ} \mathrm{N}, 69^{\circ} \mathrm{W}$ & OC278-2 (g) & 11 & 0 & R. François, P.C. \\
\hline $33^{\circ} \mathrm{N}, 69^{\circ} \mathrm{W}$ & OC278-3 (h) & 10 & 0 & R. François, P.C. \\
\hline $36^{\circ} \mathrm{N}, 68^{\circ} \mathrm{W}$ & OC278-4 (i) & 11 & 0 & R. François, P.C. \\
\hline $38^{\circ} \mathrm{N}, 70^{\circ} \mathrm{W}$ & $\mathrm{OC} 278-5(\mathrm{j})$ & 9 & 0 & R. François, P.C. \\
\hline $39.69^{\circ} \mathrm{N}, 69.81^{\circ} \mathrm{W}$ & GT11-01 (1) & 25 & 10 & Hayes et al. $(2015 \mathrm{~b}, \mathrm{a})$ \\
\hline $39.35^{\circ} \mathrm{N}, 69.54^{\circ} \mathrm{W}$ & GT11-02 (2) & 17 & 12 & Hayes et al. $(2015 \mathrm{~b}, \mathrm{a})$ \\
\hline $38.67^{\circ} \mathrm{N}, 69.10^{\circ} \mathrm{W}$ & GT11-03 (3) & 20 & 12 & Hayes et al. $(2015 \mathrm{~b}, \mathrm{a})$ \\
\hline $38.09^{\circ} \mathrm{N}, 68.78^{\circ} \mathrm{W}$ & GT11-04 (4) & 16 & 12 & Hayes et al. $(2015 \mathrm{~b}, \mathrm{a})$ \\
\hline $37.61^{\circ} \mathrm{N}, 68.39^{\circ} \mathrm{W}$ & GT11-06 (6) & 20 & 12 & Hayes et al. $(2015 \mathrm{~b}, \mathrm{a})$ \\
\hline $35.42^{\circ} \mathrm{N}, 66.52^{\circ} \mathrm{W}$ & GT11-08 (8) & 17 & 12 & Hayes et al. $(2015 \mathrm{~b}, \mathrm{a})$ \\
\hline $31.75^{\circ} \mathrm{N}, 64.17^{\circ} \mathrm{W}$ & GT11-10 (10) & 28 & 12 & Hayes et al. $(2015 \mathrm{~b}, \mathrm{a})$ \\
\hline $29.70^{\circ} \mathrm{N}, 56.82^{\circ} \mathrm{W}$ & GT11-10 (12) & 18 & 12 & Hayes et al. $(2015 \mathrm{~b}, \mathrm{a})$ \\
\hline
\end{tabular}

a. Value in parentheses are the corresponding labels in Figure 2.

b. personal communication

Table 1: Water column measurements of ${ }^{230} \mathrm{Th}_{d, p}$ in the study region.

\subsubsection{Beam Attenuation Coefficient, Dynamic Topography, and Bathymetry}

This study uses measurements of the beam attenuation coefficient associated with particles $\left(c_{p}\right)$ to represent the effect of benthic nepheloid layers on the scavenging of ${ }^{230} \mathrm{Th}$. These measurements were obtained from the transmissometer mounted on the GA03 rosette and have been reported by Hayes et al. (2015a). Notably, regions of enhanced $c_{p}$ were found near the bottom of stations GT11-04, GT11-06, GT11-08, and GT11-10, along the western segment of the trans-Atlantic section.

This study also relies on dynamic topography and bathymetric datasets from a variety of sources. Data of dynamic topography originate from satellite altimetry maps prepared by Ssalto/Duacs and distributed by AVISO (https://www.aviso.altimetry.fr/en/home.html). Monthly observations of sea surface height (SSH) between 1993-2012 are considered and compared to model results.

Bathymetric data (Fig. 2) are obtained from ETOPO2 version 2 (National Geophysical Data 
Center, 2006), a gridded product with 2-min resolution and derived from satellite altimeter and shipboard sounding data. In section 5.4.2, a higher resolution bathymetric data set, the Global Multi-Resolution Topography synthesis (GMRT), is used. The GMRT archive relies on satellite altimetry data and available high resolution data from multibeam swath echo sounders to derive a gridded bathymetry with local resolution as high as $\sim 100 \mathrm{~m}$, depending on swath data availability.

\subsection{Model of Ocean Circulation and ${ }^{230}$ Th Transport}

\subsubsection{Domain}

The model domain extends from $80{ }^{\circ} \mathrm{W}$ to $55.5^{\circ} \mathrm{W}$, and from $28^{\circ} \mathrm{N}$ to $45^{\circ} \mathrm{N}$ (Fig. 2). It is bounded in the west and north by the 200-m isobath, which approximately traces the shelf edge, and in the east and south by a longitude line and a latitude line, respectively. The ocean bottom is at $z=-h(x, y)$, and the ocean surface is at $z=\eta(x, y, t)$, where $x$ is the longitude coordinate, $y$ the latitude coordinate, and $z$ is depth. Thus, $h(x, y)$ is the local water depth and $\eta(x, y, t)$ is the local value of the free surface elevation. The bathymetry is obtained from ETOPO2 version 2 (National Geophysical Data Center, 2006). The horizontal resolution of the model grid is $1 / 4^{\circ}$ in both the meridional and zonal directions. Primitive-equation models run at this resolution are sometimes called "eddy-permitting", i.e., the flow can develop apparent eddies but eddies are poorly represented (e.g., Tréguier et al., 2014). As a result, model solutions are expected to be time-dependent, even with time-invariant boundary conditions. To minimize the need for downward extrapolation of temperature $(T)$ and salinity $(S)$ to model grid points where $T$ and $S$ data are not available to initialize the model (section 5.4.3), the smallest water depth among the ETOPO2 version 2 values within the model grid cell surrounding the model grid point is taken as the depth for that cell. The model uses terrain following coordinates (Blumberg and Mellor, 1987; Mellor, 2002), so that the number of vertical levels is constant throughout the domain and the vertical resolution is increased in shallow regions. A vertical level is defined as:

$$
\sigma=\frac{z-\eta(x, y, t)}{h(x, y)+\eta(x, y, t)} .
$$

The model includes $31 \sigma$ levels, and the maximum water depth in the model domain is $5715 \mathrm{~m}$. The vertical resolution in the upper part of the water column is increased (logarithmic layer) to better represent the Ekman circulation in the upper ocean. 


\subsubsection{Tracer Equations}

In this study, the Princeton Ocean Model (POM; the computer code applied here is pom2k.f) is used to numerically solve the equations of motion and the governing equations for ${ }^{230} \mathrm{Th}$ in dissolved and particulate forms (see Table A1 for physical and numerical parameters). POM is a primitive-equation, free-surface model of ocean circulation that relies on the hydrostatic and Boussinesq approximations. The model equations as well as their method of solution are described in Blumberg and Mellor (1987) and Mellor (2002). The dynamic variables of the model include the velocity $\boldsymbol{u}=[u, v, w]$, temperature $T$, salinity $S$, and pressure $p$. Density is calculated from $(T, S, p)$ from a non-linear equation of state. A turbulent closure scheme (Mellor and Yamada, 1982) is applied to obtain the vertical turbulent viscosities and diffusivities, while horizontal diffusivities and viscosities are parameterized according to Smagorinsky (1963) and a fixed turbulent Prandtl number. The equations of motion are solved using a 'mode-splitting' technique (Blumberg and Mellor, 1987; Mellor, 2002), in which an external (barotropic) mode is calculated separately from an internal (baroclinic) mode. In this study, the time-step for the external mode is 15 seconds and is a factor of 30 smaller than the time-step for the internal mode.

The governing equations for ${ }^{230} \mathrm{Th}_{d, p}$ are (prior to their transformations to $\sigma$-coordinates):

$$
\begin{aligned}
\frac{\partial A_{d}}{\partial t} & =-\boldsymbol{u} \cdot \nabla A_{d}+\frac{\partial}{\partial z}\left(\kappa_{T} \frac{\partial A_{d}}{\partial z}\right)+F_{d}+\lambda A_{\pi}+k^{*}{ }_{-1} A_{p}-k_{1} A_{d}, \\
\frac{\partial A_{p}}{\partial t}+w_{s} \frac{\partial A_{p}}{\partial z} & =-\boldsymbol{u} \cdot \nabla A_{p}+\frac{\partial}{\partial z}\left(\kappa_{T} \frac{\partial A_{p}}{\partial z}\right)+F_{p}+k_{1} A_{d}-k^{*}{ }_{-1} A_{p} .
\end{aligned}
$$

In the above equations, $\mathrm{A}_{d}$ is the ${ }^{230} \mathrm{Th}_{d}$ activity $\left(\mathrm{dpm} \mathrm{m}^{-3}\right), \mathrm{A}_{p}$ is the ${ }^{230} \mathrm{Th}_{p}$ activity (dpm $\left.\mathrm{m}^{-3}\right), \mathrm{A}_{\pi}$ is the activity of the radioactive parent, ${ }^{234} \mathrm{U}\left(\mathrm{dpm} \mathrm{m}{ }^{-3}\right), k_{1}$ is the rate constant for Th attachment (adsorption) onto particles $\left(\mathrm{yr}^{-1}\right), k_{-1}^{*}$ is the rate constant for thorium detachment (desorption + particle degradation) from particles $\left(\mathrm{yr}^{-1}\right), w_{s}$ is the particle sinking speed $\left(\mathrm{m} \mathrm{yr}^{-1}\right)$, $\nabla$ is the horizontal gradient operator, $\kappa_{T}$ is the vertical diffusivity $\left(\mathrm{m}^{2} \mathrm{~s}^{-1}\right)$, and $F_{d}\left(F_{p}\right)$ represents horizontal diffusion of ${ }^{230} \mathrm{Th}_{d}\left({ }^{230} \mathrm{Th}_{p}\right)$. The remaining symbols are $t$ for time and $z$ for depth. Note that the above equations omit the effects of sediment resuspension and dust deposition. Radioactive decay of ${ }^{230} \mathrm{Th}$ is also neglected, due to its long half-life of 75,584 yrs. The method of solution of equations $(5.2 \mathrm{a}-\mathrm{b})$ is based on a simple positive definite advection scheme with small implicit diffusion and one iterative corrective step (Smolarkiewicz, 1983); it is identical to that used for the $T$ and $S$ equations (Blumberg and Mellor, 1987; Mellor, 2002). 


\subsubsection{Initial Conditions}

The initial conditions used for $(T, S)$ are obtained from the $1 / 4^{\circ}$ resolution climatology (decadal averages, objectively analyzed) of the World Ocean Atlas (WOA, 2013). The geographic locations of model grid points coincide with points of the climatological grid, so no horizontal interpolation is necessary. On the other hand, the climatologic values, available at 102 levels, are interpolated vertically to obtain $(T, S)$ values at the model levels (linear extrapolation was also applied at a few locations, where needed). The initial values of $(u, v, w)$ are set to 0 everywhere except at the boundaries (see next section). The initial conditions for ${ }^{230} \mathrm{Th}_{d, p}$ are prescribed based on ${ }^{230} \mathrm{Th}_{d, p}$ measurements at station GT11-14 $\left(27.58^{\circ} \mathrm{N}, 49.63^{\circ} \mathrm{W}\right)$, the closest station outside of the model domain. To this end, exponential functions are fit to the ${ }^{230} \mathrm{Th}_{d, p}$ profiles measured at station GT11-14 (Fig. 10).

$$
\begin{aligned}
& A_{d}(z)=A_{d, 0}\left(1-e^{-z / l_{d}}\right) \\
& A_{p}(z)=A_{p, 0}\left(1-e^{-z / l_{p}}\right) .
\end{aligned}
$$

Here $A_{d, 0}\left(A_{p, 0}\right)$ is a maximum dissolved (particulate) ${ }^{230} \mathrm{Th}$ activity and $l_{d}$ is a vertical scale obtained by fitting $\ln \left(A_{d}(z)-A_{d, 0}\right)$ against $\left.\ln \left(-z / l_{d}\right)\right)$ using ordinary least squares (a similar procedure is used to estimate $l_{p}$ ). Note that ${ }^{230} \mathrm{Th}_{d, p}$ data between 1500 and $3000 \mathrm{~m}$ were not considered for the fit, due to the apparent influence of the TAG hydrothermal vent on these data.

\subsubsection{Boundary Conditions}

At the surface, the model is forced by annual mean wind stresses derived from the Scatterometer Climatology of the World Ocean (Risien and Chelton, 2008), and heat and freshwater fluxes are set to zero as in previous studies (Mellor and Ezer, 1991; Ezer, 2016). At the lateral boundaries, the following conditions are specified. Our regional model includes open boundaries both at the eastern and southern limits of the domain (Fig. 2). In contrast, the western and northern boundaries, which approximately follow the 200-m isobath near the shelf break, are taken as closed boundaries. As a result, exchanges between the shelf and slope regions, which are not the focus of our study, are not represented in the model. For both the internal and external modes, conditions of no slip and no normal flow are imposed at the western and northern boundaries, i.e., these boundaries act as solid walls,

$$
u_{n}=u_{t}=0 \text { and } \bar{u}_{n}=\bar{u}_{t}=0 .
$$


Here, $u_{n}$ and $u_{t}$ refer to the components of $\boldsymbol{u}$ normal to and tangent to the boundary, respectively, and overbars denote vertically averaged components.

At the eastern and southern boundaries, $u_{n}$ is calculated from a radiation condition (Mellor, 2002), whereas $u_{t}$ is set to zero,

$$
\frac{\partial u_{n}}{\partial t} \pm c_{i} \frac{\partial u_{n}}{\partial n}=0 \text { and } u_{t}=0
$$

Here, $n$ is the coordinate normal to the boundary, and $c_{i}$ is a speed for the internal mode derived from the water depth at the boundary and a maximum depth of $5000 \mathrm{~m}$.

At the eastern and southern boundaries, $\bar{u}_{n}$ is calculated from a Flather condition (Flather, 1976), whereas $\bar{u}_{t}$ is set to zero.

$$
\bar{u}_{n}=\bar{u}_{n}^{*}+\sqrt{g / h}\left(\eta-\eta^{*}\right) \text { and } \bar{u}_{t}=0 .
$$

Here, $\eta^{*}$ is the initial free-surface elevation, $g$ is the acceleration due to gravity, and $\bar{u}_{n}^{*}$ is the initial depth-averaged velocity normal to the boundary. At locations of inflow or outflow (Fig. 2), $\bar{u}_{n}^{*}$ is calculated from.

$$
\bar{u}_{n}^{*}=\int_{-H}^{0} u_{n}^{*} d z
$$

where $u_{n}^{*}$ is obtained from,

$$
\begin{array}{r}
u_{n}(z)=u_{n, \max } e^{-\left(\left(z-z^{*}\right) / l_{z^{*}}\right)^{2}} \\
u_{n, \max }=\operatorname{Tr} / A . \\
A=\iint_{A} e^{-\left(\left(z-z^{*}\right) / l_{z^{*}}\right)^{2}} d A
\end{array}
$$

Here, $A$ is the area of integration over the inflowing or outflowing surface, $z^{*}$ is the depth of the core of the inflow or outflow $(\mathrm{m}), l_{z^{*}}$ is a vertical scale for the inflow or outflow $(\mathrm{m}), \operatorname{Tr}$ is the volume transport of the inflow or outflow $\left(\mathrm{m}^{3} \mathrm{~s}^{-1}\right)$, and $u_{n, \max }$ is the maximum velocity normal to the boundary $\left(\mathrm{m} \mathrm{s}^{-1}\right)$. Table 2 contains the values assumed in this study for the inflow and outflow transports, the horizontal extent of the inflow or outflow regions, and the length scale, $l_{z^{*}}$ (see also Figure 2).

Except for a narrow segment of the eastern boundary between $43^{\circ} \mathrm{N}$ and $44.25^{\circ} \mathrm{N}$ (hereafter, 
NE segment), where Slope Water (DWBC plus western branch of NRG) flows into the domain, the activities of ${ }^{230} \mathrm{Th}_{d, p}$ at the open boundaries are assumed to be equal to the initial activities (eqs. 5.3a-5.3b). Along the NE segment, boundary values of ${ }^{230} \mathrm{Th}_{d, p}$ are prescribed as the averages of ${ }^{230} \mathrm{Th}_{d, p}$ data measured at different depths at a station in the Labrador Sea (Moran et al., 1997) (Fig. 11).

\begin{tabular}{|l|c|c|c|c|}
\hline & boundary region & transport $^{a}$ & $z^{*}$ & $l_{z^{*}}$ \\
\hline DWBC inflow & $43.5^{\circ} \mathrm{N}$ to $45^{\circ} \mathrm{N}$ & $40 \mathrm{~Sv}$ & $2000 \mathrm{~m}$ & $500 \mathrm{~m}$ \\
\hline DWBC outflow & $76.5 \mathrm{~W}^{\circ} \mathrm{N}$ to $75.5^{\circ} \mathrm{W}$ & $-20 \mathrm{~Sv}$ & $3000 \mathrm{~m}$ & $500 \mathrm{~m}$ \\
\hline GS inflow & $80.0 \mathrm{~W}^{\circ} \mathrm{N}$ to $78.0^{\circ} \mathrm{W}$ & $30 \mathrm{~Sv}$ & $0 \mathrm{~m}$ & $200 \mathrm{~m}$ \\
\hline GS outflow & $39.0 \mathrm{~N}^{\circ} \mathrm{N}$ to $41.0^{\circ} \mathrm{N}$ & $-90 \mathrm{~Sv}$ & $0 \mathrm{~m}$ & $200 \mathrm{~m}$ \\
\hline STG Inflow & $28.0 \mathrm{~N}^{\circ} \mathrm{N}$ to $39.0^{\circ} \mathrm{N}$ & $40 \mathrm{~Sv}$ & $0 \mathrm{~m}$ & $1000 \mathrm{~m}$ \\
\hline
\end{tabular}

a. Positive (negative) values indicate transport to the east or north (west or south).

Table 2: Open boundary conditions of the model.

As with ${ }^{230} \mathrm{Th}_{d, p}$, temperature and salinity along the eastern and southern boundaries are set equal to their initial values. Boundary conditions for turbulent quantities $\left(q^{2}\right.$ and $\left.q^{2} l\right)$, which are used by the model to calculate the vertical viscosities and diffusivities, are also required. $q^{2}$ and $q^{2} l$ along the eastern and southern boundaries are equal to a constant value of $10^{-9} \mathrm{~m}^{2} \mathrm{~s}^{-2}$ and $10^{-9}$ $\mathrm{m}^{3} \mathrm{~s}^{-2}$, respectively.

\subsection{Results}

Given the relatively large number of numerical experiments conducted with the model, it is convenient to define a reference experiment, which will serve as a pivot and basis of comparison. In this section, we first describe the circulation obtained from this reference experiment (section 5.5.1). Next, the ${ }^{230} \mathrm{Th}_{d, p}$ distributions obtained from the reference experiment are presented (section 5.5.2). Finally, the sensitivities of the ${ }^{230} \mathrm{Th}_{d, p}$ distributions to the boundary activities (section 5.5.3.1), the DWBC inflow and GS outflow (section 5.5.3.2), and spatial variations in scavenging (section 5.5.5) are explored.

\subsubsection{Reference Experiment: Circulation}

The reference simulation assumes the boundary conditions described in section 5.4.4. Over the course of the model integration, the kinetic energy averaged over the model domain (MKE) first shows large fluctuations over the first 1500 days and then reduced fluctuations during the remaining 
period (Fig. 12a). The MKE still shows an overall tendency to increase with time near the end of the simulation, at $t=3700$ days. Here, elements of the physical solution $(u, v$, and surface elevation) are averaged over the last 2200 days of the simulation when compared to observations, since the increase in mke with time does not appear as pronounced after $t=2200$ days.

The major features of the observed circulation in the Northwest Atlantic are reproduced by the model, including (i) the Gulf Stream flowing over the shelf, detaching near Cape Hatteras, and then meandering to the northeast along a SW-NE direction (Fig. 13a) and (ii) the Deep Western Boundary Current flowing to the southwest along the continental slope (Fig. 13b). Along the eastern boundary at $42^{\circ} \mathrm{N}$, there is also evidence of a southward flow that joins the GS to exit this boundary (Fig. 13a) and that may represent the western limb of the NRG and its confluence with the GS outflow in this region (Hogg et al., 1986).

Elements of the modeled circulation are compared below to different climatological fields derived from observations in the Northwest Atlantic. The time-span of these climatologies varies from 8 to $20 \mathrm{yrs}$, depending on the specific field. While this is much longer than the period over which the model solution is averaged, it is difficult to subsample a 2200-day period for any given field to compare with model output, since the model simulations, forced with time-invariant boundary conditions, cannot be tied to a specific historical time interval. Table 3 provides measures of the model-data comparison.

The model mean and standard deviation of sea surface heigh (SSH) (Fig. 14a,d) are compared with the observed mean and standard deviation in SSH from satellite altimetry (Fig. 14b,e). The mean values of SSH simulated by the model correlate strongly with observed values, with $r^{2}=0.81$, although the scatter plot shows a clear sign of variable variance (Fig. 14c). The relationship between modeled and observed standard deviation in SSH shows more scatter and reduced correlation $\left(r^{2}=\right.$ 0.45; Fig. 14f). Moreover, the modeled standard deviations of SSH are generally smaller than those observed. One possible cause of the under-prediction of sea level variability by the model is its relatively coarse horizontal resolution $\left(1 / 4^{\circ}\right)$, which would prevent an accurate simulation of the vigorous eddy field observed in the Northwest Atlantic.

The model mean horizontal velocities along line W (Fig. 5c,d) are compared with mean velocities observed between 2004-2014 (Fig. 5a,b). Table 3 shows that the correlation between modeled and observed velocities is poor: $r^{2}=0.01$ for $u$ and 0.13 for $v$, where $u(v)$ here refer to along-line 
(across-line) velocity. The poor agreement of model results with the data is due in large part to the fact that the locations of the GS and the DWBC are not accurately simulated along line W: the modeled GS is southeast of the observed GS and the modeled DWBC is northwest of the observed DWBC. On the other hand, the magnitude of the cross-stream velocity in the GS core as simulated by the model agrees with the observed mean speed of $\sim 0.5 \mathrm{~m} \mathrm{~s}^{-1}$. The simulated DWBC, however, is too strong compared to the observations, with a maximum speed of $0.2 \mathrm{~m} \mathrm{~s}^{-1}$ in the model vs. $0.06 \mathrm{~m} \mathrm{~s}^{-1}$ in the data. Overall, while the comparison of model results to observations suggest significant systematic errors, the model does reproduce the major elements of the Northwest Atlantic circulation (GS and DWBC) with approximately the appropriate strength (for the GS) and spatial pattern.

\begin{tabular}{|l|c|c|c|c|}
\hline & SSH & SSH standard deviation & $u$ & $v$ \\
\hline RMSE $^{a}$ & $0.51(\mathrm{~m})$ & $0.13(\mathrm{~m})$ & $8.0 \mathrm{~m} / \mathrm{s}$ & $16.4(\mathrm{~m} / \mathrm{s})$ \\
\hline bias $^{b}$ & $-0.47(\mathrm{~m})$ & $-0.12(\mathrm{~m})$ & $4.1(\mathrm{~m} / \mathrm{s})$ & $10.4(\mathrm{~m} / \mathrm{s})$ \\
\hline$r^{2}(p)$ & $0.81(<0.001)$ & $0.45(<0.001)$ & $0.01(0.15)$ & $0.13(<0.001)$ \\
\hline
\end{tabular}

a. $\mathrm{RMSE}=\sqrt{\left(\sum_{i}^{N}\left(x_{i}-x_{o b s, i}\right)^{2}\right) / N}$, where $x_{i}$ is model output at point $i, x_{o b s, i}$ is a corresponding observation, and $N$ is the total number of observations.

b. bias $=\sum_{i}^{N}\left(x_{i}-x_{o b s, i}\right) / N$

Table 3: Comparison of reference experiment with physical observations

\subsubsection{Reference Experiment: ${ }^{230} \mathrm{Th}_{d, p}$ distributions}

The reference experiment assumes horizontally and vertically uniform values of $k_{1}, k_{-1}^{*}$, and $w$ obtained from ${ }^{230} \mathrm{Th}_{d, p}$ measurements at station GT11-12 (Fig. 2). Specifically, estimates of $k_{1}$, $k^{*}-1$, and $w$ are derived from an ordinary least-square fit of the reversible exchange equations (i.e., eqs. (5.2a-5.2b) with unsteadiness and transport terms omitted) to the data in the upper $3000 \mathrm{~m}$ at that station. The values of these and other parameters for the reference experiment are included in Table 4.

\begin{tabular}{|l|c|c|}
\hline$k^{*}{ }_{1}$ & Th attachment rate constant & $0.4 \mathrm{yr}^{-1}$ \\
\hline$k_{-1}$ & Th detachment rate constant & $3.69 \mathrm{yr}^{-1}$ \\
\hline$w$ & particle sinking speed & $-959 \mathrm{~m} \mathrm{yr}^{-1}$ \\
\hline$l_{z}$ & length scale for ${ }^{230} \mathrm{Th}_{d, p}$ boundary values & $1600 \mathrm{~m}$ \\
\hline$A_{d, 0}$ & maximum ${ }^{230} \mathrm{Th}_{d}$ boundary value & $0.860 \mathrm{dpm} \mathrm{m}^{-3}$ \\
\hline$A_{p, 0}$ & maximum ${ }^{230} \mathrm{Th}_{p}$ boundary value & $0.075 \mathrm{dpm} \mathrm{m}^{-3}$ \\
\hline
\end{tabular}

Table 4: Parameters for reference experiment.

In the reference experiment, the domain-averaged ${ }^{230} \mathrm{Th}_{d, p}$ activities appear to approach steady state values after about 9 yrs or 3285 days (Fig. 12b,c). As a result, the ${ }^{230} \mathrm{Th}_{d, p}$ activities are 
averaged over the last year of each simulation when compared to the (pre-)GEOTRACES data. Note that even if the model were to contain all the processes affecting ${ }^{230} \mathrm{Th}_{d, p}$ distributions in the Northwest Atlantic, the comparison to data would still be tenuous since the solution after 9 yrs only represents an approximate steady state and the time-dependency of ${ }^{230} \mathrm{Th}_{d, p}$ at the (preGEOTRACES stations is unknown.

Figures (15) to (17) compare the simulated ${ }^{230} \mathrm{Th}_{d, p}$ profiles with the (pre-)GEOTRACES observations. Given the differences in vertical ${ }^{230} \mathrm{Th}$ gradients observed above and below $3000 \mathrm{~m}$, we diagnose the model fit to the data in both depth intervals. Measures of goodness of fit are listed in Table 5, while scatterplots of observed vs. simulated activities are shown in Figure 18. It is seen that the model generally produces an increase with depth in the activities of both phases at the (pre-)GEOTRACES stations. Below $1000 \mathrm{~m}$ both activities continue to increase with depth, but less markedly than above $1000 \mathrm{~m}$. For z $<3000 \mathrm{~m}$, the model explains $91 \%$ (40\%) of the variance of the ${ }^{230} \mathrm{Th}_{d}\left({ }^{230} \mathrm{Th}_{p}\right)$ data. For $\mathrm{z}<3000 \mathrm{~m}$, the fraction of explained variance drops to $36 \%$ and $5 \%$ for, respectively, the dissolved phase and the particulate phase. The RMSE and the bias are also noticeably greater for ${ }^{230} \mathrm{Th}_{d}$ below $3000 \mathrm{~m}$ than above. The model simulates quasi-uniform values of ${ }^{230} \mathrm{Th}_{d, p}$ below $1000 \mathrm{~m}$ at stations GT11-01 and GT11-02. The nearly uniform ${ }^{230} \mathrm{Th}_{d}$ simulated by the model appears consistent with ${ }^{230} \mathrm{Th}_{d}$ data below $1000 \mathrm{~m}$ at these stations, which also show smaller vertical gradients below $1000 \mathrm{~m}$ than above (Fig. 15). However, no such reduction in the vertical gradient in ${ }^{230} \mathrm{Th}_{p}$ is observed at these stations (Fig. 16).

As noticed above, below $3000 \mathrm{~m}$, the agreement between the model and the data appreciably deteriorates for the activities in both phases. The observed ${ }^{230} \mathrm{Th}_{d}$ deficits compared to values linearly extrapolated from data in the upper $3000 \mathrm{~m}$ and expected from reversible exchange cannot be explained by the model. Observed deficits in ${ }^{230} \mathrm{Th}_{p}$ are also apparent below $3000 \mathrm{~m}$ at GT11-06 and GT11-08, and similarly cannot be explained by the model. In fact, the model skill is weakest in explaining the ${ }^{230} \mathrm{Th}_{p}$ data within this depth interval (Table 5).

\begin{tabular}{|l|c|c|c|c|}
\hline & ${ }^{230} \mathrm{Th}_{d}(\mathrm{z}<3000 \mathrm{~m})$ & ${ }^{230} \mathrm{Th}_{d}(\mathrm{z}>3000 \mathrm{~m})$ & ${ }^{230} \mathrm{Th}_{p}(\mathrm{z}<3000 \mathrm{~m})$ & ${ }^{230} \mathrm{Th}_{p}(\mathrm{z}>3000 \mathrm{~m})$ \\
\hline RMSE & $0.08\left(\mathrm{dpm} \mathrm{m}^{-3}\right)$ & $0.22\left(\mathrm{dpm} \mathrm{m}^{-3}\right)$ & $0.02\left(\mathrm{dpm} \mathrm{m}^{-3}\right)$ & $\left.0.02(\mathrm{dpm} \mathrm{m})^{-3}\right)$ \\
\hline bias & $-0.07\left(\mathrm{dpm} \mathrm{m}^{-3}\right)$ & $-0.17\left(\mathrm{dpm} \mathrm{m}^{-3}\right)$ & $0.01\left(\mathrm{dpm} \mathrm{m}^{-3}\right)$ & $-0.01\left(\mathrm{dpm} \mathrm{m}^{-3}\right)$ \\
\hline$r^{2}(p)$ & $0.91(<0.001)$ & $0.36(<0.001)$ & $0.40(<0.001)$ & $0.05(0.46)$ \\
\hline
\end{tabular}

Table 5: Comparison of reference experiment with ${ }^{230} \mathrm{Th}_{d, p}$ data. 


\subsubsection{Sensitivity Experiments}

While the reference experiment describes most of the variance in ${ }^{230} \mathrm{Th}_{d}$ above $3000 \mathrm{~m}$, the simulated ${ }^{230} \mathrm{Th}_{d}$ activities below $3000 \mathrm{~m}$ and ${ }^{230} \mathrm{Th}_{p}$ activities at all depths display a much poorer fit to the observations. In this section, the model's ability to fit the data is explored in a variety of sensitivity experiments. Table 6 contains a brief description of each experiment. Due to the large number of experiments considered, figures depicting their results are placed in Appendix B.

\begin{tabular}{|c|c|c|c|}
\hline experiment name & Boundary values of ${ }^{230} \mathrm{Th}_{d, p}$ & Boundary fluxes of GS/DWBC & Scavenging Intensity $\left(k_{1}\right)$ \\
\hline linbnd & ${ }^{230} \mathrm{Th}$ increases linearly with $z$ & no change & uniform \\
\hline invbnd & ${ }^{230} \mathrm{Th}$ increases to $3000 \mathrm{~m}$, then decreases & no change & uniform \\
\hline smlDWBCin & no change $^{a}$ & DWBC inflow of $20 \mathrm{~Sv}$ & uniform \\
\hline larGSout & no change & GS outflow of $150 \mathrm{~Sv}$ & uniform \\
\hline$k_{1}$ var1 & no change & no change & $\begin{array}{c}\text { varying, lecc }=\sqrt{84}, \mathrm{lf}=1.6 \\
\Delta k_{1}=5.38 \mathrm{yr}^{-1}, b=4.72\end{array}$ \\
\hline$k_{1}$ var2 & no change & no change & $\begin{array}{c}\text { varying, lecc }=\sqrt{168}, \mathrm{lf}=3.2 \\
\Delta k_{1}=5.38 \mathrm{yr}^{-1}, b=4.72\end{array}$ \\
\hline$k_{1}$ var3 & no change & no change & $\begin{array}{l}\text { varying, lecc }=\sqrt{41}, \mathrm{lf}=0.8 \\
\Delta k_{1}=5.38 \mathrm{yr}^{-1}, b=4.72\end{array}$ \\
\hline$k_{1} \operatorname{var} 4$ & no change & no change & $\begin{array}{l}\text { varying, lecc }=\sqrt{84}, \mathrm{lf}=1.6 \\
\Delta k_{1}=5.38 \mathrm{yr}^{-1}, b=15.12\end{array}$ \\
\hline$k_{1} \operatorname{var} 5$ & no change & no change & $\begin{array}{c}\text { varying, lecc }=\sqrt{84}, \mathrm{lf}=1.6 \\
\Delta k_{1}=5.38 \mathrm{yr}^{-1}, b=1.72\end{array}$ \\
\hline$k_{1} \operatorname{var} 6$ & no change & no change & $\begin{array}{l}\text { varying, lecc }=\sqrt{84}, \mathrm{lf}=1.6 \\
\Delta k_{1}=57.29 \mathrm{yr}^{-1}, b=4.72\end{array}$ \\
\hline$k_{1} \operatorname{var} 7$ & no change & no change & $\begin{array}{c}\text { varying, lecc }=\sqrt{84}, \mathrm{lf}=1.6 \\
\Delta k_{1}=1.00 \mathrm{yr}^{-1}, b=4.72\end{array}$ \\
\hline
\end{tabular}

a. No change relative to reference experiment.

Table 6: Sensitivity experiments with the ocean circulation- ${ }^{230}$ Th transport model.

\subsubsection{Influence of Boundary Values of ${ }^{230} \mathbf{T h}_{d, p}$}

In this section, we explore the sensitivity of the simulated ${ }^{230} \mathrm{Th}_{d, p}$ distributions to the ${ }^{230} \mathrm{Th}_{d, p}$ activities specified at the open boundaries of the model domain (eastern and southern boundaries). In these sensitivity experiments, values of ${ }^{230} \mathrm{Th}_{d, p}$ are changed all along the eastern and southern boundaries compared to the reference experiment, except along the NE segment where, boundary values of ${ }^{230} \mathrm{Th}_{d, p}$ are kept equal to ${ }^{230} \mathrm{Th}_{d, p}$ averages derived from Labrador Sea data (Fig. 11). Figure 19 shows the vertical profiles of ${ }^{230} \mathrm{Th}_{d, p}$ assumed as open boundary conditions in different experiments. In the experiment "linbnd", ${ }^{230} \mathrm{Th}_{d, p}$ along the open boundaries increases quasi-linearly with depth. In the experiment "invbnd", ${ }^{230} \mathrm{Th}_{d, p}$ along the open boundaries linearly increase with depth above $3000 \mathrm{~m}$ and linearly decrease with depth below. These two experiments implicitly assume that different processes are responsible for the ${ }^{230} \mathrm{Th}_{d, p}$ distributions at the boundaries: whereas "linbnd" assumes these distributions are caused by reversible exchange with uniform rate 
constants, "invbnd" assumes a process that depletes ${ }^{230} \mathrm{Th}_{d, p}$ below $3000 \mathrm{~m}$, such as the intrusion of ${ }^{230}$ Th-poor waters from higher latitudes, or enhanced scavenging below this depth.

The (dis)agreement of simulated ${ }^{230} \mathrm{Th}_{d, p}$ with the observations varies for different boundary values of ${ }^{230} \mathrm{Th}_{d, p}$, but not drastically so (Table 7 ). The largest improvement appears to be the bias reduction for ${ }^{230} \mathrm{Th}_{d}$ below $3000 \mathrm{~m}$ for experiment "linbnd". However, the variance in the ${ }^{230} \mathrm{Th}_{p}$ observations above $3000 \mathrm{~m}$ explained by the model $\left(r^{2}\right)$ only improves slightly for experiment "linbnd." The variance in the ${ }^{230} \mathrm{Th}_{d}$ observations below $3000 \mathrm{~m}$ explained by the model increases for experiment "invbnd", and it decreases above and below $3000 \mathrm{~m}$ in experiment "linbnd", compared to the reference experiment. Thus, an overall improvement is obtained when considering boundary values of ${ }^{230} \mathrm{Th}_{d, p}$ for which the activities increase linearly to $3000 \mathrm{~m}$, and decrease below $3000 \mathrm{~m}$ . The simulated distributions of ${ }^{230} \mathrm{Th}_{p}$, however, do not appear to be very sensitive to the values assumed for the two phases at the eastern and southern boundaries of the domain.

\begin{tabular}{|l|c|c|c|c|}
\hline & ${ }^{230} \mathrm{Th}_{d}(\mathrm{z}<3000 \mathrm{~m})$ & ${ }^{230} \mathrm{Th}_{d}(\mathrm{z}>3000 \mathrm{~m})$ & ${ }^{230} \mathrm{Th}_{p}(\mathrm{z}<3000 \mathrm{~m})$ & ${ }^{230} \mathrm{Th}_{p}(\mathrm{z}>3000 \mathrm{~m})$ \\
\hline RMSE linbnd & $0.07\left(\mathrm{dpm} \mathrm{m}^{-3}\right)$ & $0.16\left(\mathrm{dpm} \mathrm{m}^{-3}\right)$ & $0.02\left(\mathrm{dpm} \mathrm{m}^{-3}\right)$ & $0.02\left(\mathrm{dpm} \mathrm{m} \mathrm{m}^{-3}\right)$ \\
\hline bias linbnd & $0.01\left(\mathrm{dpm} \mathrm{m}^{-3}\right)$ & $-0.04\left(\mathrm{dpm} \mathrm{m}^{-3}\right)$ & $\left.0.01(\mathrm{dpm} \mathrm{m})^{-3}\right)$ & $0.00\left(\mathrm{dpm} \mathrm{m}^{-3}\right)$ \\
\hline$r^{2}(p)$ linbnd & $0.85(<0.001)$ & $0.25(0.03)$ & $0.43(<0.001)$ & $<0.001(0.99)$ \\
\hline & & & & $0.02\left(\mathrm{dpm} \mathrm{m}^{-3}\right)$ \\
\hline RMSE invbnd & $0.07\left(\mathrm{dpm} \mathrm{m}^{-3}\right)$ & $0.16\left(\mathrm{dpm} \mathrm{m}^{-3}\right)$ & $0.02\left(\mathrm{dpm} \mathrm{m}^{-3}\right)$ & $-0.01\left(\mathrm{dpm} \mathrm{m}^{-3}\right)$ \\
\hline bias invbnd & $-0.05\left(\mathrm{dpm} \mathrm{m}^{-3}\right)$ & $-0.09\left(\mathrm{dpm} \mathrm{m}^{-3}\right)$ & $0.01\left(\mathrm{dpm} \mathrm{m}^{-3}\right)$ & $0.04(0.52)$ \\
\hline$r^{2}(p)$ invbnd & $0.91(<0.001)$ & $0.48(<0.001)$ & $0.42(<0.001)$ & 0 \\
\hline
\end{tabular}

Table 7: Comparison of sensitivity experiments with ${ }^{230} \mathrm{Th}_{d, p}$ data: Boundary values of ${ }^{230} \mathrm{Th}_{d, p}$.

\subsubsection{Influence of DWBC Inflow and GS Outflow}

Next, the sensitivity of the simulated ${ }^{230} \mathrm{Th}_{d, p}$ is tested against changes in circulation. To this end, two experiments are performed. In experiment "smlDWBCin", the inflow of Slope Water into the domain along the NE segment is lowered from 40 to $20 \mathrm{~Sv}$. Since the vertical profiles of ${ }^{230} \mathrm{Th}_{d, p}$ along the NE segment are set by the mean ${ }^{230}$ Th activities measured in the Labrador Sea (Fig. 11), this experiment tests the sensitivity of ${ }^{230} \mathrm{Th}_{d, p}$ to the inflow of low ${ }^{230} \mathrm{Th}$ waters. To maintain volume conservation, the outflow of DWBC at the southern boundary between $76.5^{\circ} \mathrm{W}$ to $75.5^{\circ} \mathrm{W}$ is lowered to 0 Sv. In experiment "larGSout", the outflow of the GS at the eastern boundary between $39^{\circ} \mathrm{N}$ and $41^{\circ} \mathrm{N}$ is raised from 90 to $150 \mathrm{~Sv}$, a value that represents the synoptic average of the GS transport along 55 W (Hogg et al., 1986). For volume conservation, the Sargasso Sea inflow at the eastern boundary between $28^{\circ} \mathrm{N}$ and $39^{\circ} \mathrm{N}$ is raised from 40 to $100 \mathrm{~Sv}$ in this experiment.

For both experiments, "smlDWBCin" and "larGSout", the major results do not deviate ap- 
preciably from those of the reference experiment (Table 8). In all three experiments (reference, "smlDWBCin", "larGSout") ${ }^{230} \mathrm{Th}_{d, p}$ have nearly the same RMSE and bias above and below 3000 m. Compared to the reference experiment, $r^{2}$ for ${ }^{230} \mathrm{Th}_{d}$ above $3000 \mathrm{~m}$ remains almost unchanged in both sensitivity experiments, and increases slightly below $3000 \mathrm{~m}$ for ${ }^{230} \mathrm{Th}_{d}$ and above $3000 \mathrm{~m}$ for ${ }^{230} \mathrm{Th}_{p}$. Thus, the model fit to the data does not appear to depend strongly on the boundary transports employed here. Consistent with this result, individual profiles of simulated ${ }^{230} \mathrm{Th}_{d, p}$ also do not show any dramatic changes when modifying either the DWBC inflow or GS outflow (compare Figs. 15-18 with Figs. B9-12 and Figs. B13-15). Overall, there appears to be little sensitivity of the simulated ${ }^{230} \mathrm{Th}_{d, p}$ distributions to the strengths of DWBC and GS transports that are assumed at the eastern and southern boundaries of the domain.

\begin{tabular}{|l|c|c|c|c|}
\hline & ${ }^{230} \mathrm{Th}_{d}(\mathrm{z}<3000 \mathrm{~m})$ & ${ }^{230} \mathrm{Th}_{d}(\mathrm{z}>3000 \mathrm{~m})$ & ${ }^{230} \mathrm{Th}_{p}(\mathrm{z}<3000 \mathrm{~m})$ & ${ }^{230} \mathrm{Th}_{p}(\mathrm{z}>3000 \mathrm{~m})$ \\
\hline RMSE smlDWBCin & $0.09\left(\mathrm{dpm} \mathrm{m}^{-3}\right)$ & $0.23\left(\mathrm{dpm} \mathrm{m}^{-3}\right)$ & $\left.0.02(\mathrm{dpm} \mathrm{m})^{-3}\right)$ & $0.02\left(\mathrm{dpm} \mathrm{m}^{-3}\right)$ \\
\hline bias smlDWBCin & $\left.-0.08(\mathrm{dpm} \mathrm{m})^{-3}\right)$ & $-0.18\left(\mathrm{dpm} \mathrm{m}^{-3}\right)$ & $0.00\left(\mathrm{dpm} \mathrm{m}^{-3}\right)$ & $-0.01\left(\mathrm{dpm} \mathrm{m}^{-3}\right)$ \\
\hline$r^{2}(p)$ smlDWBCin & $0.90(<0.001)$ & $0.42(<0.001)$ & $0.43(<0.001)$ & $0.04(0.48)$ \\
\hline & & & & \\
\hline RMSE larGSout & $0.09\left(\mathrm{dpm} \mathrm{m}^{-3}\right)$ & $0.24\left(\mathrm{dpm} \mathrm{m}^{-3}\right)$ & $0.02\left(\mathrm{dpm} \mathrm{m}^{-3}\right)$ & $0.02\left(\mathrm{dpm} \mathrm{m}^{-3}\right)$ \\
\hline bias larGSout & $-0.08\left(\mathrm{dpm} \mathrm{m}^{-3}\right)$ & $-0.20\left(\mathrm{dpm} \mathrm{m}^{-3}\right)$ & $0.00\left(\mathrm{dpm} \mathrm{m}^{-3}\right)$ & $-0.02\left(\mathrm{dpm} \mathrm{m}^{-3}\right)$ \\
\hline$r^{2}(p)$ larGSout & $0.91(<0.001)$ & $0.39(<0.001)$ & $0.43(<0.001)$ & $0.06(0.38)$ \\
\hline
\end{tabular}

Table 8: Comparison of sensitivity experiments with ${ }^{230} \mathrm{Th}_{d, p}$ : Boundary Transports.

\subsubsection{Influence of Spatial Variations in Scavenging}

Finally, we conduct seven experiments to test the sensitivity of the simulated ${ }^{230} \mathrm{Th}_{d, p}$ distributions to vertical and lateral variations in particle scavenging as described by $k_{1}$ (Table 6). These experiments are meant to capture enhanced thorium scavenging by resuspended sediment. Therefore, we assume that $k_{1}$ can only deviate from the uniform value of our reference simulation $\left(k_{1,0}\right.$ below) in regions where significant resuspended sediment concentrations have been observed. In a first experiment (" $k_{1}$ var1"), $k_{1}$ is allowed to deviate from $k_{1,0}$ within an elliptical region whose bounds roughly follow the $2000-\mu \mathrm{g} \mathrm{m}^{-2}$ suspended sediment load contour mapped from a compilation of nephelometer profiles in the western North Atlantic (Gardner et al., 2017). Since the contours in this map are constructed from a relatively small number of irregularly spaced profiles, and because our elliptical region is only a crude representation of the area of the Northwest Atlantic with elevated suspended sediment load, we test the sensitivity of our results to the size of the ellipse, by adjusting the linear eccentricity (lecc) and the length of the line segment joining two points of the ellipse and perpendicular to the semi-major axis at a focal point (for brevity, this segment will 
be referred to as lf) in two experiments (experiments " $k_{1}$ var2" and " $k_{1}$ var3"; Table 6). Thus, in total, we consider three elliptical regions within which variations in $k_{1}$ are allowed (Fig. 20).

Within each elliptical region of enhanced Th scavenging, $k_{1}$ is allowed to vary with depth according to

$$
k_{1}=k_{1,0}+\Delta k_{1}\left(\frac{z-3000 m}{h-3000 m}\right)^{b} \mathcal{H}(z-3000 m) .
$$

Here, $k_{1,0}=0.4 \mathrm{yr}^{-1}$ is the background value of $k_{1}$ in the reference experiment and outside of the bottom scavenging region, $\Delta k_{1}$ is the enhancement in $k_{1}$ caused by resuspended sediment, i.e., the difference between $k_{1}$ at $z=3000 \mathrm{~m}$ and at the seafloor, and $\mathcal{H}(z-3000 \mathrm{~m})$ is a Heaviside function which is 0 when $z<3000 \mathrm{~m}, 1 / 2$ when $z=3000 \mathrm{~m}$, and 1 when $z>3000 \mathrm{~m}$. The exponent $b$ in equation (5.9) is taken as positive, indicating that $k_{1}$ decreases with increasing height above the seabed. Since $k_{1}$ is expected to increase with particle concentration (Honeyman et al., 1988), it is assumed that $\Delta k_{1}$ and $b$ are related to the increase in particle concentration with depth that is observed below $3000 \mathrm{~m}$ in each elliptical region. A range of $b$ values are estimated from an equation similar to (5.9) but describing the vertical variations of the beam attenuation coefficient associated with particles $\left(c_{p}\right)$ below $3000 \mathrm{~m}$. Specifically, the equation for $c_{p}(z)$ is fitted to $c_{p}$ profiles measured by transmissometry along CTD casts at stations GT11-04, GT11-06, GT11-08 and GT11-10. These fits are restricted to layers of the water column where a plot of $\log _{10}$ of $c_{p}$ vs. water depth suggests a linear relationship (Fig. 21).

This approach to estimate vertical variations of $k_{1}$ involves two assumptions, i.e., that $k_{1}$ varies linearly with particle concentration, and that particle concentration varies linearly with $c_{p}$. The former assumption is supported by results from laboratory experiments (Nyffeler et al., 1984), and by analyses of Th isotope and particle data collected in the Panama and Guatemala Basins (Bacon and Anderson, 1982) and at open-ocean stations of GA03 (Lerner et al., 2017), although in this latter study the estimated relationship between $k_{1}$ and $P$ was not strictly linear. The latter assumption is supported by studies showing that $c_{p}$ presents a linear response to suspended particle concentration in multiple oceanic environments. These studies rely on transmissometry data paired with suspended particle concentrations measured (i) with a coulter counter during the survey of HEBBLE (Gardner et al., 1985) or (ii) from large volume filtration during the U.S. GEOTRACES 
Eastern Tropical South Pacific survey (Ohnemus et al., 2017), though in this latter study the response to total suspended particle concentration was not as linear as that to particulate organic matter.

A range of $\Delta k_{1}$ values is also estimated by assuming that $k_{1, \max } / k_{1,0}=c_{p, \max } / c_{p, 0}$, where $c_{p, 0}$ $\left(c_{p, \max }\right)$ is the coefficient at $z=3000 \mathrm{~m}$ (at the bottom), and $k_{1, \max }$ is maximum value of $k_{1}$ at the bottom. Simulations based on minimum, maximum, and median values of $\Delta k_{1}$ and $b$ are performed (experiments " $k_{1}$ var4", " $k_{1}$ var5", " $k_{1}$ var6", and " $k_{1}$ var'7"; Table 6). As an example, Figure 22 shows the $k_{1}$ profile at a location within the region of bottom scavenging for each of the combinations of $\Delta k_{1}$ and $b$ values considered.

The measures of the goodness of fit between the simulated and observed ${ }^{230} \mathrm{Th}_{d, p}$ are provided in Table 9. Above $3000 \mathrm{~m}$, different experiments result in only a slight change in the model fit to the observed ${ }^{230} \mathrm{Th}_{d}$ activities (RMSE, the bias, $r^{2}$ for ${ }^{230} \mathrm{Th}_{d, p}$ above 3000 among all of the experiments in Table 9). Below $3000 \mathrm{~m}$, the model explains over $50 \%$ of the variance in observed ${ }^{230} \mathrm{Th}_{d}$ activities in experiments $k_{1}$ var $1, k_{1}$ var2, $k_{1}$ var 5 , and $k_{1}$ var6. The best fit to these activities is achieved in experiment $k_{1} \operatorname{var} 1\left(r^{2}=0.56\right)$, which corresponds to in an increase in $r^{2}$ by 0.20 compared with the reference experiment. As with the reference experiment, the model fits to ${ }^{230} \mathrm{Th}_{p}$ data below $3000 \mathrm{~m}$ is not significant. One exception to this general feature is experiment $k_{1} \operatorname{var} 5$, where the relationship between the simulated and observed ${ }^{230} \mathrm{Th}_{p}$ is significant (at the $\alpha=0.10$ significance level) but negative below $3000 \mathrm{~m}\left(r^{2}=0.26, p=0.06\right)$.

Both decreasing the exponent $b$ and increasing $\Delta k_{1}$ in equation (5.9) (experiments $k_{1}$ var5 and $k_{1}$ var6 $)$ reduces the fit to ${ }^{230} \mathrm{Th}_{p}$ data below $3000 \mathrm{~m}$, as the RMSE increases in both of these experiments. Moreover, while $r^{2}$ for ${ }^{230} \mathrm{Th}_{p}$ increases in both of these experiments, it becomes negative, with the observed ${ }^{230} \mathrm{Th}_{p}$ below $3000 \mathrm{~m}$ tending to decrease as the simulated ${ }^{230} \mathrm{Th}_{p}$ increases (Fig. B40 and Fig. B44). Thus, the model severely overestimates the observed ${ }^{230} \mathrm{Th}_{p}$ below $3000 \mathrm{~m}$, with the model-data difference increasing as the simulated ${ }^{230} \mathrm{Th}_{p}$ activities increase. The simulated ${ }^{230} \mathrm{Th}_{p}$ activities in both of these experiments generally increase water depth below $3000 \mathrm{~m}$, as shown in Figs. B38 and B42. The experiment that produces the "best" fit to the data cannot be easily determined, as the goodness of fit depends on the phase considered (dissolved or particulate). If considering ${ }^{230} \mathrm{Th}_{d}$, experiments $k_{1} \operatorname{var} 1$ and $k_{1} \operatorname{var} 5$ produce the best fit to the data, while if considering ${ }^{230} \mathrm{Th}_{p}$, experiment $k_{1}$ var2 produces the best fit. However, in all sensitivity experiments 


\begin{tabular}{|c|c|c|c|c|}
\hline & ${ }^{230} \mathrm{Th}_{d}(\mathrm{z}<3000 \mathrm{~m})$ & ${ }^{230} \mathrm{Th}_{d}(\mathrm{z}>3000 \mathrm{~m})$ & ${ }^{230} \mathrm{Th}_{p}(\mathrm{z}<3000 \mathrm{~m})$ & ${ }^{230} \mathrm{Th}_{p}(\mathrm{z}>3000 \mathrm{~m})$ \\
\hline $\mathrm{RMSE}^{a} k_{1}$ var 1 & $0.08\left(\mathrm{dpm} \mathrm{m} \mathrm{m}^{-3}\right)$ & $0.14\left(\mathrm{dpm} \mathrm{m} \mathrm{m}^{-3}\right)$ & $0.02\left(\mathrm{dpm} \mathrm{m} \mathrm{m}^{-3}\right)$ & $0.03\left(\mathrm{dpm} \mathrm{m} \mathrm{m}^{-3}\right)$ \\
\hline $\operatorname{bias}^{b} k_{1} \operatorname{var} 1$ & $-0.06\left(\mathrm{dpm} \mathrm{m} \mathrm{m}^{-3}\right)$ & $-0.08\left(\mathrm{dpm} \mathrm{m} \mathrm{m}^{-3}\right)$ & $0.01\left(\mathrm{dpm} \mathrm{m} \mathrm{m}^{-3}\right)$ & $-0.02\left(\mathrm{dpm} \mathrm{m}^{-3}\right)$ \\
\hline$r^{2}(p) k_{1} \operatorname{var} 1$ & $0.91(<0.001)$ & $0.56(<0.001$ & $0.40(<0.001)$ & $0.02(0.61)^{a}$ \\
\hline RMSE $k_{1}$ var2 & $0.07\left(\mathrm{dpm} \mathrm{m}^{-3}\right)$ & $0.12\left(\mathrm{dpm} \mathrm{m} \mathrm{m}^{-3}\right)$ & $0.02\left(\mathrm{dpm} \mathrm{m} \mathrm{m}^{-3}\right)$ & $0.04\left(\mathrm{dpm} \mathrm{m} \mathrm{m}^{-3}\right)$ \\
\hline bias $k_{1}$ var2 & $-0.05\left(\mathrm{dpm} \mathrm{\textrm {m } ^ { - 3 } )}\right.$ & $0.01\left(\mathrm{dpm} \mathrm{m} \mathrm{m}^{-3}\right)$ & $0.01\left(\mathrm{dpm} \mathrm{m} \mathrm{m}^{-3}\right)$ & $-0.03\left(\mathrm{dpm} \mathrm{\textrm {m } ^ { - 3 } )}\right.$ \\
\hline$r^{2}(p) k_{1}$ var2 & $0.91(<0.001)$ & $0.54(<0.001)$ & $0.41(<0.001)$ & $0.07(0.36))$ \\
\hline RMSE $k_{1}$ var3 & $0.08\left(\mathrm{dpm} \mathrm{m} \mathrm{m}^{-3}\right)$ & $0.21\left(\mathrm{dpm} \mathrm{m} \mathrm{m}^{-3}\right)$ & $0.02\left(\mathrm{dpm} \mathrm{m} \mathrm{m}^{-3}\right)$ & $0.02\left(\mathrm{dpm} \mathrm{m}^{-3}\right)$ \\
\hline bias $k_{1}$ var3 & $-0.07\left(\mathrm{dpm} \mathrm{\textrm {m } ^ { - 3 } )}\right.$ & $-0.16\left(\mathrm{dpm} \mathrm{\textrm {m } ^ { - 3 } )}\right.$ & $0.01\left(\mathrm{dpm} \mathrm{m} \mathrm{m}^{-3}\right)$ & $-0.01\left(\mathrm{dpm} \mathrm{\textrm {m } ^ { - 3 } )}\right.$ \\
\hline$r^{2}(p) k_{1}$ var3 & $0.91(<0.001)$ & $0.38(0.003)$ & $0.40(<0.001)$ & $0.03(0.59)$ \\
\hline RMSE $k_{1}$ var4 & $0.08\left(\mathrm{dpm} \mathrm{m} \mathrm{m}^{-3}\right)$ & $0.20\left(\mathrm{dpm} \mathrm{m} \mathrm{m}^{-3}\right)$ & $0.02\left(\mathrm{dpm} \mathrm{m}^{-3}\right)$ & $0.02\left(\mathrm{dpm} \mathrm{m}^{-3}\right)$ \\
\hline bias $k_{1}$ var 4 & $-0.07\left(\mathrm{dpm} \mathrm{\textrm {m } ^ { - 3 } )}\right.$ & $-0.16\left(\mathrm{dpm} \mathrm{\textrm {m } ^ { - 3 } )}\right.$ & $0.01\left(\mathrm{dpm} \mathrm{m} \mathrm{m}^{-3}\right)$ & $-0.01\left(\mathrm{dpm} \mathrm{\textrm {m } ^ { - 3 } )}\right.$ \\
\hline$r^{2}(p) k_{1}$ var4 & $0.91(<0.001)$ & $0.49(<0.001)$ & $0.39(<0.001)$ & $0.03(0.56)$ \\
\hline RMSE $k_{1}$ var 5 & $0.08\left(\mathrm{dpm} \mathrm{m} \mathrm{m}^{-3}\right)$ & $0.12\left(\mathrm{dpm} \mathrm{m} \mathrm{m}^{-3}\right)$ & $0.02\left(\mathrm{dpm} \mathrm{m} \mathrm{m}^{-3}\right)$ & $0.06\left(\mathrm{dpm} \mathrm{m} \mathrm{m}^{-3}\right)$ \\
\hline bias $k_{1}$ var 5 & $-0.06\left(\mathrm{dpm} \mathrm{\textrm {m } ^ { - 3 } )}\right.$ & $0.01\left(\mathrm{dpm} \mathrm{m} \mathrm{m}^{-3}\right)$ & $0.01\left(\mathrm{dpm} \mathrm{m} \mathrm{m}^{-3}\right)$ & $-0.04\left(\mathrm{dpm} \mathrm{\textrm {m } ^ { - 3 } )}\right.$ \\
\hline$r^{2}(p) k_{1}$ var5 & $0.91(<0.001)$ & $0.55(<0.001)$ & $0.41(<0.001)$ & $0.26(0.06)^{a}$ \\
\hline RMSE $k_{1}$ var6 & $0.07\left(\mathrm{dpm} \mathrm{m} \mathrm{m}^{-3}\right)$ & $0.16\left(\mathrm{dpm} \mathrm{m} \mathrm{m}^{-3}\right)$ & $0.02\left(\mathrm{dpm} \mathrm{m}^{-3}\right)$ & $0.06\left(\mathrm{dpm} \mathrm{m} \mathrm{m}^{-3}\right)$ \\
\hline bias $k_{1} \operatorname{var} 6$ & $-0.06\left(\mathrm{dpm} \mathrm{m} \mathrm{m}^{-3}\right)$ & $0.06\left(\mathrm{dpm} \mathrm{m} \mathrm{m}^{-3}\right)$ & $0.01\left(\mathrm{dpm} \mathrm{m} \mathrm{m}^{-3}\right)$ & $-0.04\left(\mathrm{dpm} \mathrm{m} \mathrm{m}^{-3}\right)$ \\
\hline$r^{2}(p) k_{1} \operatorname{var} 6$ & $0.89(<0.001)$ & $0.53(<0.001)$ & $0.40(<0.001)$ & $0.19(0.12)^{a}$ \\
\hline RMSE $k_{1}$ var 7 & $0.08\left(\mathrm{dpm} \mathrm{m} \mathrm{m}^{-3}\right)$ & $0.19\left(\mathrm{dpm} \mathrm{m}^{-3}\right)$ & $0.02\left(\mathrm{dpm} \mathrm{m}^{-3}\right)$ & $0.02\left(\mathrm{dpm} \mathrm{m}^{-3}\right)$ \\
\hline bias $k_{1}$ var 7 & $-0.07\left(\mathrm{dpm} \mathrm{m^{-3 } )}\right.$ & $-0.14\left(\mathrm{dpm} \mathrm{m} \mathrm{m}^{-3}\right)$ & $0.01\left(\mathrm{dpm} \mathrm{m} \mathrm{m}^{-3}\right)$ & $-0.01\left(\mathrm{dpm} \mathrm{m} \mathrm{m}^{-3}\right)$ \\
\hline$r^{2}(p) k_{1}$ var $^{\prime}$ & $0.91(<0.001)$ & $0.48(<0.001)$ & $0.40(<0.001)$ & $0.02(0.66)$ \\
\hline
\end{tabular}

a. The relationship between observed and simulated values is negative.

Table 9: Comparison of sensitivity experiments with trace metal observations: Spatial variations in $k_{1}$.

dealing with spatial variations in particle scavenging, the fit to the ${ }^{230} \mathrm{Th}_{d}$ data is improved compared to the reference experiment, suggesting that variations in scavenging intensity are required to explain the observed ${ }^{230} \mathrm{Th}_{d}$ distribution in the Northwest Atlantic. However, explaining the ${ }^{230} \mathrm{Th}_{p}$ data is more challenging.

\subsection{Discussion}

The previous sections include results from eleven sensitivity experiments, but only a fraction of these experiments displayed an improvement in the ability of the model to describe the data (Fig 23). Below $3000 \mathrm{~m}$, the fit to ${ }^{230} \mathrm{Th}_{d}$ observations is only slightly sensitive to changes in the DWBC inflow and GS outflow. The fit to ${ }^{230} \mathrm{Th}_{d}$ data below $3000 \mathrm{~m}$ improves in experiment "invbnd," while the fit to ${ }^{230} \mathrm{Th}_{d}$ data above and below $3000 \mathrm{~m}$ deteriorates in experiment "linbnd." The largest improvements in the fit to ${ }^{230} \mathrm{Th}_{d}$ are obtained in experiments allowing spatial variations in $k_{1}$. In each of these experiments, the fits to ${ }^{230} \mathrm{Th}_{d, p}$ data above $3000 \mathrm{~m}$ change only slightly. The best fit to ${ }^{230} \mathrm{Th}_{d}$ data below $3000 \mathrm{~m}$ is for experiment " $k_{1}$ var1", for which $r^{2}$ is increased by 0.20 compared to 
the reference experiment. Experiments $k_{1}$ var2, $k_{1} \operatorname{var} 5$, and $k_{1} \operatorname{var} 6$ also show changes in $r^{2}$ that are larger by 0.17 than $r^{2}$ for the reference experiment. However, for experiments $k_{1}$ var 5 and $k_{1} v a r 6$, the corresponding correlations between simulated and observed ${ }^{230} \mathrm{Th}_{p}$ below $3000 \mathrm{~m}$ become negative. Moreover, the fit to the observed ${ }^{230} \mathrm{Th}_{p}$ in all experiments is not significant at the $\alpha=0.05$ confidence level. Therefore, all experiments suggest that the model poorly represents ${ }^{230} \mathrm{Th}_{p}$ data below $3000 \mathrm{~m}$. Thus, it appears that the model can only be brought into the closest consistency with the ${ }^{230} \mathrm{Th}_{d}$ data by allowing vertical variations in $k_{1}$, but considering these variations does not improve the model's ability to represent the observed ${ }^{230} \mathrm{Th}_{p}$ data.

Differences in the ${ }^{230} \mathrm{Th}_{d, p}$ profiles simulated in the different experiments are shown at station GT11-08 (Fig. 24). For experiments that do not consider spatial variations in $k_{1}$, the deficit in ${ }^{230} \mathrm{Th}_{d}$ observed at this station cannot be reproduced. The observed deficit is better captured when spatial variations in $k_{1}$ are considered, particularly in experiments $k_{1}$ var $1, k_{1}$ var2, $k_{1}$ var 5 , and $k_{1}$ var6. On the other hand, allowing spatial variations in $k_{1}$ leads the model to strongly overestimate ${ }^{230} \mathrm{Th}_{p}$ data below $3000 \mathrm{~m}$ (Fig. 24b), particularly in experiments $k_{1}$ var5 and $k_{1}$ var6, in which $k_{1}$ below $3000 \mathrm{~m}$ attains its largest values among the experiments considered in this study (Fig. 22). Allowing for the presence of bottom scavenging both improves the model fit to the ${ }^{230} \mathrm{Th}_{d}$ data and deteriorates the fit to the ${ }^{230} \mathrm{Th}_{p}$ data below $3000 \mathrm{~m}$, though even in the reference simulation the correlation between the modeled and observed ${ }^{230} \mathrm{Th}_{p}$ activities below $3000 \mathrm{~m}$ is not significant (Table 5).

\subsubsection{Missing Processes Impacting ${ }^{230} \mathrm{Th}_{p}$}

\subsubsection{Overestimation of ${ }^{230} \mathrm{Th}_{p}$ Below $3000 \mathrm{~m}$}

The inability of the model to explain more than $50 \%$ (10\%) of the variability in ${ }^{230} \mathrm{Th}_{p}$ above (below) $3000 \mathrm{~m}$ suggests that the model is missing one or several key processes controlling the activity of ${ }^{230} \mathrm{Th}_{p}$ in the Northwest Atlantic. It is also likely that missing processes influence ${ }^{230} \mathrm{Th}_{p}$ to a larger extent than ${ }^{230} \mathrm{Th}_{d}$, which is much better explained by the model, particularly when variable scavenging intensity is considered. Below $3000 \mathrm{~m}$, the simulation that displays that best fit to ${ }^{230} \mathrm{Th}_{d}$ data, experiment $k_{1}$ var1, overestimates ${ }^{230} \mathrm{Th}_{p}$ data in the same depth interval at stations GT11-08 (Fig. 24) and GT11-06 (Fig B39). For this experiment, the depth at which the build up of ${ }^{230} \mathrm{Th}_{p}$ in the model begins coincides with the depletion with ${ }^{230} \mathrm{Th}_{d}$, also just below $3000 \mathrm{~m}$. Processes that may drive additional removal of ${ }^{230} \mathrm{Th}_{p}$ include increased aggregation of particles 
leading to enhanced sinking velocities, and lateral removal of ${ }^{230} \mathrm{Th}_{p}$ associated with fine-grained particles (with small sinking velocities). Both processes would imply that particle dynamics below and above $3000 \mathrm{~m}$ are distinct.

\subsubsection{Underestimation of Extreme ${ }^{230} \mathbf{T h}_{p}$ Near the Seafloor}

Thus far, we have neglected the four extremely large ${ }^{230} \mathrm{Th}_{p}$ activities measured near the bottom of stations GT11-04 (below $3500 \mathrm{~m}$ ) and GT11-08 (below $4500 \mathrm{~m}$ ), exceeding the activities of ${ }^{230} \mathrm{Th}_{d}$ measured at the same stations and depths. At station GT11-08 the model both overestimates ${ }^{230} \mathrm{Th}_{p}$ between $3000-4500 \mathrm{~m}$ and underestimates of ${ }^{230} \mathrm{Th}_{p}$ below $4500 \mathrm{~m}$. The model is utterly unable to explain the extreme ${ }^{230} \mathrm{Th}_{p}$ in near-bottom samples at GT11-04 and GT11-08, drastically underestimating their activities even in experiment $k_{1} \operatorname{var} 6$, with the largest maximum values of $k_{1}$ (Fig. 25). Thus, in addition to missing a process that removes ${ }^{230} \mathrm{Th}_{p}$ below $3000 \mathrm{~m}$ at a few stations, the model appears to miss a process that supplies ${ }^{230} \mathrm{Th}_{p}$ to near-bottom waters, at least at the two stations considered here.

\subsubsection{Potential Impact of Sediment Resuspension}

${ }^{230} \mathrm{Th}_{p}$ resuspension is a process that has already been postulated to explain ${ }^{230} \mathrm{Th}_{p}$ data in the Northwest Atlantic (Cochran et al., 1987; Hayes et al., 2015a). In our model, the effect of resuspended particles is parameterized by a downward increase in $k_{1}$ in a region of enhanced scavenging. This parameterization is motivated by the general increase in $k_{1}$ for Th with particle concentration (Nyffeler et al., 1984; Honeyman et al., 1988; Lerner et al., 2017), which suggest that $k_{1}$ should increase towards the seafloor in areas with active resuspension. However, resuspended particles may carry ${ }^{230} \mathrm{Th}$, a plausible assumption for this long-lived radionuclide, so areas of active resuspension should have activities of ${ }^{230} \mathrm{Th}_{p}$ that are elevated near the seabed due to an additional sedimentary ${ }^{230} \mathrm{Th}_{p}$ source. Such a process is not represented in the model.

To understand how sediment resuspension may impact ${ }^{230} \mathrm{Th}_{p}$, consider the following governing equation for ${ }^{230} \mathrm{Th}_{p}$ in a bottom nepheloid layer (Rutgers van der Loeff and Boudreau, 1997)

$$
\kappa_{T} \frac{\partial^{2} A_{p}}{\partial z^{2}}-\left(w+w_{s}\right) \frac{\partial A_{p}}{\partial z}+\beta A_{s s} e^{-\alpha z}+k_{1} A_{d}-k_{-1} A_{p}=0
$$

The third term on the left-hand side represents a source due to sediment resuspension, where $\alpha$ is a length scale for the attenuation of resuspended sediments from the top of the resuspension zone, or 
the zone of well mixed suspended sediments, $\mathrm{A}_{s s}$ is the ${ }^{230} \mathrm{Th}$ activity per gram of sediments, and $\beta$ is the rate of ejection of particles from the resuspension zone into the nepheloid layer. The magnitude of $\beta$ is determined by the properties of the sediments, such as particle size and cohesiveness (McCave, 1984), as well as by the bottom shear stress (McCave, 1984; Mehta et al., 1989; Blaas et al., 2007). In models describing the erosion of sediments, the effects of sediment cohesion are often neglected or crudely represented, e.g., via a critical shear stress above which resuspension can occur for a single class (Ribbe and Holloway, 2001) or multiple classes (Blaas et al., 2007) of sediments. Importantly, equation (5.10) does not consider the time-dependency of sediment resuspension, which is known to be episodic in the Northwest Atlantic (Gardner et al., 2017).

Whilst the injection of resuspended ${ }^{230} \mathrm{Th}_{p}$ from the seafloor can enhance ${ }^{230} \mathrm{Th}_{p}$ activities in near-bottom waters, it is not immediately apparent how this process may lower ${ }^{230} \mathrm{Th}_{p}$ above these near-bottom waters, and lead to observed ${ }^{230} \mathrm{Th}_{p}$ that are much less than those predicted by the model. A simple explanation is that resuspended sediment may not have reached the heights above the bottom where these relatively low activity ${ }^{230} \mathrm{Th}_{p}$ samples were collected. However, this reasoning contrasts with the ${ }^{230} \mathrm{Th}_{d}$ deficits that co-exist at the same depth levels, which necessitated an increase in scavenging intensity $\left(k_{1}\right)$ to be explained by the model. Another possibility is that enhanced scavenging below $3000 \mathrm{~m}$ does occur, but the ${ }^{230} \mathrm{Th}_{p}$ is then rapidly removed, either by lateral transport or by aggregation followed by rapid sinking. McCave (1983) suggested, based on particle sizes measured by coulter counter, that lateral injection of resuspended sediments can be important for maintaining thick (up to $1500 \mathrm{~m}$ ) bottom nepheloid layers on the Scotian Rise. He proposed a lateral injection region above the bottom mixed layer, that could result from lateral transport of resuspended sediments. Moreover, as a suspension ages, aggregation can change the particle size distribution from one that is peaked (mode $<0.4 \mu \mathrm{m}$ ) to one with a "flatter" distribution, suggesting a loss of finer-grained material via aggregation (McCave, 1983, 1984). Thus, if sediment injected above the bottom mixed layer has aged sufficiently, it may consist of a higher abundance of larger, faster sinking particles. Speculatively, then, ${ }^{230} \mathrm{Th}_{p}$ in near-bottom waters may represent recently resuspended sediment of small size, and ${ }^{230} \mathrm{Th}_{p}$ at shallower depths may be represent an older resuspension in which aggregates have formed and are being removed by gravitational setting. 


\subsubsection{Mechanisms of Sediment Resuspension}

In this section, I speculate on mechanisms that can enhance bottom shear stresses and lead to resuspending sediment. One possible mechanism, particularly along continental slopes, is internal wave reflection (Cacchione and Wunsch, 1974; Cacchione et al., 2002). The reflection of internal waves on sloping topography can lead to an increase in along-slope velocities and in near-bottom shear stresses. This effect depends on the slope of the topography $(\gamma)$ relative to the slope of the internal wave characteristic, $c=\sqrt{\left(\sigma^{2}+f^{2}\right) /\left(N^{2}+\sigma^{2}\right)}$, where $\sigma$ is the internal wave frequency, $f$ is the Coriolis parameter, and $N$ is the Brunt-Väisälä frequency. The increase in bottom velocity can be particularly pronounced when $\gamma=c$, a situation in which the bottom slope is called "critical" (Wunsch, 1968; Cacchione and Drake, 1986; Cacchione et al., 2002). The M2 semi-diurnal tide contains a large portion of the internal wave kinetic energy in the ocean (Ferrari and Wunsch, 2009). In the western North Atlantic, studies have reported intermediate nepheloid layers in the vicinity of near-critical slopes, with respect to the M2 tide (Gardner, 1989; Azetsu-Scott et al., 1995).

If critical reflection of the M2 internal tide is responsible for the resuspension of sediment and ${ }^{230} \mathrm{Th}_{p}$ in the western North Atlantic, then the large activities of ${ }^{230} \mathrm{Th}_{p}$ near the bottom of station GT11-04 and GT11-08 would be expected to be adjacent to bathymetric slopes that are nearcritical. In order to address this interesting question, the distribution of $N$ along line $\mathrm{W}$ is estimated using objectively interpolated temperature and salinity fields from Hydrobase 3, a hydrographic compilation with $1 / 4^{\circ}$ resolution (Curry and Nobre, 2008). Hydrobase should offer an improved description of the climatological $\mathrm{T} / \mathrm{S}$ fields, by averaging irregularly spaced data over potential density surfaces instead of depth surfaces, which eliminates the artificial mixing of waters with different properties (Lozier et al., 1995). The bathymetry along line W is obtained from the Global Multi-Resolution Topography (GMRT) dataset, which has a resolution of $\sim 100 \mathrm{~m}$ (Ryan et al., 2009). Since the bathymetric resolution is much higher than the resolution of the $\mathrm{T} / \mathrm{S}$ gridded data, the frequency of occurrence of critical slopes along line $\mathrm{W}$ is calculated by estimating $\gamma / c$ for each bathymetric grid point inside a grid cell of the hydrographic compilation. Specifically, this frequency is calculated from $N\left(\gamma_{c}\right) / N_{t o t}$, where $N\left(\gamma_{c}\right)$ is the number of critical slopes within each grid cell of the hydrographic compilation, and $N_{t o t}$ is the total number of bathymetric grid points 
inside a grid cell (Fig. 26).

Notably, I find that stations GT11-04 and GT11-08 do not occur near regions of high critical slope occurrence. The critical slopes appear confined mostly between stations GT11-01 and GT1103, above the $3500-\mathrm{m}$ isobath. Thus, the large ${ }^{230} \mathrm{Th}_{p}$ activities near the bottom observed at stations GT11-04 and GT11-08 do not appear to be associated with M2 internal tide reflection along the bottom near these stations. Conceivably, the M2 internal tide may still contribute to these activities through critical reflection near the upslope stations, and subsequent downslope transport of particulate ${ }^{230} \mathrm{Th}$. However, under this scenario, one would also expect a dramatic increase in ${ }^{230} \mathrm{Th}_{p}$ at stations GT11-01 to GT11-03 (where more slopes are critical and the sediment should be more frequently resuspended), which is not observed. Multiple processes, however, may be occurring to almost completely or partly erode nepheloid layers generated in this upslope region, including particle aggregation and settling of the aggregates to the seabed, downslope transport of sediment, and removal of sediment by the DWBC, which has the strongest CFC signal near station GT11-03 (Smith et al., 2016). Still, there is no clear evidence that internal tides play a large role in resuspending sediment along line W, particularly near stations GT11-04 and GT1108. In fact, Gardner (1989) found that sediments resuspended due to internal tide activity near the Baltimore submarine canyon incising the western North Atlantic continental slope are rarely observed in optical measurements far from the canyon mouth, whereas along the western end of section GA03 the largest ${ }^{230} \mathrm{Th}_{p}$ activities and the second largest $c_{p}$ values (aside from station GT11-04) are located far from the continental slope at GT11-08.

Other processes have been implicated in the creation and maintenance of intense bottom nepheloid layers (BNLs). Gardner et al. (2017) found that "benthic storms", episodes characterized by high (300-4000 $\left.\mu \mathrm{g} \mathrm{L}^{-1}\right)$ suspended sediment concentrations and intense BNL development, tend to occur when bottom currents exceed $20-35 \mathrm{~cm} \mathrm{~s}^{-1}$. These regions of elevated bottom velocity appear to be associated with the position of Gulf Stream meanders and rings, perhaps through their attendant production of deep cyclones, deep anti-cyclones, and (or) topographic Rossby Waves. Gardner et al. (2017) also found that persistent BNLs and benthic storms can occur in low energy environments via the advection of suspended sediments from regions of active resuspension. For example, at a monitoring station along the continental slope near Cape Hatteras (BOM S), the latter part of a benthic storm occurred during a period when bottom currents were below $20 \mathrm{~cm}$ 
$\mathrm{s}^{-1}$, suggesting that the resuspended material had an upstream source.

As stated above, deep cyclones may be at least party responsible for the offshore transport of suspended material. Andres et al. (2016) found evidence, by combining observations from satellite altimetry with repeated shipboard measurements of velocity, water properties, and concentrations of CFC-11 and CFC-12 along line W, that deep cyclogenesis under Gulf Stream troughs can be a significant mechanism for exchanging material between the DWBC and the interior. A possible mechanism for the exchange of suspended particles between the continental slope and the interior, then, would be the erosion of sediments along the slope, likely beneath the DWBC during intermittent periods of increased velocity, and subsequent transport by cyclones beneath a GS meander or ring.

\subsubsection{Paleoceanographic Implications}

The small sensitivity of ${ }^{230} \mathrm{Th}_{d, p}$ to DWBC and GS transports in the model suggests that ${ }^{230} \mathrm{Th}_{d, p}$ is largely insensitive to circulation changes in the western North Atlantic. This finding is consistent with the assumption by $Y u$ et al. (1996) that ${ }^{230} \mathrm{Th}$ is scavenged too rapidly to be redistributed by lateral advection and diffusion. Rhein et al. (2015) found that south of $42^{\circ} \mathrm{N}$ within our study region, approximately $50 \%$ of Labrador Sea Water is younger than 40 yrs, and approximately $40 \%$ of Denmark Strait Overflow Water is younger than 20 yrs. Interestingly, the average residence time of ${ }^{230} \mathrm{Th}$ with respect to particle scavenging in seawater is estimated to be $\sim 30-40$ yrs (François, 2007). Thus, one may reasonably assume that ${ }^{230} \mathrm{Th}$ should be sensitive to the strength of the DWBC, at least along its pathway. However, experiment "smlDWBCin" suggests that ${ }^{230} \mathrm{Th}_{d, p}$ activities are not sensitive to changes in the deep current transports. Likewise, a change in the GS inflow, as incorporated in experiment "larGSout", does not dramatically alter the distributions of ${ }^{230} \mathrm{Th}_{d, p}$ simulated by the model.

While the effect of the DWBC on ${ }^{230} \mathrm{Th}_{d, p}$ appears to be small, ${ }^{230} \mathrm{Th}_{d}$ below $3000 \mathrm{~m}$ shows a noticeable response to the presence of enhanced scavenging in the lower part of the water column. For depths $>3000 \mathrm{~m}$, the best fit of the model to the ${ }^{230} \mathrm{Th}_{d}$ data occurs when $k_{1}$ increases towards the bottom. This finding suggests ${ }^{230} \mathrm{Th}$ in the western North Atlantic can be affected by an increase in scavenging intensity in BNLs. While the representation of bottom scavenging in this study is based on the apparent relationship between $k_{1}$ and particle concentration (Nyffeler et al., 1984; Honeyman et al., 1988; Lerner et al., 2017), particle composition may also potentially enhance the removal of 
particle-reactive metals in the deep water column. Elevated concentrations of particulate $\mathrm{MnO}_{2}$ in the small size fraction $(1-51 \mu \mathrm{m})$ collected by large volume in-situ filtration have been reported at GT11-04 and GT11-08, along with slight enhancements in $\mathrm{FeOH}_{3}$ at station GT11-04 (Lam et al., 2015a). Multiple studies have reported elevated values of $\mathrm{Th}_{p} /\left(\mathrm{Th}_{d} P\right)$, a ratio thought to reflect the intensity of Th scavenging, associated with Fe and Mn (oxyhydr)oxides (Anderson et al., 1992; Guo et al., 2002b; Geibert and Usbeck, 2004; Lin et al., 2014; Hayes et al., 2015a). Thus, the best fit of ${ }^{230} \mathrm{Th}_{d}$ data obtained for large $k_{1}$ values below $3000 \mathrm{~m}$ (Table 9) may reflect changes in particle concentration and (or) particle composition within bottom nepheloid layers in the western North Atlantic. Since representing the effect of the BNLs through spatial variations in $k_{1}$ only improves the fit to ${ }^{230} \mathrm{Th}_{d}$ data below $3000 \mathrm{~m}$, it is unclear whether these layers can also change the ${ }^{230} \mathrm{Th}$ activity of particulate material deposited on the seafloor. The model does show large differences in near-bottom ${ }^{230} \mathrm{Th}_{p}$ activities between experiments where the effects of BNLs are neglected and experiments where these effects are considered (Fig. 24). However, none of the experiments offer a significantly improved fit to ${ }^{230} \mathrm{Th}_{p}$ observations below $3000 \mathrm{~m}$ compared to a reference experiment where bottom scavenging is omitted. Still, given the improvement in the ability of the model to replicate the ${ }^{230} \mathrm{Th}_{d}$ data below $3000 \mathrm{~m}$ when scavenging intensity is non-uniform, and given that ${ }^{230} \mathrm{Th}_{p}$ activities in our sensitivity experiments are different in deep waters from those in the reference experiment, it seems plausible that the ${ }^{230} \mathrm{Th}_{p}$ reaching the seabed is altered by spatial and temporal changes in scavenging. This result raises questions about the applications of ${ }^{230} \mathrm{Th}$ measured on bulk sediment to paleoceanography. For example, the use of ${ }^{230} \mathrm{Th}$ to correct flux records for lateral redistribution of sediment assumes that the ${ }^{230} \mathrm{Th}_{p}$ flux at the bottom equals the rate of radioactive production in the overlying water column. Under this assumption, ${ }^{230} \mathrm{Th}_{p}$ should increase linearly with depth from the sea surface to the seafloor, a distribution inconsistent with the ${ }^{230} \mathrm{Th}_{p}$ activities simulated when the effects of BNLs are considered and with the ${ }^{230} \mathrm{Th}_{p}$ activities observed at some stations (e.g., station GT11-04 and GT11-08). This assumption is also vital for the application of the ${ }^{231} \mathrm{~Pa} /{ }^{230} \mathrm{Th}$ in bulk sediments to investigate changes in circulation in the geologic past. It is generally thought that changes in this ratio in the sediments are caused by changes in particulate ${ }^{231} \mathrm{~Pa}$ in overlying waters, while ${ }^{230} \mathrm{Th}_{p}$ in the overlying waters is assumed to be from the radioactive production of ${ }^{230} \mathrm{Th}$. While the model presented here does not include ${ }^{231} \mathrm{~Pa}$, the large increase in near-bottom ${ }^{230} \mathrm{Th}_{p}$ activities observed in some GA03 samples and simulated 
in experiments where BNL effects are considered suggest that the radionuclide ratio in sediments can be sensitive to processes taking place near the seabed, such as intensive or frequent sediment resuspensions.

\subsection{Conclusion}

In this chapter, ${ }^{230} \mathrm{Th}_{d, p}$ distributions in the western North Atlantic are simulated by embedding a reversible exchange model within the Princeton Ocean Model. The resultant circulation-scavenging model solves the primitive equations as well as the ${ }^{230} \mathrm{Th}_{d, p}$ governing equations. Quasi-steady state solutions are found for thorium in dissolved and particulate forms after 9-10 years of numerical integration. The physical aspects of the solution are confronted to a suite of oceanographic and satellite observations. Modeled sea surface height is found to correlate with dynamic topography derived from satellite altimetry, and the major elements of the general circulation (GS and DWBC) are simulated with strengths with the appropriate orders of magnitude and general spatial pattern. On the other hand, the locations of the GS and the DWBC along line W, and the strength of the DWBC along the line show systematic differences with observations made from moorings and repeated ship occupations.

To document the sensitivity of the simulated ${ }^{230} \mathrm{Th}_{d, p}$ distributions to changes in circulation and scavenging intensity, a number of numerical experiments were carried out. Compared to a reference experiment, changing the GS outflow or DWBC inflow does not remarkably alter the model fit to the ${ }^{230} \mathrm{Th}_{d, p}$ data, while changing the boundary values of ${ }^{230} \mathrm{Th}_{d, p}$ results in either a deterioration or improvement of the model fit, depending on the experiment considered. However, the best agreement with the ${ }^{230} \mathrm{Th}_{d}$ data is obtained by allowing spatial variations in $k_{1}$. Therefore, an increase in the specific adsorption rate of ${ }^{230} \mathrm{Th}$ below this depth appears necessary, absent of other processes not encapsulated in the current model, to explain the deficits in ${ }^{230} \mathrm{Th}_{d}$ observed at (pre-)GEOTRACES stations. On the other hand, ${ }^{230} \mathrm{Th}_{p}$ data are poorly reproduced by any model experiments considered here. ${ }^{230} \mathrm{Th}_{p}$ resuspension, not included in the current model, may significantly alter ${ }^{230} \mathrm{Th}_{p}$ activities in the deep ocean. However, properly describing the resuspension of ${ }^{230} \mathrm{Th}_{p}$ would require a model for sediment resuspension, which depends on a wide variety of factors, including sediment cohesion, size distribution, and bottom shear stresses induced by dynamical phenomena such as the bottom reflection of internal waves and benthic storms. Consideration of all these sedimentological 
and physical factors and processes would require a more complete model and perhaps also higher spatial resolution and computational resources than those used here. Nonetheless, considering these factors and processes will be important in order to understand the scavenging of ${ }^{230} \mathrm{Th}$ and other trace metals and isotopes in the vicinity of ocean margins. Such an understanding is essential for the application of ${ }^{230} \mathrm{Th}$ measured in sediment cores originating from the margins to a variety of paleoceanographic problems, including estimating the vertical flux of material to the seafloor, correcting for the lateral redistribution of sediments, and pairing this radioisotope with ${ }^{231} \mathrm{~Pa}$ to constrain ocean circulation in the geologic past.

Aside from missing particle resuspension, the model's representation of the circulation in the Northwest Atlantic is not completely consistent with available physical observations. In particular, the model displaces the DWBC westward (upslope) and the GS eastward compared to velocity observations along line $\mathrm{W}$. The $\mathrm{DWBC}$, carrying low-activity ${ }^{230} \mathrm{Th}_{d}$ waters from northern seas, may be an importance source of the deficits in ${ }^{230} \mathrm{Th}_{d}$ below $3000 \mathrm{~m}$, particularly at station GT1104, located near the observed DWBC core (this high velocity core is also below $3000 \mathrm{~m}$ along line $\mathrm{W})$. The current model may have difficulty capturing the effect of the DWBC on ${ }^{230} \mathrm{Th}_{d}$ at these locations, since it places this current further west of GT11-04 and above $3000 \mathrm{~m}$. The effect of this misplaced current on ${ }^{230} \mathrm{Th}$ may be better understood by restricting the comparison of observed and modeled ${ }^{230} \mathrm{Th}_{d, p}$ activities at stations GT11-02 and GT11-03 (e.g., calculating the RMSE, bias, and $r^{2}$ from ${ }^{230} \mathrm{Th}$ activities at only these stations), where the DWBC is present in the model. Similarly, restricting the comparison to station GT11-08, near the modeled GS core, may isolate the effect of the misplaced GS on ${ }^{230} \mathrm{Th}$. However, a more rigorous assessment of the effect of both these currents on ${ }^{230} \mathrm{Th}$ in the Northwest Atlantic will require the model to more accurately represent their time-averaged positions. These positions may be improved by using a higher spatial resolution and (or) considering buoyancy fluxes (from exchanges of heat between the ocean and atmosphere, and changes in salinity due to net evaporation or precipitation), not included in the current model, in the region.

\section{Acknowledgement}

I acknowledge the U.S. National Science Foundation for supporting this study (grant OCE155644) and the U.S. GEOTRACES North Atlantic section ship time, sampling, and data analysis. 
The U.S. NSF also supported the generation of ${ }^{230}$ Th data (OCE-0927064 to LDEO, OCE-O092860 to WHOI, and OCE-0927754 to UMN). I thank the chief scientists of the GA03 section (Ed Boyle, Bill Jenkins, and Greg Cutter) as well as the captain, the crew, and the scientific party on the $\mathrm{R} / \mathrm{V}$ Knorr, which completed this section. I am also grateful to the scientists and staff involved in the analysis of the ${ }^{230} \mathrm{Th}$ and particle data. I also acknowledge the U.S. NSF and WHOI's Ocean and Climate Change Institute for funding the line W observing program between 2004 and 2014. I am grateful to the scientists and staff involved in the deployment and recovery of line $\mathrm{W}$ moorings and processing and analysis of moored observations. I especially thank John Toole (WHOI) for providing access to daily subinertial-filtered velocity profiles from line $\mathrm{W}$, and both John Toole and Magdalena Andres (WHOI) for our very beneficial discussions on how to best compare modeled and observed velocities along line W. Finally, I am grateful to Roger François for sharing unpublished ${ }^{230} \mathrm{Th}$ data for the western North Atlantic. 


\section{A Appendix A}

Table A1 lists the values of each parameter used for the numerical integration of the ocean model.

\begin{tabular}{|c|c|c|c|}
\hline \multicolumn{4}{|c|}{ Physical Parameters } \\
\hline & & value & units \\
\hline$\rho_{o}$ & reference density & 1025 & $\mathrm{~kg} \mathrm{~m}{ }^{-3}$ \\
\hline$g$ & acceleration due to gravity & 9.806 & $\mathrm{~m} \mathrm{~s}^{-2}$ \\
\hline$f$ & Coriolis parameter & $10^{-4}$ & $\mathrm{~s}^{-1}$ \\
\hline$C$ & Smagorinsky coefficient & 0.2 & dimensionless \\
\hline$\kappa_{u, o}$ & background vertical viscosity & 0 & $\mathrm{~m}^{2} \mathrm{~s}^{-1}$ \\
\hline$\kappa_{T, o}$ & background vertical diffusivity & 0 & $\mathrm{~m}^{2} \mathrm{~s}^{-1}$ \\
\hline $\mathrm{P}_{r}$ & turbulent Prandtl number & 5 & dimensionless \\
\hline $\mathrm{z}_{0}$ & bottom roughness & 0.01 & $\mathrm{~m}$ \\
\hline \multicolumn{4}{|c|}{ Numerical Parameters } \\
\hline & & value & units \\
\hline$\Delta t_{\mathrm{E}}$ & time step (external mode) & 15 & $\mathrm{~s}$ \\
\hline$\Delta t_{\mathrm{I}}$ & time step (internal mode) & 450 & $\mathrm{~s}$ \\
\hline$\Delta s$ & step interval for advective terms ${ }^{a}$ & 5 & dimensionless \\
\hline$h_{\max }$ & maximum depth in radiation condition & 5000 & $\mathrm{~m}$ \\
\hline$u_{\max }$ & maximum velocity for CFL violation & 100 & $\mathrm{~m} \mathrm{~s}^{-1}$ \\
\hline$c$ & constant of Asselin filter & 0.10 & dimensionless \\
\hline$\alpha_{w}$ & weight for surface slope $\operatorname{term}^{b}$ & 0 & dimensionless \\
\hline
\end{tabular}

${ }^{a}$ Step interval during which advective terms of the external mode are not updated

${ }^{b}$ Weight used for surface slope terms in the external mode equations

Table A1: Parameters of the Princeton Ocean Model

\section{B Appendix B}

Figures B1-B44 similar to Fig.15-18 in the main text, but for all sensitivity experiments listed in Table 6. The appropriate experiments are listed in the caption in these figures. 


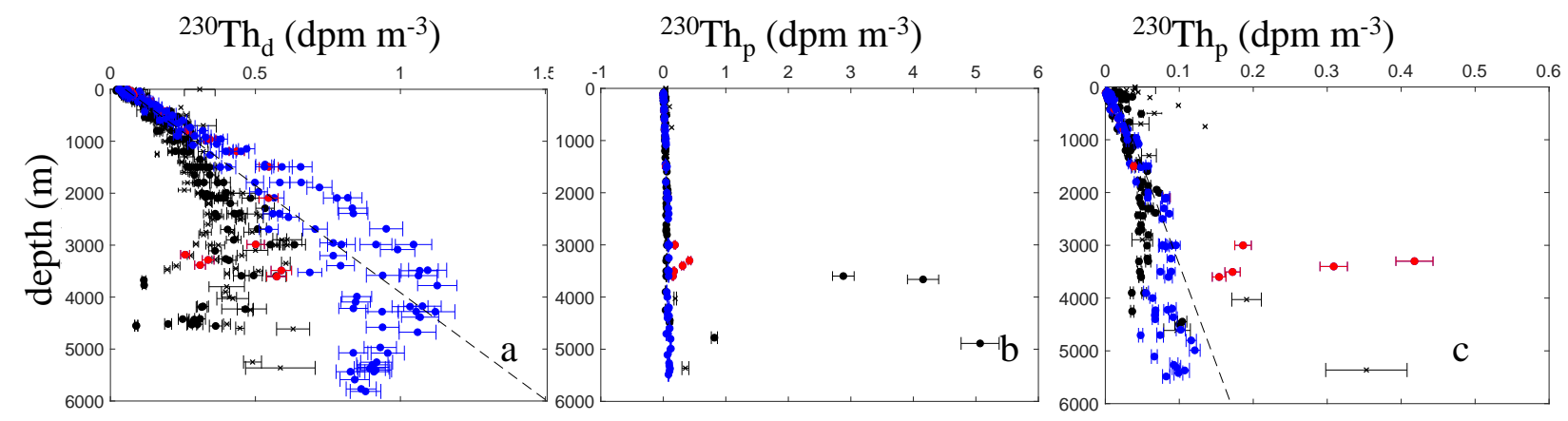

Figure 1: Panel (a) shows vertical ${ }^{230} \mathrm{Th}_{d}$ activity profiles in the North Atlantic. The blue values are data west of of Bermuda (east of $64^{\circ} \mathrm{W}$ ), the black values are data east of Bermuda, and the red values are data from station GT11-16 near the TAG hydrothermal vent. The dashed black line is the extrapolation of the OLS fit to all data in the upper $1000 \mathrm{~m}$. Panels (b) and (c) are the same as panel (a), but for (b) all ${ }^{230} \mathrm{Th}_{p}$ data and (c) ${ }^{230} \mathrm{Th}_{p}$ data $<0.6 \mathrm{dpm} \mathrm{m}{ }^{-3}$. The best fit line is only shown in panel (c). For each panel, error bars are \pm 1 standard deviation, data in the west of Bermuda are from references in Table 1, and data east of Bermuda are from Hayes et al. (2015a). 


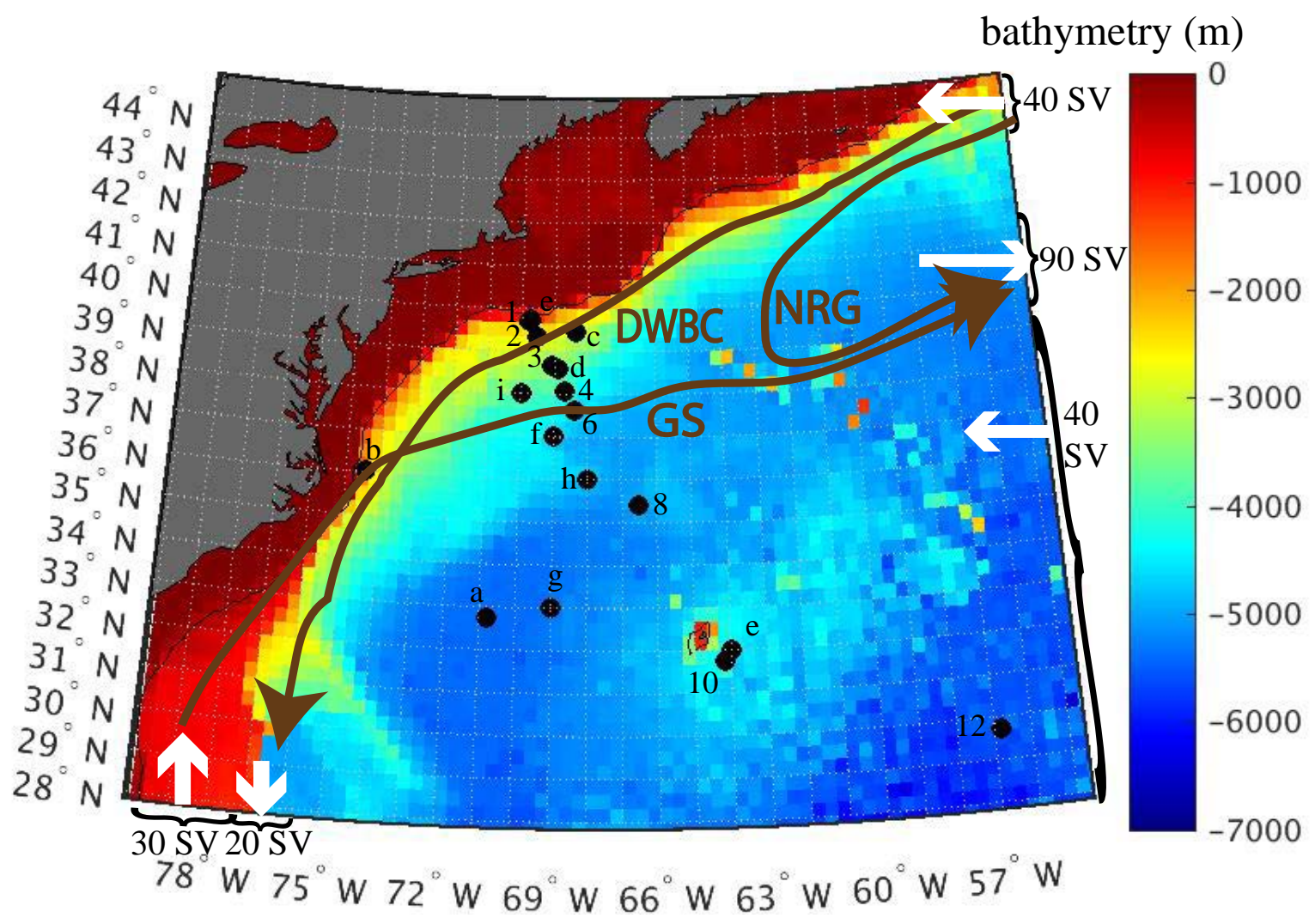

Figure 2: Bathymetry and schematic of the general circulation in the western North Atlantic. Abbreviations are as follows: GS for Gulf Stream, DWBC for Deep Western Boundary Current, and NRG for Northern Recirculation Gyre. The DWBC flows under the GS where the two flows intersect near Cape Hatteras. Labeled black circles are water column stations, with numbers corresponding to GEOTRACES stations and letters corresponding to preGEOTRACES stations (see Table 1 for station names and locations). Bathymetric data are from National Geophysical Data Center (2006). Volume transports of GS, DWBC, and subtropical water through the eastern and (or) southern boundaries of the study region are shown. The transport values for the GS and the DWBC are based on observational estimates, whereas the transport of subtropical water, assumed to occur only through the eastern boundary, is set to a value that ensures volume conservation in the study region. 


\section{Salinity (PSS-78)}

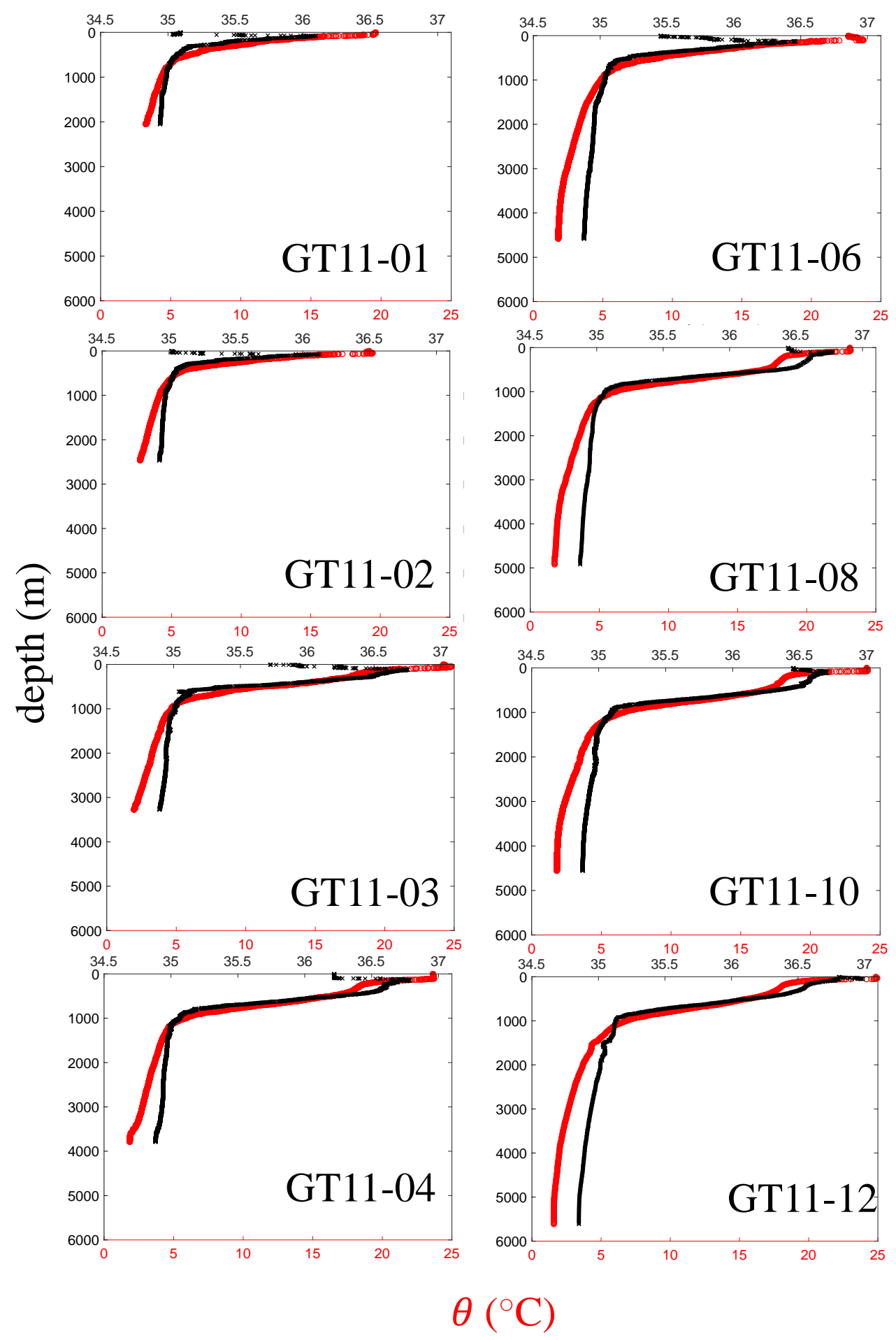

Figure 3: Profiles of potential temperature and salinity at eight stations occupied along the western segment of GA03. Data from Jenkins et al. (2015). 


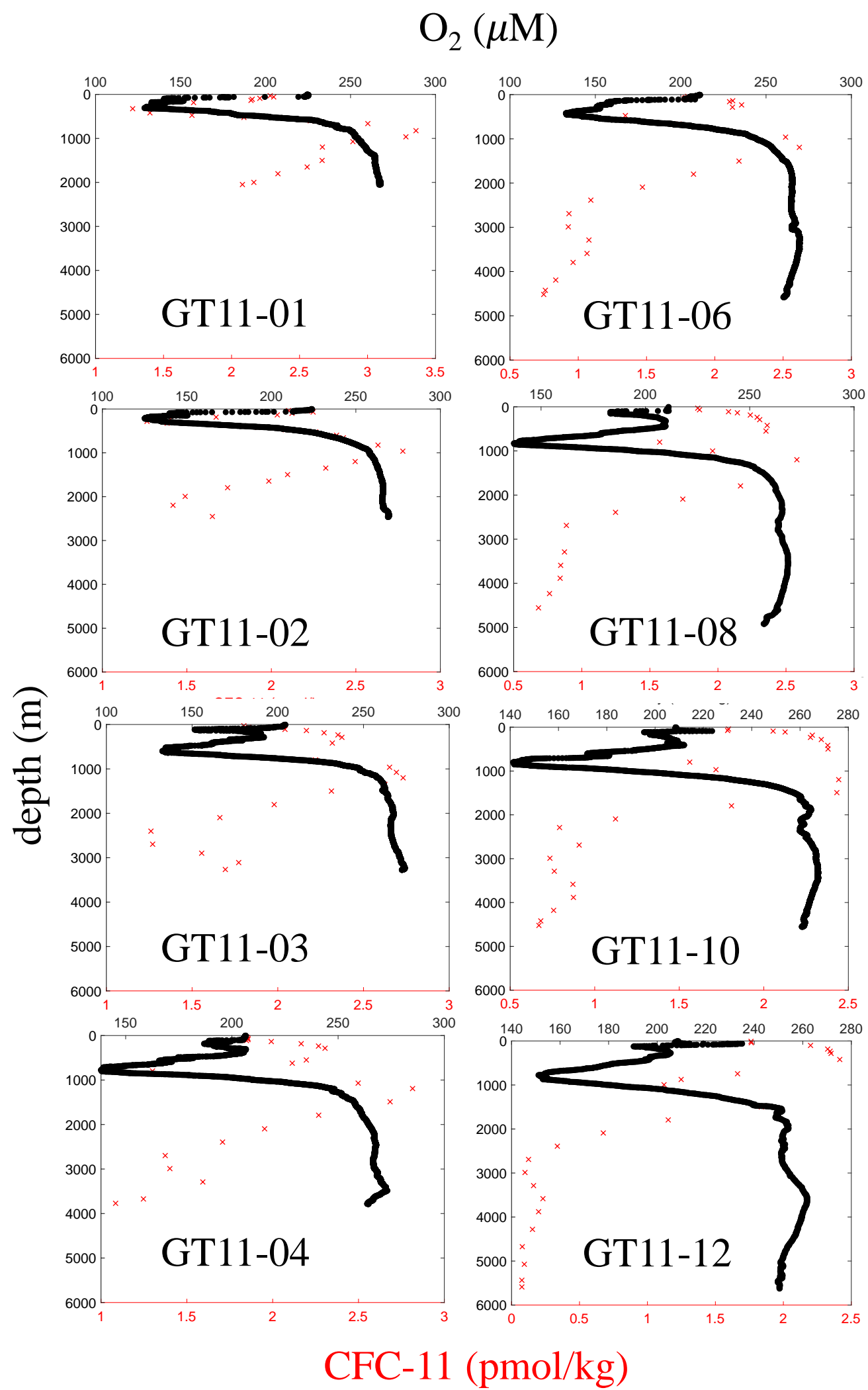

Figure 4: Profiles of CFC-11 and dissolved $\mathrm{O}_{2}$ concentrations at eight stations occupied along the western segment of GA03. $\mathrm{O}_{2}$ data from Jenkins et al. (2015), and CFC-11 data from the group of William Smethie (The GEOTRACES group, 2015) 

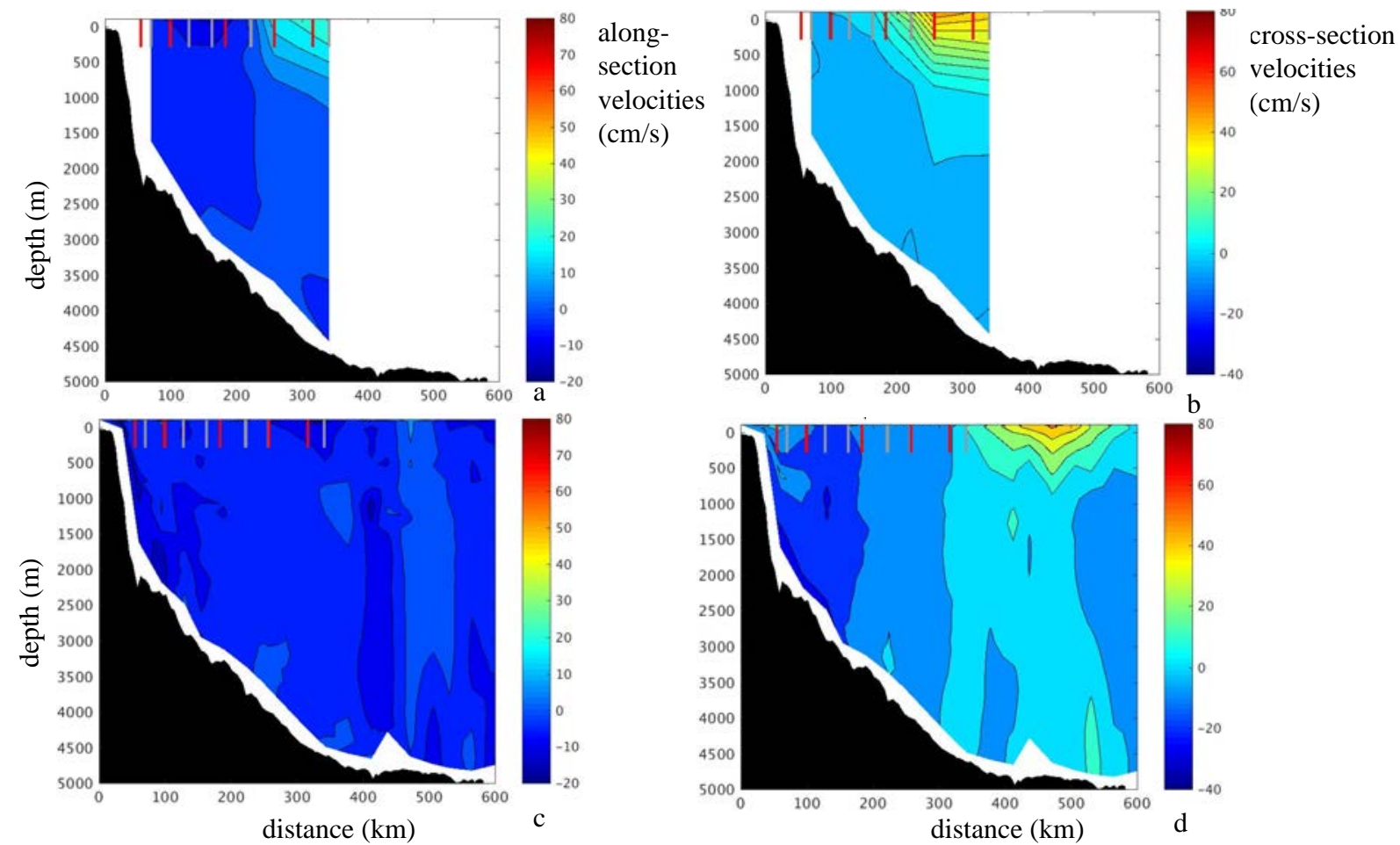

Figure 5: Panels (a) and (b) show, respectively the along-line $\mathrm{W}$ and acrossline $\mathrm{W}$ velocities, obtained from a 10-year average of daily, subinertial-filtered velocity profiles from moored instruments at six locations (data from Toole et al. (2017)). Panels (c) and (d) are the same as panels (a) and (b), but for model velocities averaged over the last 2200 days of the reference simulation. Red lines are GEOTRACES stations GT11-01 to GT11-06, and the grey lines are the mooring locations. The $\mathrm{x}$-axis is distance from $40.125^{\circ} \mathrm{N}, 70.125^{\circ} \mathrm{W}$. 


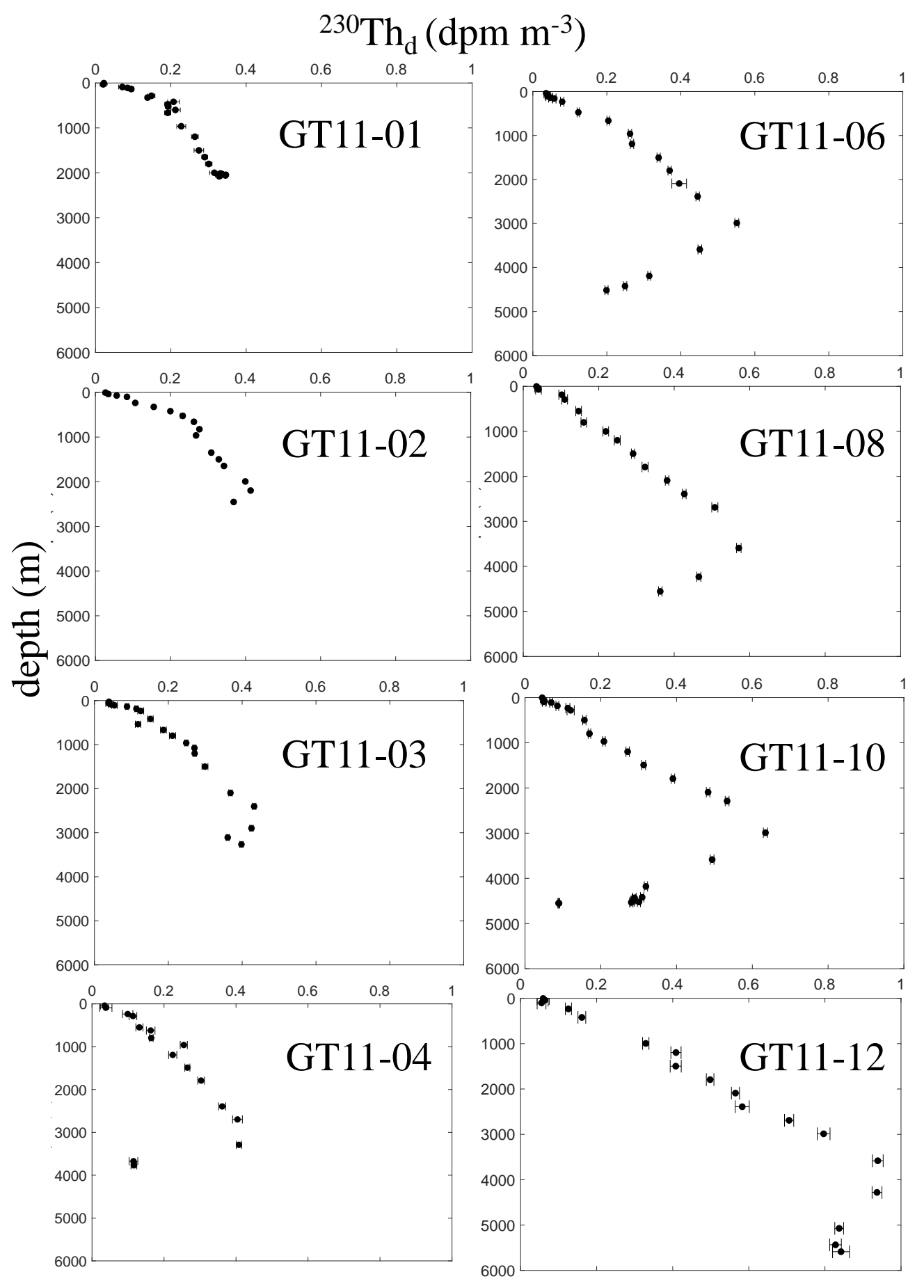

Figure 6: ${ }^{230} \mathrm{Th}_{d}$ activity profiles along the western end of section GA03. Error bars are \pm 1 standard deviation. Data from Hayes et al. (2015b) 


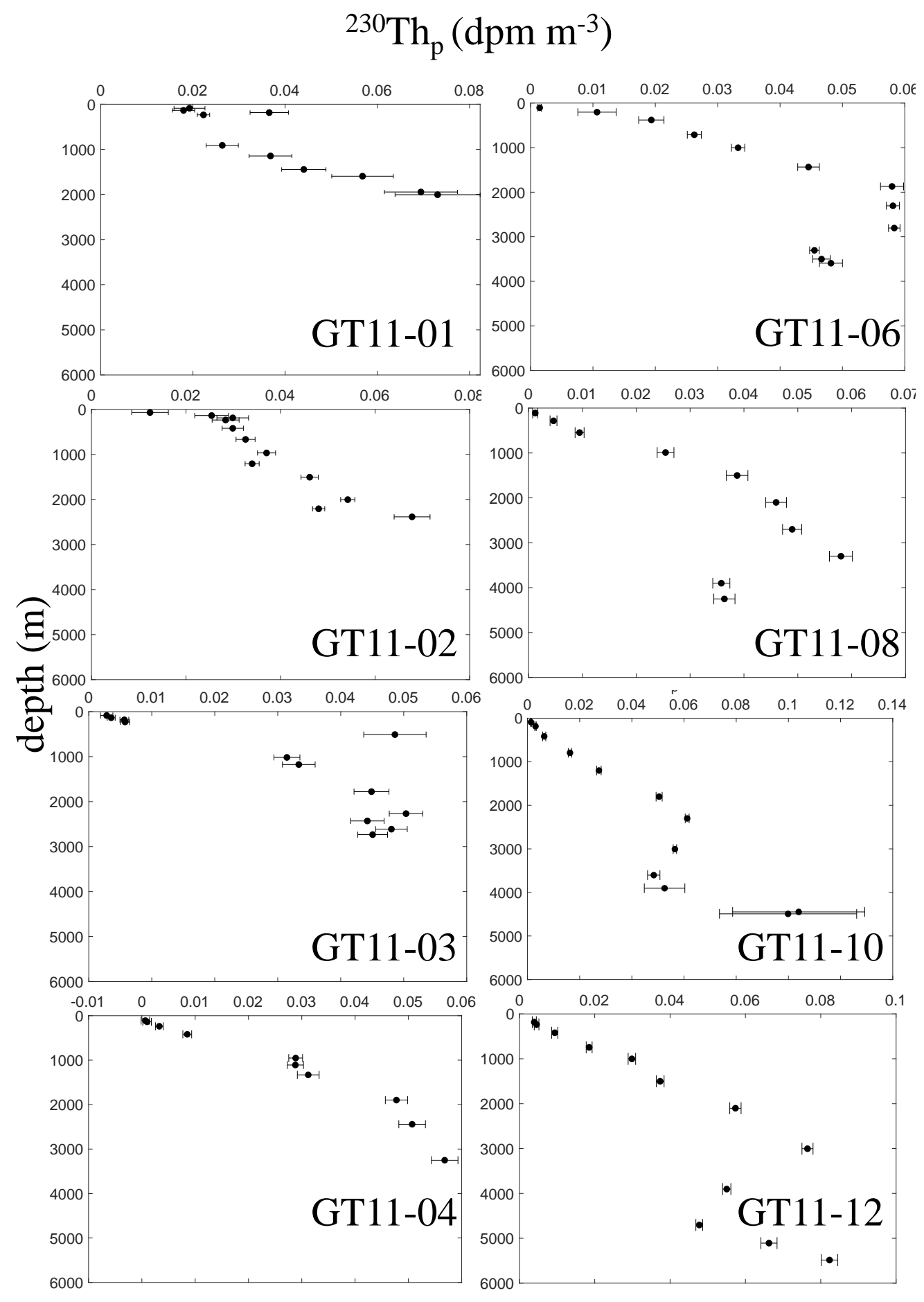

Figure 7: ${ }^{230} \mathrm{Th}_{p}$ activity profiles along the western end of section GA03. Error bars are \pm 1 standard deviation. Note that extremely large activities $(>0.6$ $\mathrm{dpm} \mathrm{m}^{3}$ ) measured on four near-bottom samples collected at stations GT11-04 and GT11-08 are not shown. Data from Hayes et al. (2015b). 


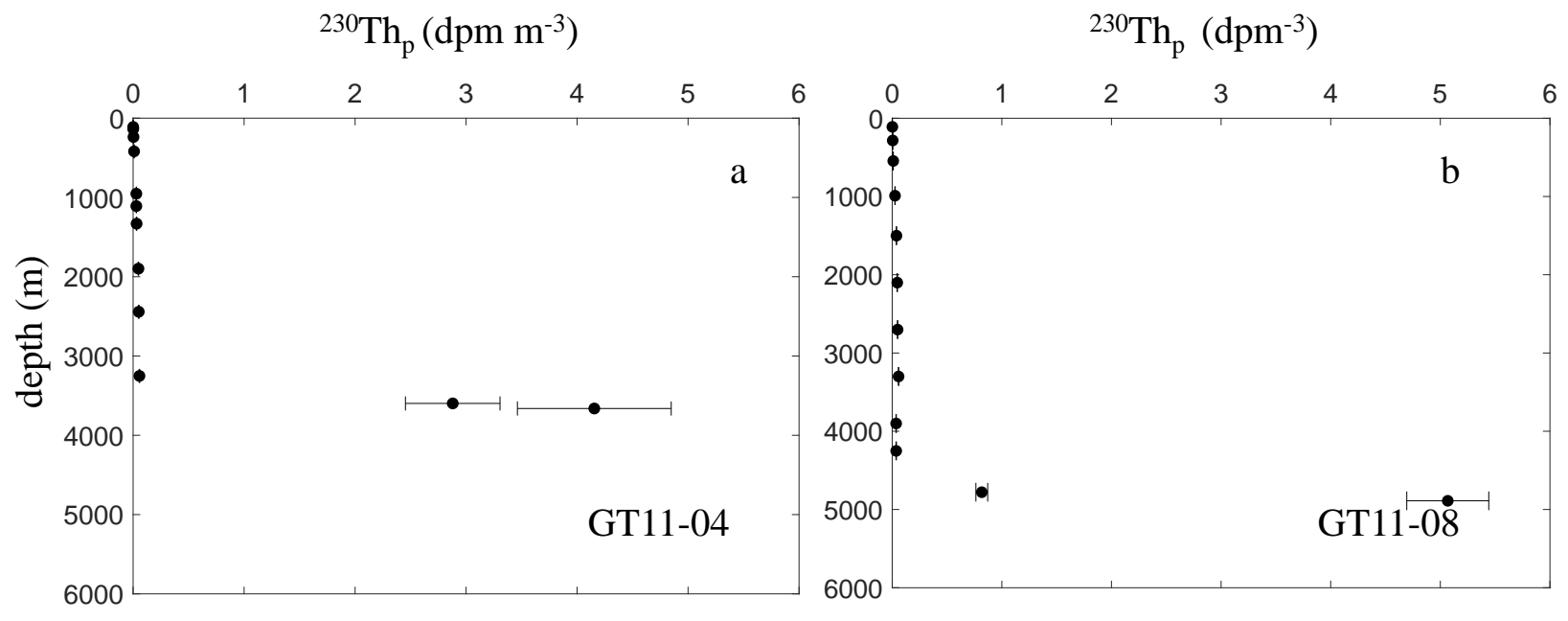

Figure 8: ${ }^{230} \mathrm{Th}_{p}$ activity profiles measured, at stations GT11-04 (a) and GT1108 (b). The errors bars are \pm 1 standard deviation. 


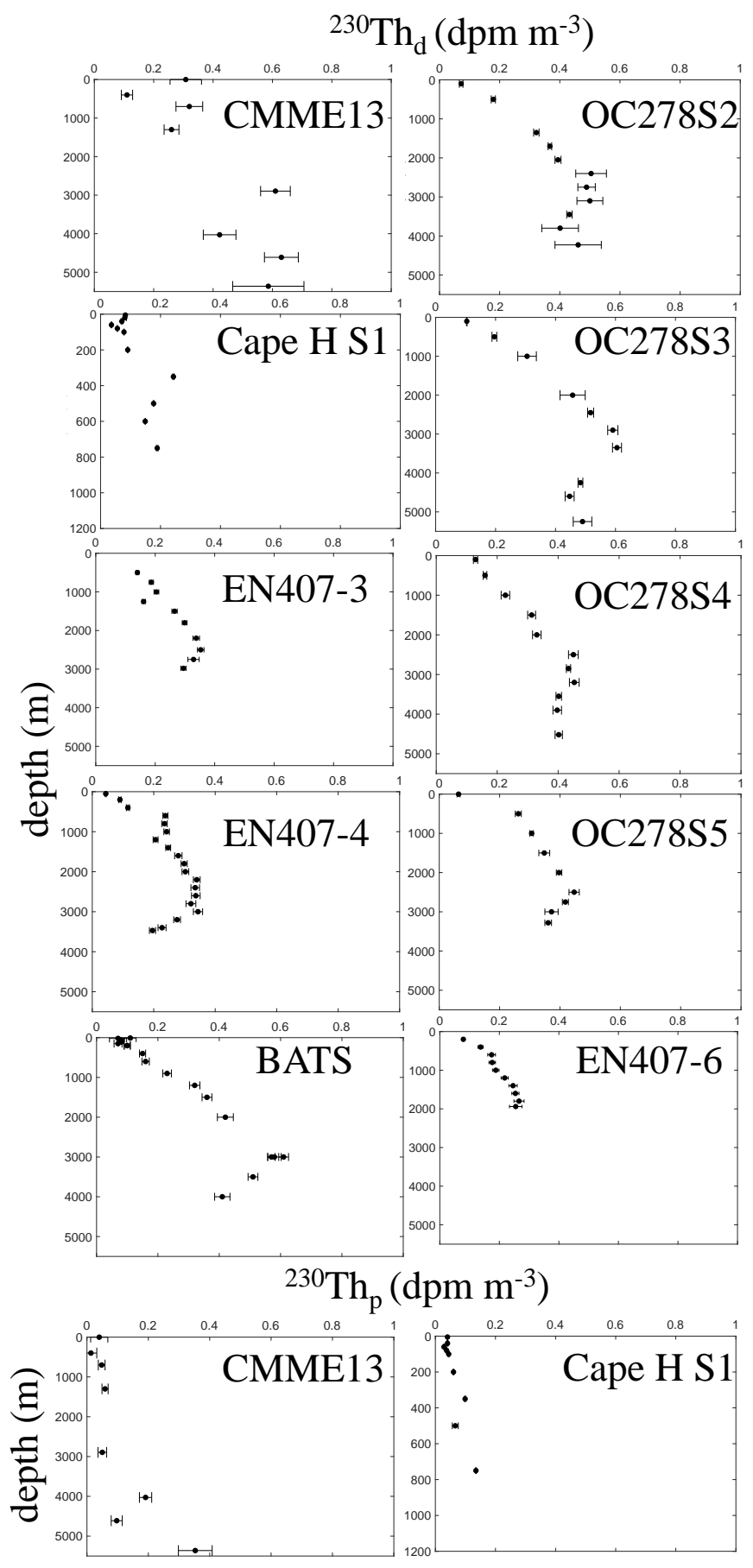

Figure 9: ${ }^{230} \mathrm{Th}_{d, p}$ activity profiles from the pre-GEOTRACES era. The eight top panels show dissolved activities and the two bottom panels show particulate activities. Error bars are \pm 1 standard devaiton. Locations of stations and sources of the data are listed in Table 2 . 

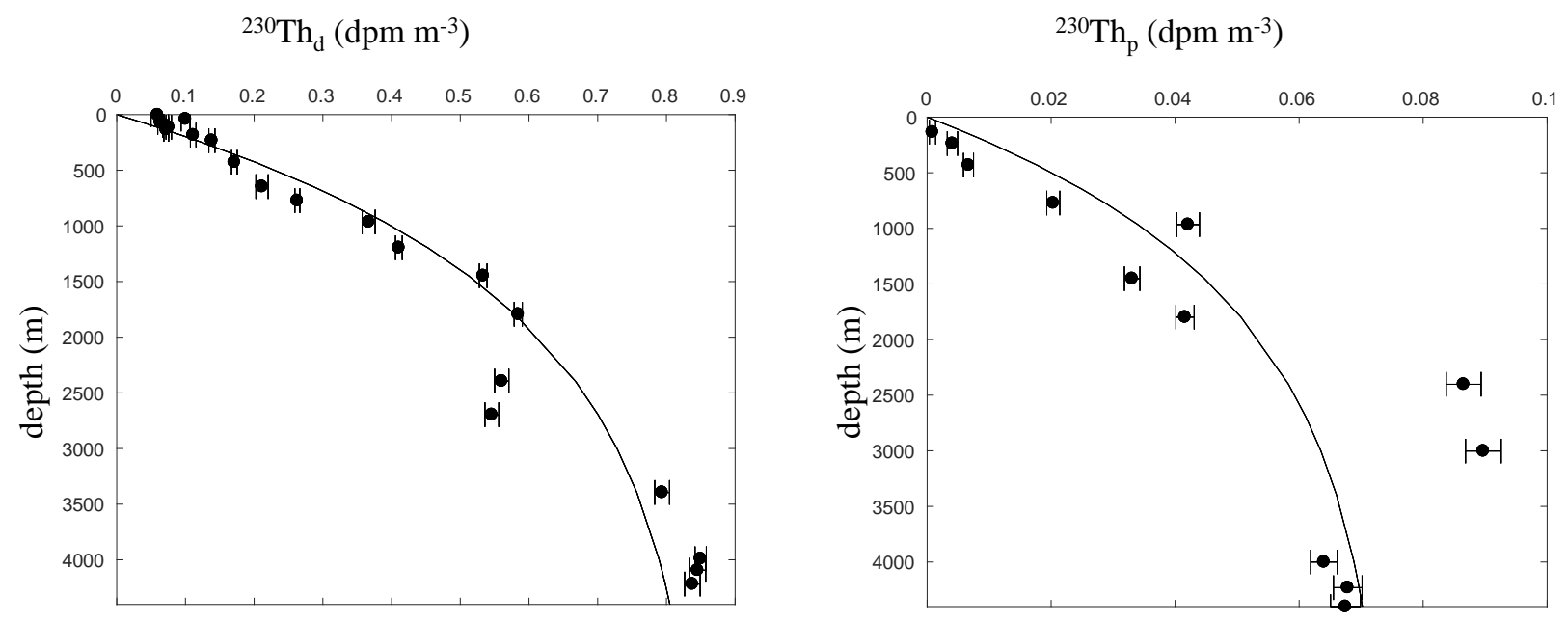

Figure 10: ${ }^{230} \mathrm{Th}_{d, p}$ activity profiles at GT11-14 with error bars showing \pm 1 standard deviation. The solid lines are exponential fits to data used as conditions imposed along all open boundaries except along a narrow segment in the northeast where Slope Water enters the domain. Data between 1500-3000 $\mathrm{m}$ water depth are suspected to be influenced by hydrothermal vents of the Middle Atlantic Ridge and are not used for the fits. 

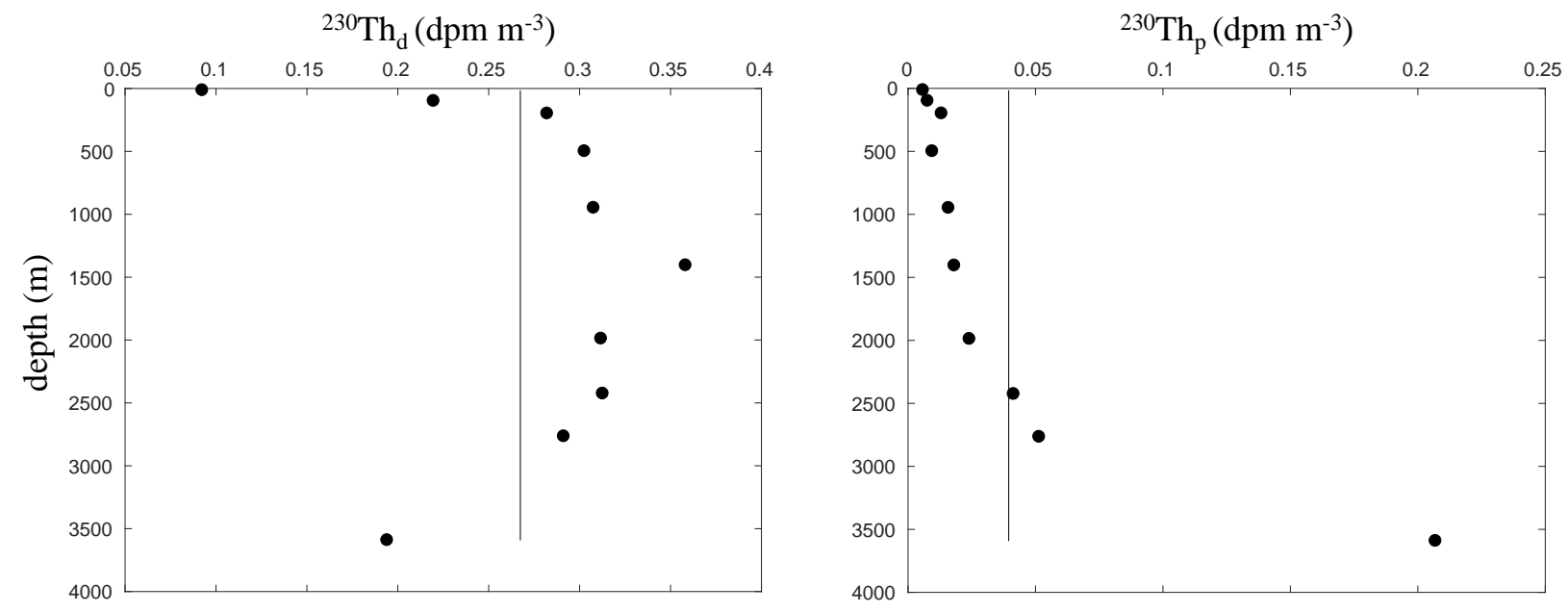

Figure 11: ${ }^{230} \mathrm{Th}_{d, p}$ activity profiles in the Labrador Sea at $54.5^{\circ} \mathrm{N}, 48.46^{\circ} \mathrm{W}$. The solid lines are the averages of the data and used as boundary activities for ${ }^{230} \mathrm{Th}_{d, p}$ along a narrow segment in the north where Slope Water enters the domain. Data from Moran et al. (1997). 

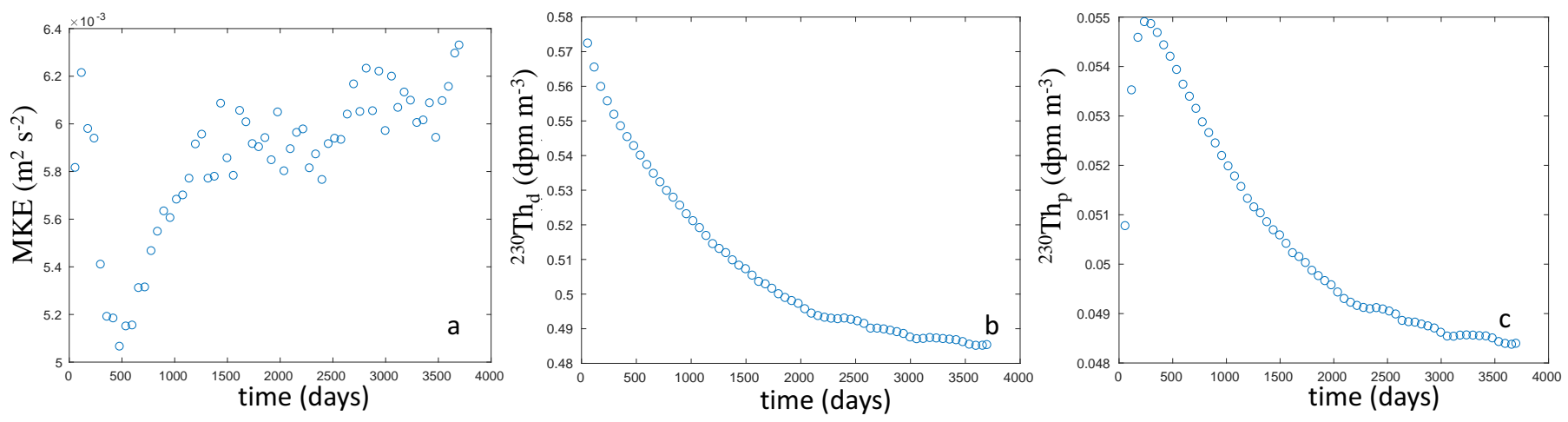

Figure 12: Evolution of the domain averages of kinetic energy (a), ${ }^{230} \mathrm{Th}_{d}$ activity (b), and ${ }^{230} \mathrm{Th}_{p}$ activity (c) in the reference simulation 

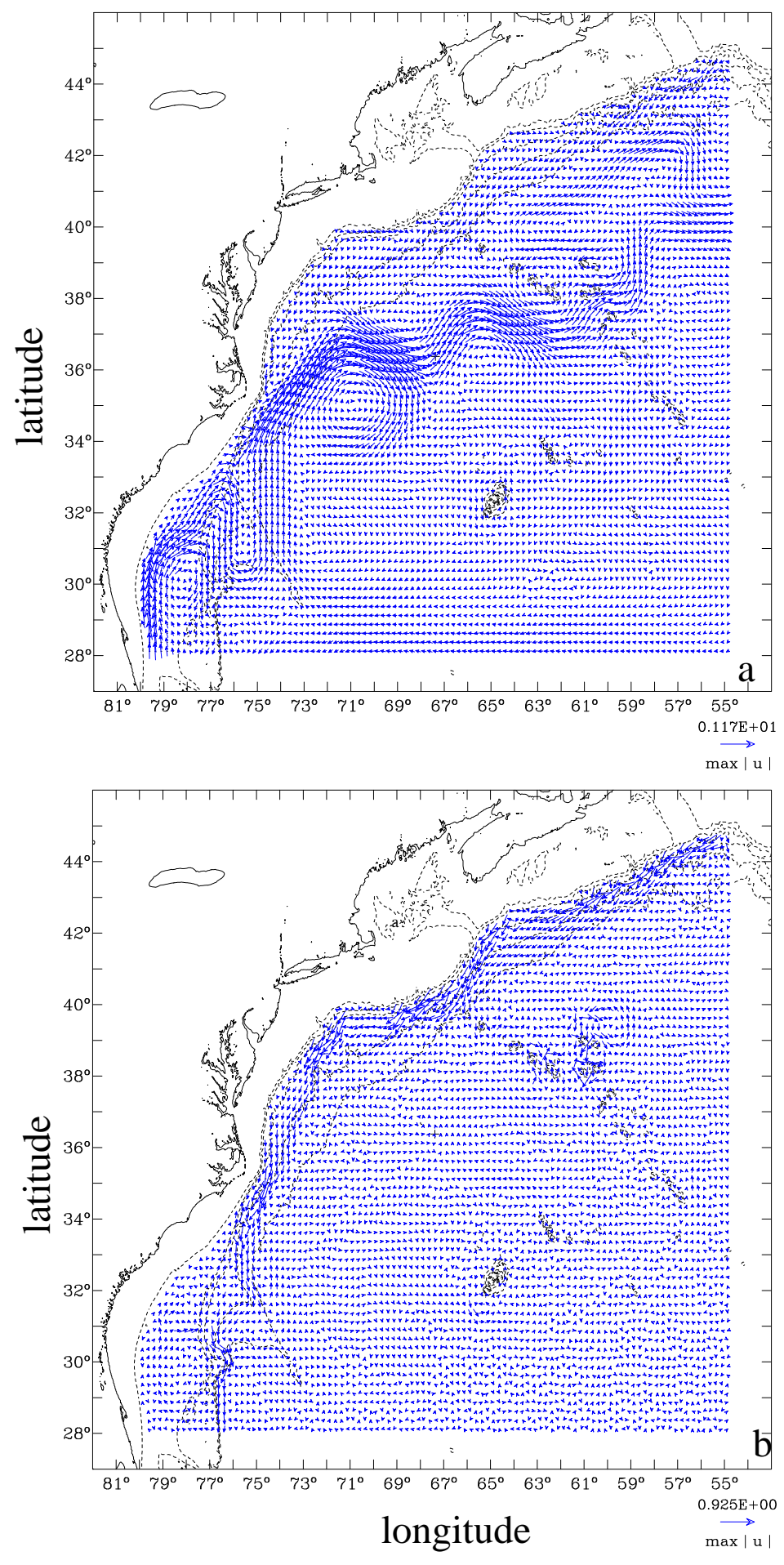

Figure 13: Horizontal velocities simulated in the reference experiment in the surface layer (a) and at $3000 \mathrm{~m}$ (b). The arrow in the bottom right corner of each panel is the magnitude of the largest velocity vector depicted (units: $\mathrm{m}$ $\mathrm{s}^{-1}$ ). The coastline is shown by the solid line, and the 200, 1000, 2000, 3000, and $4000 \mathrm{~m}$ isobaths are denoted by dashed lines. 


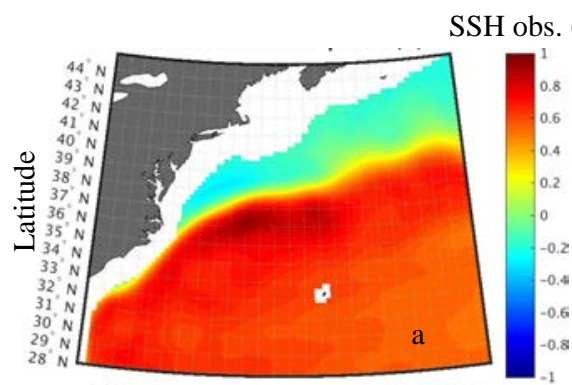

$78^{\circ}$ W $75^{\circ}$ W $72^{\circ}$ W $69^{\circ}$ W $66^{\circ}$ W $63^{\circ}$ W $60^{\circ}$ W $57^{\circ}$ W

Longitude

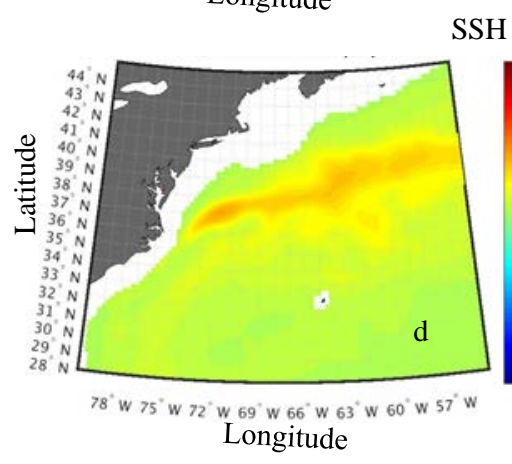

SSH std obs. (m)

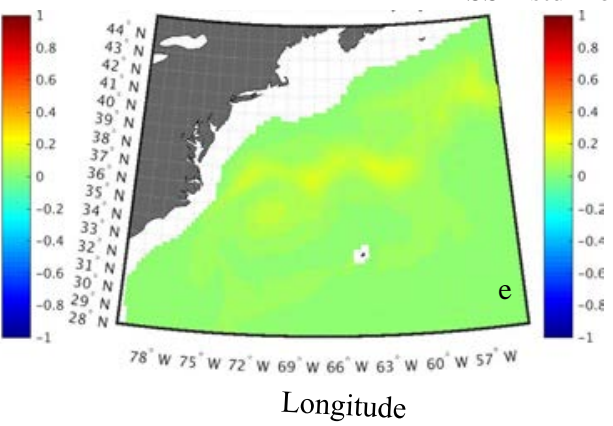

SSH model (m)

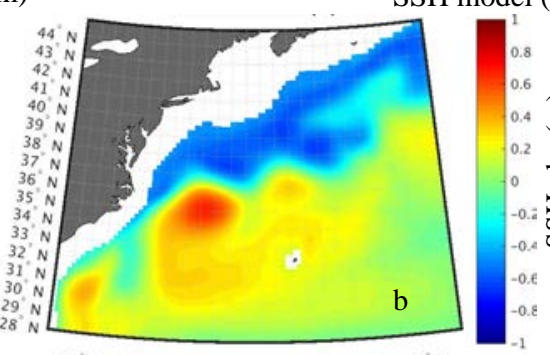

$78^{\circ}$ W $75^{\circ}$ W $72^{\circ}$ W $69^{\circ} \mathrm{W} 66^{\circ} \mathrm{W} 63^{\circ} \mathrm{W} 60^{\circ} \mathrm{W} 57^{\circ} \mathrm{W}$

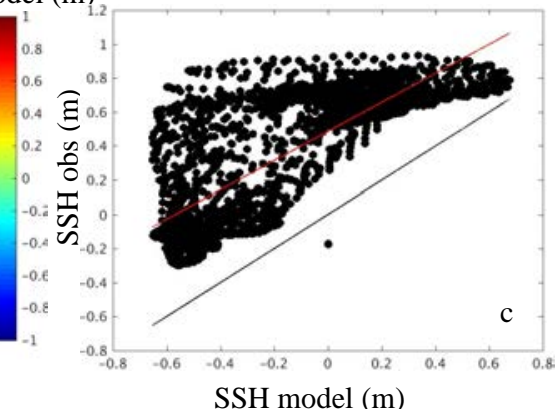

SSH model (m)

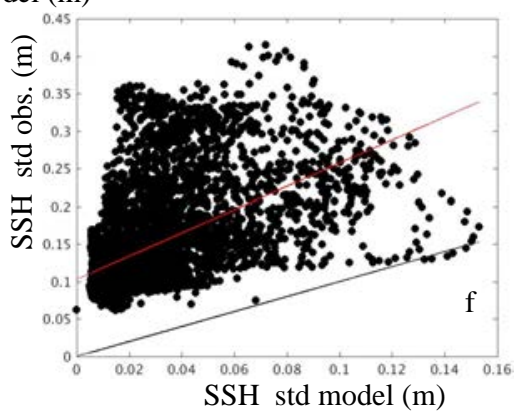

Figure 14: Panel (a) shows the AVISO SSH averaged between 1993 and 2012, panel (b) shows the model SSH averaged over the last 2200 days of the reference simulation, and panel (c) shows the scatter plot between the model and observed mean SSH. In panel (c), the black line is the line of perfect agreement, and the red line is the ordinary least-squares fit. Panels (d-e) are the same as panels (a-c), but for the standard deviation of the observed and modeled SSH. 


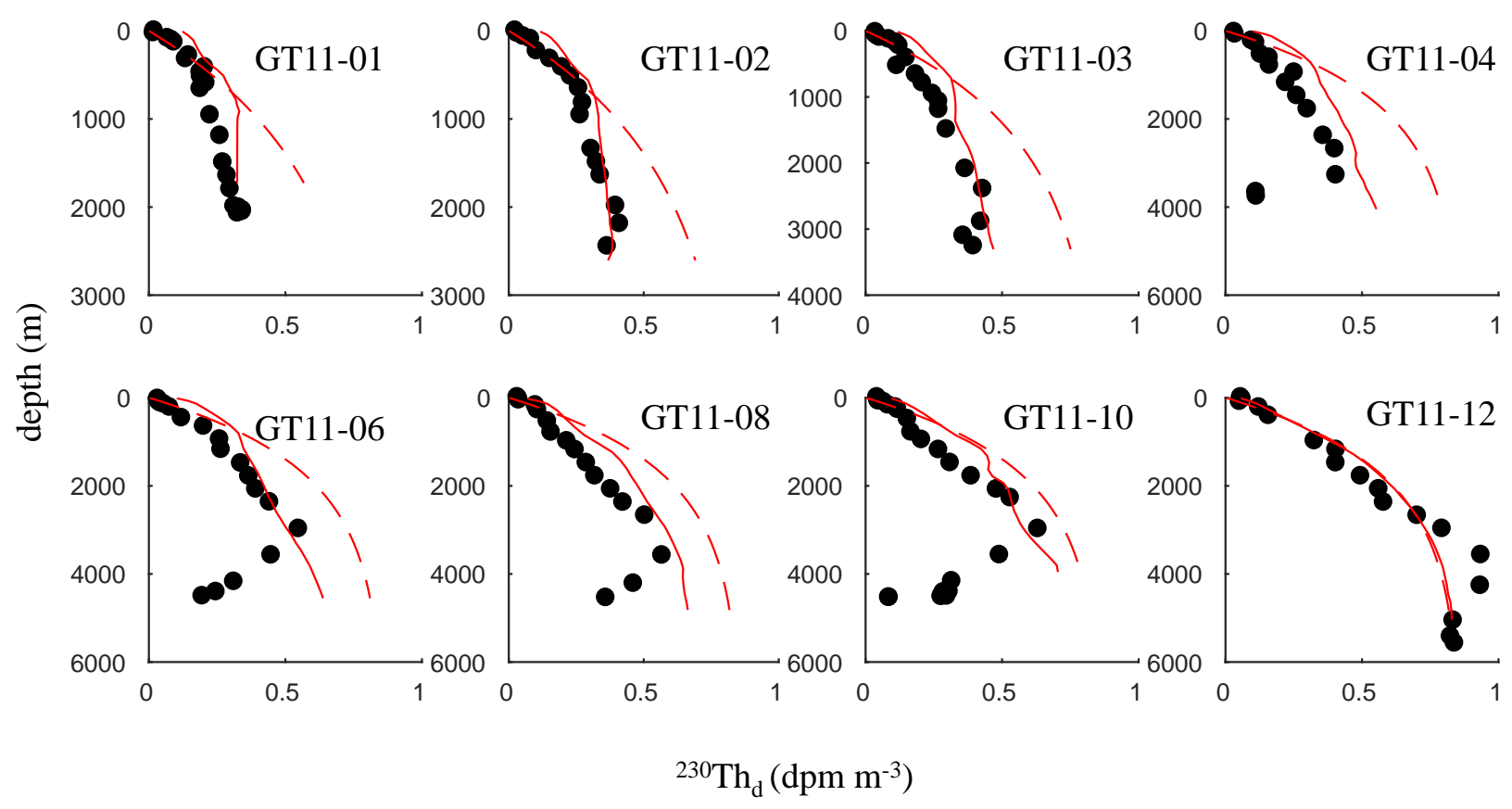

Figure 15: Simulated and observed ${ }^{230} \mathrm{Th}_{d}$ profiles at GEOTRACES stations. The black dots are the observations, the red dashed lines are the initial ${ }^{230} \mathrm{Th}_{d}$ activities assumed in the model, and the red solid lines are the final (after $t=3285$ days) ${ }^{230} \mathrm{Th}_{d}$ activities. Simulated values shown are for the reference experiment. 


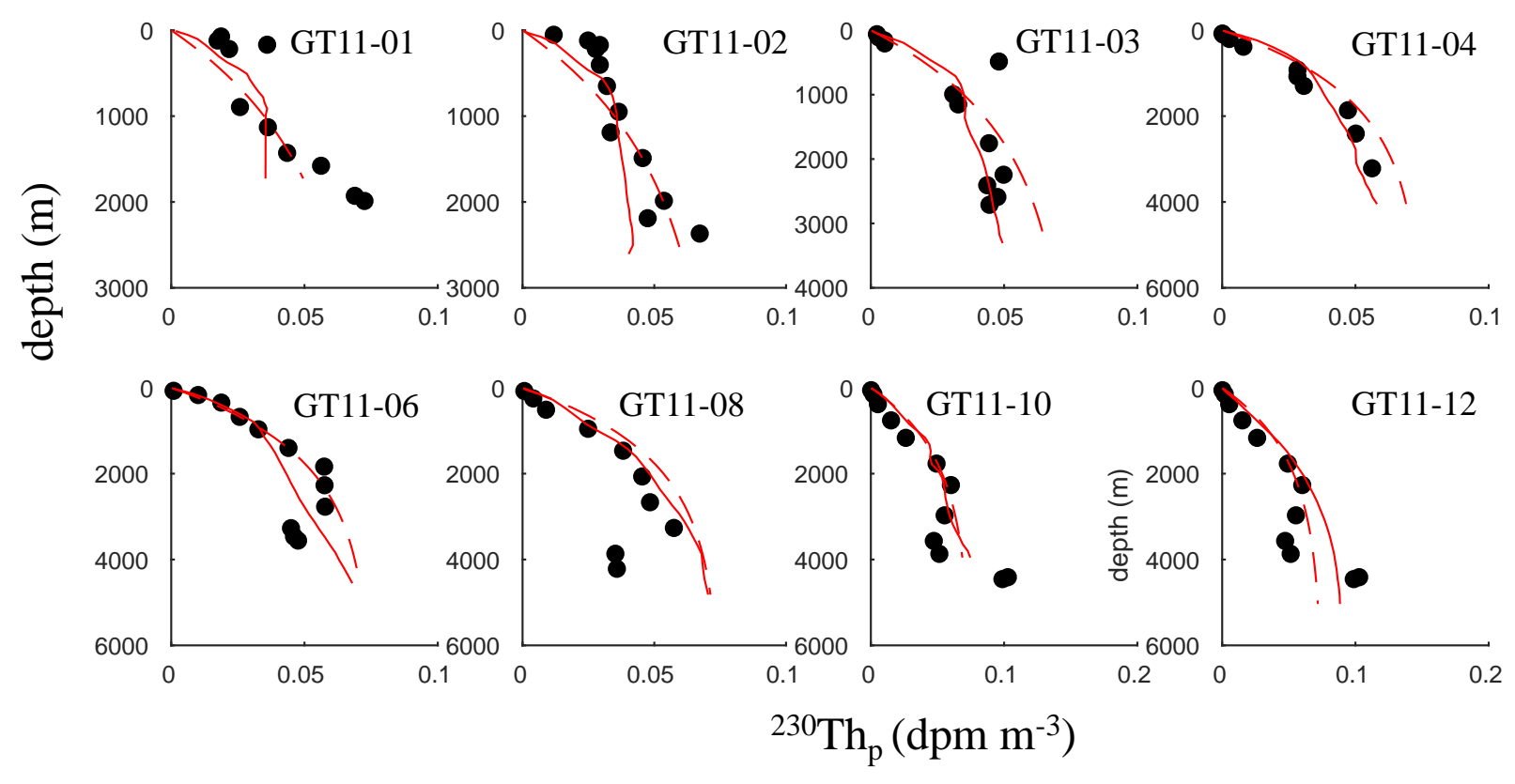

Figure 16: Same as Figure 15, but for ${ }^{230} \mathrm{Th}_{p}$ activities. Note that extremely large ${ }^{230} \mathrm{Th}_{p}$ values measured in four near-bottom samples at stations GT11-04 and GT11-08 are not shown in this figure (these activities, $>0.6 \mathrm{dpm} \mathrm{m}^{-3}$, are shown in Figs. 8 and 25). 

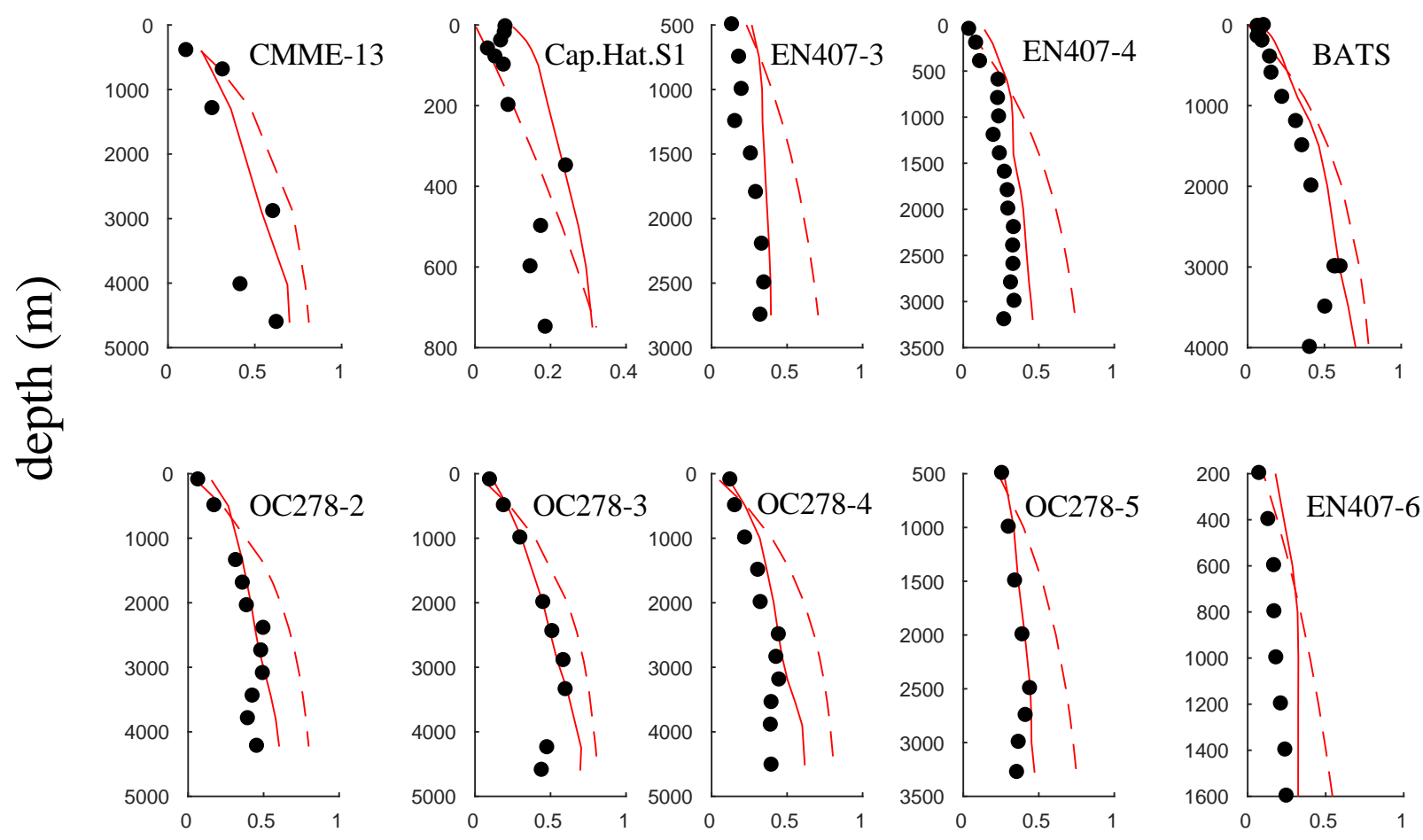

${ }^{230} \mathrm{Th}_{\mathrm{d}}\left(\mathrm{dpm} \mathrm{m} \mathrm{m}^{-3}\right)$

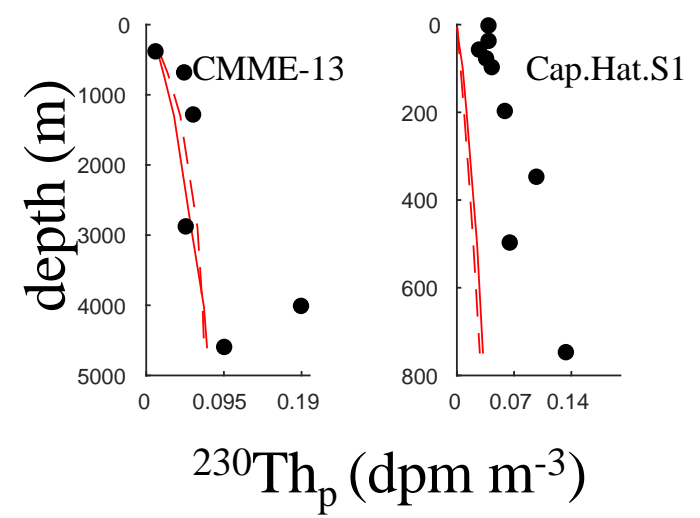

Figure 17: Same as Figures $15-16$, but for ${ }^{230} \mathrm{Th}_{d, p}$ at pre-GEOTRACES stations. The top panels are for ${ }^{230} \mathrm{Th}_{d}$, and the bottom two panels are for ${ }^{230} \mathrm{Th}_{p}$. 

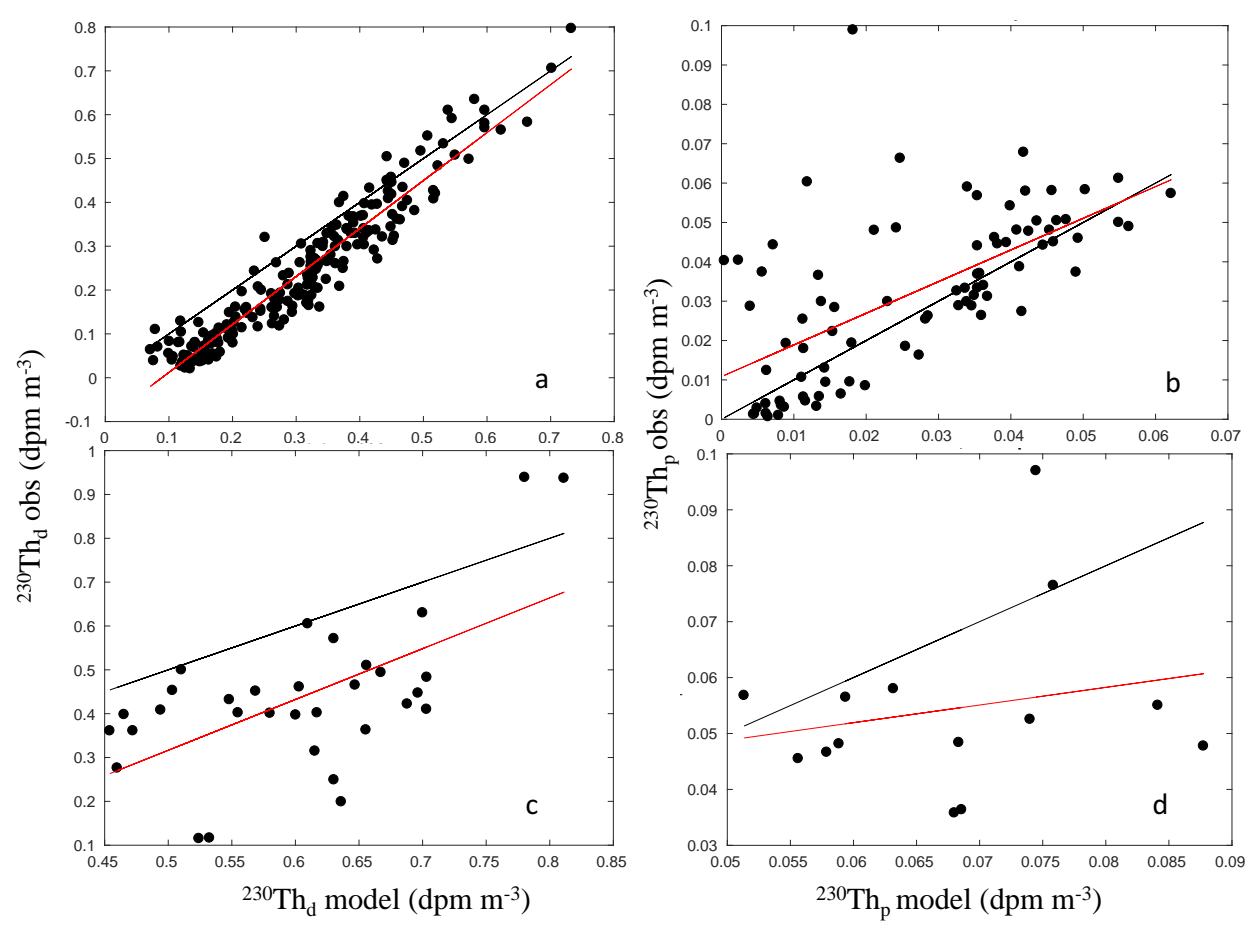

Figure 18: Observed vs. simulated (a,c) ${ }^{230} \mathrm{Th}_{d}$ and $(\mathrm{b}, \mathrm{d}){ }^{230} \mathrm{Th}_{p}$. Panels (a) and (b) show activities above $3000 \mathrm{~m}$, and panels (c) and (d) show activities below $3000 \mathrm{~m}$. In each panel, the black line is the line of perfect agreement, and the red line is the best fit obtained by ordinary least squares. Simulated values shown are for the reference experiment. 

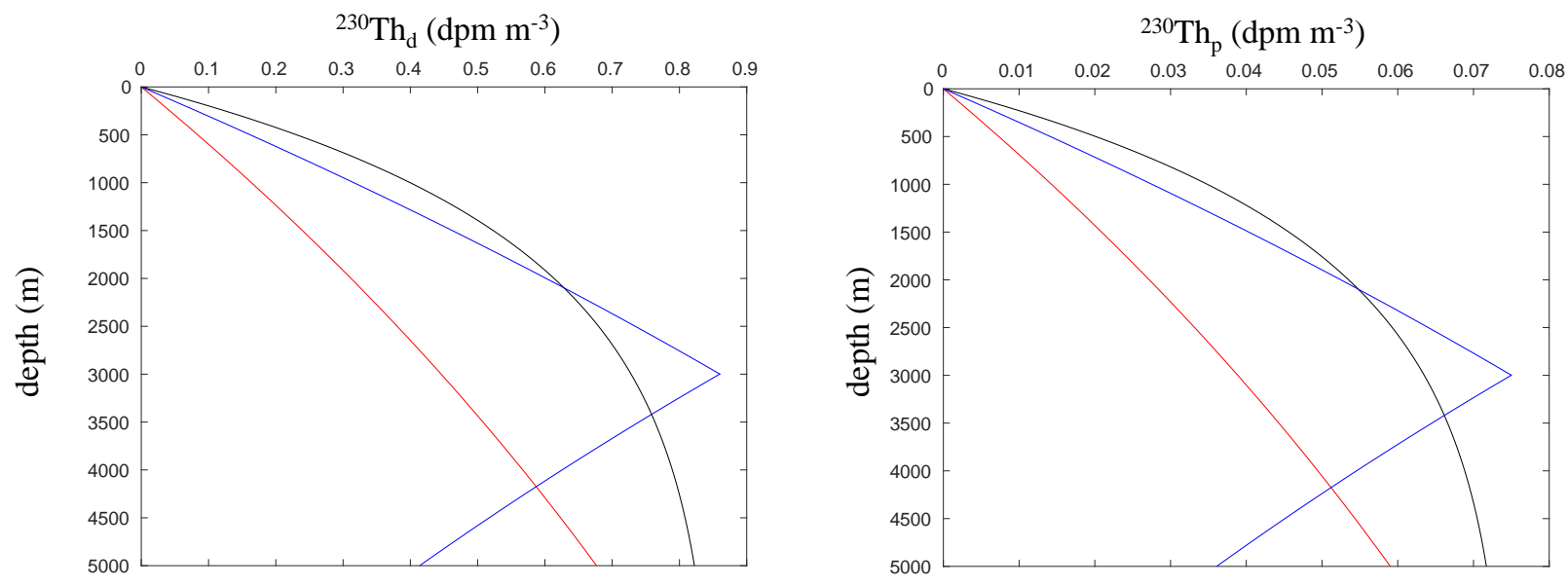

Figure 19: ${ }^{230} \mathrm{Th}_{d, p}$ profiles assumed at the open (eastern and southern) boundaries in different model experiments. The black line, red line, blue line show the activities assumed in the reference experiment, experiment linbnd, and experiment invbnd, respectively 

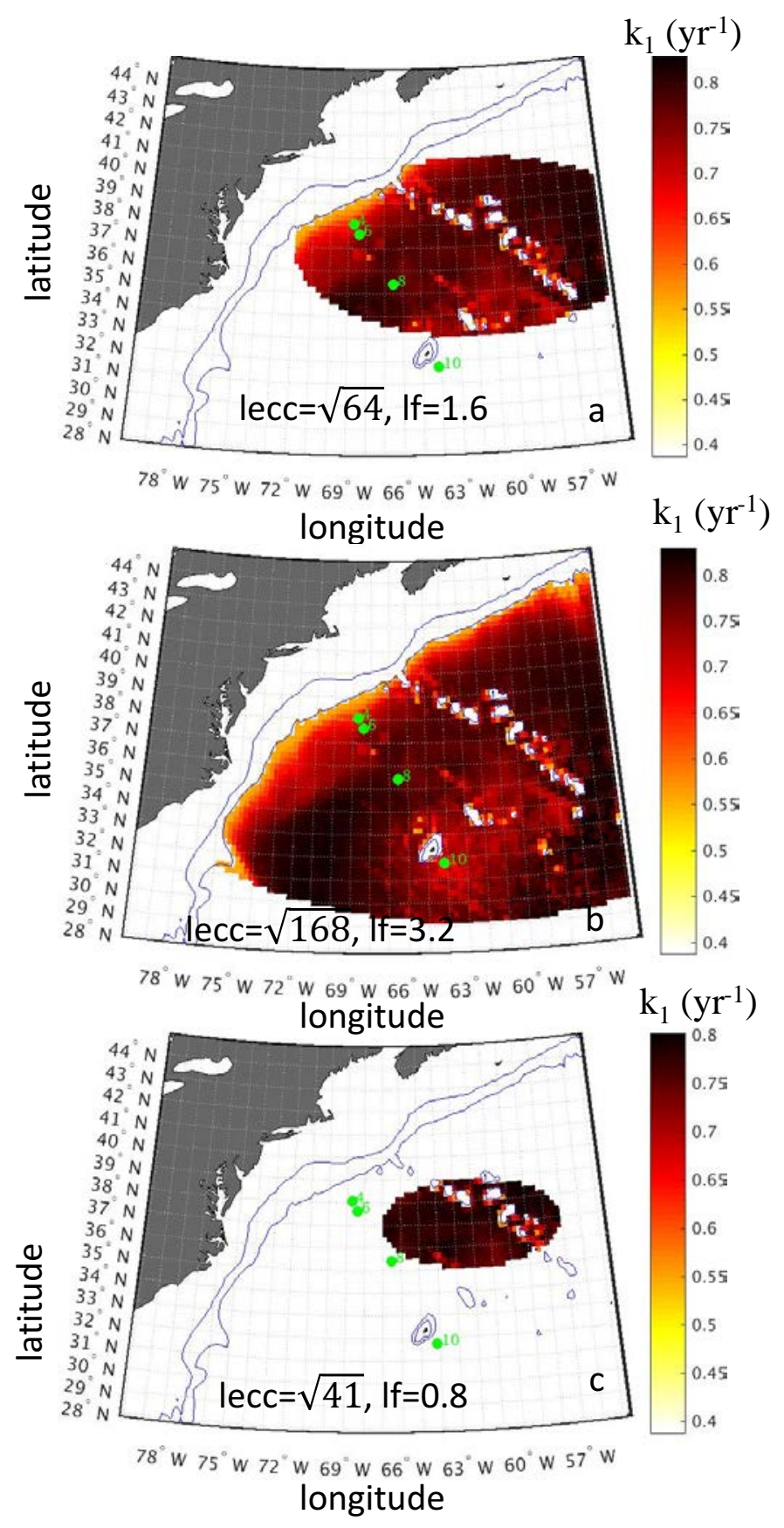

Figure 20: Vertically averaged thorium adsorption rate constant, $k_{1}\left(\mathrm{yr}^{-1}\right)$, assumed in model experiments $k_{1} \operatorname{var} 1$ (a), $k_{1} \operatorname{var} 2(\mathrm{~b})$, and $k_{1} \operatorname{var} 3$ (c). $k_{1}$ is only allowed to vary within the colored regions defined using the parameters in Table 6, and only below $3000 \mathrm{~m}$, where $k_{1}$ is calculated using equation (5.9). Outside of the colored regions, $k_{1}$ is set to a uniform value of $0.4 \mathrm{yr}^{-1}$. The solid lines are the 1000 and $3000 \mathrm{~m}$ isobaths. Shown at the bottom of each panel are values of lecc and lf, which determine the size of the colored regions. The green dots show the locations of station GT11-04, GT11-06, GT11-08, and GT11-10. 

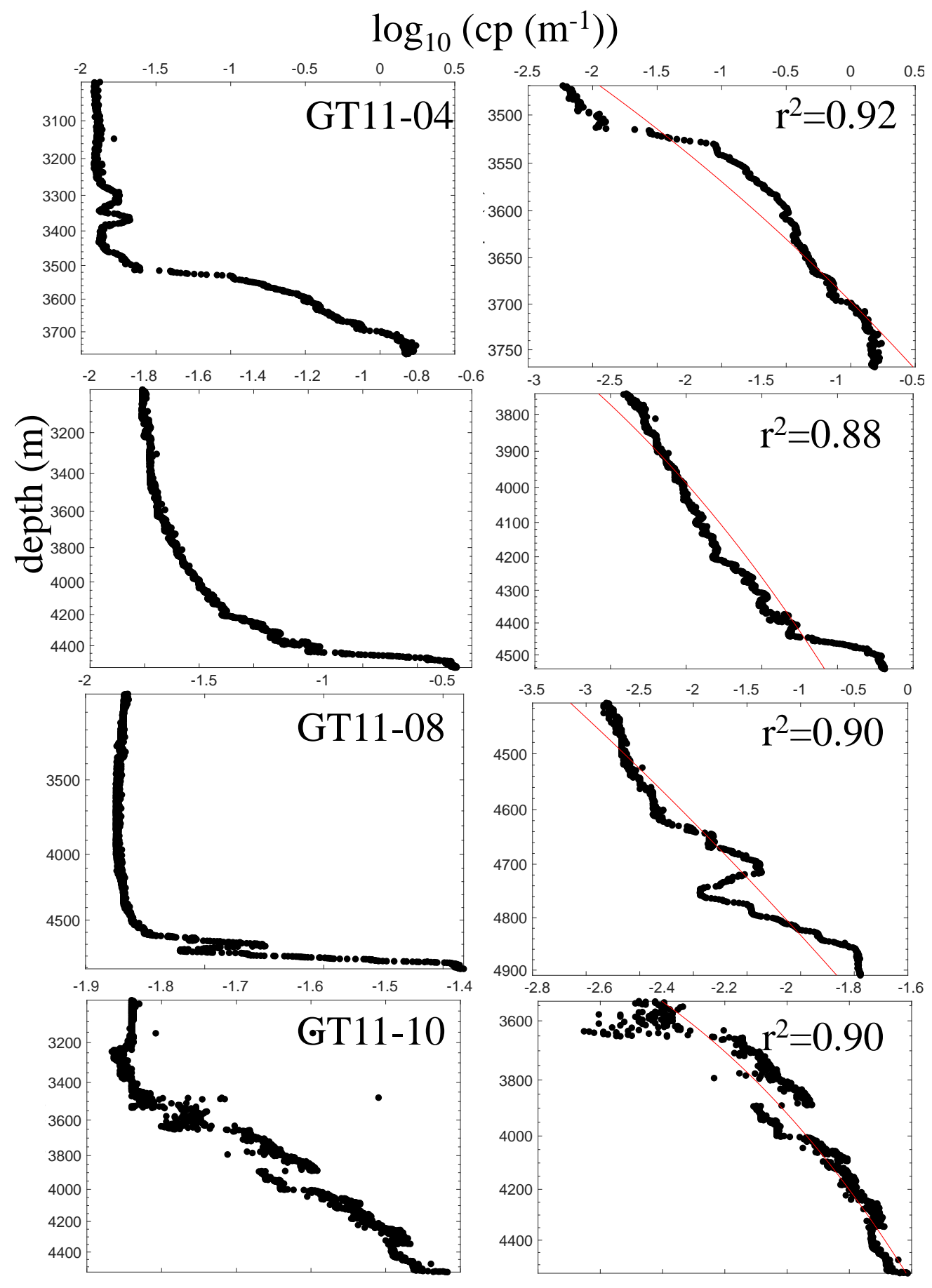

Figure 21: Profiles of the beam attenuation coefficient associated with particles, measured at four stations along the western end of GA03. The left panels show the full profiles below $3000 \mathrm{~m}$, and the right panels show depth intervals where the logarithm of $c_{p}$ varies approximately linearly with depth. The red lines in the right panels are ordinary least-squares fits from which vertical variations in $k_{1}$ are constrained in the model. Data from Hayes et al. (2015a). 

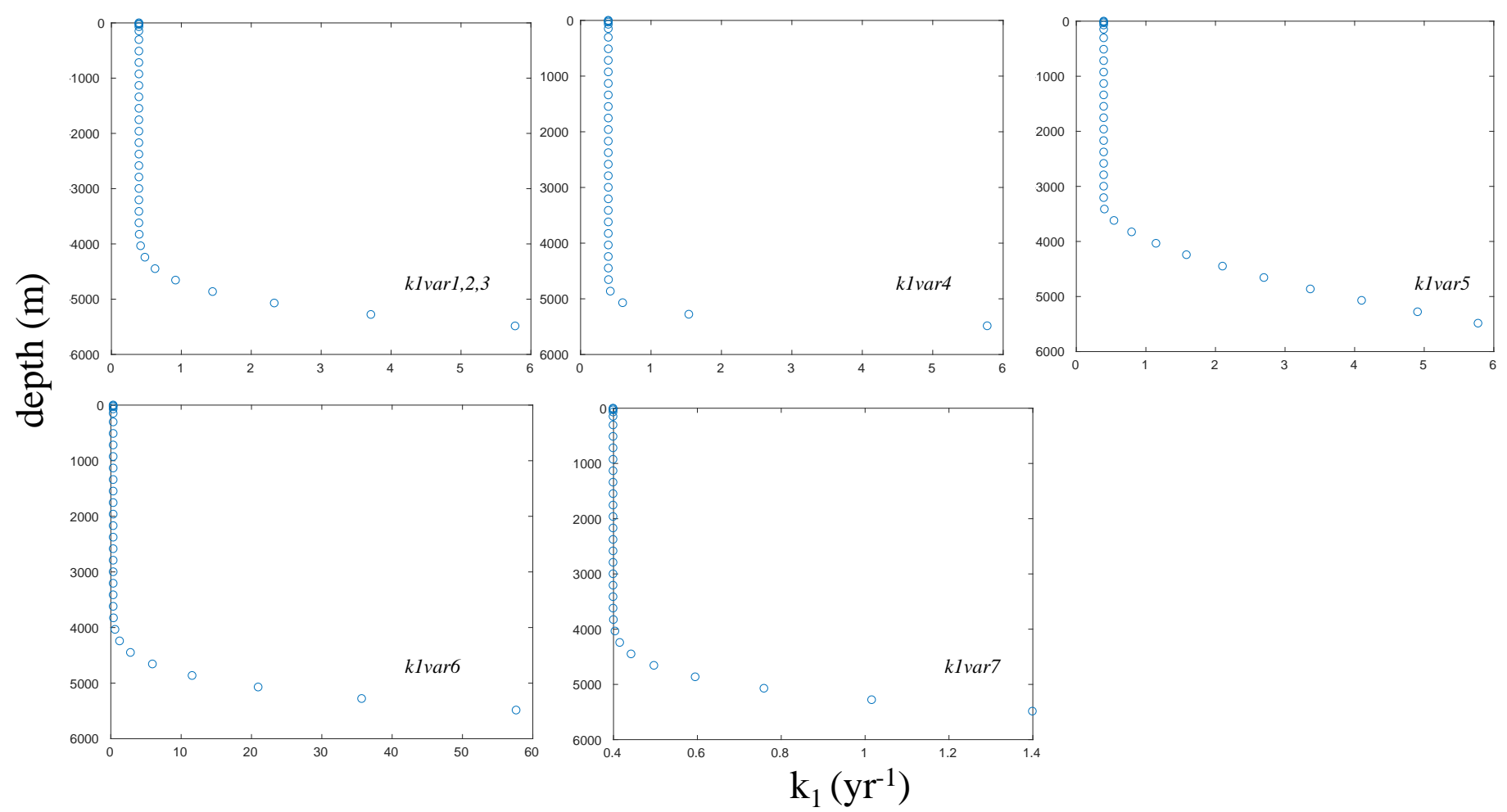

Figure 22: Vertical profiles of $k_{1}$ at $35.7^{\circ} \mathrm{N}, 67.75^{\circ} \mathrm{W}$ (within the region of bottom scavenging) assumed in sensitivity experiments. 

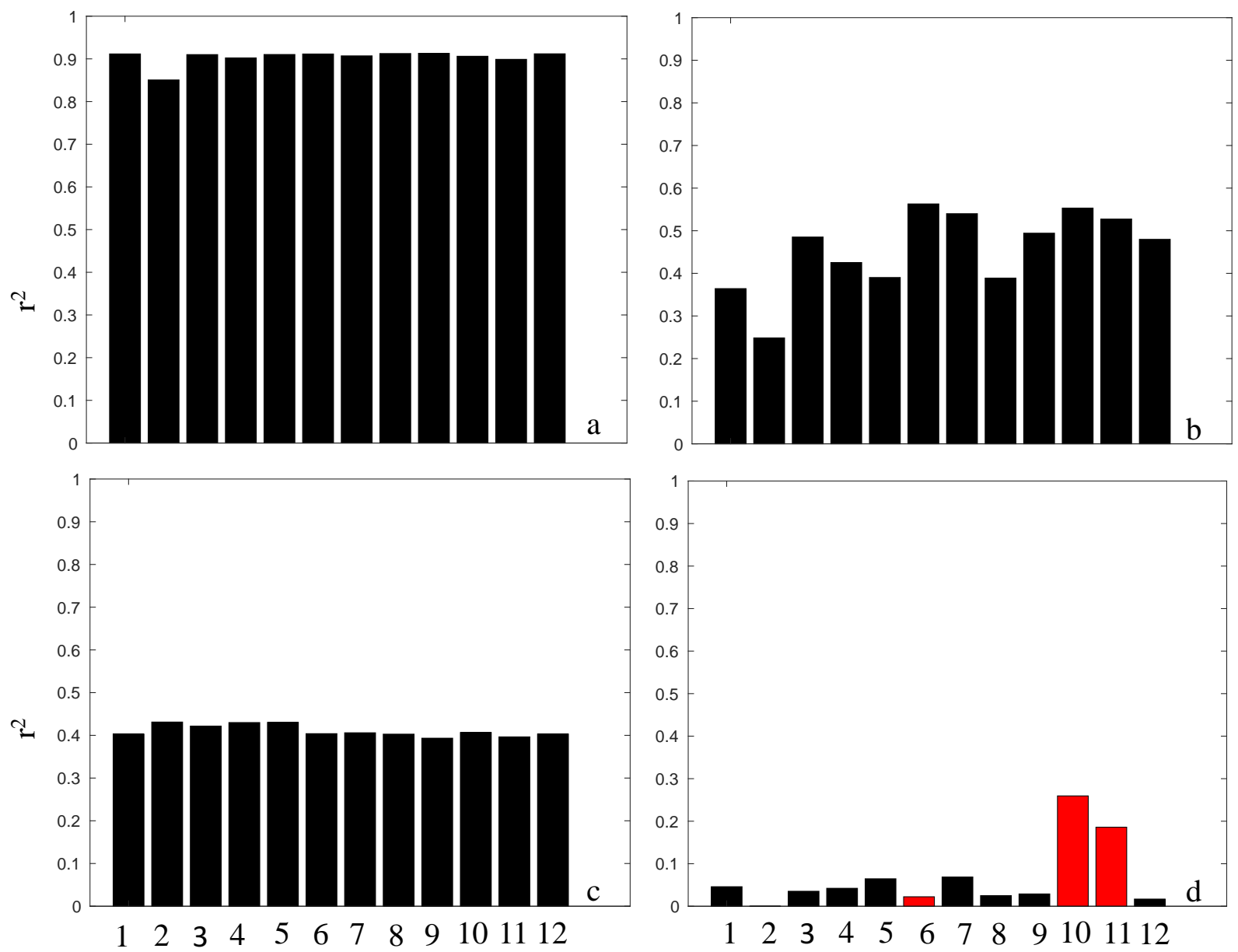

Figure 23: Squared correlation coefficient $\left(r^{2}\right)$ for each model simulation. Panels (a) and (b) show the squared correlation coefficient for ${ }^{230} \mathrm{Th}_{d}$ activities, respectively, above $3000 \mathrm{~m}$ and below $3000 \mathrm{~m}$. Panels (c) and (d) show the squared correlation coefficient for ${ }^{230} \mathrm{Th}_{p}$ activities, respectively, above $3000 \mathrm{~m}$ and below $3000 \mathrm{~m}$. Red bars denote a negative correlation, and black bars denote a positive correlation. The numbers at the bottom refer to the reference experiment (1) and sensitivity experiments (2-12; Table 10). 

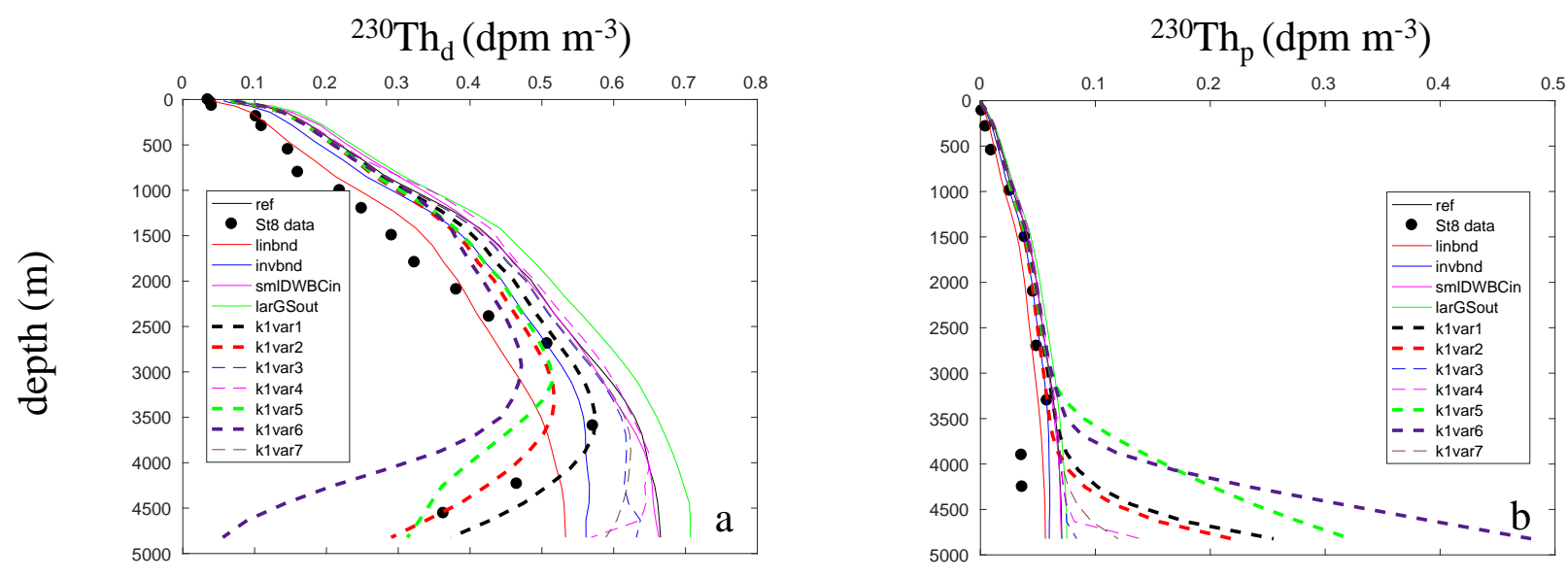

Figure 24: Panel (a) shows the modeled and observed ${ }^{230} \mathrm{Th}_{d}$ activities at station GT11-08. Panel (b) shows these activities for ${ }^{230} \mathrm{Th}_{p}$. Different colored lines are for different experiments. Dashed lines are used for experiments considering spatial variations in $k_{1}$. Thicker lines show experiments for which the model explained over $50 \%$ of the variance in ${ }^{230} \mathrm{Th}_{d}$ below $3000 \mathrm{~m}$. 

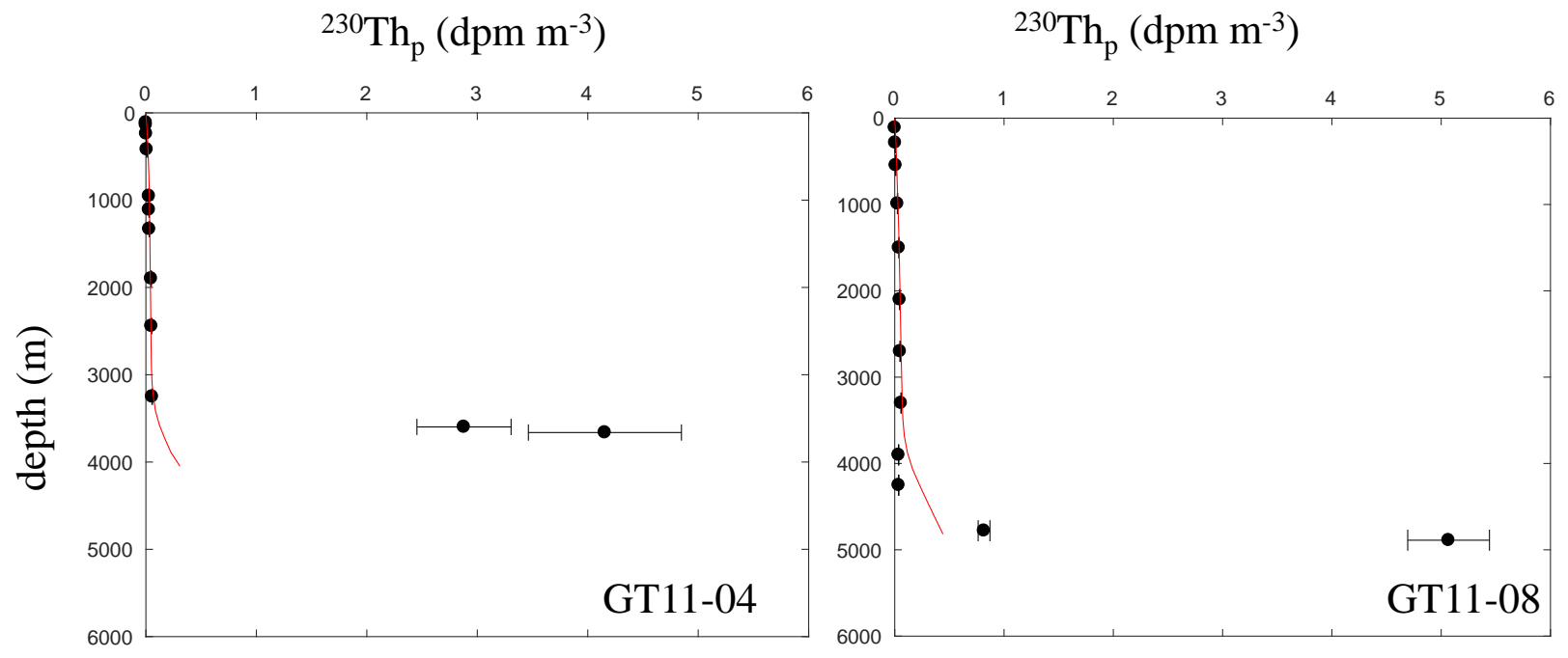

Figure 25: Same as Figure 8, but with the addition of ${ }^{230} \mathrm{Th}_{p}$ activities simulated in experiment $k_{1} \operatorname{var} 6$ (red lines). 

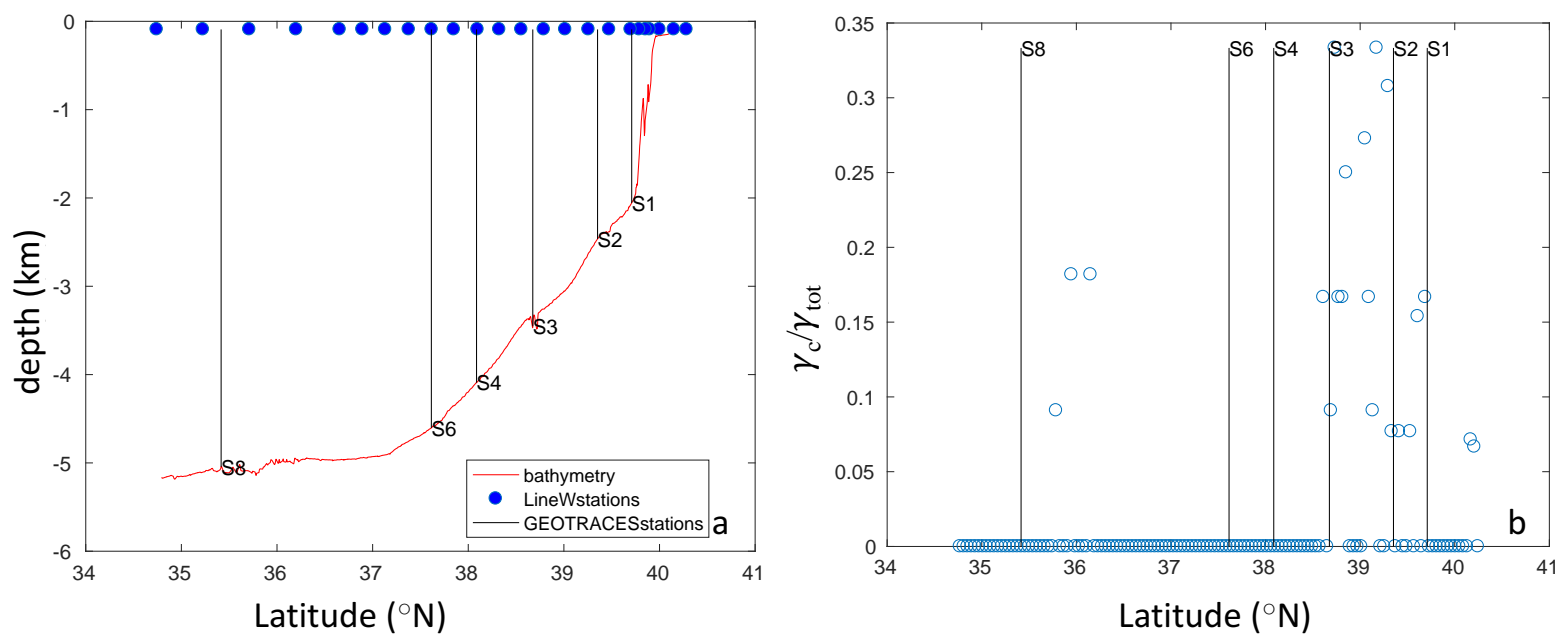

Figure 26: Panel (a) shows the bathymetry (red line) along line W obtained from the GMRT (Ryan et al., 2009), together with the locations of the GEOTRACES (vertical lines) and line W (solid blue circles) stations. Stations S1, S2, S3, S4, S6, and S8 in the panel are stations GT11-01, GT11-02, GT11-03, GT11-04, GT11-06, and GT11-08, respectively. Panel (b) shows the frequency of occurrence of critical slopes with respect to the M2 internal tide along line W. Vertical lines show the locations of GEOTRACES stations, as in panel (a). 


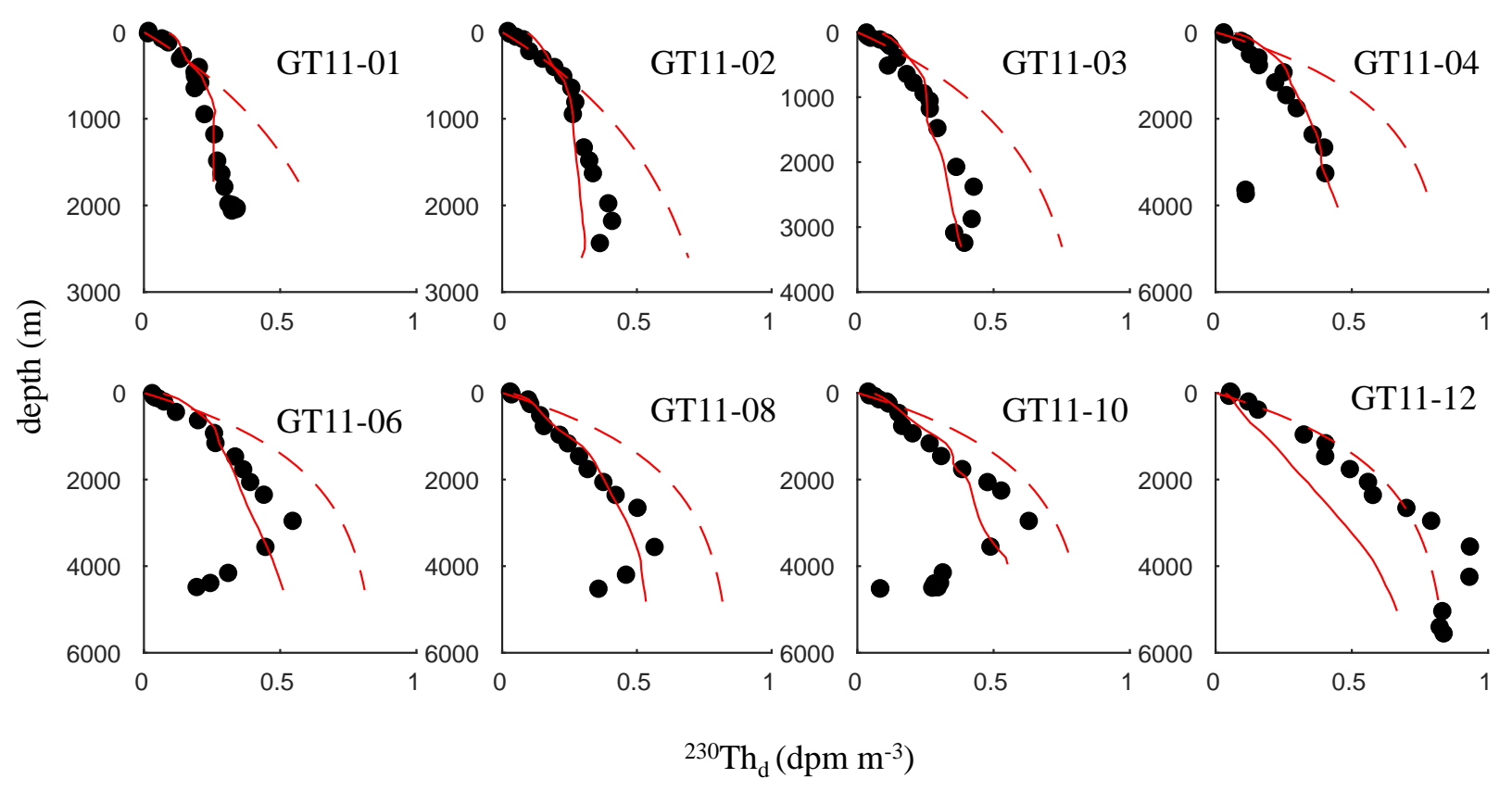

Figure B1. Simulated and observed ${ }^{230} \mathrm{Th}_{d}$ profiles at GEOTRACES stations. The black dots are the observations, the red dashed lines are the initial ${ }^{230} \mathrm{Th}_{d}$ activities assumed in the model, and the red solid lines are the final (after $t=3285$ days) ${ }^{230} \mathrm{Th}_{d}$ activities. Simulated values shown are for experiment "linbnd". 


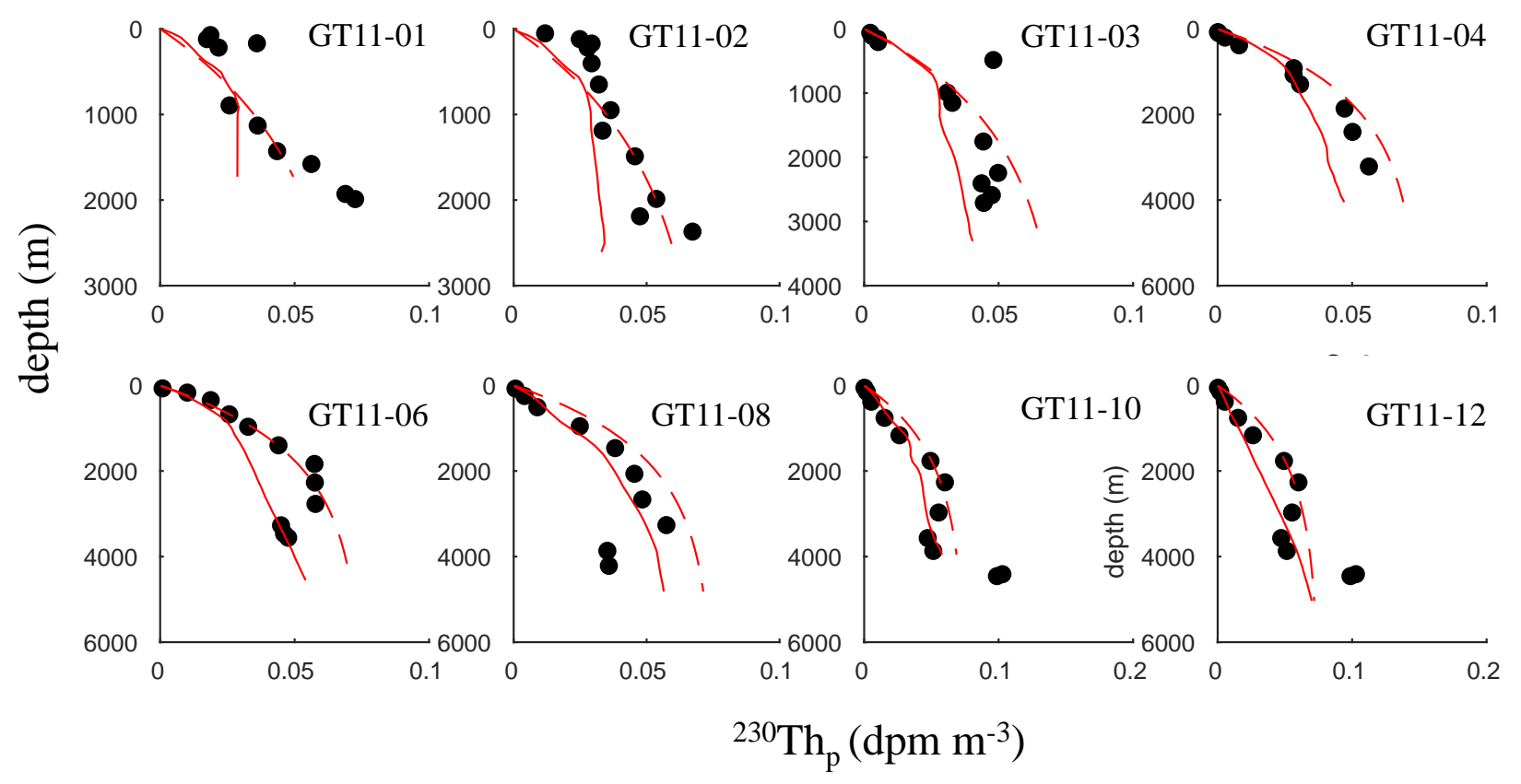

Figure B2. Same as Fig. B1, but for ${ }^{230} \mathrm{Th}_{p}$. 

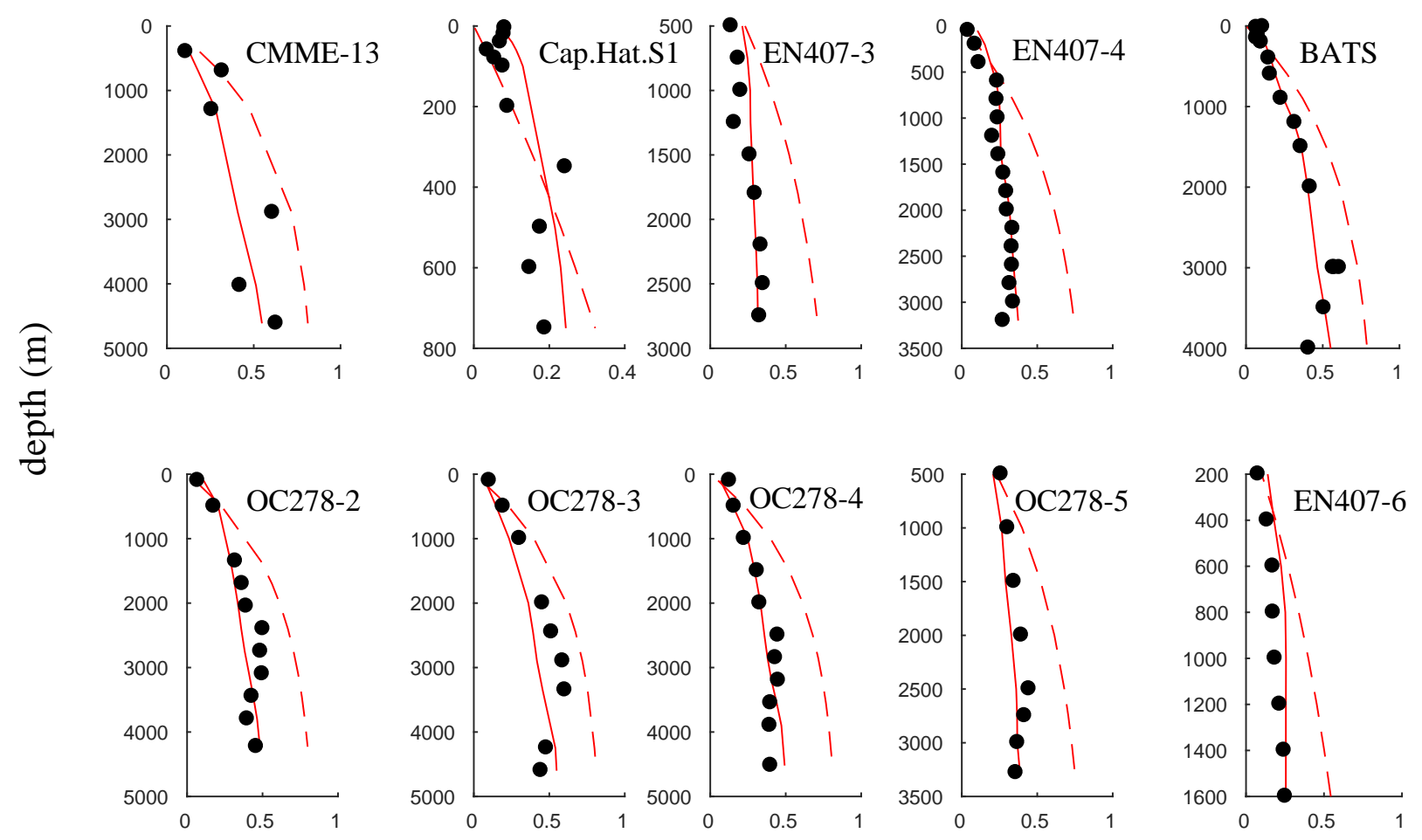

${ }^{230} \mathrm{Th}_{\mathrm{d}}\left(\mathrm{dpm} \mathrm{m}^{-3}\right)$

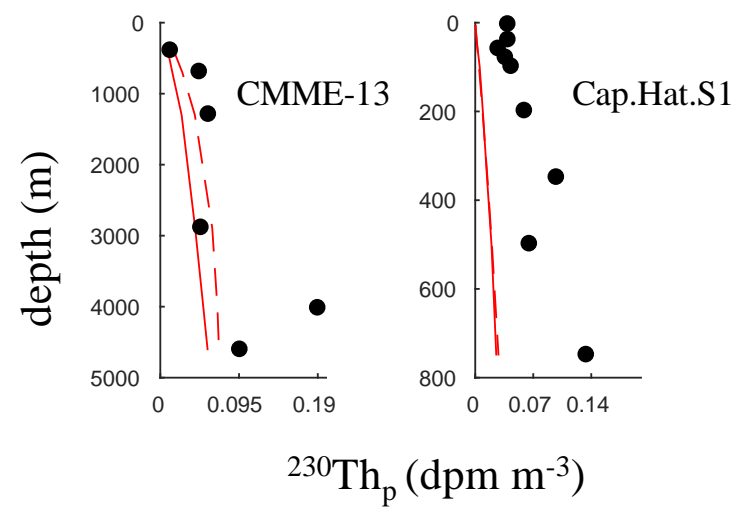

Figure B3. Sames as Figs. B1-B2 but for ${ }^{230} \mathrm{Th}_{d, p}$ at pre-GEOTRACES stations. 

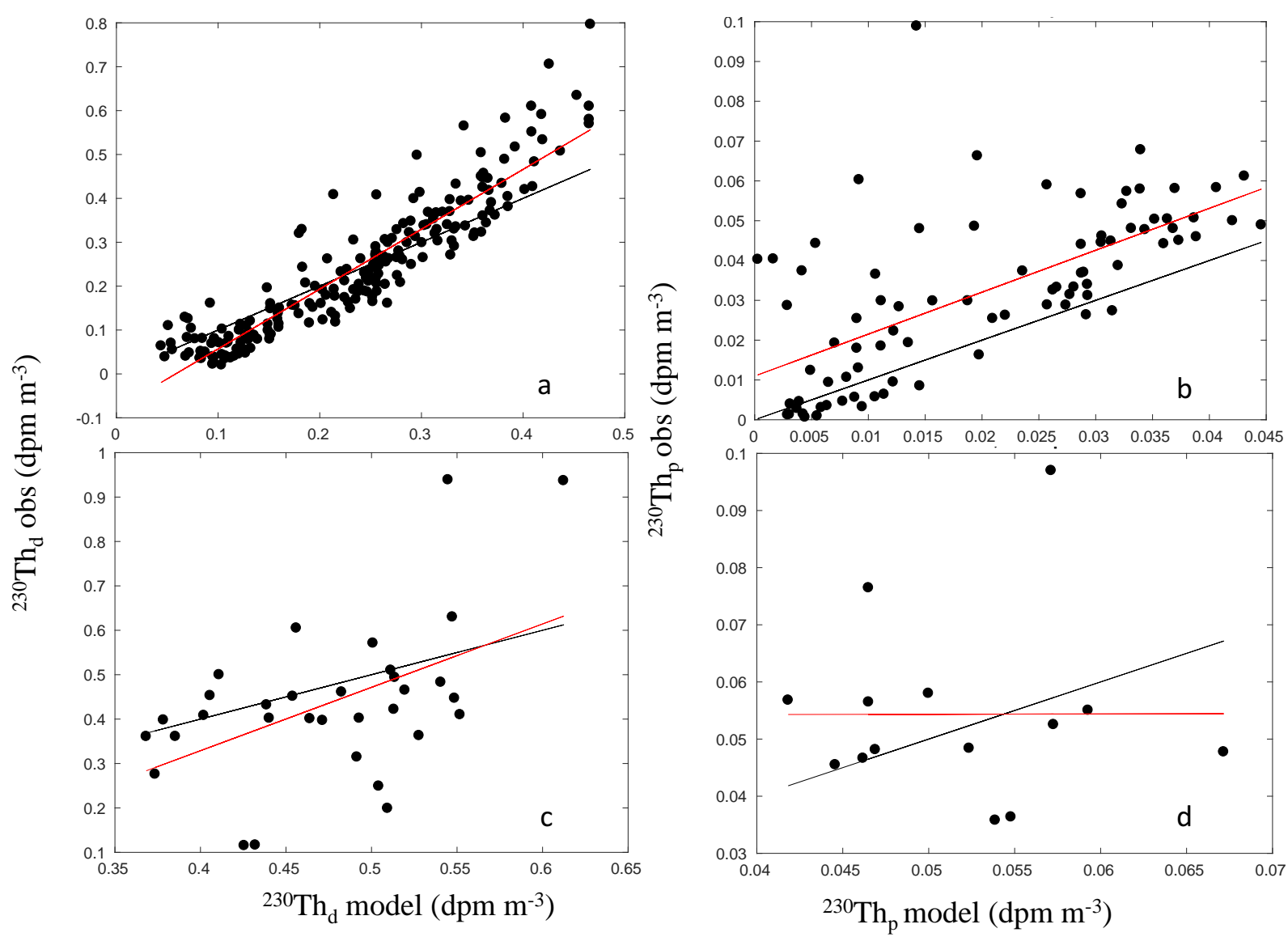

Figure B4. Observed vs. simulated (a,c) ${ }^{230} \mathrm{Th}_{d}$ and (b,d) ${ }^{230} \mathrm{Th}_{p}$. Panels (a) and (b) show activities above $3000 \mathrm{~m}$, and panels (c) and (d) show activities below $3000 \mathrm{~m}$. In each panel, the black line is the line of perfect agreement, and the red line is the best fit obtained by ordinary least squares. Simulated values shown are for experiment "linbnd". 


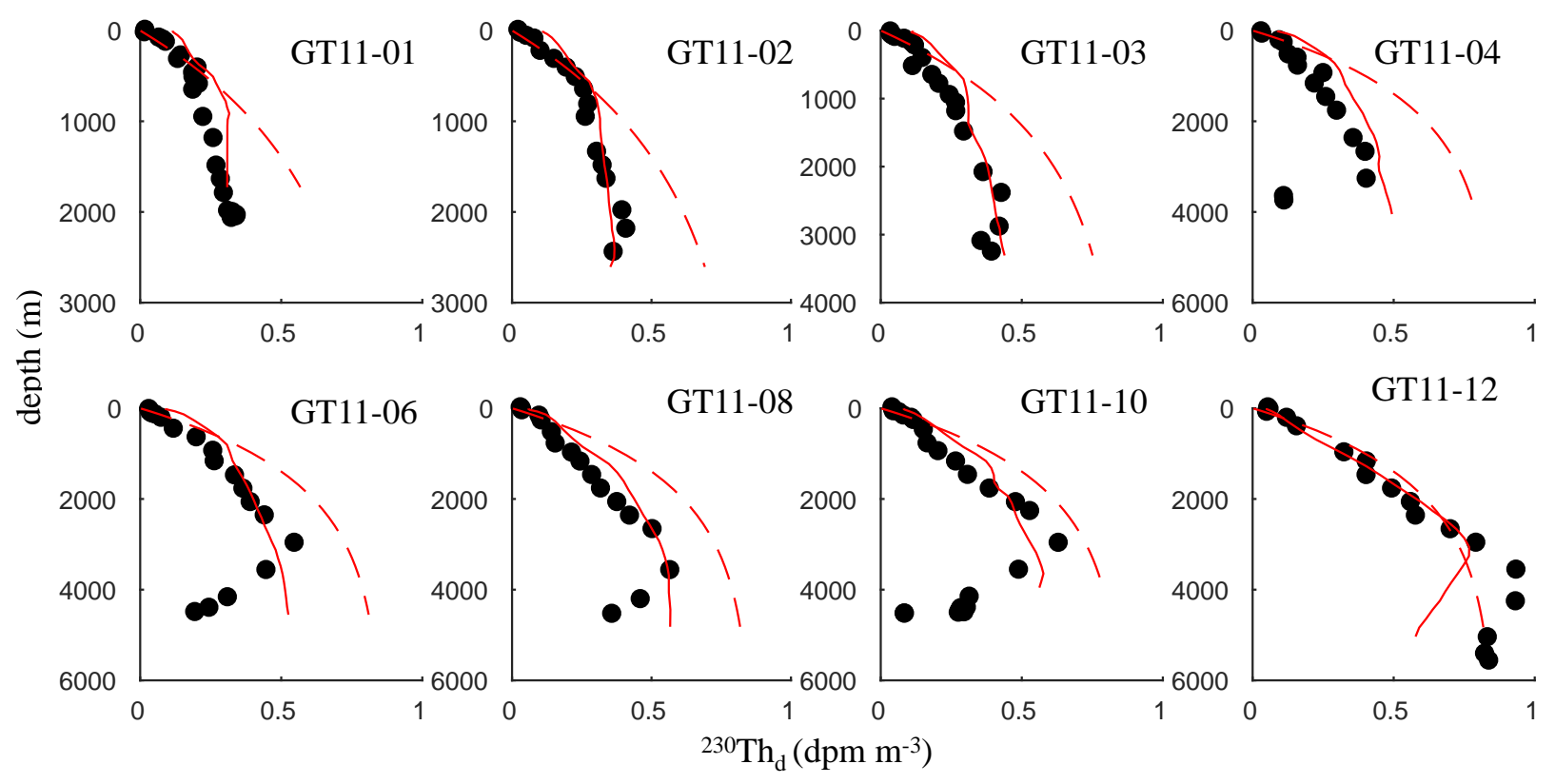

Figure B5. Simulated and observed ${ }^{230} \mathrm{Th}_{d}$ profiles at GEOTRACES stations. The black dots are the observations, the red dashed lines are the initial ${ }^{230} \mathrm{Th}_{d}$ activities assumed in the model, and the red solid lines are the final (after $t=3285$ days ${ }^{230} \mathrm{Th}_{d}$ activities. Simulated values shown are for experiment "invbnd". 


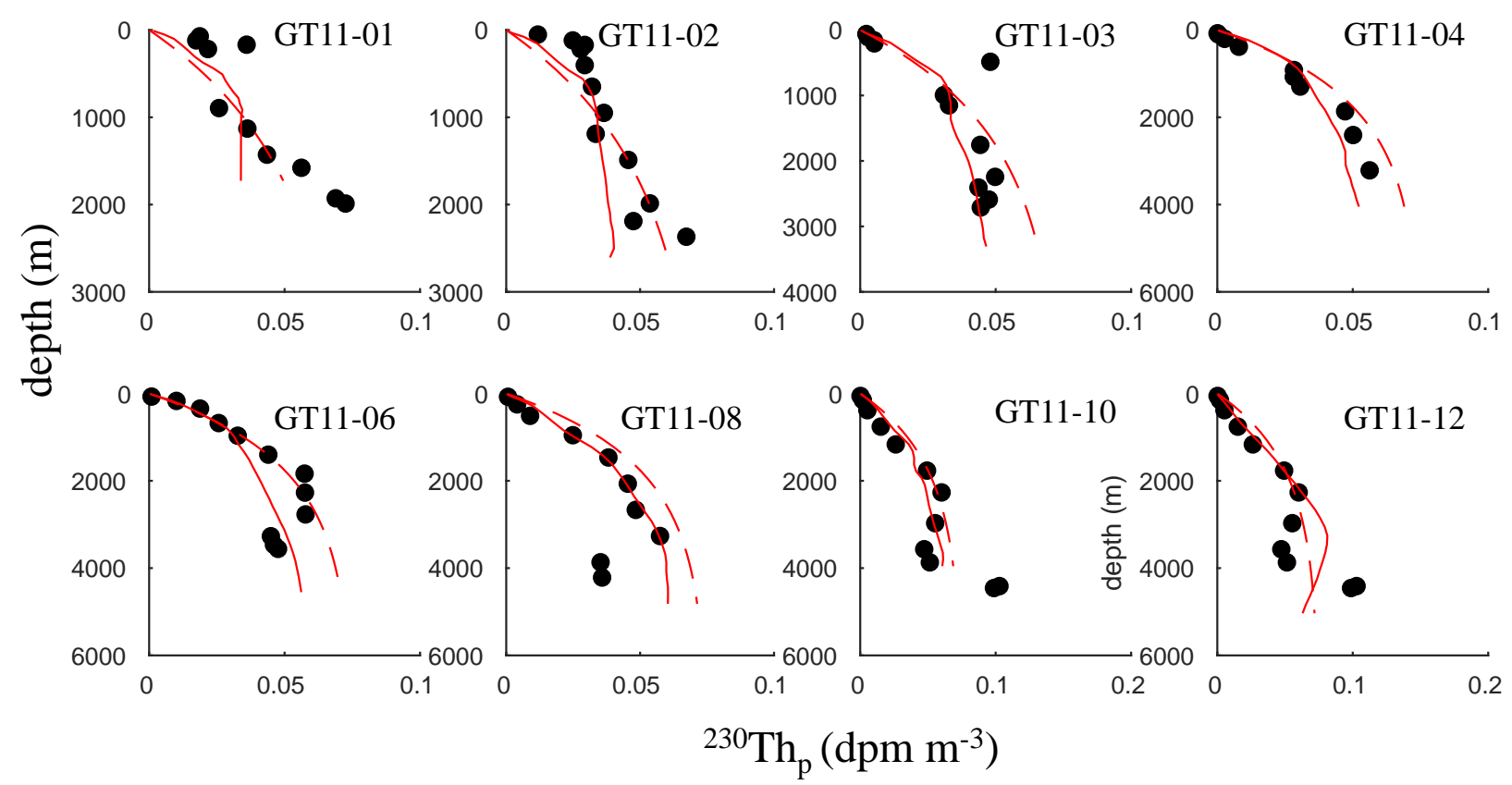

Figure B6. Same as Fig. B5, but for ${ }^{230} \mathrm{Th}_{p}$ 

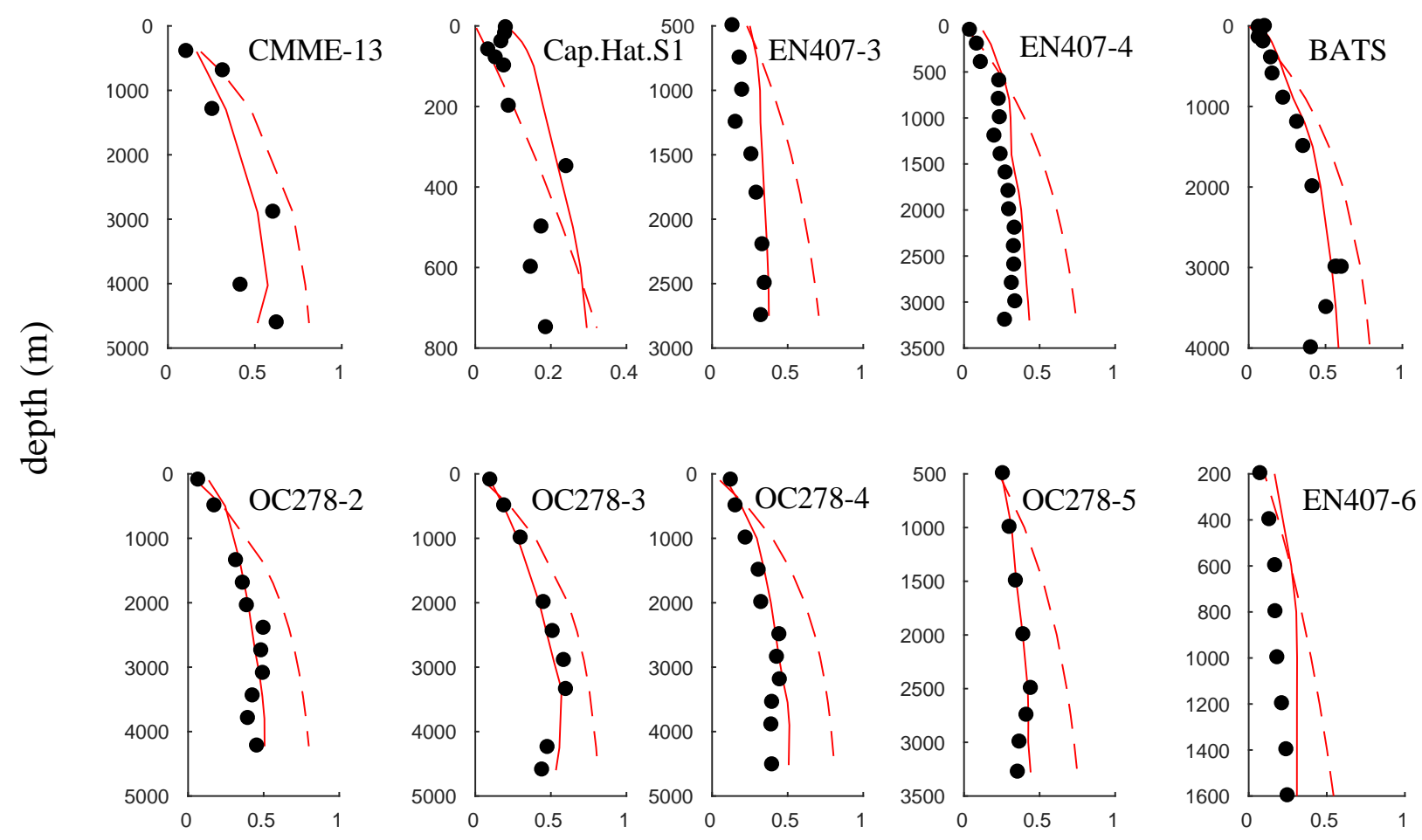

${ }^{230} \mathrm{Th}_{\mathrm{d}}\left(\mathrm{dpm} \mathrm{m} \mathrm{m}^{-3}\right)$

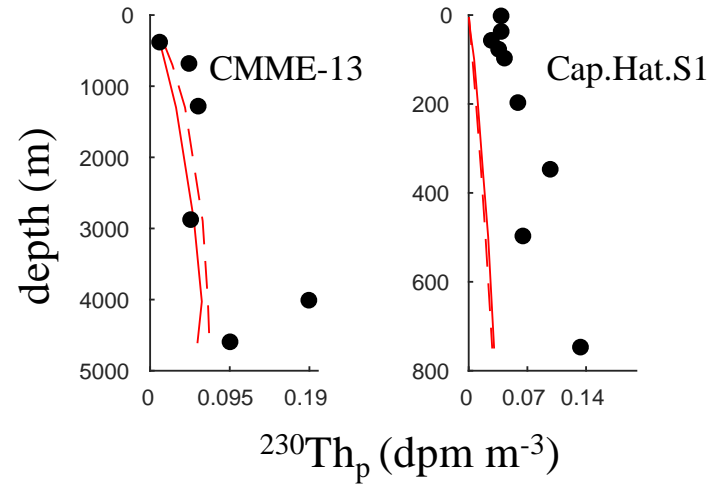

Figure B7. Same as Figs. B5-B6, but for ${ }^{230} \mathrm{Th}_{d, p}$ pre-GEOTRACES stations. 

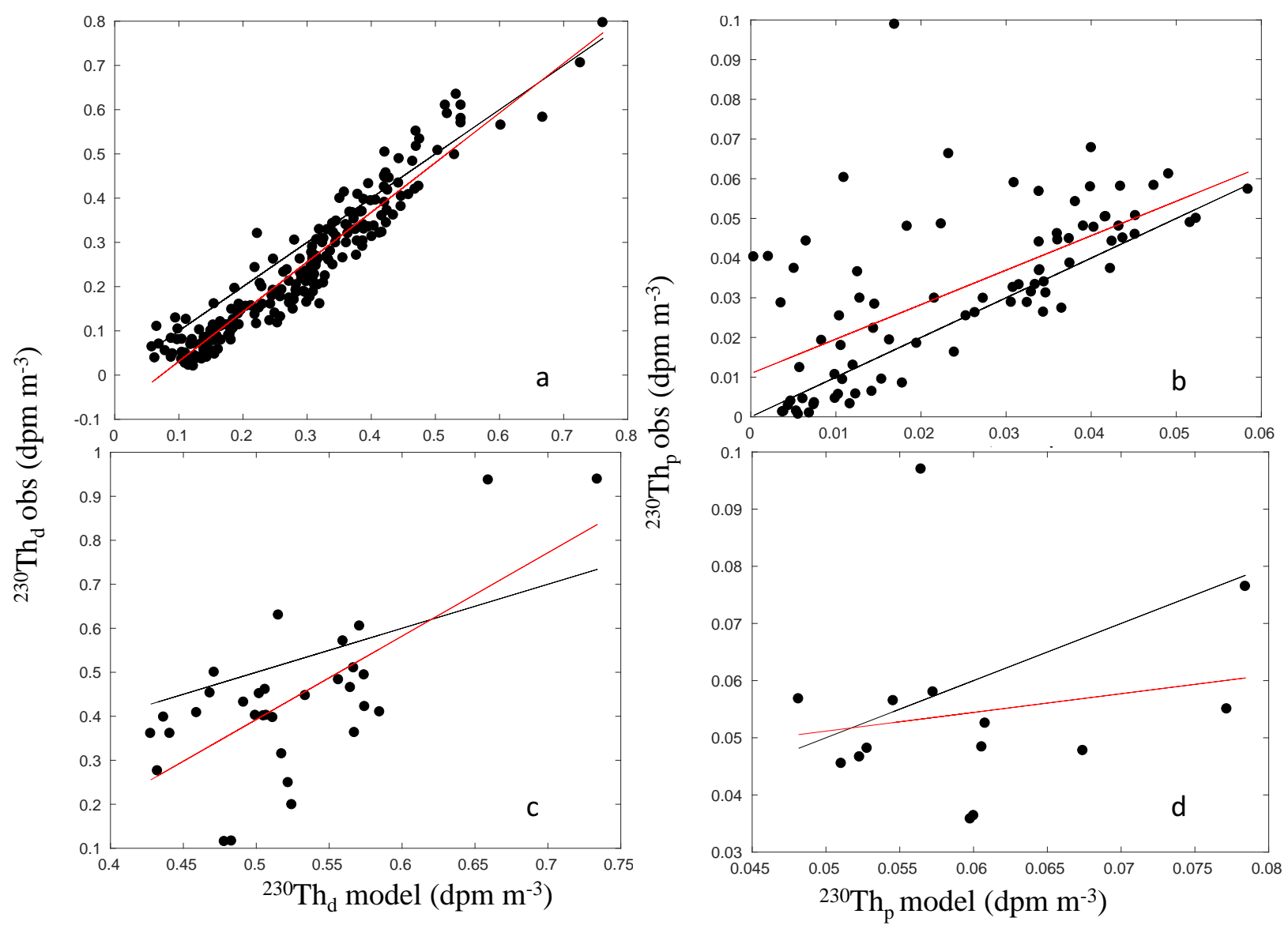

Figure B8. Observed vs. simulated (a,c) ${ }^{230} \mathrm{Th}_{d}$ and (b,d) ${ }^{230} \mathrm{Th}_{p}$. Panels (a) and (b) show activities above $3000 \mathrm{~m}$, and panels (c) and (d) show activities below $3000 \mathrm{~m}$. In each panel, the black line is the line of perfect agreement, and the red line is the best fit obtained by ordinary least squares. Simulated values shown are for experiment "invbnd". 


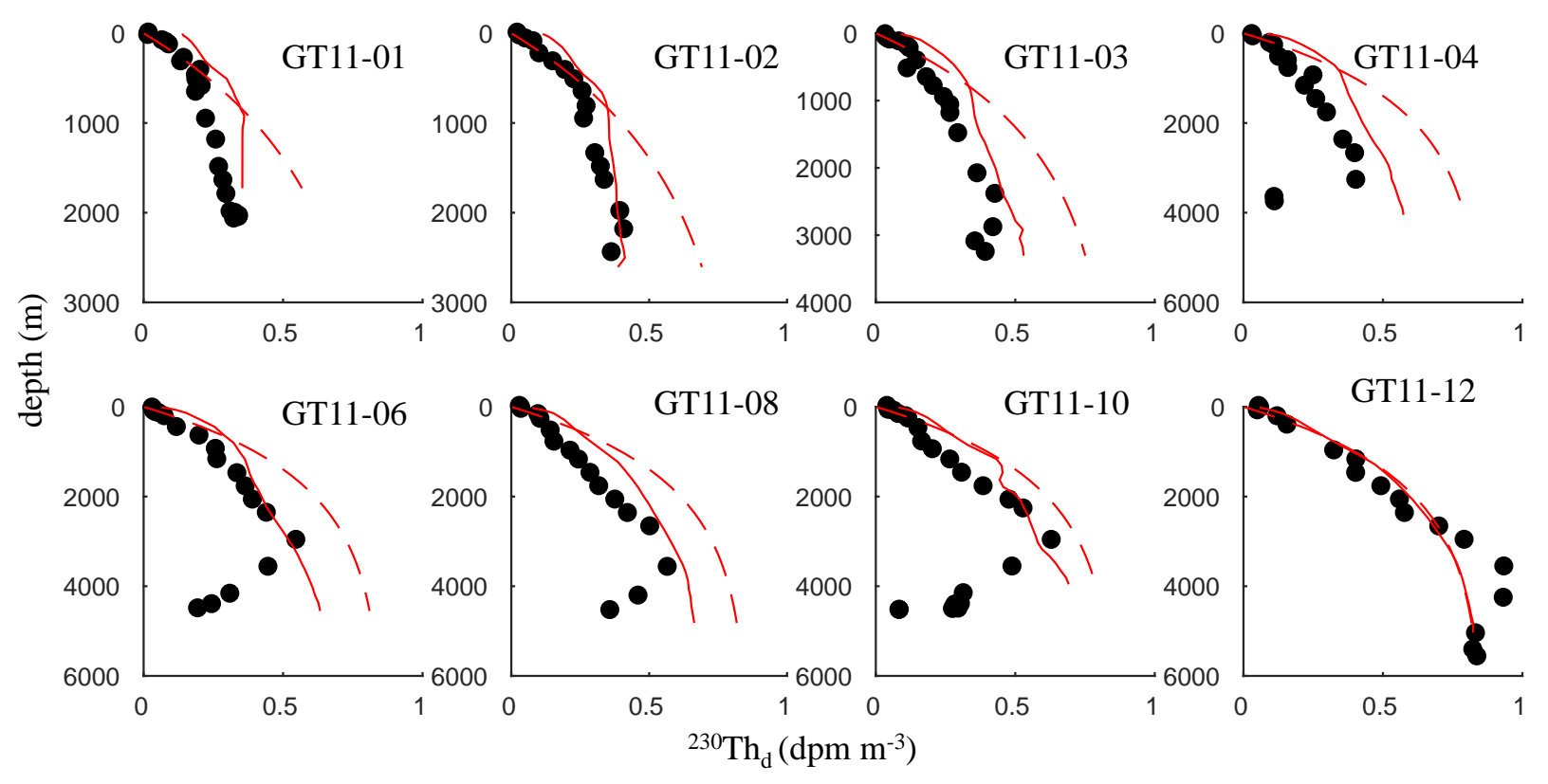

Figure B9. Simulated and observed ${ }^{230} \mathrm{Th}_{d}$ profiles at GEOTRACES stations. The black dots are the observations, the red dashed lines are the initial ${ }^{230} \mathrm{Th}_{d}$ activities assumed in the model, and the red solid lines are the final (after $t=3285$ days) ${ }^{230} \mathrm{Th}_{d}$ activities. Simulated values shown are for experiment "smlDWBC". 


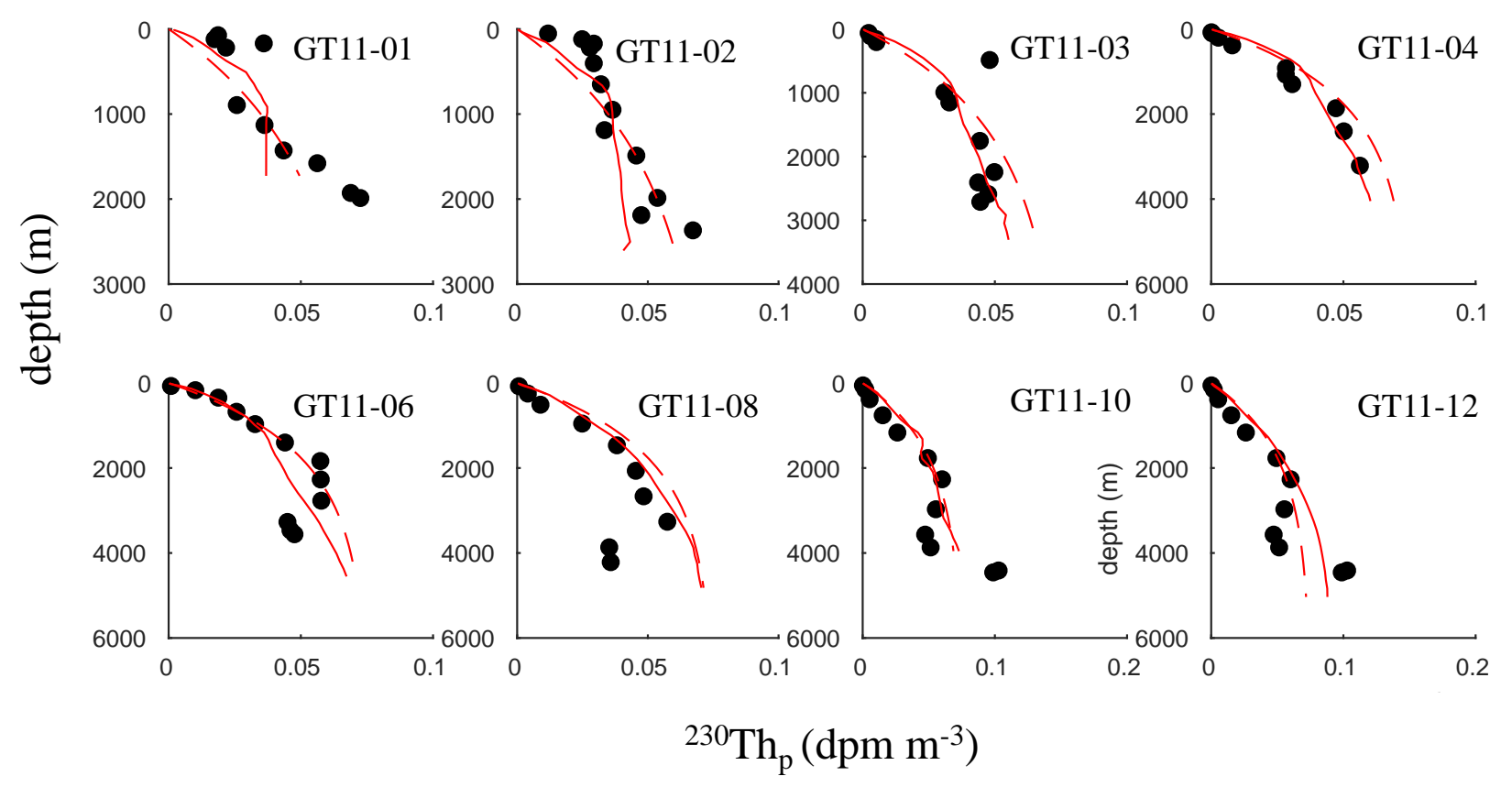

Figure B10. Same as Fig. B9, but for ${ }^{230} \mathrm{Th}_{p}$ 

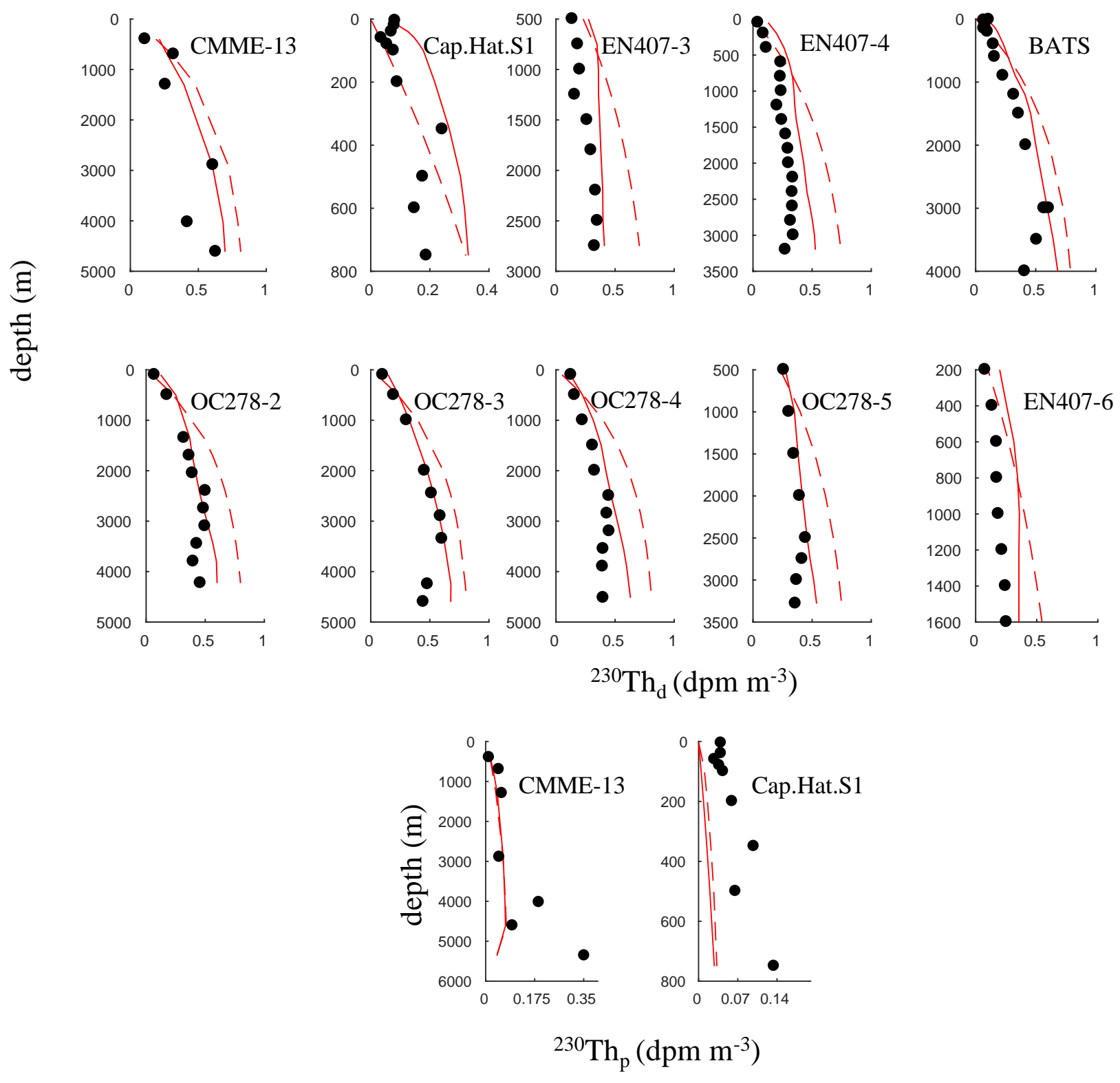

Figure B11. Same as Figs. B9-B10, but for ${ }^{230} \mathrm{Th}_{d, p}$ at pre-GEOTRACES stations 

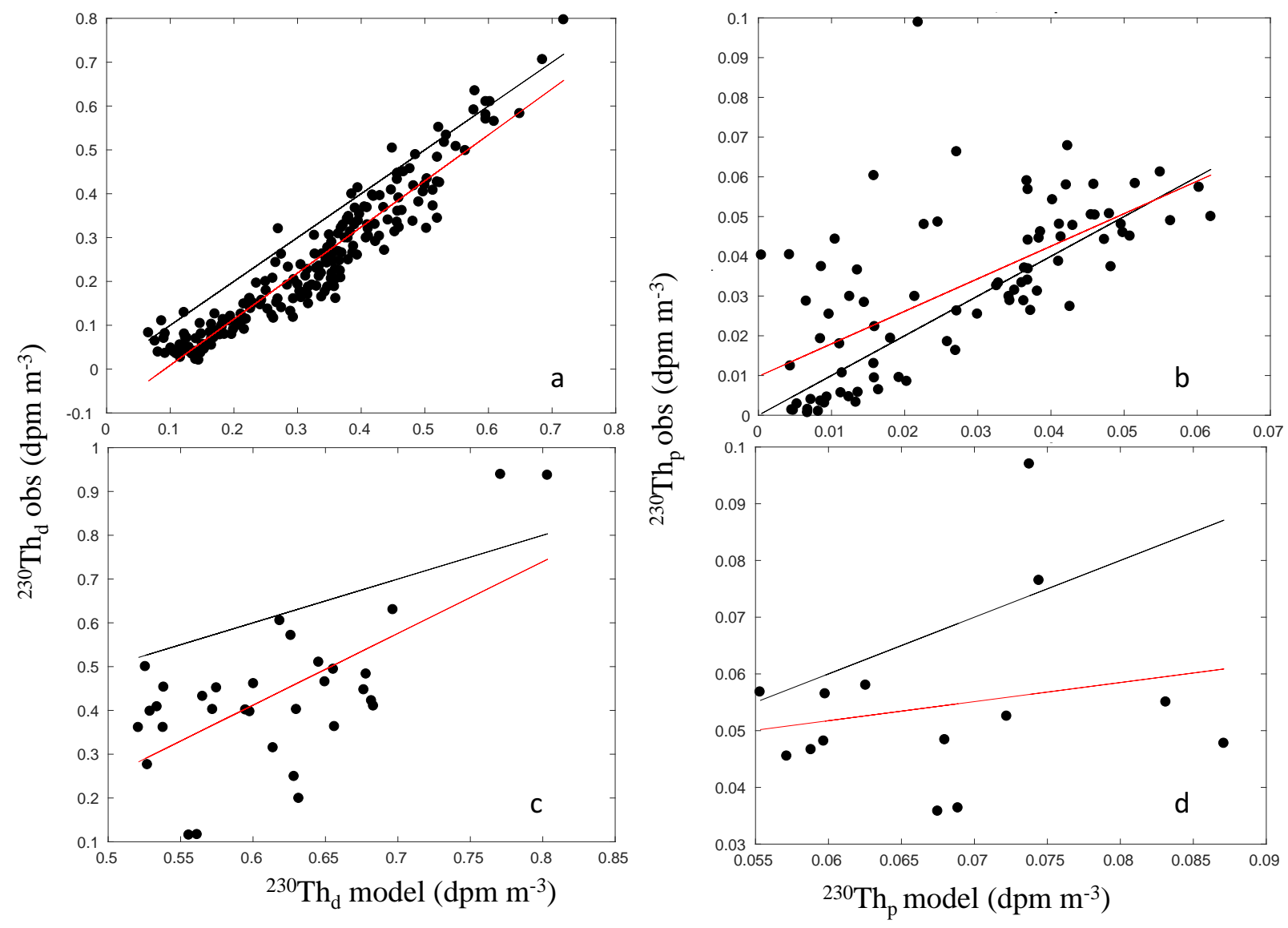

Figure B12. Observed vs. simulated (a,c) ${ }^{230} \mathrm{Th}_{d}$ and (b,d) ${ }^{230} \mathrm{Th}_{p}$. Panels (a) and (b) show activities above $3000 \mathrm{~m}$, and panels (c) and (d) show activities below $3000 \mathrm{~m}$. In each panel, the black line is the line of perfect agreement, and the red line is the best fit obtained by ordinary least squares. Simulated values shown are for experiment "smlDWBCin". 


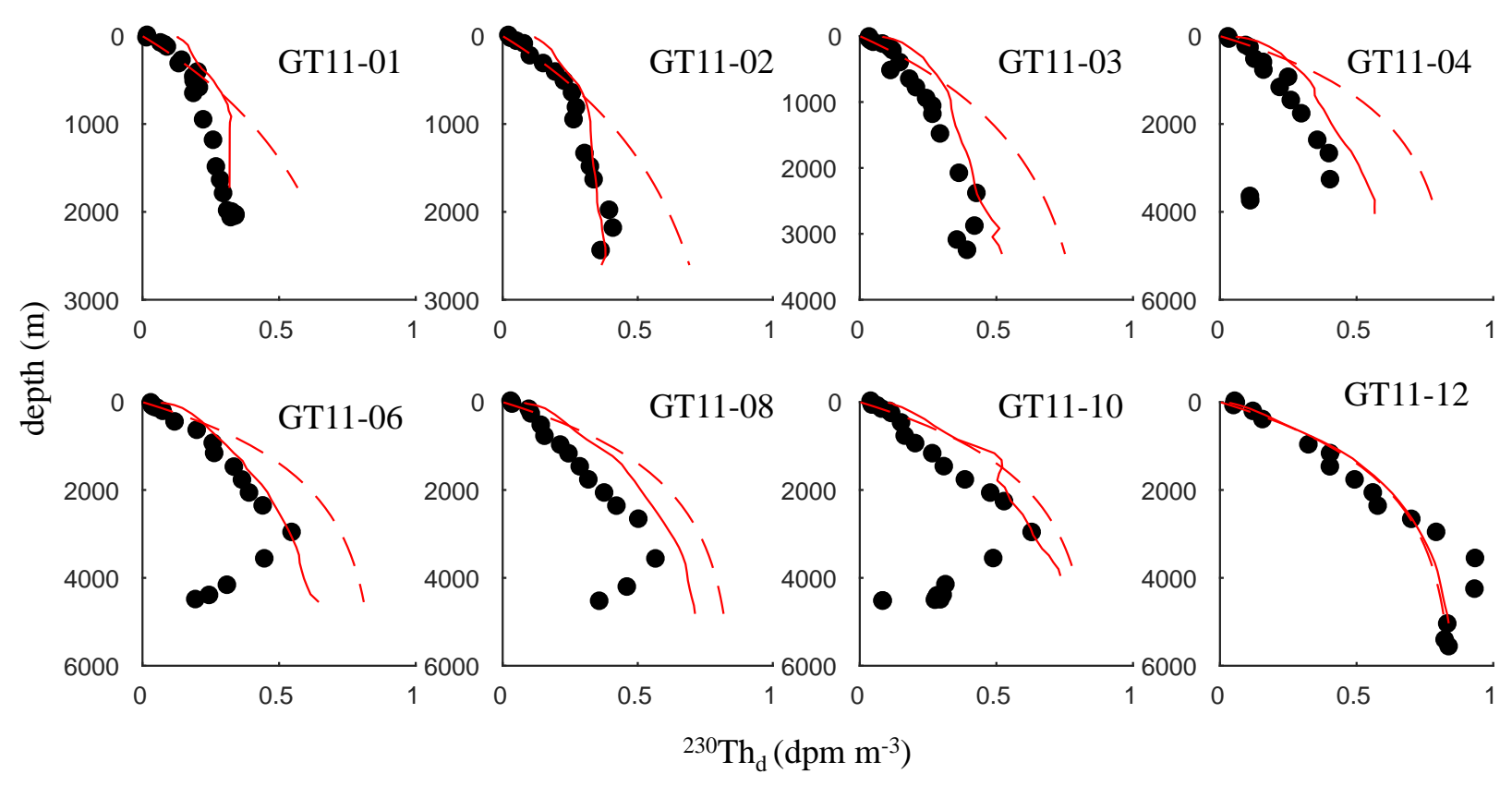

Figure B13. Simulated and observed ${ }^{230} \mathrm{Th}_{d}$ profiles at GEOTRACES stations. The black dots are the observations, the red dashed lines are the initial ${ }^{230} \mathrm{Th}_{d}$ activities assumed in the model, and the red solid lines are the final (after $t=3285$ days ${ }^{230} \mathrm{Th}_{d}$ activities. Simulated values shown are for experiment "larGSout". 


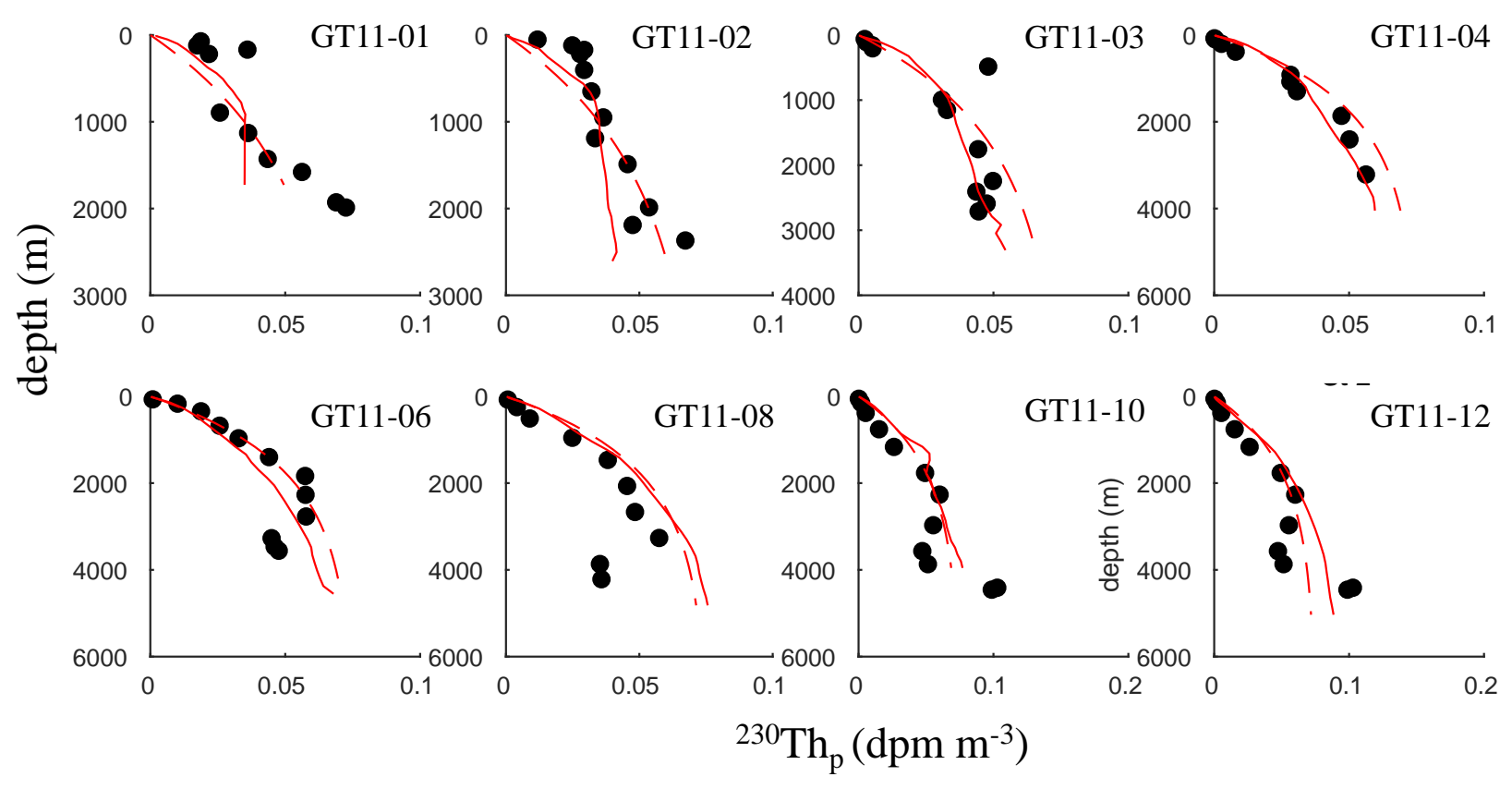

Figure B14. Same as Fig. B13, but for ${ }^{230} \mathrm{Th}_{p}$ 

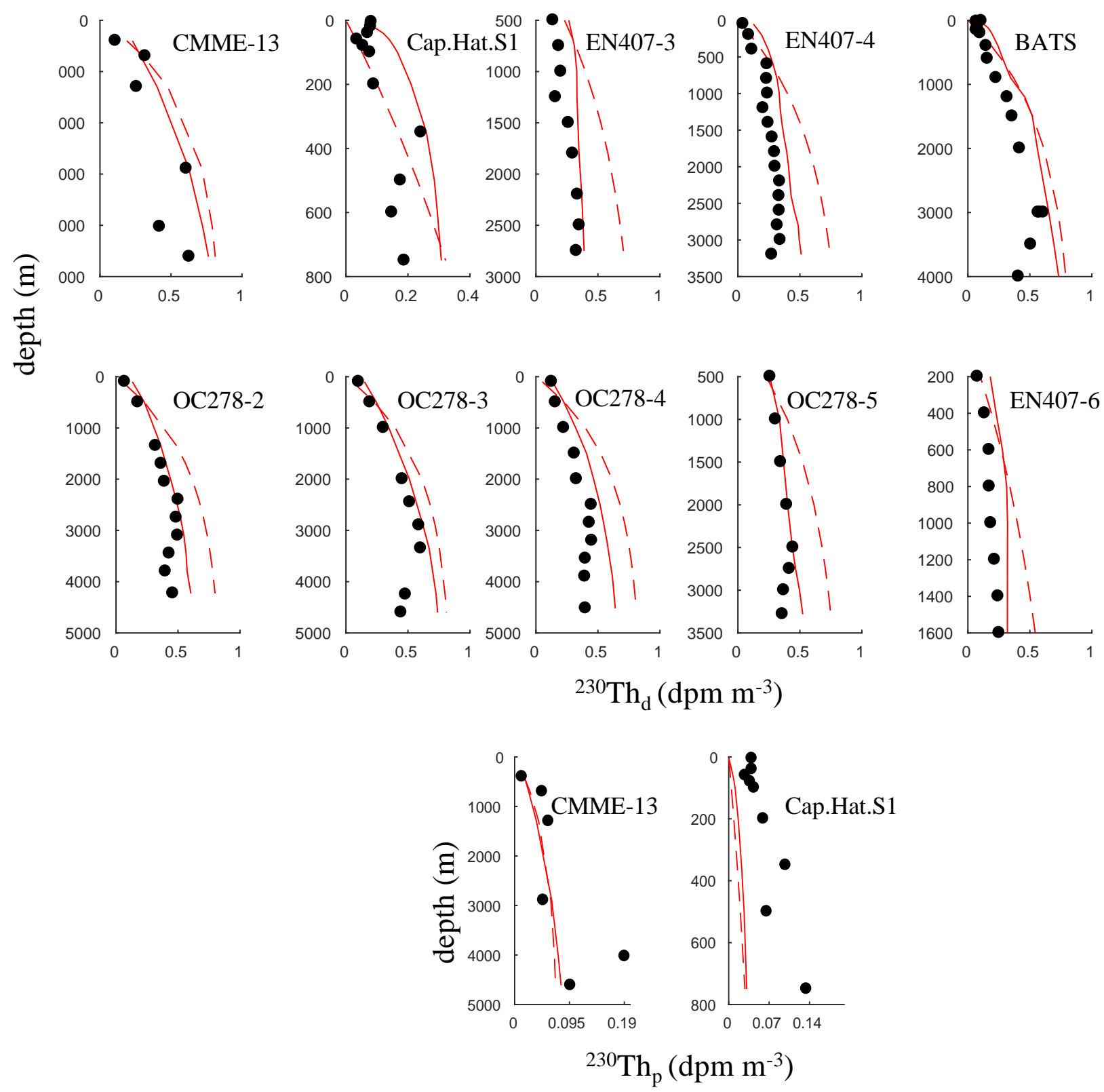

Figure B15. Same as Figs. B13-B14, but for ${ }^{230} \mathrm{Th}_{d, p}$ at pre-GEOTRACES stations 

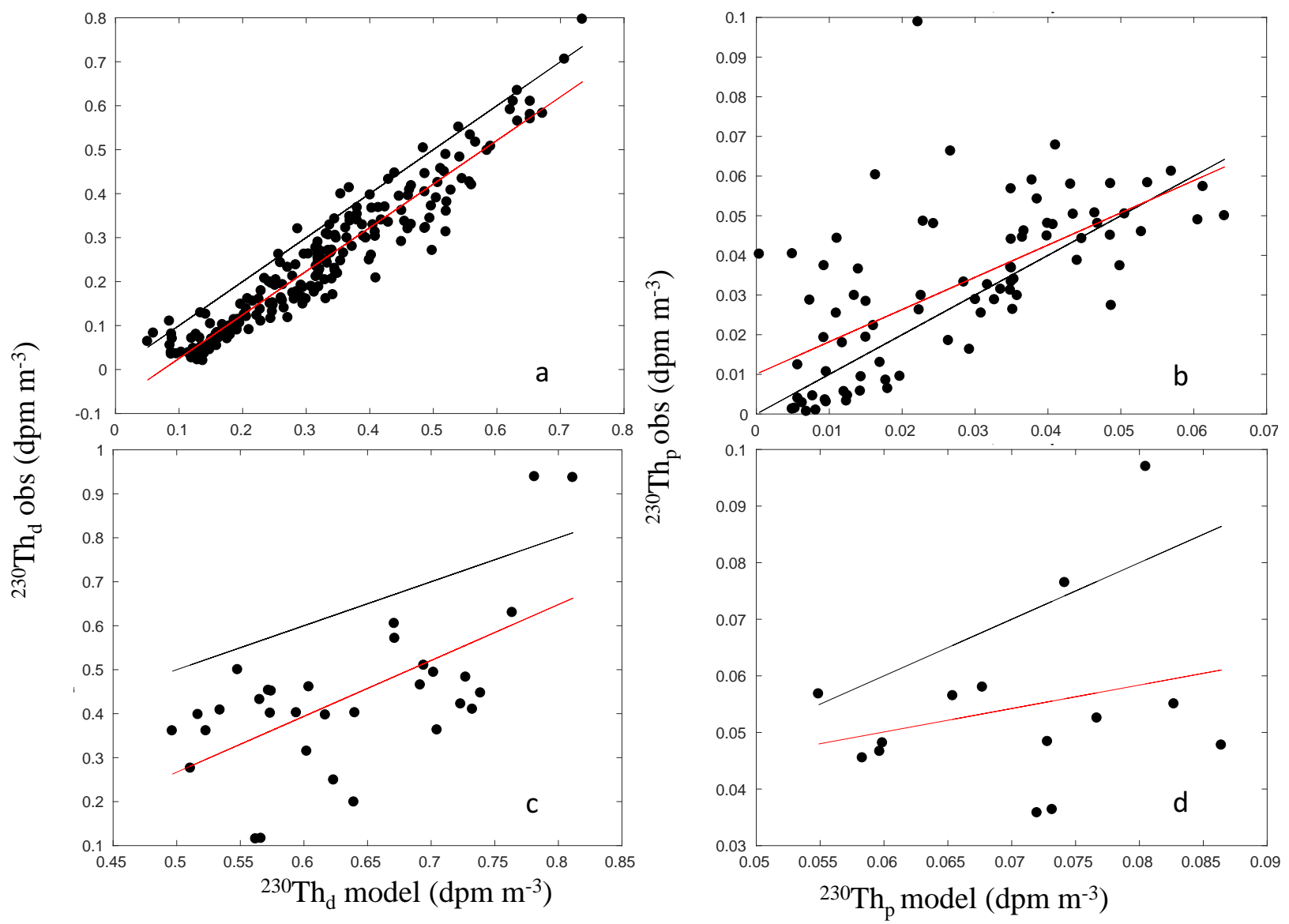

Figure B16. Observed vs. simulated (a,c) ${ }^{230} \mathrm{Th}_{d}$ and (b,d) ${ }^{230} \mathrm{Th}_{p}$. Panels (a) and (b) show activities above $3000 \mathrm{~m}$, and panels (c) and (d) show activities below $3000 \mathrm{~m}$. In each panel, the black line is the line of perfect agreement, and the red line is the best fit obtained by ordinary least squares. Simulated values shown are for experiment "larGSout". 


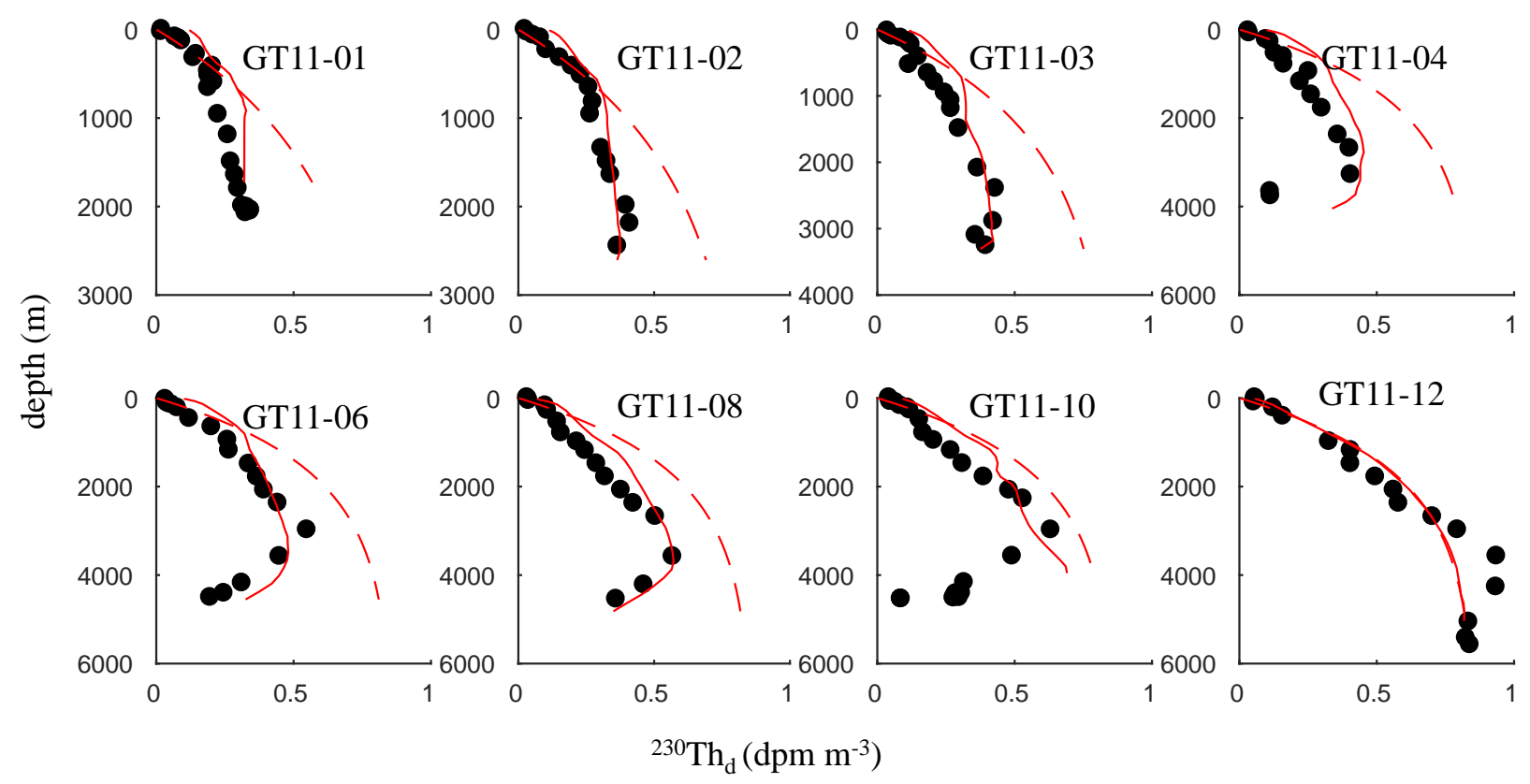

Figure B17. Simulated and observed ${ }^{230} \mathrm{Th}_{d}$ profiles at GEOTRACES stations. The black dots are the observations, the red dashed lines are the initial ${ }^{230} \mathrm{Th}_{d}$ activities assumed in the model, and the red solid lines are the final (after $t=3285$ days) ${ }^{230} \mathrm{Th}_{d}$ activities. Simulated values shown are for experiment k1var1. 

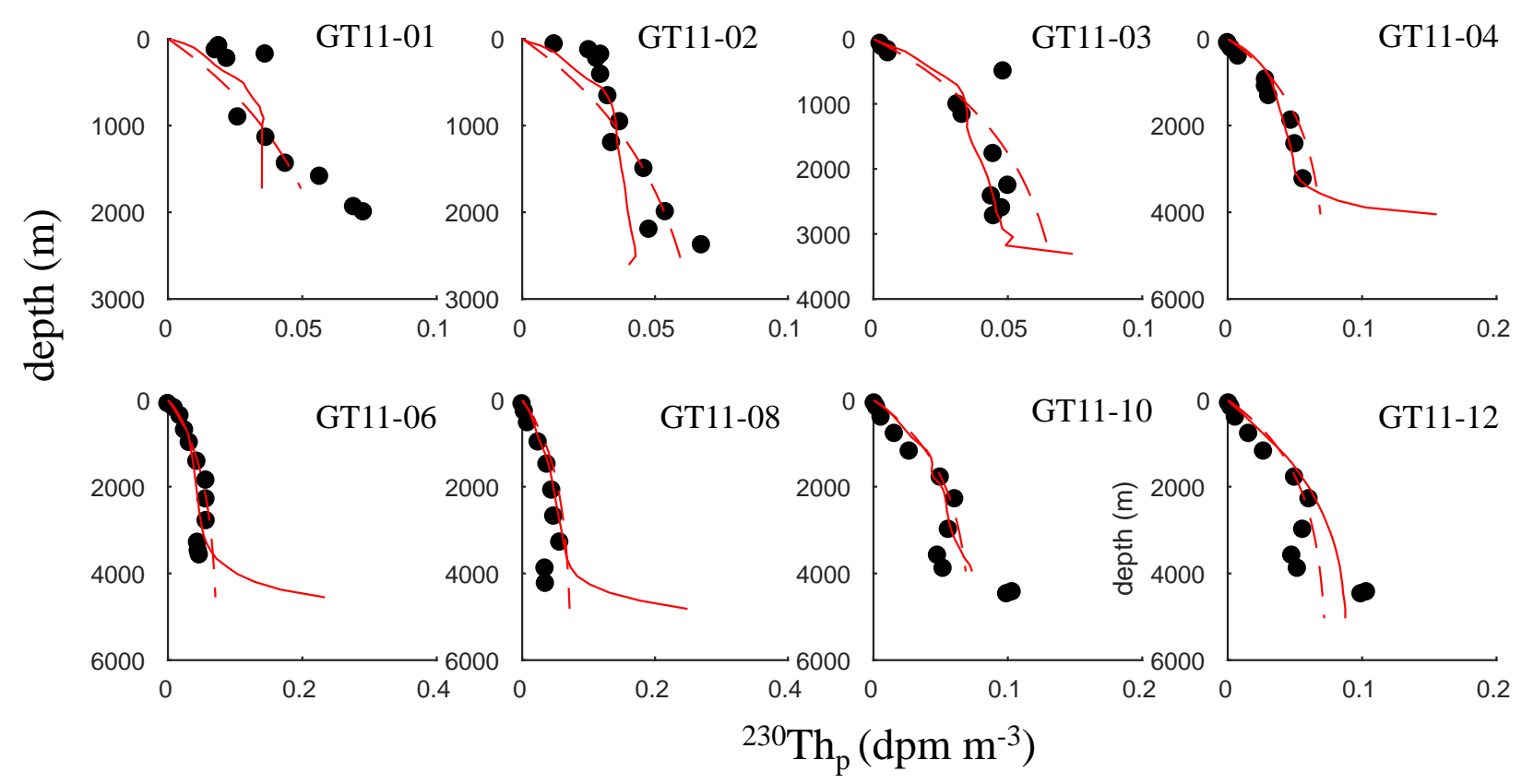

Figure B18. Same as Fig. B17, but for ${ }^{230} \mathrm{Th}_{p}$. 

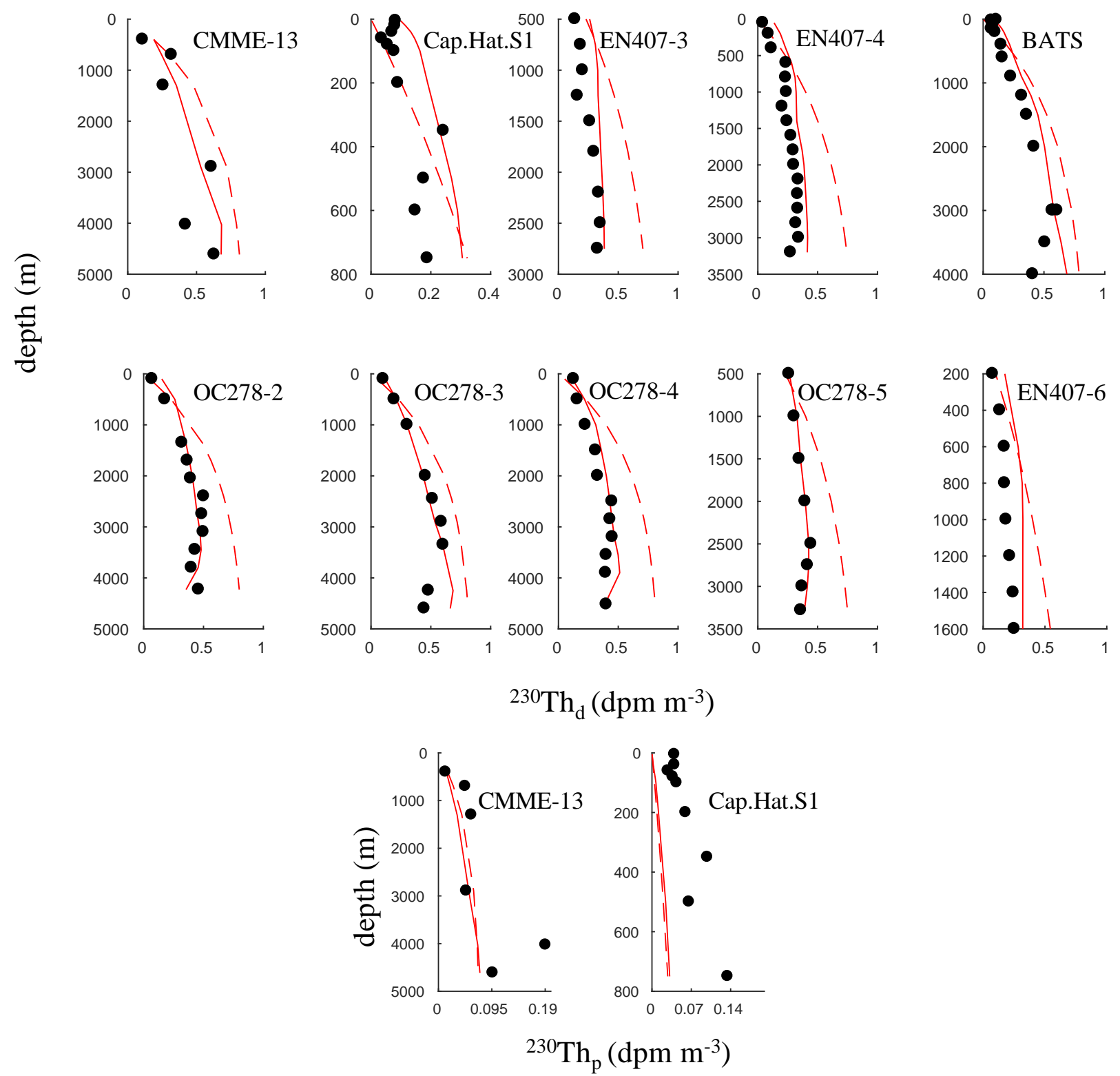

Figure B19. Same as Fig. B17-B18, but for ${ }^{230} \mathrm{Th}_{d, p}$ at pre-GEOTRACES stations. 

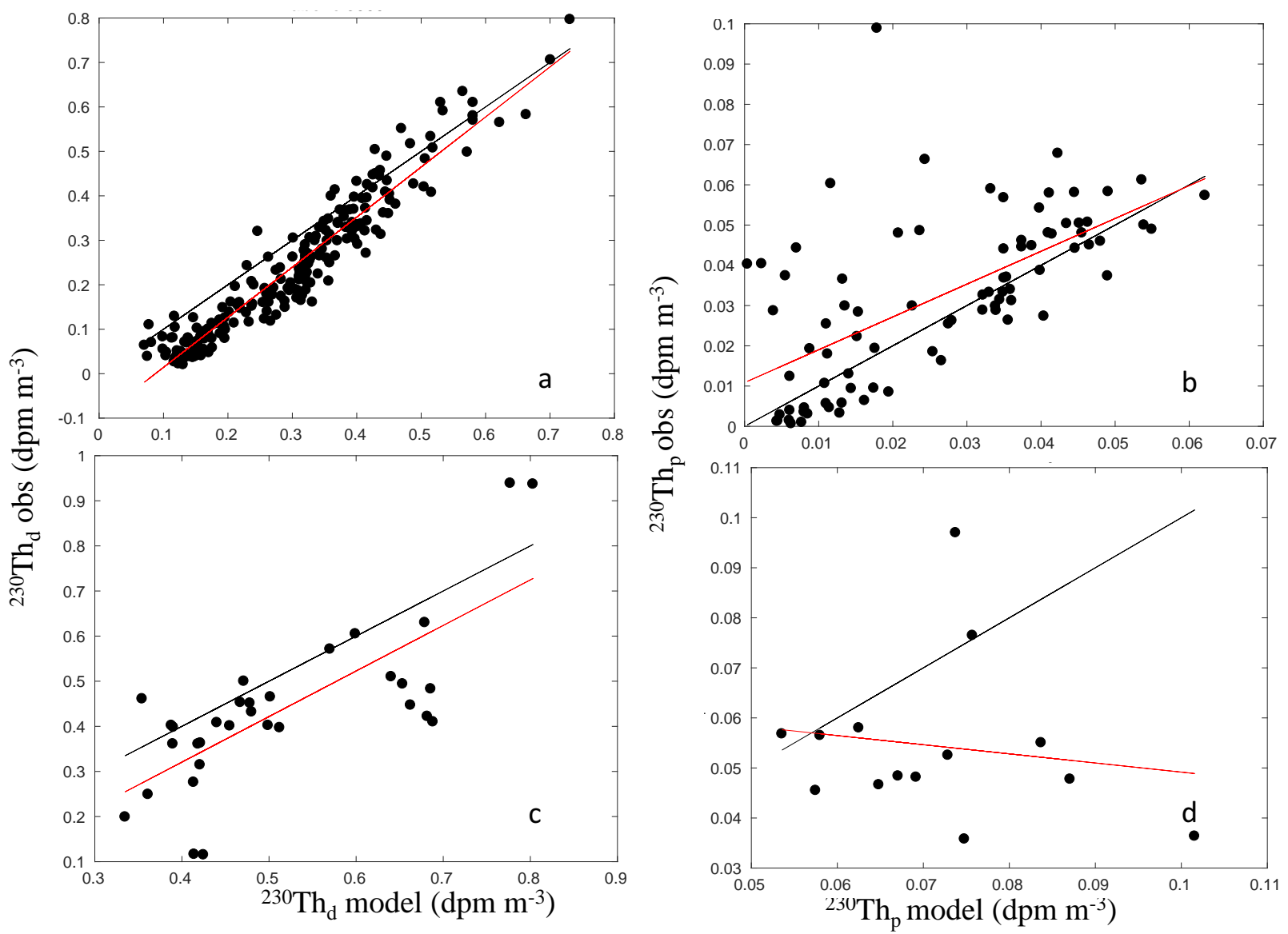

Figure B20. Observed vs. simulated (a,c) ${ }^{230} \mathrm{Th}_{d}$ and (b,d) ${ }^{230} \mathrm{Th}_{p}$. Panels (a) and (b) show activities above $3000 \mathrm{~m}$, and panels (c) and (d) show activities below $3000 \mathrm{~m}$. In each panel, the black line is the line of perfect agreement, and the red line is the best fit obtained by ordinary least squares. Simulated values shown are for experiment k1var1. 


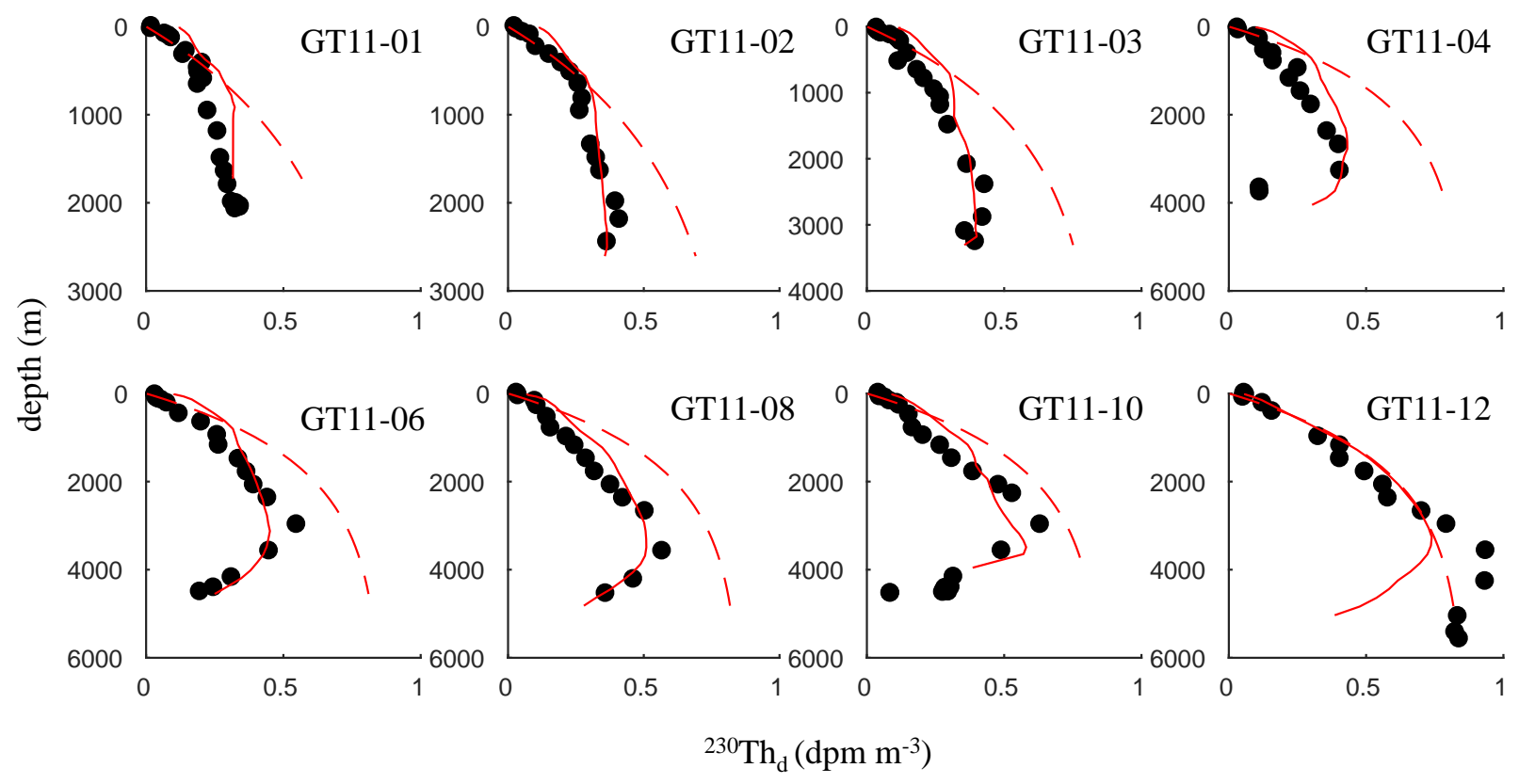

Figure B21. Simulated and observed ${ }^{230} \mathrm{Th}_{d}$ profiles at GEOTRACES stations. The black dots are the observations, the red dashed lines are the initial ${ }^{230} \mathrm{Th}_{d}$ activities assumed in the model, and the red solid lines are the final (after $t=3285$ days) ${ }^{230} \mathrm{Th}_{d}$ activities. Simulated values shown are for experiment k1var2. 


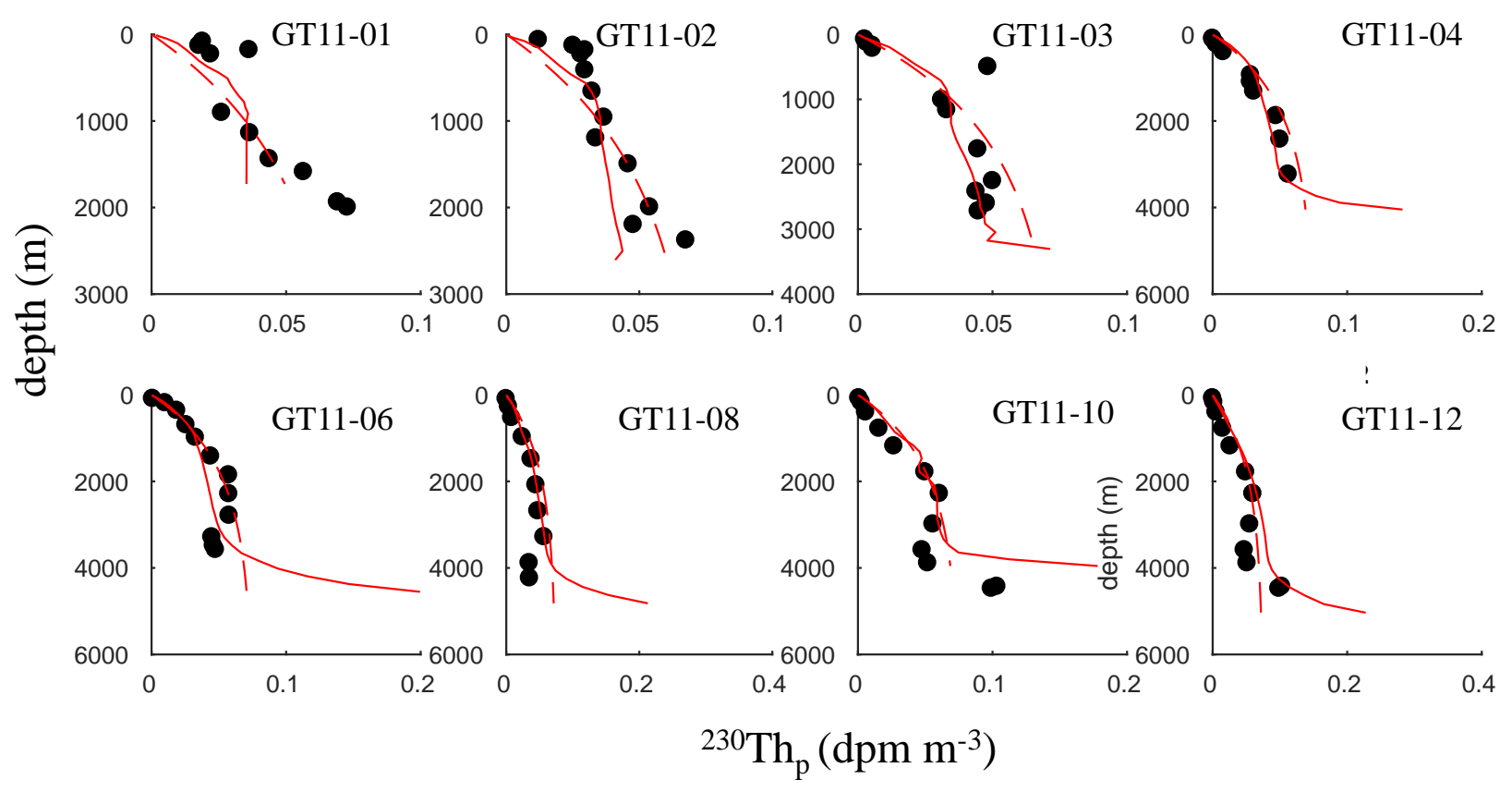

Figure B22. Same as Fig. B21, but for ${ }^{230} \mathrm{Th}_{p}$. 

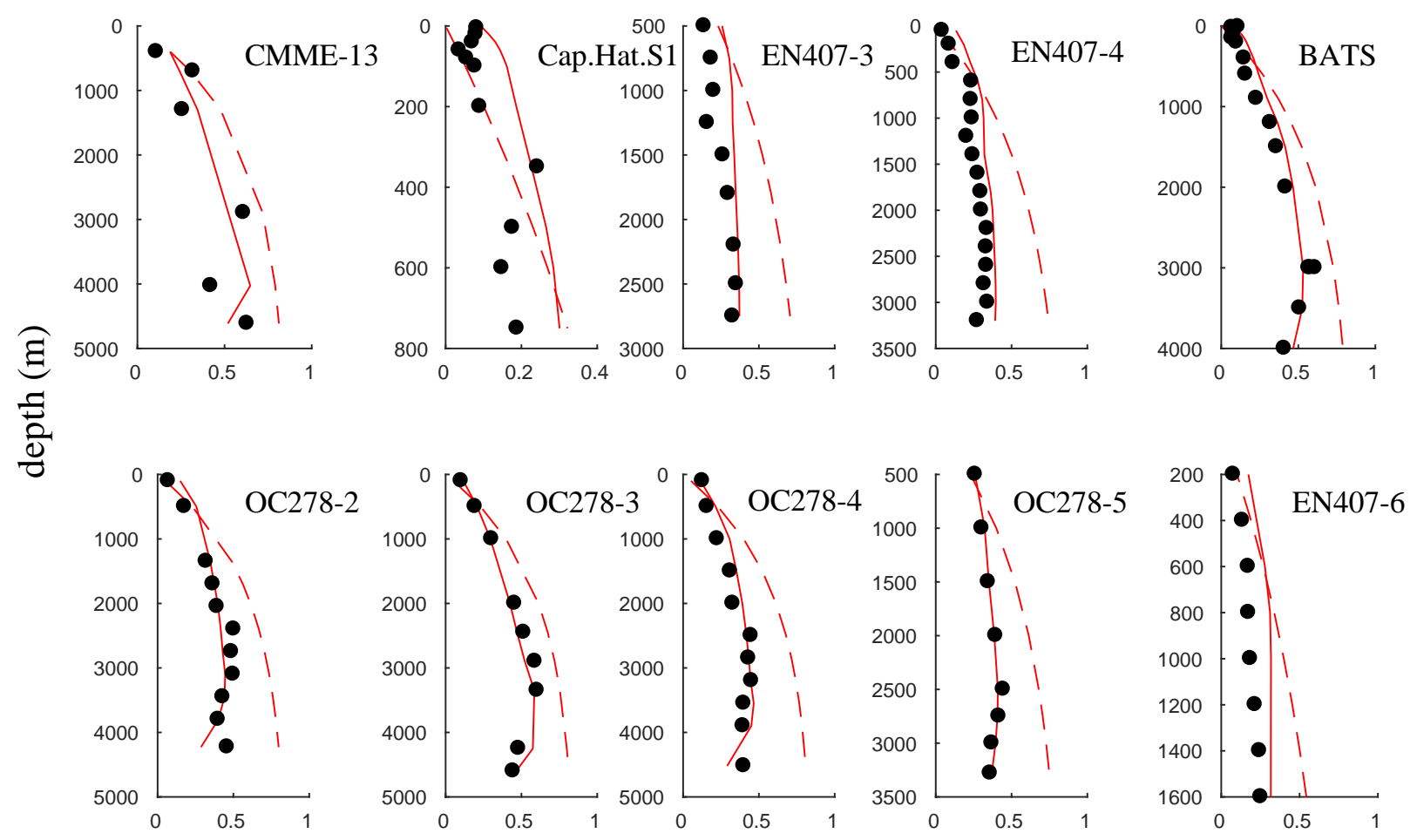

${ }^{230} \mathrm{Th}_{\mathrm{d}}\left(\mathrm{dpm} \mathrm{m}^{-3}\right)$

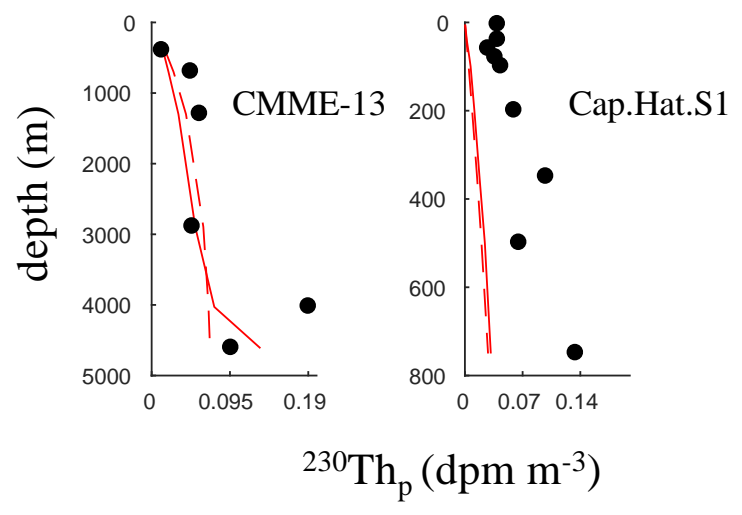

Figure B23. Same as Figs. B21-B22, but for ${ }^{230} \mathrm{Th}_{d, p}$ at pre-GEOTRACES stations. 

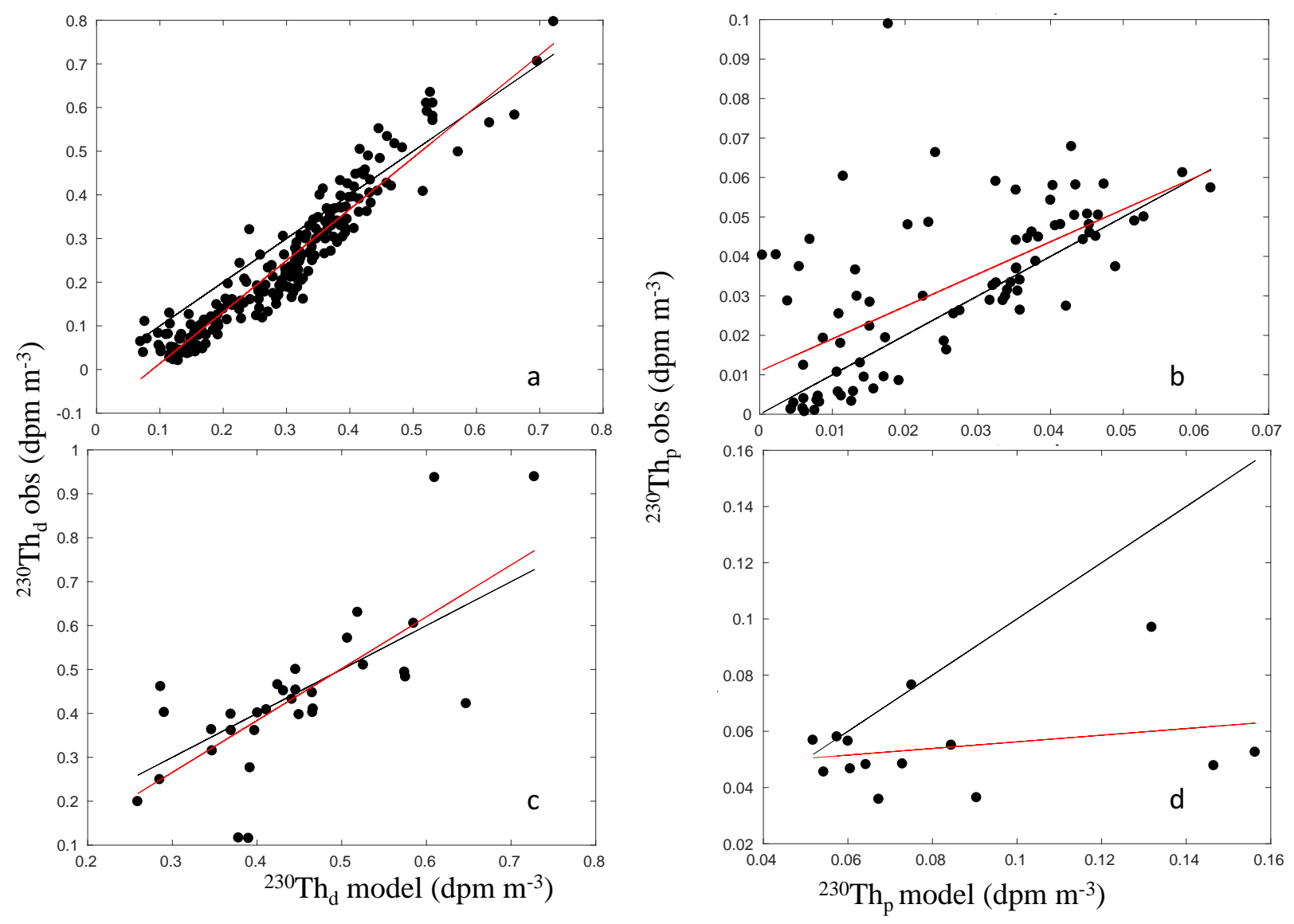

Figure B24. Observed vs. simulated (a,c) ${ }^{230} \mathrm{Th}_{d}$ and $(\mathrm{b}, \mathrm{d})^{230} \mathrm{Th}_{p}$. Panels (a) and (b) show activities above $3000 \mathrm{~m}$, and panels (c) and (d) show activities below $3000 \mathrm{~m}$. In each panel, the black line is the line of perfect agreement, and the red line is the best fit obtained by ordinary least squares. Simulated values shown are for experiment k1var2. 


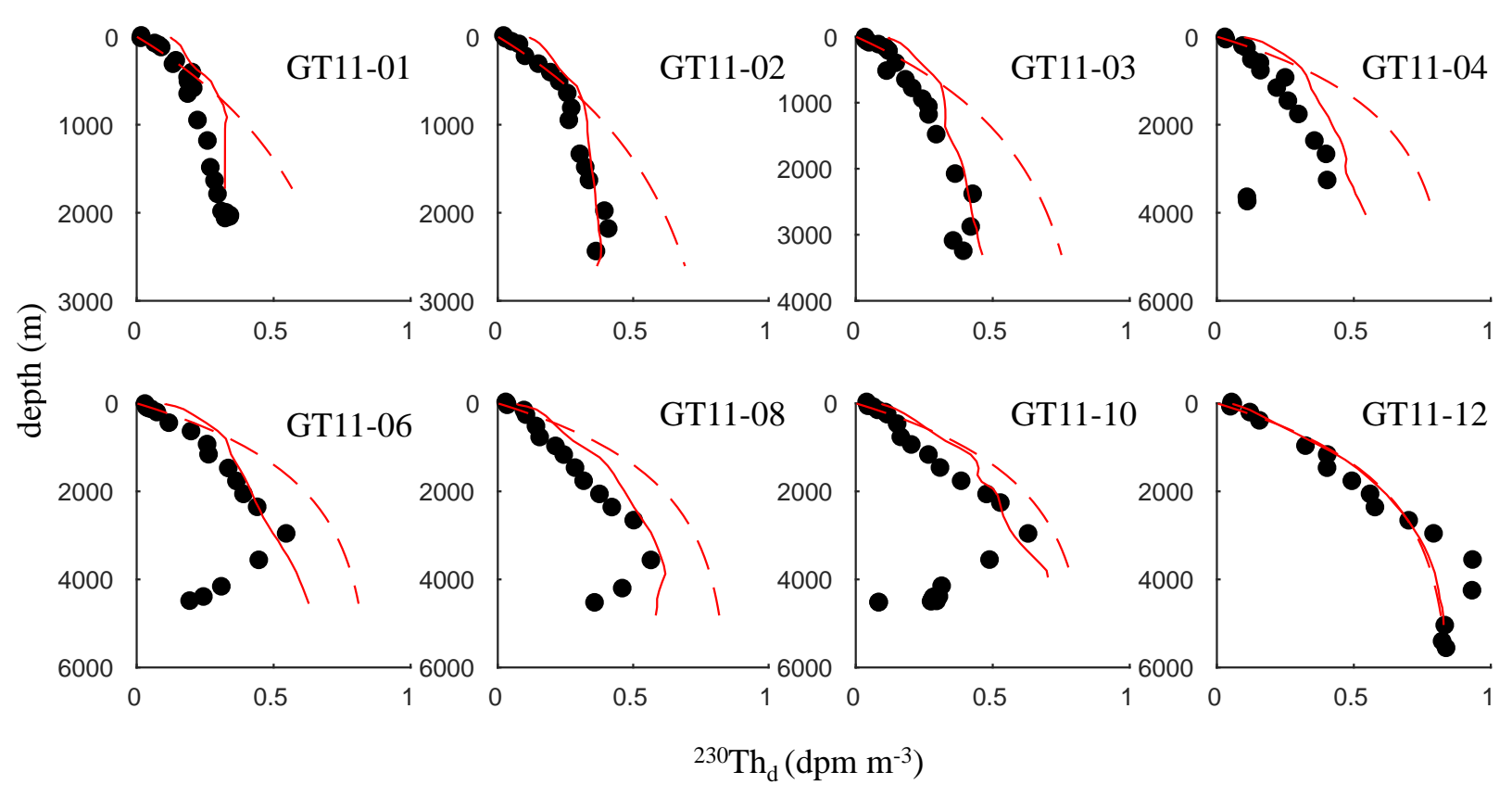

Figure B25. Simulated and observed ${ }^{230} \mathrm{Th}_{d}$ profiles at GEOTRACES stations. The black dots are the observations, the red dashed lines are the initial ${ }^{230} \mathrm{Th}_{d}$ activities assumed in the model, and the red solid lines are the final (after $t=3285$ days) ${ }^{230} \mathrm{Th}_{d}$ activities. Simulated values shown are for experiment k1var3. 


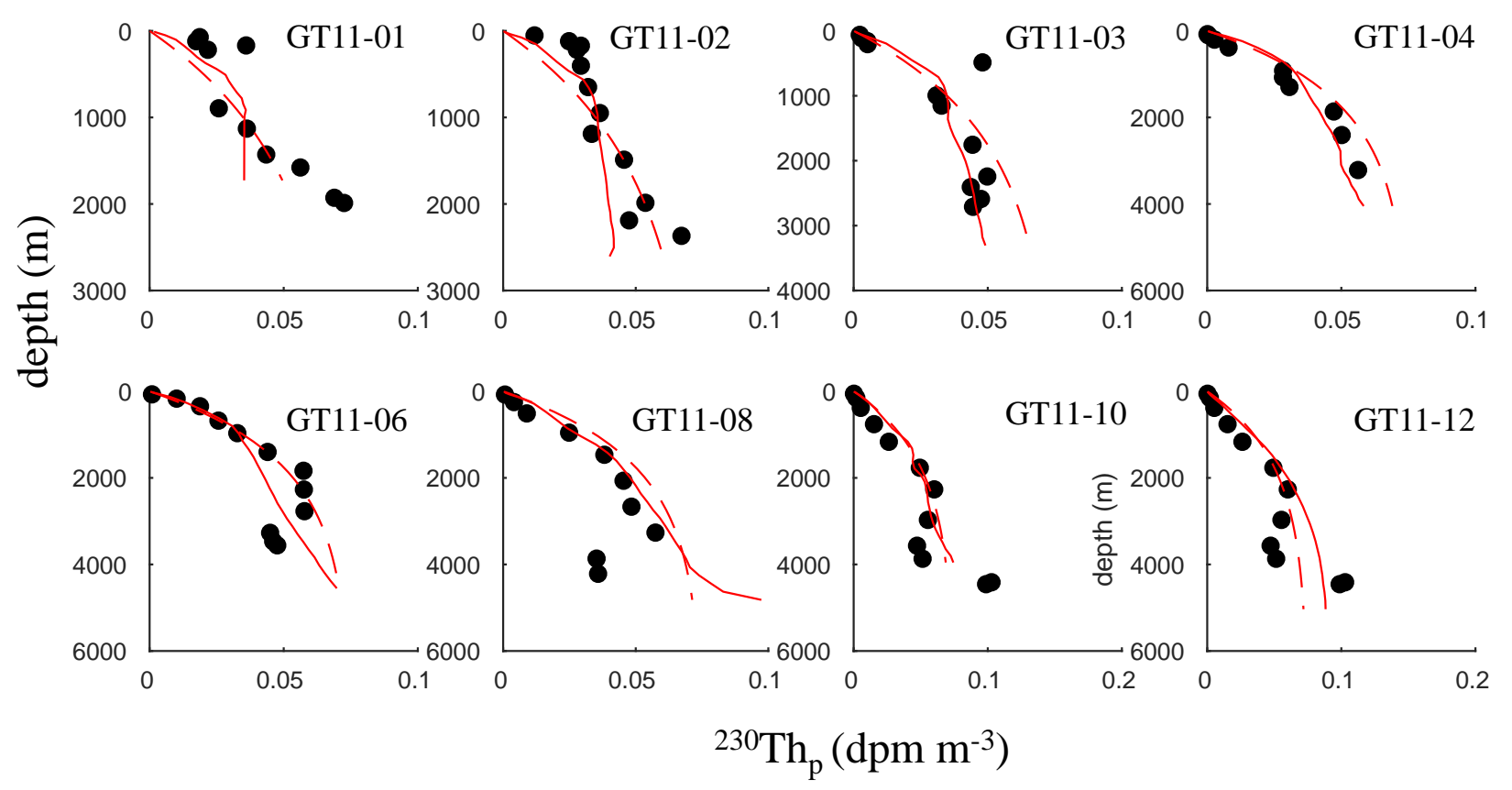

Figure B26. Same as Fig. B25, but for ${ }^{230} \mathrm{Th}_{p}$. 

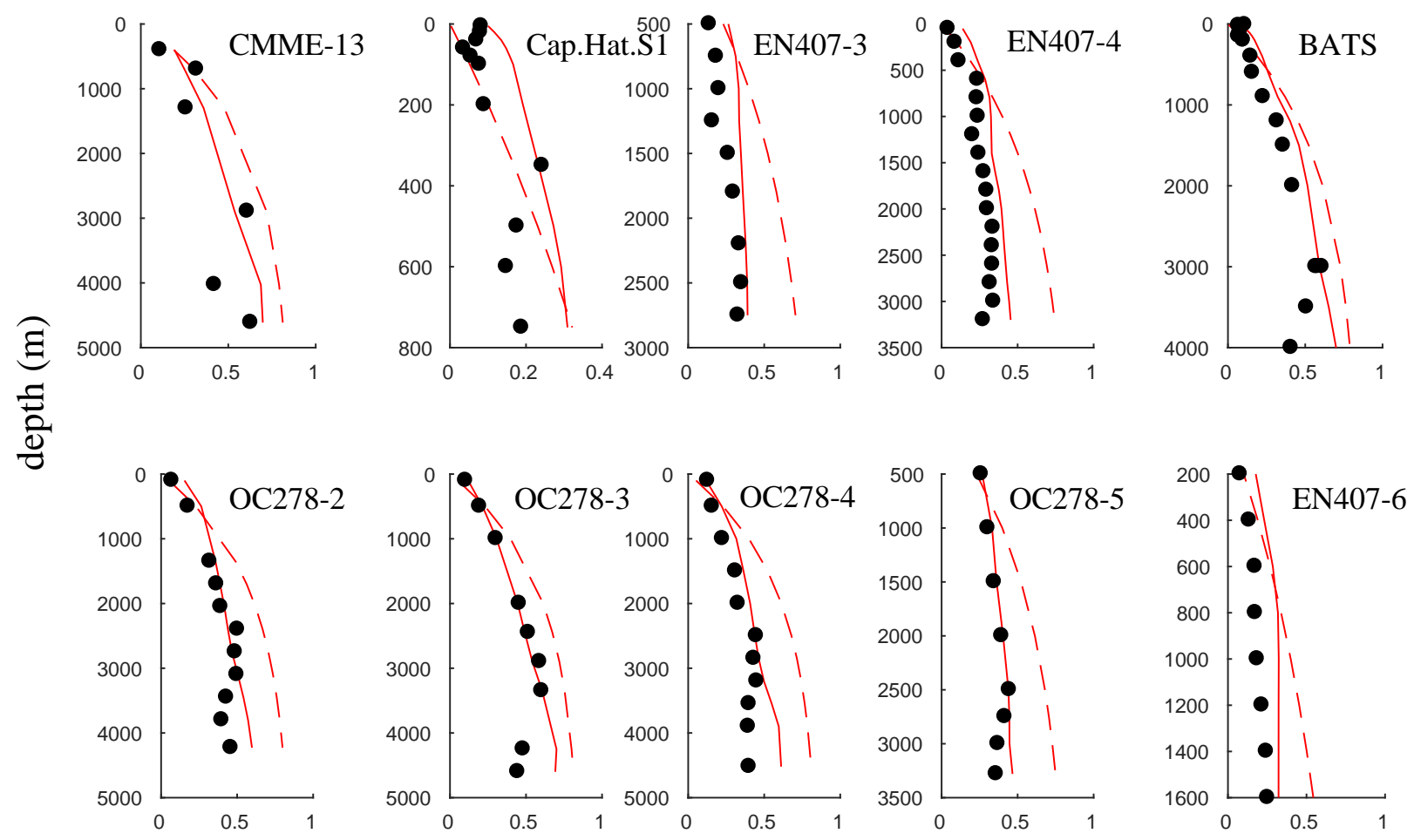

${ }^{230} \mathrm{Th}_{\mathrm{d}}\left(\mathrm{dpm} \mathrm{m}^{-3}\right)$

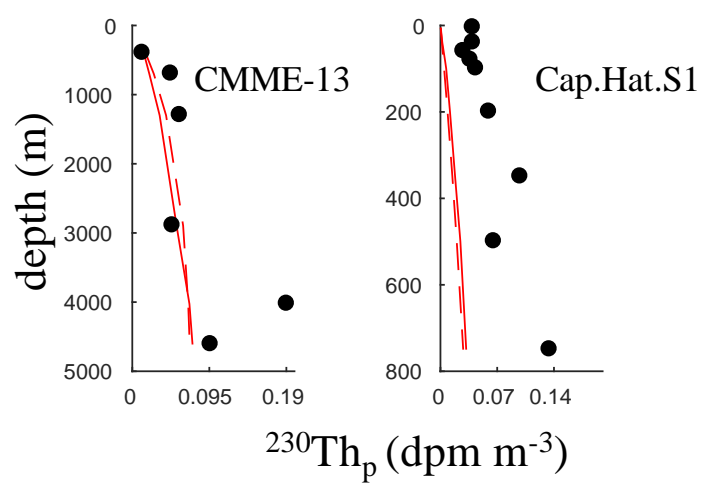

Figure B27. Same as Figs. B25-B26, but for ${ }^{230} \mathrm{Th}_{d, p}$ at pre-GEOTRACES stations. 

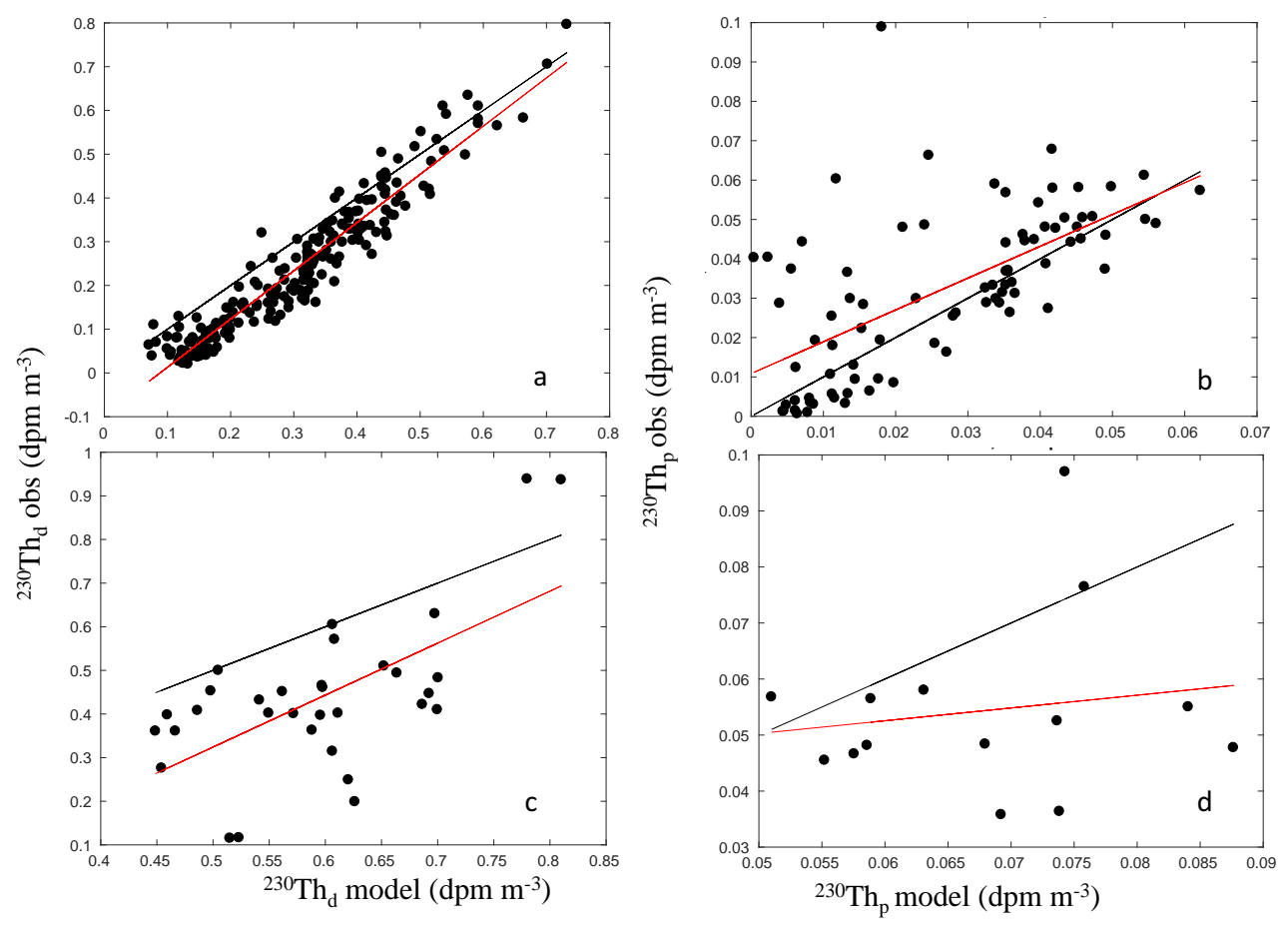

Figure B28. Observed vs. simulated (a,c) ${ }^{230} \mathrm{Th}_{d}$ and (b,d) ${ }^{230} \mathrm{Th}_{p}$. Panels (a) and (b) show activities above $3000 \mathrm{~m}$, and panels (c) and (d) show activities below $3000 \mathrm{~m}$. In each panel, the black line is the line of perfect agreement, and the red line is the best fit obtained by ordinary least squares. Simulated values shown are for experiment k1var3. 


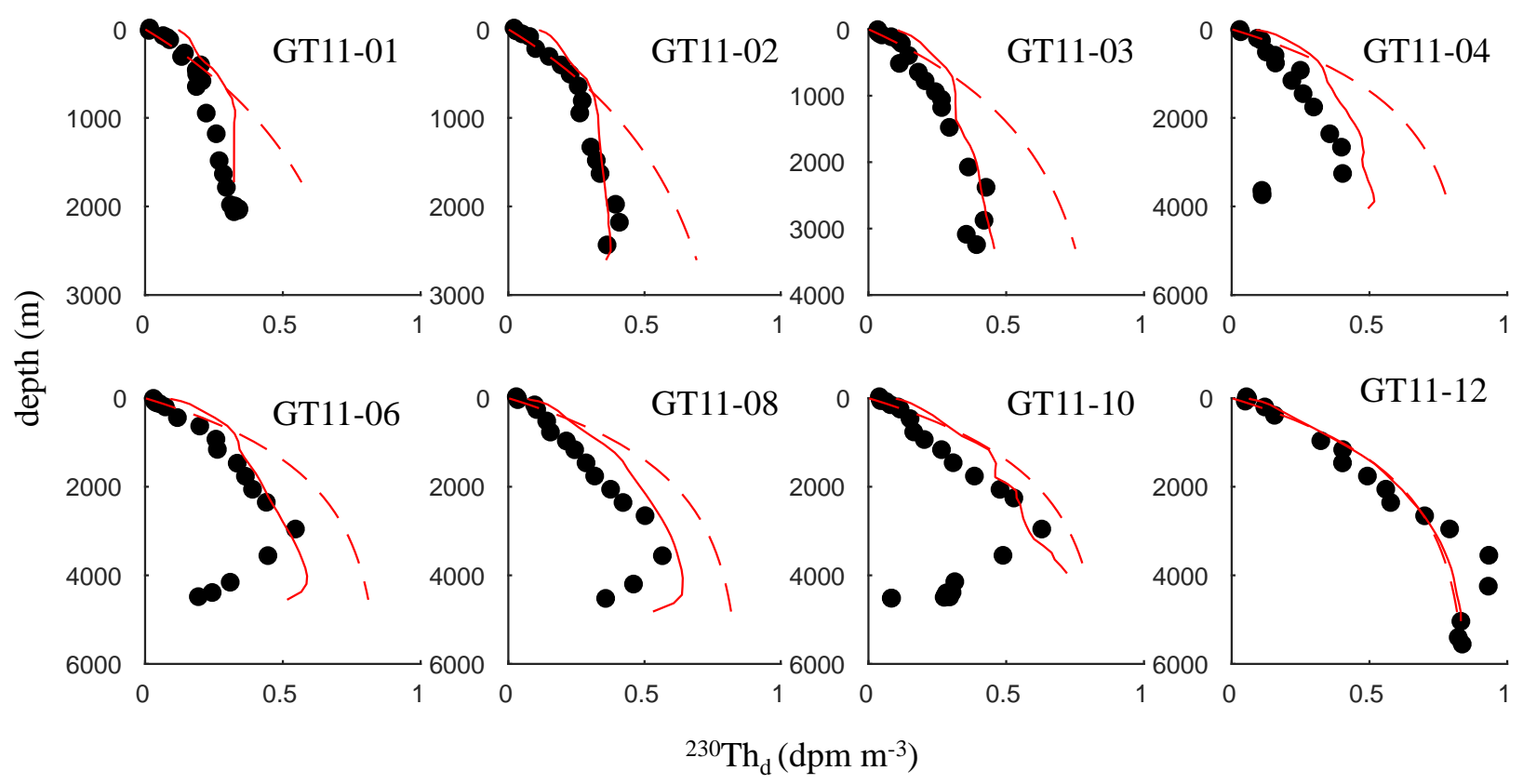

Figure B29. Simulated and observed ${ }^{230} \mathrm{Th}_{d}$ profiles at GEOTRACES stations. The black dots are the observations, the red dashed lines are the initial ${ }^{230} \mathrm{Th}_{d}$ activities assumed in the model, and the red solid lines are the final (after $t=3285$ days) ${ }^{230} \mathrm{Th}_{d}$ activities. Simulated values shown are for experiment k1var4. 


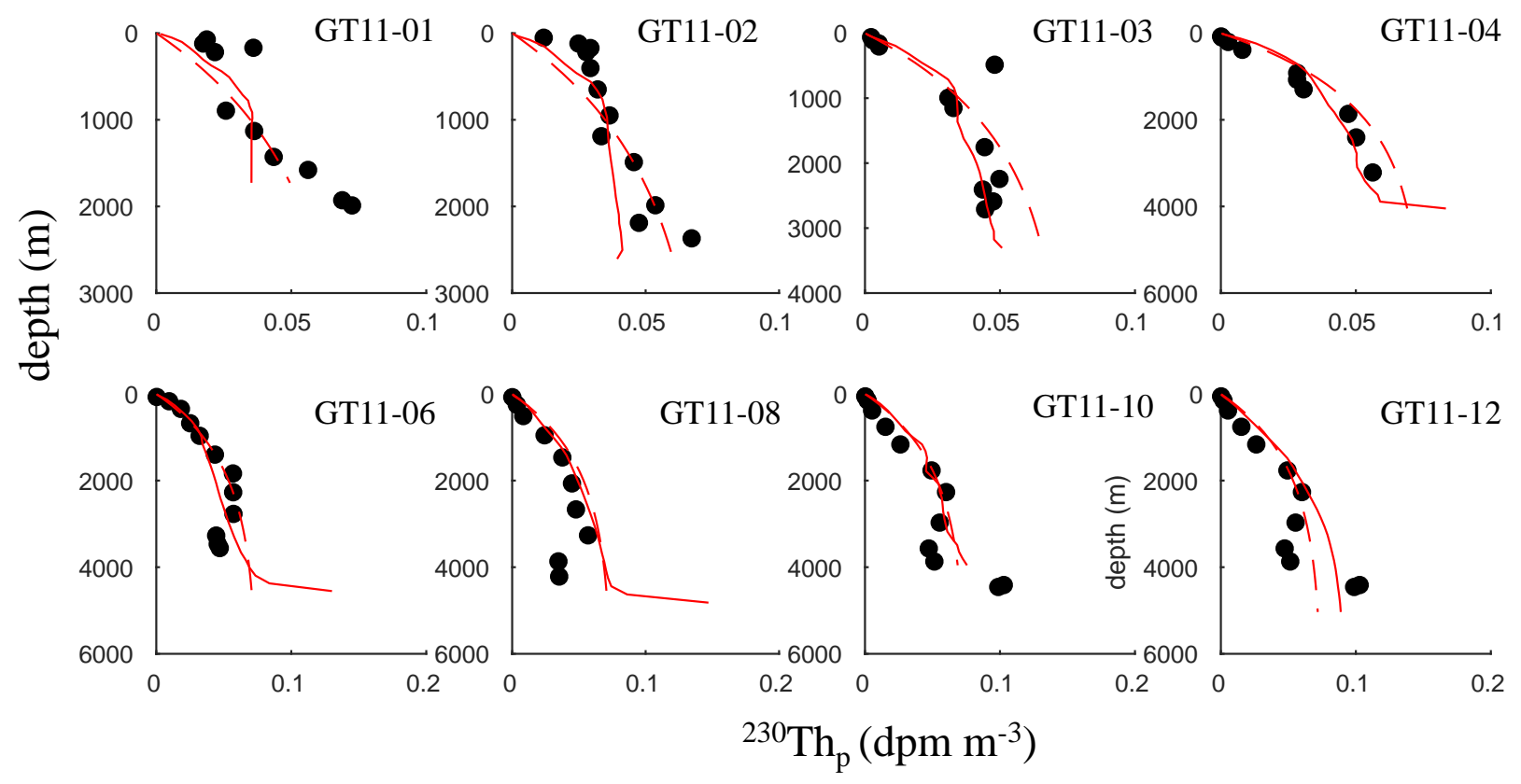

Figure B30. Same as Fig. B29, but for ${ }^{230} \mathrm{Th}_{p}$. 

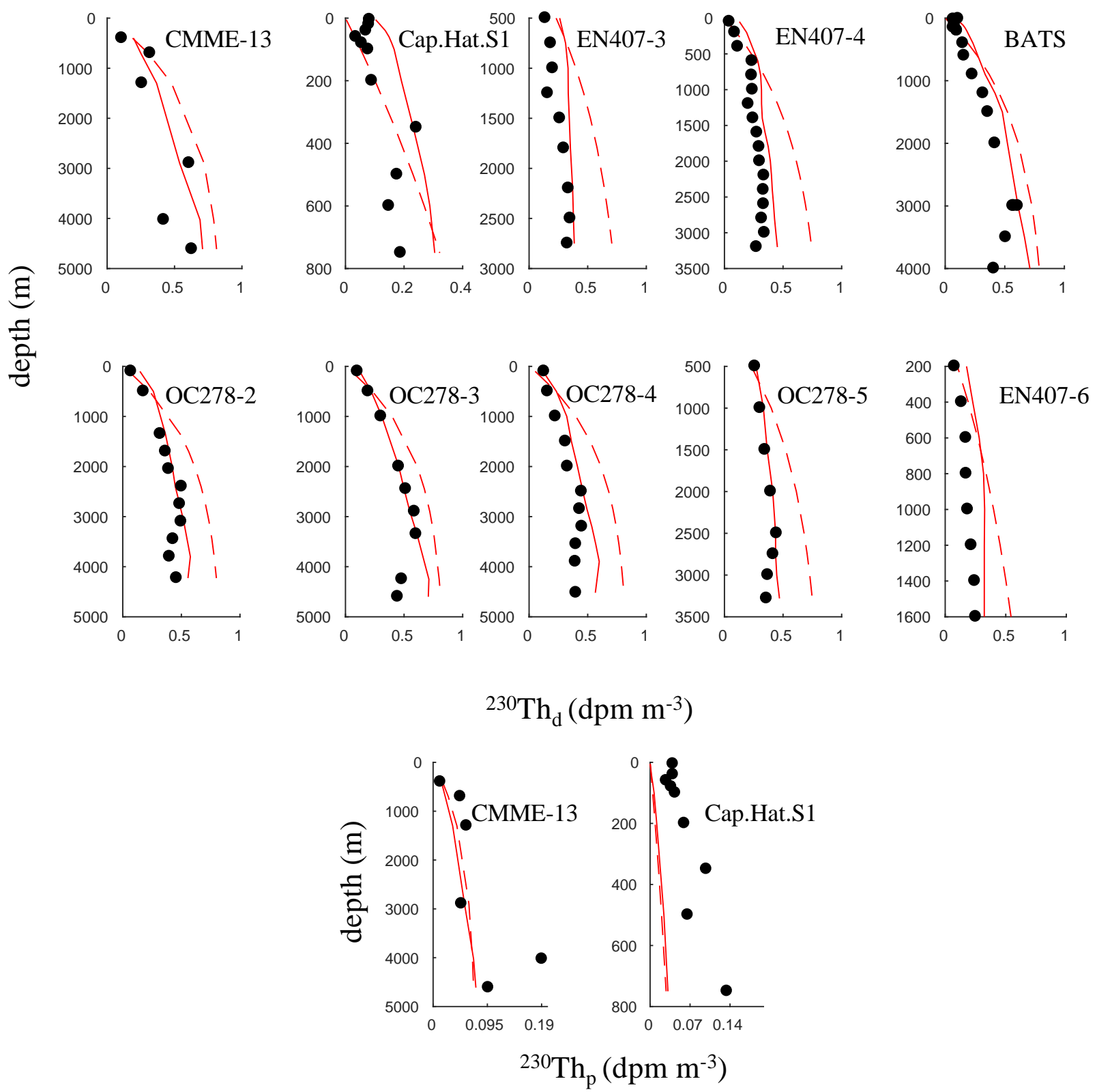

Figure B31. Same as Fig. B29-B30, but for ${ }^{230} \mathrm{Th}_{d, p}$ at pre-GEOTRACES stations. 

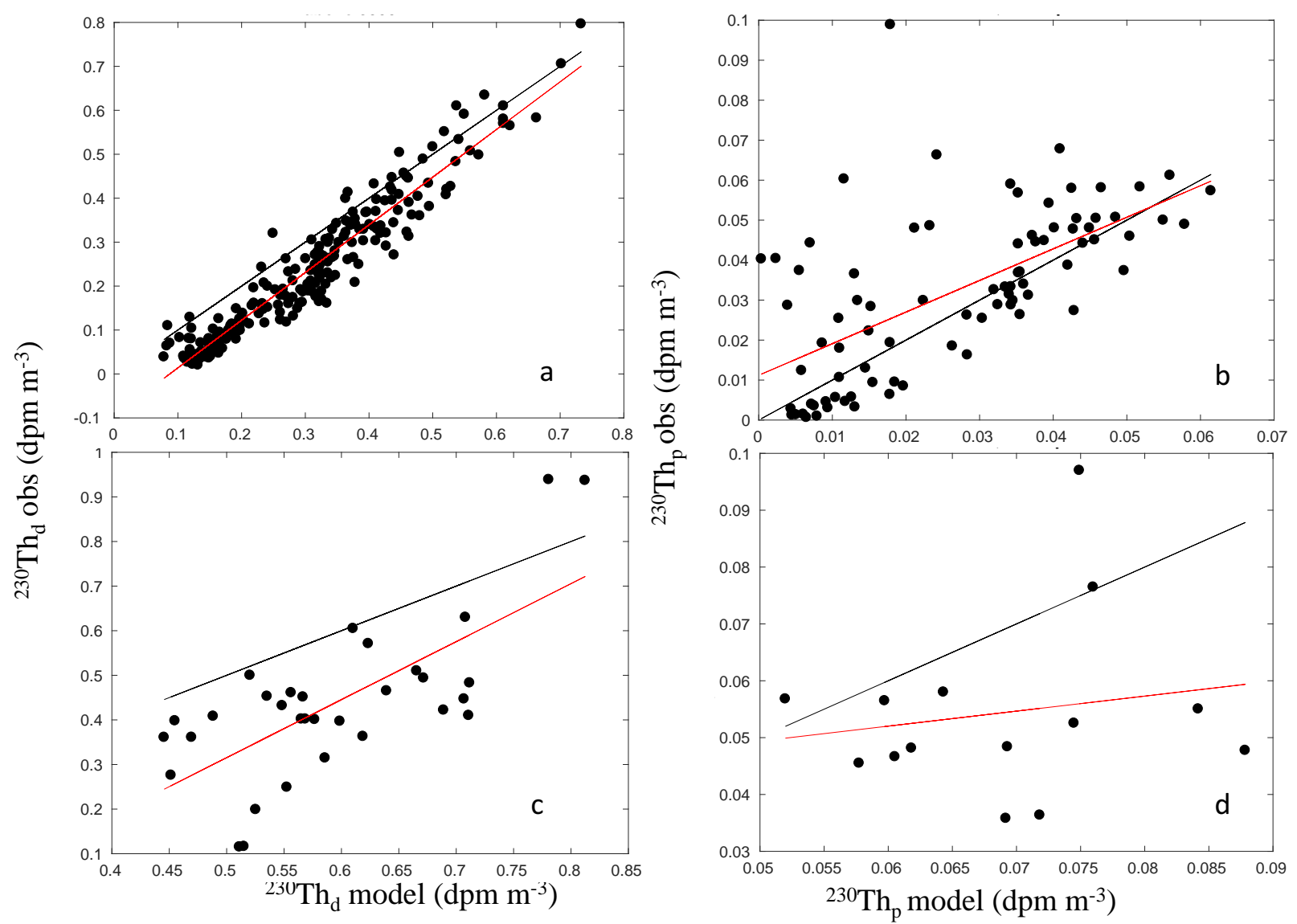

Figure B32. Observed vs. simulated (a,c) ${ }^{230} \mathrm{Th}_{d}$ and (b,d) ${ }^{230} \mathrm{Th}_{p}$. Panels (a) and (b) show activities above $3000 \mathrm{~m}$, and panels (c) and (d) show activities below $3000 \mathrm{~m}$. In each panel, the black line is the line of perfect agreement, and the red line is the best fit obtained by ordinary least squares. Simulated values shown are for experiment k1var4. 


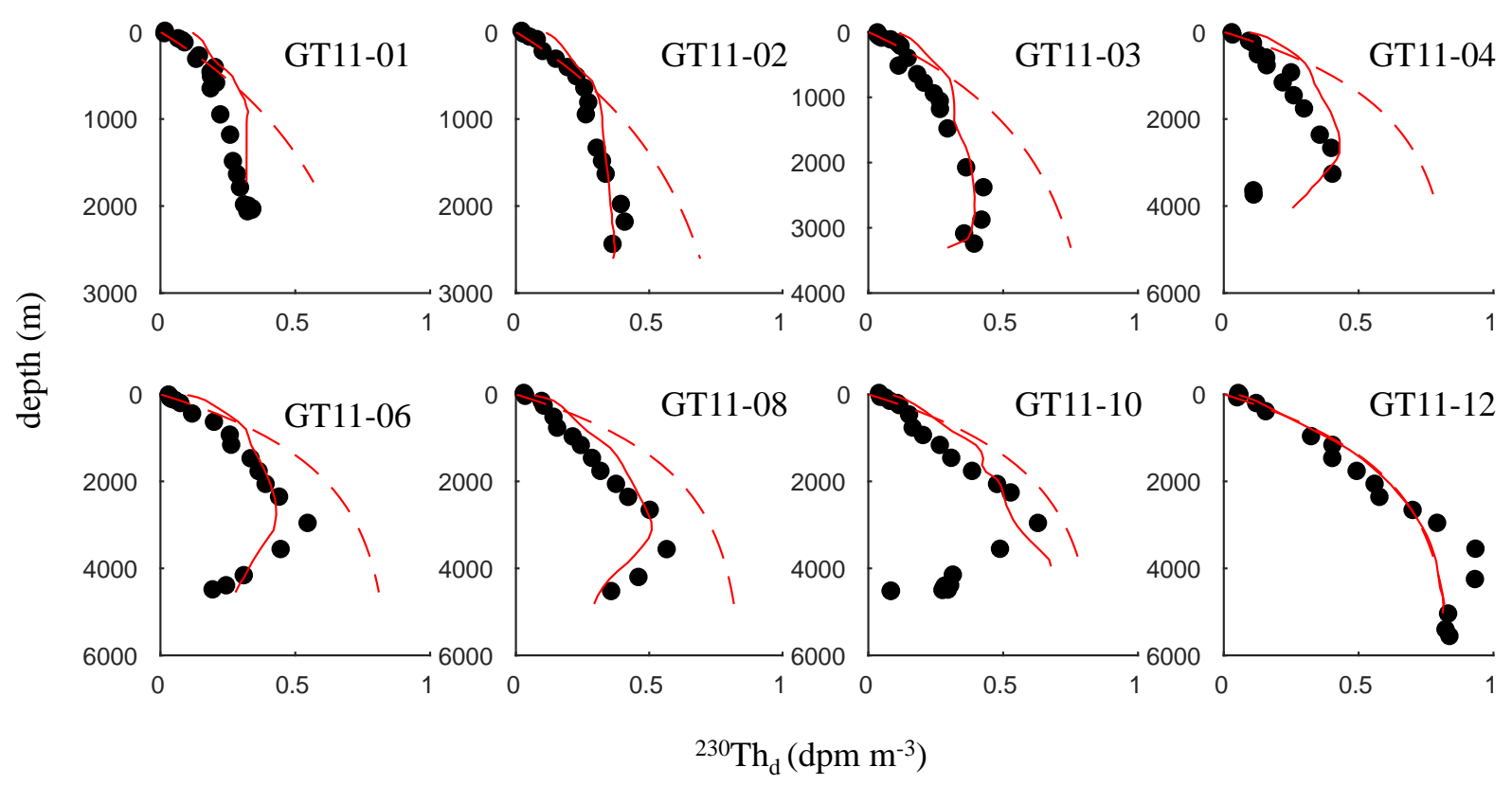

Figure B33. Simulated and observed ${ }^{230} \mathrm{Th}_{d}$ profiles at GEOTRACES stations. The black dots are the observations, the red dashed lines are the initial ${ }^{230} \mathrm{Th}_{d}$ activities assumed in the model, and the red solid lines are the final (after $t=3285$ days) ${ }^{230} \mathrm{Th}_{d}$ activities. Simulated values shown are for experiment k1var5. 


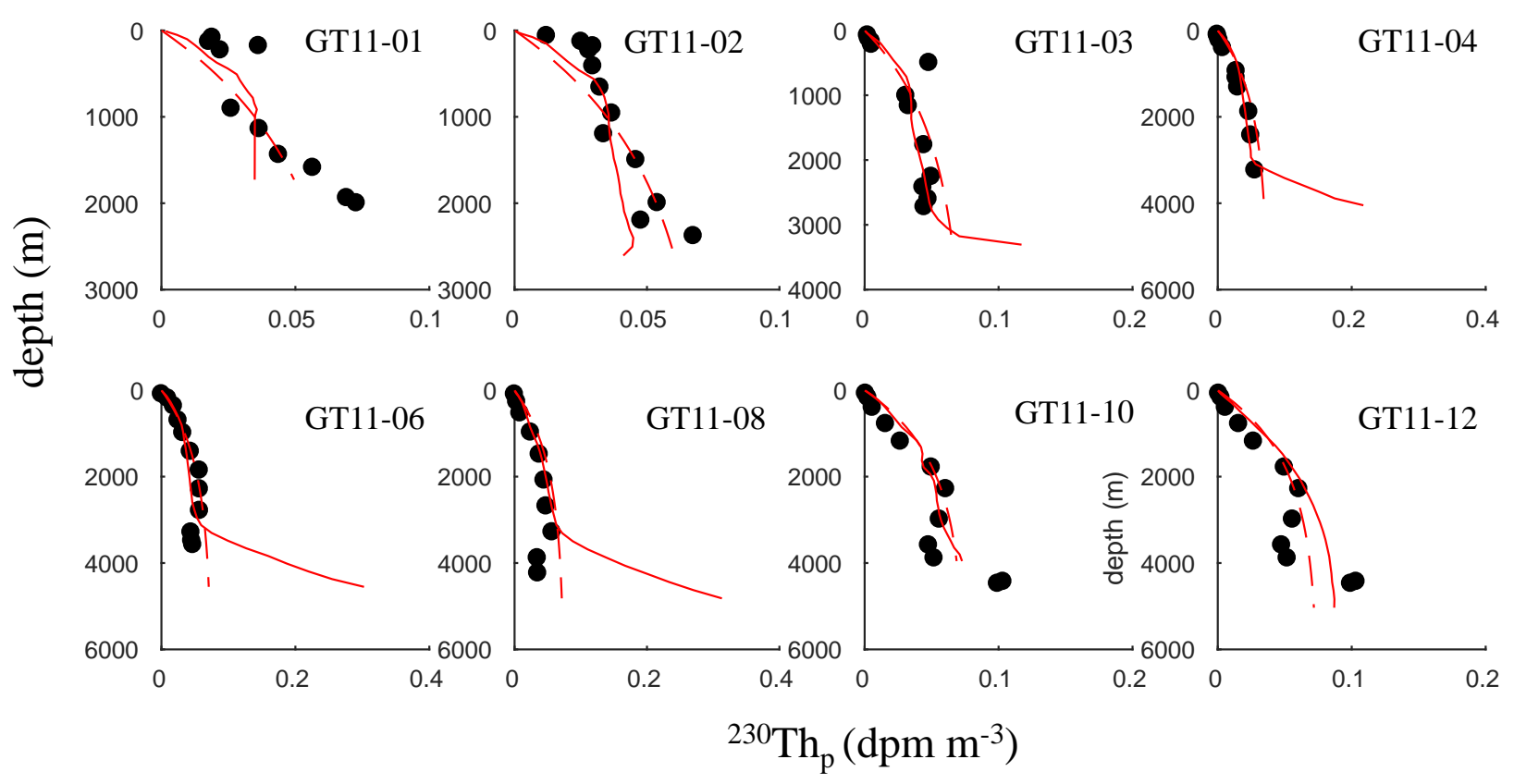

Figure B34. Same as Fig. B33, but for ${ }^{230} \mathrm{Th}_{p}$. 

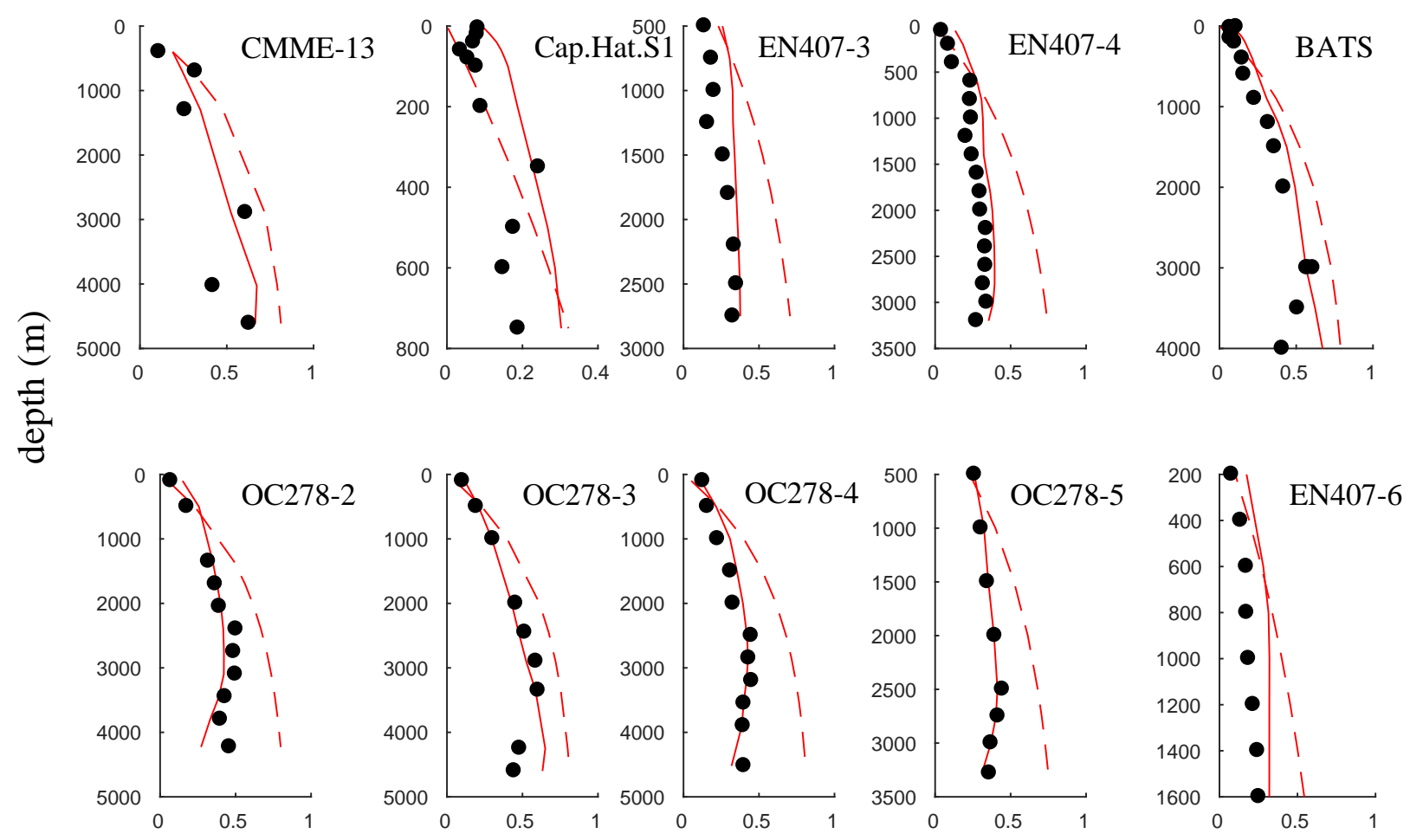

\section{${ }^{230} \mathrm{Th}_{\mathrm{d}}\left(\mathrm{dpm} \mathrm{m} \mathrm{m}^{-3}\right)$}

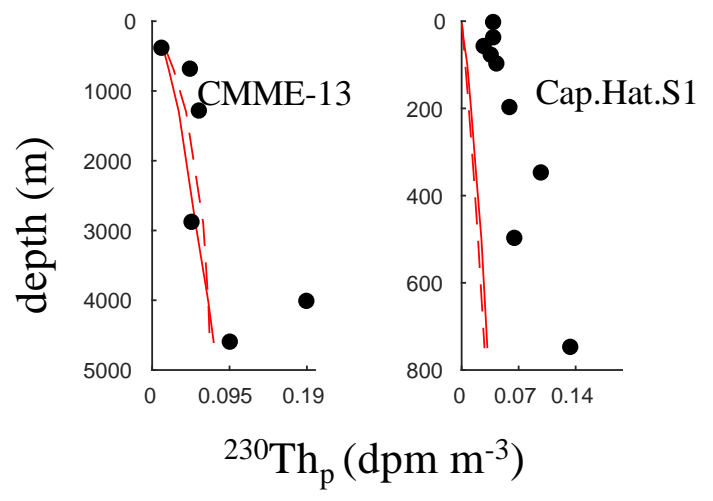

Figure B35. Same as Figs. B33-B34, but for ${ }^{230} \mathrm{Th}_{d, p}$ at pre-GEOTRACES stations. 

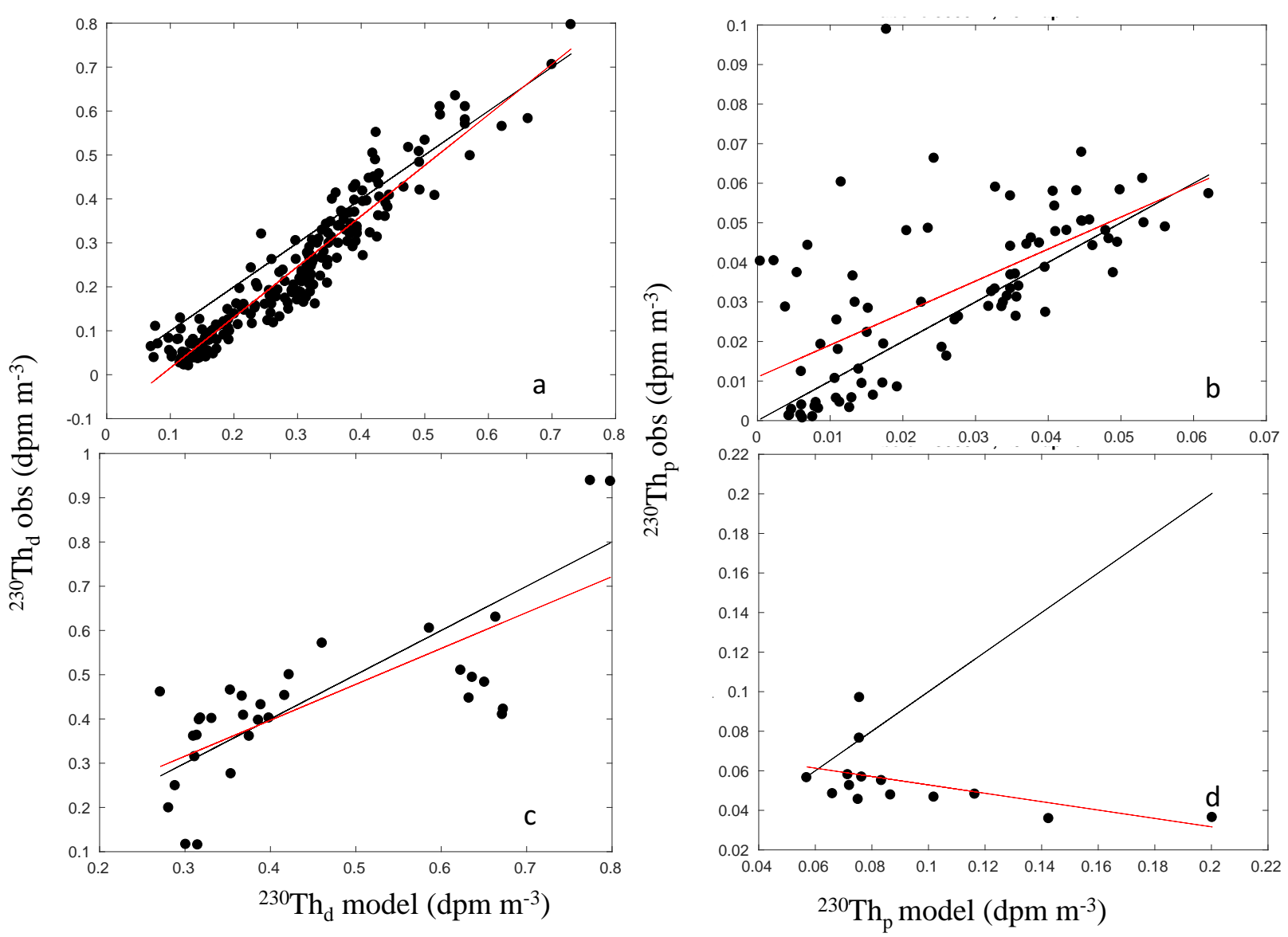

Figure B36. Observed vs. simulated (a,c) ${ }^{230} \mathrm{Th}_{d}$ and $(\mathrm{b}, \mathrm{d}){ }^{230} \mathrm{Th}_{p}$. Panels (a) and (b) show activities above $3000 \mathrm{~m}$, and panels (c) and (d) show activities below $3000 \mathrm{~m}$. In each panel, the black line is the line of perfect agreement, and the red line is the best fit obtained by ordinary least squares. Simulated values shown are for experiment k1var5. 


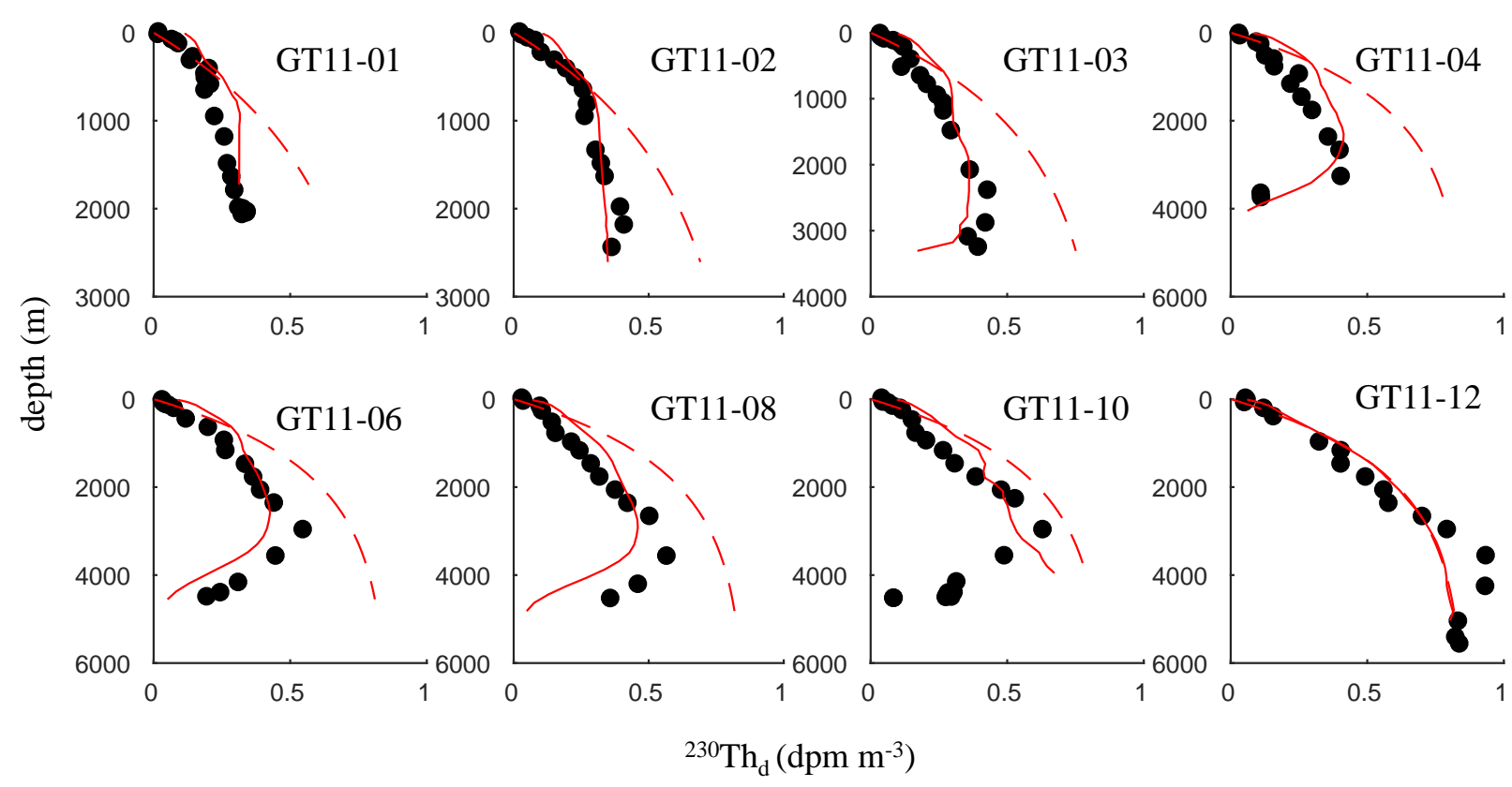

Figure B37. Simulated and observed ${ }^{230} \mathrm{Th}_{d}$ profiles at GEOTRACES stations. The black dots are the observations, the red dashed lines are the initial ${ }^{230} \mathrm{Th}_{d}$ activities assumed in the model, and the red solid lines are the final (after $t=3285$ days ${ }^{230} \mathrm{Th}_{d}$ activities. Simulated values shown are for experiment k1var6. 


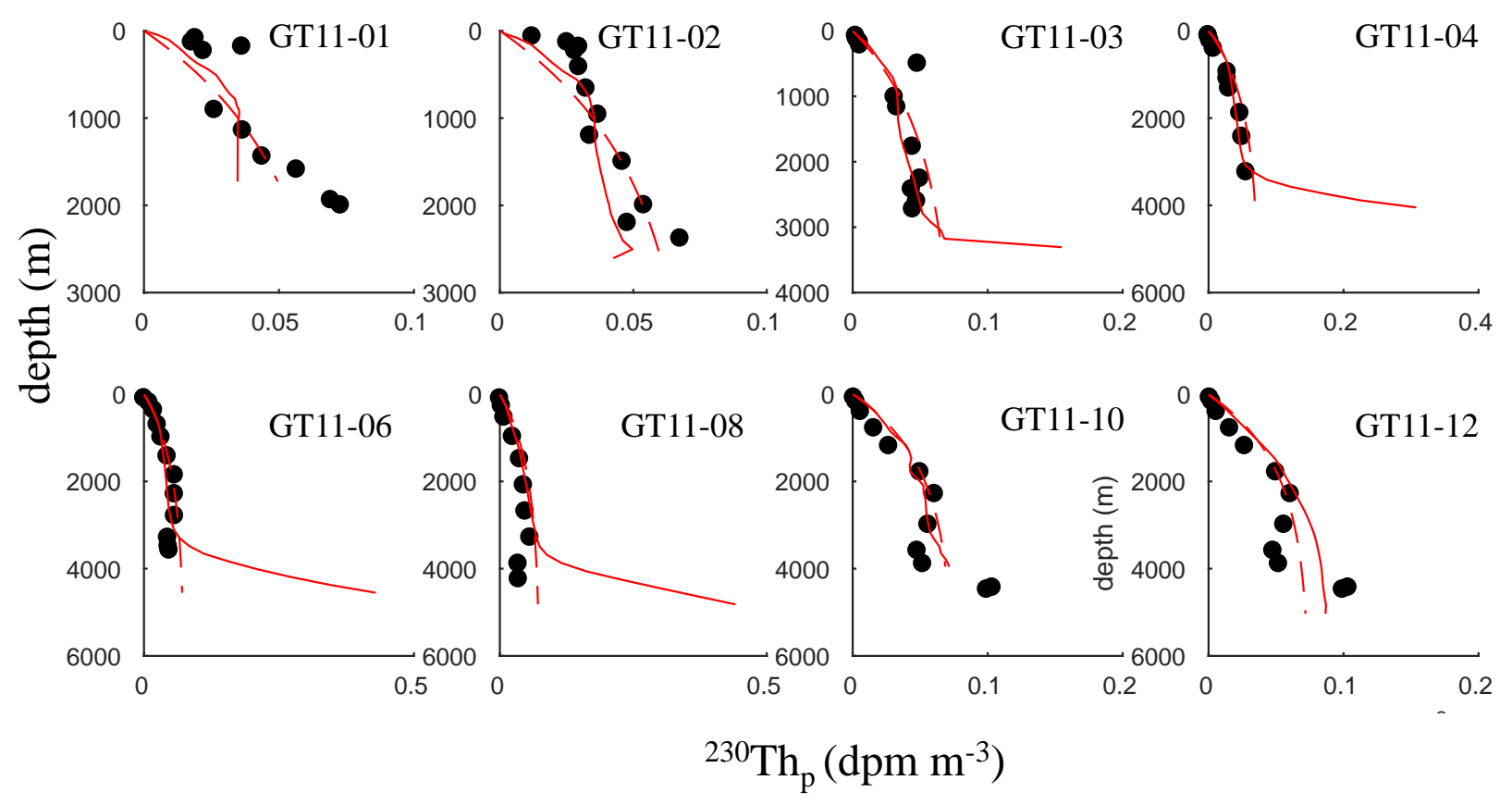

Figure B38. Same as Fig. B37, but for ${ }^{230} \mathrm{Th}_{p}$. 

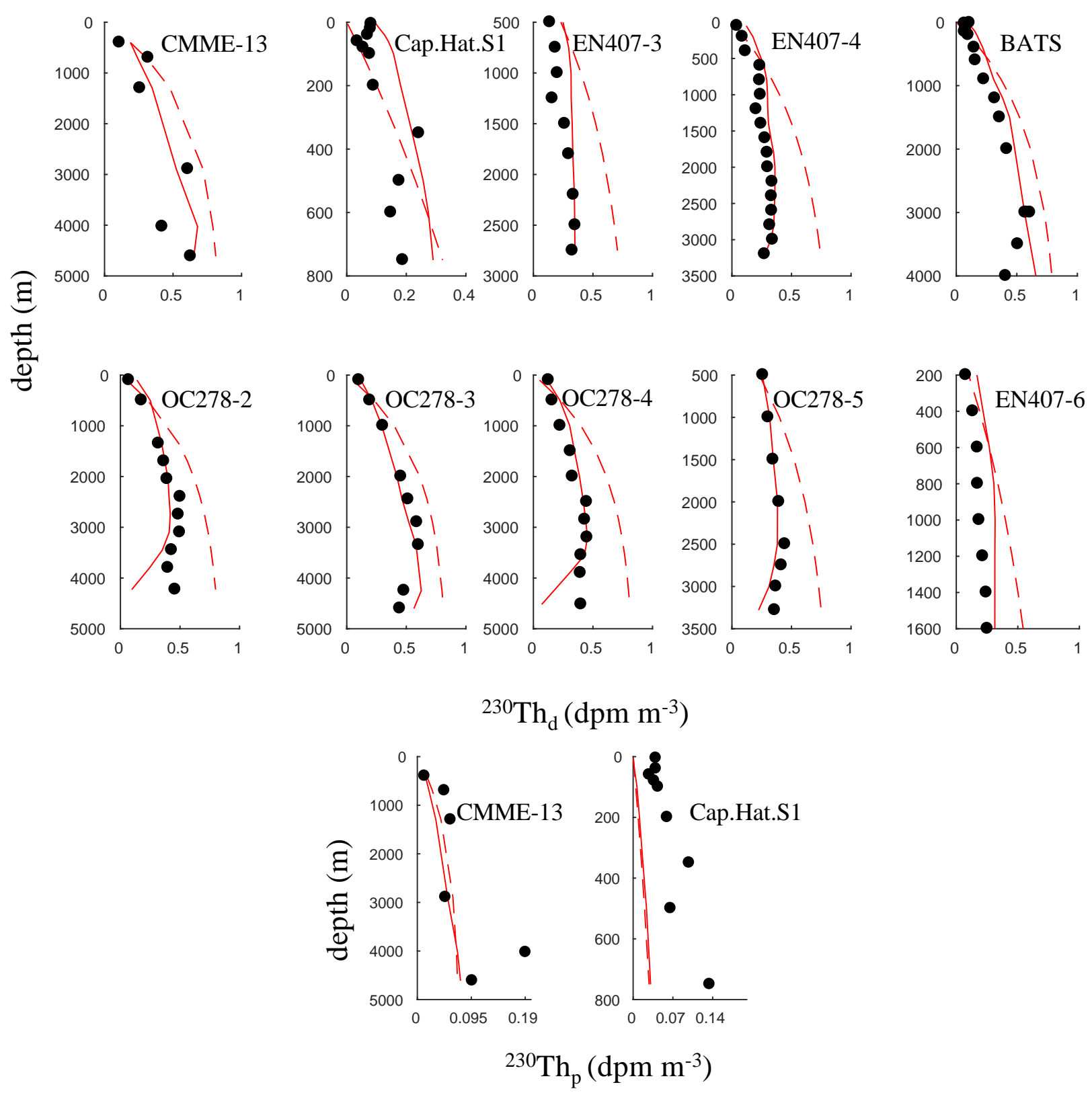

Figure B39. Same as Figs. B37-B38, but for ${ }^{230} \mathrm{Th}_{d, p}$ at pre-GEOTRACES stations. 

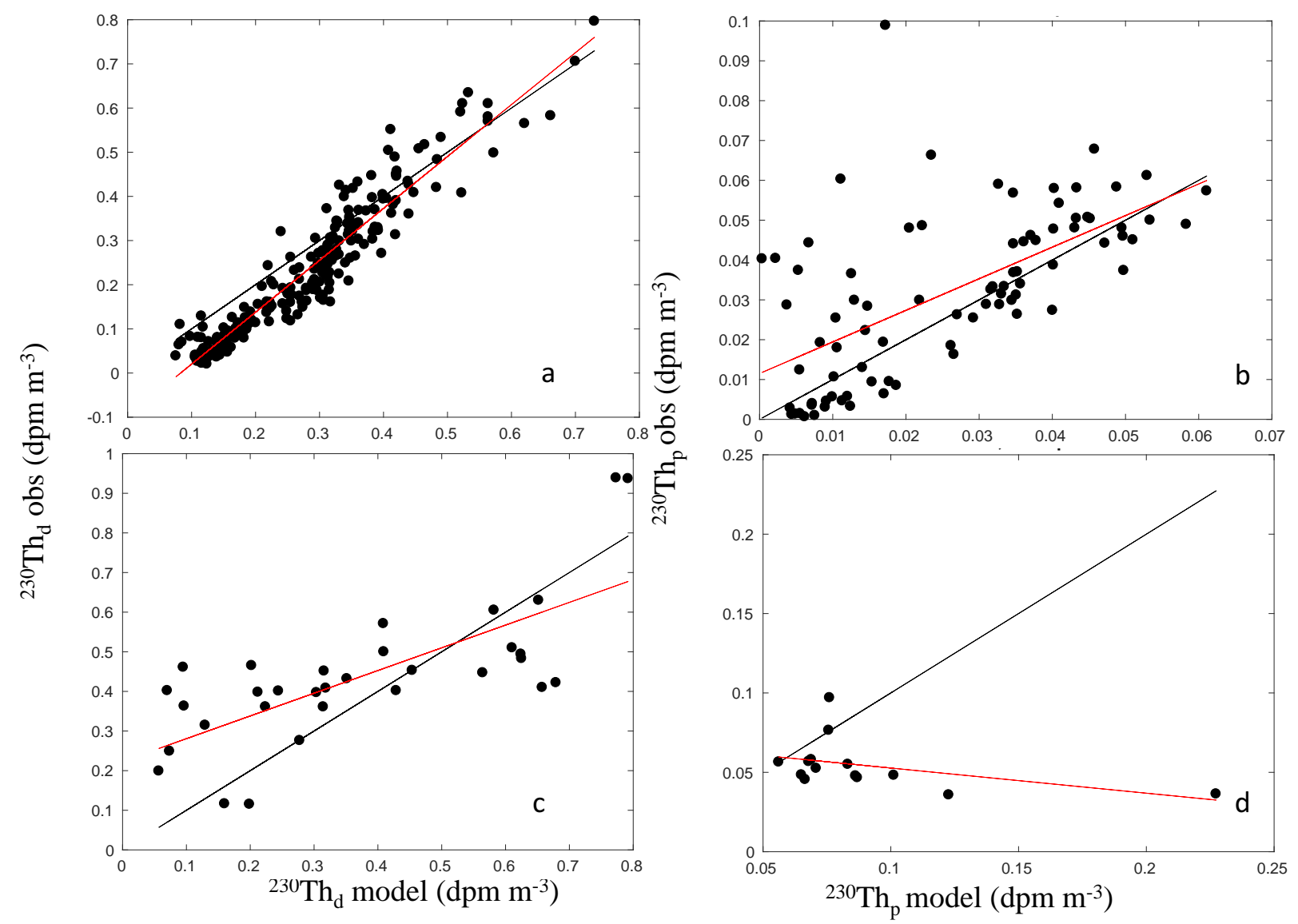

Figure B40. Observed vs. simulated (a,c) ${ }^{230} \mathrm{Th}_{d}$ and (b,d) ${ }^{230} \mathrm{Th}_{p}$. Panels (a) and (b) show activities above $3000 \mathrm{~m}$, and panels (c) and (d) show activities below $3000 \mathrm{~m}$. In each panel, the black line is the line of perfect agreement, and the red line is the best fit obtained by ordinary least squares. Simulated values shown are for experiment k1var6. 


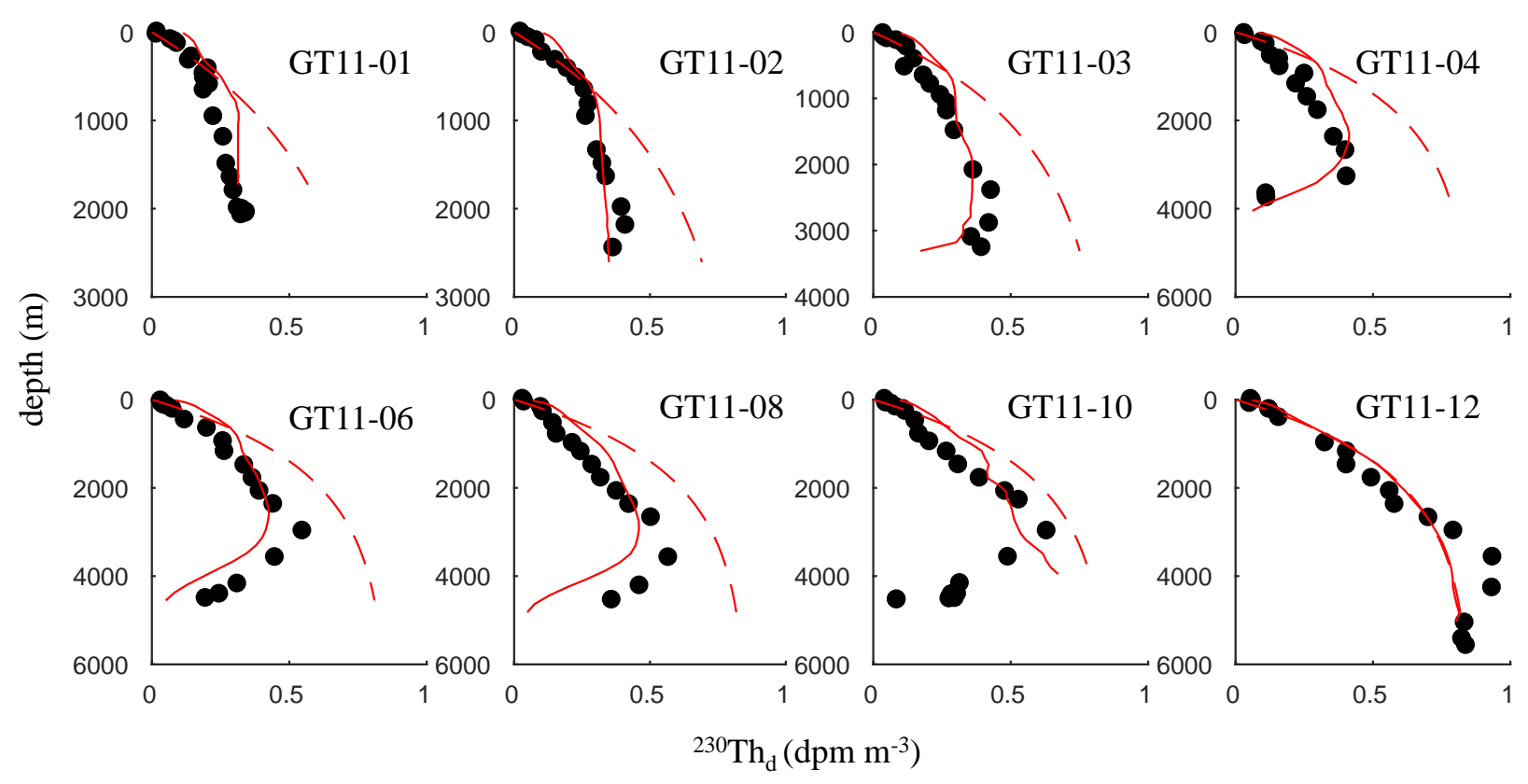

Figure B41. Simulated and observed ${ }^{230} \mathrm{Th}_{d}$ profiles at GEOTRACES stations. The black dots are the observations, the red dashed lines are the initial ${ }^{230} \mathrm{Th}_{d}$ activities assumed in the model, and the red solid lines are the final (after $t=3285$ days) ${ }^{230} \mathrm{Th}_{d}$ activities. Simulated values shown are for experiment k1var'\%. 


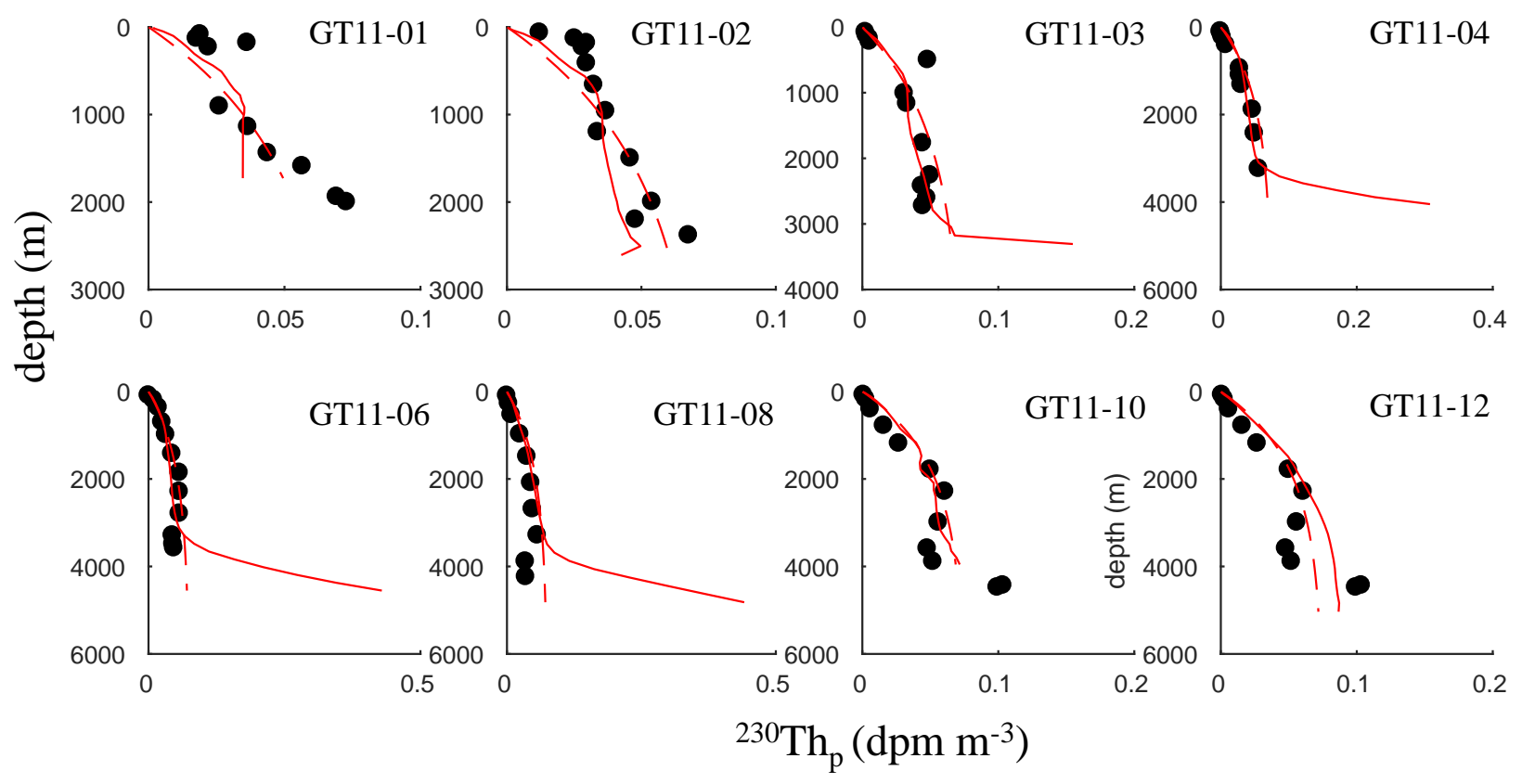

Figure B42. Same as Fig. B41, but for ${ }^{230} \mathrm{Th}_{p}$. 

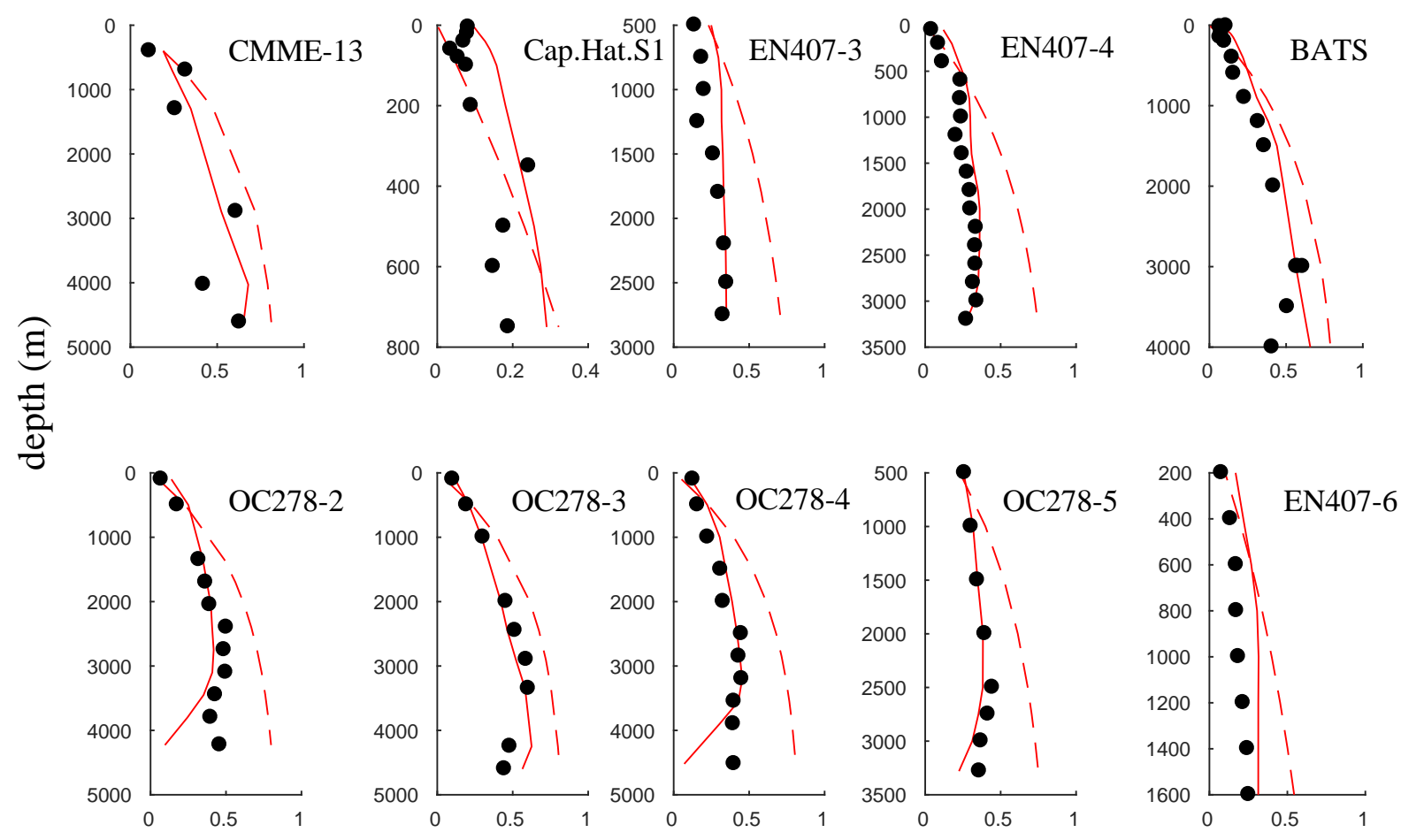

$$
{ }^{230} \mathrm{Th}_{\mathrm{d}}\left(\mathrm{dpm} \mathrm{m}{ }^{-3}\right)
$$

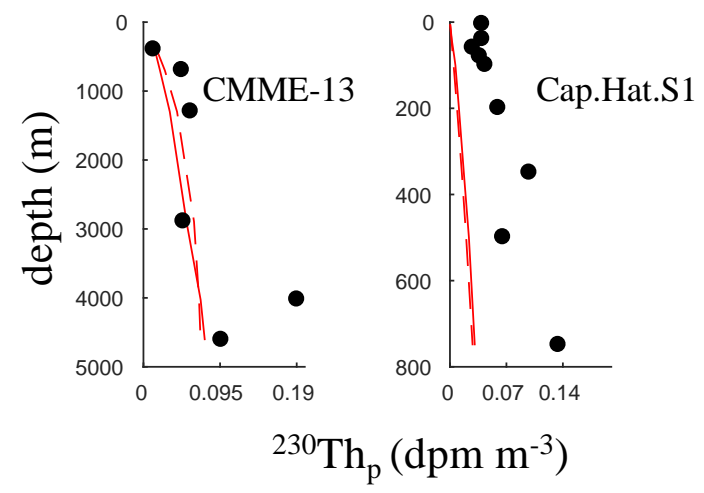

Figure B43. Same as Figs. B41-B42, but for ${ }^{230} \mathrm{Th}_{d, p}$ at pre-GEOTRACES stations. 

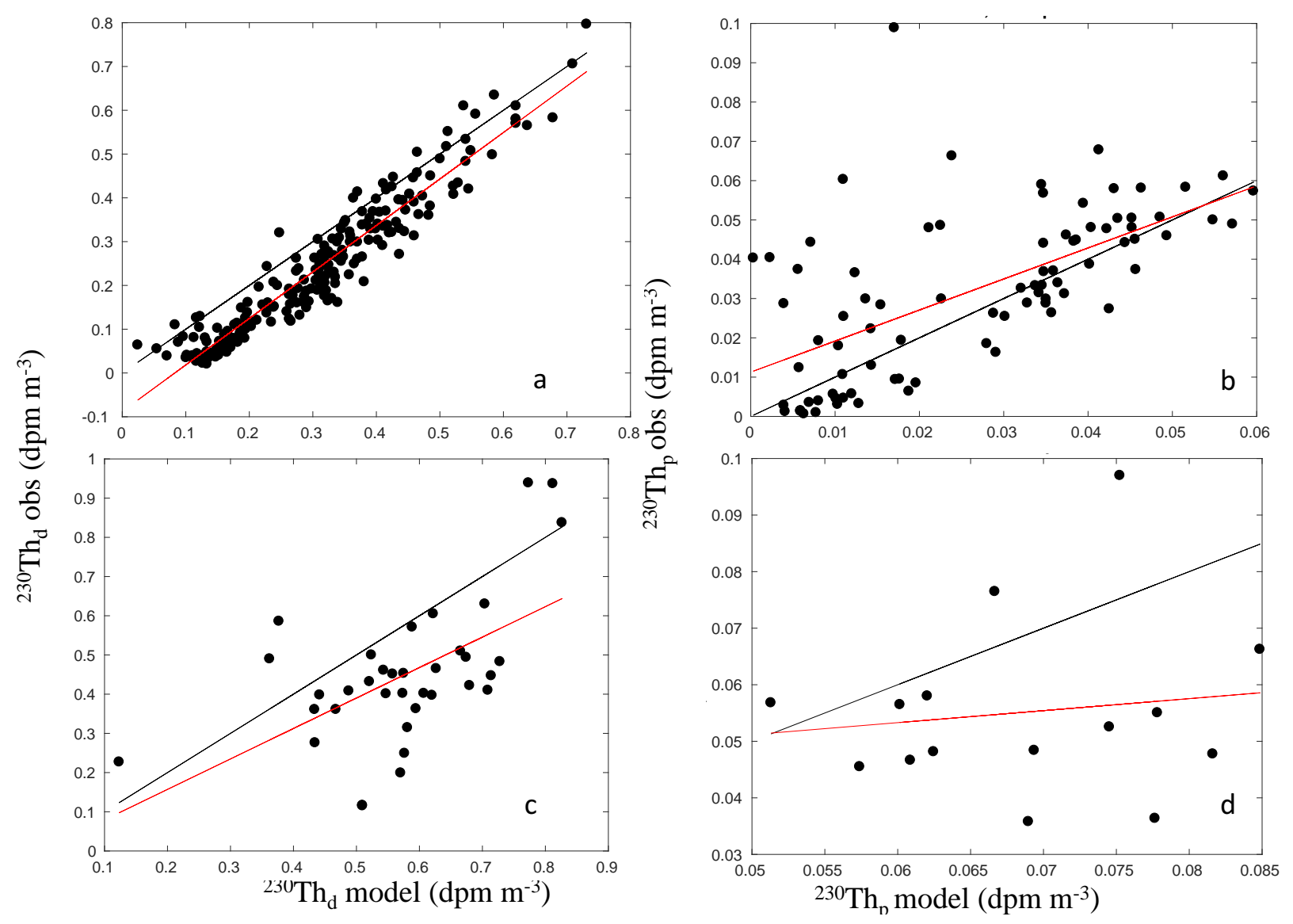

Figure B44. Observed vs. simulated (a,c) ${ }^{230} \mathrm{Th}_{d}$ and $(\mathrm{b}, \mathrm{d})^{230} \mathrm{Th}_{p}$. Panels (a) and (b) show activities above $3000 \mathrm{~m}$, and panels (c) and (d) show activities below $3000 \mathrm{~m}$. In each panel, the black line is the line of perfect agreement, and the red line is the best fit obtained by ordinary least squares. Simulated values shown are for experiment k1var\%. 


\section{Chapter 6}

\section{Conclusion}

The overarching objective of this thesis was to examine how particle concentration, particle composition, and ocean circulation affect the cycling of thorium isotopes in the North Atlantic. The objective was approached by combining the unprecedented radioisotope and particle dataset from the U.S. GEOTRACES North Atlantic Section (section GA03) with models describing the reversible exchange of thorium with slowly settling particles. Inverse techniques were used to constrain the rate parameters describing the cycling of thorium isotopes and particles at stations east of BATS, while the effects of circulation on ${ }^{230}$ Th activities in the Northwest Atlantic were investigated using a regional ocean circulation model based on the primitive equations and run at eddy-permitting resolution. Emphasis was placed throughout the thesis on the relationship between the apparent rate constant for the adsorption of thorium onto particles and particle concentration on the one hand or particle composition on the other hand. Below, the contributions of this thesis to our understanding of particle concentration, particle composition, and circulation effects on thorium in the North Atlantic are summarized and placed in the context of prior studies. A number of recommendations for future research are then suggested.

\subsection{Effect of Particle Concentration}

In Chapter 2, the ${ }^{234,238} \mathrm{U},{ }^{228} \mathrm{Ra},{ }^{228,230,234} \mathrm{Th}_{d, p}$, and particle concentration data or observational estimates from station GT11-22 of section GA03 were combined with two versions of a thorium and particle cycling model using the Algorithm of Total Inversion or ATI (Tarantola and Valette, 1982). A first version assumed that the apparent rate constants for Th adsorption onto 
particles $\left(k_{1}\right)$, Th desorption from particles $\left(k_{-1}\right)$, particle degradation $\left(\beta_{-1}\right)$, as well as the apparent particle sinking speed $(w)$ are vertically uniform, while the second version allowed them to vary along the water colum. It was shown that the second version, with depth-dependent rate parameters, displayed a significantly better fit to the data than the first one. Based on the second version, both $k_{1}$ and the rate constant ratio $K=k_{1} /\left(k_{-1}+\beta_{-1}\right)$ were shown to increase significantly with particle concentration, while $K / P$ was shown to decrease significantly with particle concentration. Moreover, a power-law relationship between $k_{1}$ and $P, k_{1}=k_{1, c} P^{b}$, was found with an exponent $b \leq 1$. Both this finding, as well as the negative correlation between $K / P$ and $P$ inferred in this chapter, are consistent with an effect of particle concentration on the adsorption rate constant for thorium due to colloidal coagulation (Honeyman et al., 1988).

In Chapter 3, an inverse method was used to estimate the distributions of $k_{1}, k_{-1}, \beta_{-1}$, and $w$ along the water column at 11 open-ocean stations (including GT11-22) of the GA03 transect. In contrast to the ATI, this method, based on concepts of nonlinear programming, allowed us to impose inequality constraints, i.e., avoid the inference of negative values for rate parameters and concentrations, although results from both methods were found to be very similar. Consistent with results from Chapter 2, an effect of particle concentration on $k_{1}$ and $K / P$ was also apparent for this group of stations. As in Chapter $2, k_{1}$ was found to be positively correlated with particle concentration, while $K / P$ was found to be negatively correlated with particle concentration. However, in contrast to the result in Chapter 2 , the power-law relationship between $k_{1}$ and $P, k_{1}=k_{1, c} P^{b}$, was shown to have an exponent $b \geq 1$, inconsistent with the effect of particle concentration due to colloidal coagulation (Honeyman et al., 1988). Despite the dependency of $k_{1}$ on $P$, the correlation between $K / P$ and $P$ remained negative, which is unexpected if only variations in $k_{1}$ are considered to drive variations in $K$. In fact, it was found that the relationship between $K / P$ and $P$ at the 11 stations is driven jointly by the increase in $k_{1}$ with $P$ and by the increase in the sum of $k_{-1}$ and $\beta_{-1}$ with $P$. This joint effect points to an influence of particle concentration, not only on $k_{1}$, but also on the sum $k_{-1}+\beta_{-1}$, and suggests that neither Th adsorption onto particles nor Th release from particles due to the combination of Th desorption from particles and particle degradation should be taken as first-order processes.

The relationship $k_{1}=k_{1, c} P^{b}$ with $b \geq 1$ is not surprising if the rate of colloidal coagulation does not limit the rate at which thorium is transferred from the truly dissolved to the filterable particle 
pools. Other studies have suggested a limited role of colloids in the exchange of $\mathrm{Th}$ between the dissolved and particulate phases in the marine environment. In waters offshore of Buzzards Bay, Moran and Buesseler (1993) found that only $1 \%$ of ${ }^{234} \mathrm{Th}$ is colloidal, and that the residence time of colloidal ${ }^{234} \mathrm{Th}$ is too short $(<0.5 \mathrm{~d})$ for colloidal coagulation to be the rate limiting step for the scavenging of thorium. Baskaran et al. (2003) found the coefficient of ${ }^{234} \mathrm{Th}$ partitioning between dissolved and colloidal forms in the Canada Basin is on average one magnitude smaller than for the partitioning between dissolved and particulate forms. More recently, at a station south of BATS in the western subtropical North Atlantic, Hayes et al. (2017) found that colloidal ${ }^{232}$ Th made up $<7 \%$ of dissolved ${ }^{232} \mathrm{Th}$, and that colloidal ${ }^{230} \mathrm{Th}$ activity was statistically indistinguishable from zero. Therefore, the relationship between $k_{1}$ and $P$ found in Chapter 3 in the North Atlantic may partially be due to the limited influence of colloids on thorium scavenging in this region. Other factors, such as the effect of particle composition, changes in the number of surface sites per mass of particles, and disaggregation of filterable particles, may also contribute to the effect of particle concentration found in this study.

These results appear to have significant implications for the modeling of Th and perhaps also other particle-reactive elements in the ocean. Previous studies have implemented a reversible exchange model for ${ }^{230} \mathrm{Th}$ within an ocean circulation model. Some of these models have considered either separate governing equations for ${ }^{230} \mathrm{Th}_{d}$ and ${ }^{230} \mathrm{Th}_{p}$ with explicit parameterizations for $k_{1}$, $k_{-1}$, and $\beta_{-1}$ (or $k_{-1}+\beta_{-1}$; e.g., Marchal et al. (2000); Rempfer et al. (2017)), or a single governing equation for total ${ }^{230} \mathrm{Th},{ }^{230} \mathrm{Th}_{\text {tot }}={ }^{230} \mathrm{Th}_{d}+{ }^{230} \mathrm{Th}_{p}$, where the term $w K_{D}^{230} \mathrm{Th}_{\text {tot }}$ accounts for particle scavenging and sinking, with $K_{D}={ }^{230} \mathrm{Th}_{p} /\left({ }^{230} \mathrm{Th}_{d} P\right)$ (e.g., Henderson et al. (1999); Siddall et al. (2005); Marchal et al. (2007); Dutay et al. (2009)). Both Marchal et al. (2000) and Rempfer et al. (2017) assumed that $k_{1}=\sum_{i=1} F_{i} * \sigma_{i}$, where $F_{i}$ is the vertical sinking flux of particle type $i\left(\mathrm{mg} \mathrm{m}^{-2} \mathrm{yr}^{-1}\right)$ and $\sigma_{i}$ is the scavenging efficiency of that particle type for thorium $\left(\mathrm{m}^{2} \mathrm{mg}^{-1}\right)$. This expression appears consistent with a linear relationship between $k_{1}$ and particle concentration, as displayed in Chapters 2 and 3, since particle concentration is linearly related to the vertical particle sinking flux by $F \equiv w P$. On the other hand, these previous studies did not consider spatial variations in the rate parameters $k_{-1}, \beta_{-1}$, and $w$.

Other model studies (e.g., Henderson et al. (1999), Siddall et al. (2005), Marchal et al. (2007), Dutay et al. (2009), and Burke et al. (2011)) simulated the distribution of either ${ }^{230} \mathrm{Th}_{\text {tot }}$ or ${ }^{230} \mathrm{Th}_{d}$, 
rather than the radionuclide in the dissolved and particulate phases separately, by making different assumptions about the partition coefficient $K_{D}={ }^{230} \mathrm{Th}_{p} /\left({ }^{230} \mathrm{Th}_{d} P\right)$. Henderson et al. (1999) and one experiment in Dutay et al. (2009) applied the relationship between $K_{D}$ and particle concentration found by Honeyman et al. (1988) to derive the $K_{D}$ values used in their model, while Burke et al. (2011) assumed $K_{D}$ to be proportional to 1/P. However, Siddall et al. (2005), Marchal et al. (2007), and four experiments by Dutay et al. (2009) assumed $K_{D}$ to be independent of particle concentration. The relationship between $K_{D}$ and $P$ found by Honeyman et al. (1988) was empirical but could be kinetically grounded assuming that (i) $K_{D}=k_{1} /\left(k_{-1}+\beta_{-1}+\lambda\right)$, where $\lambda$ is the radioactive decay constant for the considered thorium radioisotope, and (ii) the specific rate of Th release from particles $\left(k_{-1}+\beta_{-1}\right)$ is constant. Moreover, the relationship found between $K_{D}$ and $P$ by Honeyman et al. (1988) relied mostly on data collected in estuarine and coastal environments where $P>10 \mathrm{mg} \mathrm{m}^{-3}$. Contrary to assumption (ii), the relationship between $K / P$ and $P$ presented in Chapter 3 is found to involve variations in both $k_{1}$ and $k_{-1}+\beta_{-1}$, and is derived mainly from data below $\sim 100 \mathrm{~m}$ in the open ocean, where particle concentrations are much smaller $\left(\mathrm{P}<10 \mathrm{mg} \mathrm{m}^{-3}\right)$. While acknowledging that $K / P$ and $K_{D}$ are different concepts, they would be equivalent under several assumptions, including negligible contributions of radioactive production, radioactive decay, particulate thorium sinking, advection, diffusion, and time-dependency to the governing equations for ${ }^{230} \mathrm{Th}_{d, p}$. The relationship between $K / P$ and $P$ found in Chapter 3 would therefore suggest that $K_{D}$ depends on particle concentration through variations in both $k_{1}$ and $k_{-1}+\beta_{-1}$ separately. Future models of Th cycling in the ocean based on a single equation for ${ }^{230} \mathrm{Th}_{\text {tot }}$ may need to take this latter result into account.

\subsection{Effect of Particle Composition}

In Chapter 4 , the relationship between $k_{1}$ and particle composition (including $\mathrm{POC}+\mathrm{PIC}+\mathrm{bSi}$, lithogenic material, Mn (oxyhydr)oxides, and Fe (oxyhydr)oxides) at the 11 stations of GA03 considered in Chapter 3 was examined. An additive model and a multiplicative model for the effect of particle composition on $k_{1}$ were applied to three groups of stations: (i) all considered stations, (ii) stations west of the Mauritanian upwelling ("western stations"), and (iii) stations within the Mauritanian upwelling ("eastern stations"). Regardless of the model considered, particle composition

was found to explain more of the variance in $k_{1}$ within the Mauritanian upwelling than west of the 
Mauritanian upwelling. Moreover, it was shown that Mn (oxyhydr)oxides account for most of the explained variance in $k_{1}$ at the western stations, while biogenic particles account for most of the explained variance in $k_{1}$ at the eastern stations. Therefore, the results presented in Chapter 4 point to an effect of particle composition on $k_{1}$ that exhibits regional variability, with a stronger dependence on biogenic particles in eutrophic waters and a stronger dependence on Mn (oxyhydr)oxides in oligotrophic regimes.

The regressions between $k_{1}$ and particle composition were compared to regressions in previous studies of the partition coefficient, $K_{D}=T h_{p} /\left(T h_{d} P\right)$, against particle concentration $(P)$, in ocean waters. Here we inquire whether the effect of particle composition is consistent with that reported in previous studies based on laboratory experiments. Balistrieri et al. (1981) used the following regression model to predict the stability constants for the interaction of metals with a variety of types of particle surfaces:

$$
\log K_{M e}\left(\text { or } \log K_{M e O H}\right)=m \log ^{*} K_{1}^{\text {soln }}\left(\text { or } \log ^{*} \beta_{2}^{\text {soln }}\right)+b+\epsilon
$$

Here $K_{M e}$ is the stability constant for the reaction of a free-metal ion with a particle surface, $K_{\mathrm{MeOH}}$ is the stability constant for the reaction of a metal hydrolysis complex with a particle surface, ${ }^{*} K_{1}^{\text {soln }}$ is the first hydrolysis constant, ${ }^{*} \beta_{2}^{\text {soln }}$ is the second hydrolysis constant, $m$ and $b$ are the slope and intercept, and $\epsilon$ is an error. In equation (6.1), it is assumed that $\log \left(K_{M e}\right)\left(\log \left(K_{M e O H}\right)\right)$ varies linearly with $\log \left({ }^{*} K_{1}^{\text {soln }}\right)\left(\log \left({ }^{*} \beta_{2}^{\text {soln }}\right)\right)$ and that $m$ and $b$ are the same for the relationship between $K_{\mathrm{Me}}\left(K_{\mathrm{MeOH}}\right)$ and ${ }^{*} K_{1}^{\text {soln }}\left({ }^{*} \beta_{2}^{\text {soln }}\right)$. The quantities $m$ and $b$ can vary with particle type. Thus, Balistrieri et al. (1981) estimated $m$ and $b$ for a variety of particle types or organic compounds assumed to represent organic material on the surface of marine particles by fitting experimentally determined $K_{\mathrm{Me}}$ or $K_{\mathrm{MeOH}}$ values to ${ }^{*} K_{1}^{\text {soln }}$ or ${ }^{*} \beta_{2}^{\text {soln }}$ (hydrolysis constants were obtained from Baes and Mesmer (1976)). The particle types considered included $\alpha \mathrm{FeOOH}$ (Balistrieri et al., 1981), $\mathrm{SiO}_{2}$ (Schindler, 1975), $\mathrm{Al}_{2} \mathrm{O}_{3}$ (Davis et al., 1978a,b), and the organic compounds considered included acetic, lactic, salicylic (Sillen et al., 1964), and seawater humic acids (Wilson and Kinney, 1977; Mantoura et al., 1978; Huizenga and Kester, 1979). From the values for $m$ and $b$ obtained by Balistrieri et al. (1981) (see their figures 2 an 3) and the first hydrolysis constant for the formation of thorium-hydroxo complexes, ${ }^{*} K_{1}^{\text {soln }}=10^{-3.2} \mathrm{~mol} / \mathrm{mol}$ (Baes and Mesmer, 1976), I infer that the 
largest stability constants are obtained for the reaction of thorium with salicylic acid $\left(K_{T h}=10^{12.63}\right.$ $\mathrm{mol} / \mathrm{mol})$ and with marine humics $\left(K_{T h}=10^{13.43} \mathrm{~mol} / \mathrm{mol}\right)$ (the stability constants for the reaction of the first hydrolysis complex with organic substances could not be determined due to a lack of experimentally determined $K_{\mathrm{MeOH}}$ values for metal-organic reactions). Compiling a wide variety of laboratory experiments that investigated the association of Th with various organic and inorganic ligands, Santschi et al. (2006) also found the strongest stability constants to be for the complexation of Th with organic ligands, particularly EDTA and DTPA. These results are qualitatively consistent with the finding in Chapter 4 that biogenic particles, which are composed largely of particulate organic carbon, drives most of the variance in the apparent adsorption rate constant for $\mathrm{Th}, k_{1}$, at all 11 stations or at the stations within the Mauritanian upwelling.

Our above calculations of stability constants do not involve Mn (oxyhydr)oxides, since Balistrieri et al. (1981) did not conduct a regression analysis for the reaction of metals with this particle phase. However, Hunter et al. (1988) found that, when applying their experimentally derived stability constants for the adsorption of $\mathrm{Th}(\mathrm{OH})_{4}$ onto the surfaces of synthetic $\delta-\mathrm{MnO}_{2}$ at a particle concentration $<0.001 \mathrm{mg} / \mathrm{m}^{3}$, the partition between particulate and dissolved Th was 4 orders of magnitude smaller than that found by Bacon and Anderson (1982) in the Panama and Guatemala Basins. The concentration of Mn (oxyhydr)oxides west of the Mauritanian upwelling is also $<0.001 \mathrm{mg} / \mathrm{m}^{3}$ (Lam et al., 2015a), yet it is shown in Chapter 4 that $\mathrm{Mn}$ (oxyhydr)oxides contribute to the largest fraction of explained variability in $k_{1}$ in this region. One possible reason for this discrepancy is that Hunter et al. (1988) assumed that Th-organic ligand complexes would not adsorb to Mn (oxyhydr)oxides, whereas the reversible exchange model does not discriminate between different forms of thorium in the dissolved phase (e.g, inorganic vs. organic dissolved Th complexes). Other reasons for the discrepancy include potential variations in surface site concentration and (or) surface charge of Mn (oxyhydr)oxides in the ocean, and the influence of organic coatings on Mn (oxyhydr)oxides (Lerner et al., 2018).

\subsection{Circulation vs. Scavenging Intensity in the Northwest Atlantic}

In Chapter 5, the ability of a regional circulation model to reproduce ${ }^{230} \mathrm{Th}_{d, p}$ data collected from (pre-)GEOTRACES programs in the Northwest Atlantic was examined. The sensitivity of model results to boundary values of ${ }^{230} \mathrm{Th}_{d, p}$, the inflow (outflow) of the Deep Western Boundary 
Current (Gulf Stream), and vertical and lateral variations in particle scavenging as represented by $k_{1}$, was elucidated through a number of numerical experiments. The major finding was that the model could only be brought into better consistency with ${ }^{230} \mathrm{Th}_{d}$ data below $3000 \mathrm{~m}$ when spatial variations in $k_{1}$ were considered. This finding questions a key assumption in the application of ${ }^{230} \mathrm{Th}$ in paleoceanographic studies that the rate at which this radioisotope is removed from the water column does not vary between the open ocean and ocean margins. It further suggests that changes in scavenging intensity due to changes in particle concentration and (or) particle composition, rather than the circulation, determine spatial and temporal variations of ${ }^{230} \mathrm{Th}$ in the Northwest Atlantic. This finding also supports the application of the 1-d reversible exchange model to the open-ocean stations considered in chapters 2-4. Since circulation appears to have only small effects on the distribution of ${ }^{230} \mathrm{Th}$ near the western North Atlantic margin where strong boundary currents are present, I would expect circulation to be even less influential at open-ocean stations. Combined with results in chapter 3 which show that advection, diffusion, and unsteadiness appear negligible in the Th isotope and particle budgets at GA03 stations examined, the assumptions in chapters 2-4 that circulation effects can be neglected appears to be justified. Importantly, however, the model in chapter 5 may not fully represent the effects of circulation on ${ }^{230} \mathrm{Th}$ in the Northwest Atlantic. For example, the DWBC in the model is displaced up-slope compared to observations along line W. Since the DWBC is expected to carry low activities of ${ }^{230} \mathrm{Th}_{d, p}$ (Moran et al., 1997), its contribution to the observed deficits of ${ }^{230} \mathrm{Th}_{d}$ below $3000 \mathrm{~m}$ in the Northwest Atlantic may not be captured by the model. To more completely assess the effect of circulation on ${ }^{230} \mathrm{Th}$ in the Northwest Atlantic, and address the implications of this effect for other oceanic regions, the representation of the DWBC in the model needs to be improved.

Importantly, our model relied on multiple assumptions to parameterize spatial variations in $k_{1}$ in the Northwest Atlantic. First, $k_{1}$ was assumed to increase with depth below $3000 \mathrm{~m}$, similarly to light attenuation, in order to reflect the presence of a bottom nepheloid layer (BNL), a permanent feature of the deep water column in this region. Our description of vertical variations of $k_{1}$ in the model makes two assumptions: (i) $k_{1}$ increases linearly with resuspended sediment concentration (Nyffeler et al., 1984) and (ii) the particle beam attenuation coefficient $\left(c_{p}\right)$ increases linearly with the concentration of resuspended sediment. Assumption (i) has been questioned by Honeyman et al. (1988), who have proposed instead that the apparent rate constant $k_{1}$ varies with particle 
concentration according to a power law, $k_{1}=k_{1, c} P^{b}$, where $b<1$ due to colloidal coagulation limiting the transfer of thorium from truly soluble to filterable particulate pools. However, the application of this power law may be inappropriate in the North Atlantic, as we found evidence, from an analysis of open-ocean Th isotope and particle data collected along GA03, that $k_{1}$ scales with $P$ with an exponent close to, or slightly greater than, 1 (Chapter 3). Regarding assumption (ii), $c_{p}$ has been shown to vary linearly with particle concentration on the Scotian Rise (Gardner et al., 1985). More recently, $c_{p}$ has been shown to explain a larger proportion of variance in particulate organic carbon than total suspended particulate matter in the Eastern Tropical South Pacific (Ohnemus et al., 2017). However, in that study, $c_{p}$ still represented over $75 \%$ of the variance in total suspended particulate matter. Therefore, both studies suggest that our approach to constrain vertical variations in $k_{1}$ may be justified.

Second, vertical variations in $k_{1}$ were allowed only in a limited area of the model domain. In the model, the region of enhanced scavenging below $3000 \mathrm{~m}$ approximated the region of enhanced light scattering below the clear water minimum documented by two decades of nephelometer data (Gardner et al., 2017). While the spatial extent of this region does not dramatically influence model results, in particular the (dis)agreement to ${ }^{230} \mathrm{Th}_{d, p}$ data, an improved representation of the influence of BNLs, which would require the simulation of sediments resuspended from the seafloor, would clearly be desirable. The inclusion of resuspended sediments in the model could better resolve regions where the influence of nepheloid layers on thorium scavenging is important, while also improving the representation of vertical variations in $k_{1}$ by explicitly relating this parameter with the modeled particle concentration. However, there are significant challenges in modeling the resuspension and transport of sediments, including the accurate simulation of vertical velocity shears near the bottom and the consideration of sediment properties, such as texture and cohesiveness. Particularly, models able to simulate the observed "benthic storms" that are commonly observed in the Northwest Atlantic (Gardner et al., 2017) may be key to capturing the extremely large particulate ${ }^{230} \mathrm{Th}_{p}$ activities near the seafloor observed near the bottom at a few GEOTRACES stations. 


\subsection{General Outlook}

The major findings of this thesis suggest a number of future research directions in order to better understand the effects of scavenging and circulation on thorium isotopes and particle-reactive metals more generally in the ocean.

\subsubsection{Recommendations for Models}

One avenue for future investigations would be to examine the effects of particle concentration and composition on ${ }^{230} \mathrm{Th}_{d, p}$ distributions simulated by circulation models. A preliminary attempt to this end was undertaken in Chapter 5 , by a variation of $k_{1}$ with height above the seafloor based on an assumed effect of resuspended sediment on the Th adsorption rate. However, the representation of the effect of particle concentration on $k_{1}$ and hence on thorium distributions would likely be improved if particles, produced biologically in the surface waters and resuspended from the seafloor, were included in the model. Of particular interest would be differences in simulated ${ }^{230} \mathrm{Th}_{d, p}$ distributions based on (i) a model that only considers a positive correlation between $k_{1}$ and $P$, (ii) a model that also considers a positive correlation between $k_{-1}+\beta_{-1}$ and $P$ (Chapter 3), and (iii) a model that considers the correlation between $k_{1}$ and particle composition (Chapter 4). Moreover, ${ }^{230} \mathrm{Th}_{d, p}$ distributions in Chapter 5 were simulated at a relatively coarse resolution of $1 / 4^{\circ}$, and some elements of the simulated circulation were not consistent with observations (e.g., along Line W, the positions of the GS and DWBC were not the same as those observed, and the DWBC was too strong compared to the observations). The GS and DWBC positions might be improved if buoyancy fluxes from the exchange of heat and salt with the atmosphere, currently not included in the model, are considered. A higher resolution model may also better represent circulation in the Northwest Atlantic. Accurate simulations of the circulation near the bottom are particularly important for sediment resuspension, since resuspension depends partly on bottom shear stresses.

The model presented in Chapter 5 may be adapted to include ${ }^{231} \mathrm{~Pa}$, allowing the simulation of the ${ }^{231} \mathrm{~Pa} /{ }^{230} \mathrm{Th}$ ratio in particles near the seafloor, and testing the sensitivity of this ratio to changes in scavenging intensity and circulation. The GEOTRACES North Atlantic section has provided simultaneous measurements of ${ }^{230} \mathrm{Th}_{d, p},{ }^{231} \mathrm{~Pa}_{d, p}$, and concentrations of particles separated by composition. Using this dataset combined with pre-GEOTRACES measurements, Chapter 5 lays 
the groundwork for rigorous hypothesis testing regarding the ability of different types of particles to scavenge these radioisotopes in both clear and turbid waters. This chapter thus provides a critical step in deciphering sediment ${ }^{231} \mathrm{~Pa} /{ }^{230} \mathrm{Th}$ records, whose current interpretations generally make strong assumptions about the influences of particle concentration, particle composition, and circulation on both radioisotopes.

It is important to highlight that the results presented in Chapters 2 through 4 of this thesis were obtained by combining multiple thorium radioisotopes with a model describing the reversible exchange of thorium with particles. As shown in Figure 11 of Chapter 2, the conclusions drawn from fitting a reversible exchange model with a single thorium radioisotope may not be the same as those drawn when using multiple thorium isotopes. One may infer different values of $k_{1}, k_{-1}, \beta_{-1}$, and $w$, from fits to different combinations of radioisotopes. Notably, this thesis did not include ${ }^{232} \mathrm{Th}$ in the inference of the rate constants and $w$. The sources ${ }^{228,230,234} \mathrm{Th}$ in the ocean are predominantly the radioactive decay of their respective radioactive parents, which can be estimated based on salinity in the case of ${ }^{230,234} \mathrm{Th}$, or can be measured simultaneously with the daughter isotope in the case of ${ }^{228} \mathrm{Th}$. Thus, the sources of ${ }^{228,230,234} \mathrm{Th}$ are generally well known. In contrast, for ${ }^{232} \mathrm{Th}$, the predominant source, atmospheric dust deposition, is poorly constrained. As a result, the extent to which ${ }^{232} \mathrm{Th}$ data could contribute, beyond ${ }^{228,230,234} \mathrm{Th}$ data, to the estimation of Th and particle cycling rates is unclear. In summary, since different thorium isotopes should be indistinguishable in their chemical behavior, it is recommended that future studies aiming to infer $k_{1}, k_{-1}, \beta_{-1}$, and $w$ take advantage of, when possible, information provided by multiple thorium radioisotopes.

\subsubsection{Recommendations for Laboratory Experiments and Observations}

Throughout this thesis, an emphasis has been placed on the importance of spatial variations in $k_{1}$ in controlling the distributions of dissolved and particulate thorium in the North Atlantic. Most of our current estimates of $k_{1}$ come from fitting the reversible exchange model to data of Th isotope activities in the dissolved and particulate phases and (or) sediment trap data collected in various ocean regions (e.g., Bacon and Anderson (1982); Nozaki et al. (1987); Murnane (1994a)). Few laboratory studies have examined the kinetics of Th adsorption, (e.g., Nyffeler et al. (1984)), and to my knowledge, no laboratory experiments so far have estimated the effects of particle composition on the adsorption rate of thorium in aqueous media. Similarly, aside from Nyffeler et al. (1984), to my knowledge no laboratory studies have investigated the kinetics of Th desorption from particles. 
Such studies could be invaluable for developing a better understanding of the geochemical cycle of Th in the ocean.

Finally, in this thesis, the exchange of Th isotopes in solution with a single particle class is only considered. As result, important processes such as particle aggregation and particle disaggregation were not addressed. Several studies have considered the exchange of Th isotopes between three operationally defined pools: a dissolved pool, "small" (non-sinking) particles and "large" (sinking) particles (Nozaki et al., 1987; Clegg et al., 1991a; Cochran et al., 1993; Murnane, 1994a). Consideration of all three pools permits estimates of particle aggregation and disaggregation rates, two important processes that partially determine the efficiency of the biological pump to transfer carbon and other elements from the surface waters to the deep ocean (Burd and Jackson, 2009; Lam and Marchal, 2014). In exploring the ability to recover rate parameters from synthetic datasets of 228,230,234 $\mathrm{Th}$ in the dissolved, small particulate, and large particulate phases, as well as of particle concentration in the small and large fractions, Marchal and Lam (2012) found that good recoveries were obtained when (i) the relative errors of the radioisotope measurements were less than $20 \%$, (ii) vertical sampling was dense enough to resolve activity and particle concentration gradients, and (iii) model errors were negligible. Large particulate ${ }^{228,230,234} \mathrm{Th}$ activities were not considered in this thesis mainly because condition (ii) was not met: large particulate ${ }^{234} \mathrm{Th}$ was only sampled in the top $1000 \mathrm{~m}$, while large particulate ${ }^{228} \mathrm{Th}\left({ }^{230} \mathrm{Th}\right)$ was only sampled at a few depths at three (four) stations along the second leg of GA03. Increasing the sampling resolution of large particulate Th isotopes can allow for inversions similar to those performed in Chapter 2 and 3, but for a two-particle class model that includes aggregation and disaggregation. Similarly, the effect of colloidal coagulation may be more thoroughly investigated if Th isotopes are measured on the colloidal fraction in concert with those in the dissolved, small particulate, and large particulate fractions. Studies that have reported colloidal Th measurements have generally focused on ${ }^{234} \mathrm{Th}$ in coastal environments (Baskaran et al., 1992; Moran and Buesseler, 1993; Guo et al., 1997), though a few studies have also reported colloidal ${ }^{230} \mathrm{Th}$ in the open ocean, including at Station ALOHA and south of Bermuda (Hayes et al., 2015c, 2017). However, as with the other rate parameters, the inference of accurate and precise estimates of colloidal Th coagulation will probably require (i) a sampling resolution of colloidal Th that is similar to that of dissolved and particulate Th isotope samples (the resolution of ${ }^{228,230,234} \mathrm{Th}_{d, p}$ used in this study varied from $\sim 100 \mathrm{~m}$ above $1000 \mathrm{~m}$ to $\sim 500 \mathrm{~m}$ below 
this depth, and includes surface samples to near bottom samples) and (ii) colloidal Th isotopes to be collected simultaneously with soluble (i.e., truly dissolved), small particulate, and large particulate Th isotopes. Alongside Th isotopes, measurements of the colloids themselves would allow for the inclusion of a particle formation term in the governing equation for bulk particles, providing important information on the rate at which particles that cannot be measured using conventional filtering techniques aggregate to form particles that are typically filtered. Dissolved organic carbon (DOC) measurements may also be useful for estimating rates of filterable particle formation. DOC is often measured by filtering seawater collected in Niskin bottles through glass microfiber filters with pore-sizes $<1 \mu \mathrm{m}$, so that the filtrate contains both truly dissolved and colloidal organic carbon (Verdugo et al., 2004). While the second leg of section GA03 does not include DOC measurements, the first leg does (unpublished data from Joaquin Chavez and Charles McClain, also listed in the GEOTRACES Intermediate Data Product; Schlitzer et al. (2018)), and these measurements are colocated with measurements of ${ }^{228,230,234} \mathrm{Th}_{d, p}$ and particle concentration and composition in small and large size fractions at station GT10-10, GT10-11, and GT10-12. These stations may provide an opportunity for understanding how the transfer of dissolved to filterable particulate thorium is affected by DOC. The estimation of the rates of colloidal coagulation and Th adsorption onto colloids would allow for a more rigorous assessment of the effect of colloids on the transfer of thorium from the truly dissolved to filterable particulate phases. In turn, such an assessment should lead to a better understanding on the apparent rate at which thorium, and perhaps other trace metals, are adsorbed onto marine particles and ultimately removed to the sediment. 


\section{Bibliography}

Aitchison, J., and J. A. C. Brown (1957), The Lognormal Distribution (With Special Reference to Its Uses in Economics), 168 pp., Cambridge University Press, New York.

Alonso-González, I. J., J. Arístegui, C. Lee, A. Sanchez-Vidal, A. Calafat, J. Fabrés, P. Sangrá, P. Masqué, A. Hernández-Guerra, and V. Benítez-Barrios (2010), Role of slowly settling particles in the ocean carbon cycle, Geophys. Res. Lett., 37, 1-5.

Andersen, M. B., C. H. Stirling, B. Zimmermann, and A. N. Halliday (2010), Precise determination of the open ocean ${ }^{234} \mathrm{U} /{ }^{238} \mathrm{U}$ composition, Geochemistry, Geophysics, Geosystems, $11(12)$.

Anderson, H. L., R. François, and S. B. Moran (1992), Experimental evidence for differential adsorption of Th and $\mathrm{Pa}$ on different solid phases in seawater, EOS, 73, 270.

Anderson, R. F., M. P. Bacon, and P. G. Brewer (1983), Removal of ${ }^{230} \mathrm{Th}$ and ${ }^{231} \mathrm{~Pa}$ from the open ocean, Earth Planet. Sci. Lett., 62, 7-23.

Anderson, R. F., M. Q. Fleisher, P. E. Biscaye, N. Kumar, B. Dittrich, P. Kubik, and M. Suter (1994), Anomalous boundary scavenging in the Middle Atlantic Bight: evidence from ${ }^{230} \mathrm{Th}$, ${ }^{231} \mathrm{~Pa},{ }^{10} \mathrm{Be}$ and ${ }^{210} \mathrm{~Pb}$. Deep Sea Research Part II: Topical Studies in Oceanography, Deep Sea Research Part II: Topical Studies in Oceanography, 41(2-3), 537-561.

Anderson, R. F., M. Q. Fleisher, and Y. Lao (2006), Glacial-interglacial variability in the delivery of dust to the central equatorial Pacific Ocean, Earth and Planetary Science Letters, 242(3), 406-414.

Anderson, R. F., M. Q. Fleisher, L. F. Robinson, R. L. Edwards, J. Hoff, S. B. Moram, M. M. Rutgers van der Loeff, A. L. Thomas, M. Roy-Barman, and R. François (2012), GEOTRACES 
intercalibration of ${ }^{230} \mathrm{Th},{ }^{232} \mathrm{Th},{ }^{231} \mathrm{~Pa}$, and prospects for ${ }^{10} \mathrm{Be}$, Limnol. Oceanogr. Methods, 10 , $179-213$.

Anderson, R. F., M. Q. Fleisher, and C. Hayes (2013), Transmissometer_GTC, Biological and chemical oceanography data system, BCO DMO, WHOI, http://data.bco-dmo.org/ jg/info/BCO/GEOTRACES/NorthAtlanticTransect/Transmissometer_GTC $\ \%$ Bdir=data . bco-dmo.org/jg/dir/BCO/GEOTRACES/NorthAtlanticTransect/, data=data.bco-dmo.org: 80/jg/serv/BCO/GEOTRACES/NorthAtlanticTransect/GT10-11_trans_Cp_GTC.htm10 \%7D?, accessed: 22 January, 2015.

Andres, M., J. M. Toole, D. J. Torres, W. M. Smethie, T. M. Joyce, and R. G. Curry (2016), Stirring by deep cyclones and the evolution of Denmark strait overflow water observed at line W, Deep Sea Research Part I: Oceanographic Research Papers, 109, 10-26.

Armstrong, R. A., M. L. Peterson, C. Lee, and S. G. Wakeham (2009), Settling velocity spectra and the ballast ratio hypothesis, Deep Sea Research II, 56, 1470-1478.

Athias, V., P. Mazzega, and C. Jeandel (2000a), Selecting a global optimization method to estimate the oceanic particle cycling rate constants, J. Mar. Res., 58(5), 675-707.

Athias, V., P. Mazzega, and C. Jeandel (2000b), Nonlinear Inversions of a Model of the Oceanic Dissolved-Particulate Exchanges, Inverse Methods in Global Biogeochemical Cycles (eds. P. Kasibhatla, M. Heimann, P. Rynar, M. Mahowald, R. G.Prinn, and D. E. Hartley), Geoph. Monog. Series, 114, Am. Geophys. Union. 205-222.

Azen, R., and D. V. Budescu (2003), The dominance analysis approach for comparing predictors in multiple regression, Psychol. methods, 8(2), 129-148.

Azetsu-Scott, K., B. D. Johnson, and B. Petrie (1995), An intermittent, intermediate nepheloid layer in Emerald Basin, Scotian Shelf, Continental Shelf Research, 15(2-3), 281-293.

Bacon, M. P. (1984), Glacial to interglacial changes in carbonate and clay sedimentation in the Atlantic Ocean estimated from ${ }^{230} \mathrm{Th}$ measurements, Earth and planetary science letters, $46(2)$, 97-111. 
Bacon, M. P., and R. F. Anderson (1982), Distribution of thorium isotopes between dissolved and particulate forms in the deep sea, J. Geophys. Res. Oceans, 87(C3), 2045-2056.

Bacon, M. P., C. A. Huh, A. P. Fleer, and W. G. Deuser (1985), Seasonality in the flux of natural radionuclides and plutonium in the deep Sargasso Sea, Deep Sea Res. A, 32(3), 273-286.

Bacon, M. P., R. A. Belastock, M. Tecotzky, K. K. Turekian, and D. W. Spencer (1988), Lead-210 and polonium-210 in ocean water profiles of the continental shelf and slope south of New England, Continental Shelf Research, 8(5-7), 841-853.

Baes, C. F., and R. E. Mesmer (1976), Hydrolysis of Cations, John Wiley and Sons, New York City, NY.

Balistrieri, L., P. G. Brewer, and J. W. Murray (1981), Scavenging residence times of trace metals and surface chemistry of sinking particles in the deep ocean, Deep Sea Res. A, 28(2), 101-121.

Balistrieri, L. S., and J. W. Murray (1986), The surface chemistry of sediments from the Panama Basin: The influence of Mn oxides on metal adsorption, Geochimica et Cosmochimica Acta, $50(10), 2235-2243$.

Baskaran, M., P. H. Santschi, G. Benoit, and B. D. Honeyman (1992), Scavenging of thorium isotopes by colloids in seawater of the Gulf of Mexico, Geochim. Cosmochim. Acta, 56(9), 33753388.

Baskaran, M., P. H. Santschi, L. Guo, T. S. Bianchi, and C. Lambert (1996), ${ }^{234}$ Th: ${ }^{238}$ U disequilibria in the Gulf of Mexico: the importance of organic matter and particle concentration, Continental Shelf Research, 16(3), 353-380.

Baskaran, M., P. W. Swarzenski, and D. Porcelli (2003), Role of colloidal material in the removal of ${ }^{234} \mathrm{Th}$ in the Canada basin of the Arctic Ocean, Deep Sea Res. Part I Oceanogr. Res. Pap., $50(10), 1353-1373$.

Bevington, P. R., and D. K. Robinson (1992), Data Reduction and Error Analysis for the Physical Sciences, McGraw-Hill, New York. 
Bhat, S. G., S. Krishnaswami, D. Lal, and W. S. Moore (1969), ${ }^{234}$ Th and ${ }^{238} \mathrm{U}$ ratios in the ocean, Earth Planet. Sci. Lett., 5, 483-491.

Biscaye, P. E., R. F. Anderson, and B. L. Deck (1988), Fluxes of particles and constituents to the eastern United States continental slope and rise: SEEP-I, Cont. Shelf Res., 8(5-7), 855-904.

Biscaye, P. E., C. N. Flagg, and P. G. Falkowski (1994), The shelf edge exchange processes experiment, SEEP-II: an introduction to hypotheses, results and conclusions, Deep Sea Research Part II: Topical Studies in Oceanography, 41(2-3), 231-252.

Bishop, J. K. (2009), Autonomous observations of the ocean biological carbon pump, Oceaonography, 22(2), 182-193.

Bishop, J. K., and M. Q. Fleisher (1987), Particulate manganese dynamics in Gulf Stream warmcore rings and surrounding waters of the NW Atlantic., Geochimica et Cosmochimica Acta, 51 (10), $2807-2825$.

Bishop, J. K., P. J. Lam, and T. J. Wood (2012), Getting good particles: Accurate sampling of particles by large volume in-situ filtration, Limnol. Oceanogr. Methods, 10(9), 681-710.

Blaas, M., C. Dong, P. Marchesiello, J. C. McWilliams, and K. D. Stolzenbach (2007), Sedimenttransport modeling on Southern Californian shelves: A ROMS case study, Continental shelf research, $27(6), 832-853$.

Blumberg, A. F., and G. L. Mellor (1987), A description of a three-dimensional coastal ocean circulation model, Three-dimensional coastal ocean models, pp. 1-16.

Borggaard, O. K. (1983), Iron oxides in relation to aggregation of soil particles, Acta Agriculturae Scandinavica, 33(3), 257-260.

Bower, A. S., and H. D. Hunt (2000), Lagrangian observations of the deep western boundary current in the North Atlantic Ocean. Part I: Large-scale pathways and spreading rates, Journal of Physical Oceanography, 30(5), 764-783. 
Boyle, E. A., R. F. Anderson, G. A. Cutter, R. Fine, W. J. Jenkins, and M. Saito (2015), Introduction to the US GEOTRACES North Atlantic Transect (GA-03): USGT10 and USGT11 cruises, Deep Sea Res. Part II Top. Stud. Oceanogr., 116, 11-5.

Bozec, A., M. S. Lozier, E. P. Chassignet, and G. R. Halliwell (2011), On the variability of the Mediterranean Outflow Water in the North Atlantic from 1948 to 2006., J. Geophys. Res. Oceans, 116(C9), 1-18.

Bradtmiller, L. I., J. F. McManus, and L. F. Robinson (2014), ${ }^{231} \mathrm{~Pa} /{ }^{230} \mathrm{Th}$ evidence for a weakened but persistent Atlantic meridional overturning circulation during Heinrich Stadial 1, Nature communications, 5 .

Brewer, P. G., Y. Nozaki, D. W. Spencer, and A. P. Fleer (1980), Sediment trap experiments in the deep North Atlantic: isotopic and elemental fluxes, J. Mar. Res., 38(4), 703-729.

Bruland, K. W., and K. H. Coale (1986), Surface water ${ }^{234} \mathrm{Th} /{ }^{238} \mathrm{U}$ disequilibria: Spatial and temporal variations of scavenging rates within the Pacific Ocean, Dynamic processes in the chemistry of the upper ocean, pp. 159-172.

Bruland, K. W., and M. C. Lohan (2006), Controls of trace metals in seawater, The oceans and marine geochemistry, 6, 23-47.

Buesseler, K., L. Ball, J. Andrews, J. Cochran, D. Hirschberg, M. Bacon, A. Fleer, and M. Brzezinski (2001), Upper ocean export of particulate organic carbon and biogenic silica in the Southern Ocean along $170^{\circ} \mathrm{W}$, Deep-Sea Research Part II: Topical Studies in Oceanography, 48(19), 4275-4297.

Buesseler, K., S. Pike, K. Maiti, C. H. Lamborg, D. A. Siegel, and T. W. Trull (2009), Thorium-234 as a tracer of spatial, temporal and vertical variability in particle flux in the North Pacific, Deep Sea Research Part I: Oceanographic Research Paper, 56(7), 1143-1167.

Buesseler, K. O., M. P. Bacon, J. K. Cochran, and H. D. Livingston (1992), Carbon and nitrogen export during the JGOFS North Atlantic Bloom Experiment estimated from ${ }^{234} \mathrm{Th}:{ }^{238} \mathrm{U}$ disequilibria, Deep Sea Res. A, 39(7-8), 1115-1137.

Buesseler, K. O., C. R. Benitez-Nelson, S. B. Moran, A. Burd, M. A. Charette, J. K. Cochran, L. Coppola, N. S. Fisher, S. W. Fowler, W. D. Gardner, L. D. Guo, O. Gustafsson, C. Lamborg, 
P. Masqué, J. C. Miquel, U. Passow, P. H. Santschi, N. Savoye, G. Stewart, and T. Trull (2006), An assessment of particulate organic carbon to thorium-234 ratios in the ocean and their impact on the application of ${ }^{234} \mathrm{Th}$ as a POC flux proxy, Mar. Chem., $100(3), 213-233$.

Burd, A., and G. A. Jackson (2009), Predicting particle coagulation and sedimentation rates for a pulsed input, J. Geophys. Res. Oceans, 102(C5), 10,545-10,561.

Burd, A. B., S. B. Moran, and G. A. Jackson (2000), A coupled adsorption aggregation model of the POC $/{ }^{234}$ Th ratio of marine particles., Deep Sea Res. Part I Oceanogr. Res. Pap., 47(1), 103-120.

Burdige, D. J. (1993), The biogeochemistry of manganese and iron reduction in marine sediments, Earth-Science Reviews,, 35(3), 249-284.

Burke, A., O. Marchal, L. I. Bradtmiller, J. F. McManus, and R. François (2011), Application of an inverse method to interpret ${ }^{231} \mathrm{~Pa} /{ }^{230} \mathrm{Th}$ observations from marine sediments, Paleoceanography, $26(1), 1-17$.

Buse, A. (1973), Goodness of fit in generalized least squares estimations, Am. Stat., 27(3), 106-108.

Byrd, R. H., M. E. Hribar, and J. Nocedal (1999), An interior point algorithm for large-scale nonlinear programming, SIAM Journal on Optimization, 9(4), 877-900.

Byrd, R. H., J. C. Gilbert, and J. Nocedal (2000), A trust region method based on interior point techniques for nonlinear programming, Mathematical Programming, 89(1), 149-185.

Cacchione, D., and C. Wunsch (1974), Experimental study of internal waves over a slope, Journal of Fluid Mechanics, 66(2), 223-239.

Cacchione, D. A., and D. E. Drake (1986), Nepheloid layers and internal waves over continental shelves and slopes, Geo-Marine Letters, 6(3), 147-152.

Cacchione, D. A., L. F. Pratson, and A. S. Ogston (2002), The shaping of continental slopes by internal tides, Science, 296(5568), 724-727.

Charette, M. A., W. S. Moore, P. J. Morris, and P. B. Henderson (2014), GT10-11 Ra and Th, Biological and chemical oceanography data system, BCO DMO, WHOI, http://data.bco-dmo.org/ 
jg/info/BCO/GEOTRACES/NorthAtlanticTransect/Ra_Th_GT10\\%7Bdir=data.bco-dmo.org/ jg/dir/BCO/GEOTRACES/NorthAtlanticTransect/, data=data . bco-dmo . org: 80/jg/serv/ RESTRICTED/GEOTRACES/NorthAtlanticTransect/Ra_Th_GT10_joined.htm10 \%7D?, accessed: 5 April, 2014.

Charette, M. A., P. J. Morris, P. B. Henderson, and W. S. Moore (2015), Radium isotope Distributions during the U.S. GEOTRACES North Atlantic cruises, Mar. Chem., 17r, 184-195.

Chase, Z., R. F. Anderson, M. Q. Fleisher, and P. W. Kubik (2002), The influence of particle composition and particle flux on scavenging of $\mathrm{Th}, \mathrm{Pa}$ and $\mathrm{Be}$ in the ocean, Earth Planet. Sci. Lett., $204(1), 215-229$.

Chen, J. H., L. R. Edwards, and G. J. Wasserburg (1986), ${ }^{238} \mathrm{U},{ }^{234} \mathrm{U}$ and ${ }^{232} \mathrm{Th}$ in seawater, Earth Planet. Sci. Lett., 80, 241-251.

Cheng, H., R. L. Edwards, C. Shen, V. J. Polyak, Y. Asmerom, J. Woodhead, J. Hellstrom, Y. Wang, X. Kong, C. Spötl, X. Wang, and E. C. Alexandar Jr. (2013), Improvements in ${ }^{230}$ Th dating, ${ }^{230}$ Th and ${ }^{234} \mathrm{U}$ half-life values, and U-Th isotopic measurements by multi-collector inductively coupled plasma mass spectrometry., Earth Planet. Sci. Lett., 371, 82-91.

Choppin, G. R., and P. J. Wong (1998), The chemistry of actinide behavior in marine systems, Aquat. Geochem., 4(1), 77-101.

Chuang, C. Y., P. H. Santschi, Y. F. Ho, M. H. Conte, L. Guo, D. Schumann, M. Aryanov, and Y. H. Li (2013), Role of biopolymers as major carrier phases of Th, $\mathrm{Pa}, \mathrm{Pb}, \mathrm{Po}$, and $\mathrm{Be}$ radionuclides in settling particles from the Atlantic Ocean, Mar. Chem., 15\%, 131-143.

Chuang, C. Y., P. H. Santschi, Y. Jiang, Y. F. Ho, A. Quigg, L. D. Guo, M. Ayranov, and D. Schumann (2014), Important role of biomolecules from diatoms in the scavenging of particle-reactive radionuclides of thorium, protactinium, lead, polonium and beryllium in the ocean: a case study with Phaeodactylum tricornutum, Limnol. Oceanogr., 59(4), 1256-1266.

Churchill, J. H., P. E. Biscaye, and F. Aikman III (1988), The character and motion of suspended particulate matter over the shelf edge and upper slope off Cape Cod, Continental Shelf Research, $8(5-7), 789-809$. 
Clegg, S. L., and J. L. Sarmiento (1989), The hydrolytic scavenging of metal ions by marine particulate matter, Prog. Oceanogr., 23(1), 1-21.

Clegg, S. L., and M. Whitfield (1990), A generalized model for the scavenging of trace metals in the open ocean I. Particle cycling, Deep Sea Res. A, 37(5), 809-832.

Clegg, S. L., and M. Whitfield (1993), Application of a generalized scavenging model to time series ${ }^{234} \mathrm{Th}$ and particle data obtained during the JGOFS North Atlantic Bloom Experiment, Deep Sea Res. Part I Oceanogr. Res. Pap., 40(8), 1529-1545.

Clegg, S. L., M. P. Bacon, and M. Whitfield (1991a), A generalized model for the scavenging of trace metals in the open ocean-II. Thorium scavenging, Deep Sea Res. A, 38(1), 91-120.

Clegg, S. L., M. P. Bacon, and M. Whitfield (1991b), Application of a generalized scavenging model to thorium isotope and particle data at equatorial and high-latitude sites in the Pacific Ocean, $J$. Geophys. Res. Oceans, 962(C11), 20,655-20,670.

Coale, K. H., and K. W. Bruland (1987), Oceanic stratified euphotic zone as elucidated by ${ }^{234}$ Th: ${ }^{238} \mathrm{U}$ disequilibria, Limnol. Oceanogr., 32(1), 189-200.

Cochran, J. K., H. D. Livingston, D. J. Hirschberg, and L. D. Surprenant (1987), Natural and anthropogenic radionuclide distributions in the northwest Atlantic Ocean, Earth and Planetary Science Letters, 84(2-3), 135-152.

Cochran, J. K., K. O. Buesseler, M. P. Bacon, H. W. Wang, D. J. Hirschberg, L. Ball, and A. Fleer (2000), Short-lived thorium isotopes $\left({ }^{234} \mathrm{Th},{ }^{228} \mathrm{Th}\right)$ as indicators of POC export and particle cycling in the Ross Sea, Southern Ocean., Deep Sea Res. Part II Top. Stud. Oceanogr., 47(15), $3451-3490$.

Cochran, K. J., K. O. Buesseler, M. P. Bacon, and H. D. Livingston (1993), Thorium isotopes as indicators of particle dynamics in the upper ocean: Results from the JGOFS North Atlantic Bloom Experiment., Deep Sea Res. Part I Oceanogr. Res. Pap., 40(8), 1569-1595.

Colley, S., J. Thomson, and P. P. Newton (1995), Detailed ${ }^{230} \mathrm{Th},{ }^{232} \mathrm{Th}$ and ${ }^{210} \mathrm{~Pb}$ fluxes recorded by the $1989 / 90$ BOFS sediment trap time-series at $48^{\circ} \mathrm{N}, 20^{\circ} \mathrm{W}$, Deep Sea Res. Part I Oceanogr. Res. Pap., 42(6), 833-848. 
Collins, J. R., B. R. Edwards, K. Thamatrakoln, J. E. Ossolinski, G. R. DiTullio, K. D. Bidle, S. C. Doney, and B. A. Van Mooy (2015), The multiple fates of sinking particles in the North Atlantic Ocean, Global Biogeochem. Cycles, 29(9), 1471-1494.

Costa, K. M., J. F. McManus, B. Boulahanis, S. M. Carbotte, G. Winckler, P. J. Huybers, and C. H. Langmuir (2016), Sedimentation, stratigraphy and physical properties of sediment on the Juan de Fuca Ridge, Marine Geology, 380, 163-173.

Craig, H. (1974), A scavenging model for trace elements in the deep sea, Earth and Planetary Science Letters, 23(1), 149-159.

Craig, H., S. Krishnaswami, and B. L. K. Somayajulu (1973), ${ }^{210} \mathrm{~Pb}^{226} \mathrm{Ra}$ : radioactive disequilibrium in the deep sea, Earth and Planetary Science Letters, 17(2), 295-305.

Curry, R., and C. Nobre (2008), HydroBase3, Woods Hole Oceanographic Institution, Woods Hole, MA, 02543.

Davis, J. A. (1982), Adsorption of natural dissolved organic matter at the oxide/water interface, Geochim. Cosmochim. Acta, 46(11), 2381-2393.

Davis, J. A., R. O. James, and J. O. Leckie (1978a), Surface ionization and complexation at the oxide/water interface: I. Computation of electrical double layer properties in simple electrolytes, Journal of colloid and interface science, 63(3), 480-499.

Davis, J. A., R. O. James, and J. O. Leckie (1978b), Surface ionization and complexation at the oxide/water interface II. Surface properties of amorphous iron oxyhydroxide and adsorption of metal ions, Journal of colloid and interface science, 67(1), 90-107.

Dehairs, F., S. Jacquet, N. Savoye, B. A. Van Mooy, K. O. Buesseler, J. K. Bishop, C. H. Lamborg, M. Elskens, W. Baeyens, P. W. Boyd, K. L. Casciotti, and C. Monnin (2008), Barium in twilight zone suspended matter as a potential proxy for particulate organic carbon remineralization: Results for the North Pacific, Deep Sea Res. Part II Top. Stud. Oceanogr., 55(14), 1673-1683.

Delanghe, D., E. Bard, and B. Hamelin (2002), New TIMS constraints on the uranium-238 and uranium-234 in seawaters from the main ocean basins and the Mediterranean Sea, Mar. Chem., 80(1), 79-93. 
Dilling, L., J. Wilson, D. Steinberg, and A. Alldredge (1998), Feeding by the euphausiid Euphausia pacifica and the copepod Calanus pacificus on marine snow., Marine Ecology Progress Series, $170,189-201$.

Dixit, S., P. Van Capellen, and A. J. van Bennekom (2001), Processes controlling solubility of biogenic silica and pore water build-up of silicic acid in marine sediments, Mar. Chem., 73(3-4), $333-352$.

Dudewicz, E. J., and S. N. Mishra (1988), Modern Mathematical Statistics, John Wiley and Sons, Inc.

Dutay, J. C., F. Lacan, M. Roy-Barman, and L. Bopp (2009), Influence of particle size and type on ${ }^{231} \mathrm{~Pa}$ and ${ }^{230} \mathrm{Th}$ simulation with a global coupled biogeochemical-ocean general circulation model: A first approach, Geochemistry, Geophysics, Geosystems, 10(1), 1-26.

Edmonds, H. N., S. B. Moran, J. A. Hoff, J. N. Smith, and R. L. Edwards (1998), Protactinium-231 and thorium-230 abundances and high scavenging rates in the western Arctic Ocean, Science, $280(5362), 405-407$.

Efron, B., and R. J. Tibshirani (1993), An introduction to the bootstrap, Chapman and Hall, New York.

Elrod, V. A., W. M. Berelson, K. H. Coale, and K. S. Johnson (2004), The flux of iron from continental shelf sediments: A missing source for global budgets., Geophysical Research Letters, $31(12), 1-4$.

Ezer, T. (2016), Revisiting the problem of the Gulf Stream separation: on the representation of topography in ocean models with different types of vertical grids, Ocean Modelling, 104, 15-27.

Ezer, T. (2017), A modeling study of the role that bottom topography plays in Gulf Stream dynamics and in influencing the tilt of mean sea level along the US East Coast, Monthly Weather Review, $67(5), 651-664$.

Ferrari, R., and C. Wunsch (2009), Ocean circulation kinetic energy: Reservoirs, sources, and sinks, Annual Review of Fluid Mechanics, 41, 253-282. 
Flather, R. A. (1976), A tidal model of the northwest European continental shelf, Mémoires de la Soc. Royale des Sciences de Liège, 10, 141-161.

Forget, G., J. M. Campin, P. Heimbach, C. N. Hill, R. M. Ponte, and C. Wunsch (2015), ECCO version 4: an integrated framework for non-linear inverse modeling and global ocean state estimation, Geosci. Model Dev., 8, 3071-3104.

François, R. (2007), Chapter Sixteen: Paleoflux and Paleocirculation from Sediment ${ }^{230} \mathrm{Th}$ and ${ }^{231} \mathrm{~Pa} /{ }^{230} \mathrm{Th}$, Developments in Marine Geology, 1, 691-716.

François, R., and M. P. Bacon (1994), Heinrich events in the North Atlantic: radiochemical evidence, Deep Sea Research Part I: Oceanographic Research Papers, 41(2), 315-334.

François, R., M. P. Bacon, M. A. Altabet, and L. D. Labeyrie (1993), Glacial/interglacial changes in sediment rain rate in the SW Indian sector of subantarctic waters as recorded by ${ }^{230} \mathrm{Th},{ }^{231} \mathrm{~Pa}$, $\mathrm{U}$, and $\delta^{15} \mathrm{~N}$, Paleoceanography, 8(5), 611-629.

François, R., M. Frank, M. M. Rutgers van der Loeff, and M. P. Bacon (2004), ${ }^{230}$ Th normalization: An essential tool for interpreting sedimentary fluxes during the late Quaternary, Paleoceanography, $19(1)$.

Frank, M., A. Mangini, R. Gersonde, M. van der Loeff, and G. Kuhn (1996), Late Quaternary sediment dating and quantification of lateral sediment redistribution applying ${ }^{230} \mathrm{Th}_{e x}$ : A study from the eastern Atlantic sector of the Southern Ocean, International Journal of Earth Science: Geologische Rundschau, 85(3), 554-566.

Frank, M., R. Gersonde, and A. Mangini (1999), Sediment redistribution, ${ }^{230} \mathrm{Th}_{e x}$-normalization and implications for the reconstruction of particle flux and export paleoproductivity, Use of proxies in paleoceanography, pp. 409-426.

Ganachaud, A., and C. Wunsch (2002), Oceanic nutrient and oxygen transports and bounds on export production during the World Ocean Circulation Experiment, Global Biogeochemical Cycles, $16(4), 1-14$.

Gardner, W. D. (1989), Periodic resuspension in Baltimore Canyon by focusing of internal waves, Journal of Geophysical Research: Oceans, 94(C12), 18,185-18,194. 
Gardner, W. D., P. E. Biscaye, J. R. V. Zaneveld, and M. J. Richardson (1985), Calibration and comparison of the LDGO nephelometer and the OSU transmissometer on the Nova Scotian Rise, Marine Geology, 66(1-4), 323-344.

Gardner, W. D., B. E. Tucholke, M. J. Richardson, and P. E. Biscaye (2017), Benthic storms, nepheloid layers, and linkage with upper ocean dynamics in the western North Atlantic, Marine Geology, 385, 304-327.

Geibert, W., and R. Usbeck (2004), Adsorption of thorium and protactinium onto different particle types: experimental findings, Geochim. Cosmochim. Acta, 68(7), 1489-1501.

German, C. R., A. P. Fleer, M. P. Bacon, and J. M. Edmond (1991), Hydrothermal scavenging at the Mid-Atlantic Ridge: radionuclide distributions, Earth and Planetary Science Letters, 105(1-3), $170-181$.

Goldberg, E. D. (1954), Marine geochemistry 1. Chemical scavengers of the sea, The Journal of Geology, pp. 249-265.

Greene, W. H. (2012), Econometric Analysis, Pearson Education, Essex, UK.

Griffith, E. M., and A. Paytan (2012), Barite in the ocean-occurrence, geochemistry and palaeoceanographic applications, Sedimentology, 59(6), 1817-1835.

Grömping, U. (2007), Estimators of relative importance in linear regression based on variance decomposition, Am. Stat., 61(2), 139-147.

GEOTRACES Planning Group (2006), Geotraces Science Plan, Scientific Committee on Oceanic Research, Batlimore, Maryland.

Guo, L., P. H. Santschi, M. Baskaran, and A. Zindler (1995), Distribution of dissolved and particulate ${ }^{230} \mathrm{Th}$ and ${ }^{232} \mathrm{Th}$ in seawater from the Gulf of Mexico and off Cape Hatteras as measured by SIMS, Earth and Planetary Science Letters, 133(1-2), 117-128.

Guo, L., P. H. Santschi, and M. Baskaran (1997), Interactions of thorium isotopes with colloidal organic matter in oceanic environments, Colloids and Surfaces A: Physicochemical and Engineering Aspects, 120(1-3), 255-271. 
Guo, L., M. Chen, and C. Gueguen (2002a), Control of Pa/Th ratio by particulate chemical composition in the ocean, Geophys. Res. Lett., 29(20), 1-4.

Guo, L., C. C. Hung, P. H. Santschi, and I. D. Walsh (2002b), ${ }^{234}$ Th scavenging and its relationship to acid polysaccharide abundance in the Gulf of Mexico, Mar. Chem., 78(2), 103-119.

Hayes, C. T., R. F. Anderson, S. L. Jaccard, R. François, M. Q. Fleisher, M. Soon, and R. Gersonde (2013), A new perspective on boundary scavenging in the North Pacific Ocean, Earth Planet. Sci. Lett., 369, 86-97.

Hayes, C. T., R. F. Anderson, M. Q. Fleisher, S. W. Vivancos, P. J. Lam, D. C. Ohnemus, K. F. Huang, L. F. Robinson, Y. Lu, H. Cheng, R. L. Edwards, and B. S. Moran (2015a), Intensity of Th and $\mathrm{Pa}$ scavenging partitioned by particle chemistry in the North Atlantic Ocean, Mar. Chem., 170, 49-60.

Hayes, C. T., R. F. Anderson, M. Q. Fleisher, K. F. Huang, L. F. Robinson, Y. Lu, H. Cheng, L. R. Edwards, and B. S. Moran (2015b), ${ }^{230} \mathrm{Th}$ and ${ }^{231} \mathrm{~Pa}$ on GEOTRACES GA03, the U.S. GEOTRACES North Atlantic transect, and implications for modern and paleoceanographic chemical fluxes, Deep Sea Res. Part II Top. Stud. Oceanogr., 116, 29-41.

Hayes, C. T., J. N. Fitzsimmons, E. A. Boyle, D. McGee, R. Anderson, R. Weisend, and P. L. Morton (2015c), Thorium isotopes tracing the iron cycle at the Hawaii Ocean Time-series Station ALOHA, Geochimica et Cosmochimica Acta, 169, 1-16.

Hayes, C. T., J. Rosen, D. McGee, and E. A. Boyle (2017), Thorium distributions in high and low dust regions and the significance for iron supply, Global Biogeochem. Cycles, 38(2), 328-347.

Hedges, J. I., G. Eglinton, P. G. Hatcher, D. L. Kirchman, C. Arnosti, S. Derenne, R. P. Evershed, I. Kögel-Knabner, J. W. de Leeuw, R. Littke, W. Michaelis, and J. Rullkötter (2000), The molecularly-uncharacterized component of nonliving organic matter in natural environments, Organ. Geochem., $31(10), 945-958$.

Henderson, G. M., and R. F. Anderson (2003), The U-series toolbox for paleoceanography, Reviews in Mineralogy and Geochemistry, 52(1), 493-531. 
Henderson, G. M., C. Heinze, R. F. Anderson, and A. M. Winguth (1999), Global distribution of the ${ }^{230}$ Th flux to ocean sediments constrained by GCM modelling, Deep Sea Res. Part I Oceanogr. Res. Pap., 46(11), 186-1893.

Henderson, P. B., P. J. Morris, W. S. Moore, and M. A. Charette (2013), Methodological advances for measuring low-level radium isotopes in seawater, Journal of Radioanalytical and Nuclear Chemistry, 296(1), 357-362.

Hiemstra, T., and W. H. Van Riemsdijk (2009), A surface structural model for ferrihydrite I: Sites related to primary charge, molar mass, and mass density, Geochim. Cosmochim. Acta, 73(15), $4423-4436$.

Hirose, K. (2004), Chemical speciation of thorium in marine biogenic particulate matter, The Scientific World Journal, 4, 67-76.

Hirose, K., and E. Tanoue (1998), The vertical distribution of the strong ligand in particulate organic matter in the North Pacific, Mar. Chem., 59(3), 235-252.

Hogg, N. G. (1983), A note on the deep circulation of the western North Atlantic: its nature and causes, Deep Sea Research Part A. Oceanographic Research Papers, 30(9), 945-961.

Hogg, N. G. (1992), On the transport of the Gulf Stream between Cape Hatteras and the Grand Banks, Deep Sea Research Part A. Oceanographic Research Papers, 39(7-8), 1231-1246.

Hogg, N. G., and H. Stommel (1985), On the relation between the deep circulation and the Gulf Stream, Deep Sea Research Part A. Oceanographic Research Papers, 32(10), 1181-1193.

Hogg, N. G., R. S. Pickart, R. M. Hendry, and W. J. Smethie Jr (1986), The northern recirculation gyre of the Gulf Stream, Deep Sea Research Part A. Oceanographic Research Papers, 33(9), $1139-1165$.

Hollister, C. D., and I. N. McCave (1984), Sedimentation under deep-sea storms, Nature, 309(5965), 220.

Honeyman, B. D., and P. H. Santschi (1989), A Brownian-pumping model for oceanic trace metal scavenging: evidence from Th isotopes, J. Mar. Res., 47(4), 951-992. 
Honeyman, B. D., L. S. Balistrieri, and J. W. Murray (1988), Oceanic trace metal scavenging: the importance of particle concentration, Deep Sea Res. A, 35(2), 227-246.

Huizenga, D. L., and D. R. Kester (1979), The complexation of metals with humic materials in natural waters, Limnology and Oceanography, 24(1), 145-150.

Hunter, K. A., and P. S. I. Liss (1979), The surface charge of suspended particles in estuarine and coastal waters, Nature, 282(5741), 823-825.

Hunter, K. A., D. J. Hawke, and L. K. Choo (1988), Equilibrium adsorption of thorium by metal oxides in marine electrolytes, Geochimica et Cosmochimica Acta, 52(3), 627-636.

Jackson, G. A., and A. B. Burd (2015), Simulating aggregate dynamics in ocean biogeochemical models, Progress in Oceanography, 133, 55-65.

Jannasch, H. W., B. D. Honeyman, and L. S. Balistrieri (1988), Kinetics of trace element uptake by marine particles, Geochim. Cosmochim. Acta, 52(2), 567-577.

Jeandel, C., T. Arsouze, F. Lacan, P. Techine, and J. C. Dutay (2007), Isotopic Nd compositions and concentrations of the lithogenic inputs into the ocean: A compilation, with an emphasis on the margins, Chemical Geology, 239(1-2), 156-164.

Jenkins, W. J., W. M. Smethie, and E. A. Boyle (2015), Water mass analysis for the U.S. GEOTRACES North Atlantic Sections, Deep Sea Res. Part II Top. Stud. Oceanogr., 116, 6-20.

Jickells, T. D., Z. S. An, K. K. Andersen, A. R. Baker, G. Bergametti, N. Brooks, J. J. Cao, P. W. Boyd, R. A. Duce, K. A. Hunter, H. Kawahata, N. Kubilay, J. LaRoche, P. S. Liss, N. Mahowald, J. M. Prospero, A. J. Ridgwell, I. Tegen, and R. Torres (2005), Global iron connections between desert dust, ocean biogeochemistry, and climate, Science, 308(5718), 67-71.

Keir, R. S. (1990), The dissolution kinetics of biogenic calcium carbonates in seawater, Geochimi. Cosmochim. Acta, 44(2), 241-252.

Keith, T. Z. (2014), Multiple regression and beyond: An introduction to multiple regression and structural equation modeling, Routledge, New York. 
Kendall, M., and J. D. R. Gibbons (1990), Rank Correlation Methods, Oxford University Press, Oxford.

Kienast, S. S., M. Kienast, A. C. Mix, S. E. Calvert, and R. François (2007), Thorium-230 normalized particle flux and sediment focusing in the Panama Basin region during the last 30,000 years, Paleoceanography and Paleoclimatology, 22(2), 1-19.

Kirby, H. W., G. R. Grove, and D. L. Timma (2002), Neutron-capture cross section of actinium-227, Physical Review, 102(4), 1140-1141.

Knight, G. B., and R. L. Macklin (1948), Half-Life of UX $\left(\mathrm{Th}_{234}\right)$, Physical Review, $74(10), 1540-$ 1541.

Krauskopf, K. B. (1956), Factors controlling the concentrations of thirteen rare metals in sea-water, Geochim. Cosmochim. Acta, 9(1), 1-B32.

Krishnaswami, S., D. Lal, B. L. K. Somayajulu, R. F. Weiss, and H. Craig (1976), Large-volume in-situ filtration of deep Pacific waters: Mineralogical and radioisotope studies, Earth Planet. Sci. Lett., 32(2), 1-B32.

Krishnaswami, S., M. M. Sarin, and B. L. K. Somayajulu (1981), Chemical and radiochemical investigations of surface and deep particles of the Indian Ocean, Earth Planet. Sci. Lett., 51(1), $81-96$.

Kruskal, W. (1987), Relative importance by averaging over orderings, Am. Stat., 41(1), 6-10.

Ku, T. L., K. G. Knauss, and G. G. Mathieu (1977), Uranium in open ocean: concentration and isotopic composition, Deep Sea Research, 24(12), 31-39.

Kumar, N., R. Gwiazda, R. Anderson, and P. Froelich (1993), ${ }^{231} \mathrm{~Pa} /{ }^{230} \mathrm{Th}$ ratios in sediments as a proxy for past changes in Southern Ocean productivity, Nature, 362(6415), 45-48.

Kumar, N., R. Anderson, R. Mortlock, P. Froelich, P. Kubik, B. Dittrich-Hannen, and M. Suter (1995), Increased biological productivity and export production in the glacial Southern Ocean, Nature, 378(6558), 675-680. 
Lai, D. Y., and P. L. Richardson (1977), Distribution and movement of Gulf Stream rings, Journal of Physical Oceanography, 7(5), 670-683.

Lal, D. (1977), The oceanic microcosm of particles, Science, 198(4321), 997-1009.

Lam, P., and O. Marchal (2014), Insights into particle dynamics from the geochemical composition of suspended and sinking particles, Annual Review of Marine Science, 7(1), 12.1-12.26.

Lam, P. J., D. C. Ohnemus, and M. E. Auro (2015a), Size-fractionated major particle composition and concentrations from the U.S. GEOTRACES North Atlantic Zonal Transect, Deep-Sea Research II, 116, 303-320.

Lam, P. J., B. S. Twining, C. Jeandel, A. Roychoudhury, J. A. Resing, P. H. Santschi, and R. F. Anderson (2015b), Methods for analyzing the concentration and speciation of major and trace elements in marine particles, Progress in Oceanography, 133, 32-42.

Lamborg, C., K. Bowman, C. Hammerschmidt, C. Gilmour, K. Munson, and N. Selin (2014), Mercury in the Anthropocene ocean, Oceanography, 27(1), 76-87.

Lampitt, R. S., K. F. Wishner, C. M. Turley, and M. V. Angel (1993), Marine snow studies in the Northeast Atlantic Ocean: distribution, composition and role as a food source for migrating plankton, Marine Biology, 116(4), 689-702.

Ledwell, J. R., A. J. Watson, and C. S. Law (1998), Mixing of a tracer in the pycnocline, J. Geophys. Res. Oceans, $103(\mathrm{C} 10)$, 21,499-21,529.

Lee, C., M. A. Peterson, S. G. Wakeham, R. A. Armstrong, J. K. Cochran, J. C. Miquel, S. W. Fowler, D. Hirschberg, A. Beck, and J. Xue (2009), Particulate organic matter and ballast fluxes measured using time-series and settling velocity sediment traps in the northwestern Mediterranean Sea, Deep Sea Research II, 56, 1420-1436.

Lerner, P., O. Marchal, P. J. Lam, R. F. Anderson, K. Buesseler, M. A. Charette, R. L. Edwards, C. T. Hayes, K. F. Huang, Y. Lu, L. F. Robinson, and A. Solow (2016), Testing models of thorium and particle cycling in the ocean using data from station GT11-22 of the US GEOTRACES North Atlantic Section, Deep Sea Res. Part I Oceanogr. Res. Pap., 113, 57-79. 
Lerner, P., O. Marchal, P. J. Lam, K. Buesseler, and M. Charette (2017), Kinetics of thorium and particle cycling along the US GEOTRACES North Atlantic Transect, Deep Sea Res. Part I Oceanogr. Res. Pap., 25, 106-128.

Lerner, P., O. Marchal, P. J. Lam, and A. Solow (2018), Effects of particle composition on thorium scavenging in the North Atlantic, Geochimica et Cosmochimica Acta, 233, 115-134.

Li, Y. (2005), Controversy over the relationship between major components of sediment-trap materials and the bulk distribution coefficients of ${ }^{230} \mathrm{Th},{ }^{231} \mathrm{~Pa}$, and ${ }^{10} \mathrm{Be}$, Earth Planet. Sci. Lett., $233(1), 1-7$.

Lin, P., L. Guo, and M. Chen (2014), Adsorption and fractionation of thorium and protactinium on nanoparticles in seawater, Mar. Chem., 162, 50-59.

Lozier, M. S., W. B. Owens, and R. G. Curry (1995), The climatology of the North Atlantic, Progress in Oceanography, 36(1), 1-44.

Lumpkin, R., and K. Speer (2003), Large-scale vertical and horizontal circulation in the North Atlantic Ocean, Journal of Physical Oceanography, 33(9), 1902-1920.

Lumpkin, R., and K. Speer (2007), Global ocean meridional overturning, Journal of Physical Oceanography, 37(10), 2550-2562.

Luo, S., and T. L. Ku (2004), On the importance of opal, carbonate, and lithogenic clays in scavenging and fractionating ${ }^{230} \mathrm{Th},{ }^{230} \mathrm{~Pa}$ and ${ }^{10} \mathrm{Be}$ in the ocean, Earth Planet. Sci. Lett., 220(1), 201-211.

Luo, Y., R. Francois, and S. E. Allen (2010), Sediment ${ }^{231} \mathrm{~Pa} /{ }^{230} \mathrm{Th}$ as a recorder of the rate of the Atlantic meridional overturning circulation: insights from a 2-D model, Ocean Science, 6(1), 381-400.

Lutz, M., R. Dunbar, and K. Caldeira (2002), Regional variability in the vertical flux of particulate organic carbon in the ocean interior, Global Biogeochem. Cycles, 16(3), 1-18.

Macdonald, A. M., and C. Wunsch (1996), An estimate of global ocean circulation and heat fluxes, Nature, 382(6590), 436. 
Madansky, A. (1988), Linear Regression Analysis, Second Edition., Springer-Verlag, New York, NY.

Maiti, K., K. O. Buesseler, S. M. Pike, C. Benitez-Nelson, P. Cai, W. Chen, K. Cochran, M. Dai, F. Dehairs, B. Gasser, R. P. Kelley, P. Masqué, L. A. Miller, J. C. Miquel, S. B. Moran, P. J. Morris, F. Peine, F. Planchon, A. A. Renfo, M. M. Rutgers van der Loeff, P. H. Santschi, R. Turnewitsch, J. T. Waples, and C. Xu (2012), Intercalibration studies of short-lived thorium234 in the water column and marine particles, Limnol. Oceanogr. Methods, 10(9), 631-644.

Maiti, K., M. A. Charette, K. O. Buesseler, K. Zhou, P. Henderson, W. S. Moore, P. Morris, and L. Kipp (2015), Determination of particulate and dissolved ${ }^{228} \mathrm{Th}$ in seawater using a delayed coincidence counter, Mar. Chem., 177, 196-202.

Mantoura, R. F. C., A. Dickson, and J. P. Riley (1978), The complexation of metals with humic materials in natural waters, Estuarine and Coastal Marine Science, 6(4), 387-408.

Marcantonio, F., R. F. Anderson, S. Higgins, M. Stute, P. Schlosser, and P. Kubik (2001), Sediment focusing in the central equatorial Pacific Ocean, Paleoceanography, 16(3), 260-267.

Marchal, O., and P. J. Lam (2012), What can paired measurements of Th isotope activity and particle concentration tell us about particle cycling in the ocean?, Geochim. Cosmochim. Acta, 90, 126-148.

Marchal, O., R. François, T. F. Stocker, and F. Joos (2000), Ocean thermohaline circulation and sedimentary ${ }^{231} \mathrm{~Pa} /{ }^{230} \mathrm{Th}$ ratio, Paleoceanography, $15(6), 625-641$.

Marchal, O., R. François, and J. Scholten (2007), Contribution of ${ }^{230}$ Th measurements to the estimation of the abyssal circulation, Deep Sea Res. Part I Oceanogr. Res. Pap., 54(4), 557-585.

Marchitto, T. M., and W. S. Broecker (2006), Deep water mass geometry in the glacial Atlantic Ocean: A review of constraints from the paleonutrient proxy $\mathrm{Cd} / \mathrm{Ca}$, Geochemistry, Geophysics, Geosystems, 7(12), 1-16.

Marra, J. F., V. P. Lance, R. D. Vaillancourt, and B. R. Hargreaves (2014), Resolving the ocean's euphotic zone, Deep Sea Res. Part I Oceanogr. Res. Pap., 83, 45-50. 
Matlab (2016), Optimization Toolbox (r2016a), User's guide, The Mathworks, Inc., http://www . mathworks.com/help/optim/, accessed: 22 July, 2016.

McCartney, M. S. (1992), Recirculating components to the deep boundary current of the northern North Atlantic, Progress in Oceanography, 29(4), 283-383.

McCartney, M. S., S. L. Bennett, and M. E. Woodgate-Jones (1991), Eastward flow through the Mid-Atlantic Ridge at $11^{\circ} \mathrm{N}$ and its influence on the abyss of the eastern basin, Journal of Physical Oceanographys, 21(8), 1089-1121.

McCave, I. N. (1983), Particulate size spectra, behavior, and origin of nepheloid layers over the Nova Scotian continental rise, Journal of Geophysical Research: Oceans, 88(C12), 7647-7666.

McCave, I. N. (1984), Mechanics of deposition of fine-grained sediments from nepheloid layers, Geo-Marine Letters, 4 (3-4), 243-245.

McDonnell, A. M., and K. O. Buesseler (2010), Variability in the average sinking velocity of marine particles, Limnol. Oceanogr., 55(5), 2085-2096.

McDonnell, A. M., P. W. Boyd, and K. O. Buesseler (2015), Effects of sinking velocities and microbial respiration rates on the attenuation of particulate carbon fluxes through the mesopelagic zone, Global Biogeochem. Cycles, 29(2), 175-193.

McManus, J. F., R. François, J. M. Gherardi, L. D. Keigwin, and S. O. Brown-Leger (2004), Collapse and rapid resumption of Atlantic meridional circulation linked to deglacial climate changes, Nature, 428(6985), 834-837.

Mehta, A. J., E. J. Hayter, W. R. Parker, R. B. Krone, and A. M. Teeter (1989), Cohesive sediment transport. I: Process description, Journal of Hydraulic Engineering, 115(8), 1076-1093.

Mellor, G. (2002), Users guide for a three-dimensional, primitive equation, numerical ocean model, Program in Atmospheric and Oceanic Sciences, Princeton University, Princeton, NJ 08544-0710.

Mellor, G. L., and T. Ezer (1991), A Gulf Stream model and an altimetry assimilation scheme, Journal of Geophysical Research: Oceans, 96(C5), 8779-8795. 
Mellor, G. L., and T. Yamada (1982), Development of a turbulence closure model for geophysical fluid problems, Reviews of Geophysics, 30(4), 851-875.

Mercier, H. (1986), Determining the general circulation of the ocean: a nonlinear inverse problem, J. Geophys. Res. Oceans, $91(\mathrm{C} 4)$, 5103-5109.

Mercier, H. (1989), A study of the time-averaged circulation in the western North Atlantic by simultaneous nonlinear inversion of hydrographic and current meter data, Deep Sea Res. A, 36(2), $297-313$.

Mercier, H., M. Ollitrault, and P. Y. Le Traon (1993), A study of the time-averaged circulation in the western North Atlantic by simultaneous nonlinear inversion of hydrographic and current meter data, Journal of physical oceanography, 23(4), 689-715.

Moon, E. M., and C. L. Peacock (2013), Modelling Cu (II) adsorption to ferrihydrite and ferrihydritebacteria composites: deviation from additive adsorption in the composite sorption system, Geochim. Cosmochim. Acta, 104, 148-164.

Moore, W. S. (2010), The effect of submarine groundwater discharge on the ocean, Annual review of marine science, 2, 59-88.

Moran, S. B., and K. O. Buesseler (1993), Size-fractionated ${ }^{234}$ Th in continental shelf waters off New England: Implications for the role of colloids in oceanic trace metal scavenging, J. Mar. Res., $51(4), 893-922$.

Moran, S. B., M. A. Charette, J. A. Hoff, R. L. Edwards, and W. M. Landing (1997), Distribution of ${ }^{230} \mathrm{Th}$ in the Labrador Sea and its relation to ventilation, Earth Planet. Sci. Lett., 150(1), $151-160$.

Moran, S. B., C. C. Shen, H. N. Edmonds, S. E. Weinstein, J. N. Smith, and R. L. Edwards (2002), Dissolved and particulate ${ }^{231} \mathrm{~Pa}$ and ${ }^{230} \mathrm{Th}$ in the Atlantic Ocean: constraints on intermediate/deep water age, boundary scavenging, and ${ }^{231} \mathrm{~Pa} /{ }^{230} \mathrm{Th}$ fractionation, Earth Planet. Sci. Lett., $203(3), 999-1014$.

Morel, F., and P. Gschwend (1987), Aquatic Surface Chemistry: Chemical Processes at the ParticleWater Interface, John Wiley and Sons, New York. 
Morel, F., A. Milligan, and M. Saito (2003), Marine Bioinorganic Chemistry: The Role of Trace Metals in the Oceanic Cycles of Major Nutrients, Treatise on Geochemistry: The Oceans and Marine Geochemistry, 6, 113-143.

Morel, F. M. M., and N. M. Price (2003), The biogeochemical cycles of trace metals in the oceans, Science, $44(5621), 944-947$.

Morse, J. W. (1986), The surface chemistry of calcium carbonate minerals in natural waters: An overview, Marine Chemistry, 20(1), 91-112.

Murnane, R. J. (1994a), Determination of thorium and particulate matter cycling parameters at station P: A reanalysis and comparison of least squares techniques, J. Geophys. Res. Oceans, $99(\mathrm{C} 2), 3393-3405$.

Murnane, R. J., J. L. Sarmiento, and M. P. Bacon (1990), Thorium isotopes, particle cycling models, and inverse calculations of model rate constants, J. Geophys. Res. Oceans, 95(C9), 16,195-16,206.

Murnane, R. J., J. K. Cochran, and J. L. Sarmiento (1994b), Estimates of particle and thorium cycling rates in the northwest Atlantic Ocean, J. Geophys. Res. Oceans, 99(C2), 3373-3392.

Murnane, R. J., J. K. Cochran, K. O. Buesseler, and M. P. Bacon (1996), Least-squares estimates of thorium, particle, and nutrient cycling rate constants from the JGOFS North Atlantic Bloom Experiment, Deep Sea Res. Part I Oceanogr. Res. Pap., 43(2), 239-258.

National Geophysical Data Center (2006), 2-minute Gridded Global Relief Data (ETOPO2) v2, NOAA., Batlimore, Maryland, doi:10.7289/V5J1012Q.

Niehof, R., and G. I. Loeb (1972), The surface charge of particulate matter in seawater, Limn. Oceanogr., 17, 7-16.

Nowell, A., C. D. Hollister, and P. A. Jumars (1982), High energy benthic boundary layer experiment: HEBBLE, EOS, Transactions American Geophysical Union, 63(31), 594-595.

Nozaki, Y., and T. Nakanishi (1985), ${ }^{231} \mathrm{~Pa}$ and ${ }^{230} \mathrm{Th}$ profiles in the open ocean water column, Deep Sea Res. A, 32(10), 1209-1220. 
Nozaki, Y., Y. Horibe, and H. Tsubota (1981), The water column distributions of thorium isotopes in the western North Pacific, Earth Planet. Sci. Lett., 54(2), 203-216.

Nozaki, Y., H.-S. Yang, and M. Yamada (1987), Scavenging of thorium in the ocean, J. Geophys. Res. Oceans, 92(C1), 772-778.

Nyffeler, U. P., Y. H. Li, and P. H. Santschi (1984), A kinetic approach to describe trace-element distribution between particles and solution in natural aquatic systems, Geochim. Cosmochim. Acta, 48(7), 1513-1522.

Ohnemus, D. C., and P. J. Lam (2015), Cycling of lithogenic marine particulates in the US GEOTRACES North Atlantic Zonal transect, Deep Sea Research II, 116, 283-302.

Ohnemus, D. C., P. J. Lam, and B. S. Twining (2017), Optical observation of particles and responses to particle composition in the GEOTRACES GP16 section, Marine Chemistry, 202(20), 124-136.

Okubo, A., H. Obata, T. Gamo, and M. Yamada (2012), ${ }^{230}$ Th and ${ }^{232}$ Th distributions in midlatitudes of the North Pacific Ocean: effect of bottom scavenging, Earth Planet. Sci. Lett., 339, $139-150$.

Östhols, E. (1995), Thorium adsorption onto amorphous silica, Geochim. Cosmochim. Acta, 59(3), $1235-1249$.

Owens, S. A., K. O. Buesseler, and K. W. W. Sims (2011), Re-evaluating the ${ }^{238}$ U-salinity relationship in seawater: Implications for the ${ }^{238} \mathrm{U}{ }^{234} \mathrm{Th}$ disequilibrium method, Mar. Chem., 126(1), $31-39$.

Owens, S. A., S. Pike, and K. O. Buesseler (2015), Thorium-234 as a tracer of particle dynamics and upper ocean export in the Atlantic Ocean, Deep Sea Research II, 116, 42-59.

Paillet, J., and H. Mercier (1997), An inverse model of the eastern North Atlantic general circulation and thermocline ventilation, Deep Sea Res. Part I Oceanogr. Res. Pap., 44(8), 1293-1328.

Palanques, A., and P. E. Biscaye (1992), Patterns and controls of the suspended matter distribution over the shelf and upper slope south of New England, Continental Shelf Research, 12(5-6), 577600. 
Peacock, C. L., and D. M. Sherman (2007), Sorption of Ni by birnessite: Equilibrium controls on Ni in seawater, Geochim. Cosmochim. Acta, 238(1-2), 94-106.

Pickart, R. S. (1992), Water mass components of the North Atlantic deep western boundary current, Deep Sea Research Part A. Oceanographic Research Papers, 39(3), 1553-1572.

Pickart, R. S., and W. M. Smethie (1993), How does the deep western boundary current cross the Gulf Stream, Journal of Physical Oceanography, 23(12), 2602-2616.

Pickart, R. S., and D. R. Watts (1990), Deep Western Boundary Current Variability at Cape Hatteras, Journal of Marine Research, 48(4), 765-791.

Planquette, H., R. R. Sanders, P. J. Statham, P. J. Morris, and G. R. Fones (2011), Fluxes of particulate iron from the upper ocean around the Crozet Islands: A naturally ironâ $\breve{A}$ fertilized environment in the Southern Ocean, Global Biogeochemical Cycles, 25(2), 5-12.

Polzin, K., K. Speer, J. Toole, and R. Schmitt (1996), Intense mixing of Antarctic Bottom Water in the equatorial Atlantic Ocean, Nature, 380, 54-57.

Quigley, M. S., P. H. Santschi, L. Guo, and B. D. Honeyman (2001), Sorption irreversibility and coagulation behavior of ${ }^{234}$ Th with marine organic matter, Mar. Chem., 76(1), 27-45.

Quigley, M. S., P. H. Santschi, C. C. Hung, L. Guo, and B. D. Honeyman (2002), Importance of acid polysaccharides for ${ }^{234} \mathrm{Th}$ complexation to marine organic matter, Limnol. Oceanogr., $47(2)$, $367-377$.

Quiroz, N. G. A., C. C. Hung, and P. H. Santschi (2006), Binding of thorium (IV) to carboxylate, phosphate and sulfate functional groups from marine exopolymeric substances (EPS), Mar. Chem., $100(3), 337-353$.

Reinthaler, T., H. Van Aken, C. Veth, J. Arístegui, C. Robinson, P. J. t. B. Williams, P. Lebaron, and G. J. Herndl (2006), Prokaryotic respiration and production in the meso and bathypelagic realm of the eastern and western North Atlantic basin, Limnol. Oceanogr., 51(3), 1262-1273.

Rempfer, J., T. F. Stocker, F. Joos, J. Lippold, and S. L. Jaccard (2017), New insights into cycling of ${ }^{231} \mathrm{~Pa}$ and ${ }^{230} \mathrm{Th}$ in the Atlantic Ocean, Earth and planetary science letters, 468, 27-37. 
Rencher, A. C. (1998), Multivariate Statistical Inference and Applications, John Wiley and Sons, New York, NY.

Rhein, M., D. Kieke, and R. Steinfeldt (2015), Advection of North Atlantic Deep Water from the Labrador Sea to the southern hemisphere, J. Geophys. Res. Oceans, 120(4), 2471-2487.

Ribbe, J., and P. E. Holloway (2001), A model of suspended sediment transport by internal tides, Continental shelf research, 21(4), 395-422.

Richardson, P. (1983), Gulf Stream Rings, in Eddies in Marine Science, edited by A. Robinson, pp. 19-45, Spring-Verlag, Berlin.

Richardson, P., R. E. Cheney, and L. A. Mantini (1977), Tracking a Gulf Stream ring with a free drifting surface buoy, Journal of Physical Oceanography, 7(4), 580-590.

Rintoul, S. R., and C. Wunsch (1991), Mass, heat, oxygen and nutrient fluxes and budgets in the North Atlantic Ocean, Deep Sea Research Part A. Oceanographic Research Papers, 38, S355-S377.

Risien, C. M., and D. B. Chelton (2008), A Global Climatology of Surface Wind and Wind Stress Fields from Eight Years of QuikSCAT Scatterometer Data, Journal of Physical Oceanography, $38,2379-2413$.

Roberts, K. A., C. Xu, C. C. Hung, M. H. Conte, and P. H. Santschi (2009), Scavenging and fractionation of thorium vs. protactinium in the ocean, as determined from particle-water partitioning experiments with sediment trap material from the Gulf of Mexico and Sargasso Sea, Earth Planet. Sci. Lett., $286(1), 131-138$.

Roemmich, D., and C. Wunsch (1985), Two transatlantic sections: Meridional circulation and heat flux in the subtropical North Atlantic Ocean, Deep Sea Research Part A. Oceanographic Research Papers, 32(6), 619-664.

Roy-Barman, M., L. Coppola, and M. Souhaut (2002), Thorium isotopes in the western Mediterranean Sea: an insight into the marine particle dynamics, Earth Planet. Sci. Lett., 196(3), 161174. 
Roy-Barman, M., C. Jeandel, M. Souhaut, M. M. van der Loeff, I. Voege, N. Leblond, and R. Freydier (2005), The influence of particle composition on thorium scavenging in the NE Atlantic ocean (POMME experiment), Earth Planet. Sci. Lett., 240(3), 681-693.

Roy-Barman, M., C. Lemaître, S. Ayrault, C. Jeandel, M. Souhaut, and J. C. Miquel (2009), The influence of particle composition on Thorium scavenging in the Mediterranean Sea, Earth Planet. Sci. Lett., $286(3), 526-534$.

Rühlemann, C., B. Diekmann, S. Mulitza, and M. Frank (2001), Late Quaternary changes of western equatorial Atlantic surface circulation and Amazon lowland climate recorded in Ceará Rise deepsea sediments, Paleoceanography, 16(3), 293-305.

Rutgers van der Loeff, M. M., and G. W. Berger (1993), Scavenging of ${ }^{230} \mathrm{Th}$ and ${ }^{231} \mathrm{~Pa}$ near the Antarctic polar front in the South Atlantic, Deep Sea Res. Part I Oceanogr. Res. Pap., 40(2), $339-357$.

Rutgers van der Loeff, M. M., and B. P. Boudreau (1997), The effect of resuspension on chemical exchanges at the sediment-water interface in the deep sea-a modelling and natural radiotracer approach, Journal of Marine Systems, 11(3-4), 305-342.

Ryan, W. B. F., C. S. M, J. O. Coplan, S. O’Hara, A. Melkonian, R. Arko, R. A. Weissel, V. Ferrini, A. Goodwillie, F. Nitsche, J. Bonczkowski, and R. Zemsky (2009), Global multiâĂ̌rresolution topography synthesis, Geochemistry, Geophysics, Geosystems, 10(3), 1-9.

Santschi, P. H., C. C. Hung, G. Schultz, N. Alvarado-Quiroz, L. Guo, J. Pinckney, and I. Walsh (2003), Control of acid polysaccharide production and ${ }^{234} \mathrm{Th}$ and POC export fluxes by marine organisms, Geophys. Res. Lett., 30(2), 47-64.

Santschi, P. H., J. W. Murray, M. Baskaran, C. R. Benitez-Nelosn, L. D. Guo, C. C. Hung, C. Lamborg, S. B. Moran, U. Passow, and M. Roy-Barman (2006), Thorium speciation in seawater, Mar. Chem., $100(3), 250-268$.

Savidge, D. K., and J. M. Bane (1999), Cyclogenesis in the deep ocean beneath the Gulf Stream: 1. Description, ournal of Geophysical Research: Oceans, 104(C8), 18,111-18,126. 
Savoye, N., C. Benitez-Nelson, A. B. Burd, J. K. Cochran, M. Charette, K. O. Buesseler, G. A. Jackson, M. Roy-Barman, S. Schmidt, and M. Elsken (2006), ${ }^{234}$ Th sorption and export models in the water column: A review, Mar. Chem., 100, 234-249.

Schindler, P. W. (1975), Removal of trace metals from the oceans: a zero order model, Thalassia Jugosl, 11(1), 101-111.

Schlitzer, R., R. F. Anderson, E. Masferrer Dodas, et al. (2018), The GEOTRACES Intermediate Data Product 2017, Chemical Geology, 493, 210-223.

Schmitz, W. J., and M. S. McCartney (1993), On the North Atlantic circulation, Rev. Geophys., $31(1), 29-49$.

Scholten, J. C., M. M. Rutgers van der Loeff, and M. A (1995), Distribution of ${ }^{230} \mathrm{Th}$ and ${ }^{231} \mathrm{~Pa}$ in the water column in relation to the ventilation of the deep Arctic basins, Deep Sea Res. Part II Top. Stud. Oceanogr., 42(6), 1519-1531.

Scholten, J. C., J. Fietzke, S. Vogler, M. M. Rutgers van der Loeff, A. Mangini, W. Koeve, J. Waniek, P. Stoffers, A. Antia, and J. Kuss (2001), Trapping efficiencies of sediment traps from the deep Eastern North Atlantic: the ${ }^{230}$ Th calibration, Deep Sea Res. Part II Top. Stud. Oceanogr., 48(10), 2383-2408.

Scholten, J. C., J. Fietzke, A. Mangini, P. Stoffers, T. Rixen, B. Gaye-Haake, T. Blanz, V. Ramaswamy, F. Sirocko, H. Schulz, and V. Ittekkot (2005), Radionuclide fluxes in the Arabian Sea: the role of particle composition, Earth Planet. Sci. Lett., 230(3-4), 319-337.

Scholten, J. C., J. Fietzke, A. Mangini, C.-D. Garbe-Schönberg, A. Eisenhauer, R. Schneider, and P. Stoffers (2008), Advection and scavenging: Effects on ${ }^{230} \mathrm{Th}$ and ${ }^{231} \mathrm{~Pa}$ distribution off Southwest Africa, Earth Planet. Sci. Lett., 271, 159-169.

Seber, G. A. F., and A. J. Lee (1992), Linear Regression Analysis, Second Edition, John Wiley and Sons, Inc, Hoboken, NJ.

Shelley, R. U., P. L. Morton, and W. M. Landing (2015), Elemental ratios and enrichment factors in aerosols from the US-GEOTRACES North Atlantic transects, Deep Sea Research Part II: Topical Studies in Oceanography, 116, 262-272. 
Shen, C. C., C. C. Wu, H. Cheng, R. L. Edwards, Y. T. Hsieh, S. Gallet, C. C. Chang, T. Y. Li, D. D. Lam, A. Kano, M. Hori, and C. Spoötl (2012), High-precision and high-resolution carbonate ${ }^{230}$ Th dating by MC-ICP-MS with SEM protocols, Geochim. Cosmochim. Acta, 99, 71-86.

Sheridan, C. C., C. Lee, S. G. Wakeham, and J. K. B. Bishop (2002), Suspended particle organic composition and cycling in surface and midwaters of the equatorial Pacific Ocean, Deep Sea Res. Part I Oceanogr. Res. Pap., 49(11), 1983-2008.

Siddall, M., G. M. Henderson, N. R. Edwards, M. Frank, S. A. Müller, T. F. Stocker, and F. Joos (2005), ${ }^{231} \mathrm{~Pa} /{ }^{230} \mathrm{Th}$ fractionation by ocean transport, biogenic particle flux and particle type, Earth Planet. Sci. Lett., 237(1), 135-155.

Sillen, L. G., A. E. Martell, and J. Bjerrum (1964), Stability constants of metal-ion complexes, Chemical Society Special Publication, 17, 754.

Smagorinsky, J. (1963), General circulation experiments with the primitive equations: I. The basic experiment, Monthly weather review, 92(4), 99-264.

Smith, J. N., W. M. Smethie, I. Yashayev, R. Curry, and K. Azetsu-Scott (2016), Time series measurements of transient tracers and tracer-derived transport in the Deep Western Boundary Current between the Labrador Sea and the subtropical Atlantic Ocean at Line W, Journal of Geophysical Research: Oceans, 121(11), 8115-8138.

Smolarkiewicz, P. K. (1983), A simple positive definite advection scheme with small implicit diffusion, Monthly Weather Review, 111(3), 479-486.

Steinberg, D. K., B. A. Van Mooy, K. O. Buesseler, P. W. Boyd, T. Kobari, and D. M. Karl (2008), Bacterial vs. zooplankton control of sinking particle flux in the ocean's twilight zone, Limnol. Oceanogr., 53(4), 1327.

Stemmann, L., G. A. Jackson, and D. Ianson (2004), A vertical model of particle size distributions and fluxes in the midwater column that includes biological and physical processes-Part I: model formulation, Deep Sea Res. Part I Oceanogr. Res. Pap., 51(7), 865-884.

Stewart, G., J. K. Cochran, J. C. Miquel, P. Masquè, J. Szlosek, A. R. Rodrigues y Baena, S. W. Folwer, B. Gasser, and D. J. Hirschberg (2007), Comparing POC export from ${ }^{234} \mathrm{Th} /{ }^{238} \mathrm{U}$ and 
${ }^{210} \mathrm{Po} /{ }^{210} \mathrm{~Pb}$ disequilibria with estimates from sediment traps in the northwest Mediterranean, Deep Sea Research Part I: Oceanographic Research Paper, 54(9), 1549-1570.

Stramma, L., H. Sabine, and J. Schafstall (2005), Water masses and currents in the upper tropical Northeast Atlantic off Northwest Africa, J. Geophys. Res. Oceans, 110(C12), 1-18.

Stumm, W., and J. Morgan (1996), Aquatic Chemistry, 3rd ed., John Wiley and Sons, New York.

Suman, D. O., and M. P. Bacon (1989), Variations in Holocene sedimentation in the North American Basin determined from ${ }^{230}$ Th measurements, Deep Sea Research Part A. Oceanographic Research Papers, 36(6), 869-878.

Szlosek, J., J. K. Cochran, J. C. Miquel, P. Masquè, R. A. Armstrong, S. W. Fowler, B. Gasser, and D. J. Hirschberg (2009), Particulate organic carbon- ${ }^{234}$ Th relationships in particles separated by settling velocity in the northwest Mediterranean Sea, Deep Sea Research Part II: Topical Studies in Oceanography, 56(18), 1519-1532.

Tagliabue, A., L. Bopp, J.-C. Dutay, A. B. Bowie, F. Chever, P. Jean-Baptiste, E. Bucciarelli, D. Lannuzel, T. Remenyi, G. Sarthou, O. Aumont, M. Gehlen, and C. Jeandel (2010), Global iron connections between desert dust, ocean biogeochemistry, and climate, Nature Geoscience, $3(4), 252$.

Tarantola, A., and B. Valette (1982), Generalized nonlinear inverse problems solved using the least squares criterion, Rev. Geophys., 20(2), 219-232.

Taylor, S. R., and S. M. McLennan (1995), The geochemical evolution of the continental crust, Rev. Geophys., 33(2), 241-265.

The GEOTRACES group (2015), The GEOTRACES Intermediate Data Product 2014, Mar. Chem., 177, Part 1, 1-8.

Thomson, J., S. Colley, R. Anderson, G. T. Cook, A. B. MacKenzie, and D. D. Harkness (1993), Holocene sediment fluxes in the northeast Atlantic from ${ }^{230} \mathrm{Th}_{\text {excess }}$ and radiocarbon measurements, Paleoceanography, 8(5), 631-650. 
Toner, B., S. Fakra, M. Villalobos, T. Warwick, and G. Sposito (2005), Spatially resolved characterization of biogenic manganese oxide production within a bacterial biofilm, Applied and environmental microbiology, 71 (3), 1300-1310.

Toole, J. M., R. W. Schmitt, and K. L. Polzin (1994), Estimates of diapycnal mixing in the abyssal ocean, Science, 264(5162), 1120-1123.

Toole, J. M., M. Andres, I. A. Le Bras, T. M. Joyce, and M. S. McCartney (2017), Moored observations of the Deep Western Boundary Current in the NW Atlantic: 2004-2014, Journal of Geophysical Research: Oceans, 122(9), 7488-7505.

Tréguier, A. M., C. W. Boning, F. Bryan, G. Danabasoglu, H. Drange, B. Taguchi, and A. Pirani (2014), CLIVAR WGOMD Workshop on high resolution ocean climate modelling: outcomes and recommendations, CLIVAR Exchanges, 19, 3-6.

Turekian, K. K. (1977), The fate of metals in the ocean, Geochim. Cosmochim. Acta, 41(8), 11391144.

Turner, J. T. (2002), Zooplankton fecal pellets, marine snow and sinking phytoplankton blooms, Aquatic Microbial Ecology, 27(1), 57-102.

Vanmarcke, E. (1983), Random Fields: Analysis and Synthesis, MIT Press, Cambridge.

Venchiarutti, C., C. Jeandel, and M. Roy-Barman (2008), Particle dynamics study in the wake of Kerguelen Island using thorium isotopes, Deep Sea Res. Part I Oceanogr. Res. Pap., 55(10), $1343-1363$.

Verdugo, P., A. L. Alldredge, F. Azam, D. L. Kirchman, U. Passow, and P. H. Santschi (2004), The oceanic gel phase: a bridge in the DOMâĂŞPOM continuum, Marine Chemistry, 92(1-4), 67-85.

Vogler, S., J. Scholten, M. M. Rutger van der Loeff, and A. Mangini (1998), ${ }^{230} \mathrm{Th}$ in the eastern North Atlantic: the importance of water mass ventilation in the balance of ${ }^{230} \mathrm{Th}$, Earth Planet. Sci. Lett., $156(1), 61-74$.

Wakeham, S. G., C. Lee, J. I. Hedges, P. J. Hernes, and M. J. Peterson (1997), Molecular indicators of diagenetic status in marine organic matter, Geochim. Cosmochim. Acta, 61(24), 5563-5369. 
Walsh, J. J., P. E. Biscaye, and G. T. Csanady (1988), The 1983-1984 shelf edge exchange processes (SEEP)-I experiment: hypotheses and highlight, Continental Shelf Research, 8(5-7), 435-456.

Waltz, R. A., J. L. Morales, J. Nocedal, and D. Orban (2006), An interior algorithm for nonlinear optimization that combines line search and trust region steps, Mathematical Programming, 107(3), 391-408.

Watts, D. R., and W. E. Johns (1982), Gulf Stream meanders: Observations on propagation and growth, Journal of Geophysical Research: Oceans, 87(C12), 9467-9476.

Wilson, D. E., and P. Kinney (1977), Effects of polymeric charge variations on the proton-metal ion equilibria of humic material, Limnology and Oceanography, 22, 2.

Wolberg, J. R. (1967), Prediction Analysis., D. Van Nostrand, Princeton.

Wunsch, C. (1968), On the propagation of internal waves up a slope, Deep Sea Research, 15(2), $251-258$.

Wunsch, C. (1978), The North Atlantic general circulation west of $50 \mathrm{~W}$ determined by inverse methods, Reviews of Geophysics, 16(4), 583-620.

Wunsch, C. (2006), Discrete inverse and state estimation problems: with geophysical fluid applications, Cambridge University Press, Cambridge.

Yates, D. E., S. Levine, and T. W. Healy (1974), Site-binding model of the electrical double layer at the oxide/water interface, Journal of the Chemical Society, Faraday Transactions 1: Physical Chemistry in Condensed Phases, 70, 1807-1818.

Yu, E.-F., R. François, and M. P. Bacon (1996), Similar rates of modern and last-glacial ocean thermohaline circulation inferred from radiochemical data, Nature, 379, 689-694.

Zhang, W. G., and G. G. Gawarkiewicz (2015), Dynamics of the direct intrusion of Gulf Stream ring water onto the MidâĂ̌̌Atlantic Bight shelf, Geophyiscal Research Letters, 42(18), 7687-7695. 